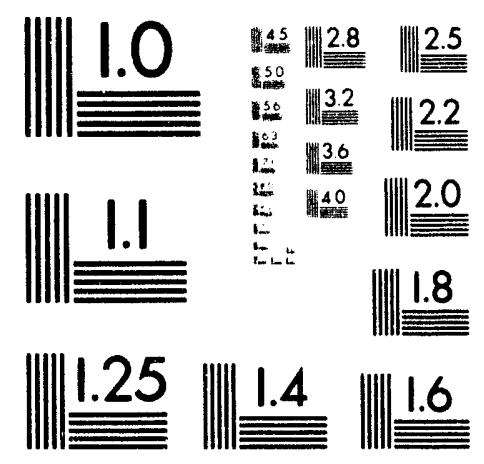



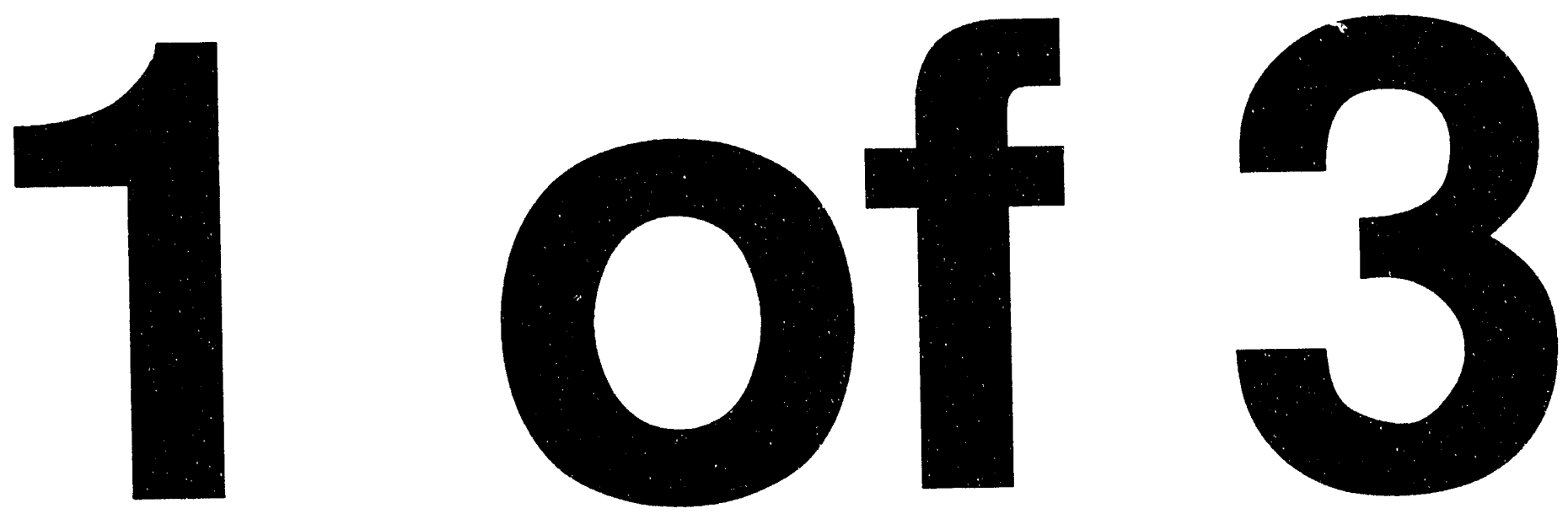
$\mathrm{DOE} / \mathrm{CE} / 23810-16$

THERMOPHYSICAL PROPERTIES<smiles>CCOC(=O)N1[C@H]2C=C[C@H]1C2C</smiles>

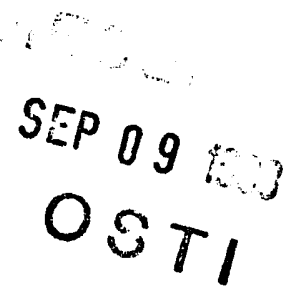

1 January 1992 - 31 March 1993

Richard F. Kayser

\section{Thermophysics Division}

Building 221, Room A105

National Institute of Standards and Technology

Gaithersburg, Maryland 20899

April 1993

\section{Prepared for \\ The Air-Conditioning and Refrigeration Technology Institute Under \\ ARTI MCLR Project Number 650-50800}

This research project is supported, in whole or in part, by U.S. Department of Energy grant number DE-FG02-91CE23810: Materials Compatibility and Lubricants Research (MCLR) on CFC-Refrigerant Substitutes. Federal funding supporting this project constitutes $93.67 \%$ of allowable costs. Funding from non-government sources supporting this project consists of direct cost sharing of $6.33 \%$ of allowable costs; and in-kind contributions from the air-conditioning and refrigeration industry. 


\section{DISCLAIMER}

The U.S. Department of Energy's and the air-conditioning industry's support for the Materials Compatibility and Lubricants Research (MCLR) program does not constitute an endorsement by the U.S. Department of Energy, nor by the air-conditioning and refrigeration industry, of the views expressed herein.

\section{NOTICE}

This report was prepared on account of work sponsored by the United States Government. Neither the United States Grovernment, nor the Department of Energy, nor the Air Conditioning and Refrigeration Technology Institute, nor any of their employees, nor any of their contractors, subcontractors, or their employees, makes any warranty, expressed or implied, or assumes any legal liability or responsibility for the accuracy, completeness, or usefulness of any information, apparatus, product or process disclosed or represents that its use would not infringe privatelyowned rights.

\section{COPYRIGHT NOTICE}

(for journal publication submissions)

By acceptance of this article, the publisher and/or recipient acknowledges the right of the U.S. Government and the Air-Conditioning and Refrigeration Technology Institute, Inc. (ARTI) to retain a nonexclusive, royalty-free license in and to any copyrights covering this paper. 
$\mathrm{DOE} / \mathrm{CE} / 23810-16$

\title{
THERMOPHYSICAL PROPERTIES
}

\author{
ARTI MCLR Project Number 650-50800 \\ Richard F. Kayser \\ Thermophysics Division \\ National Institute of Standards and Technology
}

\begin{abstract}
Numerous fluids have been identified as promising alternative refrigerants, but much of the information needed to predict their behavior as pure fluids and as components in mixtures does not exist. In particular, reliable thermophysical properties data and models are needed to predict the performance of the new refrigerants in heating and cooling equipment and to design and optimize equipment to be reliable and energy efficient. The objective of this fifteen-month project has been to provide highly accurate, selected thermophysical properties data for Refrigerants $32,123,124$, and 125 , and to use these data to fit equations of state and transport property models. The new data have filled gaps in the existing data sets and resolved problems and uncertainties that existed in and between the data sets.
\end{abstract}

\section{$\underline{\text { SCOPE }}$}

This project has involved selected measurements of the thermodynamic properties of HFC32 , HCFC-124, and HFC-125, and the development of high-accuracy modified Benedict-WebbRubin (MBWR) equations of state for each fluid. It has also included selected measurements of the transport properties of HFC-32 (viscosity and thermal conductivity) and HCFC-123 (thermal conductivity) and the development of detailed correlations for same. The experimental thermodynamic measurements have included, as appropriate, accurate determinations of the pressure-volume-temperature (PVT) behavior in the superheated vapor region; the PVT behavior of the compressed liquid; vapor pressures and saturated liquid densities; the critical temperature, pressure, and density; the vapor-phase speed of sound and the ideal-gas heat capacity; and selected measurements of the liquid-phase heat capacity. The experimental transport measurements have covered one-phase and saturated liquid and vapor states over the temperature range of interest.

\section{SIGNIFICANT RESULTS}

HFC-32

The Burnett apparatus has been used in the isochoric mode to determine the PVT relation for the vapor phase (147 data points). Eleven isochores were completed spanning the ranges 268 to $373 \mathrm{~K}\left(23\right.$ to $\left.212^{\circ} \mathrm{F}\right)$ and 0.018 to 1.3 times the critical density $\left(7.5\right.$ to $550 \mathrm{~kg} / \mathrm{m}^{3} ; 0.47$ to $\left.34.3 \mathrm{lb} / \mathrm{ft}^{3}\right)$; the highest absolute pressure was $9.7 \mathrm{MPa}(1400 \mathrm{psi})$. Two Burnett expansions were completed at $373 \mathrm{~K}\left(212^{\circ} \mathrm{F}\right)$ to establish the densities of the isochores. The locations of 
the measurements are indicated as a function of pressure and temperature by the filled circles on Figure 1, and as a function of density and pressure by the filled circles on Figure 2. The results of the measurements are given in Appendix A in Table 1: Appendix A includes all tables.

The vibrating tube densimeter has been used to determine the PVT relation for the liquid phase (654 data points). Twenty-one isotherms were completed spanning the ranges 243 to 343 $\mathrm{K}\left(-22\right.$ to $\left.158^{\circ} \mathrm{F}\right)$ and 2000 to $6500 \mathrm{kPa}(290$ to $940 \mathrm{psi})$. The locations of the measurements are indicated as a function of pressure and temperature by the open circles on Figure 1 , and as a function of density and pressure by the open circles on Figure 2 . The results of the measurements are given in Table 2.

An isochoric PVT apparatus has been used to measure the density of liquid HFC-32 at 144 points using the same sample as used in the $C_{v}$ study (see below). The temperatures ranged from 140 to $396 \mathrm{~K}\left(-208\right.$ to $\left.253^{\circ} \mathrm{F}\right)$ with pressures to $35 \mathrm{MPa}(5000 \mathrm{psi})$. The locations of the measurements are shown in Figure 3, and the results are presented in Table 3.

The Burnett apparatus has been used to measure the vapor pressure of HFC-32 at 18 temperatures in the range from $268 \mathrm{~K}\left(23^{\circ} \mathrm{F}\right)$ to the critical temperature at $351.36 \mathrm{~K}\left(172.78^{\circ} \mathrm{F}\right)$. The results of these measurements are given in Table 4. An ebulliometer has been used to measure the vapor pressure of HFC-32 at low temperatures in the range between 208 and 237 $\mathrm{K}\left(-85\right.$ and $\left.-32^{\circ} \mathrm{F}\right)$. The results are tabulated in Table 5. The NIST Burnett and ebulliometric vapor-pressure data for HFC-32 have been correlated and their deviations from the resulting correlation are shown in Figure 4. Also shown in Figure 4 are the HFC-32 data of P.F. Malbrunot, et al. [J. Chem. \& Eng. Data 13, 16 (1968)]. The uncertainties in the NIST measurements are of order $0.05 \%$.

The saturated vapor and liquid densities have been obtained by extrapolating the Burnett vapor-phase and vibrating-tube liquid-phase PVT data to the vapor pressure curve. The saturated vapor and liquid densities so obtained are given in Tables 6 and 7, respectively.

A static method has been used to perform additional measurements of the vapor pressure of HFC-32. The sample was thermostatted in the PVT cell and two additional oscillating quartzcrystal pressure transducers were used to observe the equilibrium pressures. The two transducers, with maximum pressures of $0.2 \mathrm{MPa}(30 \mathrm{psi}$ ) and $6 \mathrm{MPa}$ (900 psi), were calibrated versus a gas-lubricated piston gage with an uncertainty of $\pm 0.01 \%$. The range of the vapor pressure data is from 140 to $340 \mathrm{~K}\left(-208\right.$ to $\left.152^{\circ} \mathrm{F}\right)$. These results are given in Table 8 .

An optical cell has been used to measure the refractive index and capillary rise of HFC32 from $23^{\circ} \mathrm{C}\left(73^{\circ} \mathrm{F}\right)$ up to the critical temperature. The critical temperature was found to be $\mathrm{T}_{\mathrm{c}}=(351.36 \pm 0.02) \mathrm{K}$, which corresponds to $(172.78 \pm 0.04)^{\circ} \mathrm{F}$. The refractive index data were combined with the liquid density data to deduce the value $0.1295 \mathrm{~cm}^{3} / \mathrm{g}$ for the LorentzLorenz constant. The refractive index data and the Lorentz-Lorenz constant were used to deduce the value $\rho_{\mathrm{c}}=(419 \pm 7) \mathrm{kg} / \mathrm{m}^{3}\left[(26.13 \pm 0.44) \mathrm{lb} / \mathrm{ft}^{3}\right]$ for the critical density.

An adiabatic calorimeter has been used to measure the molar heat capacity at constant volume $\left\{\mathrm{C}_{\mathrm{v}}\right\}$ for HFC-32. In total, $79 \mathrm{C}_{\mathrm{v}}$ values were measured in the liquid state and 105 
values were measured in the vapor + liquid two-phase region. The temperatures ranged from 141 to $342 \mathrm{~K}\left(-206\right.$ to $\left.156^{\circ} \mathrm{F}\right)$, with pressures up to $35 \mathrm{MPa}(5000 \mathrm{psi})$. The measured values are given in Tables 9 through 16 for the liquid phase and in Tables 17 through 19 for the twophase region. In addition to the temperature-density-pressure (T- $\rho$-P) state conditions, the tables present estimated uncertainties of the measurements which lead to values of liquid heat capacity, $\mathrm{C}_{\mathrm{v}}$, or heat capacity of the saturated liquid, $\mathrm{C}_{\sigma}$. These measurements include the amount of sample, $N$, the calurimeter bomb volume, $V_{\text {bomb }}$, the observed temperature rise, $\Delta T$, the energy absorbed, $\mathrm{Q}_{\text {tare }}$, and the heat capacity, $\mathrm{dQ}_{\text {tare }} / \mathrm{dT}$, of the empty calorimeter bomb, gross heat capacity, $\mathrm{Q} / \Delta \mathrm{T}$, and the $\mathrm{PV}$ work done by the sample to expand the bomb during a heat capacity measurement, $W_{p v, m}$. Figure 5 illustrates the pressures and temperatures covered by this study.

A 32-term MBWR equation of state for HFC-32 has been developed. It is valid at temperatures from the triple point at $137 \mathrm{~K}\left(-213^{\circ} \mathrm{F}\right)$ to $400 \mathrm{~K}\left(260^{\circ} \mathrm{F}\right)$, and it appears to be reasonable upon extrapolation up to $500 \mathrm{~K}\left(440^{\circ} \mathrm{F}\right)$. The maximum pressure for the equation is $40 \mathrm{MPa}(6000 \mathrm{psi})$, and it appears to be reasonable upon extrapolation up to $100 \mathrm{MPa}$ (15000 psi). This equation was fit using a multiparameter linear least squares routine to the data measured under this contract as well as selected data reported in the literature. Data used in the fit included vapor pressures, saturated liquid and vapor densities, liquid and vapor phase pressure-volume-temperature (PVT) data, virial coefficients, and isochoric heat capacities at saturation and in the single-phase liquid. Table 20 gives the coefficients to the equation of state, and Table 21 tabulates the saturation properties calculated with the equation of state. Figures 6 through 10 compare the values of various properties calculated with the MBWR equation to the experimental values used in the fit. This MBWR equation for HFC-32 will be incorporated into version 4.0 of the REFPROP computer package, which will be released in the coming months; it will be available as an option in addition to the CSDM equation of state for HFC-32.

The low- and high-temperature transient hot-wire thermal conductivity instruments have been used to measure the thermal conductivity of HFC-32 at 1030 points over the region from 160 to $340 \mathrm{~K}\left(-167\right.$ to $\left.160^{\circ} \mathrm{F}\right)$ at pressures to $70 \mathrm{MPa}(10,000 \mathrm{psi})$. The results of the analysis of these 1030 transient hot-wire measurements are given in Table 22. The measurements also have been extended to include two supercritical isotherms at 365 and $380 \mathrm{~K}\left(197\right.$ and $\left.225^{\circ} \mathrm{F}\right)$; however, these measurements may have been affected by corrosion, which became a significant problem at these higher temperatures. A plot of the entire thermal conductivity surface, including the two supercritical isotherms, is provided for reference in Figure 11.

The vapor-phase thermal conductivity of HFC-32 is shown in Figure 12. The isotherms appear to be internally consistent, and a great deal of curvature is visible near the saturation boundary on the $330 \mathrm{~K}$ and $340 \mathrm{~K}$ isotherms. This curvature is attributed to the thermal conductivity critical enhancement.

The thermal conductivity data have been correlated as described in Appendix B. The fluid-specific information required for this correlation are the MBWR equation of state for HFC32 described above and the parameters provided in Table 23. Deviations of the values of thermal conductivity calculated from the correlation from the experimental data used in the fit are given in Figure 13. The uncertainty is on the order of $\pm 5 \%$. 
The shear viscosities of compressed and saturated fluid HFC-32 have been measured at temperatures between 150 and $420 \mathrm{~K}\left(-190\right.$ to $\left.297^{\circ} \mathrm{F}\right)$ at pressures to $35 \mathrm{MPa}(4400 \mathrm{psi})$ with two torsionally oscillating, quartz-crystal viscometers. The results are given in Tables 24 and 25. and their dependence on density is illustrated in Figure 14. The saturated liquid data have been correlated using the equation

$$
\eta^{-1}=406.1(\mathrm{~V}-0.0340)
$$

when $\eta$ is the viscosity in mPa-s and $\mathrm{V}$ is the molar volume in $\mathrm{mol} / \mathrm{dm}^{3}$. Most of the differences between the experimental and calculated viscosities are less than $\pm 2 \%$.

\section{HCFC-124}

The Burnett apparatus has been used in the isochoric mode to determine the PVT relation for the vapor phase of HCFC-124 at 172 points. Isochores were completed spanning the range 0.09 to $6 \mathrm{~mol} / \mathrm{L}\left(0.77 \mathrm{lb} / \mathrm{ft}^{3}\right.$ to $\left.51 \mathrm{lb} / \mathrm{ft}^{3}\right)$. The temperature range was 278 to $423 \mathrm{~K}$ (41 to $\left.302^{\circ} \mathrm{F}\right)$. A Burnett expansion was completed at $423.15 \mathrm{~K}\left(302.00^{\circ} \mathrm{F}\right)$ to establish the densities of the isochores, and 13 additional data points were acquired during this procedure. The results of these measurements are given in Table 26.

The vibrating tube densimeter has been used to determine the PVT relation for HCFC124 in the liquid phase. Twenty-two isotherms were completed spanning the ranges 275 to 372 $\mathrm{K}\left(-35\right.$ to $\left.210^{\circ} \mathrm{F}\right)$ and 396 to $6500 \mathrm{kPa}(57$ to $922 \mathrm{psi})$. The results of these liquid-phase measurements are given in Table 27.

An isochoric PVT apparatus has been used to measure densities for liquid HCFC-124 at 151 points. The temperatures ranged from 104 to $400 \mathrm{~K}\left(-272-260^{\circ} \mathrm{F}\right)$ with pressures as high as $35 \mathrm{MPa}$ (5000 psi). The pressures, temperatures, and densities are presented in Table 28.

The Burnett apparatus and the ebulliorneter have been used to measure the vapor pressure of HCFC-124, and the results are presented in Table 29. The data span the very wide pressure range from 13 to $3163 \mathrm{kPa}$ (1.9 to $458.8 \mathrm{psi}$ ) corresponding to the temperature range from 222 to $388 \mathrm{~K}\left(-60\right.$ to $\left.239^{\circ} \mathrm{F}\right)$. The 109 data points were fit with a Wagner-type vapor pressure equation and had an extraordinarily small standard deviation of $0.012 \%$. The vapor pressure equation was extrapolated to the critical temperature at $T_{c}=395.43 \mathrm{~K}$ (corresponding to $252.10^{\circ} \mathrm{F}$ ) to obtain a value of the critical pressure, $\mathrm{P}_{\mathrm{c}}=3617.9 \mathrm{kPa}(524.7 \mathrm{psi})$.

An optical cell has been used to measure the refractive index and capillary rise of HCFC124 from $23^{\circ} \mathrm{C}\left(73^{\circ} \mathrm{F}\right)$ up to the critical temperature. The critical temperature was found to be $\mathrm{T}_{\mathrm{c}}=(395.43 \pm 0.02) \mathrm{K}$, which corresponds to $(252.10 \pm 0.04)^{\circ} \mathrm{F}$. The refractive index data were combined with the liquid density data to deduce the value $0.1198 \mathrm{~cm}^{3} / \mathrm{g}$ for the LorentzLorenz constant. The refractive index data and the Lorentz-Lorenz constant were used to deduce the value $\rho_{\mathrm{c}}=(552 \pm 5) \mathrm{kg} / \mathrm{m}^{3}\left[(34.43 \pm 0.31) \mathrm{lb} / \mathrm{ft}^{3}\right]$ for the critical density.

A cylindrical acoustic resonator has been used to measure the speed of sound in HCFC124 along isotherms between 250 and $400 \mathrm{~K}\left(-9\right.$ and $\left.261^{\circ} \mathrm{F}\right)$ at pressures between 20 and 900 
$\mathrm{kPa}(3$ and $130 \mathrm{psi})$. The results are given in Table 30. The ideal-gas heat capacity, $\mathrm{C}_{\mathrm{p}}^{\mathrm{o}}$, of HCFC-124 has been obtained by analyzing the speed of sound measurements at low pressures. The results are given in Table 31. The following expressions for $C_{p}^{o}$ were obtained by fitting the data in Table 31:

$$
\mathrm{C}_{\mathrm{p}}^{\mathrm{o}}(\mathrm{HCFC}-124)=\mathrm{R}\left(\mathrm{a}_{0}+\mathrm{a}_{1} \mathrm{t}+\mathrm{a}_{2} \mathrm{t}^{2}\right)
$$

where

\section{SI UNITS}

$$
\begin{array}{ll}
\mathbf{R} & =8.314471 \mathrm{~J} / \mathrm{K} \text {-mol (universal gas constant) } \\
\mathrm{t} & =\text { temperature in }{ }^{\circ} \mathrm{C} \\
\mathrm{a}_{0} & =11.2398+/-.0035 \\
\mathrm{a}_{1} & =2.436 \times 10^{-2}+/-1.3 \times 10^{-4} \\
\mathrm{a}_{2} & =-2.13 \times 10^{-5}+/-1.3 \times 10^{-6} \\
\mathbf{M}_{\text {HCFC-124 }} & =0.136477 \mathrm{~kg} / \mathrm{mol} \text { (molar mass) }
\end{array}
$$

or

\section{PI UNITS}

$$
\begin{array}{lll}
\mathrm{R} & =0.004381042 \mathrm{Btu} / \mathrm{F}-\mathrm{mol} \text { (universal gas constant) } \\
\mathrm{t} & =\text { temperature in }{ }^{\circ} \mathrm{F} \\
\mathrm{a}_{0} & =10.8000+/-.0042 \\
\mathrm{a}_{1} & =1.395 \times 10^{-2}+/-7.7 \times 10^{-5} \\
\mathrm{a}_{2} & =-6.57 \times 10^{-6}+/-4.0 \times 10^{-7} \\
\mathrm{M}_{\mathrm{HCFC}-124} & =0.300880 \mathrm{lbm} / \mathrm{mol} \text { (molar mass) }
\end{array}
$$

The second, third, and fourth acoustic virial coefficients - beta, gamma, and delta - have been obtained by analyzing the pressure dependence of the speed of sound. The results are given in Table 32.

An adiabatic calorimeter has been used to measure the molar heat capacity at constant volume $\left\{C_{v}\right\}$ for HCFC-124. The measurements were carried out in the single-phase-liquid (74 points) and saturated-liquid (132 points) states. Five isochores have been completed. The temperatures ranged from 173 to $345 \mathrm{~K}\left(-148\right.$ to $\left.161^{\circ} \mathrm{F}\right)$ with pressures to $35 \mathrm{MPa}(5000 \mathrm{psi})$. The measurements are presented for the liquid phase in Tables 33 through 37 and for the twophase region in Table 38. The temperatures of the saturated liquid ranged from 94 to $343 \mathrm{~K}$ $\left(-290\right.$ to $\left.158^{\circ} \mathrm{F}\right)$.

A 32-term MBWR equation of state for HCFC-124 has been developed. It is valid at temperatures from 210 to $450 \mathrm{~K}\left(-82\right.$ to $\left.350^{\circ} \mathrm{F}\right)$, and it appears to be reasonable upon extrapolation up to $500 \mathrm{~K}\left(440^{\circ} \mathrm{F}\right)$; the maximum pressure is $20 \mathrm{MPa}(3000 \mathrm{psi})$. The techniques and types of data used in the fit are similar to those for HFC-32 described above. Table 39 gives the coefficients to the equation of state, and Table $\mathbf{4 0}$ gives the saturation properties calculated with the equation of state. Figures 15 and 16 compare values of density and vapor pressure calculated with the MBWR equation to the experimental values used in the fit. This MBWR equation should be adequate for ai'- refrigeration calculations involving HCFC124; however, its predictions may differ slightly from those of the MBWR equation of state that 
will be incorporated into version 4.0 of the REFPROP computer package, which will be released in the coming months.

HFC-125

The Burnett apparatus has been used in the isochoric mode to determine the PVT relation for the vapor phase of HFC-125 at 87 points. Isochores were completed at densities up to 2.9 $\mathrm{mol} / \mathrm{L}\left(21.7 \mathrm{lb} / \mathrm{ft}^{3}\right)$. The temperature range was 273 to $363 \mathrm{~K}\left(32\right.$ to $\left.194^{\circ} \mathrm{F}\right)$. A Burnett expansion was completed at $363 \mathrm{~K}\left(194^{\circ} \mathrm{F}\right)$ to establish the densities of the isochores. The results of these measurements are given in Table 41 .

The vibrating tube densimeter has been used to determine the PVT relation for HFC-125 in the liquid phase. Fifteen isotherms were completed spanning the ranges 275 to $369 \mathrm{~K}$ (36 to $205^{\circ} \mathrm{F}$ ) and 1500 to $6200 \mathrm{kPa}(230$ to $900 \mathrm{psi})$. The results of these liquid-phase measurements are given in Table 42 .

An isochoric PVT apparatus has been used to measure the density of liquid HFC-125 at 87 liquid-state conditions. The measured temperatures ranged from 174 to $398 \mathrm{~K}$ (-146 to $\left.257^{\circ} \mathrm{F}\right)$ with pressures up to $35 \mathrm{MPa}(5000 \mathrm{psi})$. The results are presented in Table 43 .

The Burnett apparatus and the glass ebulliometer have been used to measure the vapor pressure of HFC-125 at 68 points, given in Table 44 . The data span the very wide pressure range from 77 to $3313 \mathrm{kPa}$ ( 1.9 to $458.8 \mathrm{psi}$ ) corresponding to the temperature range from 218 to $335 \mathrm{~K}\left(-60\right.$ to $\left.239^{\circ} \mathrm{F}\right)$. The data were fit with a Wagner-type vapor pressure equation and had a standard deviation of $0.009 \%$. The vapor pressure equation was extrapolated to the critical temperature at $\mathrm{T}_{\mathrm{c}}=339.33 \mathrm{~K}\left(151.11^{\circ} \mathrm{F}\right)$ to obtain a value of the critical pressure, $\mathrm{P}_{\mathrm{c}}=3628.6$ $\mathrm{kPa}(525.1 \mathrm{psi})$.

A static method has been employed to measure the vapor pressure of HFC-125 at 37 temperatures ranging from 175 to $335 \mathrm{~K}\left(-145\right.$ to $\left.143^{\circ} \mathrm{F}\right)$. The results are given in Table 45 .

An optical cell has been used to measure the refractive index and capillary rise of HFC125 from $23^{\circ} \mathrm{C}\left(73^{\circ} \mathrm{F}\right)$ up to the critical temperature. The critical temperature was found to be $\mathrm{T}_{\mathrm{c}}=(339.33 \pm 0.02) \mathrm{K}$, which corresponds to $(151.11 \pm 0.04)^{\circ} \mathrm{F}$. The refractive index data were combined with the liquid density data to deduce the value $0.0984 \mathrm{~cm}^{3} / \mathrm{g}$ for the LorentzLorenz constant. The refractive index data and the Lorentz-Lorenz constant were used to deduce the value $\rho_{\mathrm{c}}=(565 \pm 9) \mathrm{kg} / \mathrm{m}^{3}\left[(35.24 \pm 0.56) \mathrm{lb} / \mathrm{ft}^{3}\right]$ for the critical density.

A cylindrical acoustic resonator has been used to measure the speed of sound in $\mathrm{HFC}-125$ [or more precisely, in a mixture of HFC-125 with a small amount of CFC-115 as an impurity] along isotherms between 240 and $380 \mathrm{~K}\left(-27.1\right.$ and $\left.224.3^{\circ} \mathrm{F}\right)$ at pressures up to $1 \mathrm{MPa}(145 \mathrm{psi})$ or $80 \%$ of the vapor pressure, whichever is less. The results are given in Table 46 . The idealgas heat capacity, $\mathrm{C}_{\mathrm{p}}^{\mathrm{o}}$, of HFC- 125 was obtained by analyzing the speed of sound measurements at low pressures and is given in Table 47. The following expressions for $C_{p}^{o}$ were obtained by fitting the data in Table 47: 


$$
\mathrm{C}_{\mathrm{p}}^{\mathrm{o}}(\mathrm{HFC}-125) \quad=\mathrm{R}\left(\mathrm{a}_{0}+\mathrm{a}_{1} \mathrm{t}+\mathrm{a}_{2} \mathrm{t}^{2}\right)
$$

where

\section{SI UNITS}

$\begin{array}{lll}\mathrm{R} & =8.314471 \mathrm{~J} / \mathrm{K} \text {-mol (universal gas constant) } \\ \mathrm{t} & =\text { temperature in }{ }^{\circ} \mathrm{C} \\ \mathrm{a}_{0} & =10.7661+/-0.0075 \\ \mathrm{a}_{1} & =0.02362+/-2.7 \times 10^{-4} \\ \mathrm{a}_{2} & =-1.42 \times 10^{-5}+/-2.7 \times 10^{-6} \\ \mathrm{M}_{\mathrm{HFC}-125} & =0.120022 \mathrm{~kg} / \mathrm{mol} \text { (molar mass) }\end{array}$

or

\section{PI UNITS}

$\begin{array}{lll}\mathbf{R} & = & 0.004381042 \mathrm{Btu} / \mathrm{F}-\mathrm{mol} \text { (universal gas constant) } \\ \mathrm{t} & =\text { temperature in }^{\circ} \mathrm{F} \\ \mathrm{a}_{0} & =10.3417+/-0.0089 \\ \mathrm{a}_{1} & =0.01313+/-1.5 \times 10^{-4} \\ \mathrm{a}_{2} & =-4.38 \times 10^{-6}+/-8.3 \times 10^{-7} \\ \mathrm{M}_{\mathrm{HFC}-125} & =0.264603 \mathrm{lbm} / \mathrm{mol} \text { (molar mass) }\end{array}$

The second, third, and fourth acoustic virial coefficients - beta, gamma, and delta - have been obtained from the pressure dependence of the speed of sound and are given in Table 48.

An adiabatic calorimeter has been used to measure molar heat capacity at constant volume $\left\{\mathrm{C}_{\mathrm{v}}\right\}$ for HFC-125. In total, $120 \mathrm{C}_{\mathrm{v}}$ values were measured in the liquid state and 100 values were measured in the vapor + liquid two-phase region. The temperatures ranged from 176 to $342 \mathrm{~K}\left(-143\right.$ to $\left.156^{\circ} \mathrm{F}\right)$ with pressures to $35 \mathrm{MPa}(5000 \mathrm{psi})$. The measured values are given in Tables 49 through 55 for the liquid phase and Tables 56 through 62 for the two-phase region.

A 32-term MBWR equation of state for HFC-125 has been developed. It is valid at temperatures from 200 to $400 \mathrm{~K}\left(-100\right.$ to $\left.260^{\circ} \mathrm{F}\right)$, and it appears to be reasonable upon extrapolation up to $500 \mathrm{~K}\left(440^{\circ} \mathrm{F}\right)$; the maximum pressure is $20 \mathrm{MPa}(3000 \mathrm{psi})$. The techniques and types of data used in the fit are similar to those for HFC-32 described above. Table 63 gives the coefficients to the equation of state, and Table 64 gives the saturation properties calculated with the equation of state. Figures 17 through 21 compare the values of various properties calculated with the MBWR equation to the experimental values used in the fit. This MBWR equation should be adequate for all refrigeration calculations involving HFC125; however, its predictions may differ slightly from those of the MBWR equation of state that will be incorporated into version 4.0 of the REFPROP computer package, which will be released in the coming months.

HCFC-123

An isochoric PVT apparatus has been used to measure the density of liquid HCFC-123 at 105 points. The temperatures ranged from 176 to $380 \mathrm{~K}\left(-143\right.$ to $\left.224^{\circ} \mathrm{F}\right)$ with pressures to $35 \mathrm{MPa}(5000 \mathrm{psi})$. The pressures, temperatures, and densities are presented in Table 65. 
An adiabatic calorimeter has been used to measure the molar heat capacity at constant volume $\left\{\mathrm{C}_{\mathrm{v}}\right\}$ for HCFC-123. The measurements were carried out in the single-phase-liquid (79 points) and saturated-liquid (92 points) states. Six isochores have been completed. The temperatures ranged from 167 to $341 \mathrm{~K}\left(-159\right.$ to $\left.155^{\circ} \mathrm{F}\right)$ with pressures up to $35 \mathrm{MPa}(5000$ psi). These measurements are presented in Tables 66 through 71 for the liquid phase and in Tables 72 and 73 for the two-phase region.

The existing MBWR equation of state for HCFC-123 has been revised and considerably improved using the recently measured data. This work was prompted by an evaluation of equations of state for HFC-134a and HCFC-123 carried out by Annex 18 of the International Energy Agency. Preliminary results of this comparison revealed weaknesses in some of the derived properties (specifically, speed of sound and heat capacity) for our previous HCFC-123 MBWR fit. The revised equation is accurate for all thermodynamic properties from just above the triple point to about $550 \mathrm{~K}\left(530^{\circ} \mathrm{F}\right)$ and at pressures up to $40 \mathrm{MPa}(6000 \mathrm{psi})$. The techniques and types of data used in the fit are similar to those for HFC-32 described above. Table 74 gives the coefficients to the equation of state. This MBWR equation for HCFC-123 will be used in version 4.0 of the REFPROP computer package, which will be released in the coming months; it will be available as an option to the CSDM equation of state for HCFC-123.

The low- and high-temperature transient hot-wire thermal conductivity instruments have been used to measure the thermal conductivity of HCFC-123 at 1618 points. Liquid-phase data have been obtained in the temperature range from 180 to $440 \mathrm{~K}\left(-136\right.$ to $\left.332^{\circ} \mathrm{F}\right)$ at pressures from the saturated vapor pressure to $70 \mathrm{MPa}(10000 \mathrm{psi})$. Vapor-phase data have been obtained from 290 to $449 \mathrm{~K}\left(62\right.$ to $\left.332^{\circ} \mathrm{F}\right)$. Supercritical data have been obtained at $480 \mathrm{~K}\left(405^{\circ} \mathrm{F}\right)$. The results of the analysis of these 1618 transient hot-wire measurements are given in Table 75 . A plot of the entire thermal conductivity surface is provided for reference in Figure 22 . The vapor-phase thermal conductivity of HCFC-123 is shown in Figure 23. It appears to be quite consistent with the supercritical isotherm.

The thermal conductivity data have been correlated as described in Appendix B. The fluid-specific information required for this correlation are the MBWR equation of state for HCFC-123 described above and the parameters provided in Table 76. Deviations of the values of thermal conductivity calculated from the correlation from the experimental data used in the fit are given in Figure 24. The deviations are generally less than $\pm 5 \%$.

\section{COMPLIANCE WITH AGREEMENT}

NIST has complied with all terms of the grant agreement modulo small shifts in the estimated level of effort from one property and/or fluid to another.

\section{PRINCIPAL INVESTIGATOR EFFORT}

Dr. Richard F. Kayser is the NIST Principal Investigator for the MCLR program. During the fifteen montns of this project, Dr. Kayser devoted approximately four weeks to monitoring and reviewing the research, and preparing the quarterly and final report. The project involves multiple researchers and capabilities in Gaithersburg, MD and Boulder, CO. 


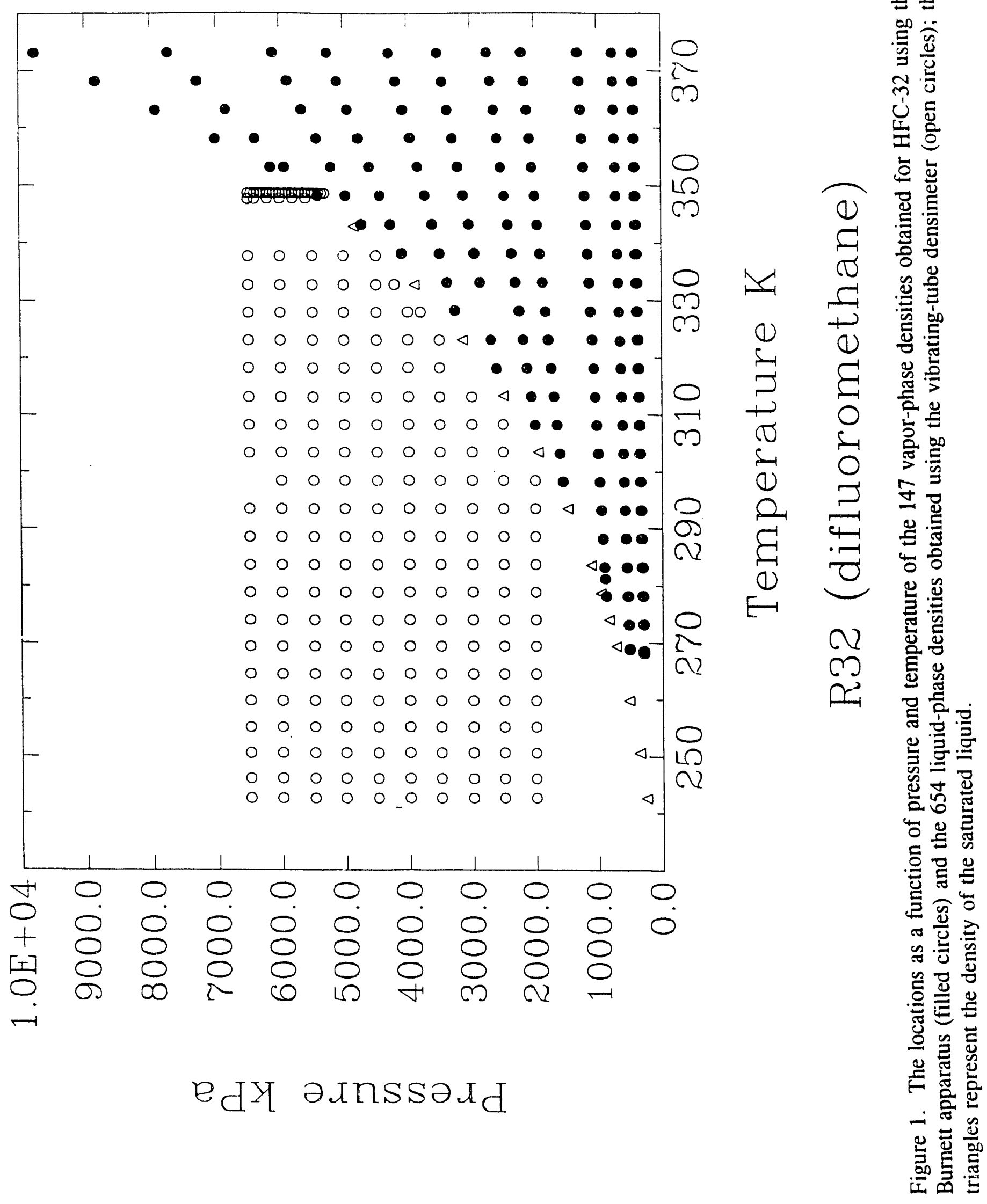




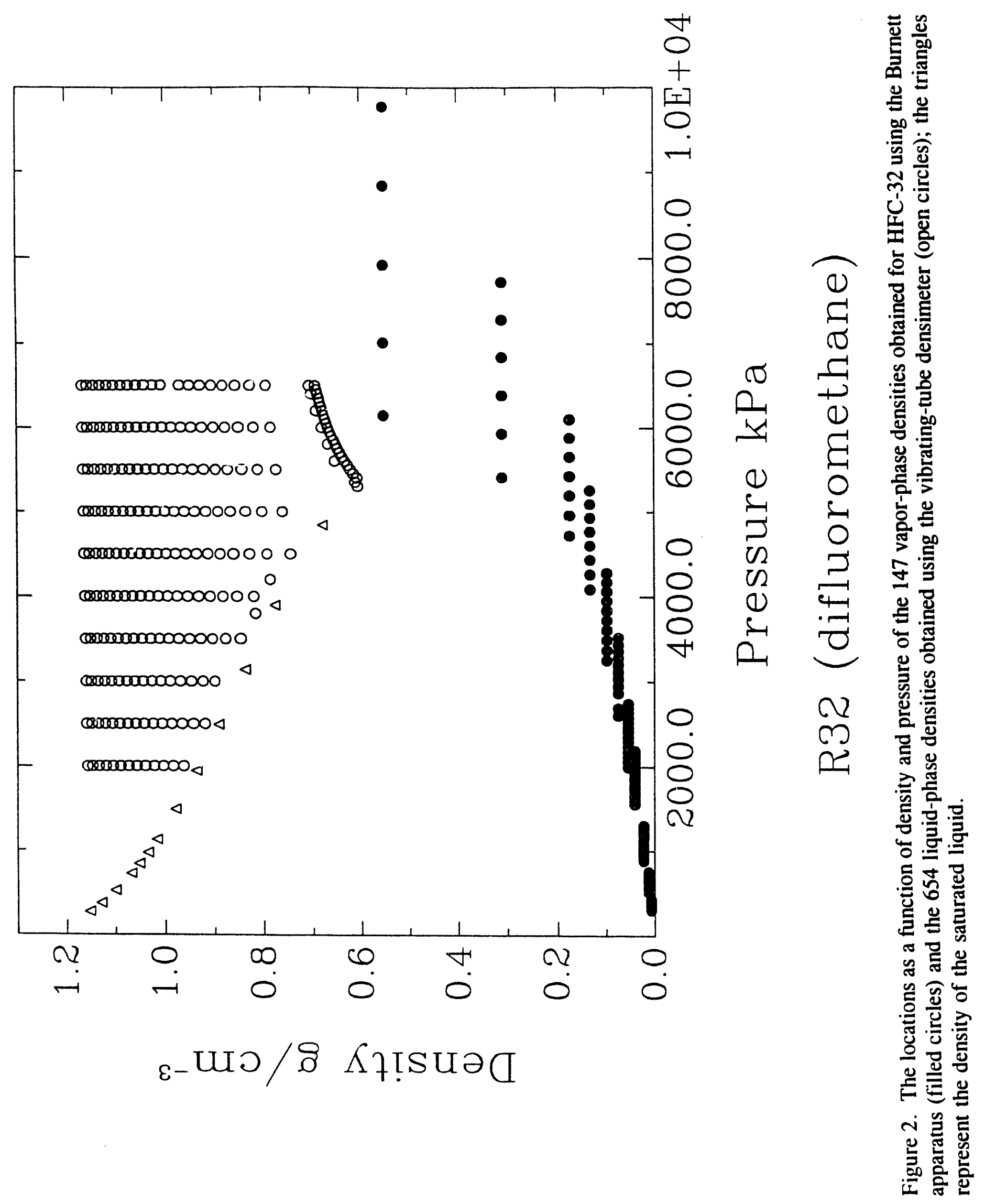




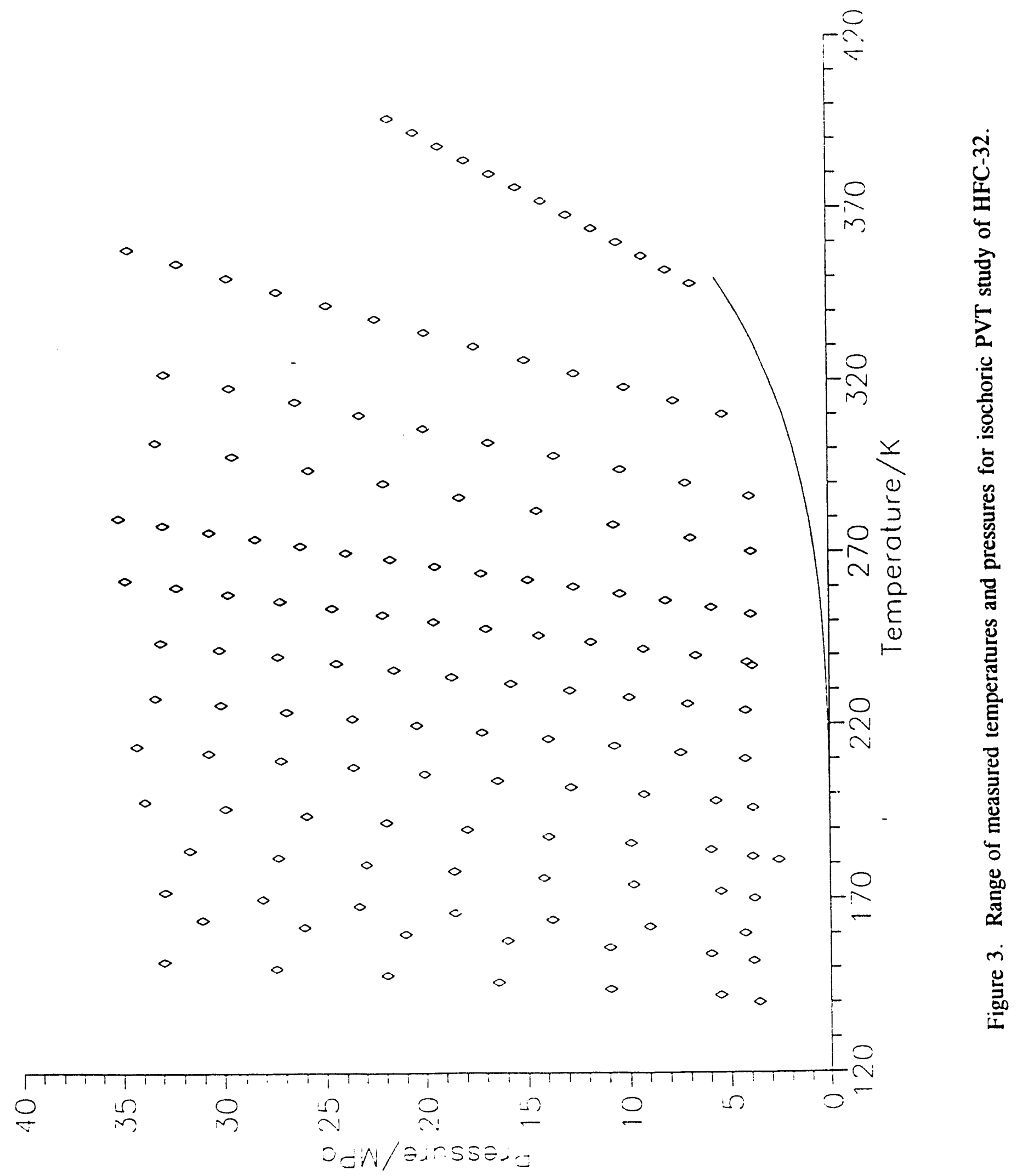



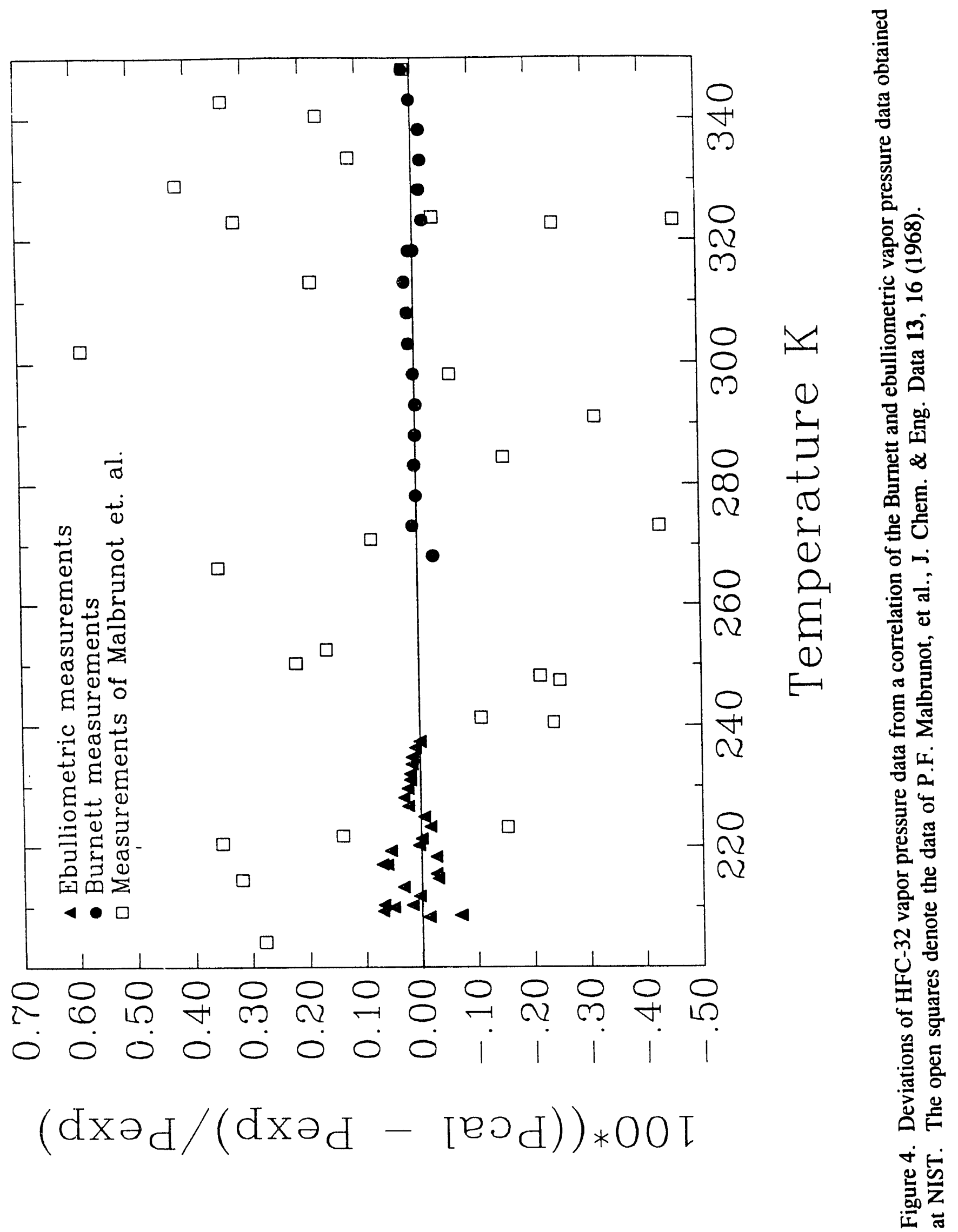


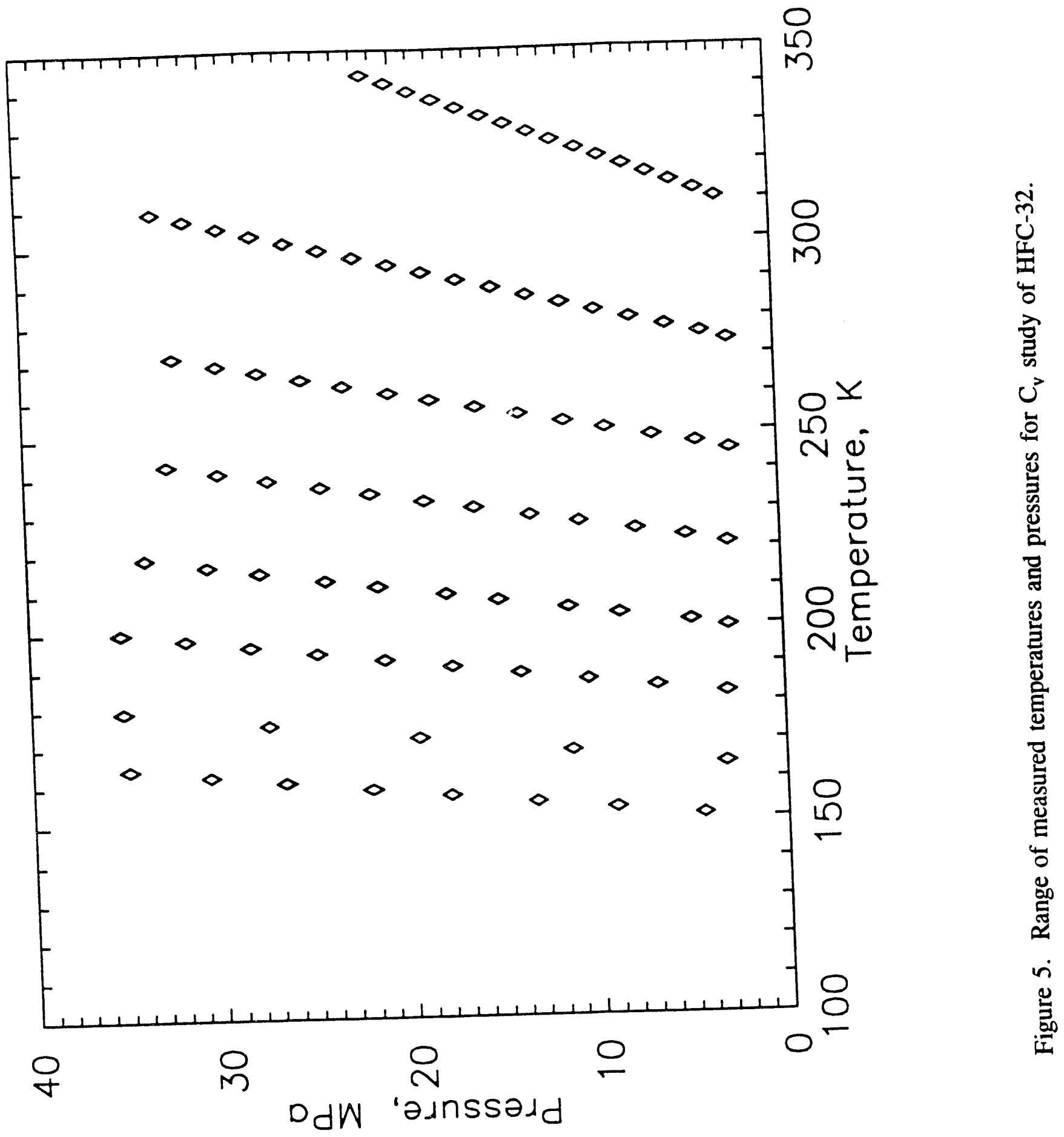




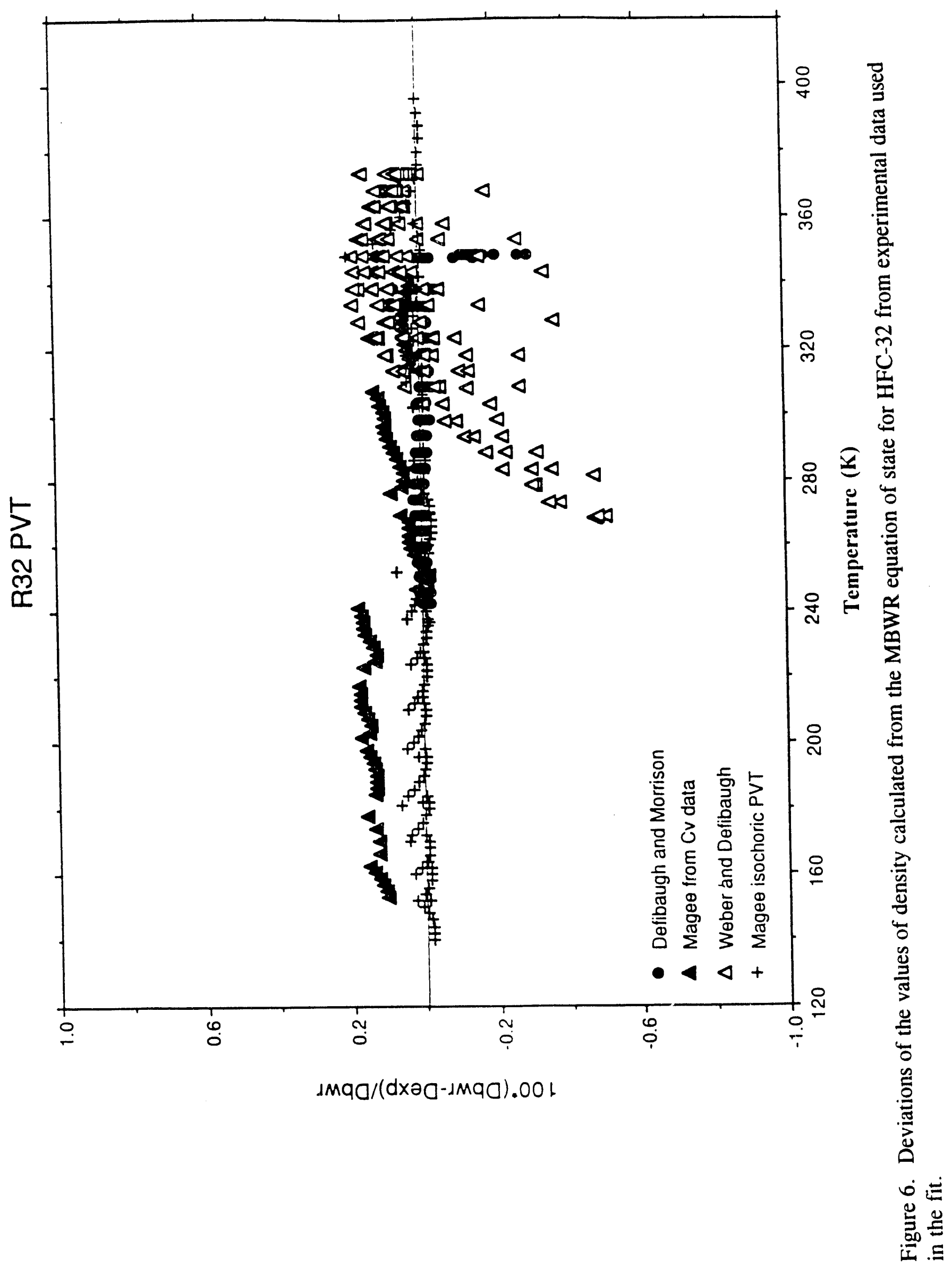




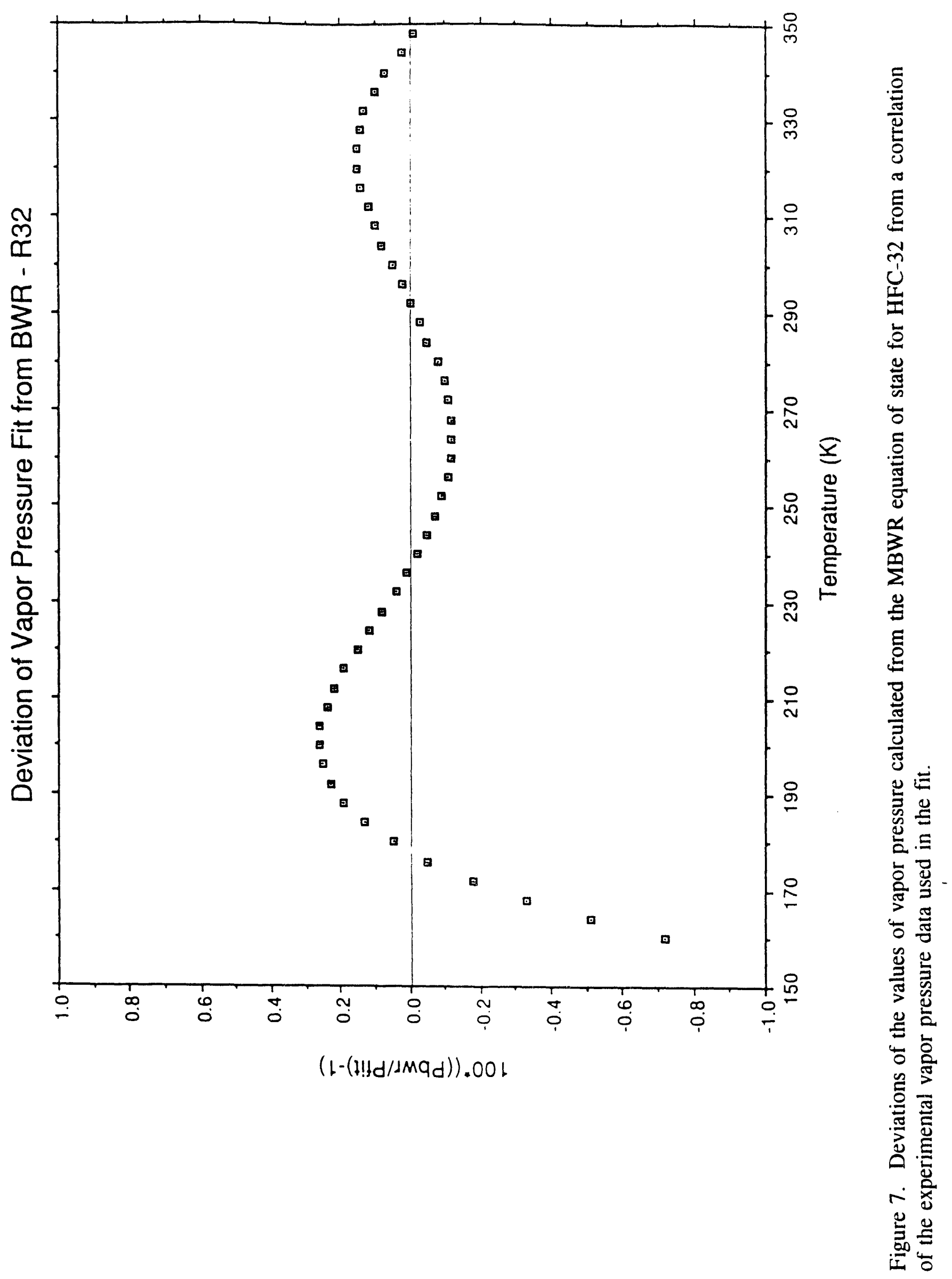




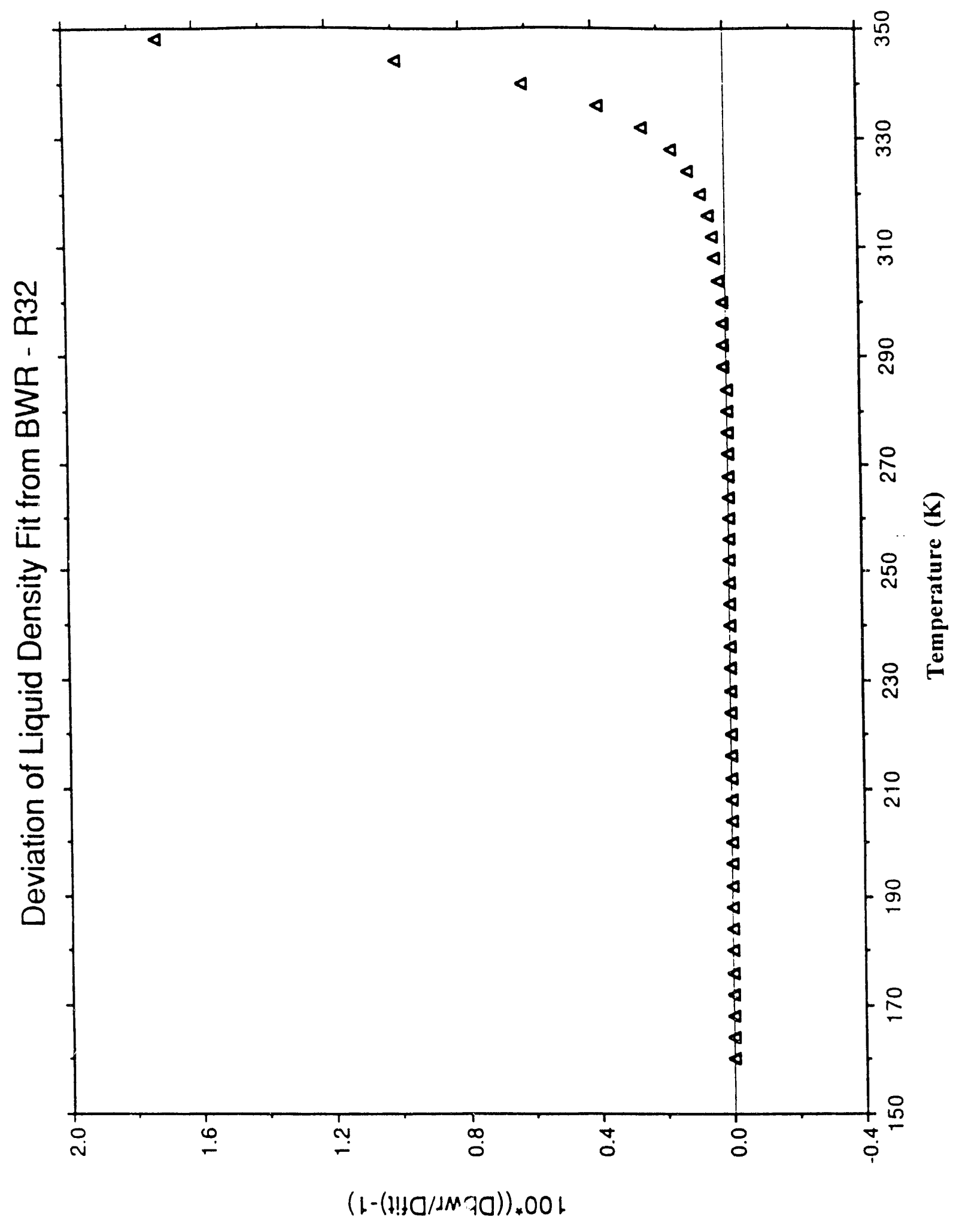

$\tau$

몽

ก

足

눙

营

4

콣

里

를

통

$\pm$

오․

马्ठ

శี

골

ปั

음

을 을

릉.

궁

정

胥

范

40

\&

宁

폴

$\pm$

$\infty$.

늠효

总 

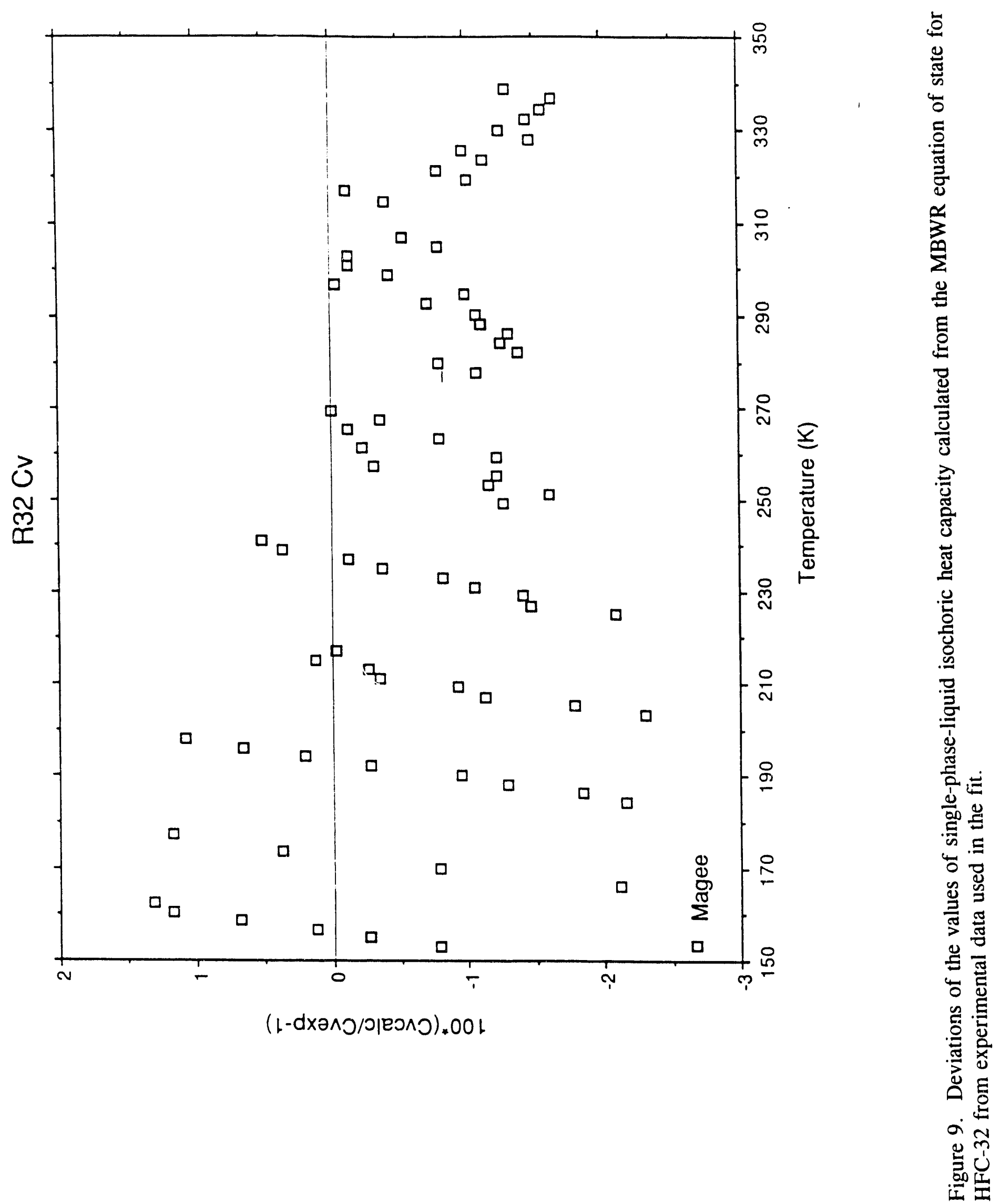


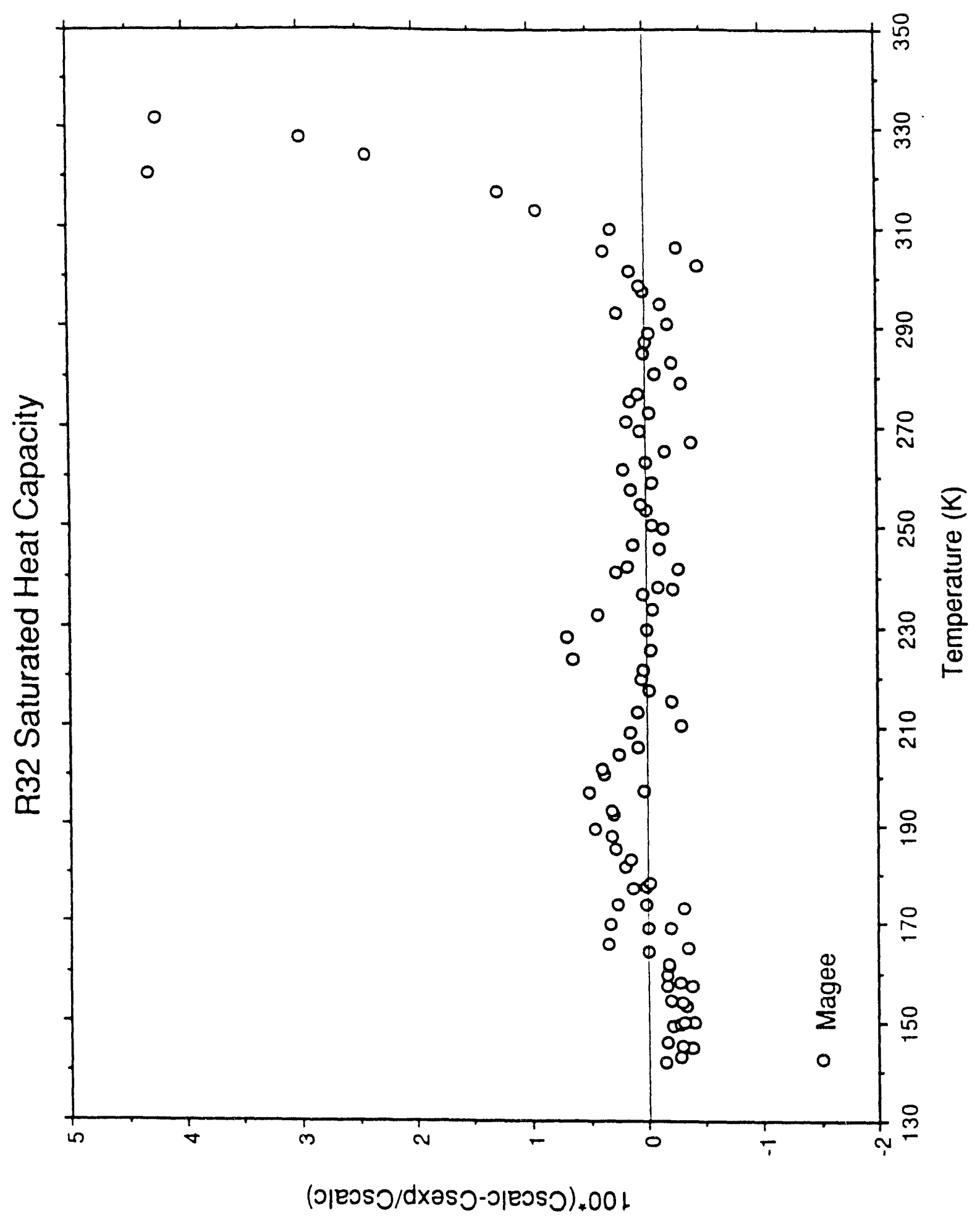

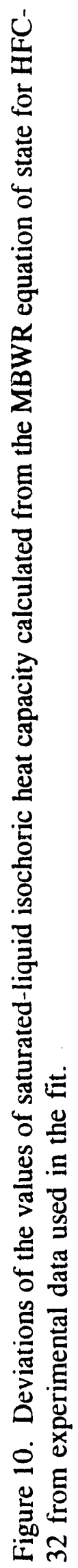




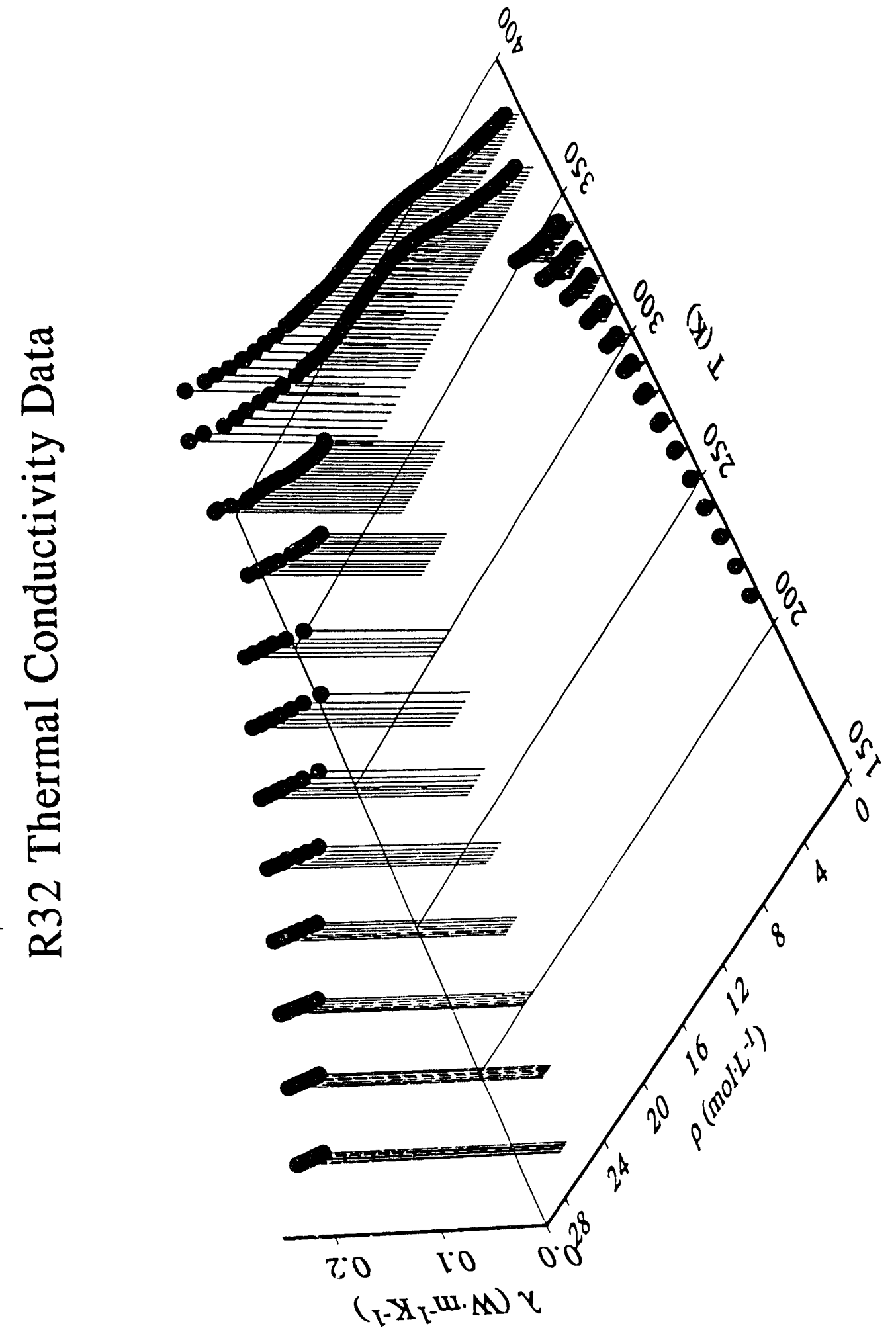

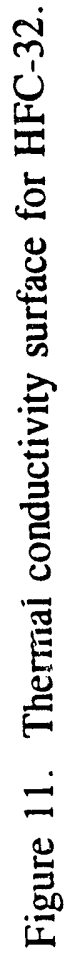




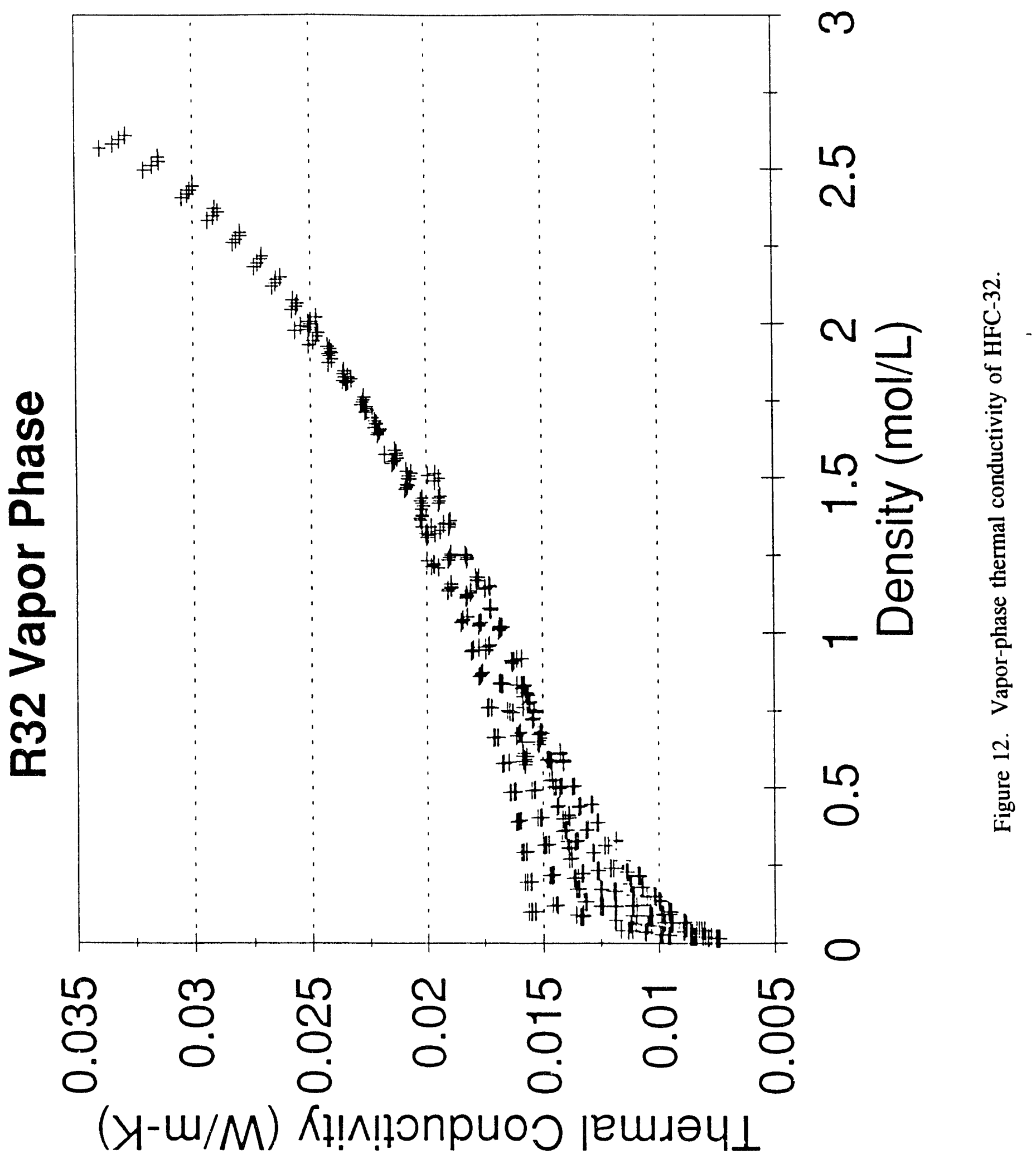




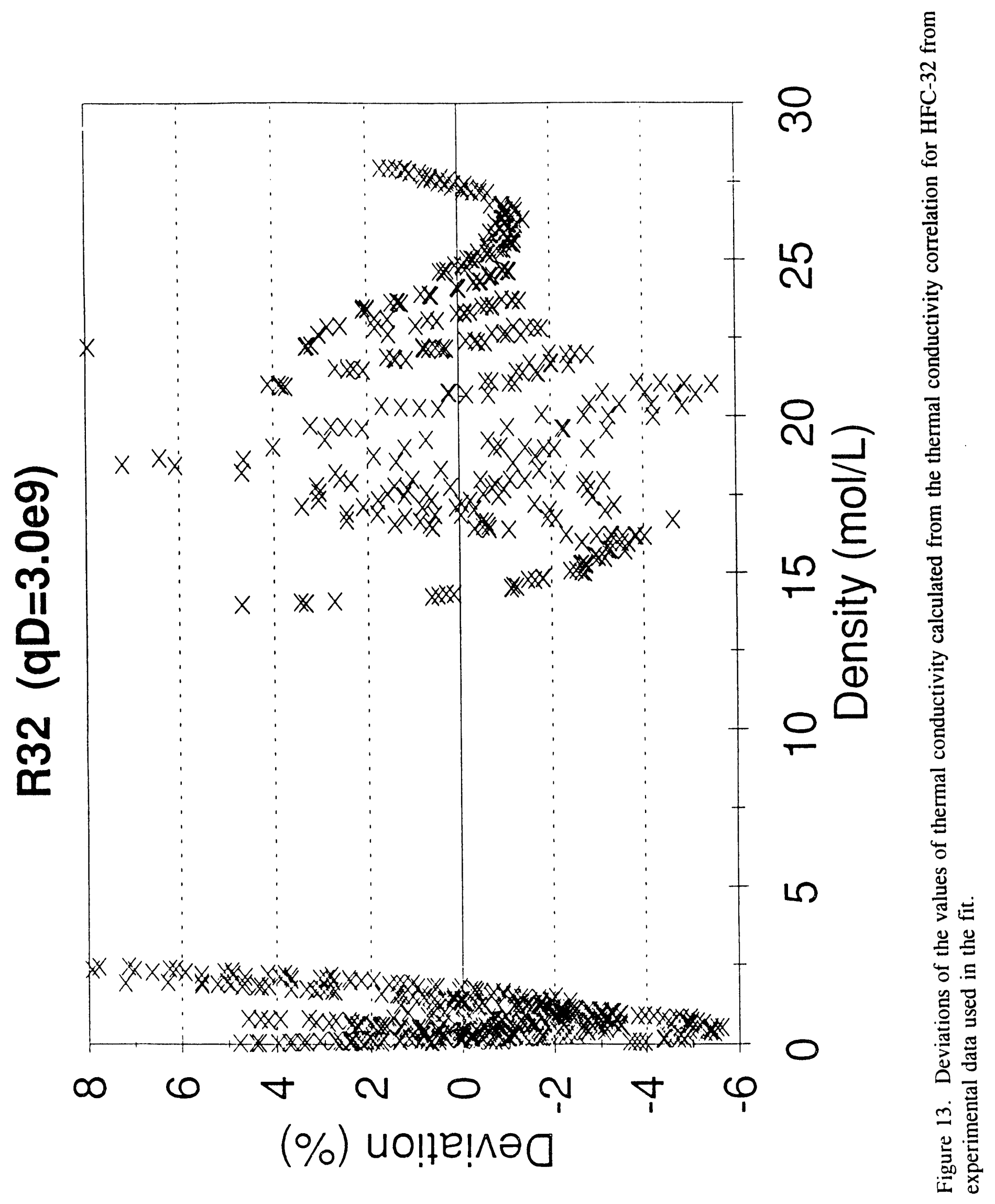




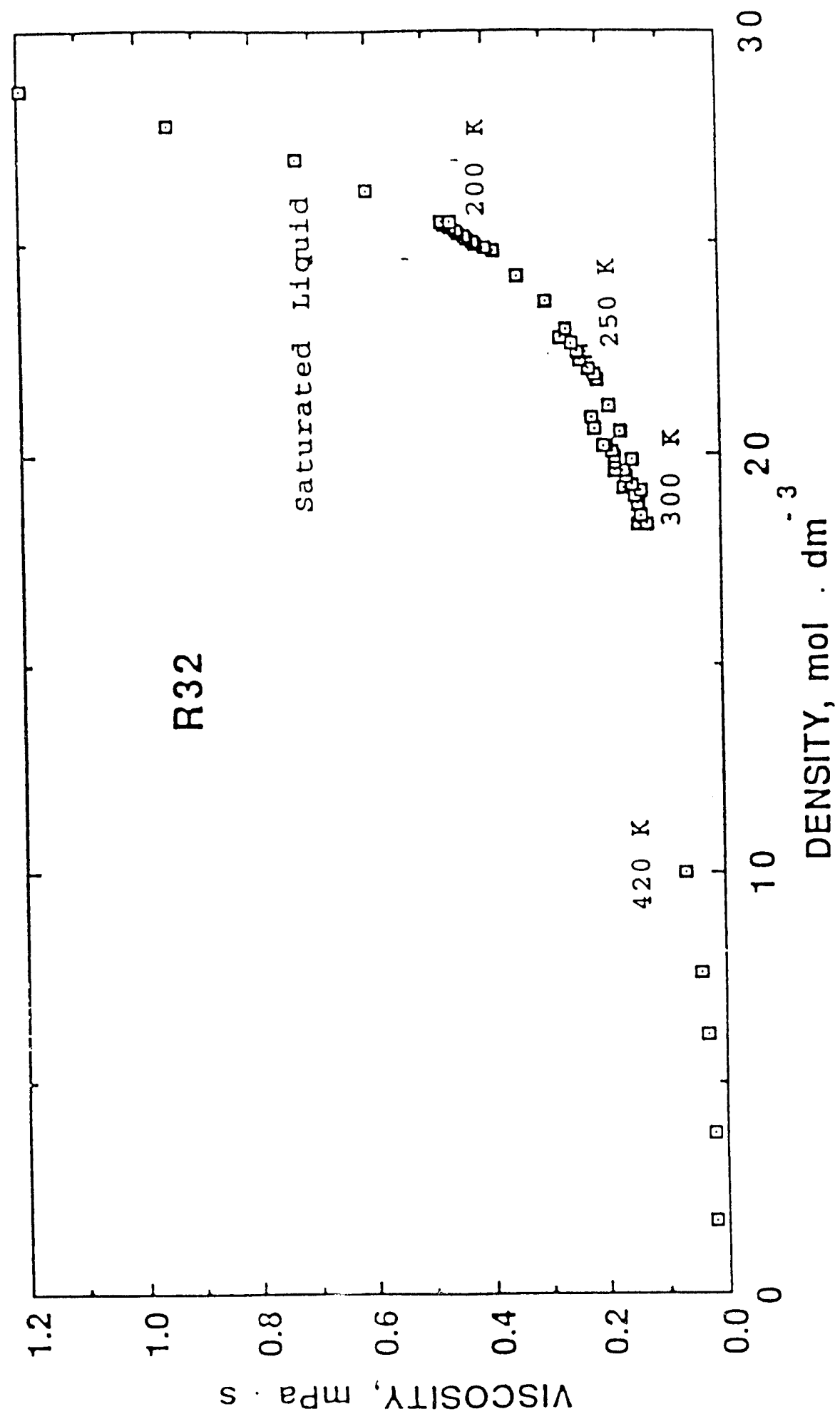

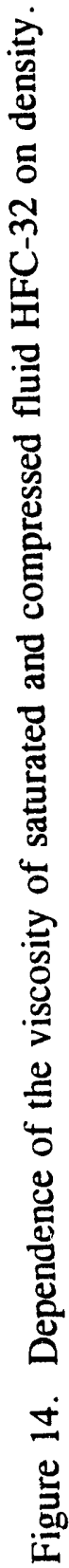



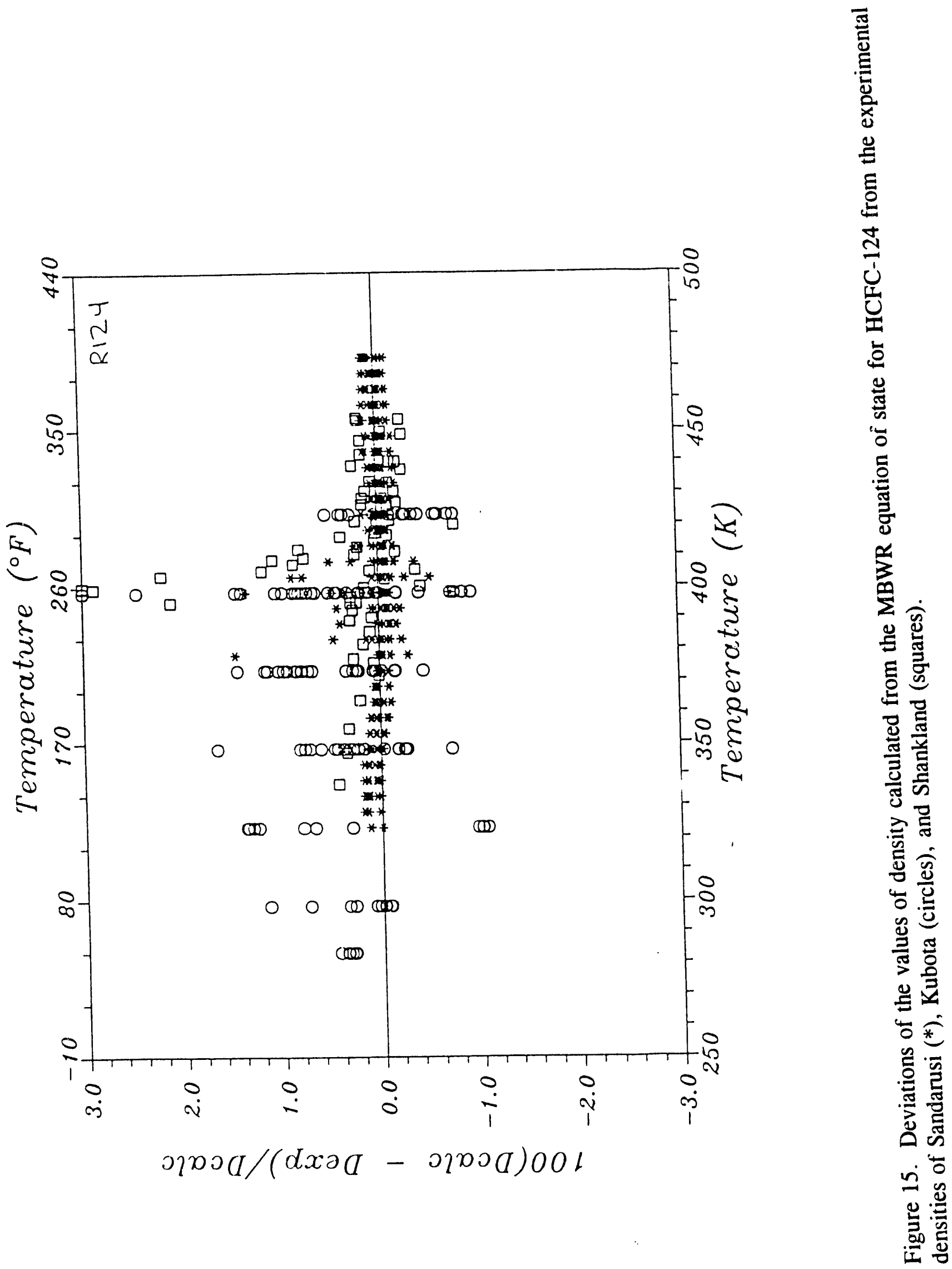


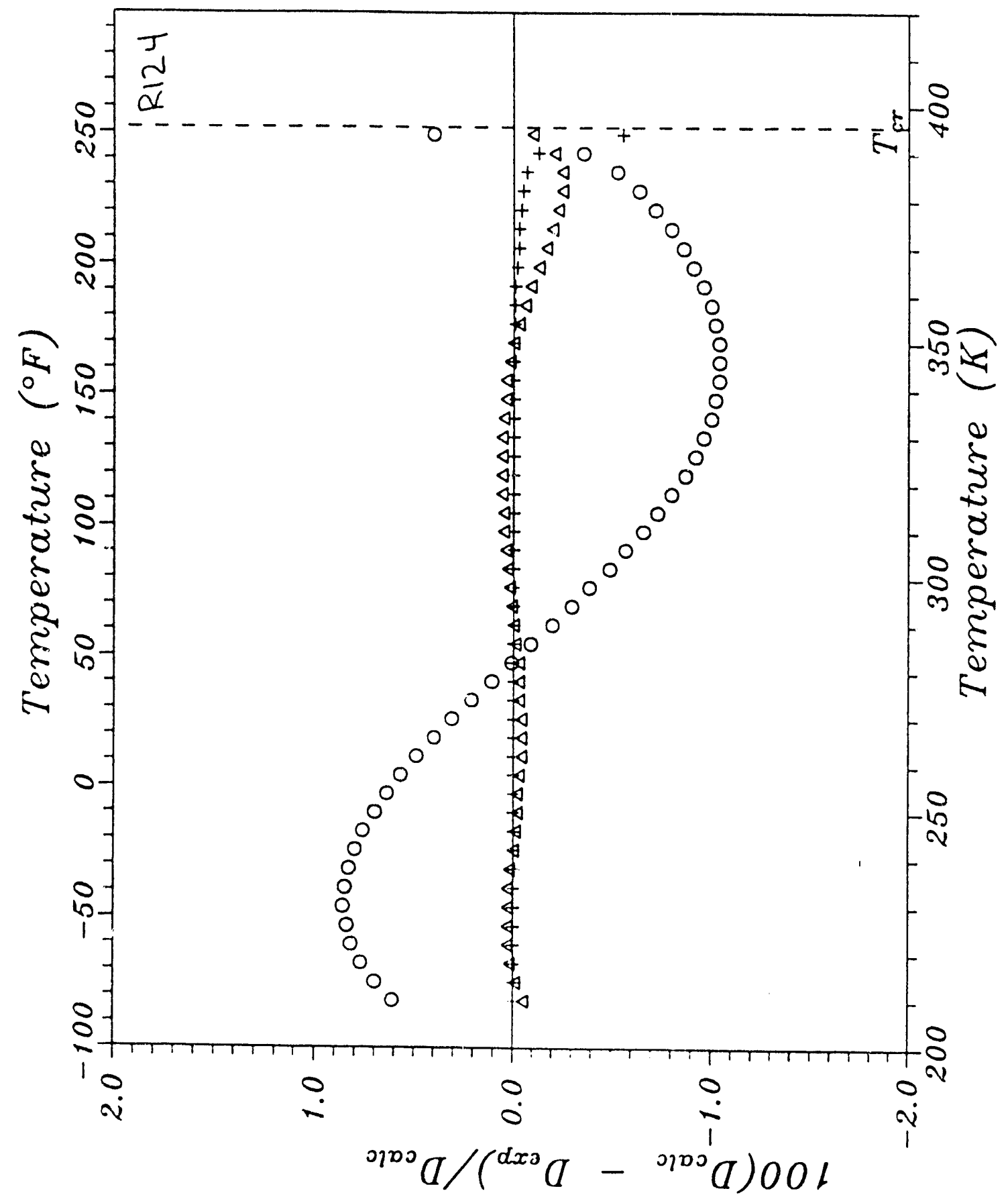




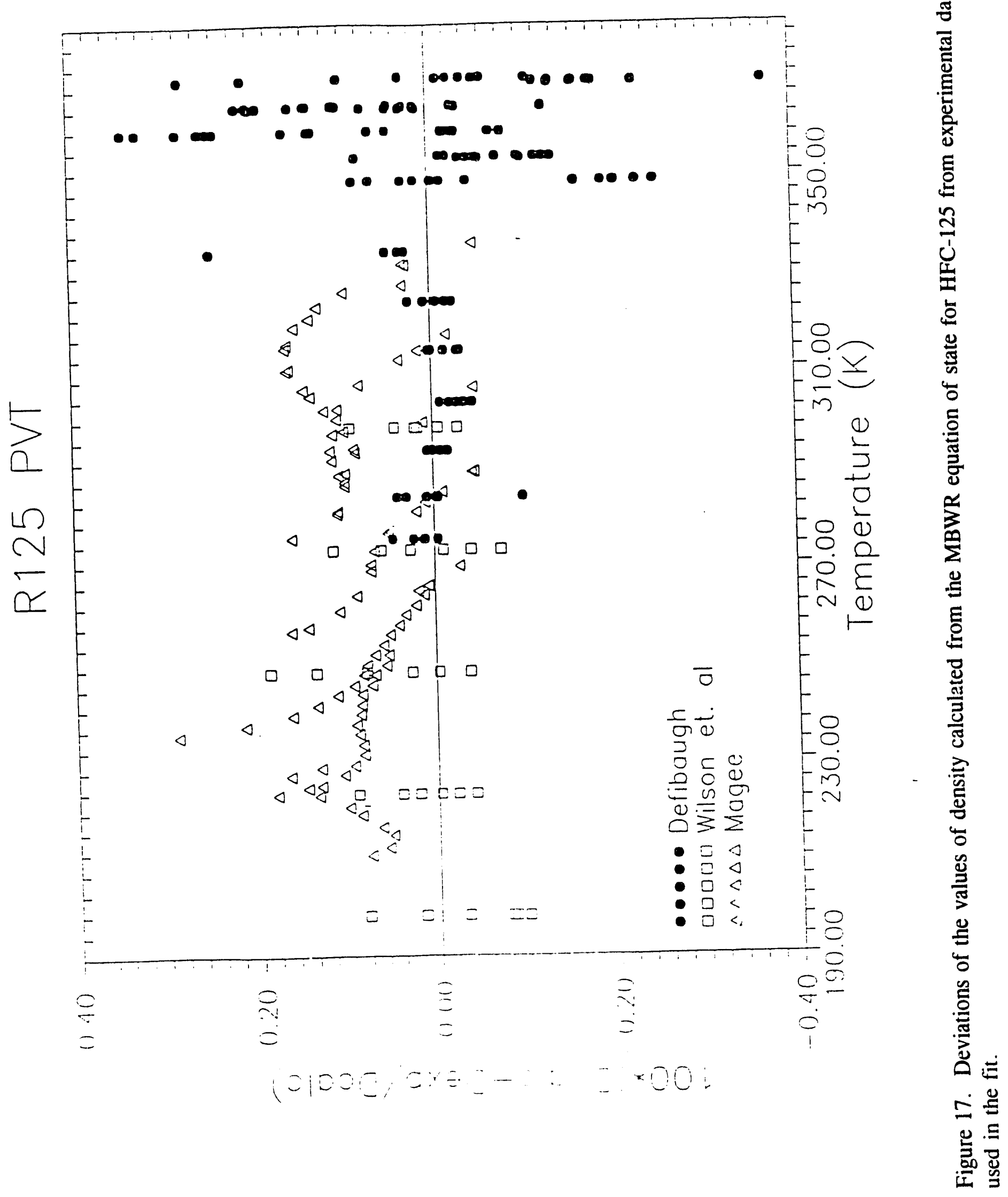



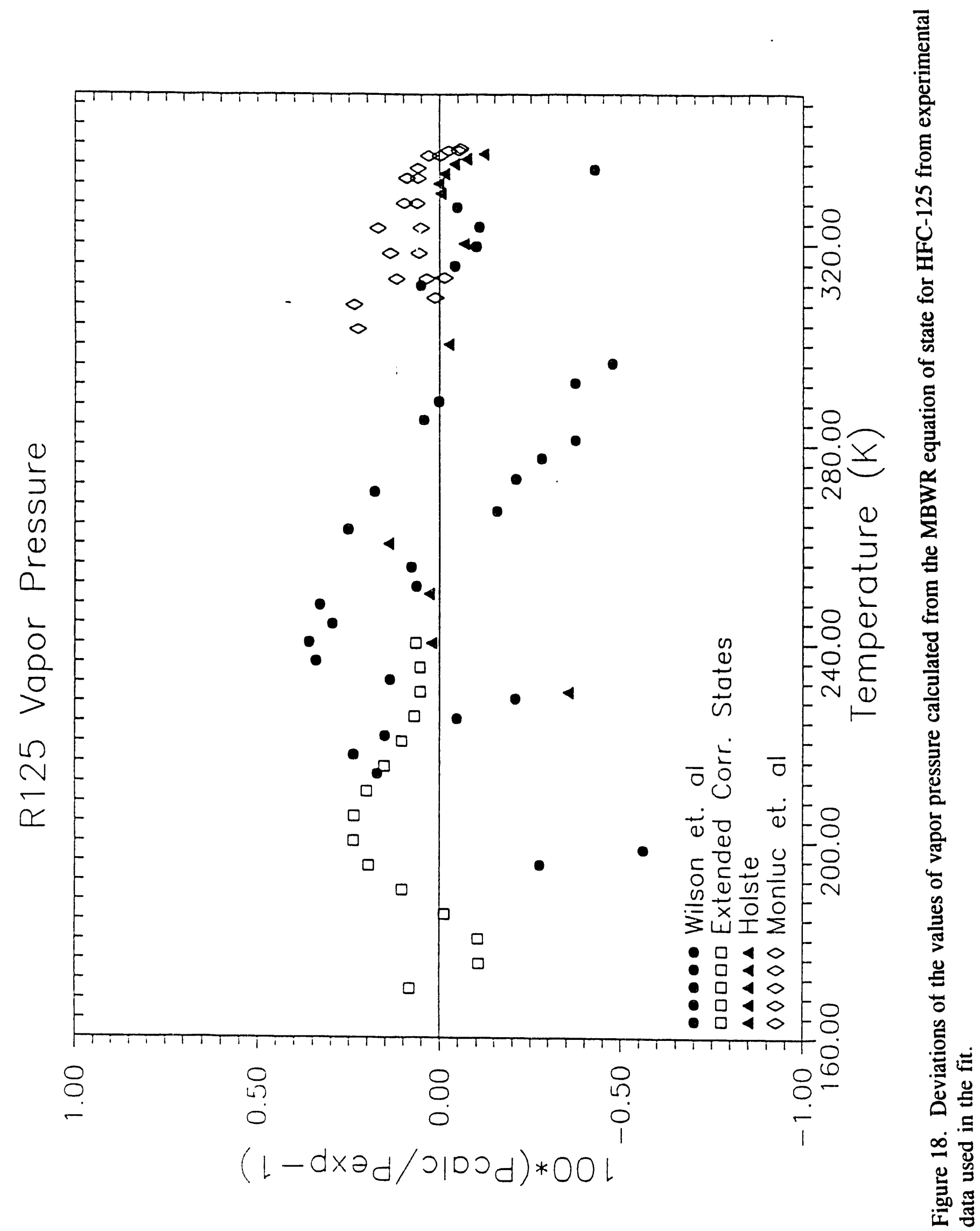


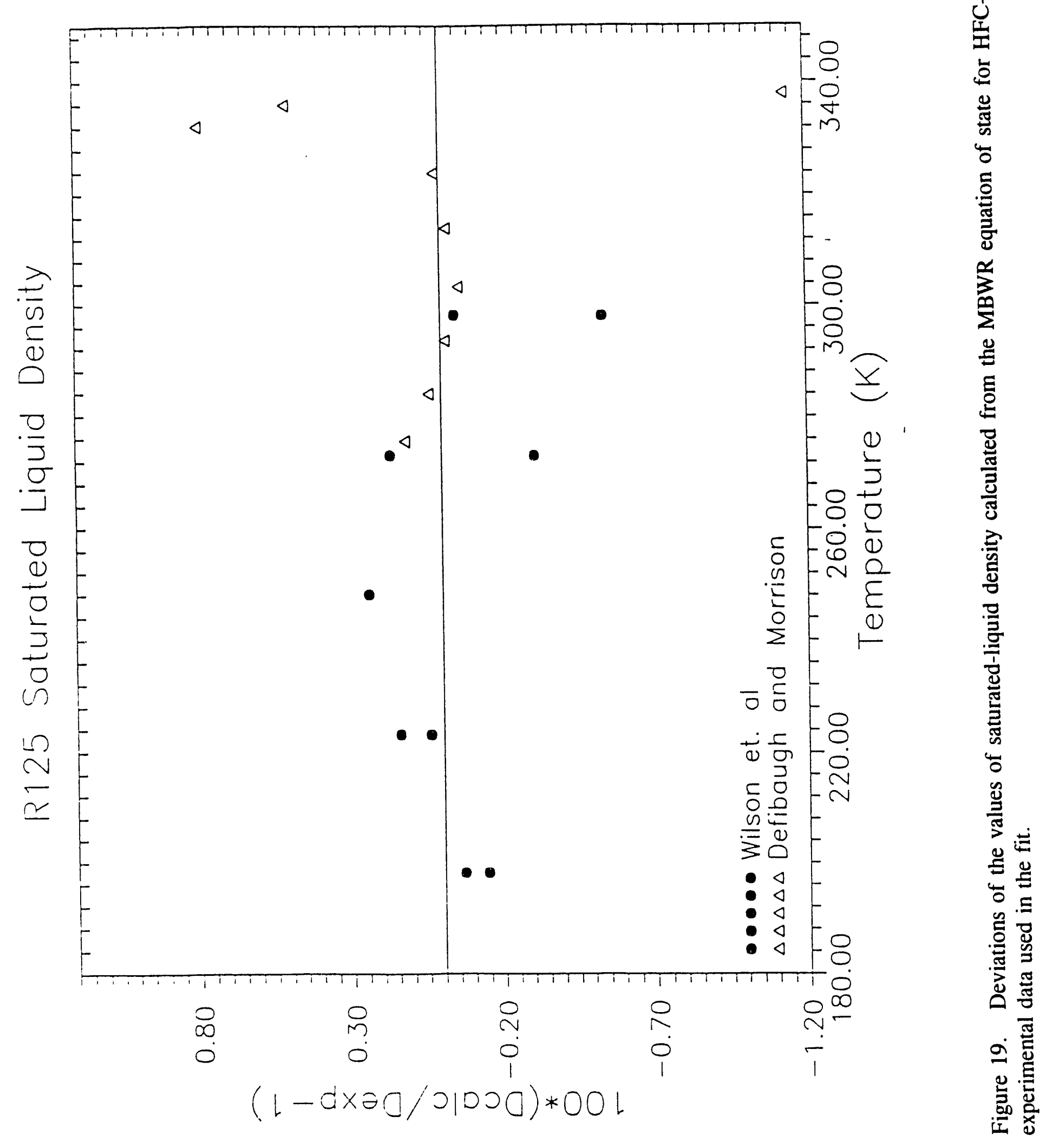




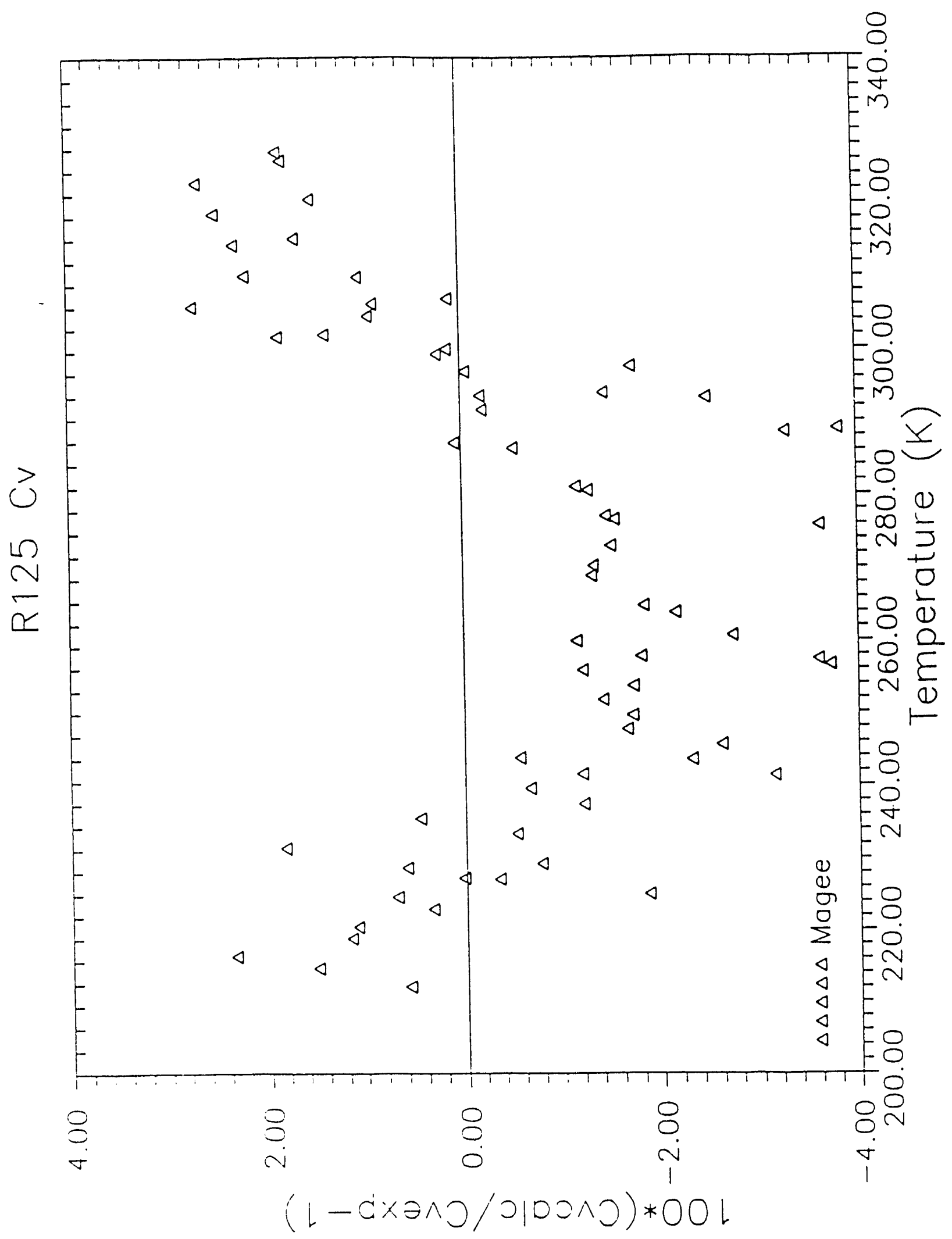

$\stackrel{5}{\circ}$

䒿

4

苂

3

$\Phi$

틍

롱

窇

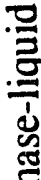




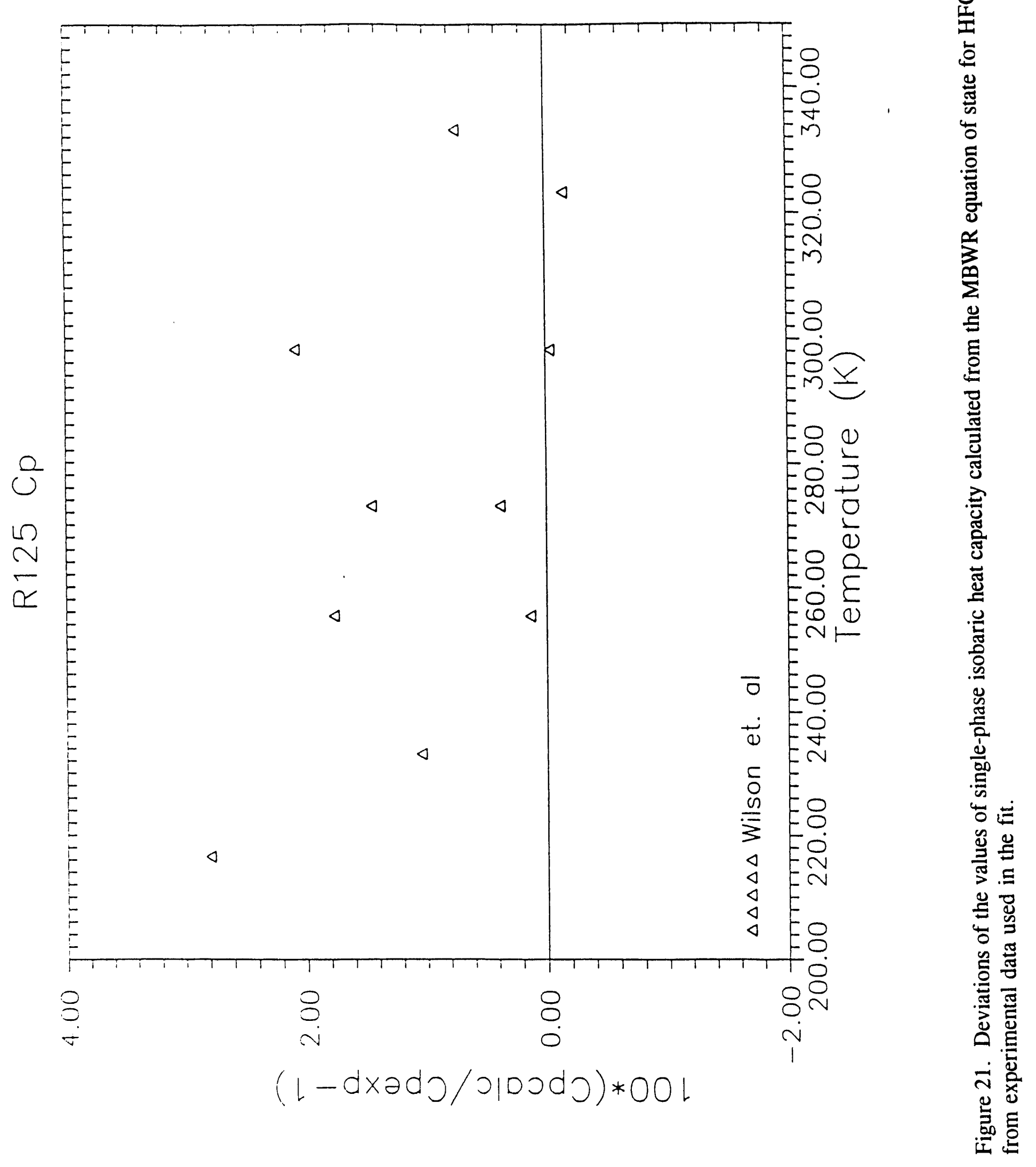




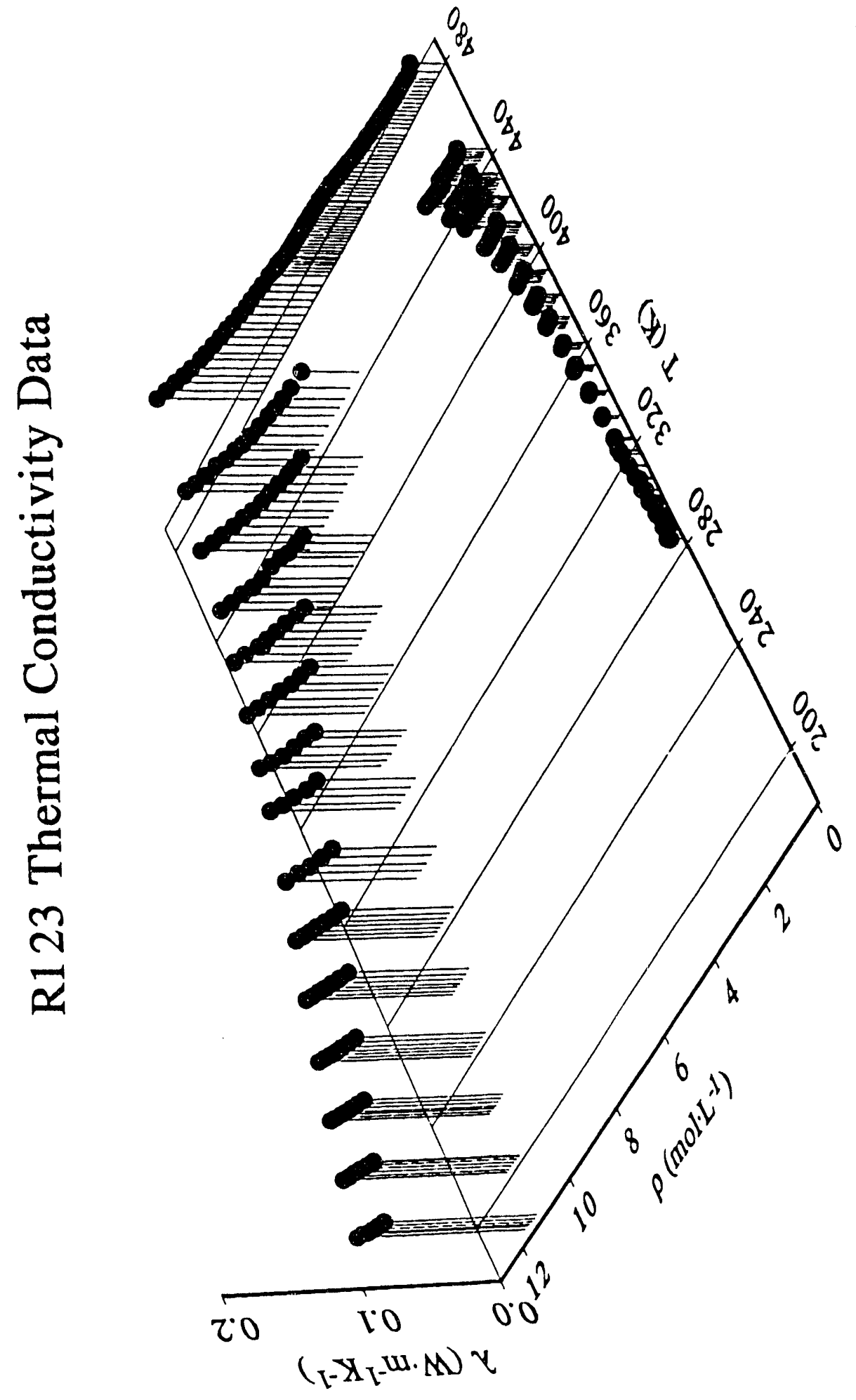

ปิ 


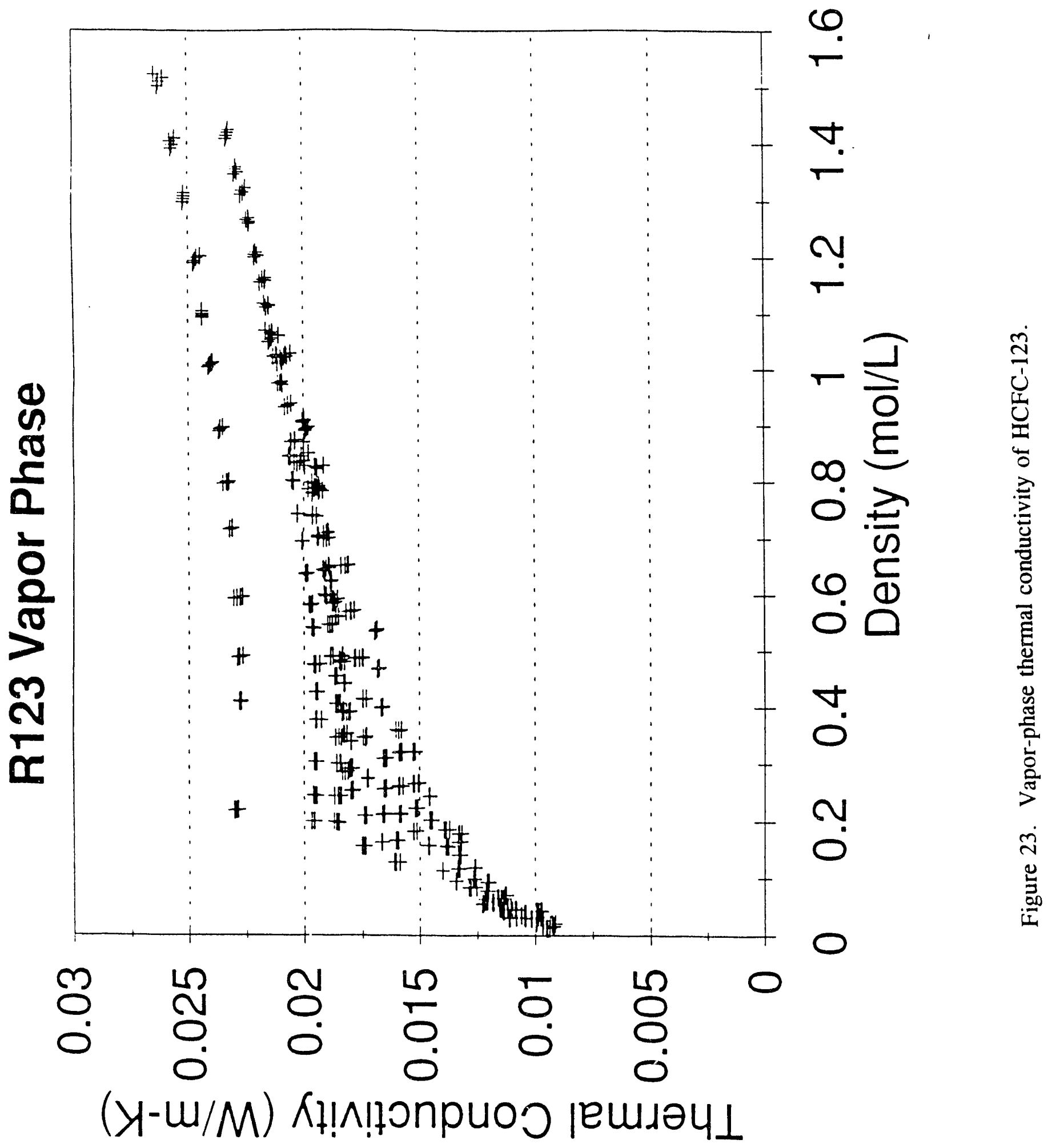



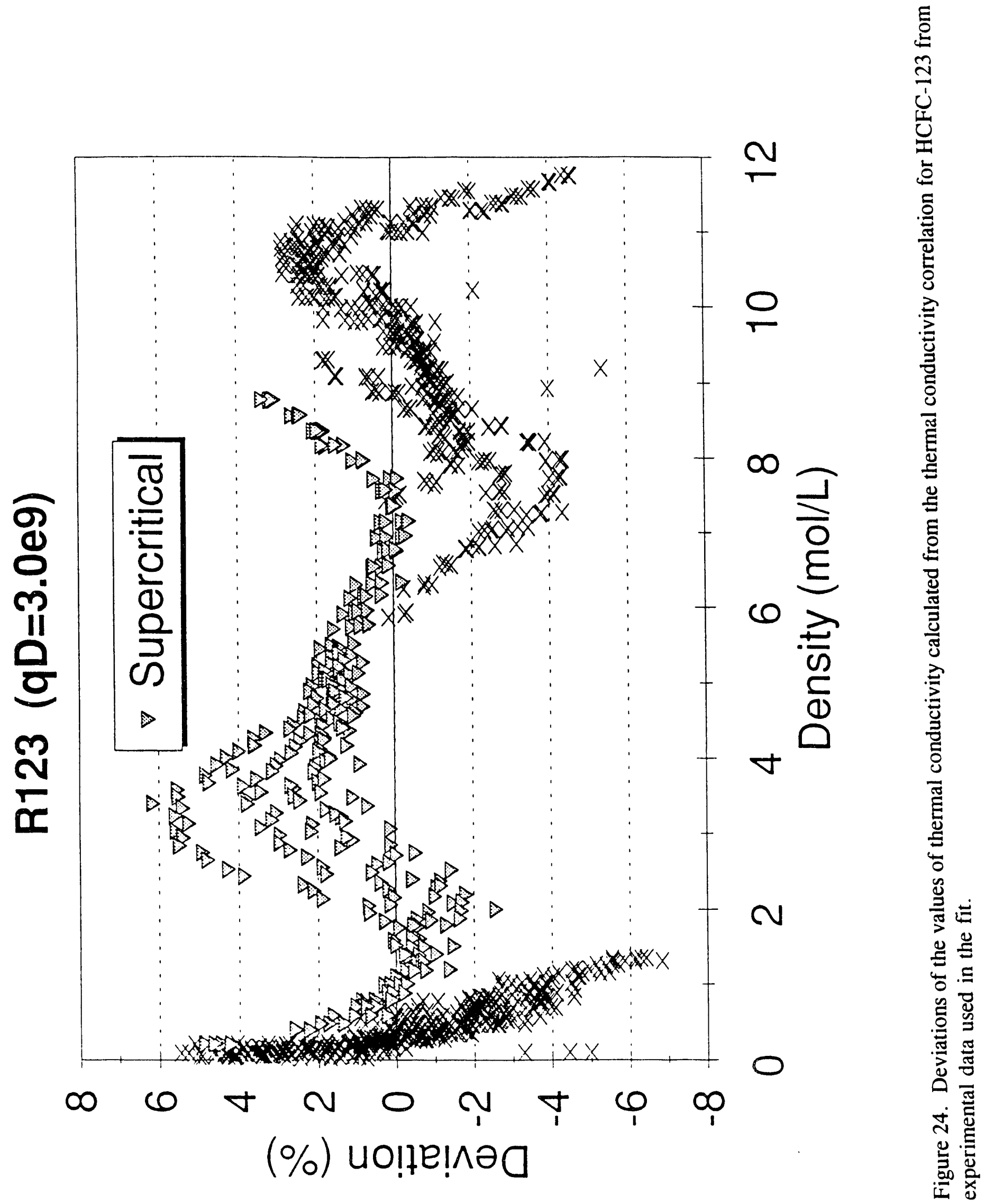
APPENDIX A:

TABLES OF THERMOPHYSICAL PROPERTIES DATA 
Table 1a. Burnett vapor-phase PVT measurements for HFC-32 (SI units)

\begin{tabular}{|c|c|c|c|c|c|c|c|c|}
\hline$T / K$ & $\rho / g / \mathrm{cm}^{3}$ & $\mathrm{P} / \mathrm{kPa}$ & $\mathrm{T} / \mathrm{K}$ & $\rho / \mathrm{g} / \mathrm{cm}^{3}$ & $\mathrm{P} / \mathrm{kPa}$ & $T / K$ & $\rho / g / \mathrm{cm}^{3}$ & $\mathrm{P} / \mathrm{kPa}$ \\
\hline 353.110 & 0.55188 & 6145.1 & 372.446 & 0.07469 & 3509.6 & 268.916 & 0.01325 & 512.1 \\
\hline 358.089 & 0.55176 & 7014.0 & 308.164 & 0.05484 & 1994.0 & 268.834 & 0.01325 & 511.8 \\
\hline 363.132 & 0.55164 & 7921.3 & 313.138 & 0.05483 & 2055.9 & 273.143 & 0.01325 & 522.6 \\
\hline 368.149 & 0.55153 & 8842.1 & 318.131 & 0.05482 & 2116.9 & 278.129 & 0.01325 & 534.9 \\
\hline 373.131 & 0.55141 & 9769.8 & 323.145 & 0.05481 & 2177.3 & 283.164 & 0.01325 & 547.2 \\
\hline 353.111 & 0.30979 & 5930.6 & 328.145 & 0.05480 & 2236.6 & 288.191 & 0.01324 & 559.4 \\
\hline 358.095 & 0.30973 & 6387.2 & 333.148 & 0.05479 & 2295.2 & 293.167 & 0.01324 & 571.3 \\
\hline 363.135 & 0.30966 & 6840.9 & 338.150 & 0.05477 & 2353.1 & 298.122 & 0.01324 & 583.1 \\
\hline 368.142 & 0.30960 & 7286.7 & 343.109 & 0.05476 & 2409.9 & 303.138 & 0.01324 & 594.9 \\
\hline 373.145 & 0.30953 & 7728.8 & 348.136 & 0.05475 & 2466.8 & 308.156 & 0.01323 & 606.7 \\
\hline 343.151 & 0.17391 & 4724.2 & 353.108 & 0.05474 & 25 & .161 & 323 & 618.4 \\
\hline 348.130 & 0.17388 & 4964.3 & 358.138 & 0.05 & .6 & 138 & 0.01323 & 630.0 \\
\hline 353.117 & 0.17384 & 519 & 363.131 & 0.05472 & 8 & 930 & 0.01322 & 641.2 \\
\hline 358.101 & 0.17380 & 542 & 368.139 & 0.05 & 8.7 & .063 & 0.01322 & 653.1 \\
\hline 363.135 & 0.17377 & 5658.7 & 373.141 & 0.05469 & 3.1 & 121 & 0.01322 & 664.7 \\
\hline 368.142 & 0.17373 & 5883.7 & 308.177 & 0.04202 & 1645.0 & .129 & 0.01322 & 676.1 \\
\hline 373.086 & 0.17369 & 6103.1 & 313.168 & 0.04201 & 1689.5 & 343.140 & 0.01321 & 687.5 \\
\hline 338.104 & 0.13323 & 4090.3 & 318.143 & 0.04201 & 1733.2 & 348.155 & 0.01321 & 698.9 \\
\hline 343.118 & 0.13320 & 4265.1 & 323.084 & 0.04200 & 1776.2 & 353.133 & 0.01321 & 710.2 \\
\hline 348.125 & 0.13318 & 4436.5 & 323.135 & 0.04200 & 1776.4 & 358.133 & 0.01321 & 721.4 \\
\hline 353.095 & 0.13315 & 4603.9 & 328.057 & 0.04199 & 1818.8 & 363.170 & 0.01320 & 732.8 \\
\hline 358.084 & 0.13312 & 4769.3 & 333.165 & 0.04198 & 1862.2 & 368.110 & 0.01320 & 743.8 \\
\hline 363.129 & 0.13309 & 4934.4 & 338.134 & 0.04197 & 1904.0 & 268.133 & 0.00744 & 299.7 \\
\hline 363.143 & 0.13309 & 4934.9 & 343.100 & 0.04196 & 1945.3 & 268.530 & 0.00744 & 300.2 \\
\hline 363.119 & 0.13309 & 4934.1 & 348.163 & 0.04195 & 1987.3 & 273.158 & 0.00744 & 306.3 \\
\hline 368.129 & 0.13306 & 5096.1 & 353.127 & 0.04194 & 2028.0 & 278.146 & 0.00744 & 312.8 \\
\hline 373.143 & 0.13304 & 5256.4 & 358.120 & 0.04194 & 2068.6 & 283.161 & 0.00743 & 319.2 \\
\hline 328.222 & 0.09765 & 3255.4 & 363.115 & 0.04193 & 2108.9 & 288.154 & 0.00743 & 325.5 \\
\hline 333.082 & 0.09763 & 3373.7 & 368.109 & 0.04192 & 2149.1 & 293.144 & 0.00743 & 331.9 \\
\hline 338.103 & 0.09761 & 3493.4 & 278.145 & 0.02361 & 882.3 & 298.176 & 0.00743 & 338.3 \\
\hline 343.124 & 0.09759 & 3611.0 & 281.187 & 0.02361 & 897.1 & 303.133 & 0.00743 & 344.6 \\
\hline 348.141 & 0.09757 & 3726.9 & 283.166 & 0.02361 & 907.1 & 308.155 & 0.00743 & 350.9 \\
\hline 353.120 & 0.09755 & 3840.4 & 288.132 & 0.02360 & 930.7 & 313.112 & 0.00742 & 357.2 \\
\hline 358.145 & 0.09753 & 3953.5 & 293.172 & 0.02360 & 954.2 & 318.110 & 0.00742 & 363.4 \\
\hline 363.133 & 0.09751 & 4064.4 & 298.125 & 0.02359 & 977.1 & 323.155 & 0.00742 & 369.8 \\
\hline 368.140 & 0.09749 & 4174.7 & 303.140 & 0.02359 & 1000.0 & 323.130 & 0.00742 & 369.9 \\
\hline 373.142 & 0.09747 & 4283.8 & 308.158 & 0.02358 & 1022.7 & 323.175 & 0.00742 & 369.8 \\
\hline 318.099 & 0.07486 & 2602.6 & 313.101 & 0.02358 & 1044.8 & 323.148 & 0.00742 & 369.8 \\
\hline 323.130 & 0.07484 & 2692.8 & 318.156 & 0.02357 & 1067.2 & 323.150 & 0.00742 & 369.8 \\
\hline 333.119 & 0.07481 & 2866.8 & 323.155 & 0.02357 & 1089.2 & 328.116 & 0.00742 & 376.1 \\
\hline 338.132 & 0.07479 & 2952.1 & 323.186 & 0.02357 & 1089.4 & 333.070 & 0.00742 & 382.3 \\
\hline 343.139 & 0.07478 & 3036.1 & 328.073 & 0.02356 & 1110.4 & 338.080 & 0.00742 & 388.5 \\
\hline 348.159 & 0.07476 & 3119.3 & 333.118 & 0.02356 & 1132.2 & 343.157 & 0.00741 & 394.8 \\
\hline 353.128 & 0.07475 & 3200.7 & 338.128 & 0.02355 & 1154.5 & 348.126 & 0.00741 & 400.9 \\
\hline 358.124 & 0.07473 & 3281.8 & 343.144 & 0.02355 & 1175.4 & 353.146 & 0.00741 & 407.0 \\
\hline 363.121 & 0.07472 & 3361.9 & 348.152 & 0.02354 & 1196.8 & 358.130 & 0.00741 & 413.1 \\
\hline 368.103 & 0.07470 & 3441.3 & 353.130 & 0.02354 & 1217.9 & 363.120 & 0.00741 & 419.3 \\
\hline 372.442 & 0.07469 & 3509.6 & 358.128 & 0.02353 & 1239.0 & 368.109 & 0.00741 & 425.4 \\
\hline
\end{tabular}


Table 1b. Burnett vapor-phase PVT measurements for HFC-32 (PI units)

\begin{tabular}{|c|c|c|c|c|c|c|c|c|}
\hline $\mathrm{T} / \mathrm{F}$ & $\rho / \mathrm{lb} / \mathrm{ft}^{3}$ & P/psia & $\mathrm{T} / \mathrm{F}$ & $\rho / \mathrm{lb} / \mathrm{ft}^{3}$ & $\mathrm{P} / \mathrm{psia}$ & $\mathrm{T} / \mathrm{F}$ & $\rho / \mathrm{lb} / \mathrm{ft}^{3}$ & P/psia \\
\hline 175.928 & 34.452 & 891.27 & 95.025 & 3.424 & 289.21 & 24.379 & 0.827 & 74.27 \\
\hline 184890 & 34.445 & 1017.30 & 103.978 & 3.423 & 298.18 & 24.231 & 0.827 & 74.24 \\
\hline 193.968 & 34.438 & 1148.89 & 112.966 & 3.422 & 307.04 & 31.987 & 0.827 & 75.79 \\
\hline 202.998 & 34.431 & 1282.43 & 121.991 & 3.422 & 315.80 & 40.962 & 0.827 & 77.58 \\
\hline 211.966 & 34.424 & 1416.99 & 130.991 & 3.421 & 324.39 & 50.025 & 0.827 & 79.37 \\
\hline 175.930 & 19.340 & 860.17 & 139.996 & 3.420 & 332.89 & 59.074 & 0.827 & 81.13 \\
\hline 184.901 & 19.336 & 926.39 & 149.000 & 3.419 & 341.29 & 68.031 & 0.827 & 82.86 \\
\hline 193.973 & 19.332 & 992.19 & 157.926 & 3.419 & 349.52 & 76.950 & 0.826 & 84.57 \\
\hline 202.986 & 19.327 & 1056.85 & 166.975 & 3.418 & 357.78 & 85.977 & 0.826 & 86.28 \\
\hline 211.991 & 19.323 & 1120.96 & 175.924 & 3.417 & 365.88 & 95.011 & 0.826 & 88.00 \\
\hline 158.002 & 10.857 & 685.19 & 184.978 & 3.417 & 373.99 & 104.020 & 0.826 & 89.70 \\
\hline 166.964 & 10.855 & 720.02 & 193.966 & 3.416 & 381.99 & 112.978 & 0.826 & 91.37 \\
\hline 175.941 & 10.853 & 754.07 & 202.980 & 3.415 & 389.96 & 121.604 & 0.826 & 93.00 \\
\hline 184.912 & 10.850 & 787.50 & 211.984 & 3.414 & 397.85 & 130.843 & 0.825 & 94.72 \\
\hline 193.973 & 10.848 & 820.72 & 95.0490 & 2.623 & 238.59 & 139.948 & 0.825 & 96.40 \\
\hline 202.986 & 10.846 & 853.35 & 104.032 & 2.623 & 245.04 & 148.962 & 0.825 & 98.06 \\
\hline 211.885 & 10.843 & 885.18 & 112.987 & 2.622 & 251.37 & 157.982 & 0.825 & 99.72 \\
\hline 148.917 & 8.317 & 593.25 & 121.881 & 2.622 & 257.62 & 167.009 & 0.825 & 101.37 \\
\hline 157.942 & 8.316 & 618.61 & 121.973 & 2.622 & 257.65 & 175.969 & 0.825 & 103.00 \\
\hline 166.955 & 8.314 & 643.47 & 130.833 & 2.621 & 263.80 & 184.969 & 0.824 & 104.63 \\
\hline 175.901 & 8.312 & 667.74 & 140.027 & 2.621 & 270.09 & 194.036 & 0.824 & 106.28 \\
\hline 184.881 & 8.310 & 691.73 & 148.971 & 2.620 & 276.15 & 202.928 & 0.824 & 107.88 \\
\hline 193.962 & 8.309 & 715.68 & 157.910 & 2.620 & 282.15 & 22.969 & 0.464 & 43.47 \\
\hline 193.987 & 8.309 & 715.74 & 167.023 & 2.619 & 288.23 & 23.684 & 0.464 & 43.55 \\
\hline 193.944 & 8.309 & 715.63 & 175.959 & 2.618 & 294.14 & 32.014 & 0.464 & 44.43 \\
\hline 202.962 & 8.307 & 739.13 & 184.946 & 2.618 & 300.03 & 40.993 & 0.464 & 45.37 \\
\hline 211.987 & 8.305 & 762.38 & 193.937 & 2.617 & 305.88 & 50.02 & 0.464 & 46.29 \\
\hline 131.130 & 6.096 & 472.16 & 202.926 & 2.617 & 311.70 & 59.007 & 0.464 & 47.21 \\
\hline 139.878 & 6.095 & 489.31 & 40.991 & 1.474 & 127.96 & 67.989 & 0.464 & 48.13 \\
\hline 148.915 & 6.094 & 506.67 & 46.467 & 1.474 & 130.12 & 77.047 & 0.464 & 49.07 \\
\hline 157.953 & 6.092 & 523.74 & 50.029 & 1.474 & 131.56 & 85.969 & 0.464 & 49.97 \\
\hline 166.984 & 6.091 & 540.54 & 58.968 & 1.473 & 134.98 & 95.009 & 0.464 & 50.90 \\
\hline 175.946 & 6.090 & 557.00 & 68.040 & 1.473 & 138.40 & 103.932 & 0.463 & 51.80 \\
\hline 184.991 & 6.088 & 573.40 & 76.955 & 1.473 & 141.71 & 112.928 & 0.463 & 52.71 \\
\hline 193.969 & 6.087 & 589.49 & 85.982 & 1.472 & 145.03 & 122.009 & 0.463 & 53.63 \\
\hline 202.982 & 6.086 & 605.49 & 95.014 & 1.472 & 148.32 & 121.964 & 0.463 & 53.65 \\
\hline 211.986 & 6.085 & 621.31 & 103.912 & 1.472 & 151.53 & 122.045 & 0.463 & 53.64 \\
\hline 112.908 & 4.673 & 377.48 & 113.011 & 1.472 & 154.79 & 121.996 & 0.463 & 53.63 \\
\hline 121.964 & 4.672 & 390.56 & 122.009 & 1.471 & 157.98 & 122.000 & 0.463 & 53.64 \\
\hline 139.944 & 4.67 & 415.79 & 122.065 & 1.471 & 158.00 & 130.939 & 0.463 & 54.55 \\
\hline 148.968 & 4.669 & 428.16 & 130.861 & 1.471 & 161.06 & 139.856 & 0.463 & 55.44 \\
\hline 157.980 & 4.668 & 440.34 & 139.942 & 1.471 & 164.20 & 148.874 & 0.463 & 56.35 \\
\hline 167.016 & 4.667 & 452.41 & 148.960 & 1.470 & 167.44 & 158.013 & 0.463 & 57.26 \\
\hline 175.960 & 4.666 & 464.23 & 157.989 & 1.470 & 170.47 & 166.957 & 0.463 & 58.14 \\
\hline 184.953 & 4.665 & 475.98 & 167.004 & 1.470 & 173.58 & 175.993 & 0.463 & 59.04 \\
\hline 193.948 & 4.664 & 487.61 & 175.964 & 1.469 & 176.64 & 184.964 & 0.463 & 59.92 \\
\hline 202.915 & 4.663 & 499.12 & 184.960 & 1.469 & 179.70 & 193.946 & 0.463 & 60.81 \\
\hline 210.726 & 4.663 & 509.03 & 193.946 & 1.469 & 182.73 & 202.926 & 0.462 & 61.70 \\
\hline
\end{tabular}


Table 2a. Vibrating-tube compressed-liquid-density measurements for HFC-32 (SI units)

\begin{tabular}{|c|c|c|c|c|c|c|c|c|}
\hline$T / K$ & $\rho / g / \mathrm{cm}^{3}$ & $\mathrm{P} / \mathrm{kPa}$ & $\mathrm{T} / \mathrm{K}$ & $\rho / g / \mathrm{cm}^{3}$ & $\mathrm{P} / \mathrm{kPa}$ & $\mathrm{T} / \mathrm{K}$ & $\rho / g / \mathrm{cm}^{3}$ & $\mathrm{P} / \mathrm{kPa}$ \\
\hline 242.593 & 1.1572 & 1999.3 & 259.779 & 1.1113 & 4502.3 & 283.647 & 1.0243 & 2500.3 \\
\hline 242.596 & 1.1583 & 2499.3 & 259.790 & 1.1126 & 5002.9 & 283.647 & 1.0264 & 3001.5 \\
\hline 242.583 & 1.1594 & 3001.3 & 259.799 & 1.1139 & 5503.2 & 283.647 & 1.0286 & 3501.6 \\
\hline 242.584 & 1.1605 & 3501.1 & 259.798 & 1.1152 & 6003.0 & 283.647 & 1.0307 & 4001.3 \\
\hline 242.583 & 1.1616 & 4001.1 & 259.806 & 1.1165 & 6503.9 & 283.643 & 1.0328 & 4502.3 \\
\hline 242.588 & 1.1626 & 4502.0 & 264.488 & 1.0891 & 2000.5 & 283.642 & 1.0348 & 5003.0 \\
\hline 242.536 & 1.1637 & 5002.4 & 264.488 & 1.0906 & 2500.5 & 283.643 & 1.0368 & 5503.4 \\
\hline 242.594 & 1.1647 & 5502.3 & 264.501 & 1.0921 & 3000.9 & 283.643 & 1.0387 & 6003.3 \\
\hline 242.588 & 1.1657 & 6002.1 & 264.507 & 1.0936 & 3501.5 & 283.643 & 1.0406 & 6504.1 \\
\hline 242.592 & 1.1667 & 6503.0 & 264.529 & 1.0950 & 4001.1 & 288.527 & 1.0033 & 2000.6 \\
\hline 246.019 & 1.1470 & 2000.8 & 264.505 & 1.0966 & 4502.0 & 288.530 & 1.0057 & 2500.5 \\
\hline 246.023 & 1.1481 & 2500.0 & 264.520 & 1.0980 & 5002.6 & 288.533 & 1.0081 & 3001.5 \\
\hline 246.033 & 1.1492 & 3000.9 & 264.518 & 1.0994 & 5503.0 & 288.526 & 1.0105 & 3501.5 \\
\hline 246.053 & 1.1503 & 3501.2 & 264.511 & 1.1008 & 6002.9 & 288.529 & 1.0128 & 4001.2 \\
\hline 246.062 & 1.1514 & 4000.9 & 264.524 & 1.1022 & 6503.9 & 288.530 & 1.0151 & 4502.2 \\
\hline 246.064 & 1.1525 & 4502.1 & 269.378 & 1.0728 & 2000.4 & 288.531 & 1.0173 & 5003.0 \\
\hline 246.077 & 1.1535 & 5003.0 & 269.382 & 1.0745 & 2500.8 & 288.530 & 1.0195 & 5503.5 \\
\hline 246.091 & 1.1546 & 5503.3 & 269.382 & 1.0761 & 3000.9 & 288.532 & 1.0217 & 6003.3 \\
\hline 246.108 & 1.1556 & 6003.1 & 269.388 & 1.0778 & 3501.1 & 288.532 & 1.0238 & 6504.2 \\
\hline 246.102 & 1.1567 & 6503.9 & 269.397 & 1.0793 & 4001.2 & 293.459 & 0.9833 & 2000.5 \\
\hline 250.555 & 1.1333 & 2000.6 & 269.398 & 1.0809 & 4502.2 & 293.460 & 0.9861 & 2500.4 \\
\hline 250.570 & 1.1344 & 2500.8 & 269.398 & 1.0825 & 5003.0 & 293.460 & 0.9888 & 3001.5 \\
\hline 250.568 & 1.1356 & 3000.9 & 269.401 & 1.0840 & 5503.1 & 293.459 & 0.9915 & 3501.5 \\
\hline 250.578 & 1.1368 & 3501.2 & 269.394 & 1.0856 & 6003.1 & 293.465 & 0.9941 & 4001.3 \\
\hline 250.581 & 1.1380 & 4001.2 & 269.397 & 1.0871 & 6503.9 & 293.463 & 0.9966 & 4502.2 \\
\hline 250.581 & 1.1392 & 4502.0 & 274.000 & 1.0569 & 2000.4 & 293.462 & 0.9991 & 5003.1 \\
\hline 250.572 & 1.1404 & 5002.9 & 274.003 & 1.0587 & 2500.6 & 293.458 & 1.0015 & 5503.4 \\
\hline 250.582 & 1.1415 & 5503.3 & 274.003 & 1.0605 & 3001.0 & 293.461 & 1.0039 & 6003.3 \\
\hline 250.587 & 1.1426 & 6003.0 & 274.005 & 1.0623 & 3501.6 & 293.461 & 1.0062 & 6504.3 \\
\hline 250.572 & 1.1438 & 6503.9 & 274.009 & 1.0640 & 4001.3 & 298.407 & 0.9622 & 2000.5 \\
\hline 255.116 & 1.1191 & 2000.5 & 274.008 & 1.0657 & 4502.2 & 298.410 & 0.9653 & 2500.4 \\
\hline 255.120 & 1.1204 & 2500.8 & 274.010 & 1.0674 & 5003.0 & 298.419 & 0.9684 & 3001.4 \\
\hline 255.125 & 1.1217 & 3001.1 & 274.005 & 1.0691 & 5503.4 & 298.415 & 0.9714 & 3501.4 \\
\hline 255.130 & 1.1230 & 3501.3 & 274.009 & 1.0707 & 6003.2 & 298.417 & 0.9743 & 4001.4 \\
\hline 255.135 & 1.1242 & 4000.9 & 274.011 & 1.0724 & 6504.0 & 298.419 & 0.9771 & 4502.3 \\
\hline 255.136 & 1.1255 & 4501.9 & 278.813 & 1.0398 & 2000.3 & 298.426 & 0.9799 & 5003.2 \\
\hline 255.141 & 1.1267 & 5002.7 & 278.813 & 1.0419 & 2500.7 & 298.425 & 0.9826 & 5503.4 \\
\hline 255.142 & 1.1280 & 5503.2 & 278.817 & 1.0438 & 3001.0 & 298.427 & 0.9852 & 6003.2 \\
\hline 255.146 & 1.1292 & 6003.2 & 278.828 & 1.0457 & 3501.7 & 303.425 & 0.9428 & 2501.4 \\
\hline 255.133 & 1.1304 & 6504.0 & 278.827 & 1.0476 & 4001.4 & 303.428 & 0.9464 & 3001.4 \\
\hline 259.752 & 1.1045 & 2000.7 & 278.834 & 1.0495 & 4502.2 & 303.425 & 0.9499 & 3501.6 \\
\hline 259.754 & 1.1059 & 2500.8 & 278.836 & 1.0513 & 5002.9 & 303.423 & 0.9532 & 4001.5 \\
\hline 259.766 & 1.1072 & 3001.0 & 278.828 & 1.0532 & 5503.2 & 303.421 & 0.9564 & 4502.4 \\
\hline 259.771 & 1.1086 & 3501.3 & 278.823 & 1.0550 & 6003.0 & 303.420 & 0.9596 & 5003.2 \\
\hline 259.771 & 1.1100 & 4001.1 & 278.816 & 1.0568 & 6504.0 & 303.418 & 0.9626 & 5503.5 \\
\hline
\end{tabular}


Table 2a. Vibrating-tube compressed-liquid-density measurements for HFC-32 (SI units) (continued)

\begin{tabular}{|c|c|c|c|c|c|}
\hline $\mathrm{T} / \mathrm{K}$ & $\rho / g / \mathrm{cm}^{3}$ & $\mathrm{P} / \mathrm{kPa}$ & $T / K$ & $\mathrm{p} / \mathrm{g} / \mathrm{cm}^{3}$ & $\mathrm{P} / \mathrm{kPa}$ \\
\hline 303.347 & 0.9687 & 6504.2 & 337.792 & 0.7594 & 5003.4 \\
\hline 308.304 & 0.9194 & 2501.2 & 337.793 & 0.7724 & 5503.7 \\
\hline 308.288 & 0.9235 & 3001.3 & 337.791 & 0.7835 & 6003.6 \\
\hline 308.254 & 0.9277 & 3501.6 & 337.793 & 0.7933 & 6504.5 \\
\hline 308.253 & 0.9316 & 4001.5 & 347.638 & 0.7057 & 6504.4 \\
\hline 308.255 & 0.9352 & 4502.4 & 347.675 & 0.6524 & 5604.4 \\
\hline 308.262 & 0.9388 & 5003.2 & 347.702 & 0.6673 & 5803.9 \\
\hline 308.269 & 0.9422 & 5503.4 & 347.683 & 0.6801 & 6003.4 \\
\hline 308.267 & 0.9455 & 6003.3 & 347.659 & 0.6912 & 6203.9 \\
\hline 308.248 & 0.9488 & 6504.2 & 347.604 & 0.7009 & 6404.0 \\
\hline 313.123 & 0.8989 & 3001.8 & 347.562 & 0.7057 & 6504.6 \\
\hline 313.118 & 0.9036 & 3501.7 & 348.531 & 0.6059 & 5303.7 \\
\hline 313.127 & 0.9081 & 4001.4 & 348.538 & 0.6104 & 5353.7 \\
\hline 313.131 & 0.9124 & 4502.4 & 348.568 & 0.6073 & 5403.5 \\
\hline 313.134 & 0.9165 & 5003.1 & 348.569 & 0.6143 & 5453.4 \\
\hline 313.144 & 0.9204 & 5503.5 & 348.592 & 0.6204 & 5503.3 \\
\hline 313.156 & 0.9242 & 6003.3 & 348.608 & 0.6260 & 5553.7 \\
\hline 313.148 & 0.9279 & 6504.2 & 348.602 & 0.6317 & 5603.6 \\
\hline 318.251 & 0.8756 & 3502.4 & 348.599 & 0.6370 & 5653.5 \\
\hline 318.255 & 0.8811 & 4001.7 & 348.601 & 0.6420 & 5703.4 \\
\hline 318.255 & 0.8863 & 4502.7 & 348.605 & 0.6466 & 5753.2 \\
\hline 318.255 & 0.8912 & 5003.6 & 348.622 & 0.6509 & 5803.8 \\
\hline 318.253 & 0.8959 & 5503.8 & 348.630 & 0.6547 & 5853.7 \\
\hline 318.253 & 0.9004 & 6003.7 & 348.567 & 0.6592 & 5903.5 \\
\hline 318.252 & 0.9046 & 6504.5 & 348.579 & 0.6632 & 5953.2 \\
\hline 323.108 & 0.8457 & 3502.4 & 348.568 & 0.6668 & 6003.0 \\
\hline 323.111 & 0.8526 & 4001.8 & 348.569 & 0.6701 & 6053.6 \\
\hline 323.111 & 0.8590 & 4502.7 & 348.578 & 0.6731 & 6103.5 \\
\hline 323.110 & 0.8650 & 5003.5 & 348.584 & 0.6760 & 6153.4 \\
\hline 323.109 & 0.8706 & 5503.8 & 348.602 & 0.6786 & 6203.4 \\
\hline 323.111 & 0.8758 & 6003.7 & 348.612 & 0.6812 & 6253.5 \\
\hline 323.112 & 0.8808 & 6504.6 & 348.609 & 0.6839 & 6304.1 \\
\hline 327.961 & 0.8162 & 3802.6 & 348.608 & 0.6864 & 6354.0 \\
\hline 327.962 & 0.8197 & 4001.9 & 348.596 & 0.6890 & 6403.9 \\
\hline 327.963 & 0.8281 & 4502.7 & 348.605 & 0.6914 & 6454.0 \\
\hline 327.964 & 0.8356 & 5003.5 & 348.595 & 0.6937 & 6504.0 \\
\hline 327.964 & 0.8426 & 5503.9 & & & \\
\hline 327.969 & 0.8490 & 6003.7 & & & \\
\hline 327.965 & 0.8550 & 6504.5 & & & \\
\hline 332.789 & 0.7850 & 4202.4 & & & \\
\hline 332.796 & 0.7918 & 4502.8 & & & \\
\hline 332.800 & 0.8020 & 5003.5 & & & \\
\hline 332.800 & 0.8110 & 5503.8 & & & \\
\hline 332.800 & 0.8191 & 6003.6 & & & \\
\hline 332.801 & 0.8266 & 6504.5 & & & \\
\hline 337.789 & 0.7435 & 4503.0 & & & \\
\hline
\end{tabular}


Table 2b. Vibrating-tube compressed-liquid-density measurements for HFC-32 (PI units)

\begin{tabular}{|c|c|c|c|c|c|c|c|c|}
\hline $\mathrm{T} / \mathrm{F}$ & $\rho / \mathrm{lb} / \mathrm{ft}^{3}$ & $\mathrm{P} / \mathrm{psia}$ & $T / F$ & $\rho / l b / \mathrm{ft}^{3}$ & P/psia & $\mathrm{T} / \mathrm{F}$ & $\rho / l b / f^{3}$ & P/psia \\
\hline-23.002 & 72.242 & 289.97 & 7.933 & 69.377 & ús3.00 & 50.895 & 63.942 & 362.64 \\
\hline-22.997 & 72.311 & 362.50 & 7.952 & 69.459 & 725.61 & 50.896 & 64.079 & 435.33 \\
\hline-23.021 & 72.382 & 435.31 & 7.969 & 69.541 & 798.17 & 50.895 & 64.212 & 507.86 \\
\hline-23.018 & 72.448 & 507.79 & 7.966 & 69.623 & 870.66 & 50.895 & 64.343 & 580.34 \\
\hline-23.021 & 72.515 & 580.30 & 7.981 & 69.702 & 943.32 & 50.887 & 64.473 & 653.00 \\
\hline-23.011 & 72.580 & 652.97 & 16.408 & 67.992 & 290.15 & 50.886 & 64.599 & 725.62 \\
\hline-23.015 & 72.646 & 725.53 & 16.408 & 68.087 & 362.67 & 50.888 & 64.723 & 798.19 \\
\hline-23.001 & 72.709 & 798.04 & 16.433 & 68.179 & 435.24 & 50.887 & 64.844 & 870.70 \\
\hline-23.012 & 72.774 & 870.53 & 16.443 & 68.272 & 507.85 & 50.888 & 64.964 & 943.35 \\
\hline-23.004 & 72.838 & 943.19 & 16.482 & 68.361 & 580.31 & 59.679 & 62.632 & 290.17 \\
\hline-16.836 & 71.605 & 290.20 & 16.439 & 68.456 & 652.96 & 59.685 & 62.786 & 362.66 \\
\hline-16.829 & 71. & 362.59 & 16.467 & 68.544 & 725.56 & 59.690 & 62.937 & 435.33 \\
\hline-16.810 & 71. & 435.25 & 16.463 & 68.633 & 798.14 & 59.677 & 63.086 & 507.85 \\
\hline-16.774 & 71. & 507.81 & 16.450 & 68.722 & 870.65 & 59.683 & 63.230 & 580.33 \\
\hline-16.759 & 71.878 & 580.28 & 16.473 & 68.807 & 943.31 & 59.684 & 63.372 & 652.99 \\
\hline-16.754 & 71.947 & 652.98 & 25.211 & 66.975 & 290.13 & 59.686 & 63.511 & 725.63 \\
\hline-16.730 & 72.014 & 725.62 & 25.218 & 67.078 & 362.70 & 59.684 & 63.647 & 798.22 \\
\hline-16.706 & 72.079 & 798.18 & 25.217 & 67.181 & 435.25 & 59.688 & 63.780 & 870.71 \\
\hline-16.675 & 72.144 & 870.68 & 25.227 & 67.282 & 507.79 & 59.687 & 63.911 & 943.36 \\
\hline-16.686 & 72.211 & 943.31 & 25.245 & 67.381 & 580.32 & 68.556 & 61.388 & 290.14 \\
\hline-8.672 & 70.747 & 290.17 & 25.247 & 67.479 & 652.98 & 68.558 & 61.561 & 362.65 \\
\hline-8.645 & 70.819 & 62.70 & 25.246 & 67.577 & 725.63 & 68.558 & 61.731 & 435.33 \\
\hline-8.647 & 70.896 & 435.24 & 25.251 & 67.674 & 798.15 & 68.557 & 61.897 & 507.85 \\
\hline-8.629 & 70.969 & 507.80 & 25.239 & 67.770 & 870.68 & 68.567 & 62.058 & 580.34 \\
\hline-8.623 & 71.043 & 580.33 & 25.244 & 67.864 & 943.31 & 68.564 & 62.216 & 653.00 \\
\hline-8.625 & 71.117 & 652.96 & 33.529 & 65.977 & 290.13 & 68.562 & 62.371 & 725.63 \\
\hline-8.640 & 71.191 & 725.61 & 33.536 & 66.091 & 362.69 & 68.555 & 62.522 & 798.21 \\
\hline-8.623 & 71.261 & 798.18 & 33.536 & 66.204 & 435.26 & 68.560 & 62.669 & 870.71 \\
\hline-8.613 & 71.332 & 870.67 & 33.538 & 66.314 & 507.87 & 68.561 & 62.814 & 943.36 \\
\hline-8.640 & 71.405 & 943.32 & 33.546 & 66.423 & 580.34 & 77.463 & 60.066 & 290.14 \\
\hline-0.461 & 69.865 & 290.14 & 33.544 & 66.530 & 652.99 & 77.467 & 60.264 & 362.66 \\
\hline-0.454 & 69.946 & 362.71 & 33.549 & 66.636 & 725.63 & 77.484 & 60.455 & 435.32 \\
\hline-0.445 & 70.026 & 435.28 & 33.539 & 66.741 & 798.20 & 77.477 & 60.643 & 507.84 \\
\hline-0.436 & 70.106 & 507.81 & 33.545 & 66.844 & 870.69 & 77.481 & 60.824 & 580.35 \\
\hline-0.428 & 70.184 & 580.28 & 33.550 & 66.945 & 943.33 & 77.484 & 61.001 & 653.01 \\
\hline-0.425 & 70.263 & 652.95 & 42.193 & 64.915 & 290.12 & 77.497 & 61.172 & 725.65 \\
\hline-0.416 & 70.340 & 725.58 & 42.194 & 65.041 & 362.70 & 77.495 & 61.340 & 798.20 \\
\hline-0.414 & 70.417 & 798.17 & 42.200 & 65.163 & 435.26 & 77.498 & 61.504 & 870.70 \\
\hline-0.407 & 70.493 & 870.69 & 42.220 & 65.282 & 507.87 & 86.495 & 58.858 & 362.80 \\
\hline-0.431 & 70.570 & 943.32 & 42.219 & 65.401 & 580.35 & 86.500 & 59.081 & 435.32 \\
\hline 7.884 & 68.950 & 290.18 & 42.231 & 65.516 & 652.98 & 86.495 & 59.298 & 507.87 \\
\hline 7.886 & 69.037 & 362.71 & 42.234 & 65.632 & 725.61 & 86.491 & 59.506 & 580.37 \\
\hline 7.909 & 69.123 & 435.26 & 42.221 & 65.746 & 798.17 & 86.488 & 59.707 & 653.02 \\
\hline 7.918 & 69.208 & 507.82 & 42.211 & 65.859 & 870.67 & 86.486 & 59.503 & 725.65 \\
\hline 7.917 & 69.294 & 580.31 & 42.198 & 65.971 & 943.33 & 86.483 & 60.093 & 798.22 \\
\hline
\end{tabular}


Table 2b. Vibrating-tube compressed-liquid-density measurements for HFC-32 (PI units) (continued)

\begin{tabular}{|c|c|c|c|c|c|}
\hline$T / F$ & $\rho / \mathrm{lb} / \mathrm{ft}^{3}$ & $\mathrm{P} / \mathrm{psia}$ & $T / F$ & $\mathrm{p} / \mathrm{lb} / \mathrm{f}^{-}$ & $\mathrm{P} / \mathrm{psia}$ \\
\hline 86.355 & 60.473 & 943.36 & 148.356 & 47.407 & 725.68 \\
\hline 95.277 & 57.393 & 362.77 & 148.358 & 48.219 & 798.25 \\
\hline 95.248 & 57.655 & 435.31 & 148.354 & 48.912 & 870.75 \\
\hline 95.186 & 57.912 & 507.86 & 148.357 & 49.522 & 943.39 \\
\hline 95.185 & 58.155 & 580.37 & 166.079 & 44.053 & 943.39 \\
\hline 95.189 & 58.385 & 653.02 & 166.145 & 40.729 & 812.84 \\
\hline 95.200 & 58.606 & 725.65 & 166.193 & 41.658 & 841.78 \\
\hline 95.214 & 58.819 & 798.21 & 166.159 & 42.459 & 870.71 \\
\hline 95.211 & 59.026 & 870.71 & 166.117 & 43.149 & 899.80 \\
\hline 95.177 & 59.234 & 943.36 & 166.017 & 43.758 & 928.82 \\
\hline 103.952 & 56.115 & 435.37 & 165.942 & 44.055 & 943.41 \\
\hline 103.943 & 56.410 & 507.87 & 167.685 & 37.824 & 769.24 \\
\hline 103.958 & 56.690 & 580.35 & 167.698 & 38.108 & 776.48 \\
\hline 103.966 & 56.958 & 653.01 & 167.753 & 37.912 & 783.71 \\
\hline 103.971 & 57.215 & 725.65 & 167.755 & 38.349 & 790.96 \\
\hline 103.989 & 57.459 & 798.22 & 167.796 & 38.727 & 798.18 \\
\hline 104.012 & 57.694 & 870.71 & 167.825 & 39.079 & 805.50 \\
\hline 103.997 & 57.924 & 943.36 & 167.814 & 39.434 & 812.74 \\
\hline 113.183 & 54.664 & 507.97 & 167.809 & 39.768 & 819.97 \\
\hline 113.190 & 55.006 & 580.40 & 167.812 & 40.078 & 827.20 \\
\hline 113.190 & 55.330 & 653.06 & 167.820 & 40.368 & 834.43 \\
\hline 113.189 & 55.637 & 725.71 & 167.850 & 40.632 & 841.77 \\
\hline 113.186 & 55.929 & 798.26 & 167.864 & 40.869 & 849.00 \\
\hline 113.186 & 56.208 & 870.77 & 167.751 & 41.156 & 856.23 \\
\hline 113.183 & 56.474 & 943.40 & 167.772 & 41.401 & 863.44 \\
\hline 121.924 & 52.793 & 507.98 & 167.752 & 41.627 & 870.66 \\
\hline 121.929 & 53.224 & 580.41 & 167.754 & 41.832 & 878.00 \\
\hline 121.929 & 53.624 & 653.07 & 167.770 & 42.019 & 885.23 \\
\hline 121.928 & 53.997 & 725.70 & 167.781 & 42.203 & 892.47 \\
\hline 121.927 & 54.347 & 798.26 & 167.313 & 42.363 & 899.73 \\
\hline 121.930 & 54.676 & 870.76 & 167.831 & 42.525 & 906.99 \\
\hline 121.931 & 54.988 & 943.41 & 167.827 & 42.693 & 914.34 \\
\hline 130.659 & 50.954 & 551.52 & 167.824 & 42.850 & 921.57 \\
\hline 130.662 & 51.175 & 580.42 & 167.803 & 43.014 & 928.81 \\
\hline 130.663 & 51.695 & 653.06 & 167.819 & 43.160 & 936.07 \\
\hline 130.665 & 52.166 & 725.70 & 167.802 & 43.308 & 943.32 \\
\hline 130.665 & 52.599 & 798.27 & & & \\
\hline 130.673 & 52.999 & 870.76 & & & \\
\hline 130.667 & 53.375 & 943.40 & & & \\
\hline 139.351 & 49.006 & 609.50 & & & \\
\hline 139.362 & 49.429 & 653.07 & & & \\
\hline 139.371 & 50.065 & 725.69 & & & \\
\hline 139.369 & 50.628 & 798.26 & & & \\
\hline 139.370 & 51.135 & 870.75 & & & \\
\hline 139.371 & 51.600 & 943.40 & & & \\
\hline 148.350 & 46.414 & 653.11 & & & \\
\hline
\end{tabular}


Table 3a. Liquid PVT Data (Isochoric Apparatus) for HFC-32 1/4

\section{Temperature K}

139.999

142.000

144.000

145.999

148.000

149.999

151.999

151.999

153.999

155.999

158.000

159.999

161.998

163.999

160.000

161.999

164.000

166.000

168.000

169.999

171.999

170.000

172.000

174.000

175.999

177.999

180.000

181.999

183.999

180.999

181.999

183.999

186.000

187.999

190.000

192.000

194.000

196.000

198.000

195.999

198.001

199.999

202.000
Pressure $\mathrm{MPa}$

3.599795

5.493646

10.929410

16.440620

21.961470

27.462020

32.947380

3.871711

5.964355

10.954690

16.001890

21.048190

26.088490

31.102130

4.299190

8.984807

13.772330

18.566270

23.358450

28.138910

32.886910

3.822186

5.465584

9.802387

14.187370

18.583860

22.973660

27.341580

31.697920

2.610451

3.896742

5.946145

9.919708

13.930580

17.946990

21.951480

25.945820

29.920680

33.885620

3.870242

5.686683

9.252136

12.849070
Density
mol $\cdot \mathrm{dm}^{-3}$

27.34983

27.28596

27.27603

27.26752

27.25969

27.25223

27.24504

26.80698

26.74380

26.73466

26.72670

26.71929

26.71226

26.70546

26.43650

26.42690

26.41888

26.41154

26.40461

26.39793

26.39142

25.96166

25.89905

25.89055

25.88314

25.87628

25.86975

25.86345

25.85728

25.41364

25.40689

25.34396

25.33614

25.32920

25.32273

25.31655

25.31054

25.30471

25.29898

24.73041

24.66777

24.66041

24.65383 
Table 3a. Liquid PVT Data (Isochoric Apparatus) for HFC-32 2/4

$\underset{\mathrm{K}}{\text { Temperature }}$

$$
\begin{aligned}
& 203.999 \\
& 206.000 \\
& 208.000 \\
& 210.000 \\
& 212.001 \\
& 213.998 \\
& 209.999 \\
& 212.000 \\
& 214.002 \\
& 216.000 \\
& 217.999 \\
& 220.000 \\
& 222.001 \\
& 224.000 \\
& 226.000 \\
& 228.000 \\
& 224.000 \\
& 226.002 \\
& 228.001 \\
& 229.999 \\
& 232.000 \\
& 234.000 \\
& 235.998 \\
& 238.000 \\
& 239.999 \\
& 242.000 \\
& 244.002 \\
& 236.999 \\
& 238.002 \\
& 239.999 \\
& 242.001 \\
& 243.999 \\
& 246.000 \\
& 248.000 \\
& 250.001 \\
& 251.999 \\
& 254.000 \\
& 256.000 \\
& 257.999 \\
& 260.001 \\
& 262.000 \\
&
\end{aligned}
$$

251.999

254.002

256.000
Pressure MPa

16.440610

20.034010

23.610620

27.177690

30.734910

34.276340

4.247910

7.448832

10.694400

13.934370

17.181560

20.423960

23.653610

26.877640

30.092390

33.291480

4.227700

7.088737

9.979462

12.871100

15.764870

18.655440

21.537230

24.418200

27.288600

30.152620

33.008000

3. 886813

4.141327

6.679945

9.248424

11.818840

14.382920

16.951710

19.515250

22.070080

24.623070

27.169940

29.711770

32.247880

34.774180

3.937722

5.883227

8.135592
Density
mol $\cdot \mathrm{dm}^{-3}$

24.64769
24.64184
24.63616
24.63064
24.62522
24.61990

24.03935

24.03186

24.02541

24.01945

24.01380

24.00835

24.00305

23.99786

23.99272

23.98769

23.32497

23.31799

23.31192

23.30631

23.30095

23.29579

23.29078

23.28585

23.28099

23.27621

23. 27148

22.63185

22.57333

22.56679

22.56107

22.55577

22.55073

22.54584

22.54109

22.53641

22.53182

22.52731

22.52281

22.51836

22.51396

21.77402

21.76836

21.76291 
Table 3a. Liquid PVT Data (Isochoric Apparatus) for HFC-32 3/4

\begin{tabular}{|c|c|c|}
\hline$\underset{\mathrm{K}}{\mathrm{T}}$ & $\begin{array}{l}\text { Pressure } \\
\text { MPa }\end{array}$ & $\begin{array}{l}\text { Density } \\
\text { mol } \cdot \mathrm{dm}^{-3}\end{array}$ \\
\hline $\begin{array}{l}258.002 \\
260.001 \\
262.000 \\
263.999 \\
266.000 \\
268.000 \\
270.001 \\
272.000 \\
274.001 \\
276.000 \\
277.999 \\
279.998\end{array}$ & $\begin{array}{l}10.396070 \\
12.657880 \\
14.918920 \\
17.171580 \\
19.427090 \\
21.677810 \\
23.922150 \\
26.161900 \\
28.400710 \\
30.632190 \\
32.859150 \\
35.076280\end{array}$ & $\begin{array}{l}21.75786 \\
21.75305 \\
21.74842 \\
21.74392 \\
21.73949 \\
21.73514 \\
21.73085 \\
21.72661 \\
21.72237 \\
21.71820 \\
21.71405 \\
21.70992\end{array}$ \\
\hline $\begin{array}{l}269.998 \\
273.998 \\
277.998 \\
282.000 \\
285.999 \\
290.001 \\
294.000 \\
297.999 \\
302.001\end{array}$ & $\begin{array}{r}3.891322 \\
6.888521 \\
10.665470 \\
14.445850 \\
18.214180 \\
21.980450 \\
25.732000 \\
29.473310 \\
33.200290\end{array}$ & $\begin{array}{l}20.70803 \\
20.64186 \\
20.63267 \\
20.62416 \\
20.61601 \\
20.60807 \\
20.60029 \\
20.59259 \\
20.58497\end{array}$ \\
\hline $\begin{array}{l}285.999 \\
290.001 \\
294.000 \\
298.001 \\
302.002 \\
306.002 \\
310.000 \\
314.001 \\
318.001 \\
321.999\end{array}$ & $\begin{array}{r}3.956490 \\
7.118600 \\
10.329430 \\
13.545260 \\
16.761990 \\
19.973720 \\
23.179760 \\
26.368710 \\
29.570930 \\
32.748360\end{array}$ & $\begin{array}{l}19.64137 \\
19.63194 \\
19.62361 \\
19.61576 \\
19.60821 \\
19.60082 \\
19.59358 \\
19.58659 \\
19.57926 \\
19.57225\end{array}$ \\
\hline $\begin{array}{l}309.999 \\
313.999 \\
317.999 \\
322.001 \\
326.001 \\
329.999 \\
334.001 \\
338.001 \\
342.002 \\
346.001 \\
350.001 \\
354.000 \\
358.000\end{array}$ & $\begin{array}{r}5.248634 \\
7.672132 \\
10.108430 \\
12.550710 \\
14.991910 \\
17.439090 \\
19.887430 \\
22.331830 \\
24.776830 \\
27.217430 \\
29.641780 \\
32.072860 \\
34.505350\end{array}$ & $\begin{array}{l}17.92306 \\
17.91547 \\
17.90840 \\
17.90160 \\
17.89498 \\
17.88849 \\
17.88205 \\
17.87572 \\
17.86935 \\
17.86303 \\
17.85696 \\
17.85112 \\
17.84499\end{array}$ \\
\hline
\end{tabular}


Table 3a. Liquid PVT Data (Isochoric Apparatus) for HFC-32 4/4

$\underset{\mathrm{K}}{\text { Temperature }}$

348.002
351.999
356.001
360.000
364.000
368.000
372.000
376.001
379.999
384.001
388.000
392.000
395.999
Pressure $\mathrm{MPa}$

6.815193

8.001572

9.202769

10.415380

11.637560

12.867150

14.103340

15.345020

16.591050

17.841610

19.094890

20.350410

21.606800
Density

13.66408

13.65927

13.65454

13.64985

13.64516

13.64053

13.63592

13.63132

13.62673

13.62213

13.61754

13.61291

13.60831 
Table 3b. Liquid PVT Data (Isochoric Apparatus) for HFC-32 1/4

\begin{tabular}{|c|c|c|}
\hline $\begin{array}{c}\text { Temperature } \\
{ }^{\circ} \mathrm{F}\end{array}$ & $\begin{array}{l}\text { Pressure } \\
\text { psia }\end{array}$ & $\begin{array}{c}\text { Density } \\
\mathrm{lb} \cdot \mathrm{ft} \mathrm{t}^{-3}\end{array}$ \\
\hline $\begin{array}{l}-207.67 \\
-204.07 \\
-200.47 \\
-196.87 \\
-193.27 \\
-189.67 \\
-186.07\end{array}$ & $\begin{array}{r}522.107 \\
796.787 \\
1585.180 \\
2384.515 \\
3185.248 \\
3983.036 \\
4778.622\end{array}$ & $\begin{array}{l}88.825 \\
88.618 \\
88.585 \\
88.558 \\
88.532 \\
88.508 \\
88.485\end{array}$ \\
\hline $\begin{array}{l}-186.07 \\
-182.47 \\
-178.87 \\
-175.27 \\
-171.67 \\
-168.07 \\
-164.47\end{array}$ & $\begin{array}{r}561.545 \\
865.058 \\
1588.846 \\
2320.882 \\
3052.787 \\
3783.822 \\
4510.991\end{array}$ & $\begin{array}{l}87.062 \\
86.857 \\
86.827 \\
86.801 \\
86.777 \\
86.754 \\
86.732\end{array}$ \\
\hline $\begin{array}{l}-171.67 \\
-168.07 \\
-164.47 \\
-160.87 \\
-157.27 \\
-153.67 \\
-150.07\end{array}$ & $\begin{array}{r}623.546 \\
1303.138 \\
1997.511 \\
2692.815 \\
3387.863 \\
4081.211 \\
4769.851\end{array}$ & $\begin{array}{l}85.859 \\
85.828 \\
85.801 \\
85.778 \\
85.755 \\
85.733 \\
85.712\end{array}$ \\
\hline $\begin{array}{l}-153.67 \\
-150.07 \\
-146.47 \\
-142.87 \\
-139.27 \\
-135.67 \\
-132.07 \\
-128.47\end{array}$ & $\begin{array}{r}554.362 \\
792.717 \\
1421.719 \\
2057.708 \\
2695.366 \\
3332.054 \\
3965.568 \\
4597.403\end{array}$ & $\begin{array}{l}84.317 \\
84.113 \\
84.086 \\
84.062 \\
84.039 \\
84.018 \\
83.998 \\
83.978\end{array}$ \\
\hline $\begin{array}{l}-133.87 \\
-132.07 \\
-128.47 \\
-124.87 \\
-121.27 \\
-117.67 \\
-114.07 \\
-110.47 \\
-106.87 \\
-103.27\end{array}$ & $\begin{array}{r}378.615 \\
565.176 \\
862.417 \\
1438.735 \\
2020.463 \\
2602.995 \\
3183.799 \\
3763.130 \\
4339.635 \\
4914.702\end{array}$ & $\begin{array}{l}82.537 \\
82.515 \\
82.310 \\
82.285 \\
82.262 \\
82.241 \\
82.221 \\
82.202 \\
82.183 \\
82.164\end{array}$ \\
\hline $\begin{array}{r}-106.87 \\
-103.27 \\
-99.67 \\
-96.07\end{array}$ & $\begin{array}{r}561.332 \\
824.785 \\
1341.911 \\
1863.603\end{array}$ & $\begin{array}{l}80.318 \\
80.114 \\
80.090 \\
80.069\end{array}$ \\
\hline
\end{tabular}


Table 3b. Liquid PVT Data (Isochoric Apparatus) for HFC-32 2/4

Temperature

${ }^{\circ} \mathrm{F}$

$-92.47$

$-88.87$

$-85.27$

$-81.67$

$-78.07$

$-74.47$

$-81.67$

$-78.07$

$-74.47$

$-70.87$

$-67.27$

$-63.67$

$-60.07$

$-56.47$

$-52.87$

$-49.27$

$-56.47$

$-52.87$

$-49.27$

$-45.67$

$-42.07$

$-38.47$

$-34.87$

$-31.27$

$-27.67$

$-24.07$

$-20.47$

$-33.07$

$-31.27$

$-27.67$

$-24.07$

$-20.47$

$-16.87$

$-13.27$

$-9.67$

$-6.07$

$-2.47$

1.13

4.73

8.33

11.93

$-6.07$

$-2.47$

1.13

4.73
Pressure psia

2384.513

2905.693

3424.437

3941.798

4457.730

4971.372

616.108

1080.364

1551.094

2021.013

2491.979

2962.250

3430.672

3898.279

4364.540

4828.529

613.177

1028.136

1447.401

1866.799

2286.505

2705.748

3123.717

3541.567

3957.884

4373.276

4787.414

563.736

600.650

968.846

1341.373

1714.181

2086.070

2458.642

2830.453

3201.000

3571.281

3940.674

4309.336

4677.168

5043.577

$$
\begin{array}{r}
571.119 \\
853.291 \\
1179.970 \\
1507.825
\end{array}
$$

Density
Ib $\mathrm{ft}^{-3}$

80.049

80.030

80.012

79.994

79.976

79.959

78.073

78.049

78.028

78.009

77.990

77.973

77.956

77.939

77.922

77.906

75.753

75.731

75.711

75.693

75.675

75.659

75.642

75.626

75.610

75.595

75.580

73.502

73.312

73.291

73.272

73.255

73.239

73.223

73.207

73.192

73.177

73.163

73.148

73.134

73.119

70.716

70.698

70.680

70.664 
Table 3b. Liquid PVT Data (Isochoric Apparatus) for HFC-32 3/4

\begin{tabular}{|c|c|c|}
\hline $\begin{array}{c}\text { Temperature } \\
{ }^{\circ} \mathrm{F}\end{array}$ & $\begin{array}{c}\text { Pressure } \\
\text { psia }\end{array}$ & $\begin{array}{l}\text { Density } \\
1 b \cdot f t^{-3}\end{array}$ \\
\hline $\begin{array}{l}8.33 \\
11.93 \\
15.53 \\
19.13 \\
22.73 \\
26.33 \\
29.93 \\
33.53 \\
37.13 \\
40.73 \\
44.33 \\
26.33 \\
33.53 \\
40.73 \\
47.93 \\
55.13 \\
62.33 \\
69.53 \\
76.73 \\
83.93\end{array}$ & $\begin{array}{r}1835.874 \\
2163.810 \\
2490.532 \\
2817.666 \\
3144.106 \\
3469.621 \\
3794.470 \\
4119.182 \\
4442.831 \\
4765.825 \\
5087.393 \\
564.390 \\
999.097 \\
1546.898 \\
2095.197 \\
2641.748 \\
3188.000 \\
3732.118 \\
4274.750 \\
4815.303\end{array}$ & $\begin{array}{l}70.648 \\
70.633 \\
70.618 \\
70.604 \\
70.590 \\
70.576 \\
70.562 \\
70.548 \\
70.535 \\
70.521 \\
70.508 \\
67.254 \\
67.039 \\
67.009 \\
66.982 \\
66.955 \\
66.930 \\
66.904 \\
66.879 \\
66.854\end{array}$ \\
\hline $\begin{array}{r}55.13 \\
62.33 \\
69.53 \\
76.73 \\
83.93 \\
91.13 \\
98.33 \\
105.53 \\
112.73 \\
119.93\end{array}$ & $\begin{array}{r}573.841 \\
1032.467 \\
1498.160 \\
1964.577 \\
2431.125 \\
2896.948 \\
3361.946 \\
3824.465 \\
4288.908 \\
4749.756\end{array}$ & $\begin{array}{l}63.790 \\
63.759 \\
63.732 \\
63.707 \\
63.682 \\
63.658 \\
63.635 \\
63.612 \\
63.588 \\
63.565\end{array}$ \\
\hline $\begin{array}{r}98.33 \\
105.53 \\
112.73 \\
119.93 \\
127.13 \\
134.33 \\
141.53 \\
148.73 \\
155.93 \\
163.13 \\
170.33 \\
177.53 \\
184.73\end{array}$ & $\begin{array}{r}761.251 \\
1112.751 \\
1466.106 \\
1820.330 \\
2174.397 \\
2529.331 \\
2884.433 \\
3238.964 \\
3593.582 \\
3947.561 \\
4299.184 \\
4651.783 \\
5004.587\end{array}$ & $\begin{array}{l}58.209 \\
58.185 \\
58.162 \\
58.140 \\
58.118 \\
58.097 \\
58.076 \\
58.056 \\
58.035 \\
58.014 \\
57.995 \\
57.976 \\
57.956\end{array}$ \\
\hline $\begin{array}{l}166.73 \\
173.93\end{array}$ & $\begin{array}{r}988.462 \\
1160.532\end{array}$ & $\begin{array}{l}44.377 \\
44.362\end{array}$ \\
\hline
\end{tabular}




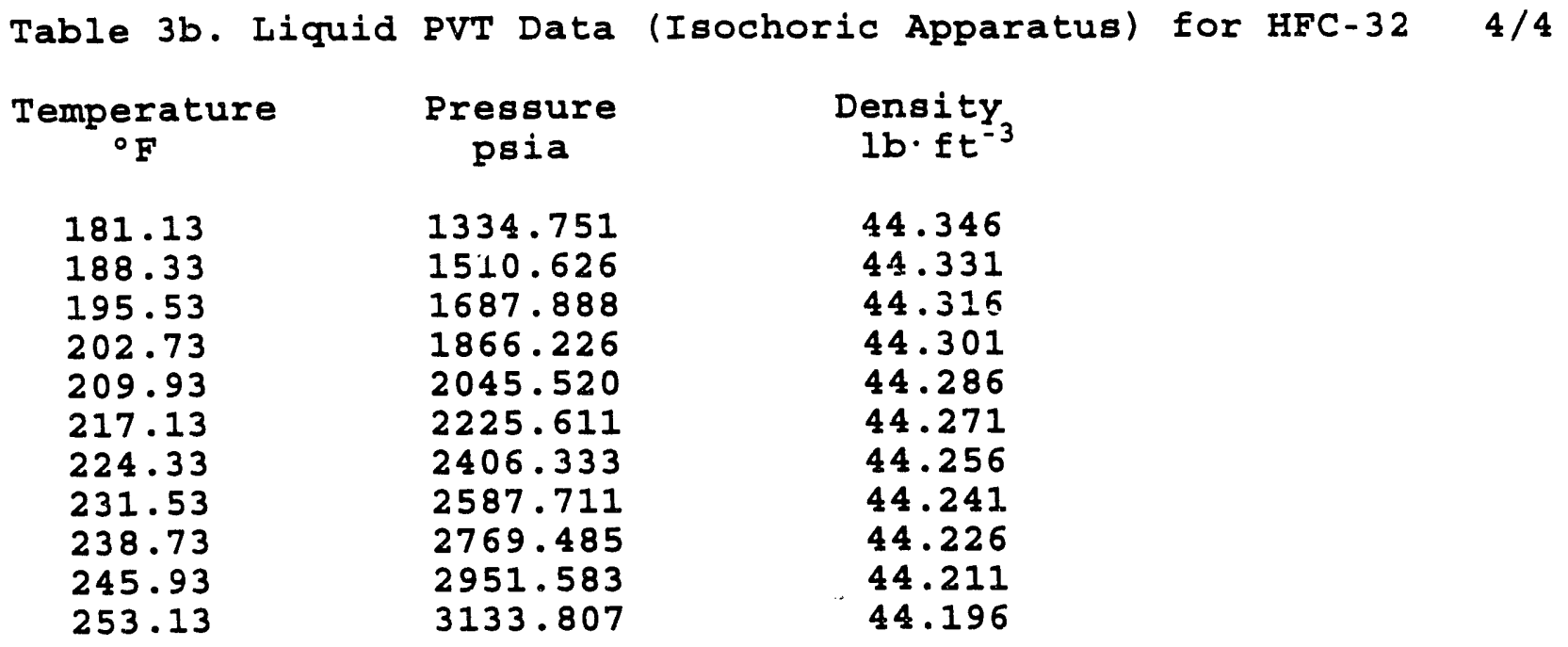


Table 4. Vapor pressures of HFC-32 obtained using the NIST Burnett apparatus

$\begin{array}{lccc}\mathrm{T} / \mathrm{K} & \mathrm{P} / \mathrm{kPa} & \mathrm{T} / \mathrm{F} & \mathrm{P} / \mathrm{psia} \\ 268.154 & 690.56 & 23.007 & 100.15 \\ 273.163 & 813.62 & 32.023 & 118.00 \\ 278.137 & 951.22 & 40.976 & 137.96 \\ 283.184 & 1108.22 & 50.061 & 160.73 \\ 288.129 & 1280.21 & 58.962 & 185.67 \\ 293.121 & 1473.50 & 67.947 & 213.71 \\ 298.174 & 1690.81 & 77.043 & 245.23 \\ 303.122 & 1926.26 & 85.949 & 279.38 \\ 308.143 & 2189.50 & 94.987 & 317.56 \\ 313.122 & 2476.63 & 103.949 & 359.20 \\ 318.154 & 2794.65 & 113.007 & 405.32 \\ 318.169 & 2795.42 & 113.034 & 405.44 \\ 323.161 & 3140.65 & 122.019 & 455.51 \\ 328.202 & 3522.52 & 131.093 & 510.89 \\ 333.105 & 3927.61 & 139.919 & 569.65 \\ 338.150 & 4382.59 & 149.000 & 635.64 \\ 343.110 & 4871.98 & 157.928 & 706.62 \\ 348.081 & 5409.01 & 166.875 & 784.51\end{array}$


Table 5. Vapor pressures of HFC-32 obtained using the NIST ebulliometer

$\begin{array}{llll}\mathrm{T} / \mathrm{K} & \mathrm{P} / \mathrm{kPa} & \mathrm{T} / \mathrm{F} & \mathrm{P} / \mathrm{psia} \\ 208.357 & & & \\ 208.650 & 49.434 & -84.627 & 7.170 \\ 209.314 & 50.260 & -84.100 & 7.290 \\ 209.933 & 52.312 & -82.904 & 7.587 \\ 210.318 & 54.206 & -81.790 & 7.862 \\ 210.350 & 55.400 & -81.097 & 8.035 \\ 211.812 & 55.530 & -81.039 & 8.054 \\ 213.306 & 60.305 & -78.408 & 8.747 \\ 214.697 & 65.582 & -75.719 & 9.512 \\ 215.477 & 70.762 & -73.215 & 10.263 \\ 217.018 & 73.835 & -71.811 & 10.709 \\ 217.067 & 80.287 & -69.037 & 11.645 \\ 218.323 & 80.504 & -68.949 & 11.676 \\ 219.303 & 85.961 & -66.688 & 12.468 \\ 220.256 & 90.560 & -64.924 & 13.135 \\ 221.371 & 95.099 & -63.209 & 13.793 \\ 223.331 & 100.691 & -61.202 & 14.604 \\ 224.944 & 111.147 & -57.674 & 16.121 \\ 226.732 & 120.413 & -54.770 & 17.464 \\ 228.109 & 131.412 & -51.552 & 19.060 \\ 229.618 & 140.395 & -49.073 & 20.363 \\ 231.030 & 150.769 & -46.357 & 21.867 \\ 232.024 & 161.017 & -43.815 & 23.354 \\ 233.598 & 168.561 & -42.026 & 24.448 \\ 234.757 & 181.069 & -39.193 & 26.262 \\ 236.369 & 190.739 & -37.107 & 27.664 \\ 237.380 & 204.848 & -34.205 & 29.711 \\ & 214.096 & -32.385 & 31.052\end{array}$


Table 6. Saturated vapor densities for HFC-32 (NIST Burnett apparatus + ebulliometer)

$\begin{array}{lrlrrr}\mathrm{T} / \mathrm{K} & \mathrm{P} / \mathrm{Bar} & \mathrm{\rho} / \mathrm{mol} / \mathrm{L} & \mathrm{T} / \mathrm{F} & \mathrm{P} / \mathrm{psia} & \mathrm{\rho} / \mathrm{lbs} / \mathrm{ft}^{3} \\ & & & & & \\ 219 & 0.890 & 0.0509 & -65.469 & 12.921 & 0.1653 \\ 224 & 1.149 & 0.0647 & -56.469 & 16.669 & 0.2101 \\ 229 & 1.464 & 0.0813 & -47.469 & 21.234 & 0.2640 \\ 234 & 1.843 & 0.1010 & -38.469 & 26.737 & 0.3280 \\ 239 & 2.296 & 0.1244 & -29.469 & 33.303 & 0.4040 \\ 244 & 2.831 & 0.1519 & -20.469 & 41.067 & 0.4933 \\ 249 & 3.459 & 0.1840 & -11.469 & 50.170 & 0.5975 \\ 254 & 4.189 & 0.2214 & -2.469 & 60.760 & 0.7190 \\ 259 & 5.032 & 0.2645 & 6.530 & 72.993 & 0.8590 \\ 264 & 6.000 & 0.3142 & 15.530 & 87.029 & 1.0204 \\ 269 & 7.104 & 0.3713 & 24.530 & 103.037 & 1.2058 \\ 274 & 8.355 & 0.4367 & 33.530 & 121.190 & 1.4182 \\ 279 & 9.767 & 0.5114 & 42.530 & 141.668 & 1.6608 \\ 284 & 11.352 & 0.5967 & 51.530 & 164.646 & 1.9378 \\ 289 & 13.124 & 0.6941 & 60.530 & 190.347 & 2.2542 \\ 294 & 15.097 & 0.8054 & 69.530 & 218.963 & 2.6156 \\ 299 & 17.284 & 0.9326 & 78.530 & 250.683 & 3.0287 \\ 304 & 19.702 & 1.0785 & 87.530 & 285.753 & 3.5026 \\ 309 & 22.367 & 1.2466 & 96.530 & 324.405 & 4.0485 \\ 314 & 25.296 & 1.4414 & 105.530 & 366.887 & 4.6812 \\ 319 & 28.508 & 1.6690 & 114.530 & 413.473 & 5.4203 \\ 324 & 32.023 & 1.9385 & 123.530 & 464.454 & 6.2956 \\ 329 & 35.865 & 2.2631 & 132.530 & 520.177 & 7.3498 \\ 334 & 40.059 & 2.6649 & 141.530 & 581.006 & 8.6547 \\ 336 & 41.843 & 2.8552 & 145.130 & 606.881 & 9.2728 \\ 339 & 44.639 & 3.1841 & 150.530 & 647.434 & 10.3409 \\ 340 & 45.604 & 3.3084 & 152.330 & 661.430 & 10.7446 \\ 343 & 48.604 & 3.7403 & 157.730 & 704.941 & 12.1473 \\ & & & & & \end{array}$


Table 7. Saturated liquid densities for HFC-32 (NIST Burnett apparatus + ebulliometer)

$\begin{array}{lccccc}\mathrm{T} / \mathrm{K} & \mathrm{P} / \mathrm{Bar} & \rho / \mathrm{mol} / \mathrm{L} & \mathrm{T} / \mathrm{F} & \mathrm{P} / \mathrm{psia} & \mathrm{\rho} / \mathrm{lbs} / \mathrm{ft}^{3} \\ & & & & & \\ 242.589 & 2.671 & 22.168 & -23.009 & 38.739 & 71.994 \\ 246.063 & 3.079 & 21.974 & -16.756 & 44 . f, 50 & 71.364 \\ 250.574 & 3.677 & 21.706 & -8.636 & 53.230 & 70.494 \\ 255.132 & 4.370 & 21.433 & -0.432 & 63.381 & 69.607 \\ 259.778 & 5.175 & 21.148 & 7.930 & 75.057 & 68.682 \\ 264.509 & 6.106 & 20.853 & 16.446 & 88.560 & 67.724 \\ 269.391 & 7.197 & 20.539 & 25.233 & 104.383 & 66.704 \\ 274.006 & 8.357 & 20.231 & 33.540 & 121.208 & 65.704 \\ 278.823 & .9 .715 & 19.908 & 42.211 & 140.904 & 64.655 \\ 283.645 & 11.234 & 19.568 & 50.890 & 162.935 & 63.550 \\ 288.530 & 12.950 & 19.216 & 59.684 & 187.823 & 62.407 \\ 293.461 & 14.874 & 18.845 & 68.559 & 215.729 & 61.202 \\ 298.418 & 17.018 & 18.457 & 77.482 & 246.825 & 59.942 \\ 303.414 & 19.407 & 18.044 & 86.475 & 281.474 & 58.601 \\ 308.266 & 21.960 & 17.620 & 95.208 & 318.502 & 57.224 \\ 313.135 & 24.770 & 17.178 & 103.973 & 359.258 & 55.788 \\ 318.253 & 28.010 & 16.670 & 113.185 & 406.250 & 54.139 \\ 323.110 & 31.374 & 16.147 & 121.928 & 455.041 & 52.440 \\ 327.963 & 35.041 & 15.582 & 130.663 & 508.226 & 50.605 \\ 332.797 & 39.017 & 14.955 & 139.364 & 565.893 & 48.569 \\ 337.791 & 43.494 & 14.175 & 148.353 & 630.827 & 46.036\end{array}$




$\begin{array}{cc}\text { Table } 8 a . & \text { Vapor Pressures (Static Method) for HFC-32 } \\ \text { Temperature } & \text { Pressure } \\ \text { K } & \text { MPa } \\ 140.00 & 0.0000889 \\ 150.00 & 0.0003294 \\ 160.00 & 0.0011685 \\ 170.00 & 0.0030297 \\ 180.00 & 0.0071181 \\ 190.00 & 0.0152322 \\ 200.00 & 0.0299572 \\ 210.00 & 0.0547286 \\ 215.00 & 0.0721023 \\ 220.00 & 0.0939875 \\ 225.00 & 0.1208910 \\ 230.00 & 0.1536646 \\ 235.00 & 0.1931371 \\ 225.00 & 0.1209475 \\ 230.00 & 0.1537230 \\ 235.00 & 0.1931801 \\ 240.00 & 0.2399032 \\ 250.00 & 0.3599517 \\ 260.00 & 0.5216263 \\ 270.00 & 0.7338014 \\ 280.00 & 1.005735 \\ 290.00 & 1.348668 \\ 300.00 & 1.772857 \\ 310.00 & 2.29115 \\ 320.00 & 2.91673 \\ 330.00 & 3.66563 \\ 340.00 & 4.55863 \\ \end{array}$


Table 8b. Vapor Pressures (Static Method) for HFC-32

$\begin{array}{cc}\begin{array}{c}\text { Temperature } \\ \text { oF }\end{array} & \begin{array}{c}\text { Pressure } \\ \text { psia }\end{array} \\ -207.67 & 0.01290 \\ -189.67 & 0.04778 \\ -171.67 & 0.16947 \\ -153.67 & 0.43942 \\ -135.67 & 1.03239 \\ -117.67 & 2.20925 \\ -99.67 & 4.34493 \\ -81.67 & 7.93771 \\ -72.67 & 10.45756 \\ -63.67 & 13.63173 \\ -54.67 & 17.53375 \\ -45.67 & 22.28717 \\ -36.67 & 28.01217 \\ -54.67 & 17.54195 \\ -45.67 & 22.29563 \\ -36.67 & 28.01840 \\ -27.67 & 34.79502 \\ -9.67 & 52.20658 \\ 8.33 & 75.6555 \\ 26.33 & 106.4289 \\ 44.33 & 145.86955 \\ 62.33 & 195.6077 \\ 80.33 & 257.1311 \\ 98.33 & 332.303 \\ 116.33 & 423.036 \\ 134.33 & 531.654 \\ 152.33 & 661.174 \\ & \end{array}$


Table 9 (a). Experimental liquid heat capacity data for HFC-32.

\begin{tabular}{|c|c|c|c|c|c|c|c|c|c|}
\hline $\mathbf{T}$ & $\delta T$ & $\bar{p}$ & $\delta p$ & $\bar{P}$ & $\delta P$ & $C_{v, \exp }$ & $\delta C_{v}$ & $\delta C_{v}, g$ & $\overline{\text { Run }}$ \\
\hline \multicolumn{2}{|c|}{$\mathbf{R}$} & mol & $\mathrm{dm}^{-3}$ & \multicolumn{2}{|c|}{ MPa } & $\mathrm{J}$ mol & $-1 k^{-1}$ & & \\
\hline $\begin{array}{l}201.4668 \\
203.1891 \\
205.3522 \\
207.1265 \\
209.2809 \\
211.0429 \\
213.1814 \\
214.9430 \\
217.0617\end{array}$ & $\begin{array}{l}0.0031 \\
0.0031 \\
0.0031 \\
0.0033 \\
0.0031 \\
0.0030 \\
0.0031 \\
0.0031 \\
0.0031\end{array}$ & $\begin{array}{l}24.463 \\
24.451 \\
24.437 \\
24.424 \\
24.409 \\
24.397 \\
24.383 \\
24.371 \\
24.357\end{array}$ & $\begin{array}{l}0.023 \\
0.025 \\
0.028 \\
0.031 \\
0.035 \\
0.037 \\
0.040 \\
0.043 \\
0.046\end{array}$ & $\begin{array}{r}5.477 \\
8.169 \\
11.744 \\
14.723 \\
18.306 \\
21.173 \\
24.573 \\
27.330 \\
30.654\end{array}$ & $\begin{array}{l}0.107 \\
0.107 \\
0.107 \\
0.107 \\
0.107 \\
0.107 \\
0.107 \\
0.107 \\
0.107\end{array}$ & $\begin{array}{l}52.76 \\
49.77 \\
49.68 \\
49.51 \\
49.62 \\
49.52 \\
49.72 \\
49.73 \\
50.07\end{array}$ & $\begin{array}{l}0.20 \\
0.17 \\
0.14 \\
0.13 \\
0.12 \\
0.11 \\
0.11 \\
0.11 \\
0.11\end{array}$ & $\begin{array}{l}0.39 \\
0.34 \\
0.28 \\
0.26 \\
0.24 \\
0.23 \\
0.22 \\
0.22 \\
0.21\end{array}$ & $\begin{array}{l}a \\
b \\
a \\
b \\
a \\
b \\
a \\
b \\
a\end{array}$ \\
\hline
\end{tabular}

Table 9 (b). Experimental liquid heat capacity data for HFC-32.

\begin{tabular}{|c|c|c|c|c|c|c|c|c|c|}
\hline$T$ & $\delta T$ & $\rho$ & $\delta p$ & $\mathbf{P}$ & $\delta \mathrm{P}$ & $C_{v, \text { Qxp }}$ & $\delta \mathrm{C}_{v}$ & $\delta C_{v}, q$ & Run \\
\hline \multicolumn{2}{|c|}{${ }^{\circ} \mathrm{F}$} & \multicolumn{2}{|l|}{$1 b$} & \multicolumn{2}{|c|}{ poia } & \multicolumn{4}{|c|}{ Btu $1 b^{-1}{ }^{\circ} F^{-1}$} \\
\hline $\begin{array}{l}-97.030 \\
-93.930 \\
-90.036 \\
-86.842 \\
-82.964 \\
-79.793 \\
-75.944 \\
-72.773 \\
-68.959\end{array}$ & $\begin{array}{l}0.005 \\
0.006 \\
0.005 \\
0.006 \\
0.006 \\
0.005 \\
0.006 \\
0.006 \\
0.006\end{array}$ & $\begin{array}{l}79.45 \\
79.41 \\
79.36 \\
79.32 \\
79.28 \\
79.24 \\
79.19 \\
79.15 \\
79.11\end{array}$ & $\begin{array}{l}0.07 \\
0.08 \\
0.09 \\
0.10 \\
0.11 \\
0.12 \\
0.13 \\
0.14 \\
0.15\end{array}$ & $\begin{array}{r}794.3 \\
1184.8 \\
1703.3 \\
2135.4 \\
2655.1 \\
3070.9 \\
3564.0 \\
3963.9 \\
4446.0\end{array}$ & $\begin{array}{l}15.6 \\
15.6 \\
15.6 \\
15.6 \\
15.6 \\
15.6 \\
15.6 \\
15.6 \\
15.6\end{array}$ & $\begin{array}{l}0.24239 \\
0.22864 \\
0.22822 \\
0.22747 \\
0.22797 \\
0.22749 \\
0.22843 \\
0.22848 \\
0.23001\end{array}$ & $\begin{array}{l}0.00094 \\
0.00077 \\
0.00063 \\
0.00058 \\
0.00054 \\
0.00053 \\
0.00051 \\
0.00051 \\
0.00049\end{array}$ & $\begin{array}{l}0.39 \\
0.34 \\
0.28 \\
0.26 \\
0.24 \\
0.23 \\
0.22 \\
0.22 \\
0.21\end{array}$ & $\begin{array}{l}a \\
b \\
a \\
b \\
a \\
b \\
a \\
b \\
a\end{array}$ \\
\hline
\end{tabular}


Table $10(a)$. Experimental liquid heat capacity data for HFC-32.

\begin{tabular}{|c|c|c|c|c|c|c|c|c|c|}
\hline$T$ & $\delta \mathbf{T}$ & $\rho$ & $\delta p$ & $\mathbf{P}$ & $8 \mathrm{P}$ & $C_{v, \exp }$ & $\delta C_{v}$ & $\delta C_{v}, \frac{q}{\delta}$ & $\overline{\text { Run }}$ \\
\hline K & 8 & mol & $d m^{-3}$ & $\mathbf{M P}$ & & J mol- & $-1 \mathbf{K}^{-1}$ & & \\
\hline $\begin{array}{l}223.0399 \\
225.1392 \\
226.9877 \\
229.1349 \\
231.0003 \\
233.1008 \\
234.9878 \\
237.0561 \\
238.9540 \\
240.9935\end{array}$ & $\begin{array}{l}0.0031 \\
0.0032 \\
0.0031 \\
0.0034 \\
0.0032 \\
0.0033 \\
0.0032 \\
0.0033 \\
0.0032 \\
0.0034\end{array}$ & $\begin{array}{l}23.372 \\
23.360 \\
23.349 \\
23.336 \\
23.325 \\
23.312 \\
23.301 \\
23.289 \\
23.278 \\
23.266\end{array}$ & $\begin{array}{l}0.021 \\
0.024 \\
0.026 \\
0.029 \\
0.031 \\
0.034 \\
0.036 \\
0.038 \\
0.041 \\
0.043\end{array}$ & $\begin{array}{r}5.067 \\
7.844 \\
10.421 \\
13.473 \\
16.121 \\
19.063 \\
21.658 \\
24.455 \\
26.999 \\
29.745\end{array}$ & $\begin{array}{l}0.059 \\
0.059 \\
0.059 \\
0.059 \\
0.059 \\
0.059 \\
0.059 \\
0.059 \\
0.059 \\
0.059\end{array}$ & $\begin{array}{l}52.53 \\
48.98 \\
48.78 \\
48.90 \\
48.87 \\
48.93 \\
48.88 \\
48.96 \\
48.92 \\
49.06\end{array}$ & $\begin{array}{l}0.20 \\
0.15 \\
0.13 \\
0.12 \\
0.11 \\
0.11 \\
0.10 \\
0.10 \\
0.10 \\
0.10\end{array}$ & $\begin{array}{l}0.39 \\
0.32 \\
0.27 \\
0.24 \\
0.22 \\
0.22 \\
0.21 \\
0.21 \\
0.20 \\
0.20\end{array}$ & $\begin{array}{l}a \\
b \\
a \\
b \\
a \\
b \\
a \\
b \\
a \\
b\end{array}$ \\
\hline
\end{tabular}

Table 10 (b). Experimental liquid heat capacity data for HFC-32.

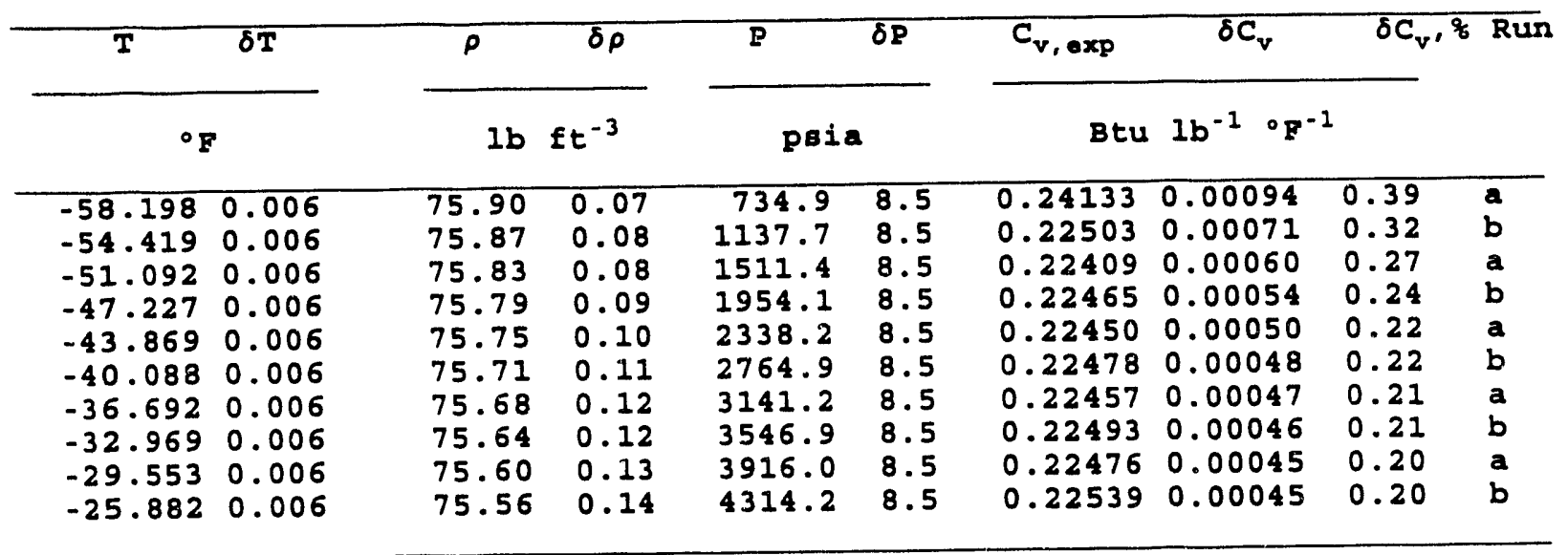


Table 11(a). Experimental liquid heat capacity data for HFC-32.

\begin{tabular}{|c|c|c|c|c|c|c|c|c|c|}
\hline $\mathbf{T}$ & $\delta \mathbf{T}$ & $p$ & $\delta \rho$ & P & $\delta P$ & $C_{v, \exp }$ & $\delta C_{v}$ & $\delta C_{v}, \xi$ & $\overline{\text { Run }}$ \\
\hline \multicolumn{2}{|c|}{$\mathbf{K}$} & mol & $d m^{-3}$ & \multicolumn{2}{|c|}{$\mathbf{M P a}$} & $\mathrm{J}$ mol & $-1 \mathrm{k}^{-1}$ & & \\
\hline $\begin{array}{l}247.0969 \\
249.1753 \\
251.1224 \\
253.2549 \\
255.1851 \\
257.3175 \\
259.2450 \\
261.3647 \\
263.2759 \\
265.3986 \\
267.3116 \\
269.4156\end{array}$ & $\begin{array}{l}0.0031 \\
0.0032 \\
0.0031 \\
0.0035 \\
0.0031 \\
0.0032 \\
0.0031 \\
0.0033 \\
0.0031 \\
0.0032 \\
0.0032 \\
0.0033\end{array}$ & $\begin{array}{l}22.066 \\
22.056 \\
22.047 \\
22.036 \\
22.026 \\
22.016 \\
22.006 \\
21.996 \\
21.987 \\
21.977 \\
21.968 \\
21.957\end{array}$ & $\begin{array}{l}0.019 \\
0.021 \\
0.023 \\
0.025 \\
0.027 \\
0.029 \\
0.031 \\
0.033 \\
0.035 \\
0.037 \\
0.038 \\
0.040\end{array}$ & $\begin{array}{r}3.950 \\
6.199 \\
8.420 \\
10.912 \\
13.175 \\
15.653 \\
17.859 \\
20.243 \\
22.361 \\
24.696 \\
26.807 \\
29.167\end{array}$ & $\begin{array}{l}0.054 \\
0.054 \\
0.054 \\
0.054 \\
0.054 \\
0.054 \\
0.054 \\
0.054 \\
0.054 \\
0.054 \\
0.054 \\
0.054\end{array}$ & $\begin{array}{l}52.43 \\
48.46 \\
48.69 \\
48.56 \\
48.69 \\
48.37 \\
48.94 \\
48.61 \\
49.04 \\
48.88 \\
49.17 \\
49.19\end{array}$ & $\begin{array}{l}0.21 \\
0.15 \\
0.12 \\
0.11 \\
0.10 \\
0.09 \\
0.09 \\
0.09 \\
0.09 \\
0.09 \\
0.09 \\
0.09\end{array}$ & $\begin{array}{l}0.40 \\
0.31 \\
0.25 \\
0.23 \\
0.21 \\
0.20 \\
0.19 \\
0.18 \\
0.18 \\
0.18 \\
0.18 \\
0.18\end{array}$ & $\begin{array}{l}a \\
b \\
a \\
b \\
a \\
b \\
a \\
b \\
a \\
b \\
a \\
b\end{array}$ \\
\hline
\end{tabular}

Table 11 (b). Experimental liquid heat capacity data for HFC-32.

\begin{tabular}{|c|c|c|c|c|c|c|c|c|c|}
\hline$T$ & $\delta \mathrm{T}$ & $\rho$ & $\delta \rho$ & P & $\delta p$ & $\overline{C_{v, \exp }}$ & $\delta C_{v}$ & \multirow[t]{2}{*}{$\delta C_{v}, 8$} & $\overline{R u n}$ \\
\hline \multicolumn{2}{|c|}{${ }^{\circ} \mathrm{E}$} & $1 b$ & $f t^{-3}$ & \multicolumn{2}{|c|}{ poia } & Btu & $1 b^{-1} \circ E^{-1}$ & & \\
\hline $\begin{array}{r}-14.896 \\
-11.154 \\
-7.650 \\
-3.811 \\
-0.337 \\
3.502 \\
6.971 \\
10.786 \\
14.227 \\
18.047 \\
21.491 \\
25.278\end{array}$ & $\begin{array}{l}0.006 \\
0.006 \\
0.006 \\
0.006 \\
0.006 \\
0.006 \\
0.006 \\
0.006 \\
0.006 \\
0.006 \\
0.006 \\
0.006\end{array}$ & $\begin{array}{l}71.67 \\
71.63 \\
71.60 \\
71.57 \\
71.54 \\
71.50 \\
71.47 \\
71.44 \\
71.41 \\
71.38 \\
71.35 \\
71.31\end{array}$ & $\begin{array}{l}0.06 \\
0.07 \\
0.07 \\
0.08 \\
0.09 \\
0.09 \\
0.10 \\
0.11 \\
0.11 \\
0.12 \\
0.12 \\
0.13\end{array}$ & $\begin{array}{r}572.9 \\
899.1 \\
1221.3 \\
1582.5 \\
1910.8 \\
2270.3 \\
2590.2 \\
2936.0 \\
3243.2 \\
3581.9 \\
3888.0 \\
4230.3\end{array}$ & $\begin{array}{l}7.8 \\
7.8 \\
7.8 \\
7.8 \\
7.8 \\
7.8 \\
7.8 \\
7.8 \\
7.8 \\
7.8 \\
7.8 \\
7.8\end{array}$ & $\begin{array}{l}0.24089 \\
0.22264 \\
0.22370 \\
0.22309 \\
0.22370 \\
0.22220 \\
0.22483 \\
0.22333 \\
0.22530 \\
0.22457 \\
0.22589 \\
0.22600\end{array}$ & $\begin{array}{l}0.00096 \\
0.00070 \\
0.00057 \\
0.00050 \\
0.00046 \\
0.00044 \\
0.00042 \\
0.00041 \\
0.00040 \\
0.00040 \\
0.00040 \\
0.00040\end{array}$ & $\begin{array}{l}0.40 \\
0.31 \\
0.25 \\
0.23 \\
0.21 \\
0.20 \\
0.19 \\
0.18 \\
0.18 \\
0.18 \\
0.18 \\
0.18\end{array}$ & $\begin{array}{l}a \\
b \\
a \\
b \\
a \\
b \\
a \\
b \\
a \\
b \\
a \\
b\end{array}$ \\
\hline
\end{tabular}


Table 12 (a). Experimental liquid heat capacity data for HFC-32.

\begin{tabular}{|c|c|c|c|c|c|c|c|c|c|}
\hline $\bar{T}$ & $\delta T$ & $p$ & $\delta \rho$ & P & $\delta P$ & $C_{v, \exp }$ & $\delta C_{v}$ & $\delta C_{v}, \%$ & $\overline{\text { Run }}$ \\
\hline \multicolumn{2}{|c|}{$\mathbf{K}$} & mol & $d m^{-3}$ & \multicolumn{2}{|c|}{ MPa } & J mol & $-1 \mathrm{~K}^{-1}$ & & \\
\hline $\begin{array}{l}275.8475 \\
277.9247 \\
279.9747 \\
282.0959 \\
284.1547 \\
286.2690 \\
288.3385 \\
290.4273 \\
292.5119 \\
294.5814 \\
296.6696 \\
298.7243 \\
300.8062 \\
302.8544 \\
304.9452 \\
306.9722\end{array}$ & $\begin{array}{l}0.0034 \\
0.0033 \\
0.0035 \\
0.0033 \\
0.0033 \\
0.0033 \\
0.0032 \\
0.0032 \\
0.0031 \\
0.0032 \\
0.0033 \\
0.0034 \\
0.0032 \\
0.0032 \\
0.0035 \\
0.0034\end{array}$ & $\begin{array}{l}20.321 \\
20.314 \\
20.306 \\
20.298 \\
20.290 \\
20.282 \\
20.274 \\
20.266 \\
20.258 \\
20.250 \\
20.242 \\
20.235 \\
20.227 \\
20.219 \\
20.211 \\
20.203\end{array}$ & $\begin{array}{l}0.018 \\
0.019 \\
0.021 \\
0.022 \\
0.023 \\
0.025 \\
0.026 \\
0.028 \\
0.029 \\
0.030 \\
0.032 \\
0.033 \\
0.034 \\
0.036 \\
0.037 \\
0.038\end{array}$ & $\begin{array}{l}4.042 \\
5.795 \\
7.567 \\
9.429 \\
11.251 \\
13.126 \\
14.959 \\
16.801 \\
18.627 \\
20.428 \\
22.232 \\
23.996 \\
25.776 \\
27.524 \\
29.309 \\
31.047\end{array}$ & $\begin{array}{l}0.040 \\
0.040 \\
0.040 \\
0.040 \\
0.040 \\
0.040 \\
0.040 \\
0.040 \\
0.040 \\
0.040 \\
0.040 \\
0.040 \\
0.040 \\
0.040 \\
0.040 \\
0.040\end{array}$ & $\begin{array}{l}52.42 \\
49.05 \\
48.91 \\
49.22 \\
49.19 \\
49.27 \\
49.24 \\
49.31 \\
49.22 \\
49.47 \\
49.12 \\
49.44 \\
49.43 \\
49.58 \\
50.07 \\
50.10\end{array}$ & $\begin{array}{l}0.21 \\
0.15 \\
0.12 \\
0.10 \\
0.09 \\
0.09 \\
0.08 \\
0.08 \\
0.08 \\
0.08 \\
0.07 \\
0.08 \\
0.07 \\
0.07 \\
0.08 \\
0.07\end{array}$ & $\begin{array}{l}0.39 \\
0.30 \\
0.24 \\
0.20 \\
0.18 \\
0.17 \\
0.16 \\
0.16 \\
0.15 \\
0.15 \\
0.15 \\
0.15 \\
0.15 \\
0.15 \\
0.15 \\
0.15\end{array}$ & $\begin{array}{l}a \\
b \\
a \\
b \\
a \\
b \\
a \\
b \\
a \\
b \\
a \\
b \\
a \\
b \\
a \\
b\end{array}$ \\
\hline
\end{tabular}

Table 12 (b). Experimental liquid heat capacity data for HFC-32.

\begin{tabular}{|c|c|c|c|c|c|c|c|c|c|}
\hline$T$ & $\delta \mathbf{T}$ & $\rho$ & $\delta \rho$ & P & $\delta \mathrm{P}$ & $C_{v, \exp }$ & $\delta c_{v}$ & $\delta \mathrm{C}_{\mathrm{v}}, \frac{8}{8}$ & $\overline{R u n}$ \\
\hline \multicolumn{2}{|c|}{${ }^{\circ} \mathrm{F}$} & $1 b$ & $f t^{-3}$ & \multicolumn{2}{|c|}{ psia } & Btu & $1 b^{-1} \circ F^{-1}$ & & \\
\hline $\begin{array}{l}36.855 \\
40.594 \\
44.285 \\
48.103 \\
51.808 \\
55.614 \\
59.339 \\
63.099 \\
66.851 \\
70.577 \\
74.335 \\
78.034 \\
81.781 \\
85.468 \\
89.231 \\
92.880\end{array}$ & $\begin{array}{l}0.006 \\
0.006 \\
0.006 \\
0.006 \\
0.006 \\
0.006 \\
0.006 \\
0.006 \\
0.006 \\
0.006 \\
0.006 \\
0.006 \\
0.006 \\
0.006 \\
0.006 \\
0.006\end{array}$ & $\begin{array}{l}66.00 \\
65.97 \\
65.95 \\
65.92 \\
65.90 \\
65.87 \\
65.84 \\
65.82 \\
65.79 \\
65.77 \\
65.74 \\
65.72 \\
65.69 \\
65.67 \\
65.64 \\
65.62\end{array}$ & $\begin{array}{l}0.06 \\
0.06 \\
0.07 \\
0.07 \\
0.08 \\
0.08 \\
0.09 \\
0.09 \\
0.09 \\
0.10 \\
0.10 \\
0.11 \\
0.11 \\
0.12 \\
0.12 \\
0.12\end{array}$ & $\begin{array}{r}586.3 \\
840.5 \\
1097.5 \\
1367.6 \\
1631.8 \\
1903.8 \\
2169.6 \\
2436.7 \\
2701.6 \\
2962.8 \\
3224.5 \\
3480.4 \\
3738.6 \\
3992.0 \\
4251.0 \\
4503.0\end{array}$ & $\begin{array}{l}5.8 \\
5.8 \\
5.8 \\
5.8 \\
5.8 \\
5.8 \\
5.8 \\
5.8 \\
5.8 \\
5.8 \\
5.8 \\
5.8 \\
5.8 \\
5.8 \\
5.8 \\
5.8\end{array}$ & $\begin{array}{l}0.24084 \\
0.22532 \\
0.22468 \\
0.22611 \\
0.22598 \\
0.22635 \\
0.22621 \\
0.22652 \\
0.22612 \\
0.22726 \\
0.22568 \\
0.22715 \\
0.22710 \\
0.22778 \\
0.23001 \\
0.23017\end{array}$ & $\begin{array}{l}0.00094 \\
0.00067 \\
0.00053 \\
0.00046 \\
0.00042 \\
0.00039 \\
0.00037 \\
0.00036 \\
0.00035 \\
0.00035 \\
0.00034 \\
0.00034 \\
0.00034 \\
0.00034 \\
0.00035 \\
0.00034\end{array}$ & $\begin{array}{l}0.39 \\
0.30 \\
0.24 \\
0.20 \\
0.18 \\
0.17 \\
0.16 \\
0.16 \\
0.15 \\
0.15 \\
0.15 \\
0.15 \\
0.15 \\
0.15 \\
0.15 \\
0.15\end{array}$ & $\begin{array}{l}a \\
b \\
a \\
b \\
a \\
b \\
a \\
b \\
a \\
b \\
a \\
b \\
a \\
b \\
a \\
b\end{array}$ \\
\hline
\end{tabular}


Table 13 (a). Experimental liquid heat capacity data for HFC-32.

\begin{tabular}{|c|c|c|c|c|c|c|c|c|c|}
\hline$T$ & $\delta T$ & $\rho$ & $\delta p$ & P & $\delta P$ & $C_{v, \text { exp }}$ & $\delta C_{v}$ & $\delta \mathrm{C}_{v}, \frac{q}{8}$ & Run \\
\hline \multicolumn{2}{|c|}{$\mathbf{R}$} & mol & $\mathrm{dm}^{-3}$ & \multicolumn{2}{|c|}{ MPa } & J mol & ${ }^{-1} \mathrm{~K}^{-1}$ & & \\
\hline $\begin{array}{l}312.5690 \\
314.7177 \\
316.9402 \\
319.1431 \\
321.3493 \\
323.5644 \\
325.7710 \\
327.9769 \\
330.1818 \\
332.3890 \\
334.5961 \\
336.8003 \\
339.0053 \\
341.2046\end{array}$ & $\begin{array}{l}0.0035 \\
0.0035 \\
0.0038 \\
0.0033 \\
0.0035 \\
0.0034 \\
0.0035 \\
0.0036 \\
0.0035 \\
0.0035 \\
0.00339 \\
0.0033 \\
0.0035 \\
0.0034\end{array}$ & $\begin{array}{l}17.508 \\
17.502 \\
17.497 \\
17.491 \\
17.486 \\
17.480 \\
17.475 \\
17.469 \\
17.464 \\
17.458 \\
17.453 \\
17.447 \\
17.442 \\
17.436\end{array}$ & $\begin{array}{l}0.016 \\
0.016 \\
0.017 \\
0.018 \\
0.019 \\
0.020 \\
0.020 \\
0.021 \\
0.022 \\
0.023 \\
0.024 \\
0.024 \\
0.025 \\
0.026\end{array}$ & $\begin{array}{r}4.054 \\
5.241 \\
6.484 \\
7.726 \\
8.975 \\
10.231 \\
11.483 \\
12.733 \\
13.980 \\
15.227 \\
16.471 \\
17.713 \\
18.956 \\
20.198\end{array}$ & $\begin{array}{l}0.012 \\
0.012 \\
0.012 \\
0.012 \\
0.012 \\
0.012 \\
0.012 \\
0.012 \\
0.012 \\
0.012 \\
0.012 \\
0.012 \\
0.012 \\
0.012\end{array}$ & $\begin{array}{l}53.52 \\
50.89 \\
50.63 \\
51.00 \\
50.81 \\
50.94 \\
50.82 \\
51.06 \\
50.94 \\
51.05 \\
51.13 \\
51.21 \\
51.08 \\
51.26\end{array}$ & $\begin{array}{l}0.16 \\
0.12 \\
0.10 \\
0.08 \\
0.08 \\
0.07 \\
0.07 \\
0.07 \\
0.07 \\
0.07 \\
0.07 \\
0.06 \\
0.06 \\
0.06\end{array}$ & $\begin{array}{l}0.29 \\
0.23 \\
0.19 \\
0.16 \\
0.15 \\
0.14 \\
0.14 \\
0.13 \\
0.13 \\
0.13 \\
0.13 \\
0.12 \\
0.13 \\
0.12\end{array}$ & $\begin{array}{l}b \\
a \\
b \\
a \\
b \\
a \\
b \\
a \\
b \\
a \\
b \\
a \\
b \\
a\end{array}$ \\
\hline
\end{tabular}

Table $13(b)$. Experimental liquid heat capacity data for HFC-32.

\begin{tabular}{|c|c|c|c|c|c|c|c|c|c|}
\hline $\mathrm{T}$ & $\delta \mathbf{T}$ & $\rho$ & $\delta \rho$ & P & $\overline{\delta P}$ & $C_{v, \exp }$ & $\delta C_{v}$ & \multirow[t]{2}{*}{$\delta C_{v}, 8$} & \multirow[t]{2}{*}{$\overline{\text { Run }}$} \\
\hline \multicolumn{2}{|c|}{${ }^{\circ} \mathrm{F}$} & $1 \mathrm{~b}$ & $f t^{-3}$ & \multicolumn{2}{|c|}{ psia } & \multicolumn{2}{|c|}{ Btu $1 b^{-1}{ }^{\circ} F^{-1}$} & & \\
\hline $\begin{array}{l}102.954 \\
106.822 \\
110.822 \\
114.788 \\
118.759 \\
122.746 \\
126.718 \\
130.688 \\
134.657 \\
138.630 \\
142.603 \\
146.570 \\
150.539 \\
154.498\end{array}$ & $\begin{array}{l}0.006 \\
0.006 \\
0.007 \\
0.006 \\
0.006 \\
0.006 \\
0.006 \\
0.006 \\
0.006 \\
0.006 \\
0.007 \\
0.006 \\
0.006 \\
0.006\end{array}$ & $\begin{array}{l}56.86 \\
56.84 \\
56.83 \\
56.81 \\
56.79 \\
56.77 \\
56.75 \\
56.74 \\
56.72 \\
56.70 \\
56.68 \\
56.67 \\
56.65 \\
56.63\end{array}$ & $\begin{array}{l}0.05 \\
0.05 \\
0.06 \\
0.06 \\
0.06 \\
0.06 \\
0.07 \\
0.07 \\
0.07 \\
0.07 \\
0.08 \\
0.08 \\
0.08 \\
0.08\end{array}$ & $\begin{array}{r}587.9 \\
760.2 \\
940.5 \\
1120.6 \\
1301.7 \\
1484.0 \\
1665.5 \\
1846.8 \\
2027.7 \\
2208.4 \\
2388.9 \\
2569.0 \\
2749.3 \\
2929.5\end{array}$ & $\begin{array}{l}1.7 \\
1.7 \\
1.7 \\
1.7 \\
1.7 \\
1.7 \\
1.7 \\
1.7 \\
1.7 \\
1.7 \\
1.7 \\
1.7 \\
1.7 \\
1.7\end{array}$ & $\begin{array}{l}0.24587 \\
0.23379 \\
0.23260 \\
0.23430 \\
0.23342 \\
0.23403 \\
0.23348 \\
0.23456 \\
0.23403 \\
0.23452 \\
0.23492 \\
0.23527 \\
0.23469 \\
0.23549\end{array}$ & $\begin{array}{l}0.00071 \\
0.00054 \\
0.00045 \\
0.00038 \\
0.00036 \\
0.00033 \\
0.00032 \\
0.00031 \\
0.00030 \\
0.00030 \\
0.00031 \\
0.00029 \\
0.00029 \\
0.00029\end{array}$ & $\begin{array}{l}0.29 \\
0.23 \\
0.19 \\
0.16 \\
0.15 \\
0.14 \\
0.14 \\
0.13 \\
0.13 \\
0.13 \\
0.13 \\
0.12 \\
0.13 \\
0.12\end{array}$ & $\begin{array}{l}b \\
a \\
b \\
a \\
b \\
a \\
b \\
a \\
b \\
a \\
b \\
a \\
b \\
a\end{array}$ \\
\hline
\end{tabular}


Table 14(a). Experimental liquid heat capacity data for HFC-32.

\begin{tabular}{|c|c|c|c|c|c|c|c|c|c|}
\hline $\mathbf{T}$ & $\delta \mathrm{T}$ & $\rho$ & $\delta \rho$ & $\mathbf{P}$ & $\delta P$ & $C_{v, \exp }$ & $\delta C_{v}$ & $\delta C_{v}, \frac{9}{6}$ & Run \\
\hline \multicolumn{2}{|c|}{$\mathbf{K}$} & mol & $\mathrm{dm}^{-3}$ & \multicolumn{2}{|c|}{$\mathrm{MPa}$} & \multicolumn{4}{|c|}{$\mathrm{J} \operatorname{mol}^{-1} \mathrm{k}^{-1}$} \\
\hline $\begin{array}{l}152.9366 \\
154.9359 \\
156.7176 \\
158.7148 \\
160.4805 \\
162.4557\end{array}$ & $\begin{array}{l}0.0029 \\
0.0029 \\
0.0030 \\
0.0029 \\
0.0030 \\
0.0030\end{array}$ & $\begin{array}{l}26.811 \\
26.793 \\
26.776 \\
26.758 \\
26.742 \\
26.724\end{array}$ & $\begin{array}{l}0.028 \\
0.033 \\
0.037 \\
0.042 \\
0.046 \\
0.051\end{array}$ & $\begin{array}{r}8.918 \\
13.597 \\
17.743 \\
22.343 \\
26.357 \\
30.788\end{array}$ & $\begin{array}{l}0.029 \\
0.029 \\
0.029 \\
0.029 \\
0.029 \\
0.029\end{array}$ & $\begin{array}{l}53.60 \\
53.40 \\
53.31 \\
53.21 \\
53.16 \\
53.34\end{array}$ & $\begin{array}{l}0.19 \\
0.16 \\
0.15 \\
0.14 \\
0.14 \\
0.13\end{array}$ & $\begin{array}{l}0.35 \\
0.30 \\
0.28 \\
0.26 \\
0.26 \\
0.25\end{array}$ & $\begin{array}{l}a \\
b \\
a \\
b \\
a \\
c\end{array}$ \\
\hline
\end{tabular}

Table $14(b)$. Experimental liquid heat capacity data for HFC-32.

\begin{tabular}{|c|c|c|c|c|c|c|c|c|c|}
\hline $\mathbf{T}$ & $\delta \mathbf{T}$ & $p$ & $\delta \rho$ & P & $\delta P$ & $C_{v, \exp }$ & $\delta C_{v}$ & \multirow[t]{2}{*}{$\delta C_{v}, 9$} & \multirow[t]{2}{*}{ Run } \\
\hline \multicolumn{2}{|c|}{${ }^{\circ} \boldsymbol{F}$} & \multicolumn{2}{|c|}{ Ib $f t^{-3}$} & \multicolumn{2}{|c|}{ psia } & \multicolumn{2}{|c|}{ Btu $1 b^{-1} \circ F^{-1}$} & & \\
\hline $\begin{array}{l}-184.384 \\
-180.785 \\
-177.578 \\
-173.983 \\
-170.805 \\
-167.250\end{array}$ & $\begin{array}{l}0.005 \\
0.005 \\
0.005 \\
0.005 \\
0.005 \\
0.005\end{array}$ & $\begin{array}{l}87.08 \\
87.02 \\
86.96 \\
86.90 \\
86.85 \\
86.79\end{array}$ & $\begin{array}{l}0.09 \\
0.11 \\
0.12 \\
0.14 \\
0.15 \\
0.16\end{array}$ & $\begin{array}{l}1293.5 \\
1972.1 \\
2573.4 \\
3240.6 \\
3822.8 \\
4465.4\end{array}$ & $\begin{array}{l}4.3 \\
4.3 \\
4.3 \\
4.3 \\
4.3 \\
4.3\end{array}$ & $\begin{array}{l}0.24624 \\
0.24531 \\
0.24490 \\
0.24445 \\
0.24420 \\
0.24505\end{array}$ & $\begin{array}{l}0.00086 \\
0.00073 \\
0.00068 \\
0.00064 \\
0.00062 \\
0.00061\end{array}$ & $\begin{array}{l}0.35 \\
0.30 \\
0.28 \\
0.26 \\
0.26 \\
0.25\end{array}$ & $\begin{array}{l}a \\
b \\
a \\
b \\
a \\
c\end{array}$ \\
\hline
\end{tabular}


Table 15 (a). Experimental liquid heat capacity data for HFC-32.

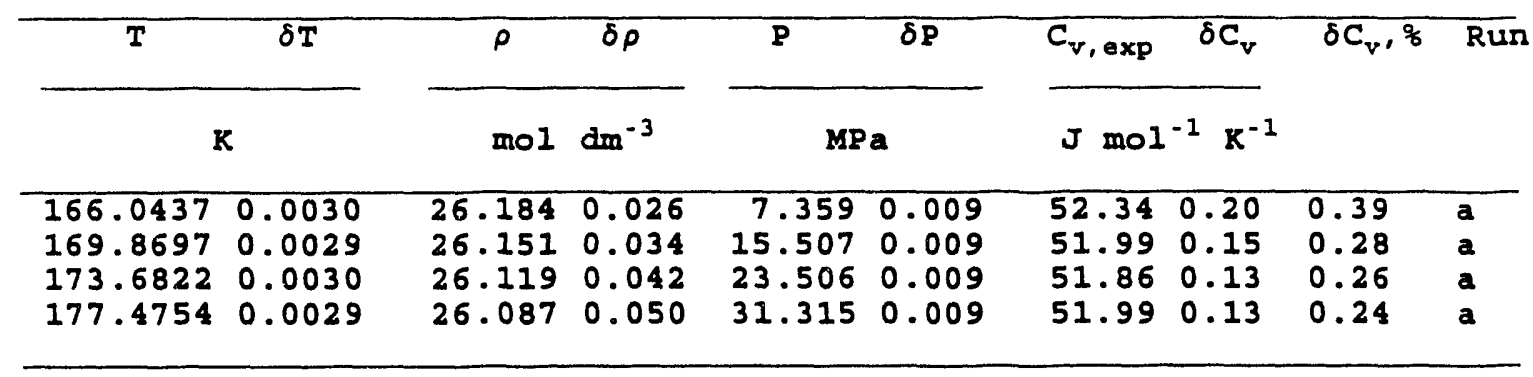

Table $15(b)$. Experimental liquid heat capacity data for HFC-32.

\begin{tabular}{|c|c|c|c|c|c|c|c|c|c|}
\hline \multicolumn{2}{|r|}{$\delta \mathrm{T}$} & \multicolumn{2}{|r|}{$\delta p$} & \multicolumn{2}{|r|}{$\delta \mathbf{P}$} & $C_{v, \exp }$ & $\delta C_{v}$ & $\delta \mathrm{C}_{\mathrm{v}}, 8$ & Run \\
\hline${ }^{\circ} \mathrm{F}$ & & $1 b$ & $f t^{-3}$ & psi & & Btu & $1 b^{-1} \circ F^{-1}$ & & \\
\hline $\begin{array}{l}-160.791 \\
-153.904 \\
-147.042 \\
-140.214\end{array}$ & $\begin{array}{l}0.005 \\
0.005 \\
0.005 \\
0.005\end{array}$ & $\begin{array}{l}85.04 \\
84.93 \\
84.83 \\
84.72\end{array}$ & $\begin{array}{l}0.08 \\
0.11 \\
0.14 \\
0.16\end{array}$ & $\begin{array}{l}1067.3 \\
2249.0 \\
3409.2 \\
4541.8\end{array}$ & $\begin{array}{l}1 \cdot 3 \\
1 \cdot 3 \\
1 \cdot 3 \\
1.3\end{array}$ & $\begin{array}{l}0.24047 \\
0.23886 \\
0.23826 \\
0.23886\end{array}$ & $\begin{array}{l}0.00093 \\
0.00067 \\
0.00061 \\
0.00058\end{array}$ & $\begin{array}{l}0.39 \\
0.28 \\
0.26 \\
0.24\end{array}$ & $\begin{array}{l}\mathbf{a} \\
\mathbf{a} \\
\mathbf{a} \\
\mathbf{a}\end{array}$ \\
\hline
\end{tabular}


Table $16(a)$. Experimental liquid heat capacity data for HFC-32.

\begin{tabular}{|c|c|c|c|c|c|c|c|c|}
\hline $\mathbf{T}$ & $\delta \mathrm{T}$ & $\rho$ & $\delta \rho$ & P & $\delta \mathbf{P}$ & $C_{v, \exp } \delta C_{v}$ & $\delta C_{v}, 9$ & $\overline{R u \Omega}$ \\
\hline \multicolumn{2}{|c|}{$\mathbf{K}$} & mol & $d m^{-3}$ & \multicolumn{2}{|c|}{ MPa } & $\mathrm{J} \operatorname{mol}^{-1} \mathrm{~K}^{-1}$ & & \\
\hline $\begin{array}{l}184.3432 \\
186.3054 \\
188.2313 \\
190.1865 \\
192.1069 \\
194.0462 \\
195.9596 \\
197.8910\end{array}$ & $\begin{array}{l}0.0030 \\
0.0030 \\
0.0030 \\
0.0030 \\
0.0030 \\
0.0031 \\
0.0030 \\
0.0031\end{array}$ & $\begin{array}{l}25.322 \\
25.307 \\
25.292 \\
25.277 \\
25.262 \\
25.248 \\
25.233 \\
25.219\end{array}$ & $\begin{array}{l}0.025 \\
0.028 \\
0.032 \\
0.035 \\
0.039 \\
0.042 \\
0.045 \\
0.049\end{array}$ & $\begin{array}{r}6.772 \\
10.466 \\
14.071 \\
17.707 \\
21.252 \\
24.802 \\
28.274 \\
31.747\end{array}$ & $\begin{array}{l}0.010 \\
0.010 \\
0.010 \\
0.010 \\
0.010 \\
0.010 \\
0.010 \\
0.010\end{array}$ & 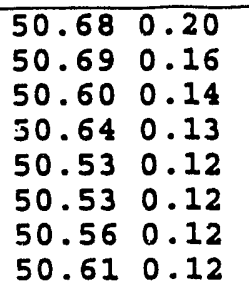 & $\begin{array}{l}0.40 \\
0.31 \\
0.28 \\
0.26 \\
0.25 \\
0.24 \\
0.23 \\
0.23\end{array}$ & $\begin{array}{l}a \\
b \\
a \\
b \\
a \\
b \\
a \\
b\end{array}$ \\
\hline
\end{tabular}

Table $16(b)$. Experimental liquid heat capacity data for HFC-32.

\begin{tabular}{|c|c|c|c|c|c|c|c|c|c|}
\hline$T$ & $\delta \mathrm{T}$ & $\rho$ & $\delta p$ & $\mathbf{P}$ & $\delta P$ & $C_{v, \exp }$ & $\delta C_{v}$ & $\delta C_{v}, \%$ & $\overline{\text { Run }}$ \\
\hline \multicolumn{2}{|c|}{${ }^{\circ} \mathrm{F}$} & $1 b$ & $f t^{-3}$ & \multicolumn{2}{|c|}{ psia } & \multicolumn{3}{|c|}{ Btu $1 b^{-1} \circ F^{-1}$} & \\
\hline $\begin{array}{l}-127.852 \\
-124.320 \\
-120.854 \\
-117.334 \\
-113.878 \\
-110.387 \\
-106.943 \\
-103.466\end{array}$ & $\begin{array}{l}0.005 \\
0.005 \\
0.005 \\
0.005 \\
0.005 \\
0.006 \\
0.005 \\
0.006\end{array}$ & $\begin{array}{l}82.24 \\
82.19 \\
82.14 \\
82.09 \\
82.05 \\
82.00 \\
81.95 \\
81.90\end{array}$ & $\begin{array}{l}0.08 \\
0.09 \\
0.10 \\
0.11 \\
0.13 \\
0.14 \\
0.15 \\
0.16\end{array}$ & $\begin{array}{r}982.2 \\
1517.9 \\
2040.9 \\
2568.2 \\
3082.4 \\
3597.3 \\
4100.8 \\
4604.5\end{array}$ & $\begin{array}{l}1.5 \\
1.5 \\
1.5 \\
1.5 \\
1.5 \\
1.5 \\
1.5 \\
1.5\end{array}$ & $\begin{array}{l}0.23285 \\
0.23288 \\
0.23248 \\
0.23265 \\
0.23215 \\
0.23216 \\
0.23230 \\
0.23249\end{array}$ & $\begin{array}{l}0.00092 \\
0.00073 \\
0.00064 \\
0.00060 \\
0.00057 \\
0.00056 \\
0.00055 \\
0.00054\end{array}$ & $\begin{array}{l}0.40 \\
0.31 \\
0.28 \\
0.26 \\
0.25 \\
0.24 \\
0.23 \\
0.23\end{array}$ & $\begin{array}{l}a \\
b \\
a \\
b \\
a \\
b \\
a \\
b\end{array}$ \\
\hline
\end{tabular}


Table 17 (a). Experimental two-phase heat capacity data for HFC-32.

\begin{tabular}{|c|c|c|c|c|c|c|c|c|c|c|c|}
\hline $\mathbf{T}$ & $\delta \mathbf{T}$ & & \multirow{2}{*}{$\begin{array}{l}\mathbf{P}_{\sigma} \\
\mathbf{M P a}\end{array}$} & \multirow[t]{2}{*}{$C_{v}^{(2)}$} & $\delta C_{v}{ }^{(2)}$ & $\delta C_{v}{ }^{(2)}$ & $c_{\sigma}$ & \multicolumn{2}{|c|}{$\delta \mathrm{C}_{\sigma}$} & \multicolumn{2}{|c|}{$\delta C_{\sigma}, 8$} \\
\hline $\mathbf{K}$ & & mol $\mathrm{dm}^{-3}$ & & & & $\mathrm{~J} \mathrm{~mol}^{-}$ & $-1 \mathbf{k}^{-1}$ & & & & \\
\hline $\begin{array}{l}141.7357 \\
145.8061 \\
149.8458 \\
153.8710 \\
157.8716 \\
161.8533 \\
165.8155 \\
169.7495 \\
173.6717 \\
177.5690 \\
181.4505 \\
185.3072 \\
189.1516 \\
192.9702 \\
196.7737 \\
200.5483 \\
204.5556 \\
208.7849 \\
212.9828 \\
217.1603 \\
221.3115 \\
225.4342 \\
229.5376 \\
233.6094 \\
237.6613 \\
241.6846 \\
245.6850 \\
249.6596 \\
253.6128 \\
257.5429 \\
261.4430 \\
265.3195 \\
269.1857 \\
273.0159 \\
276.8306 \\
280.6208 \\
284.6251 \\
288.8352 \\
293.0161 \\
297.1666 \\
301.2947 \\
305.3859\end{array}$ & $\begin{array}{l}0 \\
0.0030 \\
0.0029 \\
0.0029 \\
0.0030 \\
0.0029 \\
0.0029 \\
0.0030 \\
0.0030 \\
0.0029 \\
0.0030 \\
0.0030 \\
0.0030 \\
0.003 \\
0.0030 \\
0.0029 \\
0.0030 \\
0.0030 \\
0.0030 \\
0.0030 \\
0.0030 \\
0.0030 \\
0.0030 \\
0.0030 \\
0.0031 \\
0.0031\end{array}$ & 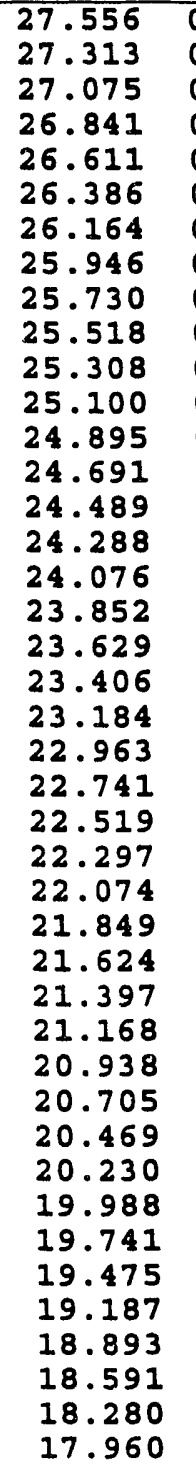 & 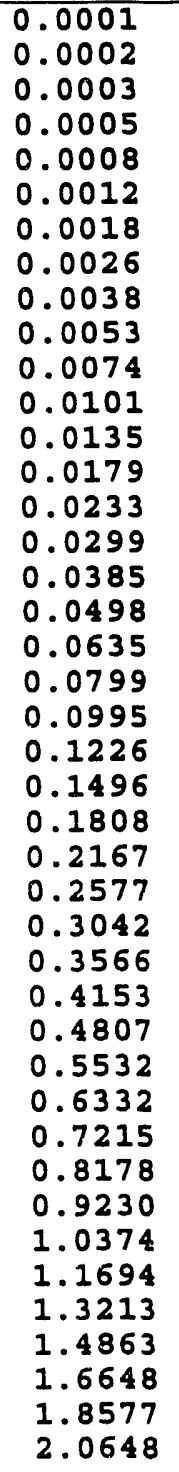 & $\begin{array}{l}82.41 \\
82.07 \\
82.03 \\
81.83 \\
81.75 \\
81.61 \\
81.16 \\
81.18 \\
81.23 \\
81.45 \\
81.33 \\
81.29 \\
81.20 \\
81.39 \\
81.33 \\
81.55 \\
81.80 \\
82.07 \\
82.34 \\
82.66 \\
82.91 \\
83.27 \\
83.58 \\
84.01 \\
84.57 \\
85.05 \\
85.38 \\
85.90 \\
86.31 \\
86.74 \\
87.28 \\
88.21 \\
88.67 \\
89.41 \\
90.05 \\
90.93 \\
91.68 \\
92.66 \\
93.41 \\
94.70 \\
95.73 \\
96.80\end{array}$ & $\begin{array}{l}0.06 \\
0.06 \\
0.06 \\
0.06 \\
0.06 \\
0.06 \\
0.06 \\
0.06 \\
0.06 \\
0.06 \\
0.07 \\
0.07 \\
0.06 \\
0.06 \\
0.07 \\
0.07 \\
0.06 \\
0.06 \\
0.06 \\
0.06 \\
0.06 \\
0.06 \\
0.07 \\
0.07 \\
0.07 \\
0.07 \\
0.07 \\
0.07 \\
0.07 \\
0.07 \\
0.07 \\
0.07 \\
0.07 \\
0.08 \\
0.08 \\
0.08 \\
0.07 \\
0.07 \\
0.07 \\
0.08 \\
0.08 \\
0.08\end{array}$ & $\begin{array}{l}\quad 0.08 \\
0.07 \\
0.08 \\
0.08 \\
0.08 \\
0.08 \\
0.08 \\
0.08 \\
0.08\end{array}$ & $\begin{array}{r}82.41 \\
82.06 \\
82.02 \\
81.81 \\
81.72 \\
81.58 \\
81.12 \\
81.12 \\
81.15 \\
81.35 \\
81.21 \\
81.15 \\
81.03 \\
81.19 \\
81.09 \\
81.27 \\
81.48 \\
81.71 \\
81.92 \\
82.20 \\
82.40 \\
82.72 \\
82.99 \\
83.37 \\
83.90 \\
84.36 \\
84.68 \\
85.20 \\
85.63 \\
86.10 \\
86.69 \\
87.70 \\
88.27 \\
89.16 \\
89.97 \\
91.07 \\
92.11 \\
93.47 \\
94.68 \\
96.54 \\
98.28 \\
100.15\end{array}$ & $\begin{array}{l}0.06 \\
0.06 \\
0.06 \\
0.06 \\
0.06 \\
0.06 \\
0.06 \\
0.07 \\
0.07 \\
0.07 \\
0.07 \\
0.08 \\
0.09 \\
0.09 \\
0.10 \\
0.11 \\
0.12 \\
0.14 \\
0.16 \\
0.17 \\
0.19 \\
0.21 \\
0.23 \\
0.26 \\
0.28 \\
0.30 \\
0.31 \\
0.33 \\
0.35 \\
0.36 \\
0.38 \\
0.39 \\
0.39 \\
0.39 \\
0.39 \\
0.39 \\
0.39 \\
0.33 \\
0.34 \\
0.31 \\
0.28 \\
0.28\end{array}$ & $\begin{array}{l}0.08 \\
0.07 \\
0.08 \\
0.08 \\
0.08 \\
0.08 \\
0.08 \\
0.08 \\
0.08 \\
0.09 \\
0.09 \\
0.10 \\
0.11 \\
0.11 \\
0.13 \\
0.14 \\
0.15 \\
0.17 \\
0.19 \\
0.21 \\
0.24 \\
0.26 \\
0.28 \\
0.31 \\
0.33 \\
0.35 \\
0.37 \\
0.39 \\
0.41 \\
0.42 \\
0.43 \\
0.44 \\
0.44 \\
0.44 \\
0.44 \\
0.43 \\
0.41 \\
0.39 \\
40.36 \\
0.32 \\
0.29 \\
00.28\end{array}$ & $\begin{array}{l}\mathbf{a} \\
\mathbf{a} \\
\mathbf{a} \\
\mathbf{a} \\
\mathbf{a} \\
\mathbf{a} \\
\mathbf{a} \\
\mathbf{a} \\
\mathbf{a} \\
\mathbf{a} \\
\mathbf{a} \\
\mathbf{a} \\
\mathbf{a} \\
\mathbf{a} \\
\mathbf{a} \\
\mathbf{a} \\
\mathbf{a}\end{array}$ & $\begin{array}{l}\mathbf{a} \\
\mathbf{a} \\
\mathbf{a} \\
\mathbf{a} \\
\mathbf{a} \\
\mathbf{a} \\
\mathbf{a} \\
\mathbf{a} \\
\mathbf{a} \\
\mathbf{a} \\
\mathbf{a} \\
\mathbf{a} \\
\mathbf{a} \\
\mathbf{a} \\
\mathbf{a} \\
\mathbf{a} \\
\mathbf{a} \\
\mathbf{a} \\
\mathbf{a} \\
\mathbf{a} \\
\mathbf{a} \\
\mathbf{a} \\
\mathbf{a} \\
\mathbf{a} \\
\mathbf{a} \\
\mathbf{a} \\
\mathbf{a} \\
\mathbf{a} \\
\mathbf{a} \\
\mathbf{a} \\
\mathbf{a} \\
\mathbf{a} \\
\mathbf{a} \\
\mathbf{a} \\
\mathbf{a} \\
\mathbf{a} \\
\mathbf{a} \\
\mathbf{a} \\
\mathbf{a} \\
\mathbf{a} \\
\mathbf{a}\end{array}$ \\
\hline
\end{tabular}


Table 17 (b). Experimental two-phase heat capacity data for HFC-32.

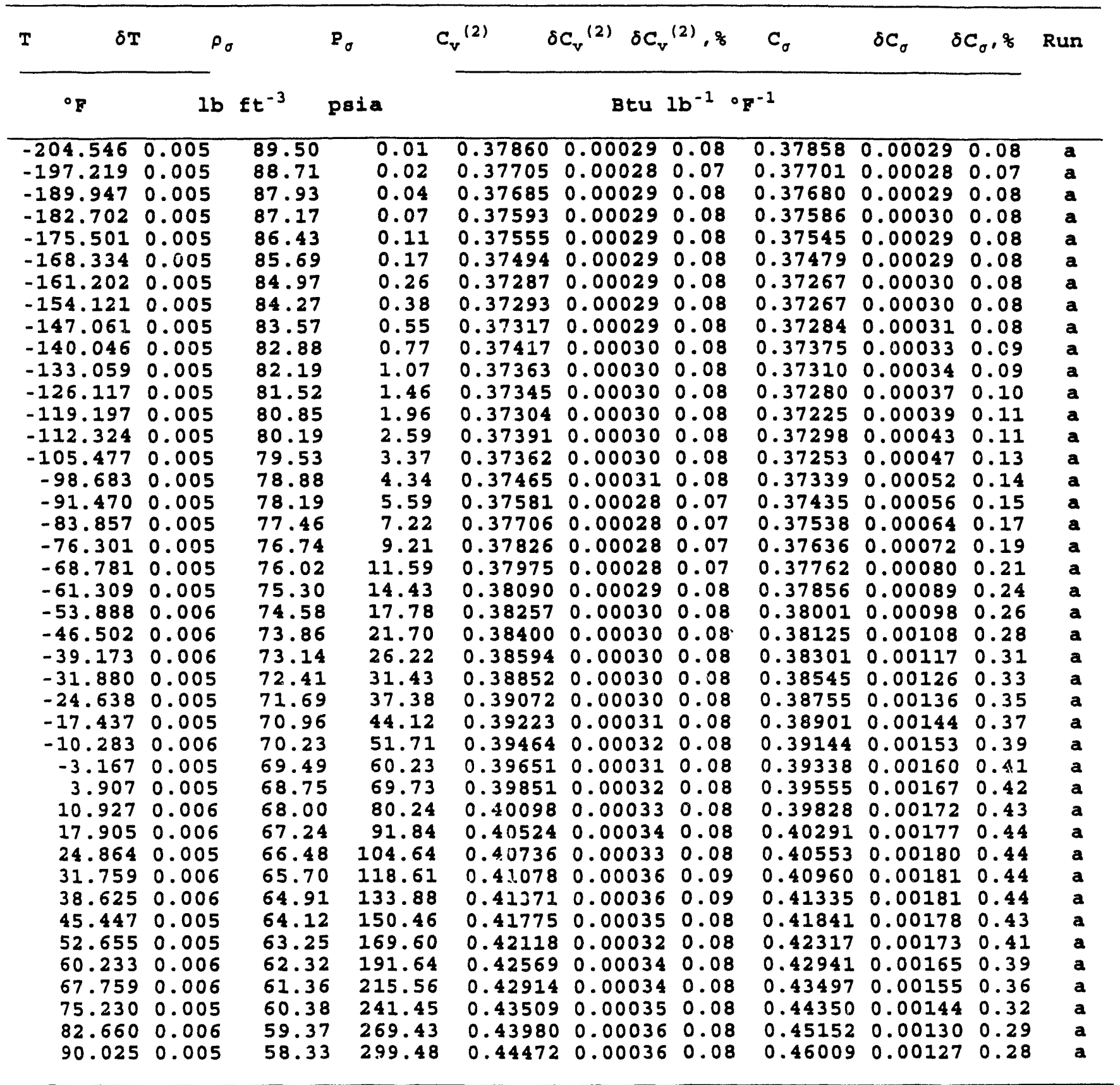


Table 18 (a). Experimental two-phase heat capacity data for HFC-32.

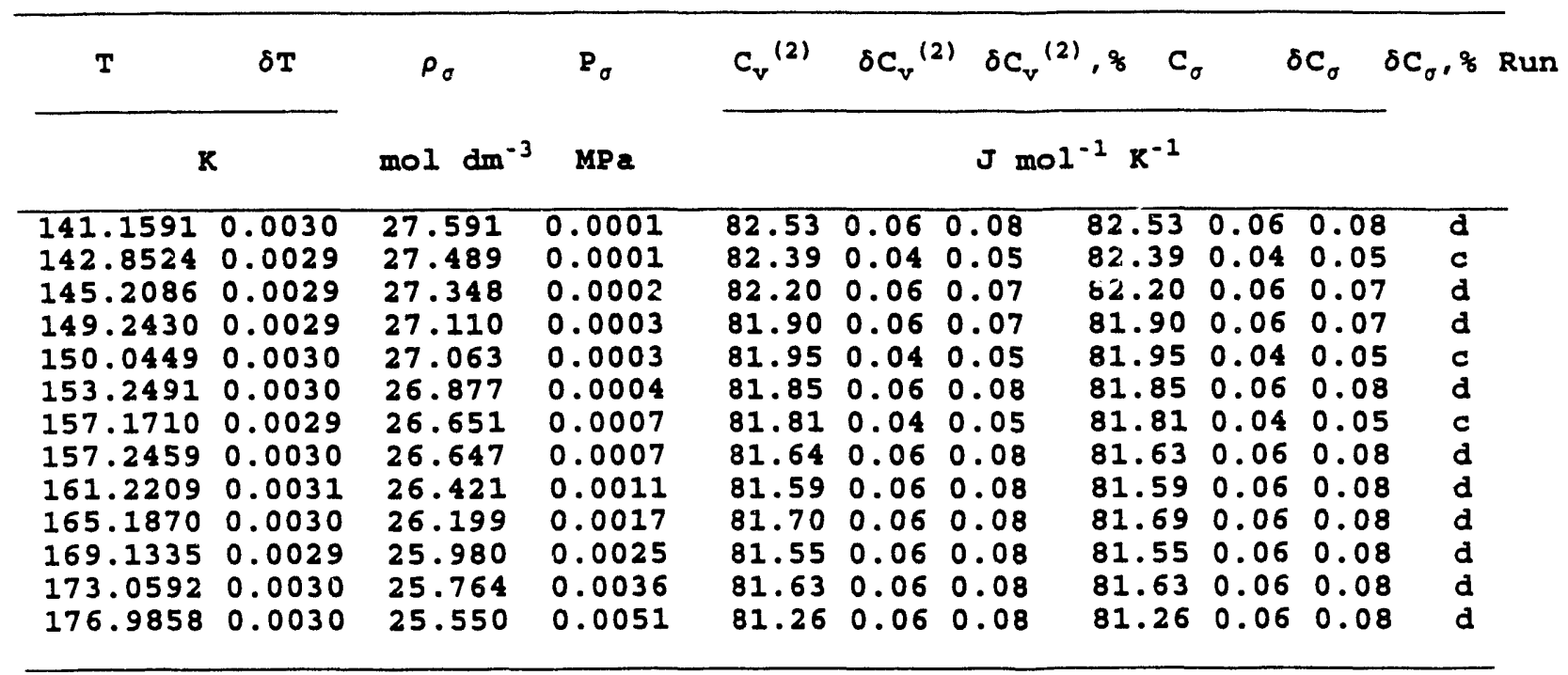

Table $18(b)$. Experimental two-phase heat capacity data for HFC-32.

\begin{tabular}{|c|c|c|c|c|c|c|c|c|c|c|}
\hline $\mathbf{T}$ & $\delta \mathrm{T}$ & $\rho_{\sigma}$ & $\mathbf{P}_{\sigma}$ & $C_{v}{ }^{(2)}$ & $\delta C_{v}{ }^{(2)}$ & $\delta C_{v}$ & .8 & $\delta C_{\sigma}$ & \multicolumn{2}{|c|}{$\delta C_{\sigma}, \%$} \\
\hline \multicolumn{2}{|c|}{${ }^{\circ} \mathrm{F}$} & $1 b f t^{-3}$ & psia & & & Btu $1 b^{-}$ & $-1 \circ F^{-1}$ & & & \\
\hline $\begin{array}{l}-205.584 \\
-202.536 \\
-198.294 \\
-191.033 \\
-189.589 \\
-183.822 \\
-176.762 \\
-176.627 \\
-169.472 \\
-162.333 \\
-155.230 \\
-148.164 \\
-141.096\end{array}$ & $\begin{array}{l}0.005 \\
0.005 \\
0.005 \\
0.005 \\
0.005 \\
0.005 \\
0.005 \\
0.005 \\
0.005 \\
0.005 \\
0.005 \\
0.005 \\
0.005\end{array}$ & $\begin{array}{l}89.61 \\
89.28 \\
88.82 \\
88.05 \\
87.89 \\
87.29 \\
86.56 \\
86.54 \\
85.81 \\
85.09 \\
84.38 \\
83.67 \\
82.98\end{array}$ & $\begin{array}{l}0.01 \\
0.02 \\
0.02 \\
0.04 \\
0.04 \\
0.06 \\
0.10 \\
0.10 \\
0.16 \\
0.24 \\
0.36 \\
0.52 \\
0.74\end{array}$ & $\begin{array}{l}0.37916 \\
0.37850 \\
0.37764 \\
0.37628 \\
0.37649 \\
0.37602 \\
0.37585 \\
0.37505 \\
0.37484 \\
0.37532 \\
0.37466 \\
0.37503 \\
0.37331\end{array}$ & $\begin{array}{l}0.00029 \\
0.00017 \\
0.00028 \\
0.00028 \\
0.00017 \\
0.00029 \\
0.00017 \\
0.00029 \\
0.00030 \\
0.00029 \\
0.00029 \\
0.00029 \\
0.00029\end{array}$ & $\begin{array}{l}0.08 \\
0.05 \\
0.07 \\
0.07 \\
0.05 \\
0.08 \\
0.05 \\
0.08 \\
0.08 \\
0.08 \\
0.08 \\
0.08 \\
0.08\end{array}$ & $\begin{array}{l}0.37915 \\
0.37850 \\
0.37763 \\
0.37628 \\
0.37649 \\
0.37602 \\
0.37584 \\
0.37504 \\
0.37483 \\
0.37532 \\
0.37466 \\
0.37504 \\
0.37333\end{array}$ & $\begin{array}{l}0.00029 \\
0.00017 \\
0.00028 \\
0.00028 \\
0.00017 \\
0.00029 \\
0.00017 \\
0.00029 \\
0.00030 \\
0.00029 \\
0.00029 \\
0.00029 \\
0.00029\end{array}$ & $\begin{array}{l}0.08 \\
0.05 \\
0.07 \\
0.07 \\
0.05 \\
0.08 \\
0.05 \\
0.08 \\
0.08 \\
0.08 \\
0.08 \\
0.08 \\
0.08\end{array}$ & $\begin{array}{l}\text { d } \\
c \\
d \\
d \\
c \\
d \\
c \\
d \\
d \\
d \\
d \\
d \\
d\end{array}$ \\
\hline
\end{tabular}


Table 19(a). Experimental two-phase heat capacity data for HFC-32.

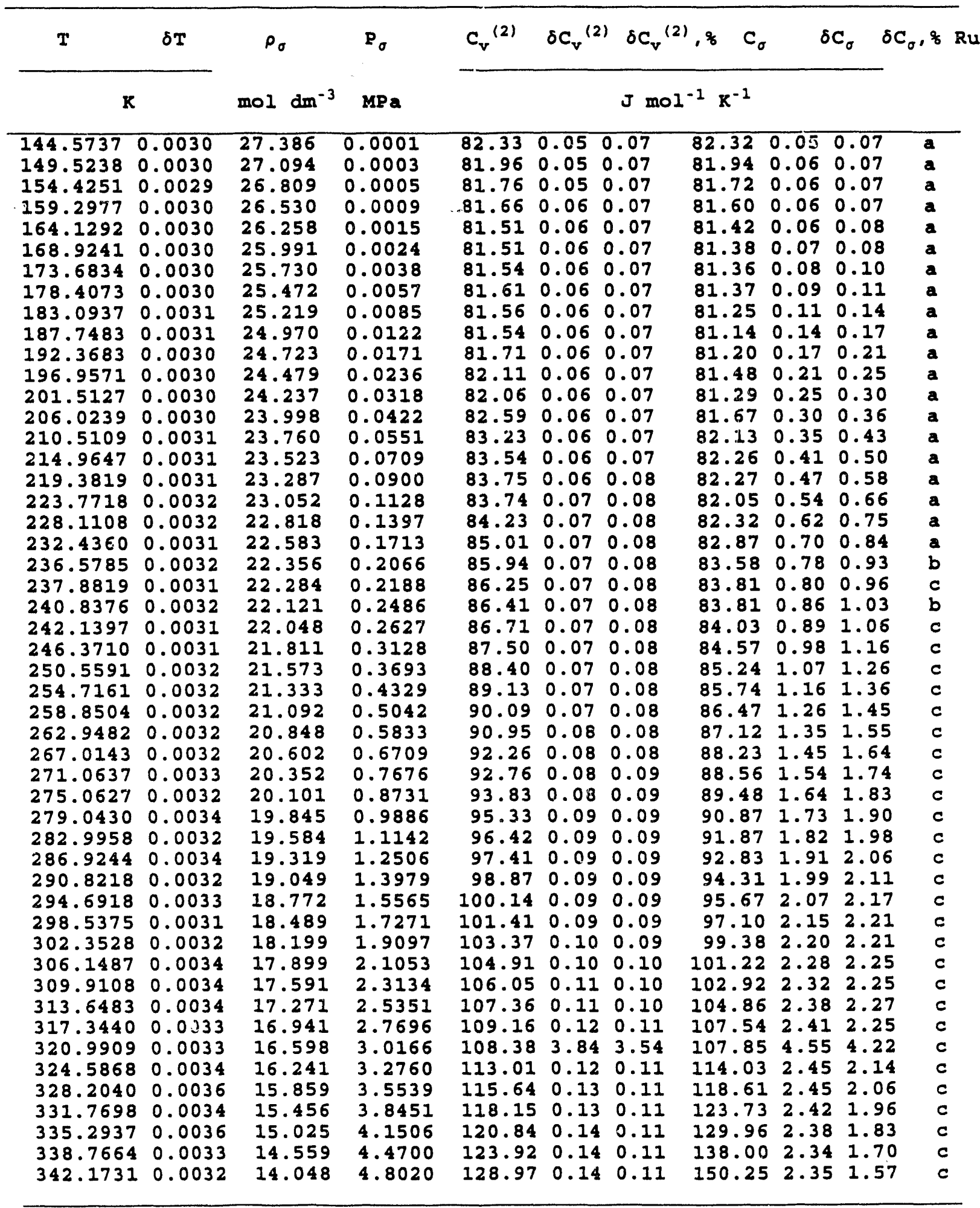


Table 19 (b). Experimental two-phase heat capacity data for HFC-32.

\begin{tabular}{|c|c|c|c|c|c|c|c|c|c|c|}
\hline$T$ & $\delta \mathbf{T}$ & & \multirow{2}{*}{$\begin{array}{l}P_{\sigma} \\
\text { paia }\end{array}$} & $C_{v}{ }^{(2)}$ & $\delta C_{v}^{(2)}$ & $\delta C_{v}^{(2)}$ & $\mathrm{C}_{\sigma}$ & $\delta C_{\sigma}$ & \multicolumn{2}{|c|}{$\delta \mathrm{C}_{\sigma}, \%$} \\
\hline$\circ \mathrm{E}$ & & $1 b f t^{-3}$ & & & & Btu $1 b^{-}$ & $-1 \cdot \mathrm{B}^{-1}$ & & & \\
\hline $\begin{array}{r}-199.437 \\
-190.527 \\
-181.705 \\
-172.934 \\
-164.237 \\
-155.607 \\
-147.040 \\
-138.537 \\
-130.101 \\
-121.723 \\
-113.407 \\
-105.147 \\
-96.947 \\
-88.827 \\
-80.750 \\
-72.734 \\
-64.783 \\
-56.881 \\
-49.071 \\
-41.285 \\
-33.829 \\
-31.483 \\
-26.162 \\
-23.819 \\
-16.202 \\
-8.664 \\
-1.181 \\
6.261 \\
13.637 \\
20.956 \\
28.245 \\
35.443 \\
42.607 \\
49.722 \\
56.794 \\
63.809 \\
70.775 \\
77.697 \\
84.565 \\
91.398 \\
158.169 \\
104.897 \\
111.549 \\
118.114 \\
124.586 \\
131.097 \\
137.516 \\
143.859 \\
-156.242\end{array}$ & $\begin{array}{l}0.005 \\
0.005 \\
0.005 \\
0.005 \\
0.005 \\
0.005 \\
0.005 \\
0.005 \\
0.006 \\
0.005 \\
0.005 \\
0.005 \\
0.005 \\
0.005 \\
0.006 \\
0.006 \\
0.006 \\
0.006 \\
0.006 \\
0.006 \\
0.006 \\
0.006 \\
0.006 \\
0.006 \\
0.006 \\
0.006 \\
0.006 \\
0.006 \\
0.006 \\
0.006 \\
0.006 \\
0.006 \\
0.006 \\
0.006 \\
0.006 \\
0.006 \\
0.006 \\
0.006 \\
0.006 \\
0.006 \\
0.006 \\
0.006 \\
0.006 \\
0.006 \\
0.006 \\
0.006 \\
0.006 \\
0.0066 \\
0.006\end{array}$ & $\begin{array}{l}88.94 \\
87.99 \\
87.07 \\
86.16 \\
85.28 \\
84.41 \\
83.56 \\
82.73 \\
81.91 \\
81.10 \\
80.29 \\
79.50 \\
78.72 \\
77.94 \\
77.17 \\
76.40 \\
75.63 \\
74.87 \\
74.11 \\
73.34 \\
72.61 \\
72.37 \\
71.84 \\
71.61 \\
70.84 \\
70.06 \\
69.29 \\
68.50 \\
67.71 \\
66.91 \\
66.10 \\
65.28 \\
64.45 \\
63.60 \\
62.74 \\
61.87 \\
60.97 \\
60.05 \\
59.11 \\
58.13 \\
57.13 \\
56.09 \\
55.02 \\
53.91 \\
52.75 \\
51.51 \\
50.20 \\
48.80 \\
17.28 \\
45.62\end{array}$ & $\begin{array}{l}0.02 \\
0.04 \\
0.07 \\
0.13 \\
0.22 \\
0.35 \\
0.55 \\
0.83 \\
1.23 \\
1.77 \\
2.48 \\
3.42 \\
4.61 \\
6.12 \\
7.99 \\
10.28 \\
13.05 \\
16.36 \\
20.26 \\
24.85 \\
29.97 \\
31.74 \\
36.06 \\
38.10 \\
45.36 \\
53.57 \\
62.79 \\
73.13 \\
84.60 \\
97.30 \\
111.33 \\
126.63 \\
143.38 \\
161.61 \\
181.39 \\
202.74 \\
225.75 \\
250.49 \\
276.98 \\
305.34 \\
335.54 \\
367.69 \\
401.70 \\
437.53 \\
475.15 \\
515.45 \\
557.68 \\
602.00 \\
648.32 \\
696.48\end{array}$ & $\begin{array}{l}0.37824 \\
0.37655 \\
0.37561 \\
0.37514 \\
0.37447 \\
0.37447 \\
0.37459 \\
0.37491 \\
0.37470 \\
0.37461 \\
0.37537 \\
0.37721 \\
0.37699 \\
0.37943 \\
0.38236 \\
0.38381 \\
0.38475 \\
0.38473 \\
0.38695 \\
0.39056 \\
0.39484 \\
0.39625 \\
0.39699 \\
0.39836 \\
0.40197 \\
0.40614 \\
0.40950 \\
0.41389 \\
0.41786 \\
0.42384 \\
0.42614 \\
0.43106 \\
0.43797 \\
0.44296 \\
0.44751 \\
0.45421 \\
0.55924 \\
0.46008 \\
0.4565 \\
0.54\end{array}$ & $\begin{array}{l}0.00025 \\
0.00025 \\
0.00025 \\
0.00026 \\
0.00026 \\
0.00026 \\
0.00026 \\
0.00026 \\
0.00027 \\
0.00027 \\
0.00027 \\
0.00027 \\
0.00027 \\
0.00028 \\
0.00028 \\
0.00029 \\
0.00029 \\
0.00030 \\
0.00031 \\
0.00031 \\
0.00031 \\
0.00031 \\
0.00032 \\
0.00031 \\
0.00032 \\
0.00033 \\
0.00034 \\
0.00034 \\
0.00035 \\
0.00036 \\
0.00037 \\
0.00037 \\
0.00040 \\
0.00039 \\
0.00042 \\
0.00041 \\
0.000043 \\
0.00042 \\
0.000044 \\
0.00048 \\
0.000049 \\
0.00051 \\
0.00053 \\
0.01764 \\
0.00055 \\
0.00060 \\
0.00059 \\
0.00063 \\
0.00062 \\
0.00064\end{array}$ & $\begin{array}{l}0.07 \\
0.07 \\
0.07 \\
0.07 \\
0.07 \\
0.07 \\
0.07 \\
0.07 \\
0.07 \\
0.07 \\
0.07 \\
0.07 \\
0.07 \\
0.07 \\
0.07 \\
0.07 \\
0.08 \\
0.08 \\
0.08 \\
0.08 \\
0.08 \\
0.08 \\
0.08 \\
0.08 \\
0.08 \\
0.08 \\
0.08 \\
0.08 \\
0.08 \\
0.08 \\
0.09 \\
0.09 \\
0.09 \\
0.09 \\
0.09 \\
0.09 \\
0.09 \\
0.09 \\
0.09 \\
0.10 \\
0.10 \\
0.10 \\
0.11 \\
3.54 \\
0.11 \\
0.11 \\
0.11 \\
0.111 \\
0.11 \\
0.11\end{array}$ & 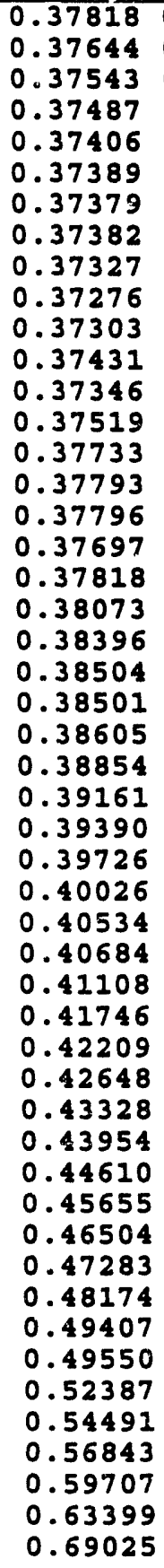 & $\begin{array}{l}0.00025 \\
0.00025 \\
0.00026 \\
0.00027 \\
0.00029 \\
0.00031 \\
0.00036 \\
0.00043 \\
0.00052 \\
0.00063 \\
0.00077 \\
0.00094 \\
0.00114 \\
0.00136 \\
0.00161 \\
0.00188 \\
0.00218 \\
0.00250 \\
0.00284 \\
0.00320 \\
0.00357 \\
0.00368 \\
0.00396 \\
0.00408 \\
0.00450 \\
0.00492 \\
0.00534 \\
0.00578 \\
0.00622 \\
0.00666 \\
0.00709 \\
0.00752 \\
0.00795 \\
0.00836 \\
0.00877 \\
0.00915 \\
0.00952 \\
0.00986 \\
0.01010 \\
0.01048 \\
0.01065 \\
0.01093 \\
0.01109 \\
0.02092 \\
0.01124 \\
0.01124 \\
0.01113 \\
0.01096 \\
0.01077 \\
0.01081\end{array}$ & $\begin{array}{l}0.07 \\
0.07 \\
0.07 \\
0.07 \\
0.08 \\
0.08 \\
0.10 \\
0.11 \\
0.14 \\
0.14 \\
0.17 \\
0.21 \\
0.25 \\
0.30 \\
0.36 \\
0.43 \\
0.50 \\
0.50\end{array}$ & $\begin{array}{l}a \\
a \\
a \\
a \\
a \\
a \\
a \\
a \\
a \\
a \\
a \\
a \\
a \\
a \\
a \\
a \\
a \\
a \\
a \\
a \\
b \\
c \\
b \\
c \\
c \\
c \\
c \\
c \\
c \\
c \\
c \\
c \\
c \\
c \\
c \\
c \\
c \\
c \\
c \\
c \\
c \\
c \\
c \\
c \\
c \\
c \\
c \\
c \\
c \\
c \\
c \\
a\end{array}$ \\
\hline
\end{tabular}


Table 20. Coefficients to the MBWR equation of state for HFC-32 (units are $\mathrm{K}, \mathrm{bar}, \mathrm{L}$, mol)

$$
\begin{aligned}
\mathrm{p}=\sum_{\mathrm{n}=1}^{9} \mathrm{a}_{\mathrm{n}} \rho^{\mathrm{n}}+\exp \left(-\rho^{2} / \rho_{\mathrm{c}}^{2}\right) \sum_{n=10}^{15} \mathrm{a}_{\mathrm{n}} \rho^{2 \mathrm{n}-17} \\
\rho_{\mathrm{c}}=8.1245 \mathrm{~mol} / \mathrm{L} \\
\mathrm{a}_{1}=\mathrm{RT} \\
\mathrm{a}_{2}=\mathrm{b}_{1} \mathrm{~T}+\mathrm{b}_{2} \mathrm{~T}^{0.5}+\mathrm{b}_{3}+\mathrm{b}_{4} / \mathrm{T}+\mathrm{b}_{5} / \mathrm{T}^{2} \\
\mathrm{a}_{3}=\mathrm{b}_{6} \mathrm{~T}+\mathrm{b}_{7}+\mathrm{b}_{8} / \mathrm{T}+\mathrm{b}_{9} / \mathrm{T}^{2} \\
\mathrm{a}_{4}=\mathrm{b}_{10} \mathrm{~T}+\mathrm{b}_{11}+\mathrm{b}_{12} / \mathrm{T} \\
\mathrm{a}_{5}=\mathrm{b}_{13} \\
\mathrm{a}_{6}=\mathrm{b}_{14} / \mathrm{T}+\mathrm{b}_{15} / \mathrm{T}^{2} \\
\mathrm{a}_{7}=\mathrm{b}_{16} / \mathrm{T} \\
\mathrm{a}_{8}=\mathrm{b}_{17} / \mathrm{T}+\mathrm{b}_{18} / \mathrm{T}^{2} \\
\mathrm{a}_{9}=\mathrm{b}_{19} / \mathrm{T}^{2} \\
\mathrm{a}_{10}=\mathrm{b}_{20} / \mathrm{T}^{2}+\mathrm{b}_{21} / \mathrm{T}^{3} \\
\mathrm{a}_{11}=\mathrm{b}_{22} / \mathrm{T}^{2}+\mathrm{b}_{23} / \mathrm{T}^{4} \\
\mathrm{a}_{12}=\mathrm{b}_{24} / \mathrm{T}^{2}+\mathrm{b}_{25} / \mathrm{T}^{3} \\
\mathrm{a}_{13}=\mathrm{b}_{26} / \mathrm{T}^{2}+\mathrm{b}_{27} / \mathrm{T}^{4} \\
\mathrm{a}_{14}=\mathrm{b}_{28} / \mathrm{T}^{2}+\mathrm{b}_{29} / \mathrm{T}^{3} \\
\mathrm{a}_{15}=\mathrm{b}_{30} / \mathrm{T}^{2}+\mathrm{b}_{31} / \mathrm{T}^{3}+\mathrm{b}_{32} / \mathrm{T}^{4} \\
\mathrm{i}
\end{aligned}
$$

$1-0.184799147712 \mathrm{E}-01$

$2 \quad 0.199258716261 \mathrm{E}+01$

$3-0.450818142855 \mathrm{E}+02$

$4 \quad 0.517320130169 \mathrm{E}+04$

$5-0.770847082500 \mathrm{E}+06$

$6-0.170184611963 \mathrm{E}-03$

$7-0.143023459131 \mathrm{E}+01$

$80.606314008455 \mathrm{E}+03$

$9 \quad 0.192559574847 \mathrm{E}+06$

$10-0.596044051707 \mathrm{E}-04$

$11 \quad 0.297147086969 \mathrm{E}+00$

$12-0.104964078480 \mathrm{E}+03$

$13-0.775008265186 \mathrm{E}-02$

$14 \quad 0.222564856042 \mathrm{E}+00$

$15-0.330783818273 \mathrm{E}+02$

$16-0.313533565119 \mathrm{E}-02$ 
Table 20. Coefficients to the MBWR equation of state for HFC-32 (units are $\mathrm{K}$, bar, $\mathrm{L}$, mol) (continued)

$17-0.399464119357 \mathrm{E}-04$

$18 \quad 0.653548292730 \mathrm{E}-01$

$19-0.119312200130 \mathrm{E}-02$

$20-0.896057555372 \mathrm{E}+05$

$21-0.218872108921 \mathrm{E}+08$

$22-0.189705435851 \mathrm{E}+04$

$23 \quad 0.310718784685 \mathrm{E}+08$

$24-0.126638710844 \mathrm{E}+02$

$25 \quad 0.246519270465 \mathrm{E}+04$

$26-0.231516734828 \mathrm{E}-01$

$27-0.438977929243 \mathrm{E}+04$

$28-0.315318636002 \mathrm{E}-03$

$29 \quad 0.139459067806 \mathrm{E}+00$

$30 \quad 0.163298486259 \mathrm{E}-06$

$31-0.326147254524 \mathrm{E}-03$

$32 \quad 0.342233333783 \mathrm{E}-01$ 


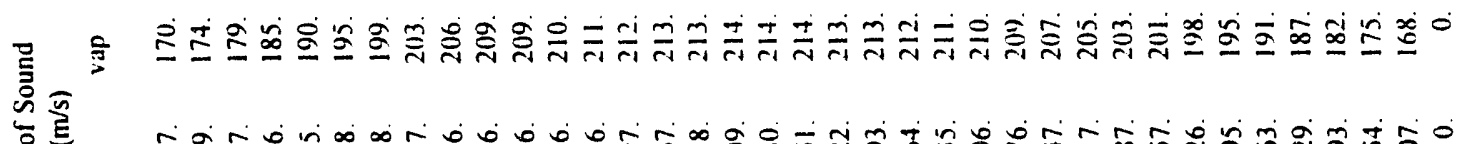

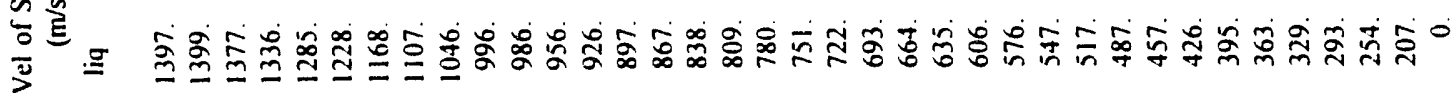

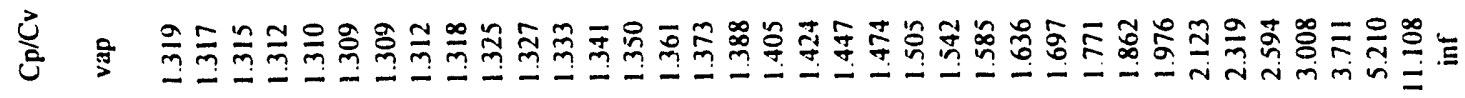

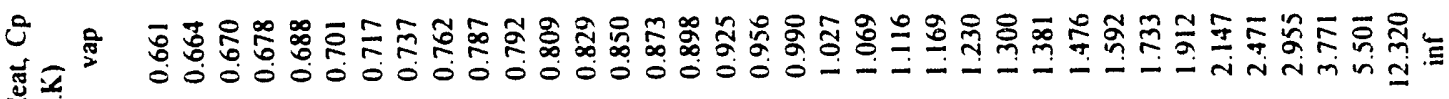

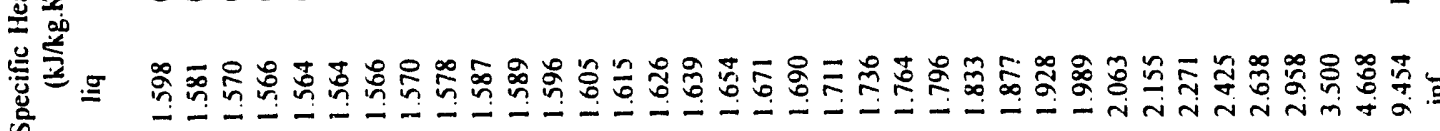

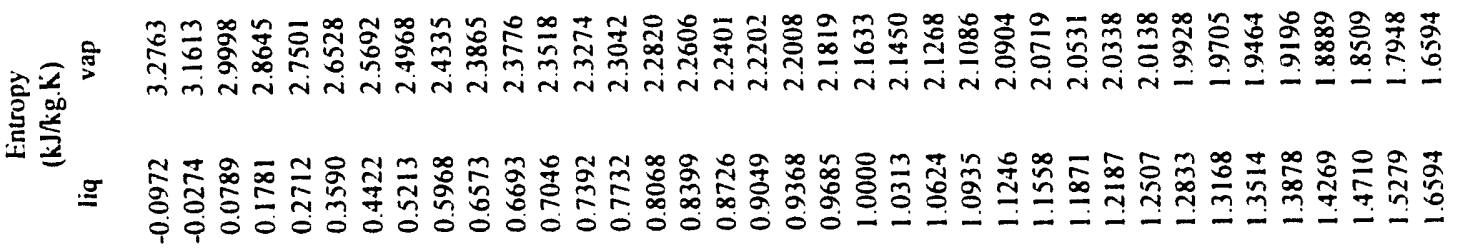

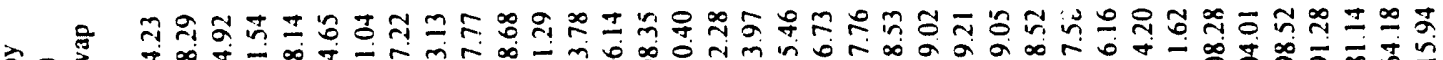

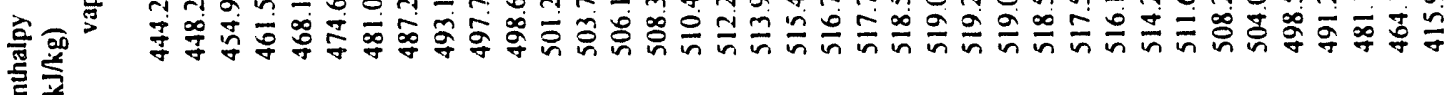

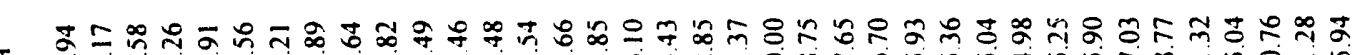

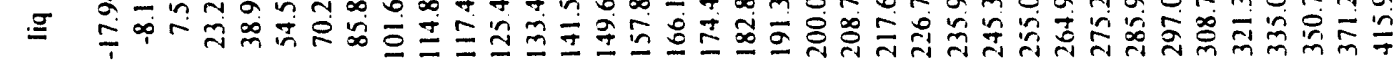

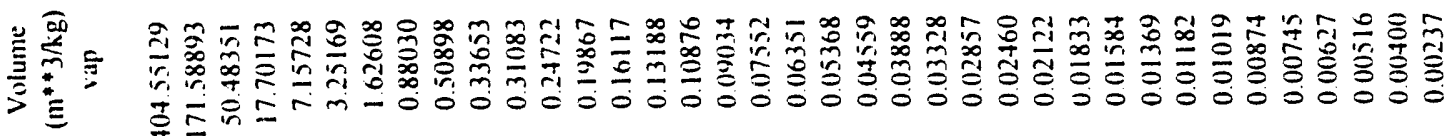

包

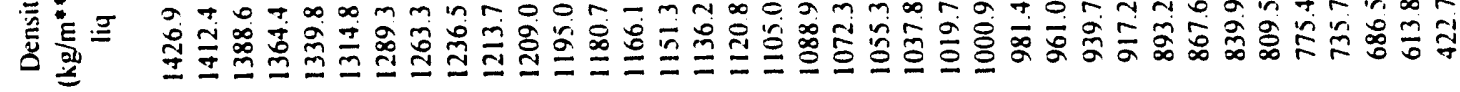

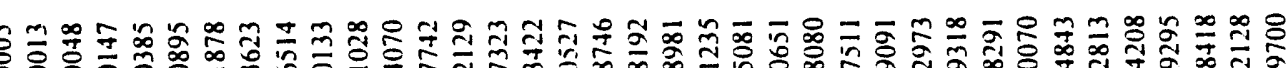

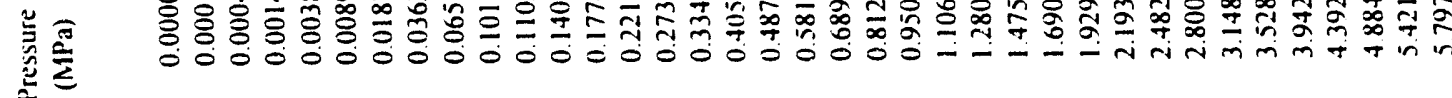

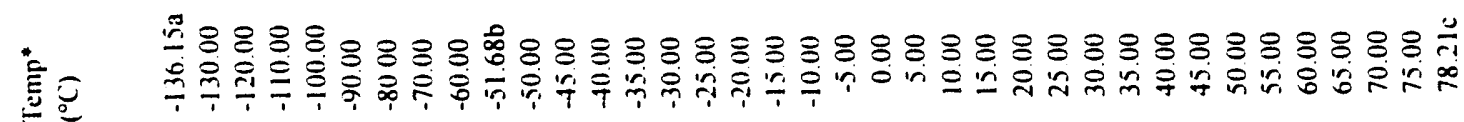




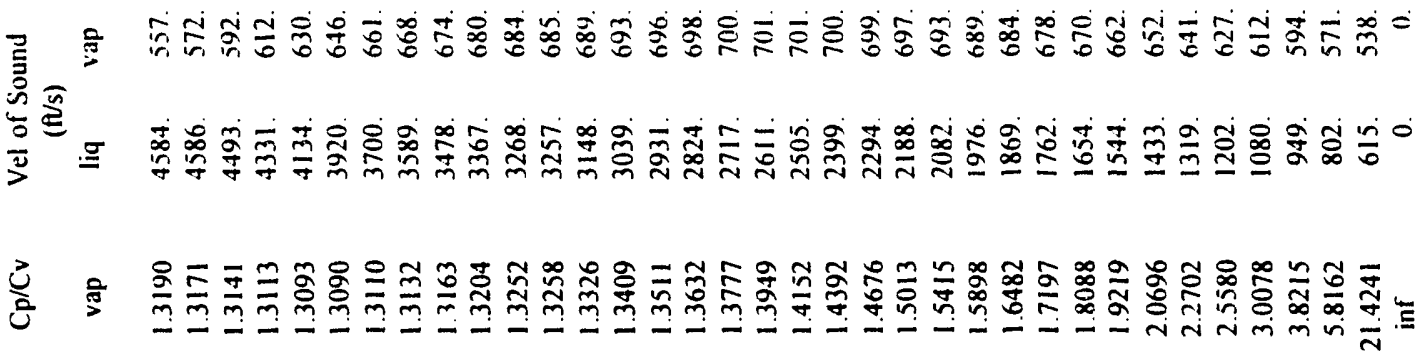

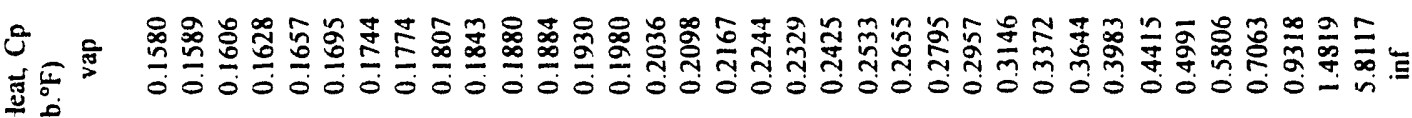

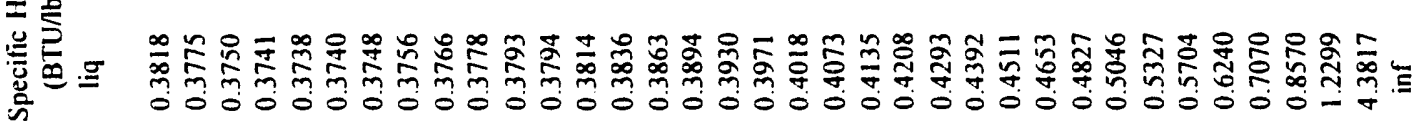

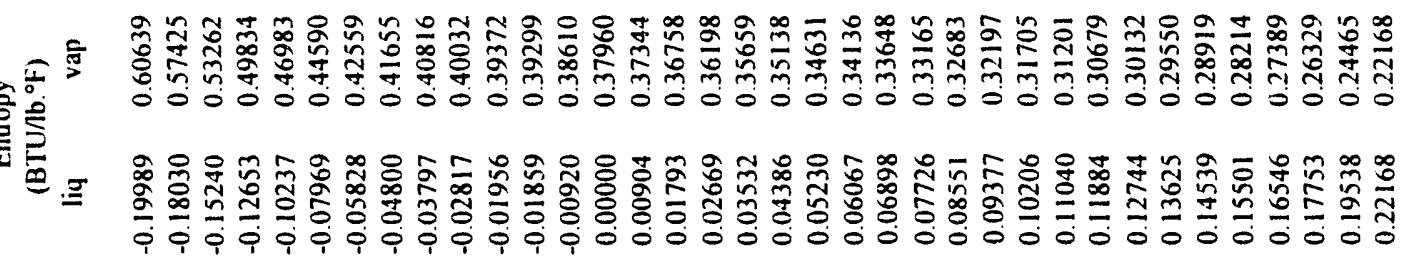

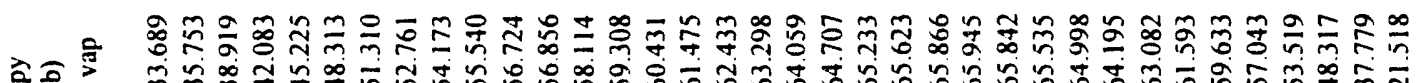

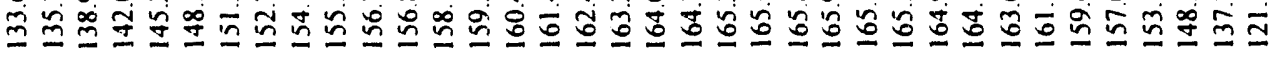

突

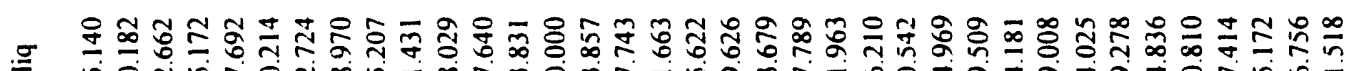

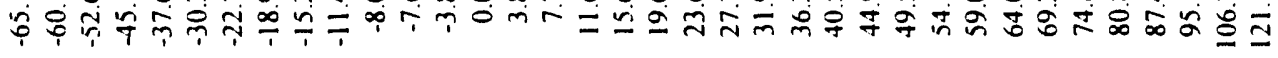

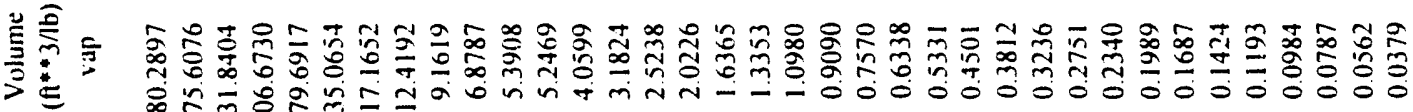

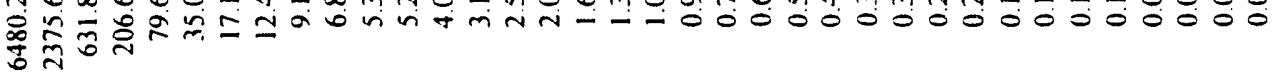

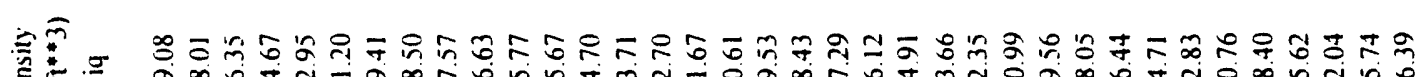

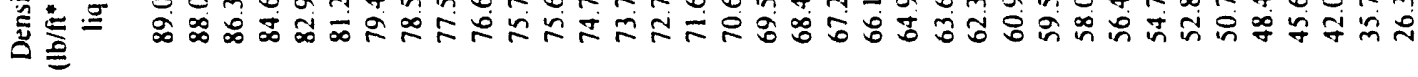

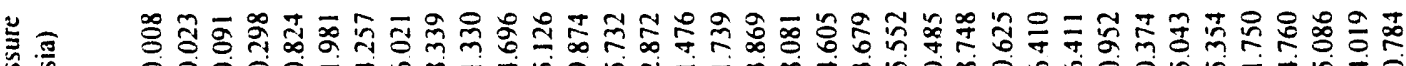

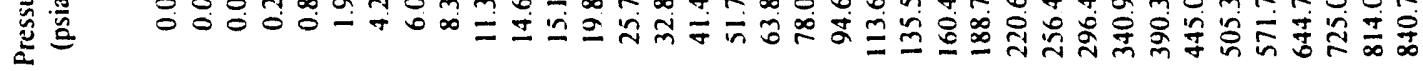

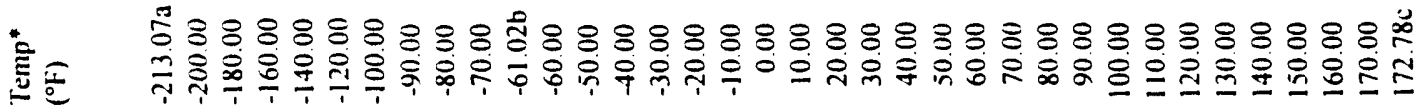


Table 22. Transient Hot-Wire Thermal Conductivity Data for HFC-32

\begin{tabular}{|c|c|c|c|c|c|c|}
\hline $\begin{array}{l}\text { Point } \\
\text { Number }\end{array}$ & $\begin{array}{l}T_{\text {Exp. }} \\
\text { (K) }\end{array}$ & $\begin{array}{l}\mathrm{P}_{\text {Cell }} \\
(\mathrm{MPa})\end{array}$ & $\begin{array}{c}\lambda_{\text {Exp. }} \\
(W / m-K)\end{array}$ & $\mathrm{T}_{\text {Exp. }}$ & $\begin{array}{l}P_{\text {Cell }} \\
\text { (psia) }\end{array}$ & $\begin{array}{c}\lambda_{\text {Exp. }} \\
(B T U / f t-h r-F)\end{array}$ \\
\hline 1001 & 162.295 & 65.155 & 0.24648 & -167.539 & 9449.98 & 0.14251 \\
\hline 1002 & 162.022 & 65.154 & 0.24693 & -168.030 & 9449.83 & 0.14277 \\
\hline 1003 & 161.770 & 65.160 & 0.24726 & -168.484 & 9450.73 & 0.14296 \\
\hline 1004 & 161.535 & 65.149 & 0.24772 & -168.907 & 9449.10 & 0.14323 \\
\hline 1005 & 162.355 & 54.444 & 0.24401 & -167.431 & 7896.47 & 0.14108 \\
\hline 1006 & 162.077 & 54.444 & 0.24449 & -167.931 & 7896.47 & 0.14136 \\
\hline 1007 & 161.819 & 54.433 & 0.24447 & -168.396 & 7894.88 & 0.14135 \\
\hline 1008 & 161.576 & 54.411 & 0.24487 & -168.833 & 7891.73 & 0.14158 \\
\hline 1009 & 162.402 & 42.614 & 0.24081 & -167.346 & 6180.71 & 0.13923 \\
\hline 1010 & 162.117 & 42.598 & 0.24134 & -167.859 & 6178.27 & 0.13954 \\
\hline 1011 & 161.859 & 42.580 & 0.24160 & -168.324 & 6175.71 & 0.13969 \\
\hline 1012 & 161.619 & 42.609 & 0.24174 & -168.756 & 6179.88 & 0.13977 \\
\hline 1013 & 161.950 & 31.583 & 0.23833 & -168.160 & 4580.71 & 0.13780 \\
\hline 1014 & 161.693 & 31.579 & 0.23877 & -168.623 & 4580.14 & 0.13805 \\
\hline 1015 & 161.453 & 31.574 & 0.23925 & -169.055 & 4579.41 & 0.13833 \\
\hline 1016 & 161.244 & 31.572 & 0.23960 & -169.431 & 4579.20 & 0.13853 \\
\hline 1017 & 161.994 & 21.269 & 0.23540 & -168.081 & 3084.86 & 0.13610 \\
\hline 1018 & 161.736 & 21.283 & 0.23597 & -168.545 & 3086.92 & 0.13643 \\
\hline 1019 & 161.473 & 21.284 & 0.23644 & -169.019 & 3087.00 & 0.13670 \\
\hline 1020 & 161.254 & 21.295 & 0.23686 & -169.413 & 3088.65 & 0.13695 \\
\hline 1021 & 161.997 & 11.839 & 0.23259 & -168.075 & 1717.07 & 0.13448 \\
\hline 1022 & 161.745 & 11.843 & 0.23324 & -168.529 & 1717.70 & 0.13485 \\
\hline 1023 & 161.498 & 11.850 & 0.23359 & -168.974 & 1718.72 & 0.13506 \\
\hline 1024 & 161.271 & 11.861 & 0.23413 & -169.382 & 1720.31 & 0.13537 \\
\hline 1025 & 162.046 & 1.765 & 0.22980 & -167.987 & 255.94 & 0.13287 \\
\hline 1026 & 161.786 & 1.765 & 0.23025 & -168.455 & 256.04 & 0.13313 \\
\hline 1027 & 161.539 & 1.759 & 0.23068 & -168.900 & 255.06 & 0.13337 \\
\hline 1028 & 161.316 & 1.758 & 0.23122 & -169.301 & 254.94 & 0.13369 \\
\hline 2001 & 182.783 & 65.593 & 0.23898 & -130.661 & 9513.46 & 0.13817 \\
\hline 2002 & 182.461 & 65.570 & 0.23966 & -131.240 & 9510.16 & 0.13857 \\
\hline 2003 & 182.159 & 65.563 & 0.23987 & -131.784 & 9509.18 & 0.13869 \\
\hline 2004 & 181.884 & 65.556 & 0.24049 & -132.279 & 9508.08 & 0.13905 \\
\hline 2005 & 182.853 & 54.418 & 0.23583 & -130.535 & 7892.63 & 0.13635 \\
\hline 2006 & 182.523 & 54.423 & 0.23636 & -131.129 & 7893.45 & 0.13666 \\
\hline 2007 & 182.221 & 54.432 & 0.23665 & -131.672 & 7894.67 & 0.13683 \\
\hline 2008 & 181.940 & 54.440 & 0.23681 & -132.178 & 7895.89 & 0.13692 \\
\hline 2009 & 182.631 & 42.987 & 0.23275 & -130.934 & 6234.70 & 0.13457 \\
\hline 2010 & 182.316 & 43.015 & 0.23316 & -131.501 & 6238.77 & 0.13481 \\
\hline 2011 & 182.025 & 43.024 & 0.23335 & -132.025 & 6240.07 & 0.13492 \\
\hline 2012 & 181.761 & 43.035 & 0.23338 & -132.500 & 6241.74 & 0.13494 \\
\hline 2013 & 182.702 & 31.577 & 0.22850 & -130.806 & 4579.93 & 0.13211 \\
\hline 2014 & 182.381 & 31.579 & 0.22909 & -131.384 & 4580.10 & 0.13246 \\
\hline 2015 & 182.089 & 31.592 & 0.22938 & -131.910 & 4582.12 & 0.13262 \\
\hline 2016 & 181.806 & 31.589 & 0.22993 & -132.419 & 4581.65 & 0.13294 \\
\hline 2017 & 182.460 & 21.233 & 0.22549 & -131.242 & 3079.64 & 0.13037 \\
\hline 2018 & 182.157 & 21.239 & 0.22603 & -131.787 & 3080.51 & 0.13069 \\
\hline 2019 & 181.869 & 21.254 & 0.22630 & -132.306 & 3082.68 & 0.13084 \\
\hline 2020 & 181.609 & 21.265 & 0.22696 & -132.774 & 3084.17 & 0.13122 \\
\hline $202 i$ & i 82.476 & $\mathrm{i} \mathrm{i} .5 \mathrm{i} 4$ & 0.22203 & -131.213 & 1669.91 & 0.12837 \\
\hline
\end{tabular}


Table 22. Transient Hot-Wire Thermal Conductivity Data for HFC-32 (Continued)

\begin{tabular}{|c|c|c|c|c|c|c|}
\hline $\begin{array}{l}\text { Point } \\
\text { Number }\end{array}$ & $\begin{array}{l}T_{\text {Exp. }} \\
(K)\end{array}$ & $\begin{array}{l}\mathrm{P}_{\mathrm{Cell}} \\
(\mathrm{MPa})\end{array}$ & $\begin{array}{c}\lambda_{\operatorname{Exp}_{.}} \\
(W / m-K)\end{array}$ & $\mathrm{T}_{\text {Exp. }}$ & $\begin{array}{l}\mathrm{P}_{\text {Cell }} \\
(\mathrm{p}, \mathrm{i})\end{array}$ & $\begin{array}{c}\lambda_{\operatorname{Exp}} \\
(B T U / f t-h r-F)\end{array}$ \\
\hline 2022 & 182.165 & 11.518 & 0.22253 & -131.773 & 1670.62 & 0.12866 \\
\hline 2023 & 181.877 & 11.528 & 0.22304 & -132.291 & 1672.06 & 0.12896 \\
\hline 2024 & 181.609 & 11.517 & 0.22321 & -132.774 & 1670.40 & 0.12906 \\
\hline 2025 & 182.520 & 1.718 & 0.21865 & -131.134 & 249.24 & 0.12642 \\
\hline 2026 & 182.200 & 1.717 & 0.21886 & -131.710 & 249.06 & 0.12654 \\
\hline 2027 & 181.912 & 1.719 & 0.21949 & -132.228 & 249.26 & 0.12690 \\
\hline 2028 & 181.637 & 1.717 & 0.21993 & -132.723 & 249.07 & 0.12716 \\
\hline 3001 & 202.724 & 66.681 & 0.23047 & -94.767 & 9671.29 & 0.13325 \\
\hline 3002 & 202.364 & 66.680 & 0.23085 & -95.415 & 9671.20 & 0.13347 \\
\hline 3003 & 202.031 & 66.673 & 0.23116 & -96.014 & 9670.13 & 0.13365 \\
\hline 3004 & 201.724 & 66.664 & 0.23197 & -96.567 & 9668.81 & 0.13412 \\
\hline 3005 & 202.767 & 56.361 & 0.22669 & -94.689 & 8174.43 & 0.13107 \\
\hline 3006 & 200.167 & 56.363 & 0.24185 & -99.369 & 8174.81 & 0.13983 \\
\hline 3007 & 199.839 & 56.372 & 0.26476 & -99.960 & 8176.13 & 0.15308 \\
\hline 3009 & 202.763 & 56.369 & 0.22665 & -94.697 & 8175.69 & 0.13104 \\
\hline 3010 & 202.404 & 56.372 & 0.22703 & -95.343 & 8176.07 & 0.13126 \\
\hline 3011 & 202.067 & 56.373 & 0.22745 & -95.949 & 8176.17 & 0.13151 \\
\hline 3012 & 201.750 & 56.381 & 0.22860 & -96.520 & 8177.38 & 0.13217 \\
\hline 3013 & 202.775 & 43.982 & 0.22221 & -94.675 & 6379.13 & 0.12848 \\
\hline 3014 & 202.406 & 43.984 & 0.22260 & -95.339 & 6379.39 & 0.12870 \\
\hline 3015 & 202.066 & 43.984 & 0.22298 & -95.951 & 6379.42 & 0.12892 \\
\hline 3016 & 201.748 & 44.006 & 0.22367 & -96.524 & 6392.55 & 0.12932 \\
\hline 3017 & 202.493 & 33.201 & 0.21838 & -95.183 & 4815.46 & 0.12626 \\
\hline 3018 & 202.137 & 33.216 & 0.21882 & -95.823 & 4817.65 & 0.12652 \\
\hline 3019 & 201.810 & 33.215 & 0.21913 & -96.412 & 4817.49 & 0.12670 \\
\hline 3020 & 201.506 & 33.222 & 0.21977 & -96.959 & 4818.48 & 0.12707 \\
\hline 3021 & 202.531 & 22.349 & 0.21412 & -95.114 & 3241.47 & 0.12380 \\
\hline 3022 & 202.172 & 22.366 & 0.21457 & -95.760 & 3243.89 & 0.12406 \\
\hline 3023 & 201.837 & 22.367 & 0.21495 & -96.363 & 3244.00 & 0.12428 \\
\hline 3024 & 201.532 & 22.377 & 0.21536 & -96.912 & 3245.48 & 0.12452 \\
\hline 3025 & 202.570 & 11.345 & 0.20912 & -95.044 & 1645.50 & 0.12091 \\
\hline 3026 & 202.203 & 11.352 & 0.20965 & -95.705 & 1646.43 & 0.12122 \\
\hline 3027 & 201.863 & 11.315 & 0.20997 & -96.317 & 1641.07 & 0.12140 \\
\hline 3028 & 201.548 & 11.318 & 0.21051 & -96.884 & 1641.52 & 0.12171 \\
\hline 3029 & 202.598 & 1.759 & 0.20500 & -94.994 & 255.17 & 0.11853 \\
\hline 3030 & 202.222 & 1.758 & 0.20556 & -95.670 & 255.01 & 0.11885 \\
\hline 3031 & 201.882 & 1.756 & 0.20566 & -96.282 & 254.69 & 0.11891 \\
\hline 3032 & 201.562 & 1.750 & 0.20579 & -96.858 & 253.85 & 0.11898 \\
\hline 10003 & 212.786 & 0.039 & 0.00778 & -76.655 & 5.59 & 0.00450 \\
\hline 10004 & 212.457 & 0.039 & 0.00760 & -77.247 & 5.59 & 0.00439 \\
\hline 10005 & 212.152 & 0.039 & 0.00733 & -77.796 & 5.61 & 0.00424 \\
\hline 10006 & 211.865 & 0.039 & 0.00705 & -78.313 & 5.59 & 0.00408 \\
\hline 10007 & 211.597 & 0.039 & 0.00682 & -78.795 & 5.59 & 0.00394 \\
\hline 10008 & 212.798 & 0.036 & 0.00815 & -76.634 & 5.25 & 0.00471 \\
\hline 10009 & 212.464 & 0.036 & 0.00814 & -77.235 & 5.25 & 0.00471 \\
\hline 10010 & 212.157 & 0.036 & 0.00815 & -77.787 & 5.25 & 0.00471 \\
\hline 10011 & 211.872 & 0.036 & 0.00812 & -78.300 & 5.27 & 0.00469 \\
\hline 10012 & 211.611 & 0.036 & 0.00803 & -78.770 & 5.28 & 0.00464 \\
\hline 10013 & 212.829 & 0.031 & 0.00790 & .76 .578 & 4.51 & 0.00457 \\
\hline
\end{tabular}


Table 22. Transient Hot-Wire Thermal Conductivity Data for HFC-32 (Continued)

\begin{tabular}{|c|c|c|c|c|c|c|}
\hline $\begin{array}{l}\text { Point } \\
\text { Number }\end{array}$ & $\begin{array}{l}T_{\text {Exp. }} \\
(\mathrm{K})\end{array}$ & $\begin{array}{l}\mathrm{P}_{\mathrm{Cell}} \\
(\mathrm{MPa})\end{array}$ & $\underset{(W / m-K)}{\lambda_{\text {Exp }}}$ & $\mathrm{T}_{\text {Exp. }}$ & $\begin{array}{l}P_{\text {Cell }} \\
\text { (psia) }\end{array}$ & $\begin{array}{c}\lambda_{\text {Exp. }} \\
\text { (BTU/ft-hr-F) }\end{array}$ \\
\hline 10014 & 212.489 & 0.031 & 0.00794 & -77.190 & 4.53 & 0.00459 \\
\hline 10015 & 212.182 & 0.032 & 0.00791 & -77.742 & 4.58 & 0.00457 \\
\hline 10016 & 211.887 & 0.032 & 0.00792 & -78.273 & 4.59 & 0.00458 \\
\hline 10017 & 211.618 & 0.032 & 0.00791 & -78.758 & 4.63 & 0.00457 \\
\hline 10018 & 212.450 & 0.027 & 0.00757 & -77.260 & 3.94 & 0.00438 \\
\hline 10019 & 212.155 & 0.027 & 0.00752 & -77.791 & 3.93 & 0.00435 \\
\hline 10020 & 211.862 & 0.027 & 0.00752 & -78.318 & 3.90 & 0.00435 \\
\hline 10021 & 211.583 & 0.027 & 0.00744 & -78.821 & 3.87 & 0.00430 \\
\hline 10022 & 211.317 & 0.027 & 0.00740 & -79.299 & 3.86 & 0.00428 \\
\hline 4001 & 223.147 & 65.496 & 0.21851 & -58.005 & 9499.49 & 0.12634 \\
\hline 4002 & 222.749 & 65.497 & 0.21883 & -58.722 & 9493.58 & 0.12652 \\
\hline 4003 & 222.386 & 65.492 & 0.21922 & -59.375 & 9498.90 & 0.12675 \\
\hline 4004 & 222.043 & 65.494 & 0.21952 & -59.993 & 9499.11 & 0.12692 \\
\hline 4005 & 223.179 & 55.074 & 0.21470 & -57.948 & 7987.87 & 0.12414 \\
\hline 4006 & 222.781 & 55.082 & 0.21492 & -58.664 & 7988.98 & 0.12426 \\
\hline 4007 & 222.410 & 55.084 & 0.21502 & -59.332 & 7989.34 & 0.12432 \\
\hline 4008 & 222.065 & 55.088 & 0.21537 & -59.953 & 7989.85 & 0.12452 \\
\hline 4009 & 223.209 & 44.988 & 0.21052 & -57.894 & 6524.93 & 0.12172 \\
\hline 4010 & 222.806 & 44.997 & 0.21052 & -58.619 & 6526.32 & 0.12172 \\
\hline 4011 & 222.434 & 44.998 & 0.21068 & -59.289 & 6526.40 & 0.12181 \\
\hline 4012 & 222.086 & 45.000 & 0.21124 & -59.915 & 6526.67 & 0.12213 \\
\hline 4013 & 223.249 & 34.030 & 0.20548 & -57.822 & 4935.69 & 0.11880 \\
\hline 4014 & 222.831 & 34.035 & 0.20577 & -58.574 & 4936.41 & 0.11897 \\
\hline 4015 & 222.444 & 34.038 & 0.20608 & -59.271 & 4936.78 & 0.11915 \\
\hline 4016 & 222.099 & 34.044 & 0.20640 & -59.892 & 4937.61 & 0.11934 \\
\hline 4018 & 222.937 & 22.298 & 0.20024 & -58.383 & 3234.11 & 0.11577 \\
\hline 4019 & 222.534 & 22.295 & 0.20065 & -59.109 & 3233.58 & 0.11601 \\
\hline 4020 & 222.171 & 22.291 & 0.20094 & -59.762 & 3233.11 & 0.11618 \\
\hline 4021 & 221.830 & 22.299 & 0.20160 & -60.376 & 3234.15 & 0.11656 \\
\hline 4022 & 222.975 & 10.963 & 0.19447 & -58.315 & 1590.07 & 0.11244 \\
\hline 4023 & 222.562 & 10.971 & 0.19528 & -59.058 & 1591.22 & 0.11291 \\
\hline 4024 & 222.187 & 10.970 & 0.19529 & -59.733 & 1591.04 & 0.11291 \\
\hline 4025 & 221.838 & 10.979 & 0.19565 & -60.362 & 1592.37 & 0.11312 \\
\hline 4026 & 223.009 & 1.713 & 0.18974 & -58.254 & 248.51 & 0.10970 \\
\hline 4027 & 222.591 & 1.715 & 0.18994 & -59.006 & 248.80 & 0.10982 \\
\hline 4028 & 222.206 & 1.717 & 0.19030 & -59.699 & 248.96 & 0.11003 \\
\hline 4029 & 221.853 & 1.718 & 0.19079 & -60.335 & 249.15 & 0.11031 \\
\hline 11001 & 222.899 & 0.034 & 0.00811 & -58.452 & 4.92 & 0.00469 \\
\hline 11002 & 220.500 & 0.034 & 0.00789 & -62.770 & 4.92 & 0.00456 \\
\hline 11003 & 220.084 & 0.034 & 0.00644 & -63.519 & 4.91 & 0.00372 \\
\hline 11004 & 221.822 & 0.034 & 0.00794 & -60.390 & 4.90 & 0.00459 \\
\hline 11005 & 225.449 & 0.034 & 0.00819 & -53.862 & 4.90 & 0.00474 \\
\hline 11006 & 223.555 & 0.041 & 0.00858 & -57.271 & 6.00 & 0.00496 \\
\hline 11007 & 223.137 & 0.040 & 0.00852 & -58.023 & 5.84 & 0.00493 \\
\hline 11008 & 222.748 & 0.039 & 0.00844 & -58.724 & 5.70 & 0.00488 \\
\hline 11009 & 222.373 & 0.039 & 0.00840 & -59.399 & 5.59 & 0.00486 \\
\hline 11011 & 223.054 & 0.076 & 0.00841 & -58.173 & 11.09 & 0.00486 \\
\hline 11012 & 222.666 & 0.076 & 0.00830 & -58.871 & 11.09 & 0.00480 \\
\hline & $222.31 \mathrm{i}$ & 0.076 & 0.00819 & $-59.5 \mathrm{io}$ & 11.08 & 0.00474 \\
\hline
\end{tabular}


Table 22. Transient Hot-Wire Thermal Conductivity Data for HFC-32 (Continued)

\begin{tabular}{|c|c|c|c|c|c|c|}
\hline $\begin{array}{l}\text { Point } \\
\text { Number }\end{array}$ & $\begin{array}{l}\mathrm{T}_{\text {Exp. }} \\
(\mathrm{K})\end{array}$ & $\begin{array}{l}\mathrm{P}_{\mathrm{Cell}} \\
(\mathrm{MPa})\end{array}$ & $\begin{array}{c}\lambda_{\text {Exp. }} \\
(W / m-K)\end{array}$ & $\mathrm{T}_{\text {Exp. }}$ & $\begin{array}{r}\mathrm{P}_{\text {Cell }} \\
\text { (psia) }\end{array}$ & $\begin{array}{c}\lambda_{\text {Exp. }} \\
\text { (BTU/ft-hr-F) }\end{array}$ \\
\hline 11014 & 221.977 & 0.076 & 0.00806 & -60.111 & 11.08 & 0.00466 \\
\hline 11015 & 222.968 & 0.059 & 0.00865 & -58.328 & 8.49 & 0.00500 \\
\hline 11016 & 222.581 & 0.059 & 0.00857 & -59.024 & 8.52 & 0.00496 \\
\hline 11017 & 222.211 & 0.059 & 0.00859 & -59.690 & 8.54 & 0.00497 \\
\hline 11018 & 221.901 & 0.059 & 0.00857 & -60.248 & 8.52 & 0.00496 \\
\hline 12001 & 233.092 & 0.127 & 0.00895 & -40.104 & 18.35 & 0.00517 \\
\hline 12002 & 232.683 & 0.126 & 0.00890 & -40.841 & 18.33 & 0.00515 \\
\hline 12003 & 232.309 & 0.126 & 0.00890 & -41.514 & 18.34 & 0.00515 \\
\hline 12004 & 231.976 & 0.126 & 0.00889 & -42.113 & 18.33 & 0.00514 \\
\hline 12005 & 233.119 & 0.117 & 0.00892 & -40.056 & 16.93 & 0.00516 \\
\hline 12006 & 232.693 & 0.116 & 0.00890 & -40.823 & 16.86 & 0.00515 \\
\hline 12007 & 232.317 & 0.116 & 0.00890 & -41.499 & 16.82 & 0.00515 \\
\hline 12008 & 231.974 & 11.116 & 0.00885 & -42.117 & 16.79 & 0.00512 \\
\hline 12009 & 233.180 & 0.105 & 0.00895 & -39.946 & 15.21 & 0.00517 \\
\hline 12010 & 232.757 & 0.105 & 0.00891 & -40.707 & 15.21 & 0.00515 \\
\hline 12011 & 232.379 & 0.105 & 0.00889 & -41.388 & 15.21 & 0.00514 \\
\hline 12012 & 232.031 & 0.105 & 0.00887 & -42.014 & 15.20 & 0.00513 \\
\hline 12013 & 233.206 & 0.094 & 0.00894 & -39.899 & 13.70 & 0.00517 \\
\hline 12014 & 232.781 & 0.095 & 0.00893 & -40.664 & 13.73 & 0.00516 \\
\hline 12015 & 232.405 & 0.095 & 0.00889 & -41.341 & 13.73 & 0.00514 \\
\hline 12016 & 232.050 & 0.095 & 0.00883 & -41.980 & 13.75 & 0.00511 \\
\hline 12017 & 233.275 & 0.072 & 0.00898 & -39.775 & 10.39 & 0.00519 \\
\hline 12018 & 232.836 & 0.072 & 0.00894 & -40.565 & 10.44 & 0.00517 \\
\hline 12019 & 232.449 & 0.072 & 0.00890 & -41.262 & 10.48 & 0.00515 \\
\hline 12020 & 232.081 & 0.073 & 0.00892 & -41.924 & 10.53 & 0.00516 \\
\hline 12021 & 233.409 & 0.043 & 0.00870 & -39.534 & 6.17 & 0.00503 \\
\hline 12022 & 232.962 & 0.042 & 0.00865 & -40.338 & 6.14 & 0.00500 \\
\hline 12023 & 232.549 & 0.042 & 0.00865 & -41.082 & 6.11 & 0.00500 \\
\hline 12024 & 232.157 & 0.042 & 0.00861 & -41.787 & 6.10 & 0.00498 \\
\hline 12025 & 231.813 & 0.042 & 0.00860 & -42.407 & 6.09 & 0.00497 \\
\hline 5001 & 243.398 & 66.849 & 0.20715 & -21.554 & 9695.72 & 0.11977 \\
\hline 5002 & 242.975 & 66.848 & 0.20767 & -22.315 & 9695.47 & 0.12007 \\
\hline 5003 & 242.591 & 66.841 & 0.20790 & -23.006 & 9694.49 & 0.12020 \\
\hline 5004 & 242.231 & 66.848 & 0.20853 & -23.654 & 9695.54 & 0.12057 \\
\hline 5005 & 243.450 & 56.194 & 0.20279 & -21.460 & 8150.33 & 0.11725 \\
\hline 5006 & 243.015 & 56.182 & 0.20350 & -22.243 & 8148.56 & 0.11766 \\
\hline 5007 & 242.618 & 56.211 & 0.20328 & -22.958 & 8152.77 & 0.11753 \\
\hline 5008 & 242.251 & 56.192 & 0.20371 & -23.618 & 8149.95 & 0.11778 \\
\hline 5009 & 243.474 & 45.386 & 0.19798 & -21.417 & 6582.67 & 0.11447 \\
\hline 5010 & 243.029 & 45.397 & 0.19859 & -22.218 & 6584.23 & 0.11482 \\
\hline 5011 & 242.628 & 45.394 & 0.19851 & -22.940 & 6583.81 & 0.11477 \\
\hline 5012 & 242.255 & 45.412 & 0.19850 & -23.611 & 6586.51 & 0.11477 \\
\hline 5013 & 243.123 & 34.152 & 0.19258 & -22.049 & 4953.35 & 0.11135 \\
\hline 5014 & 242.688 & 34.172 & 0.19329 & -22.832 & 4956.18 & 0.11176 \\
\hline 5015 & 242.299 & 34.160 & 0.19528 & -23.532 & 4954.56 & 0.11291 \\
\hline 5016 & 241.951 & 34.154 & 0.19605 & -24.158 & 4953.61 & 0.11335 \\
\hline 5017 & 243.133 & 24.483 & 0.18882 & -22.031 & 3551.01 & 0.10917 \\
\hline 5018 & 242.691 & 24.473 & 0.19113 & -22.826 & 3549.45 & 0.11051 \\
\hline 5019 & 242.310 & 24.472 & 0.19083 & $-23.5 i 2$ & 3549.33 & $0 . \mathrm{iic}$ \\
\hline
\end{tabular}


Table 22. Transient Hot-Wire Thermal Conductivity Data for HFC-32 (Continued)

\begin{tabular}{|c|c|c|c|c|c|c|}
\hline $\begin{array}{l}\text { Point } \\
\text { Number }\end{array}$ & $\begin{array}{l}\mathrm{T}_{\text {Exp. }} \\
(\mathrm{K})\end{array}$ & $\begin{array}{l}\mathrm{P}_{\mathrm{Cell}} \\
(\mathrm{MPa})\end{array}$ & $\underset{(W / m-K)}{\lambda_{\operatorname{Exp}}}$ & $\mathrm{T}_{\text {Exp. }}$ & $\begin{array}{r}\mathrm{P}_{\text {Cell }} \\
\text { (psia) }\end{array}$ & $\begin{array}{c}\lambda_{\text {Exp. }} \\
\text { (BTU/fthr-F) }\end{array}$ \\
\hline 5020 & 241.968 & 24.495 & 0.18795 & -24.128 & 3552.78 & 0.10867 \\
\hline 5021 & 243.149 & 14.148 & 0.18501 & -22.002 & 2052.05 & 0.10697 \\
\hline 5022 & 242.689 & 14.147 & 0.18948 & -22.830 & 2051.84 & 0.10955 \\
\hline 5023 & 242.322 & 14.153 & 0.18417 & -23.490 & 2052.75 & 0.10648 \\
\hline 5024 & 241.963 & 14.154 & 0.18165 & -24.137 & 2052.92 & 0.10503 \\
\hline 5026 & 243.211 & 1.802 & 0.18262 & -21.890 & 261.36 & 0.10559 \\
\hline 5027 & 242.786 & 1.804 & 0.17420 & -22.655 & 261.68 & 0.10072 \\
\hline 5028 & 242.347 & 1.798 & 0.17451 & -23.445 & 260.76 & 0.10090 \\
\hline 5029 & 241.961 & 1.802 & 0.17469 & -24.140 & 261.42 & 0.10100 \\
\hline 13001 & 245.033 & 0.184 & 0.00953 & -18.611 & 26.63 & 0.00551 \\
\hline 13002 & 244.559 & 0.181 & 0.00958 & -19.464 & 26.32 & 0.00554 \\
\hline 13003 & 244.102 & 0.180 & 0.00956 & -20.286 & 26.15 & 0.00553 \\
\hline 13004 & 243.660 & 0.179 & 0.00958 & -21.082 & 26.01 & 0.00554 \\
\hline 13005 & 245.025 & 0.201 & 0.00964 & -18.625 & 29.08 & 0.00557 \\
\hline 13006 & 244.544 & 0.198 & 0.00959 & -19.491 & 28.72 & 0.00554 \\
\hline 13007 & 244.098 & 0.196 & 0.00956 & -20.294 & 28.40 & 0.00553 \\
\hline 13008 & 243.655 & 0.194 & 0.00949 & -21.091 & 28.11 & 0.00549 \\
\hline 13009 & 245.414 & 0.131 & 0.00955 & -17.925 & 18.97 & 0.00552 \\
\hline 13010 & 244.901 & 0.130 & 0.00951 & -18.848 & 18.80 & 0.00550 \\
\hline 13011 & 244.411 & 0.129 & 0.00949 & -19.730 & 18.67 & 0.00549 \\
\hline 13012 & 243.943 & 0.128 & 0.00946 & -20.573 & 18.51 & 0.00547 \\
\hline 13013 & 243.495 & 0.127 & 0.00943 & -21.379 & 18.36 & 0.00545 \\
\hline 13014 & 243.085 & 0.126 & 0.00942 & -22.117 & 18.23 & 0.00545 \\
\hline 13015 & 244.335 & 0.053 & 0.00965 & -19.867 & 7.72 & 0.00558 \\
\hline 13016 & 243.846 & 0.053 & 0.00960 & -20.747 & 7.71 & 0.00555 \\
\hline 13017 & 243.387 & 0.053 & 0.00958 & -21.573 & 7.70 & 0.00554 \\
\hline 13018 & 242.952 & 0.053 & 0.00955 & -22.356 & 7.71 & 0.00552 \\
\hline 14001 & 255.208 & 0.303 & 0.01026 & -0.296 & 43.99 & 0.00593 \\
\hline 14002 & 254.754 & 0.301 & 0.01020 & -1.113 & 43.63 & 0.00590 \\
\hline 14003 & 254.306 & 0.298 & 0.01017 & -1.919 & 43.26 & 0.00588 \\
\hline 14004 & 253.881 & 0.296 & 0.01014 & -2.684 & 42.91 & 0.00586 \\
\hline 14005 & 253.040 & 0.276 & 0.01003 & -4.198 & 40.04 & 0.00580 \\
\hline 14006 & 252.677 & 0.272 & 0.01000 & -4.851 & 39.47 & 0.00578 \\
\hline 14007 & 252.328 & 0.269 & 0.00997 & -5.480 & 38.95 & 0.00576 \\
\hline 14008 & 252.000 & 0.265 & 0.00993 & -6.070 & 38.48 & 0.00574 \\
\hline 14009 & 253.193 & 0.192 & 0.00993 & -3.923 & 27.84 & 0.00574 \\
\hline 14010 & 252.816 & 0.189 & 0.00987 & -4.601 & 27.48 & 0.00571 \\
\hline 14011 & 252.444 & 0.187 & 0.00984 & -5.271 & 27.10 & 0.00569 \\
\hline 14012 & 252.094 & 0.185 & 0.00980 & -5.901 & 26.76 & 0.00567 \\
\hline 14013 & 253.326 & 0.140 & 0.00984 & -3.683 & 20.31 & 0.00569 \\
\hline 14014 & 252.927 & 0.138 & 0.00982 & -4.401 & 19.97 & 0.00568 \\
\hline 14015 & 252.534 & 0.135 & 0.00975 & -5.109 & 19.65 & 0.00564 \\
\hline 14016 & 252.174 & 0.133 & 0.00980 & -5.757 & 19.32 & 0.00567 \\
\hline 14017 & 253.705 & 0.061 & 0.00998 & -3.001 & 8.85 & 0.00577 \\
\hline 14018 & 253.254 & 0.060 & 0.00993 & -3.813 & 8.68 & 0.00574 \\
\hline 14019 & 252.830 & 0.059 & 0.00987 & -4.576 & 8.51 & 0.00571 \\
\hline 14020 & 252.442 & 0.057 & 0.00984 & -5.274 & 8.33 & 0.00569 \\
\hline 6001 & 263.141 & 67.169 & 0.19662 & 13.984 & 9742.08 & 0.11368 \\
\hline 6002 & 262.685 & 67.171 & 0.19707 & 13.163 & 9742.33 & $0.1 ! 394$ \\
\hline
\end{tabular}


Table 22. Transient Hot-Wire Thermal Conductivity Data for HFC-32 (Continued)

\begin{tabular}{|c|c|c|c|c|c|c|}
\hline $\begin{array}{l}\text { Point } \\
\text { Number }\end{array}$ & $\begin{array}{l}\mathrm{T}_{\text {Exp. }} \\
(\mathrm{K})\end{array}$ & $\begin{array}{l}\mathrm{P}_{\mathrm{Cell}} \\
(\mathrm{MPa})\end{array}$ & $\begin{array}{c}\lambda_{\text {Exp. }} \\
(W / m-K)\end{array}$ & ${ }^{T_{\text {Exp. }}}$ & $\begin{array}{l}\mathrm{P}_{\text {Cell }} \\
\text { (psia) }\end{array}$ & $\begin{array}{c}\lambda_{\text {Exp. }} \\
(B T U / f t-h r-F)\end{array}$ \\
\hline 6003 & 262.261 & 67.177 & 0.19761 & 12.400 & 9743.18 & 0.11425 \\
\hline 6004 & 261.880 & 67.178 & 0.19836 & 11.714 & 9743.36 & 0.11469 \\
\hline 6005 & 263.243 & 57.133 & 0.19177 & 14.167 & 8286.47 & 0.11088 \\
\hline 6006 & 262.768 & 57.138 & 0.19257 & 13.312 & 8287.25 & 0.11134 \\
\hline 6007 & 262.341 & 57.140 & 0.19292 & 12.544 & 8287.46 & 0.11154 \\
\hline 6008 & 261.954 & 57.146 & 0.19358 & 11.847 & 82.88 .42 & 0.11192 \\
\hline 6009 & 263.290 & 46.867 & 0.18697 & 14.252 & 6797.50 & 0.10810 \\
\hline 6010 & 262.819 & 46.873 & 0.18752 & 13.404 & 6798.37 & 0.10842 \\
\hline 6011 & 262.387 & 46.874 & 0.18785 & 12.627 & 6798.46 & 0.10861 \\
\hline 6012 & 261.987 & 46.886 & 0.18843 & 11.907 & 6800.23 & 0.10895 \\
\hline 6013 & 262.838 & 36.467 & 0.18207 & 13.438 & 5289.10 & 0.10527 \\
\hline 6014 & 262.386 & 36.478 & 0.18285 & 12.625 & 5290.77 & 0.10572 \\
\hline 6015 & 261.968 & 36.479 & 0.18324 & 11.872 & 5290.85 & 0.10595 \\
\hline 6016 & 261.596 & 36.505 & 0.18354 & 11.203 & 5294.67 & 0.10612 \\
\hline 6017 & 262.892 & 25.459 & 0.17547 & 13.536 & 3692.51 & 0.10145 \\
\hline 6018 & 262.430 & 25.465 & 0.17615 & 12.704 & 3693.33 & 0.10185 \\
\hline 6019 & 262.006 & 25.467 & 0.17648 & 11.941 & 3693.70 & 0.10204 \\
\hline 6020 & 261.626 & 25.469 & 0.17699 & 11.257 & 3693.96 & 0.10233 \\
\hline 6021 & 262.984 & 14.826 & 0.16829 & 13.701 & 2150.38 & 0.09730 \\
\hline 6022 & 262.505 & 14.830 & 0.16917 & 12.839 & 2150.92 & 0.09781 \\
\hline 6023 & 262.065 & 14.835 & 0.16924 & 12.047 & 2151.61 & 0.09785 \\
\hline 6024 & 261.673 & 14.839 & 0.17019 & 11.341 & 2152.24 & 0.09840 \\
\hline 6025 & 263.066 & 2.029 & 0.15848 & 13.849 & 294.32 & 0.09163 \\
\hline 6026 & 262.572 & 2.036 & 0.15900 & 12.960 & 295.23 & 0.09193 \\
\hline 6027 & 262.107 & 2.033 & 0.15947 & 12.123 & 294.81 & 0.09220 \\
\hline 6028 & 261.690 & 2.031 & 0.16017 & 11.372 & 294.51 & 0.09261 \\
\hline 6029 & 262.758 & 38.075 & 0.18303 & 13.294 & 5522.35 & 0.10582 \\
\hline 6030 & 262.306 & 38.057 & 0.18338 & 12.481 & 5519.72 & 0.10603 \\
\hline 6031 & 261.901 & 38.059 & 0.18391 & 11.752 & 5520.00 & 0.10633 \\
\hline 6032 & 261.522 & 38.064 & 0.18463 & 11.070 & 5520.67 & 0.10675 \\
\hline 15001 & 263.200 & 0.441 & 0.01092 & 14.090 & 63.90 & 0.00631 \\
\hline 15002 & 262.837 & 0.438 & 0.01090 & 13.437 & 63.52 & 0.00630 \\
\hline 15003 & 262.552 & 0.435 & 0.01082 & 12.924 & 63.15 & 0.00626 \\
\hline 15004 & 262.233 & 0.432 & 0.01086 & 12.349 & 62.71 & 0.00628 \\
\hline 15005 & 263.424 & 0.369 & 0.01074 & 14.493 & 53.59 & 0.00621 \\
\hline 15006 & 262.967 & 0.369 & 0.01075 & 13.671 & 53.56 & 0.00622 \\
\hline 15007 & 262.618 & 0.369 & 0.01072 & 13.042 & 53.56 & 0.00620 \\
\hline 15008 & 262.262 & 0.369 & 0.01069 & 12.402 & 53.54 & 0.00618 \\
\hline 15009 & 263.907 & 0.311 & 0.01054 & 15.363 & 45.06 & 0.00609 \\
\hline 15010 & 263.342 & 0.311 & 0.01054 & 14.346 & 45.10 & 0.00609 \\
\hline 15011 & 262.733 & 0.311 & 0.01054 & 13.249 & 45.12 & 0.00609 \\
\hline 15012 & 262.359 & 0.311 & 0.01054 & 12.576 & 45.15 & 0.00609 \\
\hline 15013 & 263.950 & 0.255 & 0.01039 & 15.440 & 36.93 & 0.00601 \\
\hline 15014 & 263.281 & 0.254 & 0.01040 & 14.236 & 36.91 & 0.00601 \\
\hline 15015 & 262.933 & 0.254 & 0.01040 & 13.609 & 36.90 & 0.00601 \\
\hline 15016 & 262.476 & 0.254 & 0.01040 & 12.787 & 36.88 & 0.00601 \\
\hline 15017 & 264.102 & 0.189 & 0.01047 & 15.714 & 27.47 & 0.00605 \\
\hline 15018 & 263.499 & 0.188 & 0.01042 & 14.628 & 27.33 & 0.00602 \\
\hline 15019 & 262.973 & 0.187 & 0.01035 & $i 3.68 i$ & 27.18 & 0.00598 \\
\hline
\end{tabular}


Table 22. Transient Hot-Wire Thermal Conductivity Data for HFC-32 (Continued)

\begin{tabular}{|c|c|c|c|c|c|c|}
\hline $\begin{array}{l}\text { Point } \\
\text { Number }\end{array}$ & $\begin{array}{l}T_{\text {Exp. }} \\
(\mathrm{K})\end{array}$ & $\begin{array}{l}\mathrm{P}_{\mathrm{Cell}} \\
(\mathrm{MPa})\end{array}$ & $\begin{array}{c}\lambda_{\text {Exp. }} \\
(\mathrm{W} / \mathrm{m}-\mathrm{K})\end{array}$ & $\mathrm{T}_{\text {Exp. }}$ & $\begin{array}{l}P_{\text {Cell }} \\
\text { (psia) }\end{array}$ & $\frac{\lambda_{\text {Exp. }}}{(\mathrm{BTU} / \mathrm{ft}-\mathrm{hr}-\mathrm{F})}$ \\
\hline 15020 & 262.577 & 0.187 & 0.01030 & 12.969 & 27.10 & 0.00596 \\
\hline 15021 & 264.386 & 0.125 & 0.01053 & 16.225 & 18.09 & 0.00609 \\
\hline 15022 & 263.590 & 0.124 & 0.01055 & 14.792 & 17.99 & 0.00610 \\
\hline 15023 & 263.115 & 0.123 & 0.01051 & 13.937 & 17.88 & 0.00608 \\
\hline 15024 & 262.708 & 0.123 & 0.01035 & 13.204 & 17.79 & 0.00598 \\
\hline 15025 & 264.367 & 0.081 & 0.01063 & 16.191 & 11.72 & 0.00615 \\
\hline 15026 & 263.716 & 0.080 & 0.01064 & 15.019 & 11.63 & 0.00615 \\
\hline 15027 & 263.234 & 0.079 & 0.01060 & 14.151 & 11.52 & 0.00613 \\
\hline 15028 & 262.825 & 0.079 & 0.01056 & 13.415 & 11.46 & 0.00611 \\
\hline 16001 & 272.887 & 0.671 & 0.01187 & 31.527 & 97.30 & 0.00686 \\
\hline 16002 & 272.547 & 0.667 & 0.01184 & 30.915 & 96.80 & 0.00685 \\
\hline 16003 & 272.229 & 0.664 & 0.01187 & 30.342 & 96.25 & 0.00686 \\
\hline 16004 & 271.934 & 0.660 & 0.01186 & 29.811 & 95.73 & 0.00686 \\
\hline 16005 & 272.959 & 0.550 & 0.01160 & 31.656 & 79.72 & 0.00671 \\
\hline 16006 & 272.599 & 0.550 & 0.01158 & 31.008 & 79.71 & 0.00670 \\
\hline 16007 & 272.291 & 0.550 & 0.01153 & 30.454 & 79.74 & 0.00667 \\
\hline 16008 & 271.977 & 0.550 & 0.01155 & 29.889 & 79.74 & 0.00668 \\
\hline 16009 & 273.107 & 0.479 & 0.01142 & 31.923 & 69.54 & 0.00660 \\
\hline 16010 & 272.674 & 0.479 & 0.01141 & 31.143 & 69.51 & 0.00660 \\
\hline 16011 & 272.362 & 0.479 & 0.01137 & 30.582 & 69.48 & 0.00657 \\
\hline 16012 & 272.060 & 0.479 & 0.01135 & 30.038 & 69.48 & 0.00656 \\
\hline 16013 & 273.166 & 0.408 & 0.01129 & 32.029 & 59.12 & 0.00653 \\
\hline 16014 & 272.772 & 0.407 & 0.01127 & 31.320 & 59.04 & 0.00652 \\
\hline 16015 & 272.445 & 0.407 & 0.01121 & 30.731 & 58.99 & 0.00648 \\
\hline 16016 & 272.130 & 0.407 & 0.01121 & 30.164 & 58.96 & 0.00648 \\
\hline 16017 & 273.331 & 0.333 & 0.01117 & 32.326 & 48.35 & 0.00646 \\
\hline 16018 & 272.894 & 0.333 & 0.01112 & 31.539 & 48.28 & 0.00643 \\
\hline 16019 & 272.539 & 0.332 & 0.01111 & 30.900 & 48.22 & 0.00642 \\
\hline 16020 & 272.199 & 0.332 & 0.01108 & 30.288 & 48.18 & 0.00641 \\
\hline 16021 & 273.412 & 0.265 & 0.01120 & 32.472 & 38.38 & 0.00648 \\
\hline 16022 & 273.004 & 0.264 & 0.01116 & 31.737 & 38.26 & 0.00645 \\
\hline 16023 & 272.634 & 0.263 & 0.01111 & 31.071 & 38.21 & 0.00642 \\
\hline 16024 & 272.345 & 0.263 & 0.01097 & 30.551 & 38.17 & 0.00634 \\
\hline 16025 & 273.557 & 0.197 & 0.01119 & 32.733 & 28.64 & 0.00647 \\
\hline 16026 & 273.104 & 0.197 & 0.01111 & 31.917 & 28.57 & 0.00642 \\
\hline 16027 & 272.730 & 0.196 & 0.01111 & 31.244 & 28.44 & 0.00642 \\
\hline 16028 & 272.401 & 0.196 & 0.01098 & 30.652 & 28.37 & 0.00635 \\
\hline 16029 & 273.656 & 0.141 & 0.01122 & 32.911 & 20.43 & 0.00649 \\
\hline 16030 & 273.233 & 0.140 & 0.01126 & 32.149 & 20.29 & 0.00651 \\
\hline 16031 & 272.868 & 0.139 & 0.01121 & 31.492 & 20.18 & 0.00648 \\
\hline 16032 & 272.542 & 0.138 & 0.01101 & 30.906 & 20.07 & 0.00637 \\
\hline 16033 & 273.776 & 0.094 & 0.01137 & 33.127 & 13.67 & 0.00657 \\
\hline 16034 & 273.349 & 0.094 & 0.01165 & 32.358 & 13.63 & 0.00674 \\
\hline 16035 & 272.944 & 0.094 & 0.01133 & 31.629 & 13.59 & 0.00655 \\
\hline 16036 & 272.573 & 0.093 & 0.01117 & 30.961 & 13.52 & 0.00646 \\
\hline 7001 & 282.873 & 67.315 & 0.18737 & 49.501 & 9763.30 & 0.10833 \\
\hline 7002 & 282.428 & 67.305 & 0.18832 & 48.700 & 9761.79 & 0.10888 \\
\hline 7003 & 282.025 & 67.304 & 0.18809 & 47.975 & 9761.68 & 0.10875 \\
\hline 7004 & $28 ! .640$ & 67.303 & 0.18928 & 47.282 & 9761.52 & 0.10944 \\
\hline
\end{tabular}


Table 22. Transient Hot-Wire Thermal Conductivity Data for HFC-32 (Continued)

\begin{tabular}{|c|c|c|c|c|c|c|}
\hline $\begin{array}{l}\text { Point } \\
\text { Number }\end{array}$ & $\begin{array}{l}\mathrm{T}_{\text {Exp. }} \\
(\mathrm{K})\end{array}$ & $\begin{array}{l}\mathrm{P}_{\mathrm{Cell}} \\
(\mathrm{MPa})\end{array}$ & $\begin{array}{c}\lambda_{\text {Exp. }} \\
(W / m-K)\end{array}$ & $\mathrm{T}_{\text {Exp. }}$ & $\begin{array}{l}\mathrm{P}_{\text {Cell }} \\
\text { (psia) }\end{array}$ & $\begin{array}{c}\lambda_{\text {Exp. }} \\
\text { (BTU/ft-hr-F) }\end{array}$ \\
\hline 7005 & 282.886 & 55.555 & 0.18103 & 49.525 & 8057.60 & 0.10467 \\
\hline 7006 & 282.419 & 55.553 & 0.18186 & 48.684 & 8057.35 & 0.10515 \\
\hline 7007 & 282.001 & 55.558 & 0.18207 & 47.932 & 8058.04 & 0.10527 \\
\hline 7008 & 281.622 & 55.560 & 0.18301 & 47.250 & 8058.28 & 0.10581 \\
\hline 7009 & 282.877 & 45.226 & 0.175 .55 & 49.509 & 6559.43 & 0.10150 \\
\hline 7010 & 282.414 & 45.229 & 0.17632 & 48.675 & 6559.96 & 0.10194 \\
\hline 7011 & 281.987 & 45.233 & 0.17599 & 47.907 & 6560.50 & 0.10175 \\
\hline 7012 & 281.596 & 45.233 & 0.17688 & 47.203 & 6560.55 & 0.10227 \\
\hline 7013 & 282.895 & 34.958 & 0.16907 & 49.541 & 5070.28 & 0.09775 \\
\hline 7014 & 282.421 & 34.964 & 0.16947 & 48.688 & 5071.14 & 0.09798 \\
\hline 7015 & 281.965 & 34.971 & 0.17028 & 47.867 & 5072.14 & 0.09845 \\
\hline 7016 & 281.563 & 34.972 & 0.17066 & 47.143 & 5072.35 & 0.09867 \\
\hline 7017 & 282.611 & 24.274 & 0.16150 & 49.030 & 3520.69 & 0.09338 \\
\hline 7018 & 282.139 & 24.277 & 0.16247 & 48.180 & 3521.10 & 0.09394 \\
\hline 7019 & 281.712 & 24.277 & 0.16325 & 47.412 & 3521.07 & 0.09439 \\
\hline 7020 & 281.326 & 24.284 & 0.16350 & 46.717 & 3522.08 & 0.09453 \\
\hline 7021 & 282.621 & 13.595 & 0.15320 & 49.048 & 1971.85 & 0.08858 \\
\hline 7022 & 282.134 & 13.593 & 0.15407 & 48.171 & 1971.46 & 0.08908 \\
\hline 7023 & 281.692 & 13.593 & 0.15494 & 47.376 & 1971.49 & 0.08958 \\
\hline 7024 & 281.291 & 13.596 & 0.15580 & 46.654 & 1971.88 & 0.09008 \\
\hline 7025 & 282.813 & 1.920 & 0.14201 & 49.393 & 278.54 & 0.08211 \\
\hline 7026 & 282.302 & 1.920 & 0.14285 & 48.474 & 278.48 & 0.08259 \\
\hline 7027 & 281.826 & 1.919 & 0.14362 & 47.617 & 278.37 & 0.08304 \\
\hline 7028 & 281.406 & 1.920 & 0.14454 & 46.861 & 278.43 & 0.08357 \\
\hline 17001 & 283.270 & 0.915 & 0.01295 & 50.216 & 132.72 & 0.00749 \\
\hline 17002 & 282.955 & 0.915 & 0.01292 & 49.649 & 132.69 & 0.00747 \\
\hline 17003 & 282.664 & 0.914 & 0.01286 & 49.125 & 132.63 & 0.00744 \\
\hline 17004 & 282.342 & 0.915 & 0.01294 & 48.546 & 132.72 & 0.00748 \\
\hline 17005 & 283.390 & 0.810 & 0.01269 & 50.432 & 117.44 & 0.00734 \\
\hline 17006 & 283.068 & 0.811 & 0.01263 & 49.852 & 117.58 & 0.00730 \\
\hline 17007 & 282.748 & 0.811 & 0.01264 & 49.276 & 117.68 & 0.00731 \\
\hline 17008 & 282.429 & 0.812 & 0.01260 & 48.702 & 117.72 & 0.00729 \\
\hline 17009 & 283.514 & 0.672 & 0.01232 & 50.655 & 97.52 & 0.00712 \\
\hline 17010 & 283.166 & 0.672 & 0.01235 & 50.029 & 97.46 & 0.00714 \\
\hline 17011 & 282.847 & 0.672 & 0.01231 & 49.455 & 97.40 & 0.00712 \\
\hline i7012 & 282.515 & 0.671 & 0.01221 & 48.857 & 97.36 & 0.00706 \\
\hline 17013 & 283.528 & 0.529 & 0.01211 & 50.680 & 76.66 & 0.00700 \\
\hline 17014 & 283.145 & 0.528 & 0.01212 & 49.991 & 76.59 & 0.00701 \\
\hline 17015 & 282.807 & 0.527 & 0.01207 & 49.383 & 76.50 & 0.00698 \\
\hline 17016 & 282.467 & 0.527 & 0.01198 & 48.771 & 76.43 & 0.00693 \\
\hline 17017 & 283.707 & 0.375 & 0.01198 & 51.003 & 54.43 & 0.00693 \\
\hline 17018 & 283.324 & 0.374 & 0.01196 & 50.313 & 54.19 & 0.00692 \\
\hline 17019 & 282.961 & 0.373 & 0.01194 & 49.660 & 54.06 & 0.00690 \\
\hline 17020 & 282.614 & 0.372 & 0.01187 & 49.035 & 53.92 & 0.00686 \\
\hline 17021 & 283.993 & 0.273 & 0.01193 & 51.517 & 39.59 & 0.00690 \\
\hline 17022 & 283.598 & 0.272 & 0.01186 & 50.806 & 39.43 & 0.00686 \\
\hline 17023 & 283.196 & 0.270 & 0.01187 & 50.083 & 39.22 & 0.00686 \\
\hline 17024 & 282.820 & 0.269 & 0.01185 & 49.406 & 39.06 & 0.00685 \\
\hline 17025 & 283.740 & 0.174 & 0.01186 & 51.062 & 25.23 & 0.00686 \\
\hline
\end{tabular}


Table 22. Transient Hot-Wire Thermal Conductivity Data for HFC-32 (Continued)

\begin{tabular}{|c|c|c|c|c|c|c|}
\hline $\begin{array}{l}\text { Point } \\
\text { Number }\end{array}$ & $\begin{array}{l}\mathrm{T}_{\text {Exp. }} \\
(\mathrm{K})\end{array}$ & $\begin{array}{l}\mathrm{P}_{\mathrm{Cell}} \\
(\mathrm{MPa})\end{array}$ & $\begin{array}{c}\lambda_{\operatorname{Exp}} \\
(W / m-K)\end{array}$ & $\mathrm{T}_{\text {Exp. }}$ & $\begin{array}{l}\mathrm{P}_{\text {Cell }} \\
\text { (psia) }\end{array}$ & $\begin{array}{c}\lambda_{\text {Exp. }} \\
(B T U / \hat{t} \cdot h r-F)\end{array}$ \\
\hline 17026 & 283.317 & 0.173 & 0.01192 & 50.301 & 25.14 & 0.00689 \\
\hline 17027 & 282.930 & 0.173 & 0.01186 & 49.604 & 25.07 & 0.00686 \\
\hline 17028 & 282.564 & 0.172 & 0.01190 & 48.945 & 24.98 & 0.00688 \\
\hline 17029 & 283.379 & 0.117 & 0.01208 & 50.412 & 17.02 & 0.00698 \\
\hline 17030 & 282.995 & 0.117 & 0.01205 & 49.721 & 16.99 & 0.00697 \\
\hline 17031 & 282.615 & 0.117 & 0.01198 & 49.037 & 16.99 & 0.00693 \\
\hline 17032 & 282.250 & 0.117 & 0.01206 & 48.380 & 16.96 & 0.00697 \\
\hline 18001 & 293.104 & 1.264 & 0.01426 & 67.917 & 183.35 & 0.00824 \\
\hline 18002 & 292.778 & 1.259 & 0.01425 & 67.330 & 182.64 & 0.00824 \\
\hline 18003 & 292.485 & 1.255 & 0.01427 & 66.803 & 181.98 & 0.00825 \\
\hline 18004 & 292.208 & 1.250 & 0.01427 & 66.304 & 181.31 & 0.00825 \\
\hline 18005 & 293.064 & 1.222 & 0.01414 & 67.845 & 177.28 & 0.00818 \\
\hline 18006 & 292.792 & 1.217 & 0.01411 & 67.356 & 176.54 & 0.00816 \\
\hline 18007 & 292.489 & 1.212 & 0.01410 & 66.810 & 175.73 & 0.00815 \\
\hline 18008 & 292.207 & 1.206 & 0.01411 & 66.303 & 174.93 & 0.00816 \\
\hline 18009 & 293.367 & 1.070 & 0.01373 & 68.391 & 155.16 & 0.00794 \\
\hline 18010 & 292.966 & 1.069 & 0.01376 & 67.669 & 155.10 & 0.00796 \\
\hline 18011 & 292.692 & 1.069 & 0.01372 & 67.176 & 155.04 & 0.00793 \\
\hline 18012 & 292.398 & 1.069 & 0.01365 & 66.646 & 155.03 & 0.00789 \\
\hline 18013 & 293.417 & 0.948 & 0.01347 & 68.481 & 137.43 & 0.00779 \\
\hline 18014 & 293.075 & 0.948 & 0.01346 & 67.865 & 137.55 & 0.00778 \\
\hline 18015 & 292.760 & 0.949 & 0.01344 & 67.298 & 137.61 & 0.00777 \\
\hline 18016 & 292.454 & 0.949 & 0.01336 & 66.747 & 137.62 & 0.00772 \\
\hline 18017 & 293.638 & 0.800 & 0.01315 & 68.878 & 116.10 & 0.00760 \\
\hline 18018 & 293.264 & 0.800 & 0.01314 & 68.205 & 116.10 & 0.00760 \\
\hline 18019 & 292.933 & 0.801 & 0.01312 & 67.609 & 116.22 & 0.00759 \\
\hline 18020 & 292.612 & 0.803 & 0.01307 & 67.032 & 116.49 & 0.00756 \\
\hline 18021 & 293.391 & 0.653 & 0.01287 & 68.434 & 94.78 & 0.00744 \\
\hline 18022 & 293.009 & 0.653 & 0.01289 & 67.746 & 94.72 & 0.00745 \\
\hline 18023 & 292.672 & 0.653 & 0.01284 & 67.140 & 94.64 & 0.00742 \\
\hline 18024 & 292.351 & 0.652 & 0.01280 & 66.562 & 94.61 & 0.00740 \\
\hline 18025 & 293.123 & 0.532 & 0.01269 & 67.951 & 77.19 & 0.00734 \\
\hline 18026 & 292.810 & 0.532 & 0.01267 & 67.388 & 77.15 & 0.00733 \\
\hline 18027 & 292.493 & 0.532 & 0.01260 & 66.817 & 77.13 & 0.00729 \\
\hline 18028 & 292.177 & 0.532 & 0.01259 & 66.249 & 77.09 & 0.00728 \\
\hline 18029 & 293.354 & 0.401 & 0.01254 & 68.367 & 58.12 & 0.00725 \\
\hline 18030 & 292.950 & 0.400 & 0.01252 & 67.640 & 58.02 & 0.00724 \\
\hline 18031 & 292.609 & 0.400 & 0.01246 & 67.026 & 57.96 & 0.00720 \\
\hline 18032 & 292.303 & 0.399 & 0.01245 & 66.475 & 57.91 & 0.00720 \\
\hline 18033 & 293.064 & 0.281 & 0.01256 & 67.845 & 40.75 & 0.00726 \\
\hline 18034 & 292.684 & 0.279 & 0.01252 & 67.161 & 40.49 & 0.00724 \\
\hline 18035 & 292.360 & 0.278 & 0.01249 & 66.578 & 40.28 & 0.00722 \\
\hline 18036 & 292.051 & 0.276 & 0.01241 & 66.022 & 40.06 & 0.00718 \\
\hline 18037 & 293.240 & 0.180 & 0.01263 & 68.162 & 26.12 & 0.00730 \\
\hline 18038 & 292.883 & 0.179 & 0.01256 & 67.519 & 26.03 & 0.00726 \\
\hline 18039 & 292.524 & 0.179 & 0.01255 & 66.873 & 25.92 & 0.00726 \\
\hline 18040 & 292.185 & 0.178 & 0.01256 & 66.263 & 25.81 & 0.00726 \\
\hline 18041 & 293.413 & 0.098 & 0.01297 & 68.473 & 14.22 & 0.00750 \\
\hline 18042 & 293.012 & 0.098 & 0.01294 & 67.752 & 14.15 & 0.00748 \\
\hline
\end{tabular}


Table 22. Transient Hot-Wire Thermal Conductivity Data for HFC-32 (Continued)

\begin{tabular}{|c|c|c|c|c|c|c|}
\hline $\begin{array}{l}\text { Point } \\
\text { Number }\end{array}$ & $\begin{array}{l}\mathrm{T}_{\text {Exp. }} \\
(\mathrm{K})\end{array}$ & $\begin{array}{l}\mathrm{P}_{\mathrm{Cell}} \\
(\mathrm{MPa})\end{array}$ & $\begin{array}{c}\lambda_{\operatorname{Exp}} \\
(W / m-x)\end{array}$ & $\mathrm{T}_{\text {Exp. }}$ & $\begin{array}{l}\mathrm{P}_{\text {Cell }} \\
\text { (psia) }\end{array}$ & $\begin{array}{c}\lambda_{\text {Exp. }} \\
\text { (BTU/ft-hr-F) }\end{array}$ \\
\hline 18043 & 292.649 & 0.097 & 0.01280 & 67.098 & 14.10 & 0.00740 \\
\hline 18044 & 292.287 & 0.097 & 0.01282 & 66.447 & 14.04 & 0.00741 \\
\hline 8001 & 303.054 & 65.111 & 0.17664 & 85.827 & 9443.64 & 0.10213 \\
\hline 8002 & 302.588 & 65.110 & 0.17769 & 84.988 & 9443.47 & 0.10274 \\
\hline 8003 & 302.143 & 65.113 & 0.17865 & 84.187 & 9443.92 & 0.10329 \\
\hline 8004 & 301.764 & 65.113 & 0.17958 & 83.505 & 9443.93 & 0.10383 \\
\hline 8005 & 303.041 & 54.392 & 0.17061 & 85.804 & 7888.95 & 0.09864 \\
\hline 8006 & 302.544 & 54.389 & 0.17144 & 84.909 & 7888.47 & 0.09912 \\
\hline 8007 & 302.098 & 54.403 & 0.17259 & 84.106 & 7890.53 & 0.09979 \\
\hline 8008 & 301.712 & 54.401 & 0.17421 & 83.412 & 7890.20 & 0.10072 \\
\hline 8009 & 303.054 & 43.225 & 0.16350 & 85.827 & 6269.23 & 0.09453 \\
\hline 8010 & 302.527 & 43.225 & 0.16464 & 84.879 & 6269.25 & 0.09519 \\
\hline 8011 & 302.072 & 43.230 & 0.16585 & 84.060 & 6269.98 & 0.09589 \\
\hline 8012 & 301.672 & 43.230 & 0.16699 & 83.340 & 6269.98 & 0.09655 \\
\hline 8013 & 302.860 & 33.917 & 0.15743 & 85.478 & 4919.32 & 0.09102 \\
\hline 8014 & 302.284 & 33.918 & 0.15904 & 84.441 & 4919.43 & 0.09195 \\
\hline 8015 & $301 . \varepsilon, 5$ & 33.922 & 0.15995 & 83.669 & 4920.04 & 0.09248 \\
\hline 8016 & 301.464 & 33.924 & 0.16150 & 82.965 & 4920.25 & 0.09338 \\
\hline 8017 & 302.160 & 23.570 & 0.15010 & 84.218 & 3418.51 & 0.08678 \\
\hline 8018 & 301.638 & 23.570 & 0.15159 & 83.278 & $34: 8.60$ & 0.08765 \\
\hline 8019 & 301.181 & 23.563 & 0.15177 & 82.456 & 3417.49 & 0.08775 \\
\hline 8020 & 300.752 & 23.567 & 0.15369 & 81.684 & 3418.05 & 0.08886 \\
\hline 8021 & 302.360 & 12.635 & 0.13912 & 84.578 & 1832.53 & 0.08044 \\
\hline 8022 & 301.789 & 12.638 & 0.14062 & 83.550 & 1833.00 & 0.08130 \\
\hline 8023 & 301.288 & 12.626 & 0.14135 & 82.648 & 1831.19 & 0.08173 \\
\hline 8024 & 300.852 & 12.628 & 0.14226 & 81.864 & 1831.60 & 0.08225 \\
\hline 9001 & 303.730 & 1.638 & 0.01588 & 87.044 & 237.61 & 0.00918 \\
\hline 9002 & 302.805 & 1.637 & 0.01565 & 85.379 & 237.44 & 0.00905 \\
\hline 9003 & 301.984 & 1.637 & 0.01569 & 83.901 & 237.39 & 0.00907 \\
\hline 9004 & 301.307 & 1.637 & 0.01575 & 82.683 & 237.41 & 0.00911 \\
\hline 9005 & 303.783 & 1.553 & 0.01539 & 87.139 & 225.20 & 0.00890 \\
\hline 9006 & 302.811 & 1.555 & 0.01538 & 85.390 & 225.49 & 0.00889 \\
\hline 9007 & 301.989 & 1.558 & 0.01556 & 83.910 & 225.93 & 0.00900 \\
\hline 9008 & 301.291 & 1.562 & 0.01585 & 82.654 & 226.54 & 0.00916 \\
\hline 9009 & 303.499 & 1.436 & 0.01509 & 86.628 & 208.33 & 0.00872 \\
\hline 9010 & 302.681 & 1.435 & 0.01507 & 85.156 & 208.14 & 0.00871 \\
\hline 9011 & 301.966 & 1.434 & 0.01510 & 83.869 & 207.99 & 0.00873 \\
\hline 9012 & 301.365 & 1.433 & 0.01510 & 82.787 & 207.89 & 0.00873 \\
\hline 9013 & 303.778 & 1.270 & 0.01465 & 87.130 & 184.15 & 0.00847 \\
\hline 9014 & 302.906 & 1.270 & 0.01465 & 85.561 & 184.26 & 0.00847 \\
\hline 9015 & 302.151 & 1.271 & 0.01463 & 84.202 & 184.39 & 0.00846 \\
\hline 9016 & 301.510 & 1.272 & 0.01466 & 83.048 & 184.44 & 0.00848 \\
\hline 9017 & 303.496 & 1.110 & 0.01428 & 86.623 & 161.03 & 0.00826 \\
\hline 9018 & 302.648 & 1.110 & 0.01426 & 85.096 & 161.01 & 0.00824 \\
\hline 9019 & 301.905 & 1.110 & 0.01426 & 83.759 & 160.96 & 0.00824 \\
\hline 9020 & 301.282 & 1.110 & 0.01428 & 82.638 & 160.93 & 0.00826 \\
\hline 9021 & 303.711 & 0.932 & 0.01393 & 87.010 & 135.15 & 0.00805 \\
\hline 9022 & 302.805 & 0.931 & 0.01393 & 85.379 & 135.07 & 0.00805 \\
\hline 9023 & 302.023 & 0.931 & 0.01388 & 83.971 & 134.99 & 0.00803 \\
\hline
\end{tabular}


Table 22. Transient Hot-Wire Thermal Conductivity Data for HFC-32 (Continued)

\begin{tabular}{|c|c|c|c|c|c|c|}
\hline $\begin{array}{l}\text { Point } \\
\text { Number }\end{array}$ & $\begin{array}{l}\mathrm{T}_{\text {Exp. }} \\
(\mathrm{K})\end{array}$ & $\begin{array}{l}\mathrm{P}_{\text {Cell }} \\
(\mathrm{MPa})\end{array}$ & $\begin{array}{c}\lambda_{\text {Exp }} \\
(W / m-K)\end{array}$ & $\mathrm{T}_{\text {Exp. }}$ & $\begin{array}{l}\mathrm{P}_{\text {Cell }} \\
\text { (psia) }\end{array}$ & $\frac{\lambda_{\text {Exp. }}}{(B T U / \mathrm{ft}-\mathrm{hr}-\mathrm{F})}$ \\
\hline 9024 & 301.351 & 0.930 & 0.01392 & 82.762 & 134.88 & 0.00805 \\
\hline 9025 & 303.587 & 0.756 & 0.01365 & 86.787 & 109.70 & 0.00789 \\
\hline 9026 & 302.731 & 0.756 & 0.01358 & 85.246 & 109.62 & 0.00785 \\
\hline 9027 & 301.962 & 0.756 & 0.01396 & 83.862 & 109.61 & 0.00807 \\
\hline 9028 & 301.319 & 0.755 & 0.01350 & 82.704 & 109.56 & 0.00781 \\
\hline 9029 & 303.860 & 0.533 & 0.01334 & 87.278 & 77.35 & 0.00771 \\
\hline 9030 & 303.098 & 0.533 & 0.01326 & 85.906 & 77.31 & 0.00767 \\
\hline 9031 & 302.349 & 0.533 & 0.01329 & 84.558 & 77.26 & 0.00768 \\
\hline 9032 & 301.742 & 0.532 & 0.01330 & 83.466 & 77.23 & 0.00769 \\
\hline 9033 & 304.127 & 0.324 & 0.01312 & 87.759 & 47.02 & 0.00759 \\
\hline 9034 & 303.296 & 0.324 & 0.01326 & 86.263 & 46.95 & 0.00767 \\
\hline 9035 & 302.619 & 0.323 & 0.01318 & 85.044 & 46.90 & 0.00762 \\
\hline 9036 & 301.994 & 0.323 & 0.01314 & 83.919 & 46.84 & 0.00760 \\
\hline 9037 & 303.523 & 0.143 & 0.01356 & 86.671 & 20.68 & 0.00784 \\
\hline 9038 & 302.918 & 0.141 & 0.01348 & 85.582 & 20.46 & 0.00779 \\
\hline 9039 & 302.366 & 0.140 & 0.01343 & 84.589 & 20.28 & 0.00776 \\
\hline 9040 & 301.837 & 0.139 & 0.01341 & 83.637 & 20.11 & 0.00775 \\
\hline 19001 & 312.996 & 2.250 & 0.01747 & 103.723 & 326.28 & 0.01010 \\
\hline 19004 & 312.387 & 2.248 & 0.01745 & 102.627 & 326.05 & 0.01009 \\
\hline 19007 & 311.871 & 2.247 & 0.01725 & 101.698 & 325.96 & 0.00997 \\
\hline 19010 & 311.436 & 2.247 & 0.01729 & 100.915 & 325.93 & 0.01000 \\
\hline 19013 & 313.243 & 2.069 & 0.01686 & 104.167 & 300.08 & 0.00975 \\
\hline 19016 & 312.597 & 2.066 & 0.01681 & 103.005 & 299.58 & 0.00972 \\
\hline 19019 & 312.034 & 2.064 & 0.01674 & 101.991 & 299.32 & 0.00968 \\
\hline 19022 & 311.546 & 2.062 & 0.01685 & 101.113 & 299.14 & 0.00974 \\
\hline 19025 & 312.738 & 1.904 & 0.01626 & 103.258 & 276.15 & 0.00940 \\
\hline 19028 & 312.144 & 1.903 & 0.01638 & 102.189 & 276.00 & 0.00947 \\
\hline 19031 & 311.639 & 1.902 & 0.01612 & 101.280 & 275.93 & 0.00932 \\
\hline 19034 & 311.203 & 1.902 & 0.01591 & 100.495 & 275.89 & 0.00920 \\
\hline 19037 & 312.843 & 1.772 & 0.01586 & 103.447 & 257.08 & 0.00917 \\
\hline 19040 & 312.224 & 1.770 & 0.01593 & 102.333 & 256.65 & 0.00921 \\
\hline 19043 & 311.705 & 1.767 & 0.01579 & 101.399 & 256.31 & 0.00913 \\
\hline 19046 & 311.261 & 1.764 & 0.01612 & 100.600 & 255.91 & 0.00932 \\
\hline 19049 & 312.968 & 1.673 & 0.01566 & 103.672 & 242.62 & 0.00905 \\
\hline 19052 & 312.292 & 1.672 & 0.01558 & 102.456 & 242.56 & 0.00901 \\
\hline 19055 & 311.733 & 1.673 & 0.01559 & 101.449 & 242.66 & 0.00901 \\
\hline 19058 & 311.316 & $\therefore .674$ & 0.01556 & 100.699 & 242.79 & 0.00900 \\
\hline 19061 & 313.072 & 1.495 & 0.01514 & 103.860 & 216.80 & 0.00875 \\
\hline 19064 & 312.403 & 1.495 & 0.01522 & 102.655 & 216.80 & 0.00880 \\
\hline 19067 & 311.822 & 1.495 & 0.01509 & 101.610 & 216.82 & 0.00872 \\
\hline 19073 & 311.342 & 1.495 & 0.01502 & 100.746 & $\therefore 16.81$ & 0.00868 \\
\hline 19073 & 312.879 & 1.337 & 0.01482 & 103.512 & 193.86 & 0.00857 \\
\hline 19076 & 312.353 & 1.337 & 0.01470 & 102.565 & 193.91 & 0.00850 \\
\hline 19079 & 311.851 & 1.337 & 0.01484 & 101.662 & 193.89 & 0.00858 \\
\hline 19082 & 311.466 & 1.337 & 0.01477 & 100.969 & 193.90 & 0.00854 \\
\hline 19085 & 312.978 & 1.164 & 0.01456 & 103.690 & 168.89 & 0.00842 \\
\hline 19089 & 312.425 & 1.165 & 0.01444 & 102.695 & 168.05 & 0.00835 \\
\hline 19093 & 311.944 & 1.165 & 0.01448 & 101.829 & 168.98 & 0.00837 \\
\hline 19097 & 311.489 & 1.165 & 0.01418 & 101.010 & 168.99 & 0.00820 \\
\hline
\end{tabular}


Table 22. Transient Hot-Wire Thermal Conductivity Data for HFC-32 (Continued)

\begin{tabular}{|c|c|c|c|c|c|c|}
\hline $\begin{array}{l}\text { Point } \\
\text { Number }\end{array}$ & $\begin{array}{l}T_{\text {Exp. }} \\
(\mathrm{K})\end{array}$ & $\begin{array}{l}\mathrm{P}_{\mathrm{Cell}} \\
(\mathrm{MPa})\end{array}$ & $\begin{array}{c}\lambda_{\operatorname{Exp}_{.}} \\
(W / m-K)\end{array}$ & $\mathrm{T}_{\text {Exp. }}$ & $\begin{array}{l}P_{\text {Cell }} \\
\text { (psia) }\end{array}$ & $\begin{array}{c}\lambda_{\text {Exp. }} \\
(B T U / f t-h r-F)\end{array}$ \\
\hline 19101 & 313.114 & 0.947 & 0.01413 & 103.935 & 137.30 & 0.00817 \\
\hline 19105 & 312.522 & 0.947 & 0.01416 & 102.870 & 137.35 & 0.00819 \\
\hline 19109 & 312.012 & 0.947 & 0.01402 & 101.952 & 137.37 & 0.00811 \\
\hline 19113 & 311.551 & 0.947 & 0.01386 & 101.122 & 137.40 & 0.00801 \\
\hline 19117 & 312.818 & 0.738 & 0.01392 & 103.402 & 107.09 & 0.00805 \\
\hline 19121 & 312.260 & 0.739 & 0.01388 & 102.398 & 107.24 & 0.00803 \\
\hline 19125 & 311.762 & 0.740 & 0.01393 & 101.502 & 107.27 & 0.00805 \\
\hline 19129 & 311.356 & 0.740 & 0.01379 & 100.771 & 107.33 & 0.00797 \\
\hline 19133 & 312.958 & 0.516 & 0.01366 & 103.654 & 74.83 & 0.00790 \\
\hline 19137 & 312.368 & 0.516 & 0.01371 & 102.592 & 74.90 & 0.00793 \\
\hline 19141 & 311.839 & 0.517 & 0.01349 & 101.640 & 74.96 & 0.00780 \\
\hline 19145 & 311.379 & 0.517 & 0.01361 & 100.812 & 74.93 & 0.00787 \\
\hline 19149 & 313.231 & 0.230 & 0.01360 & 104.146 & 33.43 & 0.00786 \\
\hline 19153 & 312.572 & 0.232 & 0.01358 & 102.960 & 33.60 & 0.00785 \\
\hline 19157 & 311.984 & 0.232 & 0.01332 & 101.901 & 33.65 & 0.00770 \\
\hline 19161 & 311.499 & 0.233 & 0.01330 & 101.028 & 33.73 & 0.00769 \\
\hline 19165 & 313.804 & 0.037 & 0.01198 & 105.177 & 5.32 & 0.00693 \\
\hline 19169 & 312.995 & 0.037 & 0.01200 & 103.721 & 5.39 & 0.00694 \\
\hline 19173 & 312.287 & 0.038 & 0.01203 & 102.447 & 5.45 & 0.00696 \\
\hline 19177 & 311.701 & 0.038 & 0.01195 & 101.392 & 5.50 & 0.00691 \\
\hline 20001 & 322.140 & 73.373 & 0.19292 & 120.182 & 10641.92 & 0.11154 \\
\hline 20002 & 322.313 & 73.392 & 0.21264 & 120.493 & 10644.58 & 0.12294 \\
\hline 20003 & 322.42 .6 & 73.437 & 0.20858 & 120.697 & 10651.13 & 0.12060 \\
\hline 20004 & 322.406 & 73.445 & 0.20794 & 120.661 & 10652.40 & 0.12023 \\
\hline 20005 & 322.443 & 36.989 & 0.14423 & 120.727 & 5364.78 & 0.08339 \\
\hline 20006 & 322.482 & 39.477 & 0.16533 & 120.798 & 5725.66 & 0.09559 \\
\hline 21001 & 322.477 & 39.683 & 0.16509 & 120.789 & 5755.55 & 0.09545 \\
\hline 21002 & 322.269 & 39.691 & 0.16735 & 120.414 & 5756.78 & 0.09676 \\
\hline 21003 & 322.039 & 39.691 & 0.17112 & 120.000 & 5756.74 & 0.09894 \\
\hline 21004 & 321.856 & 39.690 & 0.17445 & 119.671 & 5756.61 & 0.10086 \\
\hline 21005 & 322.506 & 33.897 & 0.15786 & 120.841 & 4916.37 & 0.09127 \\
\hline 21006 & 322.278 & 33.897 & 0.15991 & 120.430 & 4916.38 & 0.09246 \\
\hline 21007 & 322.075 & 33.896 & 0.16311 & 120.065 & 4916.15 & 0.09431 \\
\hline 21008 & 321.857 & 33.892 & 0.16791 & 119.673 & 4915.62 & 0.09708 \\
\hline 21009 & 322.684 & 29.378 & 0.14964 & 121.161 & 4260.99 & 0.08652 \\
\hline 21010 & 322.439 & 29.380 & 0.15438 & 120.720 & 4261.27 & 0.08926 \\
\hline 21011 & 322.207 & 29.384 & 0.15993 & 120.303 & 4261.80 & 0.09247 \\
\hline 21012 & 322.002 & 29.388 & 0.16558 & 119.934 & 4262.35 & 0.09574 \\
\hline 21013 & 322.693 & 25.745 & 0.14774 & 121.177 & 3733.99 & 0.08542 \\
\hline 21014 & 322.447 & 25.748 & 0.15154 & 120.735 & 3734.43 & 0.08762 \\
\hline 21015 & 322.217 & 25.751 & 0.15545 & 120.321 & 3734.91 & 0.08988 \\
\hline 21016 & 322.010 & 25.756 & 0.16113 & 119.948 & 3735.66 & 0.09316 \\
\hline 21017 & 322.711 & 21.924 & 0.14308 & 121.210 & 3179.84 & 0.08273 \\
\hline 21018 & 322.463 & 21.927 & 0.14662 & 120.763 & 3180.30 & 0.08477 \\
\hline 21019 & 322.234 & 21.930 & 0.14981 & 120.351 & 3180.71 & 0.08662 \\
\hline 21020 & 322.004 & 21.933 & 0.15540 & 119.937 & 3181.06 & 0.08985 \\
\hline 21021 & 322.768 & $! 5.780$ & 0.13568 & $! 2 ! .3 ! 2$ & 228872 & 0.07845 \\
\hline 21022 & 322.506 & 15.781 & 0.13796 & 120.841 & 2288.91 & 0.07977 \\
\hline 21023 & 322.256 & 15.784 & 0.14064 & 120.391 & 2289.30 & 0.08132 \\
\hline
\end{tabular}


Table 22. Transient Hot-Wire Thermal Conductivity Data for HFC-32 (Continued)

\begin{tabular}{|c|c|c|c|c|c|c|}
\hline $\begin{array}{l}\text { Point } \\
\text { Number }\end{array}$ & $\begin{array}{l}\mathrm{T}_{\text {Exp. }} \\
(\mathrm{K})\end{array}$ & $\begin{array}{l}\mathrm{P}_{\mathrm{Cell}} \\
(\mathrm{MPa})\end{array}$ & $\begin{array}{c}\lambda_{\operatorname{Exp}} \\
(W / m-K)\end{array}$ & $\mathrm{T}_{\text {Exp. }}$ & $\begin{array}{l}P_{\text {Cell }} \\
\text { (psia) }\end{array}$ & $\begin{array}{c}\lambda_{\text {Exp. }} \\
(B T U / f t-h r-F)\end{array}$ \\
\hline 21024 & 322.028 & 15.786 & 0.14521 & 119.980 & 2289.57 & 0.08396 \\
\hline 21025 & 322.803 & 13.725 & 0.13259 & 121.375 & 1990.60 & 0.07666 \\
\hline 21026 & 322.543 & 13.725 & 0.13509 & 120.907 & 1990.72 & 0.07811 \\
\hline 21027 & 322.293 & 13.729 & 0.13761 & 120.457 & 1991.18 & 0.07956 \\
\hline 21028 & 322.060 & 13.732 & 0.14057 & 120.038 & 1991.60 & 0.08127 \\
\hline 21029 & 322.855 & 11.532 & 0.12977 & 121.469 & 1672.62 & 0.07503 \\
\hline 21030 & 322.590 & 11.538 & $0.1318 ?$ & 120.992 & 1673.47 & 0.07622 \\
\hline 21031 & 322.339 & 11.542 & 0.13456 & 120.540 & 1674.00 & 0.07780 \\
\hline 21032 & 322.110 & 11.545 & 0.13742 & 120.128 & 1674.47 & 0.07945 \\
\hline 21033 & 322.922 & 9.192 & 0.12530 & 121.590 & 1333.22 & 0.07245 \\
\hline 21034 & 322.639 & 9.194 & 0.12752 & 121.080 & 1333.48 & 0.07373 \\
\hline 21035 & 322.384 & 9.194 & 0.12979 & 120.621 & 1333.45 & 0.07504 \\
\hline 21036 & 322.142 & 9.195 & 0.13191 & 120.186 & 1333.59 & 0.07627 \\
\hline 21037 & 322.938 & 7.635 & 0.12323 & 121.618 & 1107.33 & 0.07125 \\
\hline 21038 & 322.666 & 7.640 & 0.12507 & 121.129 & 1108.03 & 0.07231 \\
\hline 21039 & 322.408 & 7.641 & 0.12664 & 120.664 & 1108.23 & 0.07322 \\
\hline 21040 & 322.161 & 7.640 & 0.12880 & 120.220 & 1108.08 & 0.07447 \\
\hline 21041 & 322.977 & 5.748 & 0.11962 & 121.689 & 833.66 & 0.06916 \\
\hline 21042 & 322.689 & 5.750 & 0.12273 & 121.170 & 834.03 & 0.07096 \\
\hline 21043 & 322.420 & 5.751 & 0.12305 & 120.686 & 834.05 & 0.07115 \\
\hline 21044 & 322.167 & 5.754 & 0.12703 & 120.231 & 834.54 & 0.07345 \\
\hline 21045 & 322.992 & 4.377 & 0.12150 & 121.716 & 634.84 & 0.07025 \\
\hline 21046 & 322.695 & 4.380 & 0.12149 & 121.181 & 635.20 & 0.07024 \\
\hline 21047 & 322.425 & 4.381 & 0.12320 & 120.695 & 635.47 & 0.07123 \\
\hline 21048 & 322.175 & 4.384 & 0.12427 & 120.245 & 635.78 & 0.07185 \\
\hline 22005 & 323.625 & 2.873 & 0.01966 & 122.855 & 416.73 & 0.01137 \\
\hline 22006 & 323.045 & 2.873 & 0.01946 & 121.811 & 416.76 & 0.01125 \\
\hline 22007 & 322.531 & 2.874 & 0.01994 & 120.886 & 416.78 & 0.01153 \\
\hline 22008 & 322.084 & 2.874 & 0.01963 & 120.081 & 416.82 & 0.01135 \\
\hline 22009 & 323.763 & 2.785 & 0.01947 & 123.103 & 403.92 & 0.01126 \\
\hline 22010 & 323.163 & 2.785 & 0.01944 & 122.023 & 403.89 & 0.01124 \\
\hline 22011 & 322.625 & 2.785 & 0.01947 & 121.055 & 403.87 & 0.01126 \\
\hline 22012 & 322.147 & 2.785 & 0.01940 & 120.195 & 403.86 & 0.01122 \\
\hline 22013 & 323.856 & 2.683 & 0.01903 & 123.271 & 389.13 & 0.01100 \\
\hline 22014 & 323.230 & 2.683 & 0.01902 & 122.144 & 389.18 & 0.01100 \\
\hline $220 ! 5$ & 322.670 & 2.684 & 0.01903 & 121.136 & 389.22 & 0.01100 \\
\hline 22016 & 322.184 & 2.684 & 0.01897 & 120.261 & 389.25 & 0.01097 \\
\hline 22017 & 324.097 & 2.538 & 0.01828 & 123.705 & 368.04 & 0.01057 \\
\hline $2201 \%$ & 323.433 & 2.537 & 0.01826 & 122.509 & 368.00 & 0.01056 \\
\hline 22019 & 322.838 & 2.537 & 0.01829 & 121.438 & 367.93 & 0.01057 \\
\hline 22020 & 322.324 & 2.536 & 0.01834 & 120.513 & 367.82 & 0.01060 \\
\hline 22021 & 323.548 & 2.426 & 0.01783 & 122.716 & 351.92 & 0.01031 \\
\hline 22023 & 322.948 & 2.426 & 0.01787 & 121.636 & 351.86 & 0.01033 \\
\hline 22025 & 322.496 & 2.426 & 0.01773 & 120.661 & 351.84 & 0.01025 \\
\hline 22027 & 321.935 & 2.425 & 0.01788 & 119.813 & 351.72 & 0.01034 \\
\hline 22029 & 323.689 & 2.283 & 0.01722 & 122.970 & 331.14 & 0.00996 \\
\hline 22031 & 323.062 & 2.281 & 0.01728 & 121.842 & 330.83 & 0.00999 \\
\hline 22033 & 322.492 & 2.280 & 0.01720 & 120.816 & 330.63 & 0.00994 \\
\hline 22034 & 322.482 & 2.275 & 0.01720 & 120.798 & 329.99 & 0.00994 \\
\hline
\end{tabular}


Table 22. Transient Hot-Wire Thermal Conductivity Data for HFC-32 (Continued)

\begin{tabular}{|c|c|c|c|c|c|c|}
\hline $\begin{array}{l}\text { Point } \\
\text { Number }\end{array}$ & $\begin{array}{l}T_{\text {Exp. }} \\
(\mathrm{K})\end{array}$ & $\begin{array}{l}\mathrm{P}_{\mathrm{Cell}} \\
(\mathrm{MPa})\end{array}$ & $\begin{array}{c}\lambda_{\operatorname{Exp}_{.}} \\
(W / m-K)\end{array}$ & $\mathrm{T}_{\text {Exp. }}$ & $\begin{array}{l}\mathrm{P}_{\text {Cell }} \\
\text { (psia) }\end{array}$ & ${ }_{(B T U / f t-h r-F)}^{\lambda_{\operatorname{Exp}}}$ \\
\hline 22036 & 322.004 & 2.274 & 0.01727 & 119.937 & 329.84 & 0.00999 \\
\hline 22038 & 323.817 & 2.175 & 0.01689 & 123.201 & 315.43 & 0.00977 \\
\hline 22040 & 323.151 & 2.176 & 0.01690 & 122.002 & 315.64 & 0.00977 \\
\hline 22042 & 322.567 & 2.177 & 0.01684 & 120.951 & 315.78 & 0.00974 \\
\hline 22044 & 322.056 & 2.178 & 0.01683 & 120.031 & 315.88 & 0.00973 \\
\hline 22046 & 323.974 & 1.999 & 0.01631 & 123.483 & 289.96 & 0.00943 \\
\hline 22048 & 323.275 & 2.000 & 0.01634 & 122.225 & 290.11 & 0.00945 \\
\hline 22050 & 322.664 & 2.000 & 0.01638 & 121.125 & 290.06 & 0.00947 \\
\hline 22052 & 322.124 & 2.000 & 0.01627 & 120.153 & 290.12 & 0.00941 \\
\hline 22054 & 324.117 & 1.854 & 0.01591 & 123.741 & 268.95 & 0.00920 \\
\hline 22056 & 323.393 & 1.855 & 0.01593 & 122.437 & 269.09 & 0.00921 \\
\hline 22058 & 322.764 & 1.855 & 0.01584 & 121.305 & 269.08 & 0.00916 \\
\hline 22060 & 322.204 & 1.856 & 0.01579 & 120.297 & 269.14 & 0.00913 \\
\hline 22063 & 324.276 & 1.663 & 0.01545 & 124.027 & 241.14 & 0.00893 \\
\hline 22065 & 323.529 & 1.662 & 0.01545 & 122.682 & 241.04 & 0.00893 \\
\hline 22067 & 322.859 & 1.662 & 0.01548 & 121.476 & 241.05 & 0.00895 \\
\hline 22069 & 322.278 & 1.662 & 0.01538 & 120.430 & 241.05 & 0.00889 \\
\hline 22072 & 324.404 & 1.510 & 0.01518 & 124.257 & 219.05 & 0.00878 \\
\hline 22074 & 323.615 & 1.521 & 0.01523 & 122.837 & 220.54 & 0.00881 \\
\hline 22076 & 322.921 & 1.529 & 0.01516 & 121.588 & 221.78 & 0.00877 \\
\hline 22078 & 322.315 & 1.536 & 0.01510 & 120.497 & 222.79 & 0.00873 \\
\hline 22080 & 324.651 & 1.263 & 0.01473 & 124.702 & 183.12 & 0.00852 \\
\hline 22082 & 323.827 & 1.263 & 0.01462 & 123.219 & 183.19 & 0.00845 \\
\hline 22084 & 323.086 & 1.263 & 0.01460 & 121.885 & 183.20 & 0.00844 \\
\hline 22086 & 322.446 & 1.264 & 0.01459 & 120.733 & 183.30 & 0.00844 \\
\hline 22104 & 324.835 & 1.079 & 0.01440 & 125.033 & 156.56 & 0.00833 \\
\hline 22106 & 323.977 & 1.080 & $0.0 i 440$ & 123.489 & 156.58 & 0.00833 \\
\hline 22108 & 323.200 & 1.080 & 0.01432 & 122.090 & 156.62 & 0.00828 \\
\hline 22110 & 322.534 & 1.080 & 0.01435 & 120.891 & 156.65 & 0.00830 \\
\hline 22117 & 324.095 & 0.897 & 0.01417 & 123.701 & 130.13 & 0.00819 \\
\hline 22119 & 323.294 & 0.898 & 0.01406 & 122.259 & 130.24 & 0.00813 \\
\hline 22121 & 322.609 & 0.898 & 0.01401 & 121.026 & 130.20 & 0.00810 \\
\hline 22123 & 322.013 & 0.898 & 0.01397 & 119.953 & 130.25 & 0.00808 \\
\hline 22129 & 324.280 & 0.686 & 0.01387 & 124.034 & 99.55 & 0.00802 \\
\hline 22131 & 323.437 & 0.687 & 0.01387 & 122.517 & 99.59 & 0.00802 \\
\hline 22133 & 322.701 & 0.686 & 0.01377 & 121.192 & 99.56 & 0.00796 \\
\hline 22135 & 322.075 & 0.687 & 0.01378 & 120.065 & 99.63 & 0.00797 \\
\hline 22147 & 324.491 & 0.450 & 0.01361 & 124.414 & 65.29 & 0.00787 \\
\hline 22149 & 323.600 & 0.450 & 0.01356 & 122.810 & 65.29 & 0.00784 \\
\hline 22151 & 322.822 & 0.450 & 0.01348 & 121.410 & 65.29 & 0.00779 \\
\hline 22153 & 322.153 & 0.450 & 0.01343 & 120.205 & 65.29 & 0.00776 \\
\hline 22167 & 324.922 & 0.226 & 0.01344 & 125.190 & 32.77 & 0.00777 \\
\hline 22169 & 323.936 & 0.226 & 0.01336 & 123.415 & 32.83 & 0.00772 \\
\hline 22171 & 323.079 & 0.227 & 0.01333 & 121.872 & 32.91 & 0.00771 \\
\hline 22173 & 322.334 & 0.227 & 0.01326 & 120.531 & 32.92 & 0.00767 \\
\hline 23001 & 333.740 & 3.637 & 0.02569 & 141.062 & 527.56 & 0.01485 \\
\hline 23003 & 333.174 & 3.638 & 0.02545 & 140.043 & 527.63 & 0.01471 \\
\hline 23005 & 332.647 & 3.638 & 0.02513 & 139.095 & 527.72 & 0.01453 \\
\hline 23007 & 332.163 & 3.639 & 0.02479 & 138.223 & 527.80 & 0.01433 \\
\hline
\end{tabular}


Table 22. Transient Hot-Wire Thermal Conductivity Data for HFC-32 (Continued)

\begin{tabular}{|c|c|c|c|c|c|c|}
\hline $\begin{array}{l}\text { Point } \\
\text { Number }\end{array}$ & $\begin{array}{l}T_{\text {Exp. }} \\
(\mathrm{K})\end{array}$ & $\begin{array}{l}\mathrm{P}_{\text {Cell }} \\
(\mathrm{MPa})\end{array}$ & $\underset{(W / m-K)}{\lambda_{\operatorname{Exp}}}$ & $\mathrm{T}_{\text {Exp. }}$ & $\begin{array}{l}P_{\text {Cell }} \\
\text { (psia) }\end{array}$ & $\begin{array}{c}\lambda_{\operatorname{Exp}} \\
(B T U / \mathrm{ft}-\mathrm{hr}-\mathrm{F})\end{array}$ \\
\hline 23009 & 333.858 & 3.591 & 0.02511 & 141.274 & 520.89 & 0.01452 \\
\hline 23011 & 333.270 & 3.592 & 0.02492 & 140.216 & 520.94 & 0.01441 \\
\hline 23013 & 332.731 & 3.593 & 0.02479 & 139.246 & 521.06 & 0.01433 \\
\hline 23015 & 332.239 & 3.593 & 0.02470 & 138.360 & 521.11 & 0.01428 \\
\hline 23017 & 333.398 & 3.519 & 0.02423 & 140.446 & 510.39 & 0.01401 \\
\hline 23019 & 332.840 & 3.519 & 0.02411 & 139.442 & 510.37 & 0.01394 \\
\hline 23021 & 332.335 & 3.519 & 0.02422 & 138.533 & 510.36 & 0.01400 \\
\hline 23023 & 331.883 & 3.517 & 0.02410 & 137.719 & 510.06 & 0.01393 \\
\hline 23025 & 333.512 & 3.458 & 0.02363 & 140.652 & 501.57 & 0.01366 \\
\hline 23027 & 332.938 & 3.458 & 0.02356 & 139.618 & 501.49 & 0.01362 \\
\hline 23029 & 332.418 & 3.457 & 0.02361 & 138.682 & 501.46 & 0.01365 \\
\hline 23031 & 331.962 & 3.457 & 0.02358 & 137.862 & 501.46 & 0.01363 \\
\hline 23033 & 333.671 & 3.370 & 0.02287 & 140.938 & 488.72 & 0.01322 \\
\hline 23035 & 333.079 & 3.368 & 0.02280 & 139.872 & 488.45 & 0.01318 \\
\hline 23037 & 332.538 & 3.367 & 0.02275 & 138.898 & 488.32 & 0.01315 \\
\hline 23039 & 332.054 & 3.365 & 0.02273 & 138.027 & 488.04 & 0.01314 \\
\hline 23041 & 333.844 & 3.281 & 0.02229 & 141.249 & 475.92 & 0.01289 \\
\hline 23043 & 333.218 & 3.284 & 0.02222 & 140.122 & 476.35 & 0.01285 \\
\hline 23045 & 332.643 & 3.286 & 0.02226 & 139.087 & 476.61 & 0.01287 \\
\hline 23047 & 332.148 & 3.287 & 0.02236 & 138.196 & 476.79 & 0.01293 \\
\hline 23049 & 334.028 & 3.158 & 0.02129 & 141.580 & 458.10 & 0.01231 \\
\hline 23051 & 333.381 & 3.157 & 0.02132 & 140.416 & 457.95 & 0.01233 \\
\hline 23053 & 332.786 & 3.157 & 0.02131 & 139.345 & 457.88 & 0.01232 \\
\hline 23055 & 332.263 & 3.157 & 0.02140 & 138.403 & 457.83 & 0.01237 \\
\hline 23057 & 334.145 & 3.069 & 0.02081 & 141.791 & 445.11 & 0.01203 \\
\hline 23059 & 333.477 & 3.069 & 0.02079 & 140.589 & 445.15 & 0.01202 \\
\hline 23061 & 332.864 & 3.070 & 0.02068 & 139.485 & 445.23 & 0.01196 \\
\hline 23063 & 332.320 & 3.071 & 0.02087 & 138.506 & 445.35 & 0.01207 \\
\hline 23065 & 334.293 & 2.946 & 0.02016 & 142.057 & 427.23 & 0.01156 \\
\hline 23067 & 333.613 & 2.947 & 0.02023 & 140.833 & 427.39 & 0.01170 \\
\hline 23069 & 332.969 & 2.948 & 0.02028 & 139.674 & 427.64 & 0.01173 \\
\hline 23071 & 332.399 & 2.950 & 0.02021 & 138.648 & 427.82 & 0.01169 \\
\hline 23073 & 334.457 & 2.813 & 0.01963 & 142.353 & 408.03 & 0.01135 \\
\hline 23075 & 333.736 & 2.818 & 0.01939 & 141.055 & 408.73 & 0.01121 \\
\hline 23077 & 333.055 & 2.823 & 0.01980 & 139.829 & 409.45 & 0.01145 \\
\hline 23079 & 332.471 & 2.828 & 0.01925 & 138.778 & 410.17 & 0.01113 \\
\hline 23081 & 334.652 & 2.682 & 0.01905 & 142.704 & 389.00 & 0.01101 \\
\hline $2 j 083$ & 333.911 & 2.684 & 0.01902 & 141.370 & 389.21 & 0.01100 \\
\hline 23085 & 333.226 & 2.685 & 0.01894 & 140.137 & 389.36 & 0.01095 \\
\hline 23087 & 332.602 & 2.686 & 0.01895 & 139.014 & 389.51 & 0.01096 \\
\hline 23089 & 334.893 & 2.481 & 0.01832 & 143.137 & 359.78 & 0.01059 \\
\hline 23092 & 334.112 & 2.476 & 0.01827 & 141.732 & 359.11 & 0.01056 \\
\hline 23095 & 333.388 & 2.474 & 0.01825 & 140.428 & 358.86 & 0.01055 \\
\hline 23098 & 332.746 & 2.474 & 0.01829 & 139.273 & 358.76 & 0.01057 \\
\hline 23101 & 332.173 & 2.474 & 0.01812 & 138.241 & 358.79 & 0.01048 \\
\hline 23104 & 331.668 & 2.477 & 0.01810 & 137.332 & 359.24 & 0.01047 \\
\hline 23107 & 334.237 & 2.315 & 0.01777 & 141.957 & 335.79 & 0.01027 \\
\hline 23110 & 333.503 & 2.314 & 0.001774 & i 400.635 & 335.60 & 0.01026 \\
\hline 23113 & 332.828 & 2.312 & 0.01775 & 139.420 & 335.28 & 0.01026 \\
\hline
\end{tabular}


Table 22. Transient Hot-Wire Thermal Conductivity Data for HFC-32 (Continued)

\begin{tabular}{|c|c|c|c|c|c|c|}
\hline $\begin{array}{l}\text { Point } \\
\text { Number }\end{array}$ & $\begin{array}{l}\mathrm{T}_{\text {Exp. }} \\
(\mathrm{K})\end{array}$ & $\begin{array}{l}\mathrm{P}_{\text {Cell }} \\
(\mathrm{MPa})\end{array}$ & $\underset{(W / m-K)}{\lambda_{\operatorname{Exp}}}$ & $\mathrm{T}_{\text {Exp. }}$ & $\begin{array}{l}\mathrm{P}_{\text {Cell }} \\
\text { (psia) }\end{array}$ & $\begin{array}{c}\lambda_{\text {Exp. }} \\
\text { (BTU/ft-hr-F) }\end{array}$ \\
\hline 23116 & 332.222 & 2.309 & 0.01771 & 138.330 & 334.92 & 0.01024 \\
\hline 23119 & 331.718 & 2.307 & 0.01764 & 137.422 & 334.63 & 0.01020 \\
\hline 23122 & 334.414 & 2.174 & 0.01743 & 142.275 & 315.37 & 0.01008 \\
\hline 23125 & 333.646 & 2.176 & 0.01743 & 140.893 & 315.60 & 0.01008 \\
\hline 23128 & 332.948 & 2.178 & 0.01743 & 139.636 & 315.85 & 0.01008 \\
\hline 23131 & 332.330 & 2.180 & 0.01732 & 138.524 & 316.25 & 0.01001 \\
\hline 23134 & 331.791 & 2.183 & 0.01727 & 137.554 & 316.68 & 0.00999 \\
\hline 23137 & 334.586 & 1.972 & 0.01686 & 142.585 & 286.02 & 0.00975 \\
\hline 23140 & 333.797 & 1.963 & 0.01678 & 141.165 & 284.65 & 0.00970 \\
\hline 23143 & 333.070 & 1.955 & 0.01674 & 139.856 & 283.61 & 0.00968 \\
\hline 23146 & 332.433 & 1.954 & 0.01672 & 138.709 & 283.47 & 0.00967 \\
\hline 23149 & 331.856 & 1.955 & 0.01680 & 137.671 & 283.57 & 0.00971 \\
\hline 23152 & 334.743 & 1.800 & 0.01654 & 142.867 & 261.09 & 0.00956 \\
\hline 23155 & 333.935 & 1.787 & 0.01644 & 141.413 & 259.23 & 0.00951 \\
\hline 23158 & 333.180 & 1.779 & 0.01645 & 140.054 & 257.98 & 0.00951 \\
\hline 23161 & 332.513 & 1.772 & 0.01635 & 138.853 & 256.99 & 0.00945 \\
\hline 23164 & 331.923 & 1.767 & 0.01629 & 137.791 & 256.29 & 0.00942 \\
\hline 23167 & 334.043 & 1.626 & 0.01618 & 141.607 & 235.88 & 0.00935 \\
\hline 23170 & 333.256 & 1.627 & 0.01613 & 140.191 & 235.95 & 0.00933 \\
\hline 23173 & 332.558 & 1.637 & 0.01604 & 138.934 & 237.38 & 0.00927 \\
\hline 23176 & 331.969 & 1.638 & 0.01599 & 137.874 & 237.64 & 0.00925 \\
\hline 23179 & 334.184 & 1.457 & 0.01585 & 141.861 & 211.32 & 0.00916 \\
\hline 23182 & 333.333 & 1.488 & 0.01589 & 140.329 & 215.83 & 0.00919 \\
\hline 23185 & 332.623 & 1.503 & 0.01584 & 139.051 & 217.99 & 0.00916 \\
\hline 23188 & 331.976 & 1.577 & 0.01589 & 137.887 & 228.70 & 0.00919 \\
\hline 23191 & 334.196 & 1.431 & 0.01576 & 141.883 & 207.49 & 0.00911 \\
\hline 23194 & 333.382 & 1.447 & 0.01575 & 140.418 & 209.90 & 0.00911 \\
\hline 23197 & 332.653 & 1.463 & 0.01576 & 139.105 & 212.20 & 0.00911 \\
\hline 23200 & 332.021 & 1.478 & 0.01569 & 137.968 & 214.30 & 0.00907 \\
\hline 23203 & 334.362 & 1.237 & 0.01550 & 142.182 & 179.48 & 0.00896 \\
\hline 23206 & 333.518 & 1.238 & 0.01539 & 140.662 & 179.54 & 0.00890 \\
\hline 23209 & 332.770 & 1.238 & 0.01537 & 139.316 & 179.59 & 0.00889 \\
\hline 23212 & 332.125 & 1.238 & 0.01535 & 138.155 & 179.58 & 0.00888 \\
\hline 23215 & 334.519 & 1.034 & 0.01523 & 142.464 & 149.97 & 0.00881 \\
\hline 23218 & 333.645 & 1.033 & 0.01514 & 140.891 & 149.80 & 0.00875 \\
\hline 23221 & 332.873 & 1.032 & 0.01509 & 139.501 & 149.64 & 0.00872 \\
\hline 23224 & $337 . .187$ & 1.030 & 0.01505 & 138.267 & 149.44 & 0.00870 \\
\hline 23227 & 334.724 & 0.822 & 0.01501 & 142.833 & 119.22 & 0.00868 \\
\hline 23230 & 333.806 & 0.823 & 0.01491 & 141.181 & 119.30 & 0.00862 \\
\hline 23233 & 332.996 & 0.823 & 0.01485 & 139.723 & 119.34 & 0.00859 \\
\hline 23236 & 332.280 & 0.823 & 0.01478 & 138.434 & 119.37 & 0.00855 \\
\hline 23239 & 334.955 & 0.579 & 0.01474 & $: 43.249$ & 84.05 & 0.00852 \\
\hline 23242 & 333.990 & 0.580 & 0.01467 & 141.512 & 84.10 & 0.00848 \\
\hline 23245 & 333.122 & 0.580 & 0.01459 & 139.950 & 84.17 & 0.00844 \\
\hline 23248 & 332.371 & 0.581 & 0.01456 & 138.598 & 84.22 & 0.00842 \\
\hline 23251 & 335.335 & 0.327 & 0.01459 & 143.933 & 47.49 & 0.00844 \\
\hline 23254 & 334.274 & 0.328 & 0.01447 & 142.023 & 47.57 & 0.00837 \\
\hline 23257 & 333.337 & 0.328 & 0.01440 & 140.337 & 47.58 & 0.00833 \\
\hline 23260 & 332.522 & 0.329 & 0.01439 & 138.870 & 47.68 & 0.00832 \\
\hline
\end{tabular}


Table 22. Transient Hot-Wire Thermal Conductivity Data for HFC-32 (Continued)

\begin{tabular}{|c|c|c|c|c|c|c|}
\hline $\begin{array}{l}\text { Point } \\
\text { Number }\end{array}$ & $\begin{array}{l}\mathrm{T}_{\text {Exp. }} \\
(\mathrm{K})\end{array}$ & $\begin{array}{l}\mathrm{P}_{\mathrm{Cell}} \\
(\mathrm{MPa})\end{array}$ & $\begin{array}{c}\lambda_{\operatorname{Exp}_{.}} \\
(W / m-K)\end{array}$ & $\mathrm{T}_{\text {Exp. }}$ & $\begin{array}{l}\mathrm{P}_{\text {Cell }} \\
\text { (psia) }\end{array}$ & $\begin{array}{c}\lambda_{\text {Exp. }} \\
\text { (BTU/ft-hr-F) }\end{array}$ \\
\hline 23263 & 336.180 & 0.073 & 0.01305 & 145.454 & 10.62 & 0.00755 \\
\hline 23266 & 334.957 & 0.074 & 0.01301 & 143.253 & 10.71 & 0.00752 \\
\hline 23269 & 333.865 & 0.074 & 0.01293 & 141.287 & 10.76 & 0.00748 \\
\hline 23272 & 332.919 & 0.075 & 0.01286 & 139.584 & 10.81 & 0.00744 \\
\hline 27025 & 341.121 & 57.015 & 0.20434 & 154.348 & 8269.39 & 0.11814 \\
\hline 27027 & 341.277 & 57.022 & 0.19773 & 154.629 & 8270.33 & 0.11432 \\
\hline 27029 & 341.438 & 57.022 & 0.19273 & 154.918 & 8270.35 & 0.11143 \\
\hline 27031 & 341.619 & 57.027 & 0.18850 & 155.244 & 8271.07 & 0.10898 \\
\hline 27033 & 341.105 & 51.575 & 0.20349 & 154.319 & 7480.29 & 0.11765 \\
\hline 27035 & 341.258 & 51.581 & 0.19424 & 154.594 & 7481.29 & 0.11230 \\
\hline 27037 & 341.430 & 51.586 & 0.18764 & 154.904 & 7481.95 & 0.10849 \\
\hline 27039 & 341.604 & 51.591 & 0.18390 & 155.217 & 7482.66 & 0.10632 \\
\hline 27041 & 341.113 & 51.598 & 0.19717 & 154.333 & 7483.66 & 0.11400 \\
\hline 27042 & 341.330 & 51.600 & 0.19188 & 154.724 & 7483.95 & 0.11094 \\
\hline 27043 & 341.457 & 51.603 & 0.18434 & 154.953 & 7484.43 & 0.10658 \\
\hline 27044 & 341.635 & 51.607 & 0.18045 & 155.273 & 7484.93 & 0.10433 \\
\hline 27046 & 341.618 & 46.691 & 0.18053 & 155.242 & 6772.05 & 0.10438 \\
\hline 27048 & 341.444 & 46.696 & 0.18395 & 154.929 & 6772.78 & 0.10635 \\
\hline 27050 & 341.274 & 46.698 & 0.18943 & 154.623 & 6772.96 & 0.10952 \\
\hline 27052 & 341.100 & 46.701 & 0.19454 & 154.310 & 6773.51 & 0.11248 \\
\hline 27054 & 341.648 & 42.223 & 0.17948 & 155.296 & 6124.00 & 0.10377 \\
\hline 27056 & 341.461 & 42.228 & 0.18166 & 154.960 & 6124.66 & 0.10503 \\
\hline 27058 & 341.290 & 42.232 & 0.18617 & 154.652 & 6125.20 & 0.10764 \\
\hline 27060 & 341.130 & 42.236 & 0.19182 & 154.364 & 6125.84 & 0.11090 \\
\hline 27062 & 341.690 & 38.046 & 0.16954 & 155.372 & 5518.19 & 0.09802 \\
\hline 27064 & 341.513 & 38.048 & 0.17304 & 155.053 & 5518.37 & 0.10005 \\
\hline 27066 & 341.323 & 38.049 & 0.17830 & 154.711 & 5518.64 & 0.10309 \\
\hline 27068 & 341.147 & 38.051 & 0.18373 & 154.395 & 5518.85 & 0.10623 \\
\hline 27070 & 341.757 & 34.101 & 0.15699 & 155.493 & 4946.03 & 0.09077 \\
\hline 27072 & 341.578 & 34.101 & 0.15959 & 155.170 & 4945.95 & 0.09227 \\
\hline 27074 & 341.376 & 34.102 & 0.16298 & 154.807 & 4946.12 & 0.09423 \\
\hline 27076 & 341.221 & 34.098 & 0.16558 & 154.528 & 4945.59 & 0.09573 \\
\hline 27078 & 341.769 & 34.012 & 0.15635 & 155.514 & 4933.06 & 0.09040 \\
\hline 27080 & 341.572 & 34.016 & 0.15785 & 155.160 & 4933.61 & 0.09126 \\
\hline 27082 & 341.415 & 34.020 & 0.15899 & 154.877 & 4934.22 & 0.09192 \\
\hline 27084 & 341.237 & 34.024 & 0.16051 & 154.557 & 4934.75 & 0.09280 \\
\hline 27086 & 341.830 & 30.656 & 0.15685 & I: 5.624 & 4446.24 & 0.09069 \\
\hline 27088 & 341.645 & 30.659 & 0.15825 & 155.291 & 4446.75 & 0.09149 \\
\hline 27090 & 341.468 & 30.663 & 0.16021 & 154.972 & $444^{7} .39$ & 0.09263 \\
\hline 27092 & 341.296 & 30.667 & 0.16297 & 154.663 & 4447.95 & $0 . v+22$ \\
\hline 27094 & 341.871 & 27.547 & 0.15389 & 155.698 & 3995.38 & 0.08897 \\
\hline 27096 & 341.660 & 27.550 & $0.1557 \delta$ & 155.318 & 3995.83 & 0.09007 \\
\hline 27098 & 341.490 & 27.554 & 0.15714 & 155.012 & 3996.35 & 0.09085 \\
\hline 27100 & 341.324 & 27.557 & 0.15952 & 154.713 & 3996.78 & 0.09223 \\
\hline 27102 & 341.869 & 24.682 & 0.15108 & 155.694 & 3579.79 & 0.08735 \\
\hline 27104 & 341.682 & 24.683 & 0.15235 & 155.358 & 3580.00 & 0.08808 \\
\hline 27106 & 341.487 & 24.685 & 0.15416 & 155.007 & 3580.21 & 0.08913 \\
\hline 27108 & 341.319 & 24.686 & $0.156 ! 9$ & 154.704 & 3580.48 & 0.09030 \\
\hline 27110 & 341.901 & 22.012 & 0.14829 & 155.752 & 3192.53 & 0.08574 \\
\hline
\end{tabular}


Table 22. Transient Hot-Wire Thermal Conductivity Data for HFC-32 (Continued)

\begin{tabular}{|c|c|c|c|c|c|c|}
\hline $\begin{array}{l}\text { Point } \\
\text { Number }\end{array}$ & $\begin{array}{l}\mathrm{T}_{\text {Exp. }} \\
(\mathrm{K})\end{array}$ & $\begin{array}{l}\mathrm{P}_{\mathrm{Cell}} \\
(\mathrm{MPa})\end{array}$ & $\begin{array}{c}\lambda_{\operatorname{Exp}_{.}} \\
(\mathrm{W} / \mathrm{m}-\mathrm{K})\end{array}$ & $\mathrm{T}_{\mathrm{Exp}}$ & $\begin{array}{l}\mathrm{P}_{\mathrm{Cell}} \\
\text { (psia) }\end{array}$ & $\begin{array}{c}\lambda_{\text {Exp. }} \\
(B T U / f t-h r-F)\end{array}$ \\
\hline 27112 & 341.684 & 22.014 & 0.14938 & 155.361 & 3192.83 & 0.08637 \\
\hline 27114 & 341.513 & 22.015 & 0.15135 & 155.053 & 3192.99 & 0.08751 \\
\hline 27116 & 341.327 & 22.017 & 0.15338 & 154.719 & 3193.28 & 0.08868 \\
\hline 27118 & 341.973 & 19.575 & 0.14490 & 155.881 & 2839.15 & 0.08378 \\
\hline 27120 & 341.761 & 19.575 & 0.14572 & 155.200 & 2839.19 & 0.08425 \\
\hline 27122 & 341.566 & 19.575 & 0.14759 & 155.149 & 2839.11 & 0.08533 \\
\hline 27124 & 341.388 & 19.577 & 0.14860 & 154.828 & 2839.35 & 0.08592 \\
\hline 27126 & 342.002 & 17.460 & 0.14108 & 155.934 & 2532.32 & 0.08157 \\
\hline 27128 & 341.792 & 17.464 & 0.14125 & 155.556 & 2533.01 & 0.08167 \\
\hline 27130 & 341.591 & 17.466 & 0.14316 & 155.194 & 2533.22 & 0.08277 \\
\hline 27132 & 341.404 & 17.467 & 0.14550 & 154.857 & 2533.45 & 0.08412 \\
\hline 27134 & 342.062 & 15.444 & 0.13727 & 156.042 & 2239.97 & 0.07936 \\
\hline 27136 & 341.860 & 15.444 & 0.13787 & 155.678 & 2240.00 & 0.07971 \\
\hline 27138 & 341.670 & 15.445 & 0.13833 & 155.336 & 2240.15 & 0.07998 \\
\hline 27140 & 341.475 & 15.445 & 0.13960 & 154.985 & 2240.14 & 0.08071 \\
\hline 27141 & 342.053 & 13.730 & 0.13081 & 156.025 & 1991.43 & 0.07563 \\
\hline 27143 & 341.839 & 13.732 & 0.13147 & 155.640 & 1991.69 & 0.07601 \\
\hline 27145 & 341.639 & 13.734 & 0.13193 & 155.280 & 1992.00 & 0.07628 \\
\hline 27147 & 341.451 & 13.734 & 0.13280 & 154.942 & 1992.01 & 0.07678 \\
\hline 27150 & 342.070 & 13.742 & 0.13057 & 156.056 & 1993.12 & 0.07549 \\
\hline 27152 & 341.860 & 13.744 & 0.13081 & 155.678 & 1993.39 & 0.07563 \\
\hline 27154 & 341.655 & 13.747 & 0.13122 & 155.309 & 1993.82 & 0.07587 \\
\hline 27156 & 341.463 & 13.748 & 0.13157 & 154.963 & 1994.01 & 0.07607 \\
\hline 27158 & 342.052 & 12.166 & 0.12817 & 156.024 & 1764.56 & 0.07410 \\
\hline 27160 & 341.843 & 12.168 & 0.12837 & 155.647 & 1764.81 & 0.07422 \\
\hline 27162 & 341.641 & 12.169 & 0.12872 & 155.284 & 1764.99 & 0.07442 \\
\hline 27164 & 341.460 & 12.171 & 0.12951 & 154.958 & 1765.32 & 0.07488 \\
\hline 27166 & 342.056 & 10.775 & 0.12546 & 156.031 & 1562.84 & 0.07254 \\
\hline 27168 & 341.853 & 10.777 & 0.12593 & 155.665 & 1563.12 & 0.07281 \\
\hline 27170 & 341.650 & 10.779 & 0.12606 & 155.300 & 1563.41 & 0.07288 \\
\hline 27172 & 341.451 & 10.782 & 0.12598 & 154.942 & 1563.83 & 0.07284 \\
\hline 27174 & 342.073 & 9.559 & 0.12327 & 156.061 & 1386.38 & 0.07127 \\
\hline 27176 & 341.853 & 9.561 & 0.12350 & 155.665 & 1386.67 & 0.07140 \\
\hline 27178 & 341.648 & 9.564 & 0.12369 & 155.296 & 1387.17 & 0.07151 \\
\hline 27180 & 341.450 & 9.568 & 0.12371 & 154.940 & 1387.71 & 0.07153 \\
\hline 27182 & 342.078 & 8.486 & 0.12108 & 156.070 & 1230.73 & 0.07000 \\
\hline 27184 & 341.863 & 8.490 & 0.12130 & 155.683 & 1231.33 & 0.07013 \\
\hline 27186 & 341.657 & 8.494 & 0.12134 & 155.313 & 1231.99 & 0.07015 \\
\hline 27188 & 341.456 & 8.498 & 0.12133 & 154.951 & 1232.55 & 0.07015 \\
\hline 27190 & 342.100 & 7.536 & 0.11843 & 156.110 & 1092.94 & 0.06847 \\
\hline 27192 & 341.894 & 7.540 & 0.11861 & 155.739 & 1093.57 & 0.06858 \\
\hline 27194 & 341.694 & 7.544 & 0.11900 & 155.379 & 1094.13 & 0.06880 \\
\hline 27196 & 341.495 & 7.549 & 0.11894 & 155.021 & 1094.85 & $0.0687 i$ \\
\hline 27198 & 342.094 & 6.686 & 0.11697 & 156.099 & 969.72 & 0.06763 \\
\hline 27200 & 341.875 & 6.689 & 0.11728 & 155.705 & 970.11 & 0.06781 \\
\hline 27202 & 341.669 & 6.692 & 0.11701 & 155.334 & 970.58 & 0.06765 \\
\hline 27204 & 341.466 & 6.695 & 0.11715 & 154.969 & 971.04 & 0.06773 \\
\hline 27206 & 342.106 & 5.955 & 0.11484 & $! 56.12 !$ & 863.76 & 0.06640 \\
\hline 27208 & 341.895 & 5.956 & 0.11501 & 155.741 & 863.91 & 0.06649 \\
\hline
\end{tabular}


Table 22. Transient Hot-Wire Thermal Conductivity Data for HFC-32 (Continued)

\begin{tabular}{|c|c|c|c|c|c|c|}
\hline $\begin{array}{l}\text { Point } \\
\text { Number }\end{array}$ & $\begin{array}{l}\mathrm{T}_{\text {Exp. }} \\
(\mathrm{K})\end{array}$ & $\begin{array}{l}\mathrm{P}_{\mathrm{Cell}} \\
(\mathrm{MPa})\end{array}$ & $\begin{array}{c}\lambda_{\operatorname{Exp}_{.}} \\
(W / m-K)\end{array}$ & $\mathrm{T}_{\text {Exp. }}$ & $\begin{array}{l}\mathrm{P}_{\text {Cell }} \\
\text { (psia) }\end{array}$ & $\begin{array}{c}\lambda_{\text {Exp. }} \\
\text { (BTU/ft-hr-F) }\end{array}$ \\
\hline 27210 & 341.687 & 5.957 & 0.11501 & 155.367 & 863.98 & 0.06649 \\
\hline 27212 & 341.480 & 5.957 & 0.11524 & 154.994 & 864.03 & 0.06663 \\
\hline 27214 & 342.074 & 5.346 & 0.11420 & 156.063 & 775.41 & 0.06603 \\
\hline 27216 & 341.861 & 5.345 & 0.11422 & 155.680 & 775.29 & 0.06604 \\
\hline 27218 & 341.653 & 5.345 & 0.11400 & 155.305 & 775.18 & 0.06591 \\
\hline 27220 & 341.452 & 5.344 & 0.11436 & 154.944 & 775.12 & 0.06612 \\
\hline 27222 & 342.028 & 4.844 & 0.11646 & 155.980 & 702.63 & 0.06733 \\
\hline 27224 & 341.817 & 4.844 & 0.11505 & 155.601 & 702.58 & 0.06652 \\
\hline 27226 & 341.610 & 4.844 & 0.11540 & 155.228 & 702.55 & 0.06672 \\
\hline 27228 & 341.419 & 4.844 & 0.11476 & 154.884 & 702.53 & 0.06635 \\
\hline 24009 & 342.878 & 4.443 & 0.03392 & 157.510 & 644.42 & 0.01961 \\
\hline 24011 & 342.522 & 4.442 & 0.03337 & 156.870 & 644.26 & 0.01929 \\
\hline 24013 & 342.187 & 4.442 & 0.03307 & 156.267 & 644.27 & 0.01912 \\
\hline 24015 & 341.875 & 4.442 & 0.03283 & 155.705 & 644.26 & 0.01898 \\
\hline 24017 & 343.061 & 4.392 & 0.03206 & 157.840 & 636.97 & 0.01854 \\
\hline 24019 & 342.689 & 4.392 & 0.03170 & 157.170 & 636.94 & 0.01833 \\
\hline 24021 & 342.333 & 4.392 & 0.03140 & 156.529 & 636.99 & 0.01815 \\
\hline 24023 & 342.010 & 4.392 & 0.03145 & 155.948 & 636.95 & 0.01818 \\
\hline 24025 & 343.251 & 4.320 & 0.03046 & 158.182 & 626.55 & 0.01761 \\
\hline 24027 & 342.869 & 4.318 & 0.03022 & 157.494 & 626.31 & 0.01747 \\
\hline 24029 & 342.493 & 4.318 & 0.03010 & 156.817 & 626.24 & 0.01740 \\
\hline 24031 & 342.144 & 4.317 & 0.02998 & 156.189 & 626.20 & 0.01733 \\
\hline 24033 & 343.416 & 4.256 & 0.02934 & 158.479 & 617.33 & 0.01696 \\
\hline 24035 & 342.998 & 4.256 & 0.02913 & 157.726 & 617.29 & 0.01684 \\
\hline 24037 & 342.609 & 4.256 & 0.02894 & 157.026 & 617.31 & 0.01673 \\
\hline 24039 & 342.249 & 4.256 & 0.02906 & 156.378 & 617.30 & 0.01680 \\
\hline 24041 & 343.545 & 4.189 & 0.02833 & 158.711 & 607.59 & 0.01638 \\
\hline 24043 & 343.127 & 4.188 & 0.02818 & 157.959 & 607.47 & 0.01629 \\
\hline 24045 & 342.730 & 4.188 & 0.02799 & 157.244 & 607.36 & 0.01618 \\
\hline 24047 & 342.342 & 4.187 & 0.02801 & 156.546 & 607.34 & 0.01619 \\
\hline 24049 & 343.694 & 4.115 & 0.02741 & i 58.979 & 596.90 & 0.01585 \\
\hline 24051 & 343.245 & 4.115 & 0.02727 & 158.171 & 596.79 & 0.01577 \\
\hline 24053 & 342.824 & 4.115 & 0.02712 & 157.413 & 596.76 & 0.01568 \\
\hline 24055 & 342.436 & 4.114 & 0.02707 & 156.715 & 596.71 & 0.01565 \\
\hline 24057 & 343.817 & 4.049 & 0.02665 & 159.201 & 587.28 & 0.01541 \\
\hline 24059 & 343.339 & 4.049 & 0.02650 & 158.340 & 587.22 & 0.01532 \\
\hline 24061 & 342.901 & 4.048 & 0.02648 & 157.552 & 587.17 & 0.01531 \\
\hline 24063 & 342.508 & 4.048 & 0.02629 & 156.844 & 587.14 & 0.01520 \\
\hline 24065 & 343.936 & 3.967 & 0.02582 & 159.415 & 575.41 & 0.01493 \\
\hline 24067 & 343.460 & 3.967 & 0.02566 & 158.558 & 575.32 & 0.01484 \\
\hline 24069 & 343.009 & 3.968 & 0.02558 & 157.746 & 575.45 & 0.01479 \\
\hline 24071 & 342.589 & 3.968 & 0.02578 & 156.990 & 575.44 & 0.01491 \\
\hline 24073 & 344.071 & 3.895 & 0.02513 & 159.658 & 564.91 & 0.01453 \\
\hline 24075 & 343.587 & 3.894 & 0.02506 & 158.787 & 564.75 & 0.01449 \\
\hline 24077 & 343.111 & 3.893 & 0.02503 & 157.930 & 564.64 & 0.01447 \\
\hline 24079 & 342.682 & 3.892 & 0.02499 & 157.158 & 564.55 & 0.01445 \\
\hline 24081 & 343.696 & 3.793 & 0.02426 & 158.983 & 550.13 & 0.01403 \\
\hline 24083 & 343.234 & 3.792 & 0.02420 & 158.151 & 549.95 & 0.01399 \\
\hline 24085 & 342.794 & 3.791 & 0.02417 & 157.359 & 549.84 & 0.01397 \\
\hline
\end{tabular}


Table 22. Transient Hot-Wire Thermal Conductivity Data for HFC-32 (Continued)

\begin{tabular}{|c|c|c|c|c|c|c|}
\hline $\begin{array}{l}\text { Point } \\
\text { Number }\end{array}$ & $\mathrm{T}_{\text {Exp. }}$ & $\begin{array}{l}\mathrm{P}_{\mathrm{Cell}} \\
(\mathrm{MPa})\end{array}$ & $\begin{array}{c}\lambda_{\text {Exp. }} \\
(\mathrm{W} / \mathrm{m}-\mathrm{K})\end{array}$ & $\mathrm{T}_{\text {Exp. }}$ & $\begin{array}{l}\mathrm{P}_{\text {Cell }} \\
\text { (psia) }\end{array}$ & $\begin{array}{c}\lambda_{\text {Exp. }} \\
(B T U / f t-h r-F)\end{array}$ \\
\hline 24087 & 342.381 & 3.790 & 0.02427 & 156.616 & 549.74 & 0.01403 \\
\hline 24089 & 343.850 & 3.677 & 0.02349 & 159.260 & 533.34 & 0.01358 \\
\hline 24091 & 343.369 & 3.674 & 0.02341 & 158.394 & 532.93 & 0.01354 \\
\hline 24093 & 342.909 & 3.673 & 0.02328 & 157.566 & 532.74 & 0.01346 \\
\hline 24095 & 342.476 & 3.672 & 0.02341 & 156.787 & 532.65 & 0.01354 \\
\hline 24097 & 344.012 & 3.552 & 0.02268 & 159.552 & 515.21 & 0.01311 \\
\hline 24099 & 343.503 & 3.551 & 0.02260 & 158.635 & 514.97 & 0.01307 \\
\hline 24101 & 343.019 & 3.550 & 0.02261 & 157.764 & 514.85 & 0.01307 \\
\hline 24103 & 342.571 & 3.549 & 0.02263 & 156.958 & 514.75 & 0.01308 \\
\hline 24105 & 344.140 & 3.452 & 0.02215 & 159.782 & 500.73 & 0.01281 \\
\hline 24107 & 343.602 & $3 .+51$ & 0.02213 & 158.814 & 500.57 & 0.01280 \\
\hline 24109 & 343.109 & 3.451 & 0.02207 & 157.926 & 500.50 & 0.01276 \\
\hline 24111 & 342.653 & 3.451 & 0.02203 & 157.105 & 500.49 & 0.01274 \\
\hline 24113 & 341.346 & 3.451 & 0.02217 & 154.753 & 500.48 & 0.01282 \\
\hline 24115 & 344.019 & 3.312 & 0.02151 & 159.564 & 480.43 & 0.01244 \\
\hline 24117 & 343.478 & 3.311 & 0.02145 & 158.590 & 480.27 & 0.01240 \\
\hline 24119 & 342.996 & 3.311 & 0.02140 & 157.723 & 480.22 & 0.01237 \\
\hline 24121 & 342.530 & 3.311 & 0.02140 & 156.884 & 480.19 & 0.01237 \\
\hline 24123 & 341.390 & 3.312 & 0.02183 & 154.832 & 480.30 & 0.01262 \\
\hline 24125 & 344.153 & 3.188 & 0.02094 & 159.805 & 462.42 & 0.01211 \\
\hline 24127 & 343.601 & 3.188 & 0.02088 & 158.812 & 462.36 & 0.01207 \\
\hline 24129 & 343.086 & 3.188 & 0.02081 & 157.885 & 462.33 & 0.01203 \\
\hline 24131 & 342.612 & 3.187 & 0.02089 & 157.032 & 462.28 & 0.01208 \\
\hline 24133 & 341.434 & 3.190 & 0.02073 & 154.911 & 462.65 & 0.01199 \\
\hline 24135 & 344.316 & 3.030 & 0.02027 & 160.099 & 439.51 & 0.01172 \\
\hline 24137 & 343.739 & 3.031 & 0.02031 & 159.060 & 439.63 & 0.01174 \\
\hline 24139 & 343.207 & 3.031 & 0.02021 & 158.103 & 439.65 & 0.01169 \\
\hline 24141 & 342.710 & 3.032 & 0.02020 & 157.208 & 439.81 & 0.01168 \\
\hline 24143 & 341.488 & 3.043 & 0.02023 & 155.008 & 441.37 & 0.01170 \\
\hline 24145 & 344.412 & 2.940 & 0.01999 & 160.272 & 426.45 & 0.01156 \\
\hline 24147 & $: 43.823$ & 2.943 & 0.01993 & 159.211 & 426.78 & 0.01152 \\
\hline 24149 & 343.271 & 2.944 & 0.02006 & 158.218 & 427.06 & 0.01160 \\
\hline 24151 & 342.779 & 2.946 & 0.01996 & 157.332 & 427.28 & 0.01154 \\
\hline 24153 & 341.532 & 2.949 & 0.02020 & 155.088 & 427.74 & 0.01168 \\
\hline 24155 & 344.592 & 2.773 & 0.01948 & 160.596 & 402.12 & 0.01126 \\
\hline 24157 & 343.969 & 2.772 & 0.01979 & 159.474 & 402.02 & 0.01144 \\
\hline 24159 & 343.418 & 2.772 & 0.01968 & 158.482 & 402.09 & 0.01138 \\
\hline 24161 & 342.893 & 2.772 & 0.01969 & 157.537 & 402.05 & 0.01138 \\
\hline 24163 & 341.601 & 2.772 & 0.01999 & 155.212 & 402.01 & 0.01156 \\
\hline 24165 & 344.406 & 2.635 & 0.01908 & 160.261 & 382.19 & 0.01103 \\
\hline 24167 & 343.803 & 2.636 & 0.01905 & 159.175 & 382.29 & 0.01101 \\
\hline 24169 & 343.244 & 2.637 & 0.01893 & 158.169 & 382.42 & 0.01094 \\
\hline 24171 & 342.726 & 2.638 & 0.01895 & 157.237 & 382.57 & 0.01096 \\
\hline 24173 & 341.644 & 2.639 & 0.01895 & 155.289 & 382.77 & 0.01096 \\
\hline 24175 & 344.569 & 2.443 & 0.01853 & 160.554 & 354.38 & 0.01071 \\
\hline 24177 & 343.951 & 2.445 & 0.01852 & 159.442 & 354.59 & 0.01071 \\
\hline 24179 & 343.359 & 2.446 & 0.01845 & 158.376 & 354.80 & 0.01067 \\
\hline 24181 & 342.821 & 2.448 & 0.01845 & 157.408 & 355.03 & 0.01067 \\
\hline 24183 & 341.697 & 2.451 & 0.01826 & 155.385 & 355.50 & 0.01056 \\
\hline
\end{tabular}


Table 22. Transient Hot-Wire Thermal Conductivity Data for HFC-32 (Continued)

\begin{tabular}{|c|c|c|c|c|c|c|}
\hline $\begin{array}{l}\text { Point } \\
\text { Number }\end{array}$ & $\begin{array}{l}\mathrm{T}_{\text {Exp. }} \\
\text { (K) }\end{array}$ & $\begin{array}{l}\mathrm{P}_{\mathrm{Cell}} \\
(\mathrm{MPa})\end{array}$ & $\begin{array}{c}\lambda_{\text {Exp. }} \\
(\mathrm{W} / \mathrm{m}-\mathrm{K})\end{array}$ & $\mathrm{T}_{\text {Exp. }}$ & $\begin{array}{l}\mathrm{P}_{\text {Cell }} \\
\text { (psia) }\end{array}$ & $\begin{array}{c}\lambda_{\text {Exp. }} \\
\text { (BTU/ft-hr-F) }\end{array}$ \\
\hline 24185 & 344.700 & 2.266 & 0.01811 & 160.790 & 328.60 & 0.01047 \\
\hline 24187 & 344.071 & 2.264 & 0.01807 & 159.658 & 328.41 & 0.01045 \\
\hline 24189 & 343.462 & 2.264 & 0.01806 & 158.562 & 328.37 & 0.01044 \\
\hline 24191 & 342.912 & 2.264 & 0.01799 & 157.572 & 328.37 & 0.01040 \\
\hline 24193 & 341.759 & 2.264 & 0.01773 & 155.496 & 328.40 & 0.01025 \\
\hline 24195 & 344.503 & 2.102 & 0.01777 & 160.435 & 304.89 & 0.01027 \\
\hline 24197 & 343.867 & 2.103 & 0.01769 & 159.291 & 305.06 & 0.01023 \\
\hline 24199 & 343.269 & 2.105 & 0.01765 & 158.214 & 305.36 & 0.01020 \\
\hline 24201 & 342.716 & 2.107 & 0.01764 & 157.219 & 305.53 & 0.01020 \\
\hline 24203 & 341.795 & 2.108 & 0.01758 & 155.561 & 305.75 & 0.01016 \\
\hline 24205 & 344.669 & 1.893 & 0.01739 & 160.734 & 274.52 & 0.01005 \\
\hline 24207 & 344.008 & 1.888 & 0.01733 & 159.544 & 273.87 & 0.01002 \\
\hline 24209 & 343.390 & 1.885 & 0.01719 & 158.432 & 273.37 & 0.00994 \\
\hline 24211 & 342.819 & 1.883 & 0.01731 & 157.404 & 273.12 & 0.01001 \\
\hline 24213 & 341.864 & 1.881 & 0.01731 & 155.685 & 272.87 & 0.01001 \\
\hline 24215 & 344.840 & 1.696 & 0.01710 & 161.042 & 245.92 & 0.00989 \\
\hline 24217 & 344.157 & 1.687 & 0.01703 & 159.813 & 244.68 & 0.00985 \\
\hline 24219 & 343.518 & 1.680 & 0.01694 & 158.662 & 243.68 & 0.00979 \\
\hline 24221 & 342.934 & 1.674 & 0.01692 & 157.611 & 242.86 & 0.00978 \\
\hline 2422.3 & 341.936 & 1.668 & 0.01713 & 155.815 & 241.88 & 0.00990 \\
\hline 24225 & 344.658 & 1.492 & 0.01674 & 160.714 & 216.38 & 0.00968 \\
\hline 24227 & 343.969 & 1.491 & 0.01676 & 159.474 & 216.27 & 0.00969 \\
\hline 24229 & 343.336 & 1.491 & 0.01667 & 158.335 & 216.24 & 0.00964 \\
\hline 24231 & 342.763 & 1.491 & 0.01669 & 157.303 & 216.26 & 0.00965 \\
\hline 24233 & 341.998 & 1.492 & 0.01648 & 155.926 & 216.41 & 0.00953 \\
\hline 24235 & 344.835 & 1.271 & 0.01647 & 161.033 & 184.28 & 0.00952 \\
\hline 24237 & 344.123 & 1.271 & 0.01641 & 159.751 & 184.28 & 0.00949 \\
\hline 24239 & 343.455 & 1.271 & 0.01627 & 158.549 & 184.30 & 0.00941 \\
\hline 24241 & 342.857 & 1.271 & 0.01620 & 157.473 & 184.33 & 0.00937 \\
\hline 24243 & 342.072 & 1.271 & 0.01625 & 156.060 & 184.36 & $0.009-10$ \\
\hline 24245 & 345.041 & 1.040 & 0.01619 & 161.404 & 150.86 & 0.00936 \\
\hline 24247 & 344.291 & 1.040 & 0.01613 & 160.054 & 150.90 & 0.00933 \\
\hline 24249 & 343.603 & 1.041 & 0.01608 & 158.815 & 150.92 & 0.00930 \\
\hline 24251 & 342.974 & 1.041 & 0.01604 & 157.683 & 150.97 & 0.00927 \\
\hline 24253 & 342.145 & 1.041 & 0.01595 & 156.191 & 151.04 & 0.00922 \\
\hline 24255 & 345.278 & 0.789 & 0.01596 & 161.830 & 114.49 & 0.00923 \\
\hline 24257 & 344.489 & 0.790 & 0.01588 & 160.410 & 114.53 & 0.00918 \\
\hline 24259 & 343.771 & 0.790 & 0.01584 & 159.118 & 114.61 & 0.00916 \\
\hline 24261 & 343.113 & 0.790 & 0.01573 & 157.933 & 114.60 & 0.00909 \\
\hline 24263 & 342.253 & 0.791 & 0.01571 & 156.385 & 114.67 & 0.00908 \\
\hline 24265 & 345.557 & 0.540 & 0.01578 & 162.333 & 78.36 & 0.00912 \\
\hline 24267 & 344.724 & 0.541 & 0.01569 & 160.833 & 78.45 & 0.00907 \\
\hline 24269 & 313.956 & 0.541 & 0.01556 & 159.451 & 78.51 & 0.00900 \\
\hline 24271 & 343.262 & 0.542 & 0.01552 & $1: 8.202$ & 78.55 & 0.00897 \\
\hline 24273 & 342.348 & 0.542 & 0.01551 & 156.556 & 78.63 & 0.00897 \\
\hline 24275 & 345.986 & 0.281 & 0.01565 & 163.105 & 40.82 & 0.00905 \\
\hline 24277 & 345.104 & 0.282 & 0.01553 & 161.517 & 40.94 & 0.00898 \\
\hline 24279 & 344.267 & 0.283 & 0.01550 & 160.011 & 41.02 & 0.00896 \\
\hline 24281 & 343.523 & 0.283 & 0.01537 & 158.671 & 41.08 & 0.00889 \\
\hline 24283 & 342.531 & 0.284 & 0.01535 & 156.886 & 41.14 & 0.00888 \\
\hline
\end{tabular}


Table 23. Thermal conductivity correlation parameters for HFC-32, with thermal conductivity in $\mathrm{W} / \mathrm{m}-\mathrm{K}$, temperature in $\mathrm{K}$, and density in $\mathrm{mol} / \mathrm{L}$. The parameters are explained in Appendix $\mathrm{B}$.

Refrigerant

HFC-32

Dilute Gas Terms

$a_{1}$

$a_{2}$

$a_{3}$

Excess Function rerms
$-1.17164 \times 10^{-3}$

$3.49249 \times 10^{-5}$

$3.28621 \times 10^{-8}$
$b_{1}$
$b_{2}$
$b_{3}$
$\mathrm{b}_{4}$
$b_{5}$
$b_{6}$
$b_{7}$
$b_{8}$

$5.48429 \times 10^{0}$

$-6.86183 \times 10^{-3}$

$-6.08572 \times 10^{0}$

$1.87197 \times 10^{-2}$

$4.44371 \times 10^{-1}$

$-1.33799 \times 10^{-3}$

$-8.23987 \times 10^{-3}$

$2.58946 \times 10^{-5}$

Critical Enhancement Parameters

$$
\begin{aligned}
& T_{c} \\
& P_{c} \\
& \rho_{c} \\
& q_{D}
\end{aligned}
$$

$351.6 \mathrm{~K}$

58.30 bar

$8.124 \mathrm{~mol} / \mathrm{L}$ $3.0 \times 10^{9} \mathrm{~m}^{-1}$ 
Table 24a. Viscosity of compressed tluid difluoromethane (HFC-32) (SI units).

\begin{tabular}{|c|c|c|c|}
\hline $\begin{array}{c}\text { Temperature } \\
\mathrm{K}\end{array}$ & $\begin{array}{c}\text { Pressure } \\
\mathrm{MPa}\end{array}$ & $\begin{array}{l}\text { Density } \\
\mathrm{mol} \cdot \mathrm{dm}^{-3}\end{array}$ & $\begin{array}{c}\text { Viscosity } \\
\mathrm{mPa} \cdot \mathrm{s}\end{array}$ \\
\hline \multirow[t]{10}{*}{200.00} & 35.73 & 25.44 & 0.472 \\
\hline & 30.92 & 25.36 & 0.467 \\
\hline & 28.01 & 25.31 & 0.455 \\
\hline & 24.41 & 25.25 & 0.449 \\
\hline & 20.48 & 25.18 & 0.441 \\
\hline & 17.18 & 25.12 & 0.432 \\
\hline & 13.75 & 25.06 & 0.426 \\
\hline & 10.59 & 25.00 & 0.418 \\
\hline & 7.15 & 24.94 & 0.413 \\
\hline & 3.86 & 24.88 & 0.398 \\
\hline \multirow[t]{5}{*}{250.00} & 27.76 & 22.58 & 0.252 \\
\hline & 20.93 & 22.40 & 0.242 \\
\hline & 14.58 & 22.21 & 0.234 \\
\hline & 7.09 & 21.98 & 0.221 \\
\hline & 3.64 & 21.86 & 0.213 \\
\hline 300.00 & 34.00 & 20.17 & 0.194 \\
\hline \multirow{9}{*}{ 解 } & 31.18 & 20.06 & 0.184 \\
\hline & 27.89 & 19.92 & 0.179 \\
\hline & 24.32 & 19.76 & 0.177 \\
\hline & 20.63 & 19.59 & 0.161 \\
\hline & 17.61 & 19.43 & 0.156 \\
\hline & 14.00 & 19.22 & 0.149 \\
\hline & 10.50 & 19.00 & 0.142 \\
\hline & 6.91 & 18.75 & 0.136 \\
\hline & 3.77 & 18.50 & 0.131 \\
\hline
\end{tabular}




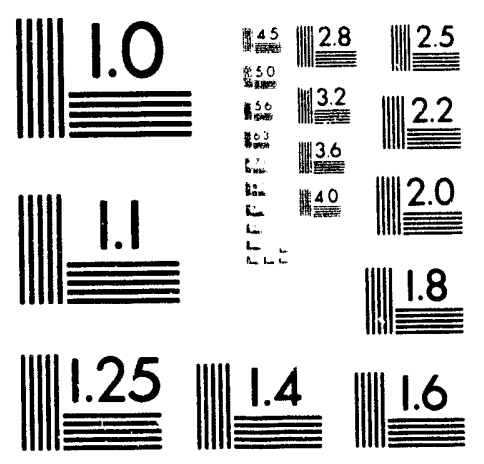



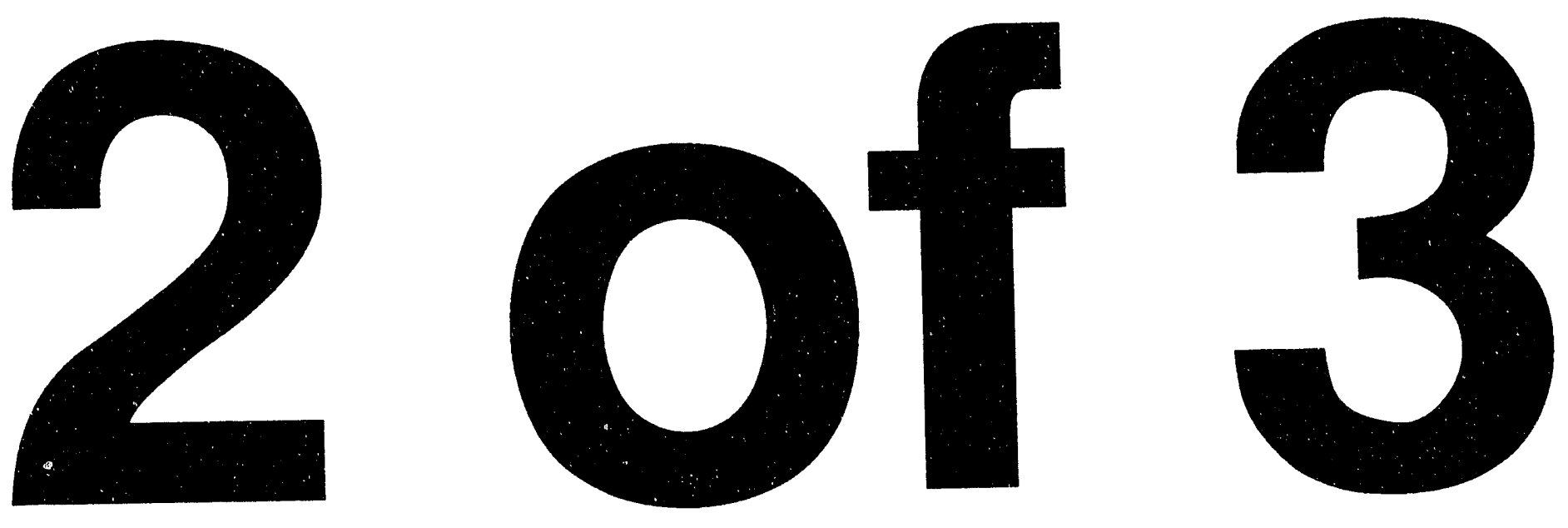


\begin{tabular}{||c|c|c|c|}
\hline $\begin{array}{c}\text { Temperature } \\
\mathrm{K}\end{array}$ & $\begin{array}{c}\text { Pressure } \\
\mathrm{MPa}\end{array}$ & $\begin{array}{c}\text { Density } \\
\mathrm{mol} \cdot \mathrm{dm}^{-3}\end{array}$ & $\begin{array}{c}\text { Viscosity } \\
\mathrm{mPa} \cdot \mathrm{s}\end{array}$ \\
\hline \hline & & & \\
\hline \hline \multirow{2}{*}{420.00} & 18.28 & 10.09 & 0.063 \\
\hline & 14.24 & 7.62 & 0.038 \\
\hline & 12.39 & 6.12 & 0.030 \\
\cline { 2 - 4 } & 9.10 & 3.78 & 0.022 \\
\cline { 2 - 5 } & 5.09 & 1.76 & 0.018 \\
\hline
\end{tabular}


Table 24b. Viscosity of compressed fluid difluoromethane (HFC-32) (PI units).

\begin{tabular}{|c|c|c|c|}
\hline $\begin{array}{l}\text { Temperature } \\
{ }^{\circ} \mathrm{F}\end{array}$ & $\begin{array}{c}\text { Pressure } \\
\text { psia }\end{array}$ & $\begin{array}{l}\text { Density } \\
\mathrm{lb} \cdot \mathrm{ft}^{-3}\end{array}$ & $\begin{array}{l}\text { Viscosity } \\
10^{-3} \mathrm{lb} / \mathrm{ft} \cdot \mathrm{s}\end{array}$ \\
\hline \multirow[t]{10}{*}{-99.67} & 5181 & 82.63 & 0.317 \\
\hline & 4483 & 82.37 & 0.314 \\
\hline & 4061. & 82.21 & 0.306 \\
\hline & 3539. & 82.01 & 0.302 \\
\hline & 2970. & 81.78 & 0.296 \\
\hline & 2491. & 81.59 & 0.290 \\
\hline & 1994. & 81.34 & 0.286 \\
\hline & 1536. & 81.20 & 0.281 \\
\hline & 1037. & 81.00 & 0.278 \\
\hline & 559.7 & 80.81 & 0.267 \\
\hline \multirow[t]{5}{*}{-9.67} & 4025. & 73.34 & 0.169 \\
\hline & 3035. & 72.76 & 0.163 \\
\hline & 2114. & 72.14 & 0.157 \\
\hline & 1028. & 71.39 & 0.149 \\
\hline & 527.8 & 71.00 & 0.143 \\
\hline \multirow[t]{10}{*}{80.33} & 4930. & 65.51 & 0.130 \\
\hline & 4521. & 65.15 & 0.124 \\
\hline & 4044. & 64.70 & 0.120 \\
\hline & 3526. & 64.14 & 0.119 \\
\hline & 2991. & 63.63 & 0.108 \\
\hline & 2553. & 63.11 & 0.105 \\
\hline & 2030. & 62.43 & 0.100 \\
\hline & 1522 & 61.71 & 0.095 \\
\hline & 1002. & 60.90 & 0.091 \\
\hline & 546.7 & 60.09 & 0.088 \\
\hline
\end{tabular}




\begin{tabular}{||c|c|c|c|}
\hline $\begin{array}{c}\text { Temperature } \\
{ }^{\circ} \mathrm{F}\end{array}$ & $\begin{array}{c}\text { Pressure } \\
\mathrm{psia}\end{array}$ & $\begin{array}{c}\text { Density } \\
\mathrm{lb} \cdot \mathrm{ft}^{-3}\end{array}$ & $\begin{array}{c}\text { Viscosity } \\
10^{-3} \mathrm{lb} / \mathrm{ft} \cdot \mathrm{s}\end{array}$ \\
\hline \hline \multirow{3}{*}{296.33} & & & \\
\hline \hline & 2651. & 32.77 & 0.042 \\
\cline { 2 - 4 } & 2065. & 24.75 & 0.026 \\
\cline { 2 - 5 } & 1797. & 19.88 & 0.020 \\
\cline { 2 - 5 } & 1320. & 12.27 & 0.015 \\
\cline { 2 - 5 } & 738. & 5.72 & 0.012 \\
\hline
\end{tabular}


Table 25a. Viscosity of saturated liquid difluoromethane (HFC-32) (SI units).

\begin{tabular}{|c|c|c|}
\hline $\begin{array}{c}\text { Temperature } \\
\mathrm{K}\end{array}$ & $\begin{array}{c}\text { Density } \\
\mathrm{mol} \cdot \mathrm{dm}^{-3}\end{array}$ & $\begin{array}{c}\text { Viscosity } \\
\mathrm{mPa} \cdot \mathrm{s}\end{array}$ \\
\hline 150.00 & 28.58 & 1.193 \\
\hline 160.00 & 27.71 & 0.945 \\
\hline 170.00 & 26.91 & 0.724 \\
\hline 180.00 & 26.16 & 0.598 \\
\hline 190.00 & 25.46 & 0.456 \\
\hline 200.00 & 24.80 & 0.384 \\
\hline 210.00 & 24.16 & 0.342 \\
\hline 220.00 & 23.55 & 0.294 \\
\hline 230.00 & 22.95 & 0.259 \\
\hline 240.00 & 22.35 & 0.227 \\
\hline 250.00 & 21.74 & 0.209 \\
\hline 260.00 & 21.12 & 0.187 \\
\hline 270.00 & 20.48 & 0.169 \\
\hline 280.00 & 19.81 & 0.149 \\
\hline 290.00 & 19.09 & 0.135 \\
\hline 300.00 & 18.31 & 0.123 \\
\hline
\end{tabular}


Table 25b. Viscosity of saturated liquid difluoromethane (HFC-32) (PI units).

\begin{tabular}{|c|c|c|}
\hline $\begin{array}{c}\text { Temperature } \\
{ }^{\circ} \mathrm{F}\end{array}$ & $\begin{array}{c}\text { Density } \\
\mathrm{lb} \cdot \mathrm{ft}^{-3}\end{array}$ & $\begin{array}{c}\text { Viscosity } \\
10^{-3} \mathrm{lb} / \mathrm{ft} \cdot \mathrm{s}\end{array}$ \\
\hline-189.67 & 92.83 & 0.802 \\
\hline-171.67 & 90.00 & 0.635 \\
\hline-153.67 & 87.40 & 0.486 \\
\hline-135.67 & 84.97 & 0.402 \\
\hline-117.67 & 82.69 & 0.306 \\
\hline-99.67 & 80.55 & 0.258 \\
\hline-81.67 & 78.47 & 0.230 \\
\hline-63.67 & 76.49 & 0.198 \\
\hline-45.67 & 74.54 & 0.174 \\
\hline-27.67 & 72.59 & 0.153 \\
\hline-9.67 & 70.61 & 0.140 \\
\hline 8.33 & 68.60 & 0.126 \\
\hline 26.33 & 66.52 & 0.114 \\
\hline 44.33 & 64.31 & 0.100 \\
\hline 62.33 & 62.00 & 0.091 \\
\hline 80.33 & 59.47 & 0.083 \\
\hline
\end{tabular}


Table 26a. Burnett vapor-phase PVT measurements for HCFC-124 (SI units).

\begin{tabular}{|c|c|c|c|c|c|c|c|c|}
\hline $\mathrm{T} / \mathrm{K}$ & $\rho / g / \mathrm{cm}^{3}$ & $\mathrm{P} / \mathrm{kPa}$ & $\mathrm{T} / \mathrm{K}$ & $\rho / g / \mathrm{cm}^{3}$ & $\mathrm{P} / \mathrm{kPa}$ & $\mathrm{T} / \mathrm{K}$ & $\rho / g / \mathrm{cm}^{3}$ & $\mathrm{P} / \mathrm{kPa}$ \\
\hline 115 & 0.8281 & 8200.801 & 378.114 & 0.1461 & 2246.522 & 333.150 & 0.0461 & 789.038 \\
\hline 23.115 & 0.8274 & 8190.710 & 373.146 & 0.1461 & 2178.006 & 323.154 & 0.0461 & 753.390 \\
\hline 418.101 & 0.8276 & 7440.368 & 368.137 & 0.1462 & 2107.447 & 423.115 & 0.0257 & 632.025 \\
\hline 408.122 & 0.8280 & 5985.352 & 423.115 & 0.0818 & 1798.808 & 423.141 & 0.0257 & 632.233 \\
\hline 403.099 & 0.8281 & 5226.898 & 418.132 & 0.0818 & 1768.495 & 418.145 & 0.0257 & 623.917 \\
\hline 398.101 & 0.8283 & 4510.935 & 413.096 & 0.8189 & 1737.613 & 413.115 & 0.0257 & 615.589 \\
\hline 423.134 & 0.4647 & 5194.508 & 408.131 & 0.0819 & 1706.952 & 408.148 & 0.0257 & 607.200 \\
\hline 423.122 & 0.4647 & 5193.894 & 403.113 & 0.0819 & 1675.852 & 403.133 & 0.0258 & 598.761 \\
\hline 423.115 & 0.4647 & 5193.637 & 398.111 & 0.8195 & 1644.584 & 398.130 & 0.0258 & 590.285 \\
\hline 418.082 & 0.4648 & 4911.935 & 393.114 & 0.0819 & 1613.187 & 393.132 & 58 & 581.876 \\
\hline 413.104 & 0.4649 & 4632.432 & 388.132 & 0.8 & 1581 & 142 & 0.0 & 573.407 \\
\hline 408.132 & 0.4650 & 435 & 383.147 & 0.8200 & 812 & 383.102 & 0.0258 & 564.863 \\
\hline 403.114 & 0.4651 & .721 & 378.114 & 0.8202 & 1517.431 & 378.133 & 0.0258 & 556.417 \\
\hline 398.114 & 0.4652 & 3779.324 & 373.148 & 0.0820 & 1485.164 & 373.108 & 0.0258 & 547.841 \\
\hline 423.117 & 0.2607 & 4057.551 & 368.131 & 0.0820 & 1452.261 & 368.152 & 0.0258 & 539.308 \\
\hline 423.115 & 0.2599 & 4050.782 & 363.126 & 0.0820 & 1419.106 & 363.148 & 0.0258 & 530.682 \\
\hline 423.115 & 0.2607 & 4057.386 & 358.132 & 0.0820 & 1385.590 & 358.145 & 0.0258 & 522.011 \\
\hline 423.108 & 0.2599 & 4050.618 & 353.137 & 0.0821 & 1351.617 & 353.149 & 0.0258 & 513.358 \\
\hline 423.106 & 0.2607 & 4057.312 & 348.159 & 0.0821 & 1317.171 & 348.118 & 0.0258 & 504.615 \\
\hline 418.121 & 0.2608 & 3926.136 & 423.115 & 0.0459 & 1083.072 & 343.145 & 0.0258 & 495.902 \\
\hline 413.092 & 0.2609 & 3792.346 & 418.124 & 0.0459 & 1067.419 & 338.112 & 0.0258 & 487.052 \\
\hline 408.127 & 0.2609 & 3658.701 & 413.097 & 0.0 & 89 & 333.101 & 0.0258 & 478.185 \\
\hline 403.111 & 0.2610 & 3521.807 & 408.131 & 0.0459 & 1035.856 & 323.168 & 0.0258 & 460.407 \\
\hline 398.110 & 0.2610 & 3383.100 & 403.112 & 0.0459 & 1019.942 & 318.127 & 0.0258 & 451.280 \\
\hline 393.113 & 0.2611 & 3241.730 & 398.109 & 0.0459 & 1003.990 & 313.143 & 0.0258 & 442.161 \\
\hline 388.125 & 0.2611 & 3096.868 & 393.116 & 0.0459 & 988.014 & 308.165 & 0.0259 & 432.976 \\
\hline 423.115 & 0.1458 & 2827.307 & 388.121 & 0.0460 & 972.011 & 303.156 & 0.0259 & 423.391 \\
\hline 423.106 & 0.1458 & 2827.303 & 383.143 & 0.0460 & 955.971 & 423.115 & 0.0144 & 362.488 \\
\hline 418.116 & 0.1459 & 2765.369 & 378.115 & 0.4602 & 939.783 & 423.115 & 0.3993 & 4899.220 \\
\hline 413.147 & 0.1459 & 2703.117 & 373.151 & 0.0460 & 923.612 & 423.115 & 0.2240 & 3734.379 \\
\hline 408.138 & 0.1459 & 2639.991 & 368.138 & 0.0460 & 907.183 & 423.115 & 0.1257 & 2535.057 \\
\hline 403.127 & 0.1460 & 2576.299 & 363.133 & 0.0460 & 890.678 & 423.115 & 0.0705 & 1585.333 \\
\hline 398.110 & 0.1460 & 2511.815 & 358.134 & 0.0460 & 874.045 & 423.115 & 0.0395 & 945.153 \\
\hline 393.108 & 0.1460 & 2446.784 & 353.140 & 0.0460 & 857.368 & 423.115 & 0.0222 & 548.573 \\
\hline 388.120 & 0.1461 & 2381.123 & 348.110 & 0.0460 & 840.451 & 423.115 & 0.0124 & 313.587 \\
\hline
\end{tabular}


Table 26b. Burnett vapor-phase PVT measurements for HCFC-124 (PI units).

\begin{tabular}{|c|c|c|c|c|c|c|c|c|}
\hline $\mathrm{T} / \mathrm{F}$ & $\rho / \mathrm{lb} / \mathrm{ft}^{3}$ & P/psia & $\mathrm{T} / \mathrm{F}$ & $\rho / \mathrm{lb} / \mathrm{ft}^{3}$ & P/psia & $\mathrm{T} / \mathrm{F}$ & $\rho / \mathrm{lb} / \mathrm{ft}^{3}$ & P'psia \\
\hline 301.937 & 1189.425 & 51.699 & 220.936 & 9.1246 & 325.830 & 140.001 & 2.8787 & 114.440 \\
\hline 301.937 & 1187.962 & 51.658 & 211.993 & 9.1265 & 315.893 & 122.007 & 2.8799 & 109.270 \\
\hline 292.911 & 1079.134 & 51.669 & 202.976 & 9.1284 & 305.659 & 301.937 & 1.6093 & 91.667 \\
\hline 274.950 & 868.102 & 51.691 & 301.937 & 5.1107 & 260.895 & 301.984 & 1.6093 & 91.698 \\
\hline 265.908 & 758.097 & 51.702 & 292.967 & 5.1118 & 256.498 & 292.990 & 1.6097 & 90.491 \\
\hline 256.912 & 654.256 & 51.713 & 283.904 & 5.1128 & 252.019 & 283.938 & 1.6100 & 89.283 \\
\hline 301.971 & 753.399 & 29.012 & 274.966 & 5.1139 & 247.572 & 274.997 & 1.6103 & 88.067 \\
\hline 301.949 & 753.310 & 29.012 & 265.934 & 5.1150 & 243.062 & 265.970 & 1.6107 & 86.843 \\
\hline 301.937 & 753.273 & 29.012 & 256.930 & 5.1161 & 238.527 & 256.964 & 1.6110 & 85.614 \\
\hline 292.877 & 712.416 & 29.018 & 247.935 & 5.1171 & 233.973 & 247.968 & 1.6113 & 84.394 \\
\hline 283.918 & 671.877 & 29.024 & 238.968 & 5.1182 & 229.395 & 238.986 & 1.6117 & 83.166 \\
\hline 274.968 & 631.175 & 29.030 & 229.995 & 5.1193 & 224.781 & 229.914 & 1.6120 & 81.926 \\
\hline 265.935 & 589.828 & 29.036 & 220.936 & 5.1203 & 220.085 & 220.970 & 1.6124 & 80.701 \\
\hline 256.935 & 548.144 & 29.043 & 211.997 & 5.1214 & 215.405 & 211.925 & 1.6127 & 79.458 \\
\hline 301.940 & 588.498 & 16.280 & 202.965 & 5.1225 & 210.633 & 203.003 & 1.6130 & 78.220 \\
\hline 301.937 & 587.516 & 16.229 & 193.956 & 5.1236 & 205.824 & 193.996 & 1.6134 & 76.969 \\
\hline 301.937 & 588.474 & 16.280 & 184.968 & 5.1246 & 200.963 & 184.991 & 1.6137 & 75.711 \\
\hline 301.924 & 587.492 & 16.229 & 175.977 & 5.1257 & 196.035 & 175.999 & 1.6141 & 74.456 \\
\hline 301.921 & 588.463 & 16.280 & 167.017 & 5.1268 & 191.039 & 166.943 & 1.6144 & 73.188 \\
\hline 292.947 & 569.438 & 16.284 & 301.937 & 2.8679 & 157.086 & $157.99 \mathrm{i}$ & 1.6147 & 71.924 \\
\hline 283.896 & 550.033 & 16.287 & 292.953 & 2.8685 & 154.816 & 148.932 & 1.6151 & 70.641 \\
\hline 274.959 & 530.650 & 16.290 & 283.905 & 2.8691 & 152.520 & 139.913 & 1.6154 & 69.355 \\
\hline 265.930 & 510.795 & 16.294 & 274.966 & 2.8697 & 150.238 & 122.033 & 1.6161 & 66.776 \\
\hline 256.928 & 490.677 & 16.297 & 265.932 & 2.8703 & 147.930 & 112.959 & 1.6164 & 65.453 \\
\hline 247.933 & 470.173 & 16.301 & 256.926 & 2.8709 & 145.618 & 103.987 & 1.6168 & 64.130 \\
\hline 238.955 & 449.163 & 16.304 & 247.939 & 2.8715 & 143.299 & 95.027 & 1.6171 & 62.798 \\
\hline 301.937 & 410.066 & 9.107 & 238.948 & 2.8721 & 140.978 & 86.012 & 1.6174 & 61.408 \\
\hline 301.921 & 410.065 & 9.107 & 229.988 & 2.8727 & 138.652 & 301.937 & 0.9031 & 52.574 \\
\hline 292.938 & 401.083 & 9.109 & 220.937 & 2.8733 & 136.304 & 301.962 & 24.9301 & 710.572 \\
\hline 283.995 & 392.054 & 9.111 & 212.002 & 2.8739 & 133.959 & 301.937 & 13.9897 & 541.626 \\
\hline 274.979 & 382.898 & 9.113 & 202.978 & 2.8745 & 131.576 & 301.937 & 7.8505 & 367.679 \\
\hline 265.959 & 373.660 & 9.115 & 193.969 & 2.8751 & 129.182 & 301.937 & 4.4054 & 229.933 \\
\hline 256.928 & 364.308 & 9.116 & 184.971 & 2.8757 & 126.769 & 301.937 & 2.4721 & 137.083 \\
\hline 247.924 & 354.876 & 9.118 & 175.982 & 2.8763 & 124.351 & 301.937 & 1.3872 & 79.564 \\
\hline 238.946 & 345.353 & 9.120 & 166.929 & 2.8769 & 121.897 & 301.937 & 0.7784 & 45.482 \\
\hline
\end{tabular}


Table 27a. Vibrating-tube compressed-liquid-density measurements for HCFC-124 (SI units)

\begin{tabular}{|c|c|c|c|c|c|c|c|c|}
\hline $\mathrm{T} / \mathrm{K}$ & $\rho / g / \mathrm{cm}^{3}$ & $\mathrm{P} / \mathrm{kPa}$ & $T / K$ & $\rho / g / \mathrm{cm}^{3}$ & $\mathrm{P} / \mathrm{kPa}$ & $\mathrm{T} / \mathrm{K}$ & $\mathrm{p} / \mathrm{g} / \mathrm{cm}^{3}$ & $\mathrm{P} / \mathrm{kPa}$ \\
\hline 275.195 & 1050.7 & 1.4369 & 303.459 & 4563.1 & 1.3573 & 337.884 & 3610.2 & 1.2288 \\
\hline 275.213 & 1575.7 & 1.4346 & 303.468 & 4979.2 & 1.3593 & 337.903 & 4136.0 & 1.2306 \\
\hline 275.159 & 2058.1 & 1.4366 & 303.479 & 5519.9 & 1.3618 & 337.876 & 4593.3 & 1.2349 \\
\hline 275.146 & 2469.2 & 1.438 & 303.488 & 6070.1 & 1.3642 & 337.877 & 5051.2 & 1.2387 \\
\hline 275.188 & 3036.4 & 1.4398 & 303.492 & 6441.2 & 1.3658 & 337.848 & 5620.8 & 1.2472 \\
\hline 275.175 & 3592.0 & 1.4417 & 308.377 & 1060.6 & 1.3259 & 337.906 & 6089.6 & 1.2471 \\
\hline 275.071 & 3951.7 & 1.4436 & 308.39 & 1599.5 & 1.3274 & 337.866 & 6586.3 & 1.251 \\
\hline 275.123 & 4507.3 & 1.4449 & 308.377 & 2077.2 & 1.3273 & 342.787 & 1588.3 & 1.1843 \\
\hline 275.18 & 4948.2 & 1.4464 & 308.416 & 2508.9 & 1.3297 & 342.733 & 2096.7 & 1.191 \\
\hline 275.143 & 5470.6 & 1.448 & 308.366 & 3062.3 & 1.333 & 342.758 & 2651.8 & 1.1974 \\
\hline 275.606 & 6023.4 & 1.448 & 308.405 & 3644.8 & 1.336 & 342.779 & 3098.2 & 1.2024 \\
\hline 275.219 & 6361.0 & 1.4505 & 308.373 & 3979.3 & 1.338 & 342.879 & 3625.9 & 1.2076 \\
\hline 278.699 & 1050.3 & 1.4258 & 308.376 & 4576.8 & 1.3412 & 342.867 & 4147.5 & 1.2105 \\
\hline 278.702 & 1578.8 & 1.4238 & 308.373 & 4986.7 & 1.3433 & 342.823 & 4601.9 & 1.2149 \\
\hline 278.723 & 2060.2 & 1.4256 & 308.387 & 5538.0 & 1.346 & 342.839 & 5065.9 & 1.2193 \\
\hline 278.695 & 2474.9 & 1.4272 & 308.391 & 6081.2 & 1.3486 & 342.808 & 5632.8 & 1.228 \\
\hline 278.725 & 3039.7 & 1.4291 & 308.394 & 6453.1 & 1.3504 & 342.877 & 6112.2 & 1.2283 \\
\hline 278.697 & 3600.1 & 1.4311 & 313.327 & 1094.1 & 1.3066 & 342.824 & 6600.7 & 1.2335 \\
\hline 278.714 & 3957.9 & 1.4324 & 313.338 & 1559.9 & 1.3098 & 347.706 & 1593.0 & 1.1587 \\
\hline 278.669 & ذّ & 1.4344 & 313.328 & 2062.6 & 1.3131 & 347.635 & 2103.5 & 1.1666 \\
\hline 278.702 & 49.46 & 1.4358 & 313.331 & 2596.0 & 1.3164 & 347.598 & 2659.2 & 1.1742 \\
\hline 278.74 & 5475.5 & 1.4374 & 313.336 & 3044.4 & 1.3192 & 347.696 & 3110.8 & 1.1797 \\
\hline 278.763 & 6022.7 & 1.4391 & 313.395 & 3568.6 & 1.322 & 347.622 & 3630.5 & 1.1863 \\
\hline 278.733 & 6371.2 & 1.4403 & 313.364 & 4091.3 & 1.3219 & 347.781 & 4155.1 & 1.1891 \\
\hline 283.621 & 1051.1 & 1.41 & 313.36 & 4537.0 & 1.3246 & 347.761 & 4615.9 & 1.1942 \\
\hline 283.635 & 1582.6 & 1.4082 & 313.363 & 4997.2 & 1.3272 & 347.76 & 5081.9 & 1.1991 \\
\hline 283.631 & 2063.0 & 1.4101 & 313.373 & 5590.8 & 1.331 & 347.738 & 5644.6 & 1.2081 \\
\hline 283.609 & 2480.0 & 1.4119 & 313.396 & 6012.6 & 1.3359 & 347.796 & 6128.0 & 1.2091 \\
\hline 283.647 & 3044.1 & 1.4139 & 313.352 & 6520.7 & 1.3354 & 347.75 & 6615.5 & 1.2141 \\
\hline 283.636 & 3608.2 & 1.4161 & 318.224 & 1097.2 & 1.2876 & $352.5 ! 7$ & 2110.0 & 1.1405 \\
\hline 283.657 & 3964.2 & 1.4174 & 318.252 & 1568.3 & 1.2911 & 352.485 & 2666.3 & 1.1496 \\
\hline 283.617 & 4522.8 & 1.4196 & 318.234 & 2068.7 & 1.2948 & 352.556 & 3116.4 & 1.1558 \\
\hline 283.625 & 4948.2 & 1.4211 & 318.236 & 2612.2 & 1.2985 & 352.64 & 3647.6 & 1.1628 \\
\hline 283.64 & 5483.9 & 1.4229 & 318.256 & 3052.0 & 1.3014 & 352.675 & 4168.0 & 1.1669 \\
\hline 283.656 & 6029.4 & 1.4247 & 318.304 & 3582.8 & 1.3046 & 352.646 & 4624.3 & 1.1726 \\
\hline 283.647 & 6384.5 & 1.426 & 318.287 & 4103.8 & 1.3047 & 352.647 & 5091.7 & 1.1781 \\
\hline 288.526 & 1052.6 & 1.3941 & 318.277 & 4549.1 & 1.3077 & 352.612 & 5658.0 & 1.1878 \\
\hline 288.538 & 1585.4 & 1.3924 & 318.283 & 5017.2 & 1.3105 & 352.626 & 6146.8 & 1.1896 \\
\hline 288.54 & 2065.9 & 1.3944 & 318.283 & 5604.9 & 1.3146 & 352.637 & 6631.1 & 1.1947 \\
\hline 288.49 & 2486.7 & 1.3964 & 318.3 & 6023.7 & 1.3165 & 357.37 & 2111.6 & 1.1122 \\
\hline 288.538 & 3043.9 & 1.3985 & 318.271 & 6533.0 & 1.3193 & 357.392 & 2673.3 & 1.1227 \\
\hline 288.568 & 3614.7 & 1.4007 & 323.093 & 1099.9 & 1.2681 & 357.28 & 3123.4 & 1.1307 \\
\hline 288.549 & 3961.5 & 1.4022 & 323.134 & 1572.8 & 1.2718 & 357.5 & 3655.8 & 1.1384 \\
\hline 288.514 & 4531.6 & 1.4046 & 323.094 & 2073.2 & 1.276 & 357.52 & 4181.5 & 1.1437 \\
\hline 288.529 & 4957.1 & 1.4062 & 323.107 & 2622.3 & 1.28 & 357.514 & 4632.3 & 1.1503 \\
\hline 288.551 & 5495.6 & 1.4082 & 323.137 & 3060.2 & 1.2833 & 357.523 & 5107.5 & 1.1562 \\
\hline 288.543 & 6038.9 & 1.4102 & 323.121 & 5592.8 & 1.2979 & 357.479 & 5670.1 & 1.1667 \\
\hline 288.555 & 6395.6 & 1.4114 & 323.184 & 3591.7 & 1.2867 & 357.498 & 6159.0 & 1.1691 \\
\hline
\end{tabular}


Table 27a. Vibrating-tube compressed-liquid-density measurements for HCFC-124 (SI units) (continued)

\begin{tabular}{|c|c|c|c|c|c|c|c|c|}
\hline TiK & $\rho / g / \mathrm{cm}^{3}$ & $\mathrm{P} / \mathrm{kPa}$ & $T / K$ & $\rho / g / \mathrm{cm}^{3}$ & $\mathrm{P} / \mathrm{kPa}$ & $\mathrm{T} / \mathrm{K}$ & $\rho / g / \mathrm{cm}^{3}$ & $\mathrm{P} / \mathrm{kPa}$ \\
\hline 293.483 & 1054.3 & 1.3776 & 323.168 & 4110.4 & 1.2871 & 357.499 & 6646.9 & 1.1748 \\
\hline 293.483 & 1589.4 & 1.3761 & 323.159 & 4557.9 & 1.2903 & 362.22 & 2117.4 & 1.0805 \\
\hline 293.517 & 1589.8 & 1.376 & 323.166 & 5023.3 & 1.2934 & 362.239 & 2683.9 & 1.0938 \\
\hline 293.488 & 2067.2 & 1.3782 & 323.184 & 6039.3 & 1.2998 & 362.286 & 3133.6 & 1.1023 \\
\hline 293.525 & 2492.4 & 1.3802 & 323.158 & 6545.9 & 1.3029 & 362.312 & 3669.5 & 1.1127 \\
\hline 293.475 & 3045.9 & 1.3827 & 327.958 & 1102.6 & 1.2476 & 362.331 & 4196.0 & 1.1191 \\
\hline 293.516 & 3624.9 & 1.3851 & 328.0 & 1577.1 & 1.2517 & 362.307 & 4648.1 & 1.1266 \\
\hline 293.484 & 3958.4 & 1.3867 & 327.979 & 2080.2 & 1.2564 & 362.335 & 5125.8 & 1.1353 \\
\hline 293.468 & 4540.6 & 1.3892 & 327.897 & 2629.4 & 1.2612 & 362.289 & 5684.1 & 1.1448 \\
\hline 293.473 & 4965.8 & 1.3909 & 328.032 & 3069.7 & 1.2644 & 362.302 & 6178.7 & 1.1482 \\
\hline 293.489 & 5505.2 & 1.3931 & 328.058 & 3596.9 & 1.2682 & 362.314 & 6663.9 & 1.1544 \\
\hline 293.49 & 6049.3 & 1.3952 & 328.042 & 41119.5 & 1.269 & 367.101 & 2693.5 & 1.0612 \\
\hline 293.49 & 6410.0 & 1.3966 & 328.031 & 4571.1 & 1.2726 & 367.106 & 3143.1 & 1.0728 \\
\hline 298.468 & 1056.2 & 1.3606 & 328.036 & 5036.0 & 1.2759 & 367.128 & 3680.5 & 1.0846 \\
\hline 298.477 & 2069.8 & 1.3616 & 328.008 & 5599.8 & 1.2807 & 367.154 & 4198.7 & 1.0951 \\
\hline 298.52 & 2497.5 & 1.3636 & 328.062 & 6060.8 & 1.2828 & 367.124 & 4651.9 & 1.1038 \\
\hline 298.471 & 3051.1 & 1.3664 & 328.023 & 6558.6 & 1.2862 & 367.128 & 5138.9 & 1.1114 \\
\hline 298.51 & 3629.1 & 1.369 & 332.79 & 1105.3 & 1.2262 & 367.101 & 5697.6 & 1.1219 \\
\hline 298.471 & 3968.2 & 1.3707 & 332.841 & 1582.3 & 1.231 & 367.126 & 6172.3 & 1.1257 \\
\hline 298.464 & 4551.2 & 1.3734 & 332.778 & 2085.4 & 1.2362 & 367.121 & 6681.5 & 1.1332 \\
\hline 298.466 & 4973.2 & 1.3753 & 332.807 & 2635.9 & 1.241 & 368.942 & 3671.9 & 1.073 \\
\hline $298.48 !$ & 5513.0 & 1.3776 & 332.836 & 3084.7 & 1.245 & 369.038 & 4200.9 & 1.0839 \\
\hline 298.486 & 6059.0 & 1.3799 & 332.884 & 3602.2 & 1.2492 & 369.063 & 4647.8 & 1.0929 \\
\hline 298.487 & 6425.9 & 1.3813 & 332.885 & 4127.8 & 1.2506 & 368.995 & 5144.2 & 1.101 \\
\hline 303.472 & 1058.4 & 1.3432 & 332.875 & 4582.7 & 1.2543 & 369.175 & 5709.0 & 1.110 \\
\hline 303.488 & 1149.6 & 1.3392 & 332.882 & 5044.0 & 1.2579 & 369.195 & 6165.5 & 1.1157 \\
\hline 303.499 & 1596.2 & 1.3418 & 332.85 & 5610.3 & 1.2636 & 368.579 & 6685.8 & 1.1263 \\
\hline 303.475 & 2073.3 & 1.3445 & 332.906 & 6077.1 & 1.2654 & 372.09 & 2694.7 & 1.0221 \\
\hline 303.515 & 2503.3 & 1.3467 & 332.87 & 6572.6 & 1.2691 & 372.366 & 3147.6 & 1.03 \\
\hline
\end{tabular}


Table 27b. Vibrating-tube compressed-liquid-density measurements for HCFC-124 (PI units)

\begin{tabular}{|c|c|c|c|c|c|c|c|c|}
\hline$T / F$ & $\mathrm{\rho} / \mathrm{lb} / \mathrm{ft}^{3}$ & $\mathrm{P} / \mathrm{psia}$ & $\mathrm{T} / \mathrm{F}$ & $\rho / \mathrm{lb} / \mathrm{ft}^{3}$ & $\mathrm{P} / \mathrm{psia}$ & $\mathrm{T} / \mathrm{F}$ & $\rho / \mathrm{lb} / \mathrm{ft}^{3}$ & $\mathrm{P} /$ psia \\
\hline 35.68 & 152.39 & 89.704 & 86.555 & 661.822 & 84.733 & 148.521 & 523.616 & 76.709 \\
\hline 35.714 & 228.529 & 89.558 & 86.572 & 722.169 & 84.856 & 148.555 & 599.876 & 76.823 \\
\hline 35.616 & 298.496 & 89.683 & 86.591 & 800.587 & $85 . \hat{v} 13$ & 148.506 & 666.208 & 77.09 \\
\hline 35.593 & 358.133 & 89.771 & 86.609 & 880.391 & 85.167 & 148.508 & 732.6144 & 77.33 \\
\hline 35.669 & 440.392 & 89.886 & 86.615 & 934.212 & 85.267 & 148.456 & 815.226 & 77.861 \\
\hline 35.645 & 520.97 & 90.005 & 95.408 & 153.821 & 82.775 & 148.56 & 883.223 & 77.853 \\
\hline 35.457 & 573.14 & 90.12 & 95.433 & 231.985 & 82.864 & 148.49 & 955.268 & 78.098 \\
\hline 35.552 & 653.728 & 90.202 & 95.408 & 301.268 & 82.864 & 157.346 & 230.365 & 73.933 \\
\hline 35.654 & 717.669 & 90.297 & 95.480 & 363.885 & 83.012 & 157.249 & 304.107 & 74.351 \\
\hline 35.587 & 793.447 & 90.394 & 95.389 & 444.156 & 83.216 & 157.295 & 384.616 & 74.749 \\
\hline 36.421 & 873.617 & 90.398 & 95.458 & 528.636 & 83.406 & 157.333 & 449.353 & 75.063 \\
\hline 35.724 & 922.591 & 90.552 & 95.401 & 577.146 & 83.528 & 157.512 & 525.609 & 75.385 \\
\hline 41.989 & 152.335 & 89.008 & 95.407 & 663.804 & 83.725 & 157.491 & 601.542 & 75.57 \\
\hline 41.993 & 228.982 & 88.883 & 95.401 & 723.261 & 83.858 & 157.412 & 667.456 & 75.842 \\
\hline 42.031 & 298.8 & 88.995 & 95.427 & 803.219 & 84.03 & 157.441 & 734.747 & 76.117 \\
\hline 41.981 & 358.958 & 89.094 & 95.434 & 882.008 & 84.193 & 157.384 & 816.963 & 76.659 \\
\hline 42.035 & 440.876 & 89.216 & 95.439 & 935.943 & 84.302 & 157.509 & 886.501 & 76.68 \\
\hline 41.984 & 522.1451 & 89.344 & 104.319 & 158.686 & 81.568 & 157.413 & 957.355 & 77.005 \\
\hline 42.015 & 574.048 & 89.422 & 104.338 & 226.239 & 81.766 & 166.201 & 49 & 72.337 \\
\hline 41.935 & 654.768 & 89.55 & 104.321 & 299.153 & 81 & 166.073 & 305.093 & 72.829 \\
\hline 41.993 & 717.15 & 89.634 & 104.326 & 376.519 & 33 & 166.007 & 385.691 & 73.305 \\
\hline 42.062 & 794.148 & 89.735 & 104.335 & 441.552 & 82.354 & 166.183 & 451.18 & 73.649 \\
\hline 42.103 & 873.514 & 89.842 & 104.441 & 517.583 & 82.532 & 166.049 & 526.561 & 74.059 \\
\hline 42.05 & 924.066 & 89.917 & 104.385 & 593.389 & 82.525 & 166.335 & 602.643 & 74.231 \\
\hline 50.848 & 152.453 & 88.023 & 104.378 & 658.035 & 82.694 & 166.3 & 669.486 & 74.549 \\
\hline 50.873 & 229.536 & 87.910 & 104.384 & 724.782 & 82.852 & 166.298 & 737.067 & 74.855 \\
\hline 50.866 & 299.216 & 88.031 & 104.401 & 810.874 & 83.091 & 166.258 & 818.685 & 75.421 \\
\hline 50.826 & 359.692 & 88.144 & 104.443 & 872.051 & 83.399 & 166.363 & 888.788 & 75.479 \\
\hline 50.894 & 441.51 & 88.268 & 104.363 & 945.746 & 83.367 & 166.281 & 959.493 & 75.795 \\
\hline 50.874 & 523.323 & 88.403 & 113.134 & 159.133 & 80.385 & 174.861 & 306.025 & 71.202 \\
\hline 50.912 & 574.959 & 88.484 & 113.184 & 227.459 & 80.6 & 174.803 & 386.715 & 71.768 \\
\hline 50.841 & 655.982 & 88.622 & 113.152 & 300.036 & 80.83 & 174.93 & 451.997 & 72.154 \\
\hline 50.856 & 717.67 & 88.714 & 113.155 & 378.855 & 81.062 & 175.081 & 529.037 & 72.593 \\
\hline 50.882 & 795.372 & 88.831 & 113.191 & 442.659 & 81.244 & 175.145 & 604.524 & 72.845 \\
\hline 50.911 & 874.495 & 88.944 & 113.277 & 519.648 & 81.444 & 175.093 & 670.697 & 73.202 \\
\hline 50.895 & 925.995 & 89.019 & $113.24 i$ & 595.202 & 81.451 & 175.095 & 738.49 & 73.544 \\
\hline 59.678 & 152.67 & 87.031 & 113.228 & 659.797 & 81.635 & 175.031 & 820.63 & 74.152 \\
\hline 59.699 & 229.937 & 86.922 & 113.239 & 727.68 & 81.813 & 175.057 & 891.523 & 74.264 \\
\hline 59.703 & 299.636 & 87.052 & 113.239 & 812.921 & 82.066 & 175.076 & 961.766 & 74.586 \\
\hline 59.612 & 360.669 & 87.177 & 113.271 & 873.658 & 82.188 & 183.595 & 306.258 & 69.432 \\
\hline 59.699 & 441.481 & 87.306 & 113.218 & 947.537 & 82.361 & 183.636 & 387.726 & 70.089 \\
\hline 59.753 & 524.266 & 87.446 & 121.898 & 159.522 & 79.162 & 183.433 & 453.01 & 70.59 \\
\hline 59.718 & 574.571 & 87.539 & 121.971 & 228.118 & 79.396 & 182.829 & 530.23 & 71.067 \\
\hline 59.656 & 657.253 & 87.686 & 121.9 & 300.692 & 79.656 & 183.867 & 606.478 & 71.397 \\
\hline 59.682 & 718.966 & 87.787 & 121.923 & 380.331 & 79.909 & 183.856 & 671.863 & 71.808 \\
\hline 59.721 & 797.069 & 87.910 & 121.975 & 443.845 & 80.113 & 183.872 & 740.775 & 72.182 \\
\hline 59.708 & 875.864 & 88.033 & 121.948 & 811.173 & 81.022 & 183.792 & 822.374 & 72.832 \\
\hline 59.729 & 927.607 & 88.111 & 122.06 & 520.936 & 80.326 & 183.827 & 893.281 & 72.983 \\
\hline 68.6 & 152.92 & 86.0 & 122.033 & 596.166 & 80.353 & 183.828 & 964.049 & 73.342 \\
\hline
\end{tabular}


Table 27b. Vibrating-tube compressed-liquid-density measurements for HCFC-124 (PI units) (continued)

\begin{tabular}{|c|c|c|c|c|c|c|c|c|}
\hline $\mathrm{T} / \mathrm{F}$ & $\rho / l b / f^{3}$ & $\mathrm{P} / \mathrm{psia}$ & $T / F$ & $\mathrm{p} / \mathrm{lb} / \mathrm{ft}^{3}$ & $\mathrm{P} / \mathrm{psia}$ & $\mathrm{T} F$ & $\rho / \mathrm{lb} / \mathrm{ft}^{3}$ & $\mathrm{P} /$ psia \\
\hline 68.599 & 230.53 & 85.907 & 122.017 & 661.06 & 80.552 & 192.326 & 307.107 & 67.453 \\
\hline 68.661 & 230.576 & 85.899 & 122.028 & 728.564 & 80.745 & 192.36 & 389.264 & 68.283 \\
\hline 68.608 & 299.816 & 86.041 & 122.062 & 875.926 & 81.144 & 192.445 & 454.492 & 68.814 \\
\hline 68.676 & 361.485 & 86.161 & 122.014 & 949.409 & 81.338 & 192.492 & 532.216 & 69.466 \\
\hline 68.586 & 441.773 & 86.318 & 130.654 & 159.918 & 77.883 & 192.526 & 608.574 & 69.865 \\
\hline 68.659 & 525.753 & 86.469 & 130.73 & 228.746 & 78.144 & 192.483 & 674.151 & 70.333 \\
\hline 68.601 & 574.114 & 86.568 & 130.691 & 301.711 & 78.432 & 192.533 & 743.427 & 70.874 \\
\hline 68.573 & 658.561 & 86.727 & 130.544 & 381.36 & 78.731 & 192.449 & 824.413 & 71.468 \\
\hline 68.582 & 720.234 & 86.834 & 130.788 & 445.225 & 78.932 & 192.473 & 896.146 & 71.679 \\
\hline 68.609 & 798.462 & 86.968 & 130.834 & 521.68 & 79.17 & 192.496 & 966.523 & 72.066 \\
\hline 68.612 & 877.373 & 87.1 & 130.805 & 597.485 & 79.224 & 201.111 & 390.658 & 66.249 \\
\hline 68.612 & 929.692 & 87.184 & 130.785 & 662.984 & 79.445 & 201.121 & 455.875 & 66.971 \\
\hline 77.572 & 153.196 & 84.941 & 130.795 & 730.417 & 79.65 & 201.161 & 533.805 & 67.711 \\
\hline 77.589 & 300.205 & 85.001 & 130.744 & 812.177 & 79.952 & 201.208 & 608.965 & 68.363 \\
\hline 77.667 & 362.237 & 85.129 & 130.841 & 879.041 & 80.081 & 201.153 & 674.695 & 68.906 \\
\hline 77.578 & 442.53 & 85.3 & 130.771 & 951.248 & 80.294 & 201.16 & 745.329 & 69.384 \\
\hline 77.647 & 526.359 & 85.463 & 139.352 & 160.316 & 76.548 & 201.111 & 826.363 & 70.037 \\
\hline 77.578 & 575.541 & 85.571 & 139.443 & 229.496 & 76.849 & 261.157 & 895.214 & 70.273 \\
\hline 77.565 & 660.091 & 85.74 & 139.331 & 302.463 & 77.171 & 201.148 & 969.063 & 70.742 \\
\hline 77.568 & 721.296 & 85.855 & 139.383 & 382.305 & 77.475 & 204.426 & 532.559 & 66.984 \\
\hline 77.596 & 799.588 & 85.999 & 139.435 & 447.394 & 77.724 & 204.598 & 609.294 & 67.663 \\
\hline 77.604 & 878.785 & 86.141 & 139.521 & 522.461 & 77.983 & 204.643 & 674.104 & 68.229 \\
\hline 77.606 & 931.996 & 86.233 & 139.522 & 598.68 & 78.071 & 204.521 & 746.106 & 68.759 \\
\hline 86.579 & 153.511 & 83.854 & 139.505 & 664.663 & 78.304 & 204.846 & 828.026 & 69.334 \\
\hline 86.608 & 166.732 & 83.601 & 139.517 & 731.564 & 78.526 & 204.881 & 894.235 & 69.651 \\
\hline 86.628 & 231.515 & 83.767 & 139.459 & 813.711 & 78.886 & 203.773 & 969.697 & 70.313 \\
\hline 86.585 & 300.71 & 83.935 & 139.562 & 881.413 & 78.999 & 210.092 & 390.833 & 63.809 \\
\hline 86.657 & 363.08 & 84.075 & 139.496 & 953.277 & 79.228 & 210.588 & 456.514 & 64.65 \\
\hline
\end{tabular}


Table 28a. Liquid PVT Data (Isochoric Apparatus) for HCFC-124 $1 / 4$

\begin{tabular}{|c|c|c|}
\hline$\underset{\mathrm{K}}{\text { Temperature }}$ & $\begin{array}{l}\text { Pressure } \\
\text { MPa }\end{array}$ & $\begin{array}{l}\text { Density } \\
\text { mol } \cdot \mathrm{dm}^{-3}\end{array}$ \\
\hline $\begin{array}{l}103.999 \\
105.999 \\
107.999 \\
109.999 \\
111.998\end{array}$ & $\begin{array}{r}6.226760 \\
12.778850 \\
19.287510 \\
25.760370 \\
32.154860\end{array}$ & $\begin{array}{l}13.91860 \\
13.91419 \\
13.91010 \\
13.90619 \\
13.90244\end{array}$ \\
\hline $\begin{array}{l}112.000 \\
113.999 \\
116.000 \\
117.998 \\
119.999 \\
121.999 \\
123.998\end{array}$ & $\begin{array}{r}.983711 \\
3.258380 \\
9.240955 \\
15.167930 \\
21.063240 \\
26.921330 \\
32.720460\end{array}$ & $\begin{array}{l}13.74957 \\
13.70930 \\
13.70497 \\
13.70103 \\
13.69729 \\
13.69369 \\
13.69021\end{array}$ \\
\hline $\begin{array}{l}124.000 \\
126.000 \\
127.999 \\
129.999 \\
131.999 \\
134.000 \\
136.000\end{array}$ & $\begin{array}{r}3.157045 \\
8.608703 \\
14.056010 \\
1.9 .471300 \\
24.844050 \\
30.174590 \\
35.472760\end{array}$ & $\begin{array}{l}13.51722 \\
13.51317 \\
13.50943 \\
13.50589 \\
13.50247 \\
13.49916 \\
13.49591\end{array}$ \\
\hline $\begin{array}{l}135.9: 29 \\
138.000 \\
139.999 \\
142.000 \\
143.999 \\
146.000 \\
147.999\end{array}$ & $\begin{array}{r}2.474208 \\
7.391337 \\
12.296560 \\
17.191640 \\
22.051510 \\
26.864480 \\
31.641030\end{array}$ & $\begin{array}{l}13.28578 \\
13.28197 \\
13.27844 \\
13.27508 \\
13.27185 \\
13.26872 \\
13.26566\end{array}$ \\
\hline $\begin{array}{l}148.000 \\
149.999 \\
151.999 \\
154.000 \\
155.998 \\
157.999 \\
159.999 \\
161.999\end{array}$ & $\begin{array}{r}2.457991 \\
6.915843 \\
11.373190 \\
15.806910 \\
20.220830 \\
24.606620 \\
28.969200 \\
33.302550\end{array}$ & $\begin{array}{l}13.06041 \\
13.05634 \\
13.05352 \\
13.05035 \\
13.04729 \\
13.04430 \\
13.04139 \\
13.03852\end{array}$ \\
\hline $\begin{array}{l}162.000 \\
164.000 \\
166.000 \\
167.999 \\
169.999 \\
172.000 \\
174.001 \\
175.999 \\
177.999\end{array}$ & $\begin{array}{r}1.892250 \\
5.875665 \\
9.856306 \\
13.815500 \\
17.758390 \\
21.683680 \\
25.588160 \\
29.463810 \\
33.319840\end{array}$ & $\begin{array}{l}12.79345 \\
12.79009 \\
12.78696 \\
12.78397 \\
12.78109 \\
12.77826 \\
12.77550 \\
12.77279 \\
12.77012\end{array}$ \\
\hline
\end{tabular}


Table 28a. Liquid PVT Data (Isochoric Apparatus) for HCFC-124 $2 / 4$

\begin{tabular}{|c|c|c|}
\hline$\underset{\mathrm{K}}{\text { Temperature }}$ & $\begin{array}{l}\text { Pressure } \\
\text { MPa }\end{array}$ & $\begin{array}{l}\text { Density } \\
\text { mol } \cdot \mathrm{dm}^{-3}\end{array}$ \\
\hline $\begin{array}{l}177.999 \\
180.000 \\
182.000 \\
184.000 \\
185.999 \\
188.001 \\
190.001 \\
191.999 \\
193.999 \\
195.999\end{array}$ & $\begin{array}{r}2.931927 \\
6.459064 \\
9.973697 \\
13.482320 \\
16.968830 \\
20.440880 \\
23.902990 \\
27.341340 \\
30.759340 \\
34.157370\end{array}$ & $\begin{array}{l}12.50169 \\
12.49865 \\
12.49577 \\
12.49299 \\
12.49030 \\
12.48767 \\
12.48507 \\
12.48252 \\
12.48001 \\
12.47752\end{array}$ \\
\hline $\begin{array}{l}194.000 \\
196.000 \\
197.999 \\
200.000 \\
202.000 \\
204.001 \\
206.000 \\
208.000 \\
210.001 \\
212.002 \\
214.000 \\
216.000\end{array}$ & $\begin{array}{r}1.931502 \\
5.033347 \\
8.122772 \\
11.209380 \\
14.286380 \\
17.348010 \\
20.397440 \\
23.429070 \\
26.452370 \\
29.403170 \\
32.396790 \\
35.375180\end{array}$ & $\begin{array}{l}12.18909 \\
12.18621 \\
12.18349 \\
12.18087 \\
12.17832 \\
12.17582 \\
12.17337 \\
12.17095 \\
12.16857 \\
12.16623 \\
12.16388 \\
12.16156\end{array}$ \\
\hline $\begin{array}{l}209.999 \\
212.000 \\
214.000 \\
216.000 \\
218.001 \\
219.999 \\
222.000 \\
224.000 \\
226.000 \\
228.000 \\
230.002 \\
232.000 \\
233.998\end{array}$ & $\begin{array}{r}2.613277 \\
5.359978 \\
8.103843 \\
10.835770 \\
13.554630 \\
16.268500 \\
18.967570 \\
21.663110 \\
24.344580 \\
27.018200 \\
29.681040 \\
32.323510 \\
34.961730\end{array}$ & $\begin{array}{l}11.89087 \\
11.88823 \\
11.88568 \\
11.88323 \\
11.88083 \\
11.87848 \\
11.87616 \\
11.87387 \\
11.87160 \\
11.86935 \\
11.86713 \\
11.86492 \\
11.86272\end{array}$ \\
\hline $\begin{array}{l}228.000 \\
229.999 \\
232.000 \\
234.001 \\
235.999 \\
238.000 \\
240.001 \\
241.999 \\
244.000 \\
245.999\end{array}$ & $\begin{array}{r}2.687304 \\
5.068540 \\
7.441756 \\
9.818677 \\
12.178040 \\
14.538120 \\
16.892600 \\
19.236050 \\
21.573870 \\
23.899430\end{array}$ & $\begin{array}{l}11.54016 \\
11.53772 \\
11.53536 \\
11.53306 \\
11.53081 \\
11.52859 \\
11.52640 \\
11.52424 \\
11.52209 \\
11.51997\end{array}$ \\
\hline
\end{tabular}


Table 28a. Liquid PVT Data (Isochoric Apparatus) for HCFC-124 $3 / 4$

\begin{tabular}{|c|c|c|}
\hline$\underset{\mathrm{K}}{\text { Temperature }}$ & $\begin{array}{l}\text { Pressure } \\
\text { MPa }\end{array}$ & $\begin{array}{l}\text { Density } \\
\text { mol } \cdot \mathrm{dm}^{-3}\end{array}$ \\
\hline $\begin{array}{l}248.001 \\
250.001 \\
252.000 \\
254.000 \\
256.001\end{array}$ & $\begin{array}{l}26.219510 \\
28.532190 \\
30.830360 \\
33.123620 \\
35.409220\end{array}$ & $\begin{array}{l}11.51786 \\
11.51576 \\
11.51367 \\
11.51159 \\
11.50952\end{array}$ \\
\hline $\begin{array}{l}247.999 \\
250.000 \\
251.999 \\
253.999 \\
256.001 \\
258.000 \\
260.000 \\
262.000 \\
263.999 \\
266.000 \\
268.000 \\
270.000 \\
271.998 \\
274.001 \\
276.000 \\
278.000 \\
279.998 \\
282.000\end{array}$ & $\begin{array}{r}.995255 \\
2.591872 \\
4.584418 \\
6.575962 \\
8.563781 \\
10.541220 \\
12.522810 \\
14.496410 \\
16.460680 \\
18.422190 \\
20.379320 \\
22.325860 \\
24.272940 \\
26.211550 \\
28.146420 \\
30.076080 \\
31.997200 \\
33.912730\end{array}$ & $\begin{array}{l}11.13293 \\
11.09319 \\
11.09096 \\
11.08878 \\
11.08665 \\
11.08456 \\
11.08249 \\
11.08045 \\
11.07843 \\
11.07642 \\
11.07443 \\
11.07245 \\
11.07047 \\
11.06851 \\
11.06655 \\
11.06460 \\
11.06266 \\
11.06071\end{array}$ \\
\hline $\begin{array}{l}276.000 \\
279.999 \\
283.999 \\
288.000 \\
292.001 \\
296.001 \\
299.998 \\
304.000 \\
308.000 \\
311.999 \\
316.000 \\
320.001\end{array}$ & $\begin{array}{r}1.215140 \\
4.364996 \\
7.507500 \\
10.637100 \\
13.762190 \\
16.872810 \\
19.970760 \\
23.058260 \\
26.131280 \\
29.185910 \\
32.226150 \\
35.252500\end{array}$ & $\begin{array}{l}10.49846 \\
10.49442 \\
10.49053 \\
10.48674 \\
10.48302 \\
10.47934 \\
10.47569 \\
10.47205 \\
10.46844 \\
10.46484 \\
10.46125 \\
10.45765\end{array}$ \\
\hline $\begin{array}{l}315.998 \\
320.000 \\
323.998 \\
328.000 \\
331.998 \\
336.000 \\
339.998 \\
343.999 \\
348.001 \\
352.000\end{array}$ & $\begin{array}{r}2.669067 \\
4.851199 \\
7.029285 \\
9.209246 \\
11.387150 \\
13.562010 \\
15.731440 \\
17.898300 \\
20.060370 \\
22.212470\end{array}$ & $\begin{array}{l}9.56622 \\
9.56284 \\
9.55950 \\
9.55621 \\
9.55294 \\
9.54969 \\
9.54645 \\
9.54321 \\
9.53999 \\
9.53679\end{array}$ \\
\hline
\end{tabular}


Table 28a. Liquid PVT Data (Isochoric Apparatus) for HCFC-124 4/4

Temperature

$\mathrm{K}$

355.998
360.001
364.002
368.000
372.001
375.998
356.001
360.000
364.000
368.000
372.001
376.001
380.000
384.000
387.998
392.001
396.001
400.000

\section{Pressure} MPa

24.357130

26.502230

28.640990

30.771150

32.893580

35.004500

2.716171

4.022936

5.335316

6.654194

7.977267

9.303176

10.633070

11.963660

13.296440

14.630800

15.964290

17.297460
Density
mol $\cdot \mathrm{dm}^{-3}$

9.53375

9.53056

9.52734

9.52411

9.52087

9.51762

8.28455

8.28178

8.27902

8.27626

8.27352

8.27078

8.26804

8.26528

8.26254

8.25977

8.25701

8.25425 
Table 28b. Liquid PVT Data (Isochoric Apparatus) for HCFC-124 $1 / 4$

$\underset{{ }_{F}}{\text { Temperature }}$

$-272.47$

$-268.87$

$-265.27$

$-261.67$

$-258.07$

$-258.07$

$-254.47$

$-250.87$

$-247.27$

$-243.67$

$-240.07$

$-236.47$

$-236.47$

$-232.87$

$-229.27$

$-225.67$

$-222.07$

$-218.47$

$-214.87$

$-214.87$

$-211.27$

$-207.67$

$-204.07$

$-200.47$

$-196.87$

$-193.27$

$-193.27$

$-189.67$

$-186.07$

$-182.47$

$-178.87$

$-175.27$

$-171.67$

$-168.07$

$-168.07$

$-164.47$

$-160.87$

$-157.27$

$-153.67$

$-150.07$

$-146.47$

$-142.87$

$-139.27$

\section{Pressure psia}

$$
\begin{array}{r}
903.117 \\
1853.419 \\
2797.422 \\
3736.232 \\
4663.676
\end{array}
$$

142.675

472.589

1340.290

2199.926

3054.970

3904.616

4745.710

457.891

1248.589

2038.655

2824.078

3603.331

4376.462

5144.898

358.854

1072.025

1783.468

2493.441

3198.307

3896.370

4589.152

356.502

1003.060

1649.545

2292.603

2932.789

3568.895

4201.635

4830.135

274.448

852.195

1429.539

2003.772

2575.641

3144.957

3711.255

4273.372

4832.643
Density
lb. $f t^{-3}$

118.586

118.548

118.513

118.480

118.448

117.145

116.802

116.765

116.732

116.700

116.669

116.640

115.166

115.131

115.099

115.069

115.040

115.012

114.984

113.194

113.162

113.131

113.103

113.075

113.049

113.023

111.274

111.243

111.215

111.188

111.162

111.137

111.112

111.087

108.999

108.971

108.944

108.919

108.894

108.870

108.846

108.823

108.801 
Table 28b. Liquid PVT Data (Isochoric Apparatus) for HCFC-124 $2 / 4$

Temperature

$\circ \mathrm{F}$

-139.27
-135.67
-132.07
-128.47
-124.87
-121.27
-117.67
-114.07
-110.47
-106.87

$-110.47$

$-106.87$

$-103.27$

$-99.67$

$-96.07$

$-92.47$

$-88.87$

$-85.27$

$-81.67$

$-78.07$

$-74.47$

$-70.87$

$-81.67$

$-78.07$

$-74.47$

$-70.87$

$-67.27$

$-63.67$

$-60.07$

$-56.47$

$-52.87$

$-49.27$

$-45.67$

$-42.07$

$-38.47$

$-49.27$

$-45.67$

$-42.07$

$-38.47$

$-34.87$

$-31.27$

$-27.67$

$-24.07$

$-20.47$

$-16.87$
Pressure psia

425.241

936.810

1446.565

1955.449

2461.125

2964.704

3466.842

3965.533

4461.273

4954.116

280.141

730.027

1178.111

1625.786

2072.068

2516.121

2958.404

3398.105

3836.599

4264.577

4698.765

5130.745

379.024

777.400

1175.365

1571.598

1965.936

2359.551

2751.018

3141.974

3530.889

3918.666

4304.879

4688.137

5070.779

389.761

735.131

1079.337

1424.081

1766.278

2108.580

2450.069

2789.958

3129.031

3466.325
Density
$1 b \cdot f t^{-3}$

106.514

106.488

106.463

106.439

106.417

106.394

106.372

106.350

106.329

106.308

103.850

103.826

103.803

103.780

103.759

103.737

103.716

103.696

103.675

103.655

103.635

103.616

101.309

101.287

101.265

101.244

101.224

101.204

101.184

101.165

101.145

101.126

101.107

101.088

101.070

98.321
98.301
98.281
98.261
98.242
98.223
98.204
98.186
98.167
98.149 
Table 28b. Liquid PVT Data (Isochoric Apparatus) for HCFC-124 $3 / 4$

\begin{tabular}{|c|c|c|}
\hline $\begin{array}{c}\text { Temperature } \\
{ }^{\circ} \mathrm{F}\end{array}$ & $\begin{array}{c}\text { Pressure } \\
\text { psia }\end{array}$ & $\begin{array}{l}\text { Density } \\
\text { lb. } f t^{-3}\end{array}$ \\
\hline $\begin{array}{r}-13.27 \\
-9.67 \\
-6.07 \\
-2.47 \\
1.13\end{array}$ & $\begin{array}{l}3802.825 \\
4138.252 \\
4471.574 \\
4804.183 \\
5135.682\end{array}$ & $\begin{array}{l}98.131 \\
98.114 \\
98.096 \\
98.078 \\
98.060\end{array}$ \\
\hline $\begin{array}{r}-13.27 \\
-9.67 \\
-6.07 \\
-2.47 \\
1.13 \\
4.73 \\
8.33 \\
11.93 \\
15.53 \\
19.13 \\
22.73 \\
26.33 \\
29.93 \\
33.53 \\
37.13 \\
40.73 \\
44.33 \\
47.93\end{array}$ & $\begin{array}{r}144.350 \\
375.920 \\
664.915 \\
953.764 \\
1242.074 \\
1528.877 \\
1816.283 \\
2102.530 \\
2387.424 \\
2671.917 \\
2955.776 \\
3238.098 \\
3520.499 \\
3801.671 \\
4082.300 \\
4362.174 \\
4640.810 \\
4918.634\end{array}$ & $\begin{array}{l}94.852 \\
94.513 \\
94.494 \\
94.476 \\
94.458 \\
94.440 \\
94.422 \\
94.405 \\
94.388 \\
94.370 \\
94.353 \\
94.337 \\
94.320 \\
94.303 \\
94.286 \\
94.270 \\
94.253 \\
94.237\end{array}$ \\
\hline $\begin{array}{r}37.13 \\
44.33 \\
51.53 \\
58.73 \\
65.93 \\
73.13 \\
80.33 \\
87.53 \\
94.73 \\
101.93 \\
109.13 \\
116.33\end{array}$ & $\begin{array}{r}176.241 \\
633.090 \\
1088.873 \\
1542.784 \\
1996.040 \\
2447.199 \\
2896.519 \\
3344.324 \\
3790.028 \\
4233.066 \\
4674.016 \\
5112.952\end{array}$ & $\begin{array}{l}89.446 \\
89.412 \\
89.379 \\
89.346 \\
89.315 \\
89.283 \\
89.252 \\
89.221 \\
89.190 \\
89.160 \\
89.129 \\
89.099\end{array}$ \\
\hline $\begin{array}{l}109.13 \\
116.33 \\
123.53 \\
130.73 \\
137.93 \\
145.13 \\
152.33 \\
159.53 \\
166.73 \\
173.93\end{array}$ & $\begin{array}{r}387.116 \\
703.608 \\
1019.513 \\
1335.691 \\
1651.569 \\
1967.007 \\
2281.657 \\
2595.934 \\
2909.516 \\
3221.652\end{array}$ & $\begin{array}{l}81.504 \\
81.475 \\
81.446 \\
81.418 \\
81.390 \\
81.363 \\
81.335 \\
81.308 \\
81.280 \\
81.253\end{array}$ \\
\hline
\end{tabular}


Table 28b. Liquid PVT Data (Isochoric Apparatus) for HCFC-124 4/4

Temperature ${ }^{\circ} \mathrm{F}$
181.13
188.33
195.53
202.73
209.93
217.13
181.13
188.33
195.53
202.73
209.93
217.13
224.33
231.53
238.73
245.93
253.13
260.33

\section{Pressure psia}

3532.709

3843.830

4154.032

4462.986

4770.819

5076.982

393.948

583.479

773.824

965.111

1157.007

1349.314

1542.199

1735.185

1928.489

2122.022

2315.429

2508.789

\section{Density
Ib. $\mathrm{ft}^{-3}$}

81.227

81.200

81.172

81.145

81.117

81.090

70.584

70.560

70.537

70.513

70.490

70.467

70.443

70.420

70.396

70.373

70.349

70.326 
Table 29a. Vapor pressures of HCFC-124 (NIST Burnett apparatus + ebulliometer) (SI units).

$\begin{array}{llllll}\mathrm{T} / \mathrm{K} & \mathrm{P} / \mathrm{kPa} & \mathrm{T} / \mathrm{K} & \mathrm{P} / \mathrm{kPa} & \mathrm{T} / \mathrm{K} & \mathrm{P} / \mathrm{kPa} \\ 388.119 & 3163.73 & 333.147 & 996.88 & 278.115 & 195.65 \\ 393.119 & 3471.42 & 333.144 & 996.39 & 278.110 & 195.68 \\ 388.132 & 3164.85 & 333.118 & 995.73 & 277.549 & 191.67 \\ 383.150 & 2882.64 & 333.112 & 995.71 & 275.821 & 179.94 \\ 383.142 & 2882.28 & 323.123 & 774.78 & 274.270 & 169.85 \\ 383.140 & 2881.88 & 318.140 & 679.38 & 272.883 & 161.18 \\ 383.137 & 2881.81 & 318.126 & 679.18 & 271.077 & 150.48 \\ 378.120 & 2619.37 & 313.139 & 592.85 & 267.404 & 130.37 \\ 378.113 & 2618.90 & 313.129 & 592.77 & 265.432 & 120.49 \\ 378.109 & 2618.91 & 308.156 & 515.04 & 263.330 & 110.59 \\ 373.150 & 2378.32 & 308.154 & 515.12 & 260.958 & 100.22 \\ 373.148 & 2378.14 & 303.189 & 445.38 & 259.892 & 95.82 \\ 373.145 & 2378.62 & 303.188 & 445.49 & 258.405 & 89.92 \\ 373.102 & 2376.06 & 303.142 & 444.87 & 257.300 & 85.73 \\ 368.152 & 2153.79 & 298.173 & 382.58 & 255.597 & 79.58 \\ 368.138 & 2153.16 & 298.169 & 382.46 & 254.414 & 75.50 \\ 368.135 & 2153.20 & 298.168 & 382.58 & 252.719 & 69.96 \\ 363.144 & 1945.56 & 293.161 & 326.67 & 251.186 & 65.23 \\ 363.134 & 1945.31 & 293.151 & 326.56 & 249.586 & 60.58 \\ 363.130 & 1945.61 & 293.148 & 326.63 & 247.695 & 55.43 \\ 358.135 & 1752.97 & 288.190 & 277.71 & 245.626 & 50.18 \\ 358.130 & 1752.87 & 288.182 & 277.57 & 243.916 & 46.16 \\ 358.122 & 1753.07 & 288.167 & 277.45 & 241.623 & 41.17 \\ 353.148 & 1575.90 & 287.859 & 274.57 & 239.026 & 36.07 \\ 353.139 & 1575.61 & 286.098 & 258.82 & 237.217 & 32.82 \\ 353.135 & 1575.96 & 283.871 & 239.75 & 235.513 & 29.98 \\ 348.156 & 1412.94 & 283.136 & 233.84 & 233.583 & 27.01 \\ 348.115 & 1411.20 & 283.128 & 233.70 & 231.986 & 24.73 \\ 348.108 & 1410.96 & 283.118 & 233.71 & 230.426 & 22.66 \\ 343.139 & 1261.34 & 282.199 & 226.25 & 228.600 & 20.42 \\ 343.133 & 1261.16 & 280.272 & 211.38 & 226.969 & 18.58 \\ 343.116 & 1260.53 & 279.073 & 202.48 & 225.082 & 16.62 \\ 338.174 & 1123.90 & 278.139 & 195.81 & 224.067 & 15.63 \\ 338.111 & 1122.25 & 278.128 & 195.71 & 222.956 & 14.61 \\ 338.109 & 1122.05 & 278.122 & 195.73 & 223.005 & 14.66\end{array}$


Table 29b. Vapor pressures of HCFC-124 (NIST Burnett apparatus + ebulliometer) (PI units).

\begin{tabular}{|c|c|c|c|c|c|}
\hline$T / F$ & P/psia & $\mathrm{T} / \mathrm{F}$ & P/psia & $T / F$ & $\mathrm{P} / \mathrm{psia}$ \\
\hline 238.944 & 458.861 & 140.006 & 144.543 & 40.938 & 28.376 \\
\hline 247.944 & 503.488 & 140.002 & 144.532 & 40.929 & 28.381 \\
\hline 238.967 & 459.023 & 139.995 & 144.585 & 39.919 & 27.799 \\
\hline 230.000 & 418.091 & 139.989 & 144.515 & 36.808 & 26.098 \\
\hline 229.985 & 418.040 & 139.943 & 144.419 & 34.016 & 24.635 \\
\hline 229.982 & 417.981 & 139.932 & 144.416 & 31.519 & 23.377 \\
\hline 229.976 & 417.972 & 121.951 & 112.373 & 28.269 & 21.825 \\
\hline 220.946 & 379.907 & 112.982 & 98.535 & 21.657 & 18.910 \\
\hline 220.933 & 379.839 & 112.957 & 98.506 & 18.108 & 17.475 \\
\hline 220.926 & 379.841 & 103.981 & 85.985 & 14.324 & 16.040 \\
\hline 212.000 & 344.947 & 103.963 & 85.974 & 10.056 & 14.536 \\
\hline 211.997 & 344.920 & 95.011 & 74.701 & 8.135 & 13.898 \\
\hline 211.991 & 344.990 & 95.007 & 74.712 & 5.459 & 13.042 \\
\hline 211.914 & 344.619 & 86.071 & 64.597 & 3.470 & 12.434 \\
\hline 203.004 & 312.381 & 86.069 & 64.613 & 0.406 & 11.542 \\
\hline 202.979 & 312.290 & 85.986 & 64.523 & -1.723 & 10.951 \\
\hline 202.973 & 312.296 & 77.042 & 55.488 & -4.774 & 10.148 \\
\hline 193.990 & 282.180 & 77.035 & 55.471 & -7.534 & 9.462 \\
\hline 193.972 & 282.143 & 77.033 & 55.489 & -10.413 & 8.787 \\
\hline 193.965 & 282.187 & 68.019 & 47.380 & -13.817 & 8.039 \\
\hline 184.974 & 254.248 & 68.001 & 47.363 & -17.542 & 7.278 \\
\hline 184.965 & 254.233 & 67.996 & 47.374 & -20.619 & 6.696 \\
\hline 184.951 & 254.261 & 59.072 & 40.279 & -24.748 & 5.972 \\
\hline 175.996 & 228.565 & 59.058 & 40.258 & -29.422 & 5.232 \\
\hline 175.980 & 228.523 & 59.031 & 40.241 & -32.678 & 4.760 \\
\hline 175.973 & 228.574 & 58.476 & 39.823 & -35.745 & 4.349 \\
\hline 167.011 & 204.929 & 55.306 & 37.540 & -39.220 & 3.917 \\
\hline 166.937 & 204.678 & 51.299 & 34.773 & -42.094 & 3.587 \\
\hline 166.925 & 204.642 & 49.975 & 33.916 & -44.901 & 3.287 \\
\hline 157.981 & 182.942 & 49.961 & 33.896 & -48.189 & 2.962 \\
\hline 157.970 & 182.916 & 49.943 & 33.897 & -51.124 & 2.694 \\
\hline 157.940 & 182.825 & 48.289 & 32.816 & -54.522 & 2.411 \\
\hline 149.043 & 163.008 & 44.821 & 30.658 & -56.348 & 2.267 \\
\hline 148.930 & 162.769 & 42.662 & 29.367 & -58.348 & 2.120 \\
\hline 148.926 & 162.739 & 40.981 & 28.400 & -58.261 & 2.126 \\
\hline 148.924 & 162.730 & 40.961 & 28.386 & -60.416 & 1.974 \\
\hline
\end{tabular}


Table 30. Speed of Sound in R-124

\begin{tabular}{|c|c|c|c|c|c|c|}
\hline $\mathbf{T} / \mathbf{K}$ & $/ \operatorname{deg} F$ & $\mathbf{p} / \mathbf{k P a}$ & p/psi & $\mathbf{u} /(\mathbf{m} / \mathbf{s})$ & $\mathbf{u} /(\mathbf{f t} / \mathbf{s})$ & $10^{6 *} \mathrm{du} / \mathrm{u}$ \\
\hline 250.010 & -9.652 & $\begin{array}{l}36.795 \\
34.212 \\
30.454 \\
27.054 \\
24.072 \\
21.407\end{array}$ & $\begin{array}{l}5.337 \\
4.962 \\
4.417 \\
3.924 \\
3.491 \\
3.105\end{array}$ & $\begin{array}{l}127.8356 \\
127.9641 \\
128.1595 \\
128.3295 \\
128.4835 \\
128.6077\end{array}$ & $\begin{array}{l}419.4081 \\
419.8297 \\
420.4708 \\
421.0285 \\
421.5338 \\
421.9413\end{array}$ & $\begin{array}{l}16.2 \\
14.8 \\
10.8 \\
10.2 \\
10.5 \\
41.1\end{array}$ \\
\hline 259.966 & 8.269 & $\begin{array}{l}58.398 \\
54.328 \\
48.125 \\
42.593 \\
39.592 \\
35.043\end{array}$ & $\begin{array}{l}8.470 \\
7.880 \\
6.980 \\
6.178 \\
5.742 \\
5.083\end{array}$ & $\begin{array}{l}129.4742 \\
129.6596 \\
129.9416 \\
130.1950 \\
130.3272 \\
130.5323\end{array}$ & $\begin{array}{l}424.7841 \\
425.3924 \\
426.3176 \\
427.1490 \\
427.5827 \\
428.2556\end{array}$ & $\begin{array}{l}29.7 \\
16.3 \\
29.2 \\
39.8 \\
21.7 \\
20.5\end{array}$ \\
\hline 269.908 & 26.164 & $\begin{array}{l}88.361 \\
78.064 \\
72.496 \\
64.077 \\
56.654 \\
46.362 \\
38.018 \\
31.151 \\
15.009\end{array}$ & $\begin{array}{r}12.816 \\
11.322 \\
10.515 \\
9.294 \\
8.217 \\
6.724 \\
5.514 \\
4.518 \\
2.177\end{array}$ & $\begin{array}{l}130.8665 \\
131.2991 \\
131.5297 \\
131.8751 \\
132.1758 \\
132.5907 \\
132.9221 \\
133.1923 \\
133.8167\end{array}$ & $\begin{array}{l}429.3520 \\
430.7713 \\
431.5279 \\
432.6611 \\
433.6476 \\
435.0089 \\
436.0961 \\
436.9826 \\
439.0312\end{array}$ & $\begin{array}{l}50.0 \\
37.2 \\
26.9 \\
13.4 \\
23.1 \\
10.1 \\
50.0 \\
21.1 \\
50.0\end{array}$ \\
\hline 279.871 & 44.098 & $\begin{array}{r}100.284 \\
100.208 \\
92.313 \\
86.020 \\
79.293 \\
73.817 \\
64.726 \\
63.412 \\
52.717 \\
52.434 \\
40.859 \\
39.183 \\
14.781\end{array}$ & $\begin{array}{r}14.545 \\
14.534 \\
13.389 \\
12.476 \\
11.501 \\
10.706 \\
9.388 \\
9.197 \\
7.646 \\
7.605 \\
5.926 \\
5.683 \\
2.144\end{array}$ & $\begin{array}{l}133.1135 \\
133.1172 \\
133.4146 \\
133.6456 \\
133.8967 \\
134.0965 \\
134.4287 \\
134.4754 \\
134.8651 \\
134.8721 \\
135.2851 \\
135.3443 \\
136.2006\end{array}$ & $\begin{array}{l}436.7241 \\
436.7362 \\
437.7119 \\
438.4698 \\
439.2936 \\
439.9491 \\
441.0390 \\
441.1923 \\
442.4708 \\
442.4938 \\
443.8488 \\
444.0430 \\
446.8524\end{array}$ & $\begin{array}{l}50.0 \\
50.0 \\
26.6 \\
50.0 \\
24.7 \\
10.6 \\
12.9 \\
10.4 \\
13.7 \\
12.1 \\
20.7 \\
11.0 \\
50.0\end{array}$ \\
\hline
\end{tabular}


Table 30. Speed of Sound in R-124 (Continued)

\begin{tabular}{|c|c|c|c|c|c|c|}
\hline$T / K$ & $/ \operatorname{deg} F$ & $\mathbf{p} / \mathbf{k P a}$ & $\mathbf{p} / \mathbf{p s i}$ & $\mathbf{u} /(\mathbf{m} / \mathbf{s})$ & $\mathbf{u} /(\mathbf{f t} / \mathbf{s})$ & $10^{6 *} \mathrm{du} / \mathrm{u}$ \\
\hline \multirow[t]{10}{*}{300.043} & 80.407 & 179.000 & 25.962 & 135.9367 & 445.9865 & 50.0 \\
\hline & & 168.320 & 24.413 & 136.2724 & 447.0879 & 50.0 \\
\hline & & 152.076 & 22.057 & 136.7828 & 448.7625 & 50.0 \\
\hline & & 111.775 & 16.212 & 138.0176 & 452.8136 & 28.7 \\
\hline & & 111.800 & 16.215 & 138.0183 & 452.8159 & 18.2 \\
\hline & & 95.020 & 13.782 & 138.5224 & 454.4698 & 10.1 \\
\hline & & 77.717 & 11.272 & 139.0341 & 456.1486 & 17.2 \\
\hline & & 57.114 & 8.284 & 139.6412 & 458.1404 & 10.6 \\
\hline & & 40.037 & 5.807 & 140.1374 & 459.7684 & 33.5 \\
\hline & & 19.710 & 2.859 & 140.7155 & 461.6650 & 22.2 \\
\hline \multirow{6}{*}{319.675} & 115.745 & 442.476 & 64.176 & 133.9481 & 439.4623 & 16.8 \\
\hline & & 378.196 & 54.853 & 135.8273 & 445.6276 & 12.4 \\
\hline & & 308.872 & 44.798 & 137.7688 & 451.9974 & 10.3 \\
\hline & & 240.381 & 34.864 & 139.6121 & 458.0449 & 15.5 \\
\hline & & 170.777 & 24.769 & 141.4163 & 463.9642 & 10.7 \\
\hline & & 102.455 & 14.860 & 143.1273 & 469.5778 & 10.0 \\
\hline \multirow[t]{7}{*}{320.269} & 116.814 & 206.351 & 29.929 & 140.6523 & 461.4577 & 52.9 \\
\hline & & 176.440 & 25.590 & 141.4174 & 463.9678 & 47.9 \\
\hline & & 145.323 & 21.077 & 142.2018 & 466.5413 & 41.3 \\
\hline & & 112.325 & 16.291 & 143.0207 & 469.2280 & 28.5 \\
\hline & & 83.718 & 12.142 & 143.7203 & 471.5233 & 10.4 \\
\hline & & 51.555 & 7.477 & 144.4987 & 474.0771 & 12.9 \\
\hline & & 20.225 & 2.933 & 145.2433 & 476.5200 & 37.6 \\
\hline \multirow[t]{11}{*}{339.740} & 151.862 & 777.171 & 112.719 & 131.8811 & 432.6808 & 12.4 \\
\hline & & 706.910 & 102.529 & 133.7857 & 438.9295 & 10.1 \\
\hline & & 638.555 & 92.614 & 1355654 & 444.7684 & 11.1 \\
\hline & & 559.584 & 81.161 & 137.5542 & 451.2933 & 12.9 \\
\hline & & 489.988 & 71.067 & 139.2476 & 456.8491 & 10.0 \\
\hline & & 418.994 & 60.770 & 140.9163 & 462.3238 & 10.5 \\
\hline & & 344.139 & 49.913 & 142.6221 & 467.9203 & 10.4 \\
\hline & & 316.415 & 45.892 & 143.2367 & 469.9367 & 55.2 \\
\hline & & 298.737 & 43.328 & 143.6284 & 471.2218 & 53.9 \\
\hline & & 277.309 & 40.220 & 144.1006 & 472.7710 & 10.5 \\
\hline & & 269.057 & 39.023 & 144.2765 & 473.3481 & 52.8 \\
\hline
\end{tabular}


Table 30. Speed of Sound in R-124 (Continued)

\begin{tabular}{|c|c|c|c|c|c|c|}
\hline $\mathbf{T} / \mathbf{K}$ & $/ \operatorname{deg} F$ & $\mathbf{p} / \mathbf{k P a}$ & $\mathrm{p} / \mathrm{psi}$ & $\mathbf{u} /(\mathbf{m} / \mathbf{s})$ & $\mathbf{u} /(\mathbf{f t} / \mathbf{s})$ & $10^{6 *} \mathrm{du} / \mathrm{u}$ \\
\hline 39.740 & 151.862 & $\begin{array}{l}242.039 \\
217.743 \\
204.751 \\
195.884 \\
176.207\end{array}$ & $\begin{array}{l}35.105 \\
31.581 \\
29.697 \\
28.411 \\
25.557\end{array}$ & $\begin{array}{l}144.8619 \\
145.3829 \\
145.6622 \\
145.8474 \\
146.2619\end{array}$ & $\begin{array}{l}475.2687 \\
476.9780 \\
477.8944 \\
478.5020 \\
479.8619\end{array}$ & $\begin{array}{l}52.9 \\
43.8 \\
10.0 \\
46.4 \\
45.5\end{array}$ \\
\hline 340.221 & 152.728 & $\begin{array}{l}294.021 \\
265.902 \\
240.398 \\
203.815 \\
172.929 \\
146.634 \\
112.631 \\
83.704 \\
55.698 \\
54.111 \\
39.741 \\
29.679\end{array}$ & $\begin{array}{l}42.644 \\
38.566 \\
34.867 \\
29.561 \\
25.081 \\
21.268 \\
16.336 \\
12.140 \\
8.078 \\
7.848 \\
5.764 \\
4.305\end{array}$ & $\begin{array}{l}143.8562 \\
144.4693 \\
145.0: 87 \\
145.7971 \\
146.4461 \\
146.9938 \\
147.6945 \\
148.2842 \\
148.8504 \\
148.8834 \\
149.1716 \\
149.3682\end{array}$ & $\begin{array}{l}471.9692 \\
473.9806 \\
475.7831 \\
478.3369 \\
480.4662 \\
482.2631 \\
484.5620 \\
486.4967 \\
488.3543 \\
488.4626 \\
489.4081 \\
490.0532\end{array}$ & $\begin{array}{l}101.3 \\
52.0 \\
50.6 \\
45.8 \\
40.9 \\
34.0 \\
22.5 \\
10.0 \\
12.2 \\
13.8 \\
20.7 \\
51.7\end{array}$ \\
\hline 355.095 & 179.501 & $\begin{array}{l}294.704 \\
267.525 \\
240.670 \\
202.915 \\
172.036 \\
146.499 \\
115.274 \\
82.394 \\
57.873 \\
55.857 \\
55.877 \\
20.316\end{array}$ & $\begin{array}{l}42.743 \\
38.801 \\
34.906 \\
29.430 \\
24.952 \\
21.248 \\
16.719 \\
11.950 \\
8.394 \\
8.101 \\
8.104 \\
2.947\end{array}$ & $\begin{array}{l}147.6819 \\
148.1941 \\
148.6976 \\
149.3982 \\
149.9658 \\
150.4310 \\
150.9955 \\
151.5862 \\
152.0232 \\
152.0600 \\
152.0603 \\
152.6843\end{array}$ & $\begin{array}{l}484.5207 \\
486.2011 \\
487.8530 \\
490.1516 \\
492.0138 \\
493.5400 \\
495.3921 \\
497.3301 \\
498.7638 \\
498.8845 \\
498.8855 \\
500.9327\end{array}$ & $\begin{array}{l}48.5 \\
46.3 \\
43.8 \\
44.7 \\
42.9 \\
29.1 \\
20.9 \\
10.8 \\
12.1 \\
25.5 \\
22.3 \\
51.2\end{array}$ \\
\hline 360.040 & 188.402 & $\begin{array}{l}861.534 \\
857.615 \\
793.765 \\
707.828 \\
622.243\end{array}$ & $\begin{array}{l}124.955 \\
124.387 \\
115.126 \\
102.662 \\
90.249\end{array}$ & $\begin{array}{l}137.6826 \\
137.7619 \\
139.1445 \\
140.9484 \\
142.6928\end{array}$ & $\begin{array}{l}451.7146 \\
451.9747 \\
456.5108 \\
462.4291 \\
468.1522\end{array}$ & $\begin{array}{l}50.0 \\
50.0 \\
50.0 \\
22.5 \\
17.8\end{array}$ \\
\hline
\end{tabular}


Table 30. Speed of Sound in R-124 (Continued)

\begin{tabular}{|c|c|c|c|c|c|c|}
\hline $\mathbf{T} / \mathbf{K}$ & $/ \operatorname{deg} F$ & $\mathbf{p} / \mathbf{k P a}$ & $\mathbf{p} / \mathbf{p s i}$ & $\mathbf{u} /(\mathbf{m} / \mathbf{s})$ & $\mathbf{u} /(\mathbf{f t} / \mathbf{s})$ & $10^{6 *} \mathrm{du} / \mathrm{u}$ \\
\hline 360.040 & 188.402 & $\begin{array}{l}534.180 \\
446.593 \\
361.880 \\
276.898 \\
189.246 \\
102.714\end{array}$ & $\begin{array}{l}77.476 \\
64.773 \\
52.486 \\
40.161 \\
27.448 \\
14.897\end{array}$ & $\begin{array}{l}144.4315 \\
146.1142 \\
147.6980 \\
149.2458 \\
150.8046 \\
152.3034\end{array}$ & $\begin{array}{l}473.8566 \\
479.3773 \\
484.5735 \\
489.6516 \\
494.7657 \\
499.6831\end{array}$ & $\begin{array}{l}10.3 \\
10.8 \\
10.9 \\
10.0 \\
10.6 \\
14.2\end{array}$ \\
\hline 365.000 & 197.330 & $\begin{array}{l}295.095 \\
275.484 \\
247.117 \\
213.316 \\
191.332 \\
160.061 \\
134.184 \\
104.816 \\
79.108 \\
49.553 \\
19.846\end{array}$ & $\begin{array}{l}42.800 \\
39.956 \\
35.841 \\
30.939 \\
27.750 \\
23.215 \\
19.462 \\
15.202 \\
11.474 \\
7.187 \\
2.878\end{array}$ & $\begin{array}{l}150.1402 \\
150.4782 \\
150.9644 \\
151.5406 \\
151.9121 \\
152.4382 \\
152.8699 \\
153.3573 \\
153.7816 \\
154.2667 \\
154.7508\end{array}$ & $\begin{array}{l}492.5860 \\
493.6949 \\
495.2900 \\
497.1804 \\
498.3993 \\
500.1253 \\
501.5417 \\
503.1407 \\
504.5328 \\
506.1243 \\
507.7126\end{array}$ & $\begin{array}{l}56.5 \\
52.2 \\
46.1 \\
42.7 \\
38.8 \\
37.0 \\
22.6 \\
15.4 \\
10.3 \\
19.4 \\
65.0\end{array}$ \\
\hline 374.900 & 215.150 & $\begin{array}{l}295.101 \\
264.599 \\
236.689 \\
204.278 \\
172.975 \\
143.965 \\
115.475 \\
82.775 \\
52.025 \\
50.877 \\
34.683 \\
20.110\end{array}$ & $\begin{array}{l}42.801 \\
38.377 \\
34.329 \\
29.628 \\
25.088 \\
20.880 \\
16.748 \\
12.006 \\
7.546 \\
7.379 \\
5.030 \\
2.917\end{array}$ & $\begin{array}{l}152.5159 \\
153.0001 \\
153.4384 \\
153.9446 \\
154.4298 \\
154.8776 \\
155.3149 \\
155.8139 \\
156.2790 \\
156.2947 \\
156.5406 \\
156.7564\end{array}$ & $\begin{array}{l}500.3803 \\
501.9688 \\
503.4068 \\
505.0676 \\
506.6594 \\
508.1286 \\
509.5633 \\
511.2005 \\
512.7264 \\
512.7779 \\
513.5846 \\
514.2927\end{array}$ & $\begin{array}{l}44.2 \\
48.3 \\
49.4 \\
44.3 \\
37.2 \\
27.5 \\
15.6 \\
10.1 \\
24.4 \\
34.4 \\
42.0 \\
56.3\end{array}$ \\
\hline 380.120 & 224.546 & $\begin{array}{l}861.545 \\
826.662 \\
734.569 \\
649.931 \\
560.832\end{array}$ & $\begin{array}{l}124.957 \\
119.897 \\
106.540 \\
94.265 \\
81.342\end{array}$ & $\begin{array}{l}144.5819 \\
145.1875 \\
146.7474 \\
148.1527 \\
149.5969\end{array}$ & $\begin{array}{l}474.3501 \\
476.3369 \\
481.4547 \\
486.0653 \\
490.8035\end{array}$ & $\begin{array}{l}70.3 \\
14.0 \\
10.8 \\
10.3 \\
10.5\end{array}$ \\
\hline
\end{tabular}


Table 30. Speed of Sound in R-124 (Continued)

\begin{tabular}{|c|c|c|c|c|c|c|}
\hline $\mathbf{T} / \mathbf{K}$ & $/ \operatorname{deg} F$ & $\mathbf{p} / \mathbf{k P a}$ & p/psi & $\mathbf{u} /(\mathbf{m} / \mathbf{s})$ & $\mathbf{u} /(\mathbf{f t} / \mathbf{s})$ & $10^{6 *} \mathrm{du} / \mathrm{u}$ \\
\hline 380.120 & 224.546 & $\begin{array}{l}467.628 \\
376.986 \\
285.789 \\
193.184 \\
102.815\end{array}$ & $\begin{array}{l}67.824 \\
54.677 \\
41.450 \\
28.019 \\
14.912\end{array}$ & $\begin{array}{l}151.0856 \\
152.4933 \\
153.8860 \\
155.2809 \\
156.6010\end{array}$ & $\begin{array}{l}495.6877 \\
500.3061 \\
504.8753 \\
509.4518 \\
513.7828\end{array}$ & $\begin{array}{l}10.5 \\
23.0 \\
12.4 \\
77.5 \\
10.1\end{array}$ \\
\hline 385.650 & 234.500 & $\begin{array}{l}295.602 \\
270.057 \\
233.661 \\
209.940 \\
173.992 \\
144.704 \\
115.415 \\
82.398 \\
51.624\end{array}$ & $\begin{array}{l}42.873 \\
39.168 \\
33.890 \\
30.449 \\
25.235 \\
20.988 \\
16.740 \\
11.951 \\
7.487\end{array}$ & $\begin{array}{l}155.0203 \\
155.3888 \\
155.9117 \\
156.2492 \\
156.7609 \\
157.1758 \\
157.5867 \\
158.0509 \\
158.4782\end{array}$ & $\begin{array}{l}508.5968 \\
509.8058 \\
511.5213 \\
512.6286 \\
514.3074 \\
515.6686 \\
517.0167 \\
518.5397 \\
519.9416\end{array}$ & $\begin{array}{l}55.8 \\
46.9 \\
45.6 \\
33.1 \\
22.5 \\
18.8 \\
15.4 \\
16.6 \\
16.3\end{array}$ \\
\hline 399.933 & 260.209 & $\begin{array}{l}294.182 \\
273.047 \\
242.256 \\
215.184 \\
191.079 \\
159.671 \\
131.874 \\
104.406 \\
76.710 \\
50.530 \\
50.574 \\
30.705 \\
19.957\end{array}$ & $\begin{array}{l}42.668 \\
39.602 \\
35.136 \\
31.210 \\
27.714 \\
23.158 \\
19.127 \\
15.143 \\
11.126 \\
7.329 \\
7.335 \\
4.453 \\
2.895\end{array}$ & $\begin{array}{l}158.2851 \\
158.5570 \\
158.9503 \\
159.2955 \\
159.6021 \\
159.9986 \\
160.3492 \\
160.6935 \\
161.0399 \\
161.3658 \\
161.3683 \\
161.6064 \\
161.7428\end{array}$ & $\begin{array}{l}519.3081 \\
520.2001 \\
521.4905 \\
522.6230 \\
523.6289 \\
524.9298 \\
526.0801 \\
527.2096 \\
528.3461 \\
529.4154 \\
529.4236 \\
530.2047 \\
530.6522\end{array}$ & $\begin{array}{l}50.0 \\
50.0 \\
50.0 \\
50.0 \\
50.0 \\
50.0 \\
50.0 \\
50.0 \\
50.0 \\
50.0 \\
50.0 \\
50.0 \\
50.0\end{array}$ \\
\hline 400.045 & 260.411 & $\begin{array}{l}874.592 \\
781.357 \\
717.790 \\
635.818 \\
560.035 \\
481.433 \\
402.900\end{array}$ & $\begin{array}{l}126.849 \\
113.326 \\
104.107 \\
92.218 \\
81.226 \\
69.826 \\
58.436\end{array}$ & $\begin{array}{l}150.4808 \\
151.7914 \\
152.6728 \\
153.7933 \\
154.8143 \\
155.8624 \\
156.8956\end{array}$ & $\begin{array}{l}493.7034 \\
498.0033 \\
500.8950 \\
504.5712 \\
507.9209 \\
511.3596 \\
514.7493\end{array}$ & $\begin{array}{l}50.0 \\
38.3 \\
51.8 \\
42.2 \\
43.2 \\
36.6 \\
40.9\end{array}$ \\
\hline
\end{tabular}


Table 30. Speed of Sound in R-124 (Continued)

\begin{tabular}{lllllll}
\hline T/K & /deg F & p/kPa & p/psi & $\mathbf{u} /(\mathbf{m} / \mathbf{s})$ & $\mathbf{u} /(\mathbf{f t} / \mathbf{s})$ & $10^{6 * \mathbf{d u} / \mathbf{u}}$ \\
\hline \multirow{3}{*}{400.045} & 260.411 & 321.068 & 46.567 & 157.9577 & 518.2339 & 34.4 \\
& & 243.643 & 35.337 & 158.9523 & 521.4970 & 26.2 \\
& & 163.068 & 23.651 & 159.9768 & 524.8583 & 15.5 \\
\hline
\end{tabular}

The following conversion factors were used to define the Engineering units:
$\mathrm{t} / \mathrm{deg} \mathrm{F}=(\mathrm{T} / \mathrm{K}-273.15) * 1.8+32$
$1 \mathrm{psi}=1 \mathrm{psia}=6894.759 \mathrm{~Pa}$
$1 \mathrm{ft} / \mathrm{s}=0.3048000 \mathrm{~m} / \mathrm{s}$
$1 \mathrm{Btu}=1054.35 \mathrm{~J}$
1 pound mass $=0.45359237 \mathrm{~kg}$ 
Table 31. Ideal-Gas Heat Capacity, $\mathbf{C}_{\mathrm{p}}^{0}$, of R-124

\begin{tabular}{lrll}
\hline & & & \\
& deg $\mathbf{F}$ & $\mathbf{C}_{\mathbf{p}}^{\mathbf{0}} \mathbf{R}$ & $\mathbf{s i g C}_{\mathbf{p}}^{\mathbf{0}} \mathbf{R}$ \\
\hline & & & \\
\hline 250.010 & -9.652 & 10.66021 & $.008^{\mathbf{a}}$ \\
259.966 & 8.269 & 10.91542 & $.006^{\mathbf{a}}$ \\
269.908 & 26.164 & 11.17243 & .017 \\
279.871 & 44.098 & 11.40686 & .014 \\
300.035 & 80.393 & 11.86296 & .015 \\
300.043 & 80.407 & 11.88922 & .009 \\
319.675 & 115.745 & 12.32617 & .009 \\
339.740 & 151.862 & 12.76547 & .010 \\
355.095 & 179.501 & 13.09448 & .011 \\
365.000 & 197.330 & 13.28091 & .017 \\
374.900 & 215.150 & 13.49625 & .012 \\
385.650 & 234.500 & 13.71336 & .019 \\
400.045 & 260.411 & 13.99065 & .008 \\
\hline
\end{tabular}

${ }^{a}$ Values of $\mathbf{C}_{\mathbf{p}}^{0} / \mathbf{R}$ on this isotherm were determined from two-parameter fits with the acoustic virial coefficient gamma held at certain fixed values. 
Table 32(a). Acoustic Viral Coefficients for R-124 (SI units)

\begin{tabular}{|c|c|c|c|}
\hline $\mathbf{T}$ & $10^{6}$ beta & $10^{9}$ gamma & $10^{12}$ delta \\
\hline$/ \mathbf{K}$ & $/\left(\mathbf{m}^{3} / \mathbf{m o l}\right)$ & $/\left(\mathbf{m}^{3} / \mathbf{m o l}-\mathbf{P a}\right)$ & $/\left(\mathbf{m}^{3} / \mathbf{m o l}-\mathbf{P a}^{2}\right)$ \\
\hline 250.010 & $-1509.83+/-5.37$ & - & - \\
\hline 259.966 & $-1367.30+/-2.55$ & - & - \\
\hline 269.908 & $-1255.04+/-6.16$ & $-0.6544+/-0.055$ & - \\
\hline 279.871 & $-1160.28+/-4.31$ & $-0.4298+/-0.032$ & - \\
\hline 300.035 & $-987.90+/-2.33$ & $-0.2438+/-0.008$ & - \\
\hline 300.043 & $-989.09+/-1.94$ & $-0.2450+/-0.010$ & - \\
\hline $319.675^{*}$ & $-854.94+/-1.62$ & $-0.1247+/-0.008$ & $-5.96 \mathrm{E}-5+/-1.1 \mathrm{E}-5$ \\
\hline 319.675 & $-863.07+/-2.77$ & $-0.0905+/-0.011$ & $-10.10 \mathrm{E}-5+/-1.3 \mathrm{E}-5$ \\
\hline 320.269 & $-855.66+/-2.99$ & $-0.1266+/-0.012$ & - \\
\hline $339.740 *$ & $-745.84+/-1.09$ & $-0.0684+/-0.004$ & $-3.40 \mathrm{E}-5+/-0.30 \mathrm{E}-5$ \\
\hline 339.740 & $-745.55+/-3.29$ & $-0.0672+/-0.008$ & $-3.58 \mathrm{E}-5+/-0.54 \mathrm{E}-5$ \\
\hline 340.221 & $-743.20+/-2.02$ & $-0.0806+/-0.006$ & - \\
\hline 355.095 & $-673.91+/-1.67$ & $-0.0528+/-0.005$ & - \\
\hline 360.040 & $-649.22+/-0.91$ & $-0.0445+/-0.002$ & $-1.53 \mathrm{E}-5+/-0.15 \mathrm{E}-5$ \\
\hline 365.000 & $-634.61+/-1.80$ & $-0.0328+/-0.006$ & - \\
\hline 374.900 & $-592.23+/-1.77$ & $-0.0336+/-0.006$ & - \\
\hline 380.120 & $-567.49+/-0.85$ & $-0.0371+/-0.001$ & - \\
\hline 385.650 & $-555.59+/-2.21$ & $-0.0193+/-0.006$ & - \\
\hline 399.933 & $-505.39+/-1.04$ & $-0.0194+/-0.003$ & - \\
\hline 400.045 & $-504.27+/-1.03$ & $-0.0198+/-0.001$ & - \\
\hline $400.045^{*}$ & $-505.64+/-0.44$ & $-0.0185+/-0.001$ & - \\
\hline
\end{tabular}

* Merged isotherm of high and low pressure data 
Table 32(b). Acoustic Viral Coefficients for R-124 (PI units)

\begin{tabular}{|c|c|c|c|}
\hline $\mathbf{t}$ & $10^{4}$ beta & $10^{6}$ gamma & $10^{9}$ delta \\
\hline$/(\operatorname{deg} \mathbf{F})$ & $/(\mathbf{b t u} / \mathbf{p s i})$ & $/\left(\mathbf{b t u} / \mathrm{psi}^{2}\right)$ & $/\left(\mathrm{btu} / \mathrm{psi}^{3}\right)$ \\
\hline-9.652 & $-98.733+/-0.351$ & - & - \\
\hline 8.269 & $-89.413+/-0.167$ & - & - \\
\hline 26.164 & $-82.072+/-0.403$ & $-29.505+/-0.248$ & - \\
\hline 44.098 & $-75.875+/-0.282$ & $-19.379+/-0.144$ & - \\
\hline 80.393 & $-64.603+/-0.152$ & $-10.992+/-0.036$ & - \\
\hline 80.407 & $-64.680+/-0.127$ & $-11.046+/-0.045$ & - \\
\hline $115.745^{*}$ & $-55.908+/-0.106$ & $-5.622+/-0.036$ & $-18.528+/-3.420$ \\
\hline 115.745 & $-56.439+/-0.181$ & $-4.080+/-0.050$ & $-31.398+/-4.041$ \\
\hline 116.814 & $-55.955+/-0.196$ & $-5.708+1-0.054$ & - \\
\hline $151.862 *$ & $-48.773+/-0.071$ & $-3.084+/-0.018$ & $-10.570+/-0.933$ \\
\hline 151.862 & $-48.754+/-0.215$ & $-3.030+/-0.036$ & $-11.129+/-1.679$ \\
\hline 152.728 & $-48.601+/-0.132$ & $-3.634+/-0.027$ & - \\
\hline 179.501 & $-44.070+/-0.109$ & $-2.381+/-0.023$ & $\begin{array}{ll}- & -\end{array}$ \\
\hline 188.402 & $-42.455+/-0.060$ & $-2.006+1-0.009$ & $-4.756+/-0.466$ \\
\hline 197.330 & $-41.500+/-0.118$ & $-1.479+/-0.027$ & - \\
\hline 215.150 & $-38.728+/-0.116$ & $-1.515+/-0.027$ & - \\
\hline 224.546 & $-37.110+/-0.056$ & $-1.673+/-0.005$ & - \\
\hline 234.500 & $-36.332+/-0.145$ & $-0.870+/-0.027$ & - \\
\hline 260.209 & $-33.049+/-0.068$ & $-0.875+/-0.014$ & - \\
\hline 260.411 & $-32.976+/-0.067$ & $-0.893+/-0.005$ & - \\
\hline $260.411^{*}$ & $-33.066+/-0.029$ & $-0.834+/-0.005$ & - \\
\hline
\end{tabular}

* Merged isotherm of high and low pressure data 
Table 33 (a). Experimental liquid heat capacity data for HCFC-124.

\begin{tabular}{|c|c|c|c|c|c|c|}
\hline \multirow{2}{*}{$\begin{array}{l}\mathbf{T} \\
\mathbf{K}\end{array}$} & $\rho$ & \multirow[b]{2}{*}{ MPa } & \multirow[t]{2}{*}{$C_{v, \exp }$} & \multicolumn{2}{|c|}{$\bar{C}_{v, c a l}$} & \multirow[t]{2}{*}{$\overline{R u n}$} \\
\hline & $\mathrm{mol} \mathrm{dm}^{-3}$ & & & mol-1 & $k^{-1}$ & \\
\hline $\begin{array}{l}173.4550 \\
175.5705 \\
177.6791 \\
179.8395 \\
181.9187 \\
184.0649 \\
186.1174 \\
188.2467\end{array}$ & $\begin{array}{l}12.5707 \\
12.5645 \\
12.5584 \\
12.5521 \\
12.5461 \\
12.5399 \\
12.5341 \\
12.5280\end{array}$ & $\begin{array}{r}7.8316 \\
10.7927 \\
13.7109 \\
16.6751 \\
19.5024 \\
22.3953 \\
25.1371 \\
27.9568\end{array}$ & $\begin{array}{l}94.78 \\
89.28 \\
89.65 \\
90.12 \\
90.30 \\
90.57 \\
91.00 \\
91.23\end{array}$ & $\begin{array}{l}74.54 \\
76.78 \\
78.70 \\
80.40 \\
81.82 \\
83.10 \\
84.18 \\
85.17\end{array}$ & $\begin{array}{r}21.35 \\
14.00 \\
12.22 \\
10.78 \\
9.39 \\
8.24 \\
7.49 \\
6.64\end{array}$ & $\begin{array}{l}\mathbf{a} \\
\mathbf{b} \\
\mathbf{a} \\
\mathbf{b} \\
\mathbf{a} \\
\mathbf{b} \\
\mathbf{a} \\
\mathbf{b}\end{array}$ \\
\hline
\end{tabular}

Table $33(b)$. Experimental liquid heat capacity data for HCFC-124.

\begin{tabular}{|c|c|c|c|c|c|c|}
\hline $\mathbf{T}$ & $p$ & $\mathbf{P}$ & $C_{v, \exp }$ & $C_{v, c a l}$ & $\delta C_{v}, \%$ & $\overline{\text { Run }}$ \\
\hline${ }^{\circ} \mathbf{F}$ & $1 b \in t^{-3}$ & paia & Bt & $1 b^{-1} \circ F^{-1}$ & & \\
\hline $\begin{array}{l}-147.451 \\
-143.643 \\
-139.848 \\
-135.959 \\
-132.216 \\
-128.353 \\
-124.659 \\
-120.826\end{array}$ & $\begin{array}{l}107.10 \\
107.05 \\
107.00 \\
106.94 \\
106.89 \\
106.84 \\
106.79 \\
106.74\end{array}$ & $\begin{array}{l}1135.9 \\
1565.4 \\
1988.6 \\
2418.5 \\
2828.6 \\
3248.2 \\
3645.8 \\
4054.8\end{array}$ & $\begin{array}{l}0.16599 \\
0.15635 \\
0.15700 \\
0.15782 \\
0.15815 \\
0.15861 \\
0.15937 \\
0.15976\end{array}$ & $\begin{array}{l}0.13054 \\
0.13446 \\
0.13783 \\
0.14081 \\
0.14330 \\
0.14554 \\
0.14742 \\
0.14915\end{array}$ & $\begin{array}{r}21.35 \\
14.00 \\
12.22 \\
10.78 \\
9.39 \\
8.24 \\
7.49 \\
6.64\end{array}$ & $\begin{array}{l}a \\
b \\
a \\
b \\
a \\
b \\
a \\
b\end{array}$ \\
\hline
\end{tabular}


Table 34 (a). Experimental liquid heat capacity data for HCFC-124.

\begin{tabular}{|c|c|c|c|c|c|c|}
\hline $\mathbf{T}$ & $p$ & P & $C_{v, \exp }$ & $C_{v, c a l}$ & $\delta C_{v}, \frac{9}{8}$ & Run \\
\hline $\mathbf{K}$ & mol $\mathrm{dm}^{-3}$ & MPa & & $\operatorname{mol}^{-1} \mathrm{~K}^{-1}$ & & \\
\hline $\begin{array}{l}207.9631 \\
209.9690 \\
212.1001 \\
214.1297 \\
216.2301 \\
218.2530 \\
220.3301 \\
222.3365 \\
224.3897 \\
226.3933 \\
228.4212 \\
230.4057\end{array}$ & $\begin{array}{l}11.9193 \\
11.9145 \\
11.9095 \\
11.9047 \\
11.8997 \\
11.8950 \\
11.8901 \\
11.8854 \\
11.8806 \\
11.8759 \\
11.8712 \\
11.8666\end{array}$ & $\begin{array}{r}5.9118 \\
8.0811 \\
10.3662 \\
12.5276 \\
14.7483 \\
16.8723 \\
19.0377 \\
21.1153 \\
23.2266 \\
25.2733 \\
27.3307 \\
29.3310\end{array}$ & $\begin{array}{l}95.64 \\
92.82 \\
93.05 \\
93.50 \\
93.83 \\
94.03 \\
94.45 \\
94.84 \\
95.30 \\
95.64 \\
96.32 \\
96.29\end{array}$ & $\begin{array}{l}90.44 \\
90.91 \\
91.39 \\
91.84 \\
92.29 \\
92.72 \\
93.16 \\
93.58 \\
94.02 \\
94.44 \\
94.86 \\
95.28\end{array}$ & $\begin{array}{l}5.43 \\
2.05 \\
1.78 \\
1.77 \\
1.64 \\
1.39 \\
1.37 \\
1.33 \\
1.35 \\
1.26 \\
1.51 \\
1.05\end{array}$ & $\begin{array}{l}a \\
b \\
a \\
b \\
a \\
b \\
a \\
b \\
a \\
b \\
a \\
b\end{array}$ \\
\hline
\end{tabular}

Table 34 (b). Experimental liquid heat capacity data for HCFC-124.

\begin{tabular}{|c|c|c|c|c|c|c|}
\hline$T$ & $p$ & P & $C_{v, \exp }$ & $C_{v, c a l}$ & $\delta C_{v}, 8$ & Run \\
\hline${ }^{\circ} \mathbf{F}$ & $I b E t^{-3}$ & psia & Btu & $1 b^{-1} \circ \mathbb{P}^{-1}$ & & \\
\hline $\begin{array}{l}-85.336 \\
-81.726 \\
-77.890 \\
-74.237 \\
-70.456 \\
-66.815 \\
-63.076 \\
-59.464 \\
-55.769 \\
-52.162 \\
-48.512 \\
-44.940\end{array}$ & $\begin{array}{l}101.55 \\
101.51 \\
101.47 \\
101.43 \\
101.38 \\
101.34 \\
101.30 \\
101.26 \\
101.22 \\
101.18 \\
101.14 \\
101.10\end{array}$ & $\begin{array}{r}857.4 \\
1172.1 \\
1503.5 \\
1817.0 \\
2139.1 \\
2447.1 \\
2761.2 \\
3062.5 \\
3368.7 \\
3665.6 \\
3964.0 \\
4254.1\end{array}$ & $\begin{array}{l}0.16749 \\
0.16255 \\
0.16296 \\
0.16374 \\
0.16433 \\
0.16467 \\
0.16542 \\
0.16610 \\
0.16690 \\
0.16750 \\
0.16868 \\
0.16864\end{array}$ & $\begin{array}{l}0.15839 \\
0.15921 \\
0.16006 \\
0.16084 \\
0.16163 \\
0.16239 \\
0.16315 \\
0.16389 \\
0.16465 \\
0.16538 \\
0.16613 \\
0.16687\end{array}$ & $\begin{array}{l}5.43 \\
2.05 \\
1.78 \\
1.77 \\
1.64 \\
1.39 \\
1.37 \\
1.33 \\
1.35 \\
1.26 \\
1.51 \\
1.05\end{array}$ & $\begin{array}{l}a \\
b \\
a \\
b \\
a \\
b \\
a \\
b \\
a \\
b \\
a \\
b\end{array}$ \\
\hline
\end{tabular}


Table 35 (a). Experimental liquid heat capacity data for HCFC-124.

\begin{tabular}{|c|c|c|c|c|c|c|}
\hline \multirow{2}{*}{$\begin{array}{l}\mathrm{T} \\
\mathrm{K}\end{array}$} & $\rho$ & \multirow{2}{*}{$\begin{array}{c}\mathrm{P} \\
\mathrm{MPa}\end{array}$} & $C_{v, \exp }$ & \multicolumn{2}{|c|}{$C_{v, c a l} \delta C_{v}, z$} & \multirow[t]{2}{*}{ Run } \\
\hline & mol $\mathrm{dm}^{-3}$ & & & $\operatorname{mol}^{-1} \mathrm{~K}^{-}$ & & \\
\hline $\begin{array}{l}252.3937 \\
254.3739 \\
256.4831 \\
258.4306 \\
260.5241 \\
262.4502 \\
264.5707 \\
266.4534 \\
268.5793 \\
270.4352 \\
272.5607 \\
274.4003 \\
276.5152 \\
278.3513 \\
280.4525 \\
282.2811\end{array}$ & $\begin{array}{l}11.0418 \\
11.0381 \\
11.0342 \\
11.0307 \\
11.0268 \\
11.0233 \\
11.0195 \\
11.0160 \\
11.0122 \\
11.0088 \\
11.0049 \\
11.0016 \\
10.9978 \\
10.9945 \\
10.9907 \\
10.9874\end{array}$ & $\begin{array}{r}3.3676 \\
4.9289 \\
6.5832 \\
8.1030 \\
9.7284 \\
11.2165 \\
12.8466 \\
14.2869 \\
15.9054 \\
17.3116 \\
18.9144 \\
20.2953 \\
21.8754 \\
23.2409 \\
24.7964 \\
26.1440\end{array}$ & $\begin{array}{r}98.19 \\
98.36 \\
98.13 \\
98.98 \\
99.02 \\
99.61 \\
99.76 \\
100.17 \\
99.87 \\
101.02 \\
100.83 \\
101.81 \\
101.76 \\
102.48 \\
102.21 \\
102.90\end{array}$ & $\begin{array}{r}99.49 \\
99.94 \\
100.42 \\
100.86 \\
101.33 \\
101.76 \\
102.23 \\
102.64 \\
103.10 \\
103.50 \\
103.95 \\
104.34 \\
104.78 \\
105.16 \\
105.59 \\
105.97\end{array}$ & $\begin{array}{l}-1.32 \\
-1.61 \\
-2.34 \\
-1.90 \\
-2.34 \\
-2.16 \\
-2.47 \\
-2.46 \\
-3.24 \\
-2.45 \\
-3.09 \\
-2.49 \\
-2.96 \\
-2.62 \\
-3.31 \\
-2.98\end{array}$ & $\begin{array}{l}a \\
b \\
a \\
b \\
a \\
b \\
a \\
b \\
a \\
b \\
a \\
b \\
a \\
b \\
a \\
b\end{array}$ \\
\hline
\end{tabular}

Table 35 (b). Experimental liquid heat capacity data for HCFC-124.

\begin{tabular}{|c|c|c|c|c|c|c|}
\hline$T$ & $\rho$ & $\mathbf{P}$ & $C_{v, \exp }$ & $\mathrm{C}_{\mathrm{v}, \mathrm{cal}}$ & $\delta C_{v}, 8$ & 8 Run \\
\hline${ }^{\circ} \mathrm{F}$ & $1 b f t^{-3}$ & psia & Btu & $1 b^{-1} \circ \mathbf{F}^{-1}$ & & \\
\hline $\begin{array}{r}-5.361 \\
-1.797 \\
2.000 \\
5.505 \\
9.273 \\
12.740 \\
16.557 \\
19.946 \\
23.773 \\
27.113 \\
30.939 \\
34.250 \\
38.057 \\
41.362 \\
45.145 \\
48.436\end{array}$ & $\begin{array}{l}94.07 \\
94.04 \\
94.01 \\
93.98 \\
93.95 \\
93.92 \\
93.88 \\
93.86 \\
93.82 \\
93.79 \\
93.76 \\
93.73 \\
93.70 \\
93.67 \\
93.64 \\
93.61\end{array}$ & $\begin{array}{r}488.4 \\
714.9 \\
954.8 \\
1175.2 \\
1411.0 \\
1626.8 \\
1863.2 \\
2072.1 \\
2306.9 \\
2510.8 \\
2743.3 \\
2943.6 \\
3172.8 \\
3370.8 \\
3596.4 \\
3791.9\end{array}$ & $\begin{array}{l}0.17196 \\
0.17226 \\
0.17185 \\
0.17335 \\
0.17340 \\
0.17444 \\
0.17471 \\
0.17542 \\
0.17489 \\
0.17692 \\
0.17659 \\
0.17829 \\
0.17821 \\
0.17947 \\
0.17900 \\
0.18020\end{array}$ & $\begin{array}{l}0.17423 \\
0.17503 \\
0.17587 \\
0.17664 \\
0.17746 \\
0.17821 \\
0.17903 \\
0.17975 \\
0.18055 \\
0.18125 \\
0.18204 \\
0.18272 \\
0.18350 \\
0.18417 \\
0.18492 \\
0.18558\end{array}$ & $\begin{array}{l}-1.32 \\
-1.61 \\
-2.34 \\
-1.90 \\
-2.34 \\
-2.16 \\
-2.47 \\
-2.46 \\
-3.24 \\
-2.45 \\
-3.09 \\
-2.49 \\
-2.96 \\
-2.62 \\
-3.31 \\
-2.98\end{array}$ & $\begin{array}{l}a \\
b \\
a \\
b \\
a \\
b \\
a \\
b \\
a \\
b \\
a \\
b \\
a \\
b \\
a \\
b\end{array}$ \\
\hline
\end{tabular}


Table 36 (a). Experimental liquid heat capacity data for HCFC-124.

\begin{tabular}{|c|c|c|c|c|c|c|}
\hline $\bar{T}$ & $\rho$ & $\bar{P}$ & $C_{v, \exp }$ & $C_{v, c a l}$ & $\delta \mathrm{C}_{\mathrm{v}}, 8$ & $\overline{\text { Run }}$ \\
\hline $\mathbf{K}$ & mol $\mathrm{dm}^{-3}$ & MPa & & $\operatorname{mol}^{-1} \mathrm{~K}^{-1}$ & & \\
\hline $\begin{array}{l}283.2097 \\
285.1525 \\
287.2912 \\
289.2119 \\
291.3544 \\
293.2499 \\
295.4023 \\
297.2712 \\
299.4244 \\
301.2852 \\
303.4346 \\
305.2795 \\
307.4236 \\
309.2492 \\
311.4093 \\
313.2210 \\
315.3686 \\
317.1919 \\
319.3270 \\
321.1285 \\
323.2708 \\
325.0592 \\
327.1984\end{array}$ & $\begin{array}{l}10.3636 \\
10.3607 \\
10.3575 \\
10.3546 \\
10.3514 \\
10.3485 \\
10.3453 \\
10.3425 \\
10.3393 \\
10.3365 \\
10.3333 \\
10.3305 \\
10.3273 \\
10.3246 \\
10.3214 \\
10.3187 \\
10.3155 \\
10.3127 \\
10.3096 \\
10.3069 \\
10.3037 \\
10.3010 \\
10.2978\end{array}$ & $\begin{array}{r}2.1384 \\
3.3478 \\
4.6739 \\
5.8607 \\
7.1795 \\
8.3423 \\
9.6579 \\
10.7962 \\
12.1031 \\
13.2288 \\
14.5245 \\
15.6331 \\
16.9170 \\
18.0066 \\
19.2915 \\
20.3657 \\
21.6347 \\
22.7087 \\
23.9621 \\
25.0162 \\
26.2657 \\
27.3054 \\
28.5449\end{array}$ & $\begin{array}{l}102.28 \\
102.35 \\
102.97 \\
103.52 \\
103.63 \\
104.01 \\
104.21 \\
104.96 \\
104.95 \\
106.07 \\
105.46 \\
105.92 \\
106.26 \\
106.31 \\
106.40 \\
107.46 \\
106.81 \\
107.87 \\
107.78 \\
108.13 \\
108.27 \\
109.14 \\
108.81\end{array}$ & $\begin{array}{l}106.13 \\
106.54 \\
106.97 \\
107.36 \\
107.78 \\
108.14 \\
108.55 \\
108.90 \\
109.30 \\
109.64 \\
110.02 \\
110.34 \\
110.71 \\
111.02 \\
111.38 \\
111.68 \\
112.03 \\
112.33 \\
112.66 \\
112.95 \\
113.27 \\
113.55 \\
113.87\end{array}$ & $\begin{array}{l}-3.77 \\
-4.10 \\
-3.88 \\
-3.71 \\
-4.01 \\
-3.97 \\
-4.17 \\
-3.76 \\
-4.14 \\
-3.36 \\
-4.32 \\
-4.17 \\
.4 .19 \\
-4.43 \\
-4.69 \\
-3.93 \\
-4.89 \\
-4.13 \\
-4.53 \\
-4.45 \\
-4.62 \\
-4.04 \\
-4.65\end{array}$ & $\begin{array}{l}a \\
b \\
a \\
b \\
a \\
b \\
a \\
b \\
a \\
b \\
a \\
b \\
a \\
b \\
a \\
b \\
a \\
b \\
a \\
b \\
a \\
b \\
a\end{array}$ \\
\hline
\end{tabular}

Table 36 (b). Experimental liquid heat capacity data for HCFC-124.

\begin{tabular}{|c|c|c|c|c|c|c|}
\hline$T$ & $\rho$ & $\mathbf{p}$ & $C_{v, \exp }$ & $\overline{C_{v, c a l}}$ & $\delta C_{v}, g$ & $\overline{R u n}$ \\
\hline${ }^{\circ} \mathrm{g}$ & Ib $E t^{-3}$ & poia & Btu & $1 b^{-1} \circ E^{-1}$ & & \\
\hline $\begin{array}{r}50.107 \\
53.604 \\
57.454 \\
60.912 \\
64.763 \\
68.180 \\
72.054 \\
75.418 \\
79.294 \\
82.643 \\
86.512 \\
89.833 \\
93.693 \\
96.979 \\
100.867 \\
104.128 \\
107.993 \\
111.275 \\
115.119 \\
118.361 \\
122.218 \\
125.437 \\
129.287\end{array}$ & $\begin{array}{l}88.30 \\
88.27 \\
88.24 \\
88.22 \\
88.19 \\
88.17 \\
88.14 \\
88.12 \\
88.09 \\
88.07 \\
88.04 \\
88.01 \\
87.99 \\
87.96 \\
87.94 \\
87.91 \\
87.89 \\
87.86 \\
87.84 \\
87.81 \\
87.79 \\
87.76 \\
87.74\end{array}$ & $\begin{array}{r}310.2 \\
485.6 \\
677.9 \\
850.0 \\
1041.3 \\
1210.0 \\
1400.8 \\
1565.9 \\
1755.4 \\
1918.7 \\
2106.6 \\
2267.4 \\
2453.6 \\
2611.6 \\
2798.0 \\
2953.8 \\
3137.9 \\
3293.6 \\
3475.4 \\
3628.3 \\
3809.5 \\
3960.3 \\
4140.1\end{array}$ & $\begin{array}{l}0.17911 \\
0.17924 \\
0.18034 \\
0.18129 \\
0.18148 \\
0.18216 \\
0.18251 \\
0.18381 \\
0.18380 \\
0.18575 \\
0.18469 \\
0.18550 \\
0.18609 \\
0.18618 \\
0.18633 \\
0.18820 \\
0.18706 \\
0.18891 \\
0.18876 \\
0.18937 \\
0.18962 \\
0.19113 \\
0.19055\end{array}$ & $\begin{array}{l}0.18587 \\
0.18658 \\
0.18734 \\
0.18801 \\
0.18875 \\
0.18939 \\
0.19011 \\
0.19072 \\
0.19141 \\
0.19200 \\
0.19267 \\
0.19324 \\
0.19389 \\
0.19443 \\
0.19507 \\
0.19559 \\
0.19620 \\
0.19672 \\
0.19731 \\
0.19780 \\
0.19838 \\
0.19885 \\
0.19941\end{array}$ & $\begin{array}{l}-3.77 \\
-4.10 \\
-3.88 \\
-3.71 \\
-4.01 \\
-3.97 \\
-4.17 \\
-3.76 \\
-4.14 \\
-3.36 \\
-4.32 \\
-4.17 \\
-4.19 \\
-4.43 \\
-4.69 \\
-3.93 \\
-4.89 \\
-4.13 \\
-4.53 \\
-4.45 \\
-4.62 \\
-4.04 \\
-4.65\end{array}$ & $\begin{array}{l}a \\
b \\
a \\
b \\
a \\
b \\
a \\
b \\
a \\
b \\
a \\
b \\
a \\
b \\
a \\
b \\
a \\
b \\
a \\
b \\
a \\
b \\
a\end{array}$ \\
\hline
\end{tabular}


Table 37 (a). Experimental liquid heat capacity data for HCFC-124.

\begin{tabular}{|c|c|c|c|c|c|c|}
\hline$T$ & $p$ & P & $C_{v, \exp }$ & $C_{v, c a l}$ & $\delta C_{v},{ }^{\circ}$ & $\overline{R u n}$ \\
\hline $\mathbf{K}$ & mol $\mathrm{dm}^{-3}$ & MPa & & $\operatorname{mol}^{-1} \mathrm{~K}^{-1}$ & & \\
\hline $\begin{array}{l}312.0861 \\
314.2179 \\
316.2227 \\
318.3765 \\
320.3513 \\
322.5148 \\
324.4604 \\
326.6482 \\
328.5609 \\
330.7650 \\
332.6574 \\
334.8760 \\
336.7578 \\
338.9813 \\
340.8491\end{array}$ & $\begin{array}{l}9.6520 \\
9.6494 \\
9.6470 \\
9.6443 \\
9.6419 \\
9.6393 \\
9.6371 \\
9.6343 \\
9.6319 \\
9.6293 \\
9.6270 \\
9.6243 \\
9.6220 \\
9.6193 \\
9.6170\end{array}$ & $\begin{array}{r}1.9472 \\
2.9748 \\
3.9394 \\
4.9736 \\
5.9201 \\
6.9551 \\
7.8841 \\
8.9268 \\
9.8367 \\
10.8832 \\
11.7800 \\
12.8295 \\
13.7179 \\
14.7657 \\
15.6441\end{array}$ & $\begin{array}{l}106.79 \\
107.24 \\
107.61 \\
107.62 \\
107.92 \\
107.91 \\
108.19 \\
108.76 \\
108.98 \\
109.06 \\
109.75 \\
109.31 \\
109.90 \\
110.04 \\
110.47\end{array}$ & $\begin{array}{l}112.14 \\
112.49 \\
112.80 \\
113.13 \\
113.43 \\
113.75 \\
114.03 \\
114.34 \\
114.61 \\
114.91 \\
115.16 \\
115.45 \\
115.69 \\
115.97 \\
116.20\end{array}$ & $\begin{array}{l}-5.01 \\
-4.89 \\
-4.83 \\
-5.12 \\
-5.11 \\
-5.41 \\
-5.40 \\
-5.13 \\
-5.17 \\
-5.36 \\
-4.93 \\
-5.61 \\
-5.27 \\
-5.39 \\
-5.18\end{array}$ & $\begin{array}{l}a \\
b \\
a \\
b \\
a \\
b \\
a \\
b \\
a \\
b \\
a \\
b \\
a \\
b \\
a\end{array}$ \\
\hline
\end{tabular}

Table $37(b)$. Experimental liquid heat capacity data for HCFC-124.

\begin{tabular}{|c|c|c|c|c|c|c|}
\hline $\mathbf{T}$ & $\rho$ & P & $C_{v, \exp }$ & $C_{v, c a l}$ & $\delta C_{v}, q$ & Run \\
\hline${ }^{\circ} \mathrm{F}$ & $1 b f t^{-3}$ & psia & Btu & $1 b^{-1} \circ \mathfrak{F}^{-1}$ & & \\
\hline $\begin{array}{l}102.085 \\
105.922 \\
109.531 \\
113.408 \\
116.962 \\
120.857 \\
124.359 \\
128.297 \\
131.740 \\
135.707 \\
139.113 \\
143.107 \\
146.494 \\
150.496 \\
153.858\end{array}$ & $\begin{array}{l}82.23 \\
82.21 \\
82.19 \\
82.17 \\
82.15 \\
82.13 \\
82.11 \\
82.08 \\
82.06 \\
82.04 \\
82.02 \\
82.00 \\
81.98 \\
81.96 \\
81.94\end{array}$ & $\begin{array}{r}282.4 \\
431.5 \\
571.4 \\
721.4 \\
858.6 \\
1008.8 \\
1143.5 \\
1294.7 \\
1426.7 \\
1578.5 \\
1708.6 \\
1860.8 \\
1989.6 \\
2141.6 \\
2269.0\end{array}$ & $\begin{array}{l}0.18701 \\
0.18780 \\
0.18845 \\
0.18847 \\
0.18900 \\
0.18898 \\
0.18948 \\
0.19048 \\
0.19085 \\
0.19100 \\
0.19220 \\
0.19144 \\
0.19246 \\
0.19271 \\
0.19347\end{array}$ & $\begin{array}{l}0.19639 \\
0.19699 \\
0.19755 \\
0.19813 \\
0.19865 \\
0.19921 \\
0.19970 \\
0.20025 \\
0.20071 \\
0.20123 \\
0.20168 \\
0.20218 \\
0.20260 \\
0.20309 \\
0.20350\end{array}$ & $\begin{array}{l}-5.01 \\
-4.89 \\
-4.83 \\
-5.12 \\
-5.11 \\
-5.41 \\
-5.40 \\
-5.13 \\
-5.17 \\
-5.36 \\
-4.93 \\
-5.61 \\
-5.27 \\
-5.39 \\
-5.18\end{array}$ & $\begin{array}{l}a \\
b \\
a \\
b \\
a \\
b \\
a \\
b \\
a \\
b \\
a \\
b \\
a \\
b \\
a\end{array}$ \\
\hline
\end{tabular}


Table 38 (a). Experimental two-phase heat capacity data for HCFC-124.

\begin{tabular}{|c|c|c|c|c|c|c|c|c|c|c|}
\hline \multirow[t]{2}{*}{$\mathbf{T}$} & $\delta \mathrm{T}$ & $\rho_{\sigma}$ & \multirow{2}{*}{$\begin{array}{l}P_{\sigma} \\
M P_{a}\end{array}$} & \multirow[t]{2}{*}{$c_{v}^{(2)}$} & $\delta C_{v}^{(2)}$ & \multicolumn{2}{|c|}{$\delta C_{v}^{(2)}, \delta$} & \multicolumn{2}{|c|}{$\delta \mathrm{C}_{\sigma}$} & \multirow[t]{2}{*}{$C_{\sigma}, \frac{8}{\delta}$} \\
\hline & & mol $\mathrm{dm}^{-3}$ & & & & J mo & $-1 \mathbf{k}^{-1}$ & & & \\
\hline $\begin{array}{l}94.2808 \\
97.8905 \\
99.6888 \\
103.2706 \\
105.0349 \\
108.5510 \\
110.2904 \\
113.7544 \\
115.4658 \\
118.8756 \\
120.4597 \\
120.5655 \\
123.9281 \\
125.4934 \\
128.9121 \\
130.4609 \\
133.0919 \\
133.8383 \\
135.3654 \\
137.9605 \\
138.7074 \\
140.2086 \\
142.7737 \\
143.5208 \\
144.9941 \\
147.5313 \\
148.2833 \\
149.7346 \\
152.2464 \\
152.9943 \\
154.4289 \\
156.9203 \\
157.6600 \\
159.0738 \\
161.5419 \\
162.2811 \\
163.6821 \\
166.1243 \\
166.8549 \\
168.2348 \\
170.6671 \\
171.3904 \\
172.7523 \\
175.1654 \\
175.8344 \\
177.2414 \\
179.6156 \\
180.2819 \\
181.6819 \\
184.0320 \\
184.7027 \\
188.4135 \\
192.0790 \\
197.0754\end{array}$ & $\begin{array}{l}\quad 0.0031 \\
0.0035 \\
0.0030 \\
0.0034 \\
0.0030 \\
0.003 \\
0.002 \\
0.003 \\
0.003 \\
0.0029 \\
0.003 \\
0.002 \\
0.002\end{array}$ & 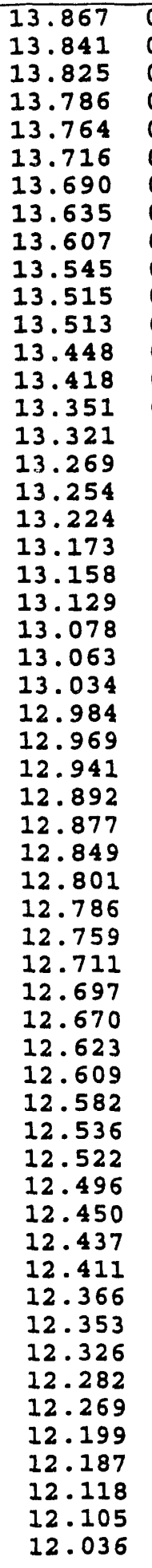 & 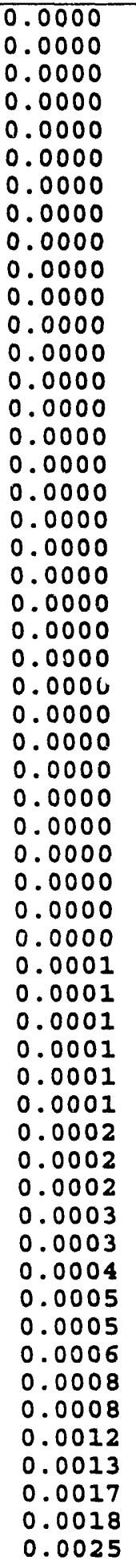 & $\begin{array}{l}126.79 \\
126.21 \\
127.15 \\
127.00 \\
127.26 \\
127.37 \\
127.12 \\
127.34 \\
127.32 \\
127.30 \\
127.17 \\
127.42 \\
127.21 \\
127.22 \\
127.21 \\
127.31 \\
127.67 \\
127.56 \\
127.56 \\
127.64 \\
127.79 \\
127.70 \\
127.87 \\
127.93 \\
127.60 \\
128.19 \\
128.27 \\
127.91 \\
128.13 \\
128.49 \\
128.20 \\
128.23 \\
128.97 \\
128.95 \\
128.88 \\
129.06 \\
129.21 \\
129.69 \\
129.62 \\
129.54 \\
130.26 \\
130.14 \\
130.20 \\
130.67 \\
130.38 \\
130.43 \\
131.17 \\
130.91 \\
130.92 \\
131.52 \\
131.33 \\
132.04 \\
132.22 \\
132.80 \\
132.76 \\
133.54\end{array}$ & 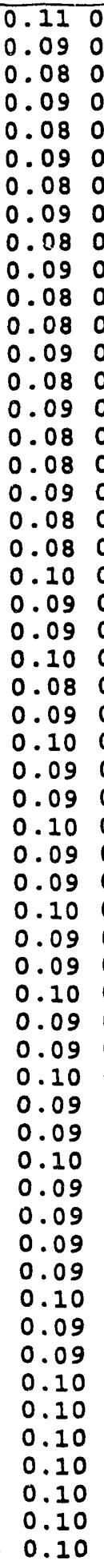 & $\begin{array}{l}\quad 0.09 \\
0.07 \\
0.06 \\
0.07 \\
0.06 \\
0.06 \\
0.07 \\
0.06 \\
0.07\end{array}$ & $\begin{array}{l}126.79 \\
126.21 \\
127.15 \\
127.00 \\
127.26 \\
127.37 \\
127.12 \\
127.34 \\
127.32 \\
127.30 \\
127.17 \\
127.42 \\
127.21 \\
127.22 \\
127.21 \\
127.31 \\
127.67 \\
127.56 \\
127.56 \\
127.64 \\
127.79 \\
127.70 \\
127.87 \\
127.93 \\
127.60 \\
128.19 \\
128.26 \\
127.91 \\
128.12 \\
128.49 \\
128.20 \\
128.23 \\
128.97 \\
128.95 \\
128.87 \\
129.06 \\
13131.29 \\
132.13 \\
132.72 \\
132.69 \\
133.45 \\
129.68 \\
129.61 \\
129.53 \\
130.25 \\
130.12 \\
130.18 \\
130.65 \\
130.36 \\
130.40 \\
131.14 \\
130.88 \\
130.89 \\
131.48\end{array}$ & 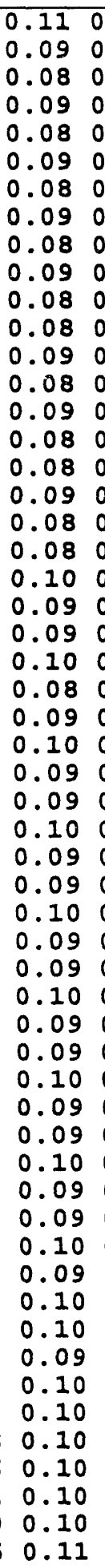 & $\begin{array}{l}\quad 0.09 \\
0.07 \\
0.06 \\
0.07 \\
0.06 \\
0.07 \\
0.06 \\
0.07 \\
0.07 \\
0.06 \\
0.07\end{array}$ & $\begin{array}{l}e \\
a \\
e \\
a \\
e \\
a \\
e \\
a \\
e \\
a \\
d \\
e \\
a \\
d \\
a \\
d \\
e \\
a \\
d \\
e \\
a \\
d \\
e \\
a \\
d \\
e \\
a \\
d \\
e \\
a \\
d \\
e \\
a \\
d \\
e \\
a \\
d \\
d \\
d \\
e \\
e \\
a \\
d \\
d \\
e \\
a \\
d \\
e \\
b \\
d \\
d \\
d\end{array}$ \\
\hline
\end{tabular}




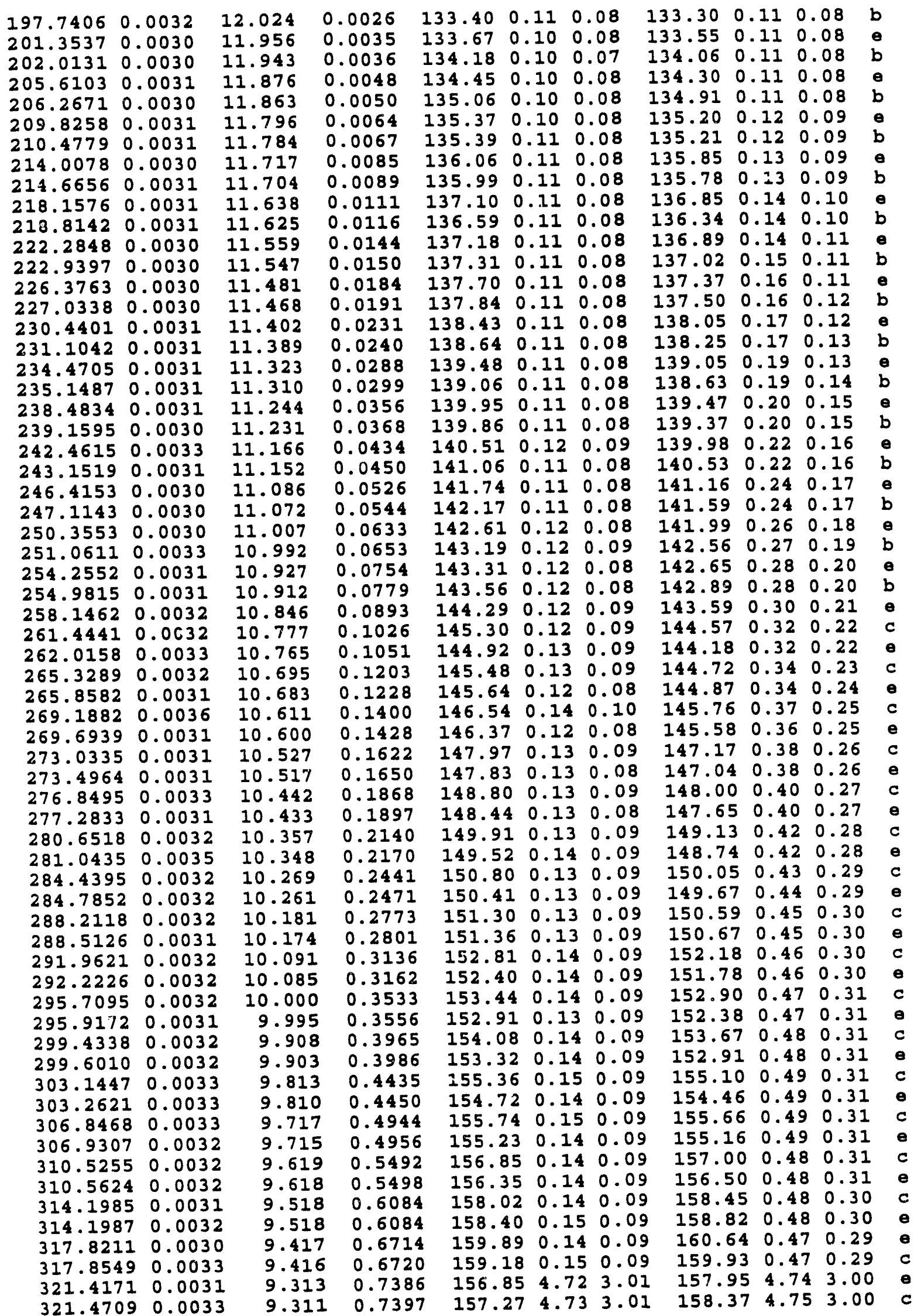




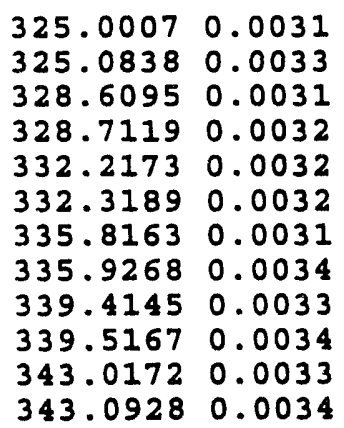

$\begin{array}{lllll}9.207 & 0.8105 & 162.74 & 0.16 & 0.10 \\ 9.204 & 0.8123 & 162.34 & 0.16 & 0.10 \\ 9.096 & 0.8881 & 164.18 & 0.16 & 0.10 \\ 9.093 & 0.8904 & 162.64 & 0.16 & 0.10 \\ 8.982 & 0.9711 & 164.69 & 0.16 & 0.10 \\ 8.979 & 0.9736 & 163.69 & 0.16 & 0.10 \\ 8.865 & 1.0596 & 165.01 & 0.16 & 0.10 \\ 8.861 & 1.0625 & 164.69 & 0.17 & 0.10 \\ 8.743 & 1.1540 & 166.56 & 0.17 & 0.10 \\ 8.739 & 1.1568 & 165.55 & 0.17 & 0.11 \\ 8.616 & 1.2547 & 167.56 & 0.17 & 0.10 \\ 8.613 & 1.2569 & 166.12 & 0.18 & 0.11\end{array}$

$\begin{array}{llll}164.29 & 0.45 & 0.27\end{array}$

$\begin{array}{llll}163.90 & 0.45 & 0.27\end{array}$

$166.250 .430 .26 \quad$ e

$164.740 .430 .26 \quad \mathrm{C}$

$167.40 \quad 0.410 .24$ e

$\begin{array}{lllll}166.41 & 0.41 & 0.24 \quad c\end{array}$

$\begin{array}{llll}168.45 & 0.39 & 0.23 & 0\end{array}$

$\begin{array}{lllll}168.15 & 0.39 & 0.23 \quad \mathrm{c}\end{array}$

$\begin{array}{llll}170.86 & 0.38 & 0.22 & \text { e }\end{array}$

$\begin{array}{lllll}169.87 & 0.38 & 0.23 \quad \mathrm{c}\end{array}$

$\begin{array}{lllll}172.89 & 0.39 & 0.23\end{array}$

343.09280 .0034

$\begin{array}{llll}171.48 & 0.40 & 0.23\end{array}$ 
Table 38 (b). Experimental two-phase heat capacity data for HCFC-124.

\begin{tabular}{|c|c|c|c|c|c|c|c|c|c|c|}
\hline $\mathbf{T}$ & $\delta \mathrm{T}$ & $\rho_{\sigma}$ & \multirow{2}{*}{$\begin{array}{l}P_{\sigma} \\
\text { psia }\end{array}$} & $C_{v}^{(2)}$ & $\delta C_{v}^{(2)}$ & $\delta C_{v}^{(2)}$ & $\mathrm{C}_{\sigma}$ & $\delta C_{\sigma}$ & \multicolumn{2}{|c|}{$\delta C_{\sigma}, \%$} \\
\hline${ }^{\circ} \mathbf{F}$ & & $I b f t^{-3}$ & & & & Btu $1 b$ & $-1 \circ F^{-1}$ & & & \\
\hline 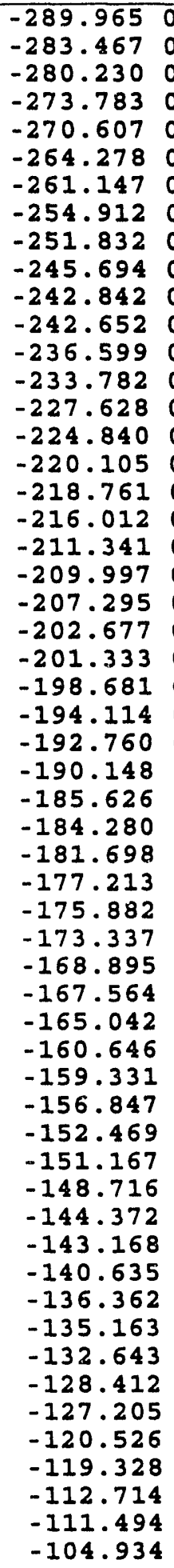 & 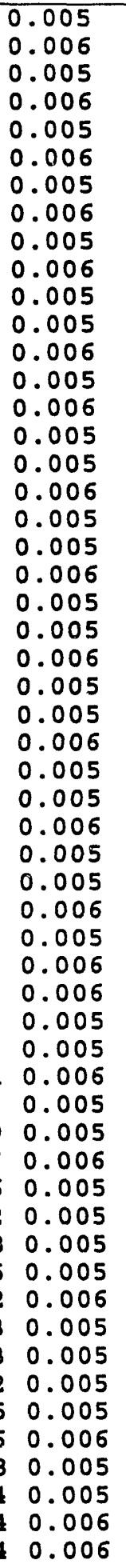 & 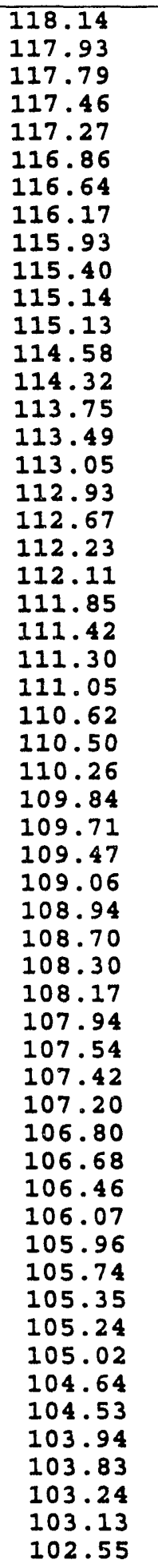 & $\begin{array}{l}\quad 0.00 \\
0.00 \\
0.00 \\
0.00 \\
0.00 \\
0.00 \\
0.00 \\
0.00 \\
0.00 \\
0.00 \\
0.00\end{array}$ & $\begin{array}{l}0.22205 \\
0.22103 \\
0.22268 \\
0.22240 \\
0.22287 \\
0.22306 \\
0.22263 \\
0.22301 \\
0.22296 \\
0.22293 \\
0.22271 \\
0.22315 \\
0.22279 \\
0.22279 \\
0.22277 \\
0.22296 \\
0.22359 \\
0.22340 \\
0.22339 \\
0.22353 \\
0.22380 \\
0.22364 \\
0.22394 \\
0.22404 \\
0.22346 \\
0.22450 \\
0.22463 \\
0.22400 \\
0.22439 \\
0.22503 \\
0.22451 \\
0.22457 \\
0.22587 \\
0.22583 \\
0.22570 \\
0.22602 \\
0.22628 \\
0.22713 \\
0.22790 \\
0.22280 \\
0.2283 \\
0.222834 \\
0.22926 \\
0.22928 \\
0.23033 \\
0.23000 \\
0.23124 \\
0.23155 \\
0.23256 \\
0.23251 \\
0.23387\end{array}$ & $\begin{array}{l}0.00019 \\
0.00015 \\
0.00014 \\
0.00015 \\
0.00013 \\
0.00015 \\
0.00014 \\
0.00016 \\
0.00014 \\
0.00016 \\
0.00014 \\
0.00014 \\
0.00016 \\
0.00014 \\
0.00016 \\
0.00014 \\
0.00015 \\
0.00017 \\
0.00015 \\
0.00015 \\
0.00017 \\
0.00015 \\
0.00015 \\
0.00017 \\
0.00015 \\
0.00015 \\
0.00017 \\
0.00015 \\
0.00015 \\
0.00017 \\
0.00016 \\
0.00016 \\
0.00018 \\
0.00016 \\
0.00016 \\
0.00018 \\
0.00016 \\
0.00016 \\
0.00018 \\
0.00016 \\
0.000016 \\
0.000018 \\
0.000016 \\
0.000018 \\
0.00016 \\
0.00017 \\
0.00016 \\
0.00017 \\
0.00017 \\
0.00016 \\
0.00017 \\
0.00017 \\
0.00017 \\
0.00017\end{array}$ & $\begin{array}{l}0.09 \\
0.07 \\
0.06 \\
0.07 \\
0.06 \\
0.07 \\
0.06 \\
0.07 \\
0.06 \\
0.07 \\
0.06 \\
0.06 \\
0.07 \\
0.06 \\
0.07 \\
0.06 \\
0.07 \\
0.07 \\
0.07 \\
0.07 \\
0.08 \\
0.07 \\
0.07 \\
0.08 \\
0.07 \\
0.07 \\
0.08 \\
0.07 \\
0.07 \\
0.08 \\
0.07 \\
0.07 \\
0.08 \\
0.07 \\
0.07 \\
0.08 \\
0.07 \\
0.07 \\
0.08 \\
0.07 \\
0.07 \\
0.08 \\
0.07 \\
0.07 \\
0.07 \\
0.07 \\
0.07 \\
0.07 \\
0.07 \\
0.07 \\
0.07 \\
0.07 \\
0.07 \\
0.07 \\
0.08 \\
0.08\end{array}$ & 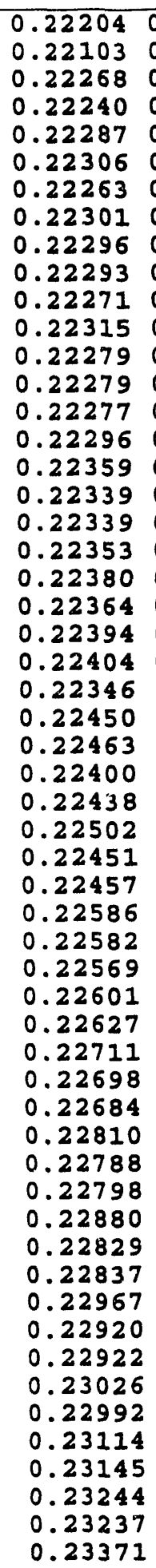 & $\begin{array}{l}0.00019 \\
0.00015 \\
0.00014 \\
0.00015 \\
0.00013 \\
0.00015 \\
0.00014 \\
0.00016 \\
0.00014 \\
0.00016 \\
0.00014 \\
0.00014 \\
0.00016 \\
0.00014 \\
0.00016 \\
0.00014 \\
0.00015 \\
0.00017 \\
0.00015 \\
0.00015 \\
0.00017 \\
0.00015 \\
0.00015 \\
0.00017 \\
0.00015 \\
0.00015 \\
0.00017 \\
0.00015 \\
0.00015 \\
0.00017 \\
0.00016 \\
0.00016 \\
0.00018 \\
0.00016 \\
0.00016 \\
0.00018 \\
0.00016 \\
0.000016 \\
0.00018 \\
0.00016 \\
0.00016 \\
0.00018 \\
0.000016 \\
0.0000018 \\
0.000016 \\
0.000016 \\
0.00017 \\
0.00016 \\
0.00017 \\
0.00017 \\
0.00017 \\
0.00017 \\
0.00017 \\
0.00017\end{array}$ & $\begin{array}{l}\quad 0.09 \\
0.07 \\
0.06 \\
0.07 \\
0.06 \\
0.06 \\
0.07\end{array}$ & 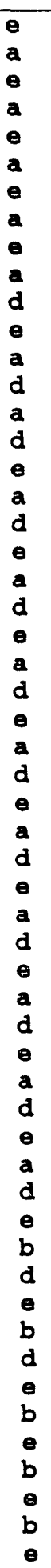 \\
\hline
\end{tabular}




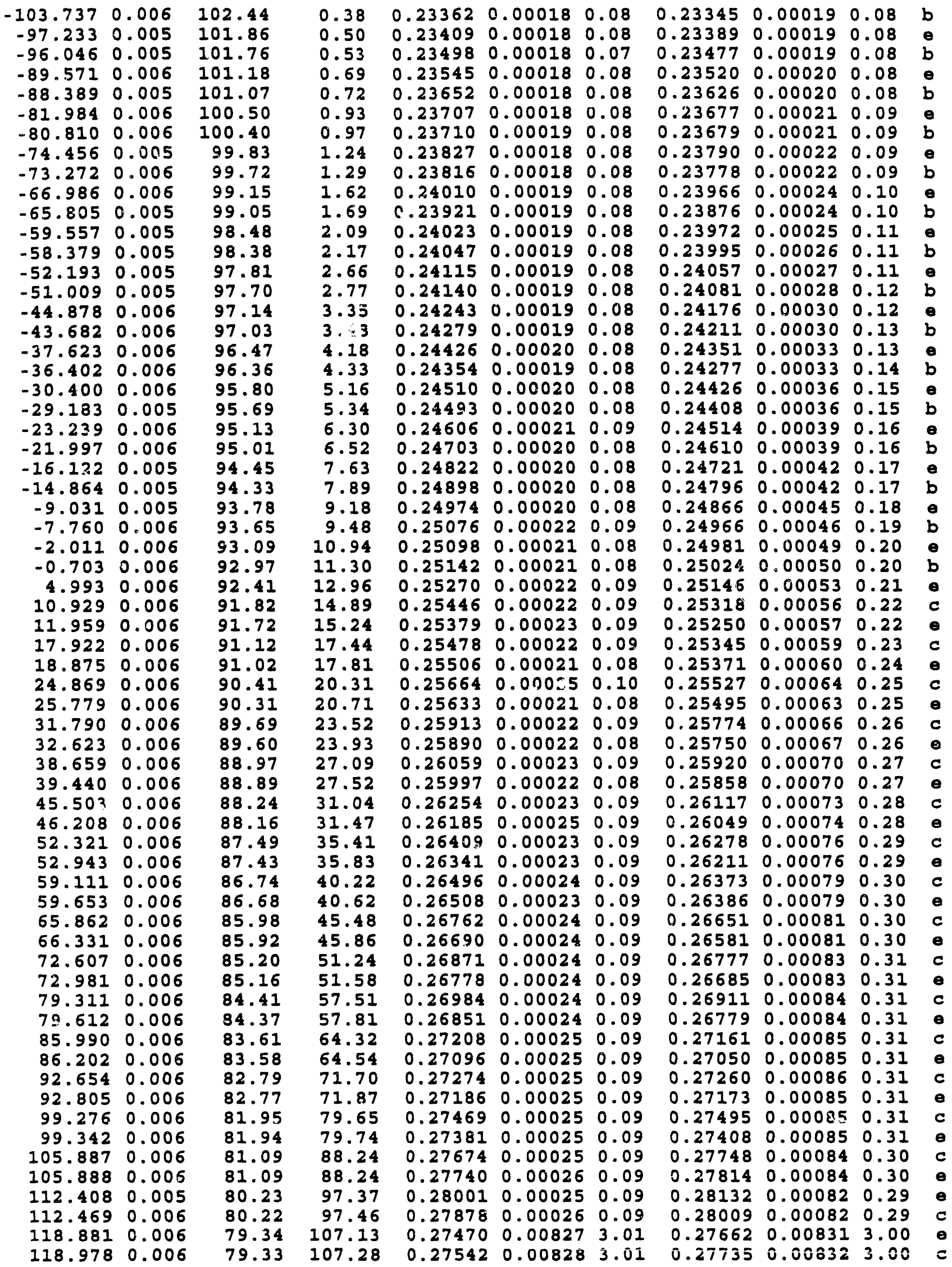


$78.44 \quad 117.56$

$78.42 \quad 117.81$

$77.50 \quad 128.81$

$77.47 \quad 129.14$

$76.53 \quad 140.85$

$76.50 \quad 141.20$

75.53

75.49

74.49

74.46

73.41

73.38

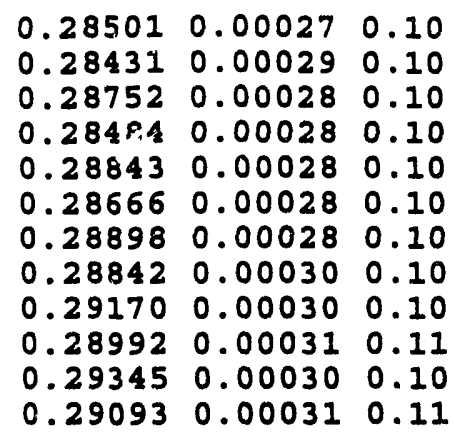

$0.287720 .00078 \quad 0.27 \quad$

$0.28703 \quad 0.000790 .27 \quad c$

0.291150 .000750 .26 e

$0.288500 .000750 .26 \quad c$

$0.293160 .000710 .24 \quad$

$0.291420 .000710 .24 \quad c$

0.295000 .000680 .23 e

$\begin{array}{llllll}0.29448 & 0.00069 & 0.23 \mathrm{c}\end{array}$

0.299230 .000670 .22 e

$\begin{array}{llllll}0.29750 & 0.00067 & 0.23 \quad c\end{array}$

0.302780 .000690 .23

$0.300300 .000700 .23 \mathrm{c}$ 
Table 39. Coefficients to the MBWR equation of state for HCFC-124 (units are K, bar, L, mol)

$$
p=\sum_{n=1}^{9} a_{n} \rho^{n}+\exp \left(-\rho^{2} / \rho_{c}^{2}\right) \sum_{n=10}^{15} a_{n} \rho^{2 n-17}
$$

$$
\begin{array}{ll}
\rho_{c}=4.10153 \mathrm{~mol} / \mathrm{L} \\
\mathrm{a}_{1}=\mathrm{RT} \\
\mathrm{a}_{2}=\mathrm{b}_{1} \mathrm{~T}+\mathrm{b}_{2} \mathrm{~T}^{0.5}+\mathrm{b}_{3}+\mathrm{b}_{4} / \mathrm{T}+\mathrm{b}_{5} / \mathrm{T}^{2} \\
\mathrm{a}_{3}=\mathrm{b}_{6} \mathrm{~T}+\mathrm{b}_{7}+\mathrm{b}_{8} / \mathrm{T}+\mathrm{b}_{9} / \mathrm{T}^{2} \\
\mathrm{a}_{4}=\mathrm{b}_{10} \mathrm{~T}+\mathrm{b}_{11}+\mathrm{b}_{12} / \mathrm{T} \\
\mathrm{a}_{5}=\mathrm{b}_{13} \\
\mathrm{a}_{6}=\mathrm{b}_{14} / \mathrm{T}+\mathrm{b}_{15} / \mathrm{T}^{2} \\
\mathrm{a}_{7}=\mathrm{b}_{16} / \mathrm{T} \\
\mathrm{a}_{8}=\mathrm{b}_{17} / \mathrm{T}+\mathrm{b}_{18} / \mathrm{T}^{2} \\
\mathrm{a}_{9}=\mathrm{b}_{19} / \mathrm{T}^{2} \\
\mathrm{a}_{10}=\mathrm{b}_{20} / \mathrm{T}^{2}+\mathrm{b}_{21} / \mathrm{T}^{3} \\
\mathrm{a}_{11}=\mathrm{b}_{22} / \mathrm{T}^{2}+\mathrm{b}_{23} / \mathrm{T}^{4} \\
\mathrm{a}_{12}=\mathrm{b}_{24} / \mathrm{T}^{2}+\mathrm{b}_{25} / \mathrm{T}^{3} \\
\mathrm{a}_{13}=\mathrm{b}_{26} / \mathrm{T}^{2}+\mathrm{b}_{27} / \mathrm{T}^{4} \\
\mathrm{a}_{14}=\mathrm{b}_{28} / \mathrm{T}^{2}+\mathrm{b}_{29} / \mathrm{T}^{3} \\
\mathrm{a}_{15}=\mathrm{b}_{30} / \mathrm{T}^{2}+\mathrm{b}_{31} / \mathrm{T}^{3}+\mathrm{b}_{32} / \mathrm{T}^{4}
\end{array}
$$

i $\quad b_{i}$

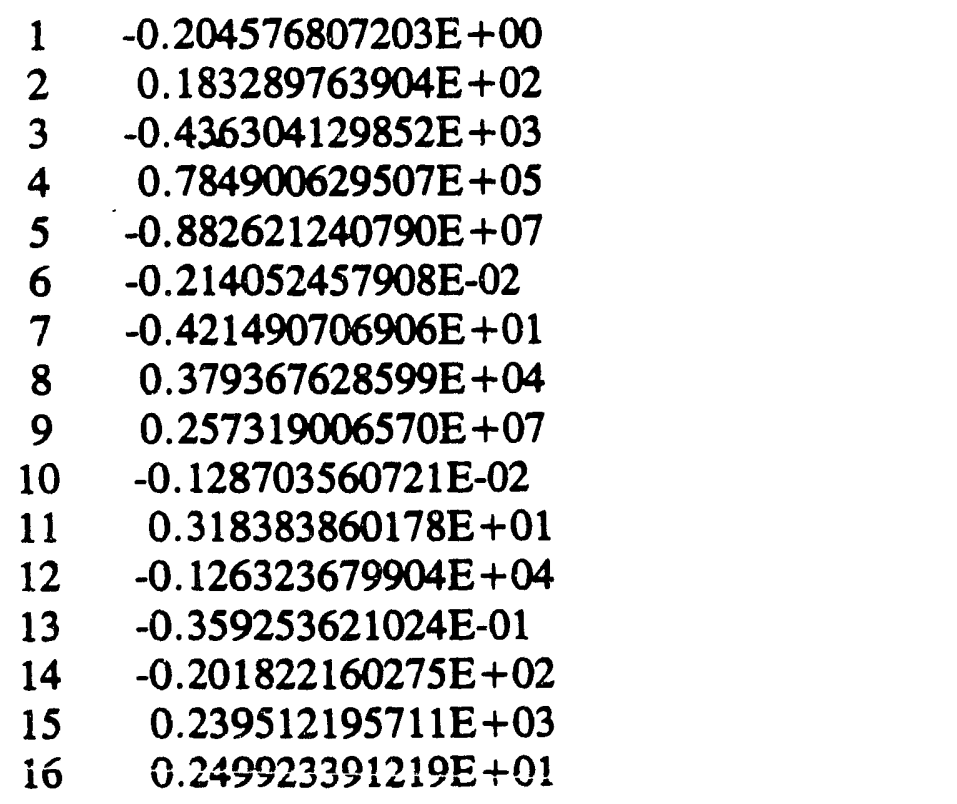


Table 39. Coefficients to the MBWR equation of state for HCFC-124 (units are K, bar, L, mol) (continued)
$17-0.688566863825 \mathrm{E}-01$
$18-0.132391812938 \mathrm{E}+02$
$19 \quad 0.667600131841 \mathrm{E}+00$
$20-0.271799858829 \mathrm{E}+07$
$21-0.111422740208 \mathrm{E}+09$
$22-0.175854504297 \mathrm{E}+06$
$23 \quad 0.566801130630 \mathrm{E}+10$
$24-0.214018815397 \mathrm{E}+04$
$25-0.327561948065 \mathrm{E}+06$
$26-0.546930696467 \mathrm{E}+02$
$27 \quad 0.931832376640 \mathrm{E}+06$
$28 \quad 0.193654970621 \mathrm{E}-02$
$29-0.110844683745 \mathrm{E}+03$
$30 \quad-0.452370482664 \mathrm{E}-02$
$31 \quad 0.163031126242 \mathrm{E}+01$
$32-0.681395650661 \mathrm{E}+03$ 


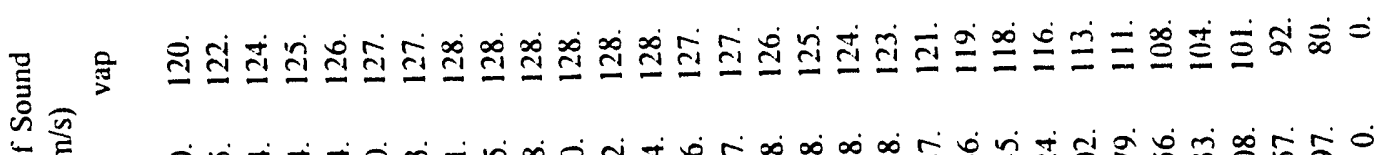

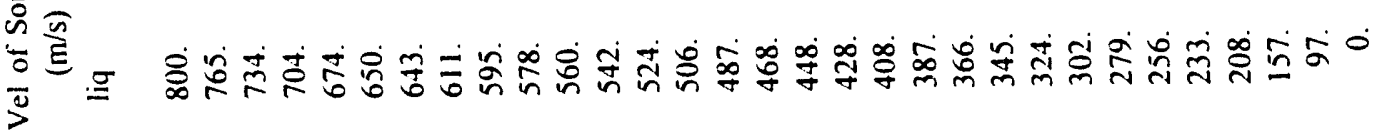

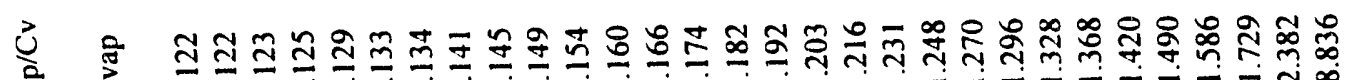

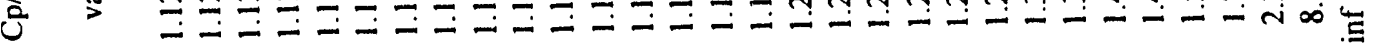

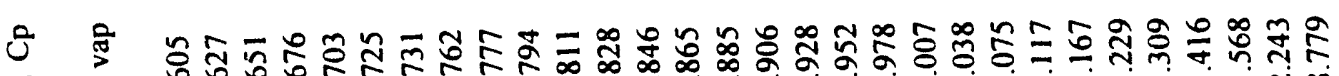

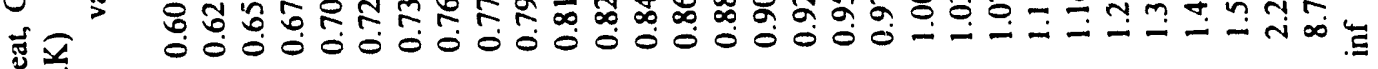

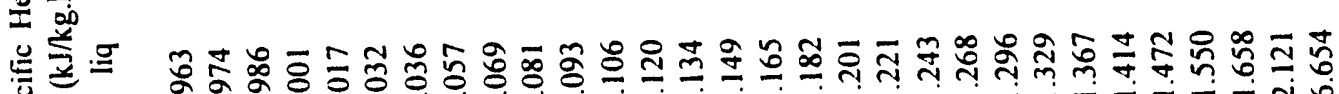

峞

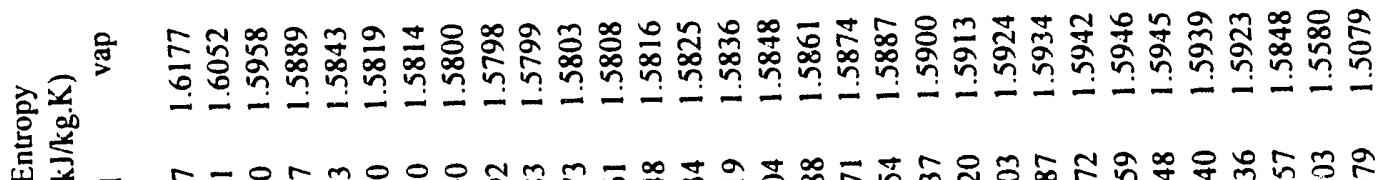

亲回

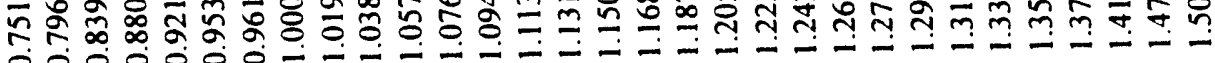

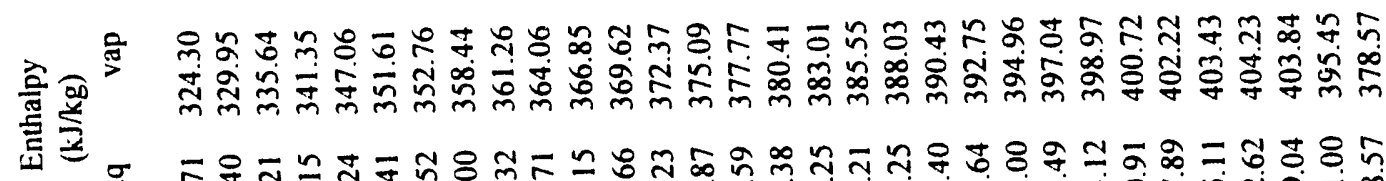

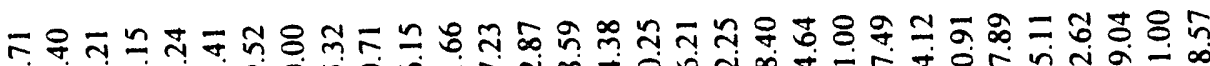

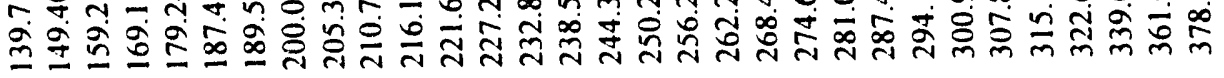

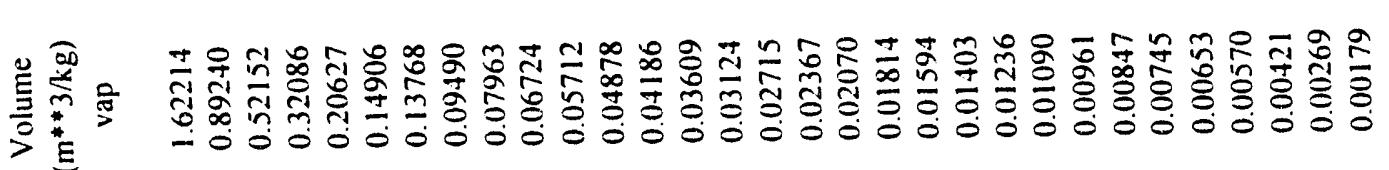

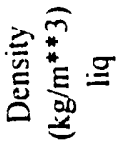

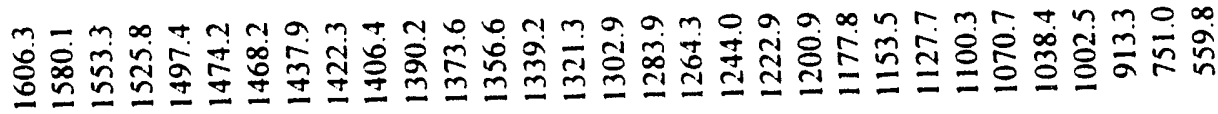

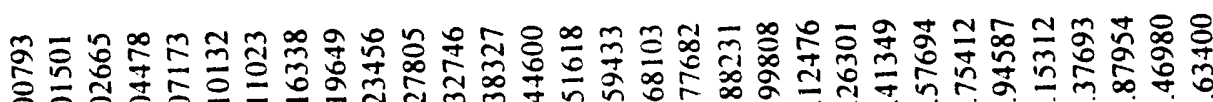

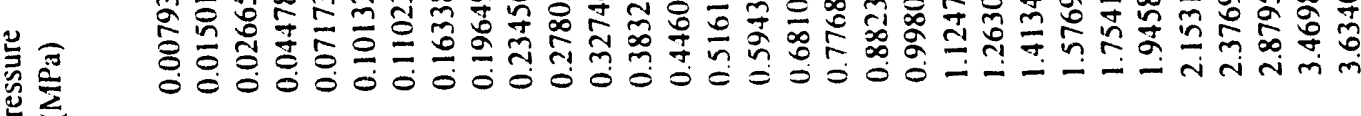
-

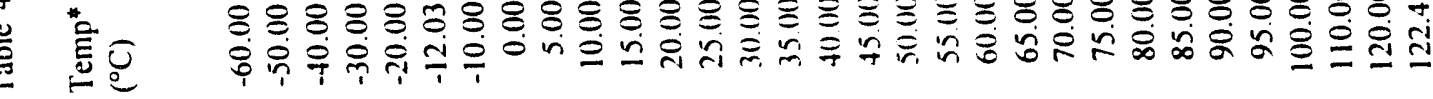




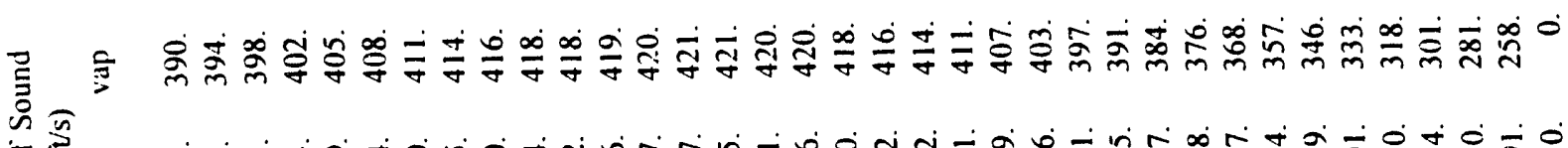

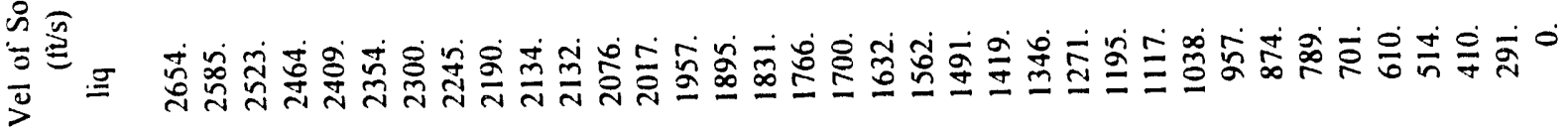

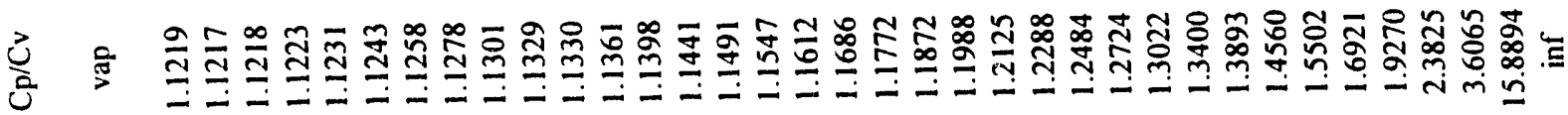

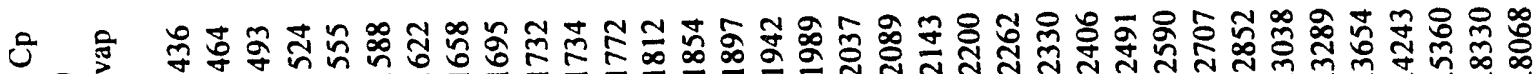

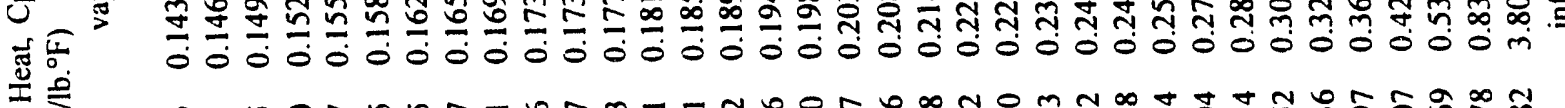

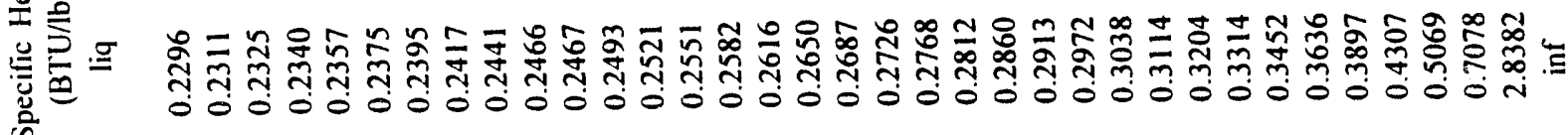

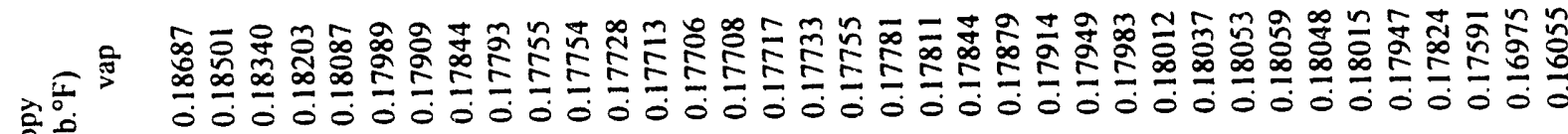

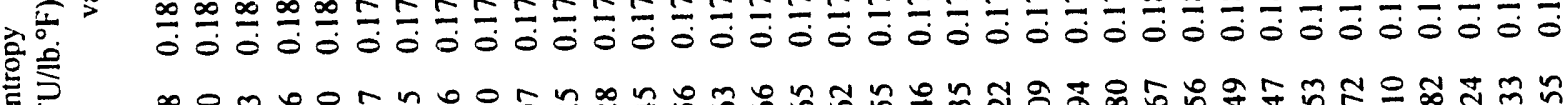

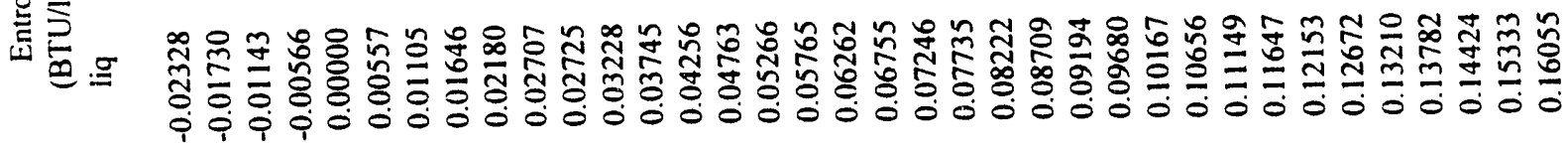

n

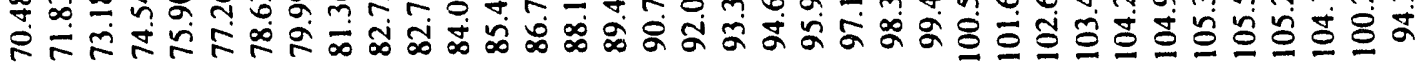

总事

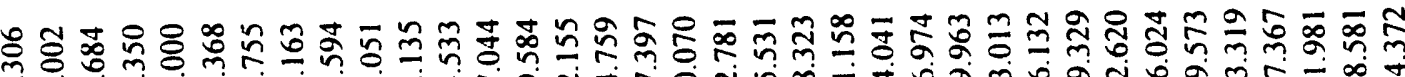

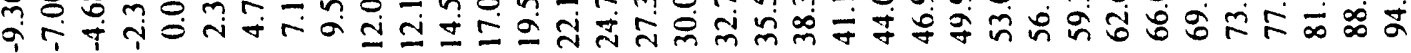

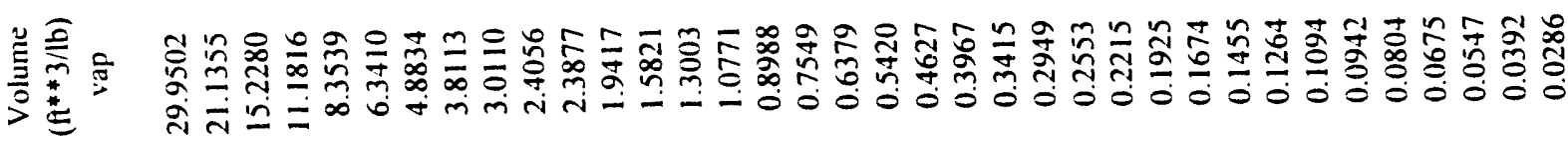

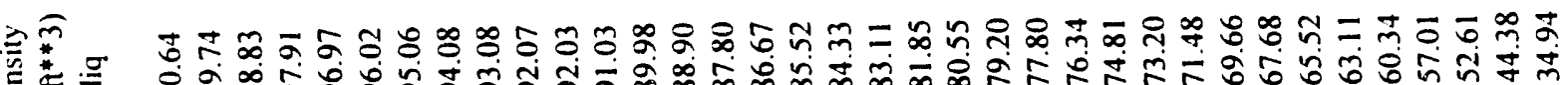

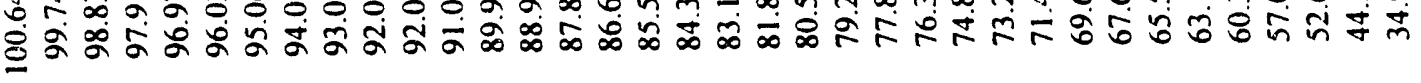

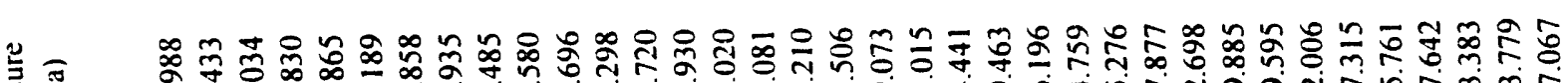

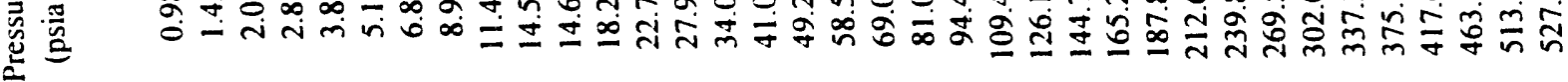

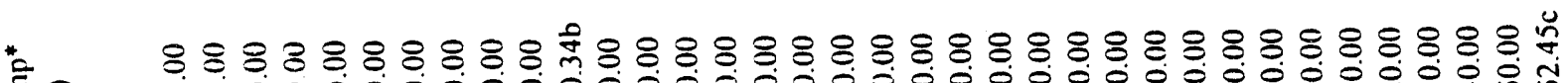

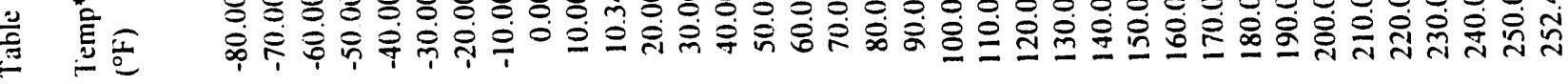


Table 41a. Burnett vapor-phase PVT measurements for HFC-125 (SI units).

\begin{tabular}{|c|c|c|c|c|c|}
\hline $\mathrm{T} / \mathrm{K}$ & $\rho / g / \mathrm{cm}^{3}$ & $\mathrm{P} / \mathrm{kPa}$ & $\Gamma / \mathrm{K}$ & $\rho / g / \mathrm{cm}^{3}$ & $\mathrm{P} / \mathrm{kPa}$ \\
\hline 363.156 & 0.3393 & 4558.61 & 358.104 & 0.0336 & 781.507 \\
\hline 363.153 & 0.3393 & 4558.23 & 353.158 & 0.0336 & 769.028 \\
\hline 363.153 & 0.3393 & 4558.17 & 348.129 & 0.0336 & 756.204 \\
\hline 363.149 & 0.3393 & 4558.39 & 343.158 & 0.0336 & 743.351 \\
\hline 358.102 & 0.3393 & 4345.19 & 338.132 & 0.0336 & 730.368 \\
\hline 353.167 & 0.3394 & 4133.97 & 332.963 & 0.0336 & 717.212 \\
\hline 348.123 & 0.3395 & 3915.00 & 323.176 & 0.0337 & 691.725 \\
\hline 343.159 & 0.3395 & 3695.21 & 318.146 & 0.0337 & 678.578 \\
\hline 363.153 & 0.1904 & 3337.93 & 313.144 & 0.0337 & 665.352 \\
\hline 358.105 & 0.1904 & 3237.66 & 308.119 & 0.0337 & 652.116 \\
\hline 353.104 & 0.1904 & 3137.14 & 303.153 & 0.0337 & 638.937 \\
\hline 348.125 & 0.1905 & 3035.77 & 298.127 & 0.0337 & 625.474 \\
\hline 343.158 & 0.1905 & 2933.29 & 293.158 & 0.0337 & 612.108 \\
\hline 338.137 & 0.1906 & 2828.07 & 288.207 & 0.0337 & 598.655 \\
\hline 333.113 & 0.1906 & 2720.88 & 283.151 & 0.0337 & 584.814 \\
\hline 363.153 & 0.1068 & 2194.52 & 278.139 & 0.0337 & 570.947 \\
\hline 363.109 & 0.1068 & 2194.30 & 273.138 & 0.0337 & 556.909 \\
\hline 358.105 & 0.1068 & 2146.30 & 323.118 & 0.0337 & 691.471 \\
\hline 353.104 & 0.1068 & 2097.98 & 363.153 & 0.0188 & 458.239 \\
\hline 348.126 & 0.1069 & 2049.53 & 358.106 & 0.0188 & 449.926 \\
\hline 343.156 & 0.1069 & 2000.74 & 353.168 & 0.0188 & 444.643 \\
\hline 338.129 & 0.1069 & 1950.91 & 348.127 & 0.0188 & 437.708 \\
\hline 333.110 & 0.1069 & 1900.59 & 343.106 & 0.0188 & 430.832 \\
\hline 323.128 & 0.1070 & 1798.70 & 338.129 & 0.0189 & 423.984 \\
\hline 318.165 & 0.1070 & 1746.98 & 333.110 & 0.0189 & 417.028 \\
\hline 313.147 & 0.1070 & 1693.83 & 323.176 & 0.0189 & 403.207 \\
\hline 308.174 & 0.1070 & 1640.06 & 323.134 & 0.0189 & 403.066 \\
\hline 323.137 & 0.1070 & 1798.70 & 318.145 & 0.0189 & 396.177 \\
\hline 328.137 & 0.1070 & 1849.94 & 313.145 & 0.0189 & 389.201 \\
\hline 363.153 & 0.0599 & 1346.59 & 308.116 & 0.0189 & 382.170 \\
\hline 358.106 & 0.0599 & 1322.23 & 303.154 & 0.0189 & 375.183 \\
\hline 353.103 & 0.0599 & 1297.92 & 298.128 & 0.0189 & 368.084 \\
\hline 348.128 & 0.0599 & 1273.59 & 293.157 & 0.0189 & 361.028 \\
\hline 343.156 & 0.0600 & 1249.15 & 288.204 & 0.0189 & 353.976 \\
\hline 338.136 & 0.0600 & 1224.39 & 283.151 & 0.0189 & 346.738 \\
\hline 333.111 & 0.0600 & 1199.40 & 278.137 & 0.0189 & 339.555 \\
\hline 323.130 & 0.0600 & 1149.26 & 273.134 & 0.0189 & 332.317 \\
\hline 318.141 & 0.0600 & 1123.90 & 363.153 & 0.2530 & 3951.636 \\
\hline 313.150 & 0.0600 & 1098.28 & 363.153 & 0.1419 & 2727.698 \\
\hline 308.173 & 0.0600 & 1072.51 & 363.153 & 0.0796 & 1723.650 \\
\hline 303.146 & 0.0601 & 1046.21 & 363.153 & 0.0447 & 1033.725 \\
\hline 298.133 & 0.0601 & 1019.65 & 363.153 & 0.0250 & 601.990 \\
\hline 293.116 & 0.0601 & 992.694 & 363.153 & 0.0140 & 344.832 \\
\hline
\end{tabular}


Table 4lb. Burnett vapor-phase PVT measurements for HFC-125 (PI units).

\begin{tabular}{|c|c|c|c|c|c|}
\hline$T / F$ & $\rho / 1 b / f^{3}$ & $\mathrm{P} / \mathrm{psia}$ & $\mathrm{T} / \mathrm{F}$ & $\rho / \mathrm{lb} / \mathrm{ft}^{3}$ & $\mathrm{P} / \mathrm{psia}$ \\
\hline 194.010 & 21.1822 & 661.171 & 194.005 & 2.1004 & 115.199 \\
\hline 194.005 & 21.1822 & 661.116 & 184.917 & 2.1009 & 113.348 \\
\hline 194.005 & 21.1822 & 661.106 & 176.015 & 2.1013 & 111.538 \\
\hline 193.998 & 21.1822 & 661.138 & 166.963 & 2.1018 & 109.678 \\
\hline 184.914 & 21.1867 & 630.217 & 158.014 & 2.1022 & 107.814 \\
\hline 176.031 & 21.1910 & 599.582 & 148.968 & 2.1026 & 105.931 \\
\hline 166.952 & 21.1955 & 567.823 & 139.664 & 2.1031 & 104.023 \\
\hline 158.016 & 21.1999 & 535.945 & 122.047 & 2.1040 & 100.326 \\
\hline 194.005 & 11.8866 & 484.126 & 112.994 & 2.1044 & 98.419 \\
\hline 184.919 & 11.8891 & 469.584 & 103.989 & 2.1048 & 96.501 \\
\hline 175.918 & 11.8916 & 455.004 & 94.944 & 2.1053 & 94.581 \\
\hline 166.956 & 11.8941 & 440.301 & 86.005 & 2.1057 & 92.670 \\
\hline 158.014 & 11.8966 & 425.439 & 76.958 & 2.1062 & 90.717 \\
\hline 148.977 & 11.8991 & 410.177 & 68.014 & 2.1066 & 88.779 \\
\hline 139.934 & 11.9016 & 394.630 & 59.103 & 2.1070 & 86.828 \\
\hline 194.005 & 6.6703 & 318.288 & 50.001 & 2.1075 & 84.820 \\
\hline 193.926 & 6.6703 & 318.257 & 40.980 & 2.1079 & 82.809 \\
\hline 184.919 & 6.6717 & 311.295 & 31.978 & 2.1084 & 80.773 \\
\hline 175.918 & 6.6731 & 304.286 & 121.943 & 2.1040 & 100.289 \\
\hline 166.958 & 6.674 .5 & 297.260 & 194.005 & 1.1787 & 66.462 \\
\hline 158.01 & 6.6759 & 290.183 & 184.921 & 1.1789 & 65.256 \\
\hline 148.962 & 6.6773 & 282.956 & 176.033 & 1.1792 & 64.490 \\
\hline 139.929 & 6.6787 & 275.658 & 166.959 & 1.1794 & 63.484 \\
\hline 121.961 & 6.6815 & 260.880 & 157.920 & 1.1797 & 62.487 \\
\hline 113.028 & 6.6829 & 253.378 & 148.962 & 1.1799 & 61.494 \\
\hline 103.994 & 6.6843 & 245.670 & 139.929 & 1.1801 & 60.485 \\
\hline 95.043 & 6.6857 & 237.872 & 122.047 & 1.1806 & 58.480 \\
\hline 121.977 & 6.6815 & 260.880 & 121.971 & 1.1806 & 58.460 \\
\hline 130.976 & 6.6801 & 268.312 & 112.992 & 1.1809 & 57.461 \\
\hline 194.005 & 3.7431 & 195.307 & 103.991 & 1.1811 & 56.449 \\
\hline 184.921 & 3.7439 & 191.774 & 94.939 & 1.1814 & 55.429 \\
\hline 175.916 & 3.7446 & 188.248 & 86.008 & 1.1816 & 54.416 \\
\hline 166.961 & 3.7454 & 184.719 & 76.960 & 1.1819 & 53.386 \\
\hline 158.01 & 3.7462 & 181.175 & 68.013 & 1.1821 & 52.363 \\
\hline 148.975 & 3.7470 & 177.584 & 59.098 & 1.1824 & 51.340 \\
\hline 139.931 & 3.7478 & 173.959 & 50.001 & 1.1826 & 50.290 \\
\hline 121.964 & 3.7494 & 166.687 & 40.976 & 1.1829 & 49.248 \\
\hline 112.985 & 3.7501 & 163.008 & 31.971 & 1.1831 & 48.198 \\
\hline 104.00 & 3.7509 & 159.292 & 194.005 & 15.796 & 573.136 \\
\hline 95.042 & 3.7517 & 155.555 & 194.005 & 8.8646 & 395.619 \\
\hline 85.994 & 3.7525 & 151.741 & 194.005 & 4.9744 & 249.994 \\
\hline 76.969 & 3.7533 & 147.888 & 194.005 & 2.7914 & 149.929 \\
\hline 67.939 & 3.7541 & 143.978 & 194.005 & 1.5664 & 87.311 \\
\hline
\end{tabular}


Table 42a. Vibrating-tube compressed-liquid-density measurements for HFC-125 (SI units)

\begin{tabular}{|c|c|c|c|c|c|c|c|c|}
\hline$T / K$ & $\rho / g / \mathrm{cm}^{3}$ & $\mathrm{P} / \mathrm{kPa}$ & $T / K$ & $\rho / g / \mathrm{cm}^{3}$ & $\mathrm{P} / \mathrm{kPa}$ & $\mathrm{T} / \mathrm{K}$ & $\rho / \mathrm{g} / \mathrm{cm}^{3}$ & $\mathrm{P} / \mathrm{kPa}$ \\
\hline 275.559 & 1.316 & 1590.1 & 340.576 & 0.9355 & 5235.1 & 358.643 & 0.389 & 4599.5 \\
\hline 275.553 & 1.3194 & 2019.8 & 340.603 & 0.9476 & 5467.7 & 358.643 & 0.389 & 4599.5 \\
\hline 275.412 & 1.3272 & 3006.5 & 340.598 & 0.9576 & 5672.5 & 358.63 & 0.415 & 4700.7 \\
\hline 275.407 & 1.3341 & 3996.3 & 340.577 & 0.9671 & 5879.3 & 358.642 & 0.4454 & 4810.0 \\
\hline 275.368 & 1.3406 & 4958.9 & 340.499 & 0.9855 & 6328.8 & 358.646 & 0.4743 & 4904.2 \\
\hline 275.374 & 1.3484 & 6213.0 & 343.332 & 0.2906 & 3542.8 & 358.652 & 0.506 & 5005.6 \\
\hline 283.966 & 1.2745 & 1607.4 & 343.338 & 0.3194 & 3645.5 & 358.661 & 0.5381 & 5105.2 \\
\hline 283.978 & 1.2783 & 2019.9 & 343.363 & 0.3633 & 3747.6 & 358.669 & 0.57 & 5208.1 \\
\hline 283.985 & 1.2873 & 3024.5 & 343.361 & 0.4748 & 3851.0 & 358.641 & 0.5927 & 5284.8 \\
\hline 283.997 & 1.2958 & 4057.0 & 343.358 & 0.6392 & 3950.3 & 358.675 & 0.6211 & 5393.9 \\
\hline 284.0 & 1.3037 & 5070.0 & 343.347 & 0.725 & 4081.3 & 358.695 & 0.6692 & 5603.8 \\
\hline 284.003 & 1.3122 & 6238.5 & 343.371 & 0.7993 & 4345.8 & 358.698 & 0.7062 & 5801.0 \\
\hline 293.477 & 1.2217 & 1586.8 & 343.346 & 0.8369 & 4570.0 & 358.675 & 0.7647 & 6212.2 \\
\hline 293.479 & 1.2275 & 2054.7 & 343.345 & 0.8883 & 5070.8 & 363.827 & 0.2826 & 4206.3 \\
\hline 293.482 & 1.2384 & 3005.2 & 343.347 & 0.9212 & 5551.8 & 363.806 & 0.295 & 4296.1 \\
\hline 293.483 & 1.2493 & 4035.0 & 343.358 & 0.9486 & 6083.4 & 363.813 & 0.308 & 4387.6 \\
\hline 293.484 & 1.2593 & 5073.5 & 348.423 & 0.2584 & 3551.5 & 363.828 & 0.3255 & 4503.4 \\
\hline 293.482 & 1.27 & 6266.3 & 348.409 & 0.2734 & 3638.6 & 363.839 & 0.3421 & 4604.7 \\
\hline 303.313 & 1.1586 & 1616.2 & 348.428 & 0.2933 & 3740.4 & 363.903 & 0.3590 & 4703.8 \\
\hline 303.323 & 1.1659 & 2026.6 & 348.412 & 0.3198 & 3854.5 & 363.254 & 0.3823 & 4799.1 \\
\hline 303.326 & 1.1824 & 3053.0 & 348.431 & 0.3445 & 3946.2 & 363.255 & 0.4029 & 4898.2 \\
\hline 303.331 & 1.196 & 4021.6 & 348.448 & 0.3778 & 4041.8 & 363.268 & 0.4248 & 4998.8 \\
\hline 303.33 & 1.2089 & 5049.6 & 348.444 & 0.4362 & 4152.0 & 363.285 & 0.4463 & 5092.2 \\
\hline 303.353 & 1.2226 & 6283.0 & 348.453 & 0.5078 & 4251.6 & 363.414 & 0.4689 & 5196.3 \\
\hline 313.94 & 1.0951 & 2496.4 & 348.448 & 0.5809 & 4348.2 & 363.675 & 0.4939 & 5310.8 \\
\hline 313.934 & 1.1094 & 3057.2 & 348.513 & 0.6915 & 4581.2 & 363.567 & 0.5210 & 5415.6 \\
\hline 313.932 & 1.1302 & 4044.2 & 348.441 & 0.7662 & 4863.6 & 363.606 & 0.5407 & 5498.2 \\
\hline 313.93 & 1.1471 & 5001.2 & 348.52 & 0.7972 & 5056.5 & 363.67 & 0.5662 & 5611.2 \\
\hline 313.935 & 1.1663 & 6263.2 & 348.439 & 0.83 & 5302.9 & 363.661 & 0.6080 & 5798.9 \\
\hline 323.873 & 1.0067 & 2811.2 & 348.517 & 0.8525 & 5542.6 & 363.655 & 0.6307 & 5911.1 \\
\hline 323.872 & 1.0178 & 3039.2 & 348.444 & 0.8778 & 5845.1 & 363.68 & 0.6471 & 6000.4 \\
\hline 323.873 & 1.0534 & 4016.5 & 348.516 & 0.8973 & 6150.1 & 363.678 & 0.6655 & 6105.5 \\
\hline 323.877 & 1.0807 & 5027.3 & 353.548 & 0.2694 & 3782.0 & 363.675 & 0.6893 & 6254.7 \\
\hline 323.872 & 1.1059 & 6212.2 & 353.57 & 0.2897 & 3905.1 & 369.108 & 0.2870 & 4411.1 \\
\hline 333.921 & 0.8857 & 3389.5 & 353.565 & 0.307 & 3999.3 & 369.134 & 0.2991 & 4509.1 \\
\hline 333.919 & 0.9498 & 4088.8 & 353.581 & 0.3287 & 4105.9 & 369.134 & 0.3111 & 4600.3 \\
\hline 333.909 & 0.9977 & 5063.8 & 353.602 & 0.3524 & 4208.7 & 368.406 & 0.4495 & 5410.4 \\
\hline 333.916 & 1.0345 & 6184.0 & 353.592 & 0.3792 & 4307.0 & 368.468 & 0.4677 & 5505.8 \\
\hline 339.059 & 0.8179 & 3816.3 & 353.549 & 0.4138 & 4411.5 & 368.565 & 0.4862 & 5604.2 \\
\hline 339.072 & 0.8618 & 4067.3 & 353.262 & 0.4579 & 4503.4 & 368.483 & 0.5089 & 5705.9 \\
\hline 339.062 & 0.9098 & 4547.7 & 353.262 & 0.502 & 4599.4 & 368.304 & 0.5302 & 5804.5 \\
\hline 339.051 & 0.944 & 5069.3 & 353.268 & 0.5425 & 4685.0 & 368.436 & 0.5477 & 5900.0 \\
\hline 339.057 & 0.9671 & 5535.5 & 353.253 & 0.5933 & 4800.1 & 368.525 & 0.5584 & 5966.2 \\
\hline 339.062 & 0.9922 & 6168.6 & 353.255 & 0.6316 & 4903.5 & 368.525 & 0.5584 & 5966.2 \\
\hline 340.499 & 0.3437 & 3556.5 & 353.265 & 0.6644 & 5008.3 & 368.578 & 0.58 & 6086.1 \\
\hline 340.504 & 0.4761 & 3645.2 & 353.278 & 0.6829 & 5075.3 & 368.586 & 0.6048 & 6226.9 \\
\hline 340.494 & 0.6451 & 3689.5 & 353.188 & 0.7399 & 5328.9 & 369.188 & 0.3249 & 4704.1 \\
\hline 340.499 & 0.7717 & 3856.1 & 353.282 & 0.7735 & 5548.4 & 369.173 & 0.3384 & 4798.6 \\
\hline
\end{tabular}


Table 42a. Vibrating-tube compressed-liquid-density measurements for HFC-125 (SI units) (continued)

$\begin{array}{lllllllll}\mathrm{T} / \mathrm{K} & \rho / \mathrm{g} / \mathrm{cm}^{3} & \mathrm{P} / \mathrm{kPa} & \mathrm{T} / \mathrm{K} & \rho / \mathrm{g} / \mathrm{cm}^{3} & \mathrm{P} / \mathrm{kPa} & \mathrm{T} / \mathrm{K} & \rho / \mathrm{g} / \mathrm{cm}^{3} & \mathrm{P} / \mathrm{kPa} \\ & & & & & & & & \\ 340.509 & 0.8272 & 4078.5 & 353.164 & 0.8116 & 5849.9 & 369.192 & 0.3568 & 4921.1 \\ 340.52 & 0.8429 & 4172.2 & 353.283 & 0.8389 & 6161.8 & 369.292 & 0.3687 & 5003.3 \\ 340.605 & 0.871 & 4403.9 & 358.631 & 0.2973 & 4129.3 & 369.292 & 0.3687 & 5003.3 \\ 340.598 & 0.8945 & 4646.5 & 358.636 & 0.3078 & 4193.6 & 369.347 & 0.3832 & 5096.6 \\ 340.594 & 0.9118 & 4865.4 & 358.652 & 0.3263 & 4301.3 & 369.361 & 0.3994 & 5194.2 \\ 340.571 & 0.9246 & 5053.0 & 358.628 & 0.3412 & 4379.2 & 369.449 & 0.4206 & 5320.5\end{array}$


Table 42b. Vibrating-tube compressed-liquid-density measurements for HFC-125 (PI units)

\begin{tabular}{|c|c|c|c|c|c|c|c|c|}
\hline $\mathrm{T} / \mathrm{F}$ & $\rho / \mathrm{lb} / \mathrm{ft}^{3}$ & P/psia & $\mathrm{T} / \mathrm{F}$ & $\rho / l b / f^{3}$ & $\mathrm{P} / \mathrm{psia}$ & $\mathrm{T} / \mathrm{F}$ & $\rho / \mathrm{lb} / \mathrm{ft}^{3}$ & P/psia \\
\hline 36.336 & 82.155 & 230.62 & 153.367 & 58.401 & 759.29 & 185.887 & 24.284 & 667.1 \\
\hline 36.325 & 82.367 & 292.95 & 153.415 & 59.157 & 793.02 & 185.887 & 24.284 & 667.1 \\
\hline 36.072 & 82.854 & 436.06 & 153.406 & 59.781 & 822.73 & 185.864 & 25.908 & 681.78 \\
\hline 36.063 & 83.285 & 579.61 & 153.369 & 60.374 & 852.72 & 185.886 & 27.805 & 697.63 \\
\hline 35.992 & 83.691 & 719.23 & 153.228 & 61.523 & 917.91 & 185.893 & 29.61 & 711.29 \\
\hline 36.003 & 84.178 & 901.12 & 158.328 & 18.142 & 513.84 & 185.904 & 31.589 & 726.0 \\
\hline 51.469 & 79.564 & 233.13 & 158.338 & 19.94 & 528.74 & 185.92 & 33.592 & 740.45 \\
\hline 51.49 & 79.802 & 292.96 & 158.383 & 22.68 & 543.54 & 185.934 & 35.584 & 755.37 \\
\hline 51.503 & 80.364 & 438.67 & 158.38 & 29.641 & 558.54 & 185.884 & 37.001 & 766.5 \\
\hline 51.525 & 80.894 & 588.42 & 158.374 & 39.904 & 572.94 & 185.945 & 38.774 & 782.32 \\
\hline 51.53 & 81.387 & 735.34 & 158.355 & 45.26 & 591.94 & 185.981 & 41.777 & 812.76 \\
\hline 51.535 & 81.918 & 904.82 & 158.398 & 49.899 & 630.3 & 185.986 & 44.087 & 841.36 \\
\hline 68.589 & 76.268 & 230.15 & 158.353 & 52.246 & 662.82 & 185.945 & 47.739 & 901.0 \\
\hline 68.592 & 76.63 & 298.01 & 158.351 & 55.455 & 735.46 & 195.219 & 17.642 & 610.07 \\
\hline 68.598 & 77.311 & 435.87 & 158.355 & 57.509 & 805.22 & 195.181 & 18.416 & 623.1 \\
\hline 68.599 & 77.991 & 585.23 & 158.374 & 59.219 & 882.32 & 195.193 & 19.228 & 636.37 \\
\hline 68.601 & 78.616 & 735.85 & 167.491 & 16.131 & 515.1 & 195.22 & 20.32 & 653.16 \\
\hline 68.598 & 79.284 & 908.85 & 167.466 & 17.068 & 527.73 & 195.24 & 21.357 & 667.86 \\
\hline 86.293 & 72.329 & 234.41 & 167.5 & 18.31 & 542.5 & 195.355 & 22.412 & 682.23 \\
\hline 86.311 & 72.785 & 293.93 & 167.472 & 19.964 & 559.05 & 194.187 & 23.866 & 696.05 \\
\hline 86.317 & 73.815 & 442.8 & 167.506 & 21.506 & 572.35 & 194.189 & 25.152 & 710.42 \\
\hline 86.325 & 74.664 & 583.28 & 167.536 & 23.585 & 586.21 & 194.212 & 26.519 & 725.01 \\
\hline 86.324 & 75.469 & 732.38 & 167.529 & 27.231 & 602.2 & 194.243 & 27.862 & 738.56 \\
\hline 86.365 & 76.324 & 911.27 & 167.545 & 31.701 & 616.64 & 194.475 & 29.272 & 753.66 \\
\hline 105.422 & 68.365 & 362.07 & 167.536 & 36.264 & 630.65 & 194.945 & 30.833 & 770.27 \\
\hline 105.411 & 69.258 & 443.41 & 167.653 & 43.169 & 664.45 & 194.751 & 32.525 & 785.47 \\
\hline 105.408 & 70.556 & 586.56 & 167.524 & 47.832 & 705.41 & 194.821 & 33.755 & 797.45 \\
\hline 105.404 & 71.611 & 725.36 & 167.666 & 49.768 & 733.38 & 194.936 & 35.347 & 813.84 \\
\hline 105.413 & 72.81 & 908.4 & 167.52 & 51.815 & 769.12 & 194.92 & 37.956 & 841.06 \\
\hline 123.301 & 62.846 & 407.73 & 167.661 & 53.22 & 803.89 & 194.909 & 39.373 & 857.33 \\
\hline 123.3 & 63.539 & 440.8 & 167.529 & 54.799 & 847.76 & 194.954 & 40.397 & 870.28 \\
\hline 123.301 & 65.762 & 582.54 & 167.659 & 56.017 & 892.0 & 194.95 & 41.546 & 885.53 \\
\hline 123.309 & 67.466 & 729.15 & 176.716 & 16.818 & 548.53 & 194.945 & 43.032 & 907.17 \\
\hline 123.3 & 69.039 & 901.0 & 176.756 & 18.085 & 566.39 & 204.724 & 17.917 & 639.78 \\
\hline 141.388 & 55.292 & 491.61 & 176.747 & 19.165 & 580.05 & 204.771 & 18.672 & 653.99 \\
\hline 141.384 & 59.294 & 593.03 & 176.776 & 20.52 & 595.51 & 204.771 & 19.421 & 667.22 \\
\hline 141.366 & 62.284 & 734.44 & 176.814 & 22.0 & 610.42 & 203.461 & 28.061 & 784.71 \\
\hline 141.379 & 64.582 & 896.91 & 176.796 & 23.673 & 624.68 & 203.572 & 29.198 & 798.55 \\
\hline 150.636 & 51.06 & 553.51 & 176.718 & 25.833 & 639.83 & 203.747 & 30.352 & 812.82 \\
\hline 150.66 & 53.8 & 589.91 & 176.202 & 28.586 & 653.16 & 203.599 & 31.77 & 827.57 \\
\hline 150.642 & 56.797 & 659.59 & 176.202 & 31.339 & 667.09 & 203.277 & 33.099 & 841.87 \\
\hline 150.622 & 58.932 & 735.24 & 176.212 & 33.867 & 679.5 & 203.515 & 34.192 & 855.72 \\
\hline 150.633 & 60.374 & 802.86 & 176.185 & 37.039 & 696.2 & 203.675 & 34.86 & 865.32 \\
\hline 150.642 & 61.941 & 894.68 & 176.189 & 39.43 & 711.19 & 203.675 & 34.86 & 865.32 \\
\hline 153.228 & 21.456 & 515.83 & 176.207 & 41.477 & 726.39 & 203.77 & 36.208 & 882.71 \\
\hline 153.237 & 29.722 & 528.69 & 176.23 & 42.632 & 736.11 & 203.785 & 37.756 & 903.14 \\
\hline 153.219 & 40.272 & 535.12 & 176.068 & 46.19 & 772.89 & 204.868 & 20.283 & 682.27 \\
\hline 153.228 & 48.176 & 559.28 & 176.238 & 48.288 & 804.73 & 204.841 & 21.126 & 695.98 \\
\hline 153.246 & 51.64 & 591.54 & 176.025 & 50.667 & 848.46 & 204.876 & 22.274 & 713.75 \\
\hline
\end{tabular}


Table 42b. Vibrating-tube compressed-liquid-density measurements for HFC-125 (PI units) (continued)

$\begin{array}{lllllllll}\mathrm{T} / \mathrm{F} & \mathrm{\rho} / \mathrm{lb} / \mathrm{ft}^{3} & \mathrm{P} / \mathrm{psia} & \mathrm{T} / \mathrm{F} & \mathrm{\rho} / \mathrm{lb} / \mathrm{ft}^{3} & \mathrm{P} / \mathrm{psia} & \mathrm{T} / \mathrm{F} & \rho / \mathrm{lb} / \mathrm{ft}^{3} & \mathrm{P} / \mathrm{psia} \\ & & & & & & & & \\ 153.266 & 52.621 & 605.13 & 176.239 & 52.371 & 893.69 & 205.056 & 23.017 & 725.67 \\ 153.419 & 54.375 & 638.73 & 185.866 & 18.56 & 598.9 & 205.056 & 23.017 & 725.67 \\ 153.406 & 55.842 & 673.92 & 185.875 & 19.215 & 608.23 & 205.155 & 23.922 & 739.2 \\ 153.399 & 56.922 & 705.67 & 185.904 & 20.37 & 623.85 & 205.18 & 24.934 & 753.36 \\ 153.358 & 57.721 & 732.88 & 185.86 & 21.3 & 635.15 & 205.338 & 26.257 & 771.67\end{array}$


Table 43a. Liquid PVT Data (Isochoric Apparatus) for HFC-125 1/2

\begin{tabular}{|c|c|c|}
\hline$\underset{\mathrm{K}}{\text { Temperature }}$ & $\begin{array}{l}\text { Pressure } \\
\text { MPa }\end{array}$ & $\begin{array}{l}\text { Density } \\
\text { mol } \cdot \mathrm{dm}^{-3}\end{array}$ \\
\hline $\begin{array}{l}174.001 \\
176.000 \\
178.000 \\
180.001 \\
182.000 \\
184.000 \\
186.001 \\
188.001 \\
190.002 \\
192.000 \\
194.001\end{array}$ & $\begin{array}{r}1.572130 \\
3.186809 \\
6.011646 \\
9.259311 \\
12.535940 \\
15.826000 \\
19.108860 \\
22.384900 \\
25.655180 \\
28.904070 \\
32.147290\end{array}$ & $\begin{array}{l}14.06755 \\
14.05714 \\
14.02831 \\
14.02319 \\
14.01906 \\
14.01535 \\
14.01189 \\
14.00860 \\
14.00543 \\
14.00235 \\
13.99934\end{array}$ \\
\hline $\begin{array}{l}185.999 \\
188.000 \\
190.000 \\
192.001 \\
194.000 \\
196.000 \\
198.000 \\
200.000 \\
202.000 \\
204.001 \\
205.999 \\
208.000\end{array}$ & $\begin{array}{r}2.588665 \\
3.931049 \\
6.785781 \\
9.737449 \\
12.702930 \\
15.672670 \\
18.642740 \\
21.605980 \\
24.559160 \\
27.505050 \\
30.429050 \\
33.352820\end{array}$ & $\begin{array}{l}13.76738 \\
13.73748 \\
13.73083 \\
13.72635 \\
13.72255 \\
13.71909 \\
13.71583 \\
13.71272 \\
13.70971 \\
13.70678 \\
13.70392 \\
13.70110\end{array}$ \\
\hline $\begin{array}{l}202.000 \\
204.000 \\
206.001 \\
208.001 \\
210.000 \\
212.000 \\
214.000 \\
216.000 \\
217.999 \\
219.999 \\
221.999 \\
223.998 \\
226.000 \\
228.000 \\
230.001\end{array}$ & $\begin{array}{r}1.547702 \\
3.168674 \\
5.107316 \\
7.612479 \\
10.149190 \\
12.695070 \\
15.243050 \\
17.786270 \\
20.321700 \\
22.850930 \\
25.373530 \\
27.893440 \\
30.405660 \\
32.905790 \\
35.396040\end{array}$ & $\begin{array}{l}13.31587 \\
13.30579 \\
13.27811 \\
13.27329 \\
13.26946 \\
13.26605 \\
13.26290 \\
13.25991 \\
13.25704 \\
13.25425 \\
13.25151 \\
13.24884 \\
13.24621 \\
13.24361 \\
13.24104\end{array}$ \\
\hline $\begin{array}{l}220.000 \\
223.999 \\
228.000 \\
232.000 \\
236.000 \\
240.000 \\
244.001 \\
248.000\end{array}$ & $\begin{array}{r}3.584159 \\
7.887842 \\
12.303330 \\
16.710760 \\
21.108060 \\
25.487380 \\
29.846050 \\
34.172920\end{array}$ & $\begin{array}{l}12.85812 \\
12.84761 \\
12.84102 \\
12.83531 \\
12.82999 \\
12.82490 \\
12.81996 \\
12.81513\end{array}$ \\
\hline
\end{tabular}


Table 43a. Liquid PVT Data (Isochoric Apparatus) for HFC-125 $2 / 2$

Temperature

$\mathrm{K}$

244.001
248.001
252.001
256.002
260.000
263.998
267.998
271.998
275.999
279.999

276.001

279.999

284.001

288.001

292.001

296.001

300.002

304.002

308.002

311.999

316.001

320.001

323.998

327.999

332.001

310.000

313.999

318.000

322.001

326.001

330.000

334.002

338.000

342.001

350.002

358.000

366.000

374.001

382.000

389.998

398.001

\section{Pressure MPa}

3.150607
6.081187
9.523219
12.978420
16.431190
19.871730
23.300320
26.721690
30.125850
33.511270

2.664308

4. 587385

6.952455

9. 330561

11.714370

14.099870

16.483800

18.866340

21.235980

23.607610

25.974380

28.340110

30.694960

33.033720

35.372100

2.472744

3.683955

5.050635

6.431671

7.823143

9.220093

10.622020

12.025280

13.435840

16.261530

19.091220

21.921170

24.747190

27.567680

30.378470

33.180550
Density
mol. $\mathrm{dm}^{-3}$

12.14012

12.10937

12.10297

12.09757

12.09262

12.08789

12.08333

12.07887

12.07448

12.07019

11.01663

10.98439

10.97823

10.97328

10.96880

10.96456

10.96048

10.95650

10.95259

10.94874

10.94491

10.94111

10.93735

10.93371

10.93014
9.38809
9.35782
9.35231
9.34799
9.34414
9.34053
9.33708
9.33402
9.33090
9. 32441
9. 31801
9. 31168
9.30537
9. 29906
9.29274
9.28639 
Table 43b. Iiquid PVT Data (Isochoric Apparatus) for HFC-125 $1 / 2$

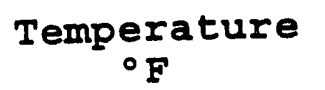

-146.47
-142.87
-139.27
-135.67
-132.07
-128.47
-124.87
-121.27
-117.67
-114.07
-110.47

$-124.87$

$-121.27$

$-117.67$

$-114.07$

$-110.47$

$-106.87$

$-103.27$

$-99.67$

$-96.07$

$-92.47$

$-88.87$

$-85.27$

$-96.07$

$-92.47$

$-88.87$

$-85.27$

$-81.67$

$-78.07$

$-74.47$

$-70.87$

$-67.27$

$-63.67$

$-60.07$

$-56.47$

$-52.87$

$-49.27$

$-45.67$

$-63.67$

$-56.47$

$-49.27$

$-42.07$

$-34.87$

$-27.67$

$-20.47$

$-13.27$

\section{Pressure \\ psia}

228.019

462.208

871.917

1342.952

1818.188

2295.371

2771.511

3246.661

3720.976

4192.188

4662.578

375.455

570.151

984.196

1412.300

1842.407

2273.133

2703.906

3133.688

3562.011

3989.277

4413.368

4837.426

224.476
459.578
740.755
1104.099
1472.018
1841.267
2210.821
2579.685
2947.419
3314.253
3680.126
4045.609
4409.976
4772.590
5133.771

519.839

1144.037

1784.450

2423.695

3061.471

3696.638

4328.811

4956.372
Density
lb $\mathrm{ft}^{-3}$

105.404

105.326

105.110

105.072

105.041

105.013

104.987

104.963

104.939

104.916

104.893

103.155

102.931

102.881

102.848

102.819

102.793

102.769

102.746

102.723

102.701

102.680

102.659

99.772

99.697

99.489

99.453

99.424

99.399

99.375

99.353

99.331

99.310

99.290

99.270

99.250

99.231

99.212

96.342

96.264

96.214

96.171

96.132

96.093

36.056

96.020 


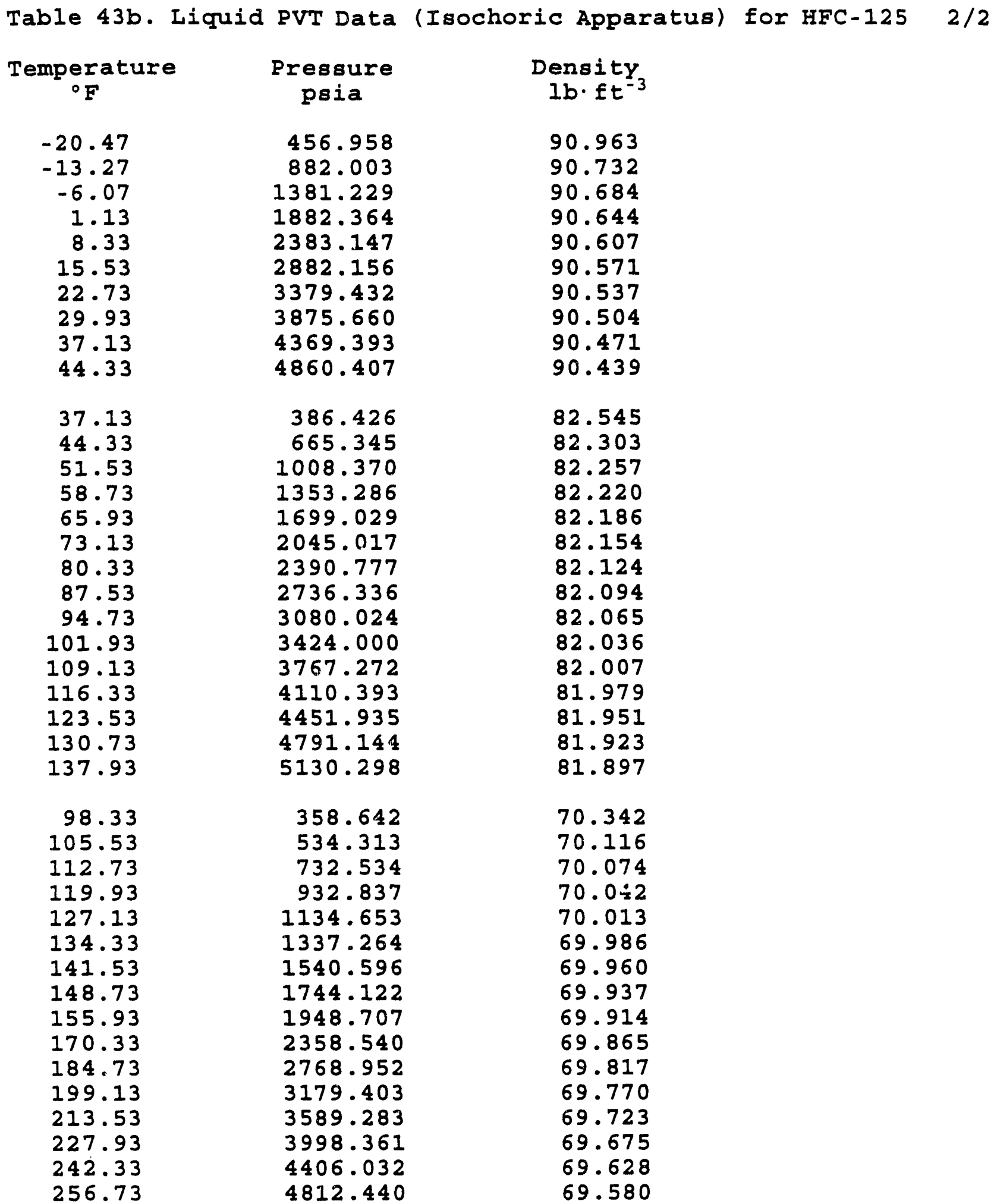


Table 44a. Vapor pressures of HFC-125 (NIST Burnett apparatus + ebulliometer) (SI units).

\begin{tabular}{|c|c|c|c|}
\hline$T / K$ & $\mathrm{P} / \mathrm{kPa}$ & $\mathrm{T} / \mathrm{K}$ & $\mathrm{P} / \mathrm{kPa}$ \\
\hline 335.160 & 3313.62 & 236.643 & 173.31 \\
\hline 334.123 & 3239.42 & 235.033 & 161.44 \\
\hline 333.115 & 3168.90 & 233.989 & 154.13 \\
\hline 328.135 & 2839.13 & 233.825 & 153.00 \\
\hline 325.628 & 2683.69 & 231.835 & 139.80 \\
\hline 323.174 & 2538.45 & 230.070 & 128.86 \\
\hline 323.145 & 2536.91 & 229.270 & 124.12 \\
\hline 320.648 & 2395.80 & 228.491 & 119.64 \\
\hline 318.146 & 2260.39 & 227.495 & 114.12 \\
\hline 315.630 & 2130.71 & 227.055 & 111.73 \\
\hline 313.140 & 2007.77 & 226.371 & 108.10 \\
\hline 310.666 & 1891.36 & 226.442 & 108.48 \\
\hline 308.122 & 1776.56 & 226.262 & 107.54 \\
\hline 305.674 & 1671.79 & 224.984 & 101.08 \\
\hline 303.154 & 1568.42 & 224.053 & 96.539 \\
\hline 300.656 & 1471.03 & 223.343 & 93.183 \\
\hline 298.126 & 1376.81 & 222.736 & 90.391 \\
\hline 295.665 & 1289.87 & 222.855 & 90.931 \\
\hline 293.161 & 1205.31 & 222.257 & 88.238 \\
\hline 290.681 & 1126.10 & 221.575 & 85.240 \\
\hline 288.117 & 1048.11 & 221.185 & 83.567 \\
\hline 285.630 & 976.663 & 220.916 & 82.419 \\
\hline 283.148 & 908.576 & 220.553 & 80.907 \\
\hline 280.658 & 844.462 & 220.381 & 80.182 \\
\hline 278.139 & 782.615 & 220.174 & 79.326 \\
\hline 275.647 & 725.350 & 220.0 & 78.623 \\
\hline 273.130 & 670.185 & 219.978 & 78.519 \\
\hline 246.589 & 262.041 & 219.444 & 76.384 \\
\hline 246.052 & 256.519 & 219.175 & 75.321 \\
\hline 245.662 & 252.551 & 218.762 & 73.712 \\
\hline 245.18 & 247.709 & & \\
\hline 244.317 & 239.246 & & \\
\hline 242.532 & 222.428 & & \\
\hline 240.801 & 207.015 & & \\
\hline 239.292 & 194.249 & & \\
\hline 238.261 & 185.870 & & \\
\hline 237.774 & 182.005 & & \\
\hline
\end{tabular}


Table 44b. Vapor pressures of HFC-125 (NIST Burnett apparatus + ebulliometer) (PI units).

\begin{tabular}{|c|c|c|c|}
\hline$T / F$ & P/psia & $T / F$ & P/psia \\
\hline 143.619 & 480.600 & -33.712 & 25.137 \\
\hline 141.751 & 469.838 & -36.610 & 23.415 \\
\hline 139.937 & 459.609 & -38.489 & 22.355 \\
\hline 130.974 & 411.780 & -38.785 & 22.191 \\
\hline 126.461 & 389.237 & -42.367 & 20.276 \\
\hline 122.043 & 368.171 & -45.544 & 18.690 \\
\hline 121.991 & 367.948 & -46.984 & 18.002 \\
\hline 117.497 & 347.481 & -48.386 & 17.352 \\
\hline 112.993 & 327.842 & -50.179 & 16.552 \\
\hline 108.464 & 309.033 & -50.971 & 16.205 \\
\hline 103.983 & 291.202 & -52.202 & 15.679 \\
\hline 99.529 & 274.318 & -52.074 & 15.734 \\
\hline 94.949 & 257.668 & -52.398 & 15.597 \\
\hline 90.544 & 242.473 & -54.698 & 14.661 \\
\hline 86.008 & 227.480 & -56.374 & 14.001 \\
\hline 81.511 & 213.355 & -57.652 & 13.515 \\
\hline 76.958 & 199.690 & -58.745 & 13.110 \\
\hline 72.527 & 187.080 & -58.531 & 13.188 \\
\hline 68.019 & 174.815 & -59.607 & 12.797 \\
\hline 63.557 & 163.328 & -60.835 & 12.363 \\
\hline 58.941 & 152.016 & -61.537 & 12.120 \\
\hline 54.463 & 141.653 & -62.021 & 11.953 \\
\hline 49.997 & 131.777 & -62.674 & 11.734 \\
\hline 45.514 & 122.478 & -62.984 & 11.629 \\
\hline 40.981 & 113.508 & -63.356 & 11.505 \\
\hline 36.495 & 105.203 & -63.670 & 11.403 \\
\hline 31.964 & 97.202 & -63.709 & 11.388 \\
\hline-15.809 & 38.005 & -64.670 & 11.078 \\
\hline-16.776 & 37.205 & -65.155 & 10.924 \\
\hline-17.478 & 36.629 & -65.898 & 10.691 \\
\hline-18.346 & 35.927 & & \\
\hline-19.899 & 34.699 & & \\
\hline-23.112 & 32.260 & & \\
\hline-26.228 & 30.025 & & \\
\hline-28.944 & 28.173 & & \\
\hline-30.800 & 26.958 & & \\
\hline-31.676 & 26.397 & & \\
\hline
\end{tabular}




Table 45a. Vapor Pressures (Static Method) for HFC-125
$\begin{array}{cc}\text { Temperature } & \text { Pressure } \\ \text { K } & \text { MPa }\end{array}$

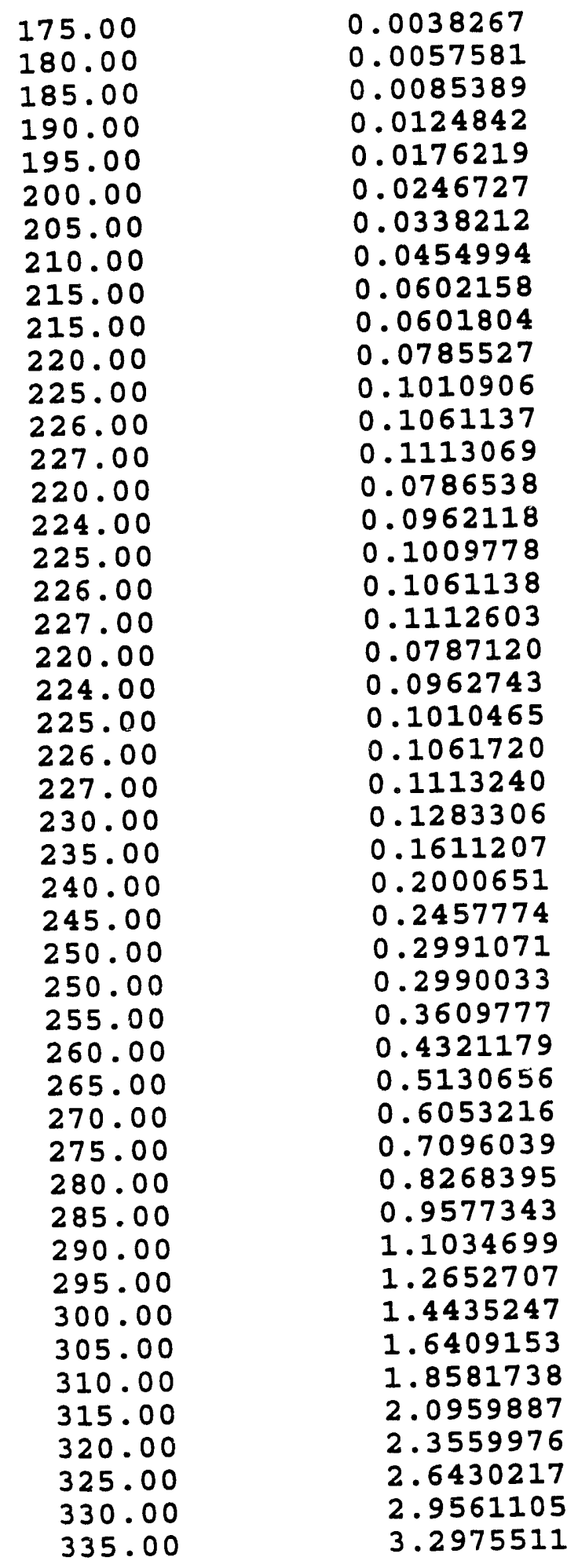


Table 45b. Vapor Pressures (Static Method) for HFC-125

\begin{tabular}{|c|c|}
\hline $\begin{array}{c}\text { Temperature } \\
{ }^{\circ} \mathrm{F}\end{array}$ & $\begin{array}{l}\text { Pressure } \\
\text { psia }\end{array}$ \\
\hline $\begin{array}{r}-144.67 \\
-135.67 \\
-126.67 \\
-117.67 \\
-108.67 \\
-99.67 \\
-90.67 \\
-81.67 \\
-72.67 \\
-72.67 \\
-63.67 \\
-54.67 \\
-52.87 \\
-51.07 \\
-63.67 \\
-56.47 \\
-54.67 \\
-52.87 \\
-51.07 \\
-63.67 \\
-56.47 \\
-54.67 \\
-52.87 \\
-51.07 \\
-45.67 \\
-36.67 \\
-27.67 \\
-18.67 \\
-9.67 \\
-9.67 \\
-0.67 \\
8.33 \\
17.33 \\
26.33 \\
35.33 \\
44.33 \\
53.33 \\
62.33 \\
71.33 \\
80.33 \\
89.33 \\
98.33 \\
107.33 \\
116.33 \\
125.33 \\
134.33 \\
143.33\end{array}$ & $\begin{array}{r}0.55501 \\
0.83514 \\
1.23847 \\
1.81069 \\
2.55584 \\
3.57847 \\
4.90535 \\
6.59913 \\
8.73357 \\
8.72843 \\
11.39311 \\
14.66196 \\
15.39048 \\
16.14371 \\
11.40778 \\
13.95434 \\
14.64559 \\
15.39051 \\
16.13694 \\
11.41621 \\
13.96340 \\
14.65556 \\
15.39894 \\
16.14619 \\
18.61278 \\
23.36858 \\
29.01700 \\
35.64700 \\
43.38182 \\
43.36675 \\
52.35539 \\
62.67339 \\
74.41387 \\
87.79448 \\
102.91934 \\
119.92293 \\
138.90761 \\
160.04477 \\
183.51200 \\
209.36556 \\
237.99464 \\
269.50532 \\
303.99745 \\
341.70855 \\
383.33789 \\
428.74757 \\
478.26934\end{array}$ \\
\hline
\end{tabular}


Table 46. Speed of Sound in HFC-125/CFC-115

(Mole Fraction HFC-125 $=\mathbf{0 . 9 9 8 5 0}$, Mole Fraction CFC-115 $=0.00150+/-0.000075)$

\begin{tabular}{|c|c|c|c|c|c|c|}
\hline $\mathbf{T} / \mathbf{K}$ & $/ \operatorname{deg} F$ & $\mathbf{p} / \mathbf{k P a}$ & p/psi & $\mathbf{u} /(\mathbf{m} / \mathbf{s})$ & $\mathbf{u} /(\mathbf{f t} / \mathbf{s})$ & $10^{6 *} \mathrm{du} / \mathrm{u}$ \\
\hline 240.007 & -27.657 & $\begin{array}{r}164.738 \\
155.341 \\
147.355 \\
133.645 \\
118.894 \\
104.137 \\
101.132 \\
98.212 \\
95.373 \\
92.631 \\
92.620 \\
86.480 \\
86.196 \\
86.026 \\
85.924 \\
85.861 \\
82.017 \\
70.758 \\
64.299 \\
53.120 \\
39.816\end{array}$ & $\begin{array}{r}23.893 \\
22.530 \\
21.372 \\
19.384 \\
17.244 \\
15.104 \\
14.668 \\
14.244 \\
13.833 \\
13.435 \\
13.433 \\
12.543 \\
12.502 \\
12.477 \\
12.462 \\
12.453 \\
11.896 \\
10.263 \\
9.326 \\
7.704 \\
5.775\end{array}$ & $\begin{array}{l}129.9841 \\
130.3539 \\
130.6612 \\
131.1830 \\
131.7378 \\
132.2778 \\
132.3896 \\
132.4972 \\
132.6016 \\
132.7023 \\
132.6993 \\
132.9213 \\
132.9330 \\
132.9339 \\
132.9404 \\
132.9433 \\
133.0813 \\
133.4821 \\
133.7143 \\
134.1130 \\
134.5672\end{array}$ & $\begin{array}{l}426.4570 \\
427.6703 \\
428.6785 \\
430.3904 \\
432.2106 \\
433.9823 \\
434.3491 \\
434.7021 \\
435.0446 \\
435.3750 \\
435.3651 \\
436.0935 \\
436.1319 \\
436.1348 \\
436.1562 \\
436.1657 \\
436.6184 \\
437.9334 \\
438.6952 \\
440.0033 \\
441.4934\end{array}$ & $\begin{array}{l}57.4 \\
72.8 \\
46.3 \\
68.8 \\
21.4 \\
48.2 \\
44.1 \\
49.9 \\
41.2 \\
45.7 \\
59.9 \\
32.7 \\
31.5 \\
38.3 \\
49.7 \\
63.6 \\
58.4 \\
33.6 \\
43.5 \\
41.4 \\
26.4\end{array}$ \\
\hline 260.023 & 8.371 & $\begin{array}{r}162.905 \\
154.233 \\
145.994 \\
138.172 \\
125.681 \\
118.900 \\
107.522 \\
97.648 \\
88.656 \\
80.111 \\
80.102 \\
68.440 \\
61.806 \\
50.379 \\
38.853\end{array}$ & $\begin{array}{r}23.627 \\
22.370 \\
21.175 \\
20.040 \\
18.228 \\
17.245 \\
15.595 \\
14.163 \\
12.858 \\
11.619 \\
11.618 \\
9.926 \\
8.964 \\
7.307 \\
5.635\end{array}$ & $\begin{array}{l}136.5707 \\
136.8219 \\
137.0625 \\
137.2884 \\
137.6465 \\
137.8388 \\
138.1589 \\
138.4388 \\
138.6899 \\
138.9311 \\
138.9308 \\
139.2506 \\
139.4352 \\
139.7521 \\
140.0576\end{array}$ & $\begin{array}{l}448.0666 \\
448.8907 \\
449.6801 \\
450.4213 \\
451.5961 \\
452.2271 \\
453.2773 \\
454.1955 \\
455.0193 \\
455.8107 \\
455.8097 \\
456.8589 \\
457.4645 \\
458.5043 \\
459.5066\end{array}$ & $\begin{array}{l}47.7 \\
52.2 \\
44.7 \\
44.9 \\
52.4 \\
42.9 \\
40.9 \\
41.0 \\
42.2 \\
54.1 \\
37.0 \\
36.8 \\
35.2 \\
79.1 \\
51.0\end{array}$ \\
\hline
\end{tabular}


Table 46. Speed of Sound in HFC-125/CFC-115 (Continued)

\begin{tabular}{|c|c|c|c|c|c|c|}
\hline $\mathbf{T} / \mathbf{K}$ & $/ \operatorname{deg} F$ & $\mathbf{p} / \mathbf{k P a}$ & p/psi & $\mathbf{u} /(\mathbf{m} / \mathbf{s})$ & $\mathbf{u} /(\mathbf{f t} / \mathbf{s})$ & $10^{6 *} \mathrm{du} / \mathrm{u}$ \\
\hline 279.988 & 44.308 & $\begin{array}{r}659.333 \\
614.809 \\
549.469 \\
485.542 \\
422.837 \\
358.699 \\
294.764 \\
231.099 \\
166.746 \\
114.415 \\
114.409 \\
102.872 \\
87.213 \\
78.424 \\
78.420 \\
70.472 \\
59.639 \\
50.526 \\
38.550\end{array}$ & $\begin{array}{r}95.628 \\
89.170 \\
79.694 \\
70.422 \\
61.327 \\
52.025 \\
42.752 \\
33.518 \\
24.184 \\
16.594 \\
16.594 \\
14.920 \\
12.649 \\
11.374 \\
11.374 \\
10.221 \\
8.650 \\
7.328 \\
5.591\end{array}$ & $\begin{array}{l}129.4841 \\
130.8155 \\
132.6964 \\
134.4609 \\
136.1270 \\
137.7722 \\
139.3577 \\
140.8860 \\
142.3859 \\
143.5739 \\
143.5755 \\
143.8318 \\
144.1832 \\
144.3783 \\
144.3763 \\
144.5548 \\
144.7897 \\
144.9861 \\
145.2484\end{array}$ & $\begin{array}{l}424.8166 \\
429.1847 \\
435.3556 \\
441.1447 \\
446.6109 \\
452.0085 \\
457.2103 \\
462.2244 \\
467.1453 \\
471.0430 \\
471.0482 \\
471.8891 \\
473.0420 \\
473.6821 \\
473.6755 \\
474.2611 \\
475.0318 \\
475.6762 \\
476.5367\end{array}$ & $\begin{array}{l}92.4 \\
86.7 \\
78.5 \\
71.5 \\
66.9 \\
64.1 \\
61.0 \\
55.8 \\
51.6 \\
49.9 \\
42.1 \\
49.8 \\
27.1 \\
38.2 \\
46.0 \\
46.3 \\
37.7 \\
36.8 \\
39.8\end{array}$ \\
\hline 300.003 & 80.335 & $\begin{array}{r}999.087 \\
910.396 \\
823.948 \\
718.298 \\
613.670 \\
513.115 \\
408.108 \\
307.469 \\
204.506 \\
102.551 \\
102.553 \\
86.100 \\
76.937 \\
64.636 \\
51.711\end{array}$ & $\begin{array}{r}144.905 \\
132.042 \\
119.504 \\
104.180 \\
89.005 \\
74.421 \\
59.191 \\
44.595 \\
29.661 \\
14.874 \\
14.874 \\
12.488 \\
11.159 \\
9.375 \\
7.500\end{array}$ & $\begin{array}{l}129.8952 \\
132.1476 \\
134.2462 \\
136.6915 \\
139.0036 \\
141.1333 \\
143.2722 \\
145.2472 \\
147.2006 \\
149.0744 \\
149.0746 \\
149.3703 \\
149.5376 \\
149.7584 \\
149.9851\end{array}$ & $\begin{array}{l}426.1654 \\
433.5551 \\
440.4403 \\
448.4629 \\
456.0486 \\
463.0358 \\
470.0532 \\
476.5328 \\
482.9416 \\
489.0892 \\
489.0899 \\
490.0600 \\
490.6089 \\
491.3333 \\
492.0771\end{array}$ & $\begin{array}{r}157.6 \\
150.0 \\
102.8 \\
92.8 \\
81.7 \\
75.8 \\
71.6 \\
65.5 \\
56.4 \\
45.5 \\
46.8 \\
46.0 \\
44.5 \\
43.4 \\
38.7\end{array}$ \\
\hline
\end{tabular}


Table 46. Speed of Sound in HFC-125/CFC-115 (Continued)

\begin{tabular}{|c|c|c|c|c|c|c|}
\hline $\mathbf{T} / \mathbf{K}$ & $/ \operatorname{deg} F$ & $\mathbf{p} / \mathbf{k P a}$ & p/psi & $\mathbf{u} /(\mathbf{m} / \mathbf{s})$ & $\mathbf{u} /(\mathbf{f t} / \mathbf{s})$ & $10^{6 *} \mathrm{du} / \mathrm{u}$ \\
\hline 319.988 & 11.6 .308 & $\begin{array}{r}1023.022 \\
840.164 \\
840.056 \\
778.982 \\
778.692 \\
778.573 \\
692.805 \\
606.223 \\
521.142 \\
435.923 \\
348.595 \\
264.250 \\
178.362 \\
103.268 \\
99.928 \\
96.191 \\
92.670 \\
89.284 \\
89.286 \\
65.168 \\
40.224\end{array}$ & $\begin{array}{r}148.377 \\
121.855 \\
121.840 \\
112.982 \\
112.940 \\
112.922 \\
100.483 \\
87.925 \\
75.585 \\
63.225 \\
50.559 \\
38.326 \\
25.869 \\
14.978 \\
14.493 \\
13.951 \\
13.441 \\
12.950 \\
12.950 \\
9.452 \\
5.834\end{array}$ & $\begin{array}{l}138.7143 \\
142.0787 \\
142.0814 \\
143.1592 \\
143.1639 \\
143.1675 \\
144.6548 \\
146.150 \\
147.5263 \\
148.9051 \\
150.2939 \\
151.6026 \\
152.9118 \\
154.0384 \\
154.0900 \\
154.1459 \\
154.1994 \\
154.2438 \\
154.2475 \\
154.6063 \\
154.9700\end{array}$ & $\begin{array}{l}455.0994 \\
466.1375 \\
466.1463 \\
469.6824 \\
469.6978 \\
469.7096 \\
474.5892 \\
479.3799 \\
484.0102 \\
488.5338 \\
493.0902 \\
497.3839 \\
501.6791 \\
505.3753 \\
505.5446 \\
505.7280 \\
505.9036 \\
506.0492 \\
506.0613 \\
507.2385 \\
508.4318\end{array}$ & $\begin{array}{r}178.7 \\
86.0 \\
132.8 \\
86.4 \\
88.3 \\
89.2 \\
82.0 \\
78.8 \\
78.1 \\
69.8 \\
53.1 \\
55.9 \\
52.4 \\
41.7 \\
39.9 \\
43.0 \\
47.3 \\
52.7 \\
59.7 \\
50.5 \\
53.7\end{array}$ \\
\hline 340.003 & 152.335 & $\begin{array}{r}1006.522 \\
911.577 \\
810.912 \\
720.791 \\
506.620 \\
405.167 \\
305.879 \\
201.865 \\
173.768 \\
136.717 \\
77.987 \\
59.946\end{array}$ & $\begin{array}{r}145.984 \\
132.213 \\
117.613 \\
104.542 \\
73.479 \\
58.764 \\
44.364 \\
29.278 \\
25.203 \\
19.829 \\
11.311 \\
8.694\end{array}$ & $\begin{array}{l}146.8144 \\
148.1678 \\
149.5761 \\
150.8154 \\
153.6838 \\
155.0068 \\
156.2808 \\
157.5952 \\
157.9451 \\
158.4074 \\
159.1366 \\
159.3592\end{array}$ & $\begin{array}{l}481.6746 \\
486.1148 \\
490.7352 \\
494.8012 \\
504.2120 \\
508.5525 \\
512.7323 \\
517.0446 \\
518.1926 \\
519.7093 \\
522.1017 \\
522.8320\end{array}$ & $\begin{array}{r}108.4 \\
92.2 \\
83.0 \\
80.0 \\
66.0 \\
57.9 \\
54.4 \\
50.7 \\
47.6 \\
45.0 \\
43.1 \\
50.7\end{array}$ \\
\hline
\end{tabular}


Table 46. Speed of Sound in HFC-125/CFC-115 (Continued)

\begin{tabular}{|c|c|c|c|c|c|c|}
\hline $\mathbf{T} / \mathbf{K}$ & /deg F & $\mathbf{p} / \mathbf{k P a}$ & p/psi & $\mathbf{u} /(\mathbf{m} / \mathbf{s})$ & $\mathbf{u} /(\mathbf{f t} / \mathbf{s})$ & $10^{6 *} \mathrm{du} / \mathrm{u}$ \\
\hline 359.958 & 188.254 & $\begin{array}{r}1002.010 \\
911.464 \\
802.369 \\
707.538 \\
615.983 \\
500.468 \\
411.293 \\
306.302 \\
203.795 \\
102.643 \\
102.651 \\
93.791 \\
83.275 \\
72.534 \\
61.698 \\
51.568 \\
42.753\end{array}$ & $\begin{array}{r}145.329 \\
132.197 \\
116.374 \\
102.620 \\
89.341 \\
72.587 \\
59.653 \\
44.425 \\
29.558 \\
14.887 \\
14.888 \\
13.603 \\
12.078 \\
10.520 \\
8.949 \\
7.479 \\
6.201\end{array}$ & $\begin{array}{l}153.6674 \\
154.6952 \\
155.9167 \\
156.9693 \\
157.9724 \\
159.2245 \\
160.1808 \\
161.2932 \\
162.3707 \\
163.4221 \\
163.4234 \\
163.5169 \\
163.6240 \\
163.7383 \\
163.8479 \\
163.9537 \\
164.0431\end{array}$ & $\begin{array}{l}504.1581 \\
507.5302 \\
511.5378 \\
514.9911 \\
518.2822 \\
522.3901 \\
525.5276 \\
529.1772 \\
532.7123 \\
536.1617 \\
536.1660 \\
536.4728 \\
536.8241 \\
537.1992 \\
537.5587 \\
537.9059 \\
538.1992\end{array}$ & $\begin{array}{l}99.0 \\
97.0 \\
87.8 \\
78.9 \\
69.9 \\
67.1 \\
62.1 \\
57.4 \\
56.8 \\
48.2 \\
45.3 \\
39.2 \\
40.0 \\
36.3 \\
47.5 \\
36.0 \\
24.2\end{array}$ \\
\hline 379.983 & 224.299 & $\begin{array}{r}999.315 \\
922.664 \\
821.681 \\
717.610 \\
613.968 \\
513.912 \\
411.334 \\
307.715 \\
205.322 \\
130.146 \\
130.129 \\
120.762 \\
112.064 \\
98.226 \\
91.150 \\
79.972 \\
70.158 \\
60.105 \\
48.982 \\
39.289\end{array}$ & $\begin{array}{r}144.938 \\
133.821 \\
119.175 \\
104.080 \\
89.049 \\
74.537 \\
59.659 \\
44.630 \\
29.779 \\
18.876 \\
18.874 \\
17.515 \\
16.254 \\
14.246 \\
13.220 \\
11.599 \\
10.176 \\
8.717 \\
7.104 \\
5.698\end{array}$ & $\begin{array}{l}159.8448 \\
160.5522 \\
161.4733 \\
162.4188 \\
163.3534 \\
164.2508 \\
165.1641 \\
166.0766 \\
166.9781 \\
167.6355 \\
167.6376 \\
167.7181 \\
167.7942 \\
167.9107 \\
167.9732 \\
168.0709 \\
168.1574 \\
168.2377 \\
168.3309 \\
168.4172\end{array}$ & $\begin{array}{l}524.4252 \\
526.7461 \\
529.7680 \\
532.8701 \\
535.9363 \\
538.8806 \\
541.8770 \\
544.8707 \\
547.8284 \\
549.9852 \\
549.9921 \\
550.2562 \\
550.5059 \\
550.8881 \\
551.0932 \\
551.4138 \\
551.6975 \\
551.9609 \\
552.2667 \\
552.5499\end{array}$ & $\begin{array}{l}95.8 \\
94.0 \\
85.6 \\
82.4 \\
79.3 \\
69.0 \\
58.6 \\
61.3 \\
42.9 \\
40.1 \\
41.7 \\
49.3 \\
42.8 \\
50.0 \\
42.9 \\
34.9 \\
32.8 \\
37.8 \\
26.0 \\
54.6\end{array}$ \\
\hline
\end{tabular}


Table 46. Speed of Sound in HFC-125/CFC-115 (Continued)

\begin{tabular}{lrrrrrr}
\hline $\mathbf{T} / \mathbf{K}$ & /deg F & $\mathbf{p} / \mathbf{k P a}$ & $\mathbf{p} / \mathbf{p s i}$ & $\mathbf{u} /(\mathbf{m} / \mathbf{s})$ & $\mathbf{u} /(\mathbf{f t} / \mathbf{s})$ & $\mathbf{1 0}^{\mathbf{6} * \mathbf{d u} / \mathbf{u}}$ \\
\hline & & & & & & \\
400.000 & 260.330 & 1016.426 & 147.420 & 165.3693 & 542.5502 & 67.9 \\
& & 935.971 & 135.751 & 165.9991 & 544.6165 & 46.2 \\
& 826.676 & 119.899 & 166.8218 & 547.3156 & 39.8 \\
& & 725.010 & 105.154 & 167.5817 & 549.8087 & 47.4 \\
& 625.623 & 90.739 & 168.3246 & 552.2461 & 58.8 \\
& 519.173 & 75.300 & 169.1190 & 554.8524 & 43.5 \\
& 415.180 & 60.217 & 169.8898 & 557.3812 & 50.5 \\
& 312.057 & 45.260 & 170.6565 & 559.8966 & 37.7 \\
& 207.025 & 30.026 & 171.4334 & 562.4455 & 31.7
\end{tabular}


Table 47. Ideal-Gas Heat Capacity, $\mathrm{C}_{\mathrm{p}}^{\mathrm{o}}$, of HFC-125

\begin{tabular}{lrrr}
\hline & & & \\
& deg $\mathbf{F}$ & $\mathbf{C}_{\mathbf{p}}^{\mathbf{0}} / \mathbf{R}$ & $\mathbf{s i g C}_{\mathbf{p}}^{\mathbf{0}} \mathbf{R}$ \\
\hline & & & \\
240.007 & -27.657 & 9.9700 & 0.020 \\
260.023 & 8.371 & 10.451 & 0.019 \\
279.988 & 44.308 & 10.925 & 0.013 \\
300.003 & 80.335 & 11.390 & 0.015 \\
319.988 & 116.308 & 11.847 & 0.013 \\
340.003 & 152.335 & 12.283 & 0.016 \\
359.958 & 188.254 & 12.706 & 0.008 \\
379.983 & 224.299 & 13.129 & 0.009 \\
\hline
\end{tabular}


Table 48(a). Acoustic Viral Coefficients for HFC-125 (SI units)

\begin{tabular}{cccc}
\hline $\mathbf{T}$ & $\mathbf{1 0 ^ { 6 }}$ beta & $\mathbf{1 0 ^ { 9 } \text { gamma }}$ & $\mathbf{1 0}^{\mathbf{1 2} \text { delta }}$ \\
\hline $\mathbf{K}$ & $/\left(\mathbf{m}^{\mathbf{3}} / \mathbf{m o l}\right)$ & $/\left(\mathbf{m}^{\mathbf{3}} / \mathbf{m o l}-\mathbf{P a}\right)$ & $/\left(\mathbf{m}^{\mathbf{3}} / \mathbf{m o l}^{\left.-\mathbf{P a}^{2}\right)}\right.$ \\
\hline & & & \\
240.007 & $-971.41+/-4.12$ & $-0.3713+/-0.0186$ & - \\
260.023 & $-811.43+/-4.31$ & $-0.1682+/-0.0204$ & - \\
279.988 & $-683.77+/-2.20$ & $-0.0754+/-0.0087$ & $-5.21+/-0.97$ \\
300.003 & $-582.40+/-1.88$ & $-0.0400+/-0.0051$ & $-2.38+/-0.39$ \\
319.988 & $-499.66+/-1.34$ & $-0.0218+/-0.0031$ & $-1.04+/-0.20$ \\
340.003 & $-430.70+/-1.44$ & $-0.0139+/-0.0025$ & - \\
359.958 & $-373.18+/-0.76$ & $-0.0072+/-0.0005$ & - \\
379.983 & $-323.18+/-0.53$ & $-0.0021+/-0.0005$ & - \\
\hline
\end{tabular}

Table 48(b). Acoustic Viral Coefficients for HFC-125 (PI units)

\begin{tabular}{|c|c|c|c|}
\hline $\mathbf{T}$ & $10^{6}$ beta & $10^{9}$ gamma & $10^{12}$ delta \\
\hline$/ \mathbf{K}$ & $/\left(\mathbf{m}^{3} / \mathbf{m o l}\right)$ & $/\left(\mathbf{m}^{3} / \mathbf{m o l}-\mathbf{P a}\right)$ & $/\left(\mathbf{m}^{3} / \mathbf{m o l}-\mathbf{P a}^{2}\right)$ \\
\hline-27.657 & $-63.524+/-0.269$ & $-16.741+/-0.839$ & - \\
\hline 8.371 & $-53.062+/-0.282$ & $-7.584+/-0.920$ & - \\
\hline 44.308 & $-44.714+/-0.144$ & $-3.400+/-0.392$ & $-16.200+/-3.0$ \\
\hline 80.335 & $-38.085+/-0.123$ & $-1.803+/-0.230$ & $-7.399+/-1.2$ \\
\hline 116.308 & $-32.674+/-0.088$ & $-0.983+/-0.140$ & $-3.233+/-0.62$ \\
\hline 152.335 & $-28.165+/-0.094$ & $-0.627+/-0.113$ & - \\
\hline 188.254 & $-24.403+/-0.050$ & $-0.325+/-0.022$ & - \\
\hline 224.299 & $-21.134+/-0.035$ & $-0.095+/-0.022$ & - \\
\hline
\end{tabular}


Table 49 (a). Experimental liquid heat capacity data for HFC-125.

\begin{tabular}{|c|c|c|c|c|c|c|c|c|c|}
\hline \multirow[t]{2}{*}{$T$} & $\rho$ & \multirow{2}{*}{$\begin{array}{c}\mathrm{P} \\
\mathrm{MPa}\end{array}$} & \multirow{2}{*}{$\begin{array}{l}\text { N } \\
\text { mol. }\end{array}$} & \multirow{2}{*}{$\begin{array}{c}V_{\text {bomb }} \\
\mathrm{cm}^{3}\end{array}$} & \multirow{2}{*}{$\begin{array}{l}\Delta \mathrm{T} \\
\mathrm{K}\end{array}$} & \multirow{2}{*}{$\begin{array}{c}Q_{\text {tare }} \\
\mathrm{J}\end{array}$} & \multirow{2}{*}{$\begin{array}{l}Q / \Delta T \\
J K^{-1}\end{array}$} & \multicolumn{2}{|c|}{$w_{p v, m}$} \\
\hline & mol $\mathrm{dm}^{-3}$ & & & & & & & J I & $1^{-1} \mathrm{~K}^{-1}$ \\
\hline $\begin{array}{l}288.4648 \\
293.8343 \\
299.2337 \\
304.5630 \\
309.9158 \\
315.2325 \\
320.5321 \\
325.8348 \\
331.0905 \\
336.3549 \\
341.6050 \\
288.8761 \\
293.1529 \\
297.4483 \\
301.7224 \\
305.9403 \\
310.1918 \\
314.4175 \\
318.6428 \\
322.8706 \\
327.0519 \\
331.2712 \\
335.4435 \\
339.6184\end{array}$ & $\begin{array}{l}10.5874 \\
10.5799 \\
10.5724 \\
10.5649 \\
10.5574 \\
10.5500 \\
10.5425 \\
10.5351 \\
10.5278 \\
10.5203 \\
10.5129 \\
10.5868 \\
10.5809 \\
10.5749 \\
10.5689 \\
10.5630 \\
10.5571 \\
10.5511 \\
10.5452 \\
10.5393 \\
10.5385 \\
10.5275 \\
10.5216 \\
10.5157\end{array}$ & $\begin{array}{r}3.7918 \\
6.5902 \\
9.4072 \\
12.1847 \\
14.9677 \\
17.7229 \\
20.4586 \\
23.1843 \\
25.8738 \\
28.5550 \\
31.2155 \\
4.0058 \\
6.2347 \\
8.4758 \\
10.7049 \\
12.9016 \\
15.1110 \\
17.3012 \\
19.4846 \\
21.6621 \\
23.8082 \\
25.9660 \\
28.0917 \\
30.2104\end{array}$ & $\begin{array}{l}0.7764 \\
0.7764 \\
0.7764 \\
0.7763 \\
0.7763 \\
0.7763 \\
0.7763 \\
0.7763 \\
0.7763 \\
0.7763 \\
0.7763 \\
0.7764 \\
0.7764 \\
0.7764 \\
0.7763 \\
0.7763 \\
0.7763 \\
0.7763 \\
0.7763 \\
0.7763 \\
0.7767 \\
0.7763 \\
0.7763 \\
0.7763\end{array}$ & $\begin{array}{l}73.332 \\
73.382 \\
73.432 \\
73.483 \\
73.533 \\
73.584 \\
73.635 \\
73.686 \\
73.737 \\
73.788 \\
73.840 \\
73.336 \\
73.376 \\
73.416 \\
73.456 \\
73.496 \\
73.536 \\
73.576 \\
73.617 \\
73.658 \\
73.698 \\
73.739 \\
73.779 \\
73.820\end{array}$ & $\begin{array}{l}5.3749 \\
5.3361 \\
5.3092 \\
5.2661 \\
5.2216 \\
5.2042 \\
5.1719 \\
5.1558 \\
5.1263 \\
5.0925 \\
5.0740 \\
4.2374 \\
4.2016 \\
4.1695 \\
4.2014 \\
4.1853 \\
4.1464 \\
4.1263 \\
4.1143 \\
4.0947 \\
4.0715 \\
4.0381 \\
4.0194 \\
4.0081\end{array}$ & $\begin{array}{l}442.907 \\
441.610 \\
441.264 \\
439.498 \\
437.561 \\
437.827 \\
436.750 \\
436.982 \\
436.020 \\
434.728 \\
434.973 \\
349.293 \\
347.535 \\
346.054 \\
349.874 \\
349.671 \\
347.534 \\
346.937 \\
346.980 \\
346.349 \\
345.362 \\
343.498 \\
342.894 \\
343.011\end{array}$ & $\begin{array}{l}160.204 \\
161.307 \\
162.038 \\
163.129 \\
164.351 \\
164.854 \\
165.785 \\
166.161 \\
166.900 \\
167.879 \\
168.337 \\
160.857 \\
161.841 \\
162.955 \\
161.580 \\
162.105 \\
163.503 \\
164.237 \\
164.761 \\
165.171 \\
166.230 \\
167.369 \\
167.970 \\
168.725\end{array}$ & $\begin{array}{l}7.527 \\
7.753 \\
7.898 \\
8.007 \\
8.123 \\
8.276 \\
8.436 \\
8.618 \\
8.777 \\
8.912 \\
9.013 \\
5.952 \\
6.093 \\
6.190 \\
6.298 \\
6.373 \\
6.440 \\
6.528 \\
6.632 \\
6.730 \\
6.834 \\
6.928 \\
7.017 \\
7.104\end{array}$ & $\begin{array}{r}98.81 \\
99.72 \\
100.17 \\
101.10 \\
102.20 \\
102.40 \\
103.15 \\
103.19 \\
103.72 \\
104.54 \\
104.64 \\
99.61 \\
100.47 \\
101.51 \\
99.36 \\
99.67 \\
101.09 \\
101.67 \\
101.99 \\
102.17 \\
103.14 \\
104.31 \\
104.74 \\
105.34\end{array}$ \\
\hline
\end{tabular}


Table 49 (b). Experimental liquid heat capacity data for HFC-125.

\begin{tabular}{|c|c|c|c|c|c|c|c|c|c|}
\hline \multirow{2}{*}{$\mathbf{T}$} & $\rho$ & \multirow{2}{*}{$\begin{array}{l}\text { P } \\
\text { psia }\end{array}$} & \multirow{2}{*}{$\begin{array}{l}\mathbf{N} \\
1 \mathrm{~b}\end{array}$} & \multirow{2}{*}{$\begin{array}{c}V_{\text {bomb }} \\
\text { in }^{3}\end{array}$} & \multirow{2}{*}{$\begin{array}{l}\Delta \mathbf{T} \\
{ }^{\circ} \mathrm{F}\end{array}$} & \multirow{2}{*}{$\begin{array}{l}Q_{\text {tare }} \\
\text { Btu }\end{array}$} & $Q / \Delta T$ & \multicolumn{2}{|c|}{$W_{p v, m} \quad C_{v, \exp }$} \\
\hline & Ib $E t^{-3}$ & & & & & & Btu ${ }^{\circ} \mathrm{F}^{-1}$ & Btu & $1 b^{-1} \circ F^{-1}$ \\
\hline $\begin{array}{r}59.567 \\
69.232 \\
78.951 \\
88.543 \\
98.178 \\
107.748 \\
117.288 \\
126.833 \\
136.293 \\
145.769 \\
155.219 \\
60.307 \\
68.005 \\
75.737 \\
83.430 \\
91.023 \\
98.675 \\
106.281 \\
113.887 \\
121.497 \\
129.023 \\
136.618 \\
144.128 \\
151.643\end{array}$ & $\begin{array}{l}79.33 \\
79.27 \\
79.21 \\
79.16 \\
79.10 \\
79.05 \\
78.99 \\
78.94 \\
78.88 \\
78.82 \\
78.77 \\
79.32 \\
79.28 \\
79.23 \\
79.19 \\
79.14 \\
79.10 \\
79.06 \\
79.01 \\
78.97 \\
78.96 \\
78.88 \\
78.83 \\
78.79\end{array}$ & $\begin{array}{r}550.0 \\
955.8 \\
1364.4 \\
1767.2 \\
2170.9 \\
2570.5 \\
2967.3 \\
3362.6 \\
3752.7 \\
4141.6 \\
4527.4 \\
581.0 \\
904.3 \\
1229.3 \\
1552.6 \\
1871.2 \\
2191.7 \\
2509.3 \\
2826.0 \\
3141.8 \\
3453.1 \\
3766.1 \\
4074.4 \\
4381.7\end{array}$ & $\begin{array}{l}0.205 \\
0.205 \\
0.205 \\
0.205 \\
0.205 \\
0.205 \\
0.205 \\
0.205 \\
0.205 \\
0.205 \\
0.205 \\
0.205 \\
0.205 \\
0.205 \\
0.205 \\
0.205 \\
0.205 \\
0.205 \\
0.205 \\
0.205 \\
0.206 \\
0.205 \\
0.205 \\
0.205\end{array}$ & $\begin{array}{l}4.475 \\
4.478 \\
4.481 \\
4.484 \\
4.487 \\
4.490 \\
4.494 \\
4.497 \\
4.500 \\
4.503 \\
4.506 \\
4.475 \\
4.478 \\
4.480 \\
4.483 \\
4.485 \\
4.487 \\
4.490 \\
4.492 \\
4.495 \\
4.497 \\
4.500 \\
4.502 \\
4.505\end{array}$ & $\begin{array}{l}9.675 \\
9.605 \\
9.557 \\
9.479 \\
9.399 \\
9.368 \\
9.309 \\
9.280 \\
9.227 \\
9.166 \\
9.133 \\
7.627 \\
7.563 \\
7.505 \\
7.563 \\
7.534 \\
7.464 \\
7.427 \\
7.406 \\
7.370 \\
7.329 \\
7.269 \\
7.235 \\
7.215\end{array}$ & $\begin{array}{l}0.420 \\
0.419 \\
0.419 \\
0.417 \\
0.415 \\
0.415 \\
0.414 \\
0.414 \\
0.414 \\
0.412 \\
0.413 \\
0.331 \\
0.330 \\
0.328 \\
0.332 \\
0.332 \\
0.330 \\
0.329 \\
0.329 \\
0.328 \\
0.328 \\
0.326 \\
0.325 \\
0.325\end{array}$ & $\begin{array}{ll}0.084 & 0.1 \\
0.085 & 0 . \\
0.085 & 0.0 \\
0.086 & 0.0 \\
0.087 & 0.0 \\
0.087 & 0 . \\
0.087 & 0 . \\
0.088 & 0 . \\
0.088 & 0 . \\
0.088 & 0 . \\
0.0889 & 0 . \\
0.085 & 0 . \\
0.0855 & 0 . \\
0.086 & 0 . \\
0.085 & 0 . \\
0.085 & 0 . \\
0.086 & 0 . \\
0.087 & 0 . \\
0.087 & 0 . \\
0.087 & 0 . \\
0.088 & 0 . \\
0.088 & 0 . \\
0.089 & 0 . \\
0.089 & 0 .\end{array}$ & $\begin{array}{l}150 \\
154 \\
157 \\
159 \\
162 \\
165 \\
168 \\
172 \\
175 \\
177 \\
0179 \\
0119 \\
0121 \\
0123 \\
0125 \\
0127 \\
0128 \\
0130 \\
0132 \\
0134 \\
0136 \\
0138 \\
0140 \\
0141\end{array}$ & $\begin{array}{l}0.1968 \\
0.1986 \\
0.1995 \\
0.2013 \\
0.2035 \\
0.2039 \\
0.2054 \\
0.2055 \\
0.2065 \\
0.2082 \\
0.2084 \\
0.1984 \\
0.2001 \\
0.2021 \\
0.1979 \\
0.1985 \\
0.2013 \\
0.2025 \\
0.2031 \\
0.2035 \\
0.2054 \\
0.2077 \\
0.2086 \\
0.2098\end{array}$ \\
\hline
\end{tabular}


Table 50(a). Experimental liquid heat capacity data for HFC-125.

\begin{tabular}{|c|c|c|c|c|c|c|c|c|c|}
\hline \multirow[t]{2}{*}{$\mathbf{T}$} & $\rho$ & \multirow{2}{*}{$\begin{array}{c}\mathbf{P} \\
\mathrm{MPa}\end{array}$} & \multirow{2}{*}{$\begin{array}{l}\mathrm{N} \\
\text { mol }\end{array}$} & \multirow{2}{*}{$\begin{array}{c}V_{\text {bomb }} \\
\mathrm{cm}^{3}\end{array}$} & \multirow{2}{*}{$\begin{array}{l}\Delta \mathrm{T} \\
\mathrm{K}\end{array}$} & \multirow{2}{*}{$\begin{array}{c}Q_{\text {tare }} \\
J\end{array}$} & \multirow{2}{*}{$\begin{array}{l}Q / \Delta T \\
J \quad K^{-1}\end{array}$} & \multicolumn{2}{|c|}{$W_{p v, m}$} \\
\hline & mol $\mathrm{dm}^{-3}$ & & & & & & & $J$ & $1^{-1} k^{-1}$ \\
\hline $\begin{array}{l}274.0725 \\
280.5589 \\
287.0378 \\
293.4970 \\
299.8980 \\
306.2467 \\
275.7181 \\
281.0479 \\
286.3325 \\
291.5642 \\
296.8015 \\
301.9998 \\
306.8817 \\
313.8655 \\
320.8002 \\
311.9560 \\
317.0758 \\
322.1551\end{array}$ & $\begin{array}{l}11.1366 \\
11.1261 \\
11.1156 \\
11.1050 \\
11.0946 \\
11.0987 \\
11.1339 \\
11.1253 \\
11.1168 \\
11.1082 \\
11.0996 \\
11.0912 \\
11.0832 \\
11.0718 \\
11.0604 \\
11.0749 \\
11.0665 \\
11.0582\end{array}$ & $\begin{array}{r}3.8317 \\
7.8191 \\
11.8615 \\
15.8752 \\
19.8149 \\
23.6876 \\
4.8303 \\
8.1235 \\
11.4215 \\
14.6779 \\
17.9139 \\
21.1005 \\
24.0734 \\
28.2995 \\
32.4633 \\
27.1470 \\
30.2318 \\
33.2717\end{array}$ & $\begin{array}{l}0.8161 \\
0.8161 \\
0.8161 \\
0.8161 \\
0.8161 \\
0.8171 \\
0.8161 . \\
0.8161 \\
0.8161 \\
0.8161 \\
0.8161 \\
0.8161 \\
0.8160 \\
0.8160 \\
0.8160 \\
0.8160 \\
0.8160 \\
0.8160\end{array}$ & $\begin{array}{l}73.285 \\
73.351 \\
73.419 \\
73.487 \\
73.554 \\
73.621 \\
73.301 \\
73.356 \\
73.411 \\
73.466 \\
73.521 \\
73.576 \\
73.628 \\
73.703 \\
73.777 \\
73.682 \\
73.737 \\
73.792\end{array}$ & $\begin{array}{l}6.5490 \\
6.5710 \\
6.5106 \\
6.4280 \\
6.3791 \\
6.3562 \\
5.3507 \\
5.3127 \\
5.2693 \\
5.2290 \\
5.1866 \\
5.1961 \\
6.9756 \\
6.9413 \\
6.8607 \\
5.0800 \\
5.0610 \\
5.0197\end{array}$ & $\begin{array}{l}533.193 \\
537.956 \\
535.873 \\
531.831 \\
530.467 \\
531.166 \\
436.253 \\
435.122 \\
433.453 \\
431.963 \\
430.252 \\
432.801 \\
583.204 \\
583.377 \\
579.473 \\
426.350 \\
426.346 \\
424.377\end{array}$ & $\begin{array}{l}162.483 \\
161.833 \\
163.151 \\
165.141 \\
166.176 \\
166.543 \\
161.405 \\
161.942 \\
163.396 \\
164.683 \\
165.748 \\
165.506 \\
167.201 \\
168.379 \\
169.739 \\
168.704 \\
169.722 \\
170.500\end{array}$ & $\begin{array}{l}12.164 \\
13.551 \\
13.879 \\
13.986 \\
14.056 \\
14.152 \\
10.505 \\
10.986 \\
11.229 \\
11.336 \\
11.376 \\
11.463 \\
15.606 \\
15.850 \\
16.085 \\
11.572 \\
11.737 \\
11.859\end{array}$ & $\begin{array}{l}97.47 \\
95.92 \\
96.93 \\
98.80 \\
99.53 \\
99.32 \\
95.90 \\
96.01 \\
97.29 \\
98.40 \\
99.26 \\
98.54 \\
100.20 \\
101.06 \\
102.16 \\
101.61 \\
102.44 \\
102.98\end{array}$ \\
\hline
\end{tabular}

Table $50(\mathrm{~b})$. Experimental liquid heat capacity data for HFC-125.

\begin{tabular}{|c|c|c|c|c|c|c|c|c|c|c|}
\hline \multirow{2}{*}{$\begin{array}{l}T \\
{ }^{\circ} \mathbf{F}\end{array}$} & $\rho$ & \multirow{2}{*}{$\begin{array}{l}\text { P } \\
\text { psia }\end{array}$} & \multirow{2}{*}{$\begin{array}{l}\text { N } \\
1 b\end{array}$} & \multirow{2}{*}{$\begin{array}{c}V_{\text {bomb }} \\
\text { in }^{3}\end{array}$} & \multirow{2}{*}{$\begin{array}{l}\Delta T \\
{ }^{\circ} \mathrm{F}\end{array}$} & $\mathbf{Q}_{\text {tare }}$ & \multicolumn{2}{|c|}{$Q / \Delta T$} & \multicolumn{2}{|c|}{$W_{p v, m} \quad C_{v, \exp }$} \\
\hline & $I b E t^{-3}$ & & & & & Btu & Btu & ${ }^{\circ} F^{-1}$ & Btu & $1 b^{-1} \circ F^{-1}$ \\
\hline $\begin{array}{r}33.660 \\
45.336 \\
56.998 \\
68.625 \\
80.146 \\
91.574 \\
36.623 \\
46.216 \\
55.728 \\
65.146 \\
74.573 \\
83.930 \\
92.717 \\
105.288 \\
117.770 \\
101.851 \\
111.066 \\
120.209\end{array}$ & $\begin{array}{l}83.44 \\
83.36 \\
83.28 \\
83.21 \\
83.13 \\
83.16 \\
83.42 \\
83.36 \\
83.29 \\
83.23 \\
83.16 \\
83.10 \\
83.04 \\
82.96 \\
82.87 \\
82.98 \\
82.92 \\
82.85\end{array}$ & $\begin{array}{r}555.7 \\
1134.1 \\
1720.4 \\
2302.5 \\
2873.9 \\
3435.6 \\
700.6 \\
1178.2 \\
1656.6 \\
2128.9 \\
2598.2 \\
3060.4 \\
3491.6 \\
4104.5 \\
4708.4 \\
3937.3 \\
4384.8 \\
4825.7\end{array}$ & $\begin{array}{l}0.216 \\
0.216 \\
0.216 \\
0.216 \\
0.216 \\
0.216 \\
0.216 \\
0.216 \\
0.216 \\
0.216 \\
0.216 \\
0.216 \\
0.216 \\
0.216 \\
0.216 \\
0.216 \\
0.216 \\
0.216\end{array}$ & $\begin{array}{l}4.472 \\
4.476 \\
4.480 \\
4.484 \\
4.489 \\
4.493 \\
4.473 \\
4.476 \\
4.480 \\
4.483 \\
4.487 \\
4.490 \\
4.493 \\
4.498 \\
4.502 \\
4.496 \\
4.500 \\
4.503\end{array}$ & $\begin{array}{r}11.788 \\
11.828 \\
11.719 \\
11.570 \\
11.482 \\
11.441 \\
9.631 \\
9.563 \\
9.485 \\
9.412 \\
9.336 \\
9.353 \\
12.556 \\
12.494 \\
12.349 \\
9.144 \\
9.110 \\
9.035\end{array}$ & $\begin{array}{l}0.506 \\
0.510 \\
0.508 \\
0.504 \\
0.503 \\
0.504 \\
0.414 \\
0.413 \\
0.411 \\
0.410 \\
0.408 \\
0.410 \\
0.553 \\
0.553 \\
0.550 \\
0.404 \\
0.404 \\
0.403\end{array}$ & $\begin{array}{l}0.086 \\
0.085 \\
0.086 \\
0.087 \\
0.088 \\
0.088 \\
0.085 \\
0.085 \\
0.086 \\
0.087 \\
0.087 \\
0.087 \\
0.088 \\
0.089 \\
0.089 \\
0.089 \\
0.089 \\
0.090\end{array}$ & $\begin{array}{ll}3 & 0.0 \\
5 & 0.1 \\
5 & 0.1 \\
7 & 0.1 \\
3 & 0.1 \\
3 & 0.1 \\
5 & 0.1 \\
5 & 0.1 \\
5 & 0.1 \\
7 & 0.1 \\
7 & 0.1 \\
7 & 0.1 \\
8 & 0.1 \\
9 & 0.1 \\
9 & 0.1 \\
9 & 0.1 \\
9 & 0.1 \\
0 & 0 .\end{array}$ & $\begin{array}{l}0242 \\
0270 \\
0276 \\
0279 \\
0280 \\
0282 \\
0209 \\
0219 \\
0224 \\
0226 \\
0227 \\
0228 \\
0321 \\
0316 \\
0320 \\
0230 \\
0234 \\
0236\end{array}$ & $\begin{array}{l}0.1941 \\
0.1910 \\
0.1930 \\
0.1968 \\
0.1982 \\
0.1978 \\
0.1910 \\
0.1912 \\
0.1937 \\
0.1960 \\
0.1977 \\
0.1962 \\
0.1995 \\
0.2013 \\
0.2034 \\
0.2023 \\
0.2040 \\
0.2051\end{array}$ \\
\hline
\end{tabular}


Table 51 (a). Experimental liquid heat capacity data for HFC-125.

\begin{tabular}{|c|c|c|c|c|c|c|c|c|c|}
\hline \multirow[t]{2}{*}{$T$} & $\rho$ & \multirow{2}{*}{$\begin{array}{c}\mathbf{P} \\
\mathrm{MPa}\end{array}$} & \multirow{2}{*}{$\begin{array}{l}\mathbf{N} \\
\text { mol }\end{array}$} & \multirow{2}{*}{$\begin{array}{c}V_{\text {bomb }} \\
\mathrm{cm}^{3}\end{array}$} & \multirow{2}{*}{$\begin{array}{l}\Delta \mathbf{T} \\
\mathrm{K}\end{array}$} & \multirow{2}{*}{$\begin{array}{c}Q_{\text {tare }} \\
J\end{array}$} & \multirow{2}{*}{$\begin{array}{l}Q / \Delta T \\
J K^{-1}\end{array}$} & \multicolumn{2}{|c|}{$W_{p v, m}$} \\
\hline & mol $\mathrm{dm}^{-3}$ & & & & & & & J II & $\operatorname{mol}{ }^{-1} \mathrm{~K}^{-1}$ \\
\hline $\begin{array}{l}252.3975 \\
256.5229 \\
260.6610 \\
264.7836 \\
268.8769 \\
272.9449 \\
276.9962 \\
281.0191 \\
285.0159 \\
288.9984 \\
257.2973 \\
263.7876 \\
270.1849 \\
276.5500 \\
282.8579 \\
289.1170\end{array}$ & $\begin{array}{l}11.8614 \\
11.8533 \\
11.8451 \\
11.8369 \\
11.8286 \\
11.8205 \\
11.8125 \\
11.8045 \\
11.7966 \\
11.7887 \\
11.8518 \\
11.8388 \\
11.8260 \\
11.8134 \\
11.8009 \\
11.7885\end{array}$ & $\begin{array}{r}3.6978 \\
6.8320 \\
10.0711 \\
13.3216 \\
16.5325 \\
19.6895 \\
22.7961 \\
25.8492 \\
28.8617 \\
31.8550 \\
7.4336 \\
12.5367 \\
17.5516 \\
22.4556 \\
27.2370 \\
31.9441\end{array}$ & $\begin{array}{l}0.8684 \\
0.8684 \\
0.8684 \\
0.8683 \\
0.8683 \\
0.8683 \\
0.8683 \\
0.8683 \\
0.8683 \\
0.8683 \\
0.8684 \\
0.8683 \\
0.8683 \\
0.8683 \\
0.8683 \\
0.8683\end{array}$ & $\begin{array}{l}73.212 \\
73.260 \\
73.309 \\
73.359 \\
73.408 \\
73.458 \\
73.506 \\
73.555 \\
73.604 \\
73.652 \\
73.269 \\
73.347 \\
73.424 \\
73.501 \\
73.577 \\
73.653\end{array}$ & $\begin{array}{l}4.1437 \\
4.1992 \\
4.1712 \\
4.1510 \\
4.1305 \\
4.1025 \\
4.0765 \\
4.0526 \\
4.0254 \\
4.0044 \\
6.5418 \\
6.4747 \\
6.4240 \\
6.3775 \\
6.3259 \\
6.2597\end{array}$ & $\begin{array}{l}330.456 \\
336.309 \\
335.444 \\
335.137 \\
334.737 \\
333.678 \\
332.728 \\
331.911 \\
330.773 \\
330.117 \\
524.330 \\
522.247 \\
521.219 \\
520.344 \\
518.881 \\
516.091\end{array}$ & $\begin{array}{l}165.195 \\
162.965 \\
164.021 \\
164.818 \\
165.572 \\
166.703 \\
167.445 \\
168.323 \\
169.294 \\
170.054 \\
163.266 \\
164.721 \\
165.906 \\
167.438 \\
168.591 \\
170.248\end{array}$ & $\begin{array}{l}9.506 \\
10.886 \\
11.216 \\
11.372 \\
11.423 \\
11.432 \\
11.457 \\
11.538 \\
11.682 \\
11.919 \\
17.177 \\
17.652 \\
17.756 \\
17.887 \\
18.171 \\
18.752\end{array}$ & $\begin{array}{l}96.10 \\
92.85 \\
93.59 \\
94.09 \\
94.58 \\
95.53 \\
96.03 \\
96.69 \\
97.44 \\
97.93 \\
93.09 \\
94.08 \\
94.86 \\
96.06 \\
96.83 \\
98.13\end{array}$ \\
\hline
\end{tabular}

Table 51(b). Experimental liquid heat capacity data for HFC- 125 .

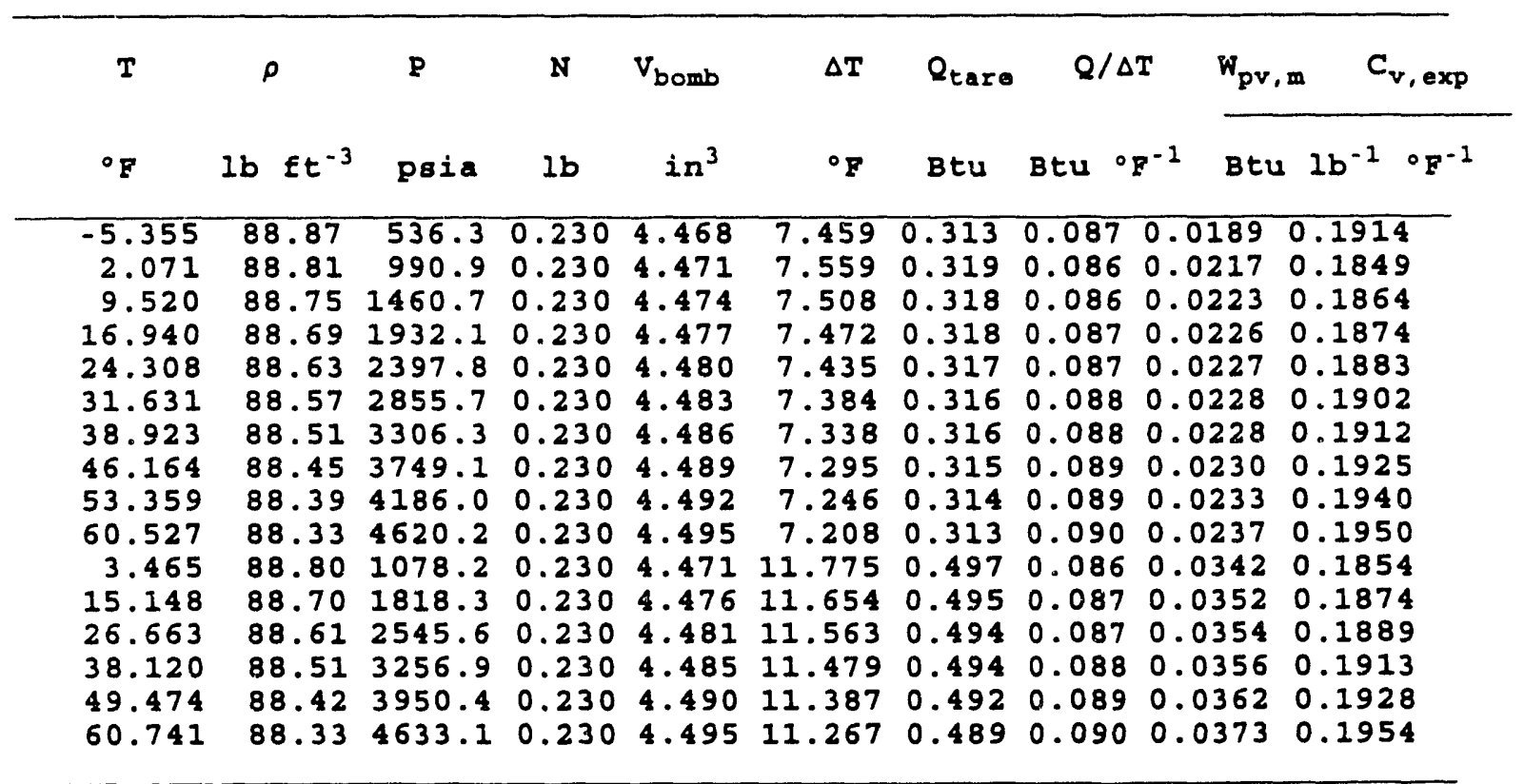


Table 52 (a). Experimental liquid heat capacity data for HFC-125.

\begin{tabular}{|c|c|c|c|c|c|c|c|c|c|}
\hline$T$ & $\rho$ & P & $\mathbf{N}$ & $V_{\text {bomb }}$ & $\Delta \mathbf{T}$ & $Q_{\text {tare }}$ & $Q / \Delta T$ & $W_{p v, m}$ & $C_{v, \exp }$ \\
\hline $\mathbf{K}$ & mol $\mathrm{dm}^{-3}$ & $\mathbf{M P a}$ & mol & $\mathrm{cm}^{3}$ & $\mathbf{K}$ & $\mathrm{J}$ & $\mathbf{J} \mathbf{K}^{-1}$ & $J$ mo & $1^{-1} k^{-1}$ \\
\hline $\begin{array}{l}235.2133 \\
239.3367 \\
243.4903 \\
247.6190 \\
251.7217 \\
255.7968 \\
259.8476 \\
263.8819 \\
267.8878 \\
237.1897 \\
241.3141 \\
245.4515 \\
249.5678 \\
253.6415 \\
257.7222 \\
261.7641 \\
265.7769 \\
269.7666\end{array}$ & $\begin{array}{l}12.3448 \\
12.3357 \\
12.3263 \\
12.3168 \\
12.3074 \\
12.2982 \\
12.2890 \\
12.2798 \\
12.2708 \\
12.3406 \\
12.3312 \\
12.3218 \\
12.3123 \\
12.3031 \\
12.2938 \\
12.2846 \\
12.2755 \\
12.2665\end{array}$ & $\begin{array}{r}2.2258 \\
5.8368 \\
9.6376 \\
13.4334 \\
17.1671 \\
20.8303 \\
24.4377 \\
28.0067 \\
31.5242 \\
3.9224 \\
7.6374 \\
11.4434 \\
15.2128 \\
18.8980 \\
22.5483 \\
26.1359 \\
29.6749 \\
33.1588\end{array}$ & $\begin{array}{l}0.9029 \\
0.9029 \\
0.9029 \\
0.9028 \\
0.9028 \\
0.9028 \\
0.9028 \\
0.9028 \\
0.9028 \\
0.9029 \\
0.9029 \\
0.9028 \\
0.9028 \\
0.9028 \\
0.9028 \\
0.9028 \\
0.9028 \\
0.9028\end{array}$ & $\begin{array}{l}73.141 \\
73.193 \\
73.247 \\
73.302 \\
73.356 \\
73.410 \\
73.464 \\
73.517 \\
73.571 \\
73.165 \\
73.218 \\
73.273 \\
73.327 \\
73.381 \\
73.435 \\
73.489 \\
73.542 \\
73.596\end{array}$ & $\begin{array}{l}4.1209 \\
4.2047 \\
4.1808 \\
4.1537 \\
4.1252 \\
4.1019 \\
4.0829 \\
4.0465 \\
4.0195 \\
4.1420 \\
4.1939 \\
4.1470 \\
4.1264 \\
4.1109 \\
4.0847 \\
4.0620 \\
4.0338 \\
4.0177\end{array}$ & $\begin{array}{l}322.210 \\
330.431 \\
330.156 \\
329.550 \\
328.741 \\
328.280 \\
328.082 \\
326.418 \\
325.453 \\
324.654 \\
330.359 \\
328.220 \\
328.085 \\
328.270 \\
327.532 \\
327.010 \\
325.976 \\
325.858\end{array}$ & $\begin{array}{ll}166.411 & 1 \\
162.981 & 1 \\
163.836 & 1 \\
164.820 & 1 \\
165.837 & 1 \\
166.626 & 1 \\
167.342 & 1 \\
168.704 & 1 \\
169.686 & 1 \\
165.199 \\
163.569 \\
164.878 \\
165.482 \\
166.577 \\
167.526 \\
168.366 \\
168.875 \\
170.021\end{array}$ & $\begin{array}{l}10.702 \\
12.876 \\
13.303 \\
13.438 \\
13.445 \\
13.440 \\
13.507 \\
13.667 \\
13.987 \\
11.549 \\
13.143 \\
13.362 \\
13.422 \\
13.459 \\
13.478 \\
13.589 \\
13.794 \\
14.220\end{array}$ & $\begin{array}{l}95.11 \\
90.41 \\
90.81 \\
91.45 \\
92.16 \\
92.64 \\
93.04 \\
94.14 \\
94.79 \\
93.37 \\
90.79 \\
91.73 \\
91.97 \\
92.79 \\
93.44 \\
93.98 \\
94.13 \\
94.95\end{array}$ \\
\hline
\end{tabular}

Table 52 (b). Experimental liquid heat capacity data for HFC-125.

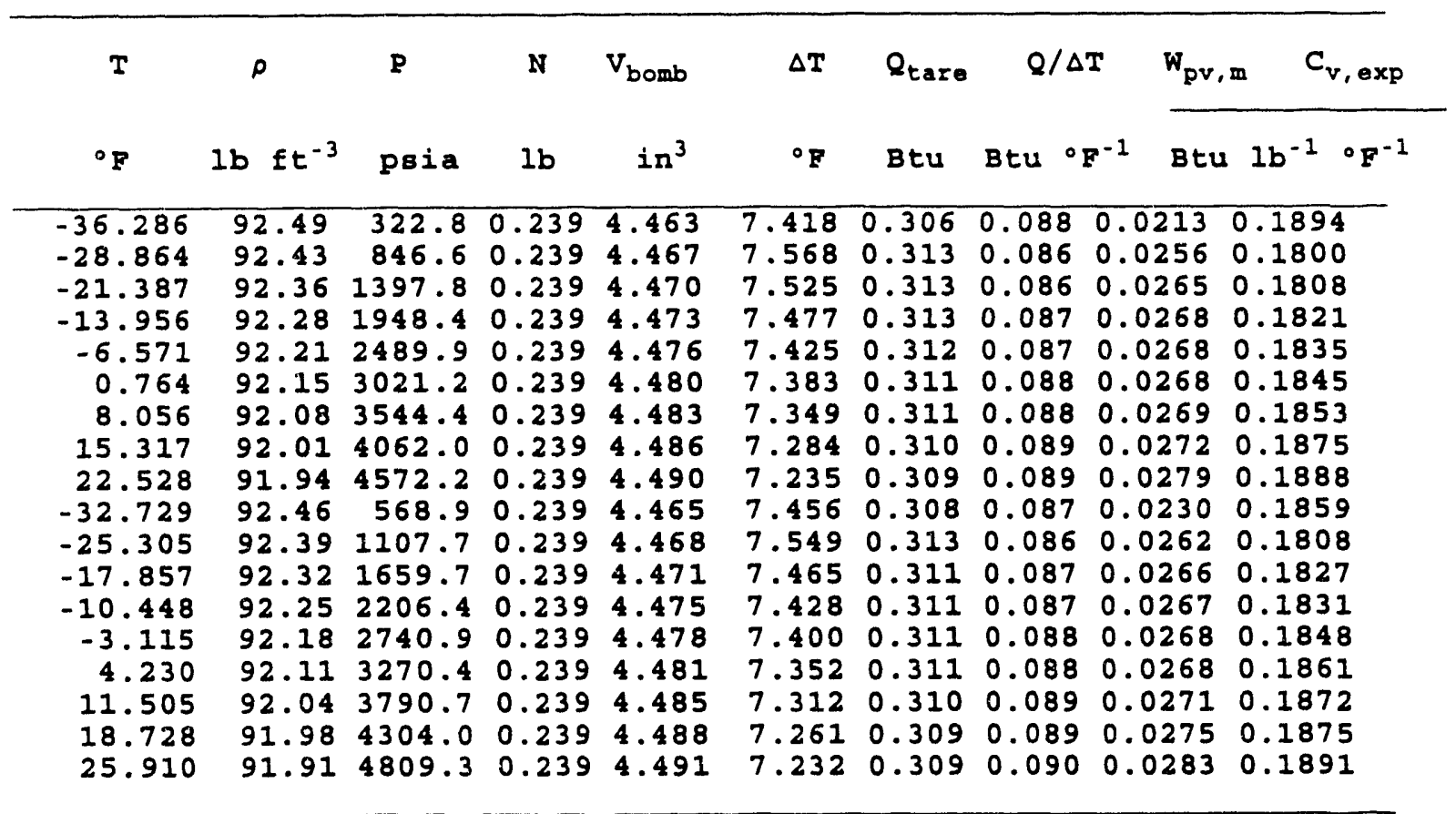


Table 53 (a). Experimental liquid heat capacity data for HFC-125.

\begin{tabular}{|c|c|c|c|c|c|c|c|c|c|}
\hline \multirow[t]{2}{*}{$\mathbf{T}$} & $\rho$ & \multirow{2}{*}{$\begin{array}{c}\mathbf{P} \\
\mathbf{M P a}\end{array}$} & \multirow{2}{*}{$\begin{array}{l}N \\
\text { mol }\end{array}$} & \multirow{2}{*}{$\begin{array}{c}V_{\text {bomb }} \\
\mathrm{cm}^{3}\end{array}$} & \multirow{2}{*}{$\begin{array}{l}\Delta \mathrm{T} \\
\mathrm{K}\end{array}$} & \multirow{2}{*}{$\begin{array}{c}Q_{\text {tare }} \\
J\end{array}$} & \multirow{2}{*}{$\begin{array}{l}Q / \Delta T \\
\mathrm{~J} \mathrm{~K}^{-1}\end{array}$} & $w_{p v, m}$ & \multirow{2}{*}{$\frac{C_{v, \exp }}{I^{-1} k^{-1}}$} \\
\hline & $\mathrm{mol} \mathrm{dm}^{-3}$ & & & & & & & $\mathrm{~J} \mathrm{mo}$ & \\
\hline $\begin{array}{l}223.1082 \\
227.2418 \\
231.3817 \\
235.5012 \\
239.5908 \\
243.6725 \\
247.6968 \\
251.7159 \\
225.0081 \\
229.1724 \\
233.3074 \\
237.4140 \\
241.4949 \\
245.5405 \\
249.5681\end{array}$ & $\begin{array}{l}12.7325 \\
12.7222 \\
12.7116 \\
12.7010 \\
12.6906 \\
12.6804 \\
12.6703 \\
12.6602 \\
12.7278 \\
12.7173 \\
12.7067 \\
12.6962 \\
12.6858 \\
12.6757 \\
12.6656\end{array}$ & $\begin{array}{r}3.2683 \\
7.4228 \\
11.7316 \\
16.0222 \\
20.2170 \\
24.3252 \\
28.3208 \\
32.3026 \\
5.1465 \\
9.4246 \\
13.7426 \\
17.9941 \\
22.1425 \\
26.1843 \\
30.1723\end{array}$ & $\begin{array}{l}0.9309 \\
0.9309 \\
0.9309 \\
0.9308 \\
0.9308 \\
0.9308 \\
0.9308 \\
0.9308 \\
0.9309 \\
0.9309 \\
0.9308 \\
0.9308 \\
0.9308 \\
0.9308 \\
0.9308\end{array}$ & $\begin{array}{l}73.113 \\
73.170 \\
73.229 \\
73.288 \\
73.347 \\
73.405 \\
73.462 \\
73.520 \\
73.139 \\
73.197 \\
73.257 \\
73.316 \\
73.374 \\
73.431 \\
73.489\end{array}$ & $\begin{array}{l}4.1108 \\
4.2287 \\
4.2257 \\
4.1678 \\
4.1185 \\
4.0995 \\
4.0560 \\
4.0208 \\
4.2173 \\
4.1969 \\
4.1653 \\
4.1232 \\
4.0971 \\
4.0723 \\
4.0472\end{array}$ & $\begin{array}{l}316.308 \\
327.236 \\
328.800 \\
325.998 \\
323.757 \\
323.805 \\
321.827 \\
320.421 \\
325.367 \\
325.619 \\
324.902 \\
323.272 \\
322.802 \\
322.342 \\
321.785\end{array}$ & $\begin{array}{l}166.385 \\
162.080 \\
162.133 \\
164.436 \\
166.326 \\
166.969 \\
168.679 \\
170.007 \\
162.644 \\
163.503 \\
164.674 \\
166.311 \\
167.142 \\
168.005 \\
168.954\end{array}$ & $\begin{array}{l}12.936 \\
15.275 \\
15.521 \\
15.489 \\
15.409 \\
15.432 \\
15.600 \\
15.979 \\
14.930 \\
15.416 \\
15.499 \\
15.421 \\
15.381 \\
15.461 \\
15.747\end{array}$ & $\begin{array}{l}92.93 \\
87.37 \\
86.91 \\
88.91 \\
90.49 \\
90.76 \\
92.13 \\
93.06 \\
88.30 \\
88.63 \\
89.39 \\
90.70 \\
91.17 \\
91.66 \\
92.21\end{array}$ \\
\hline
\end{tabular}

Table $53(b)$. Experimental liquid heat capacity data for HFC-125.

\begin{tabular}{|c|c|c|c|c|c|c|c|c|c|c|}
\hline \multirow{2}{*}{$\begin{array}{l}\mathbf{T} \\
{ }^{\circ} \mathrm{F}\end{array}$} & $\rho$ & \multirow{2}{*}{$\begin{array}{l}\text { P } \\
\text { psia }\end{array}$} & \multirow{2}{*}{$\begin{array}{l}\mathrm{N} \\
1 \mathrm{~b}\end{array}$} & \multirow{2}{*}{$\begin{array}{c}V_{\text {bomb }} \\
\text { in }^{3}\end{array}$} & \multirow{2}{*}{$\begin{array}{l}\Delta T \\
{ }^{\circ} \mathrm{F}\end{array}$} & $Q_{\text {tare }}$ & \multicolumn{2}{|c|}{$Q / \Delta T$} & \multicolumn{2}{|c|}{$W_{p v, m} \quad C_{v, \exp }$} \\
\hline & Ib $f t^{-3}$ & & & & & Btu & Btu 。 & ${ }^{\circ} \mathbf{F}^{-1}$ & Btu & $1 b^{-1} \circ F^{-1}$ \\
\hline $\begin{array}{r}-58.075 \\
-50.635 \\
-43.183 \\
-35.768 \\
-28.407 \\
-21.060 \\
-13.816 \\
-6.581 \\
-54.655 \\
-47.160 \\
-39.717 \\
-32.325 \\
-24.979 \\
-17.697 \\
-10.447\end{array}$ & $\begin{array}{l}95.40 \\
95.32 \\
95.24 \\
95.16 \\
95.09 \\
95.01 \\
94.93 \\
94.86 \\
95.36 \\
95.29 \\
95.21 \\
95.13 \\
95.05 \\
94.97 \\
94.90\end{array}$ & $\begin{array}{r}474.0 \\
1076.6 \\
1701.5 \\
2323.8 \\
2932.2 \\
3528.1 \\
4107.6 \\
4685.1 \\
746.4 \\
1366.9 \\
1993.2 \\
2609.8 \\
3211.5 \\
3797.7 \\
4376.1\end{array}$ & $\begin{array}{l}0.246 \\
0.246 \\
0.246 \\
0.246 \\
0.246 \\
0.246 \\
0.246 \\
0.246 \\
0.246 \\
0.246 \\
0.246 \\
0.246 \\
0.246 \\
0.246 \\
0.246\end{array}$ & $\begin{array}{l}4.462 \\
4.465 \\
4.469 \\
4.472 \\
4.476 \\
4.479 \\
4.483 \\
4.486 \\
4.463 \\
4.467 \\
4.470 \\
4.474 \\
4.478 \\
4.481 \\
4.485\end{array}$ & $\begin{array}{l}7.399 \\
7.612 \\
7.606 \\
7.502 \\
7.413 \\
7.379 \\
7.301 \\
7.237 \\
7.591 \\
7.554 \\
7.498 \\
7.422 \\
7.375 \\
7.330 \\
7.285\end{array}$ & $\begin{array}{l}0.300 \\
0.310 \\
0.312 \\
0.309 \\
0.307 \\
0.307 \\
0.305 \\
0.304 \\
0.309 \\
0.309 \\
0.308 \\
0.307 \\
0.306 \\
0.306 \\
0.305\end{array}$ & $\begin{array}{l}0.088 \\
0.085 \\
0.085 \\
0.087 \\
0.088 \\
0.088 \\
0.089 \\
0.090 \\
0.086 \\
0.086 \\
0.087 \\
0.088 \\
0.088 \\
0.089 \\
0.089\end{array}$ & $\begin{array}{ll}0.0 \\
0.0 \\
0.0 \\
0.0 \\
0.0 \\
0.0 \\
0.0 \\
0.0 \\
0.0 \\
5 & 0.0 \\
5 & 0.0 \\
7 & 0.0 \\
8 & 0.0 \\
8 & 0.0 \\
9 & 0.1 \\
9 & 0.1\end{array}$ & $\begin{array}{l}2580 \\
304 \\
3090 \\
3080 \\
308 \\
307 \\
307 \\
3110 \\
318 \\
319 \\
297 \\
0307 \\
0309 \\
0307 \\
0306 \\
0308 \\
0314\end{array}$ & $\begin{array}{l}0.1851 \\
0.1740 \\
0.1731 \\
0.1771 \\
0.1802 \\
0.1807 \\
0.1835 \\
0.1853 \\
0.1758 \\
0.1765 \\
0.1780 \\
0.1806 \\
0.1816 \\
0.1825 \\
0.1836\end{array}$ \\
\hline
\end{tabular}


Table 54(a). Experimental liquid heat capacity data for HFC-125.

\begin{tabular}{|c|c|c|c|c|c|c|c|c|c|}
\hline $\mathbf{T}$ & $\rho$ & $\mathbf{P}$ & $\mathbf{N}$ & $v_{\text {bomb }}$ & $\Delta \mathrm{T}$ & $Q_{\text {tare }}$ & $Q / \Delta T$ & $w_{p v, m}$ & $C_{v, \exp }$ \\
\hline $\mathbf{K}$ & mol $\mathrm{dm}^{-3}$ & $\mathbf{M P a}$ & mol & $\mathrm{cm}^{3}$ & $\mathbf{K}$ & $\mathbf{J}$ & $J K^{-1}$ & J mol & $I^{-1} k^{-1}$ \\
\hline $\begin{array}{l}210.5277 \\
214.6573 \\
218.8147 \\
222.9588 \\
227.0591 \\
231.1414 \\
235.1686 \\
212.1153 \\
216.2791 \\
220.4335 \\
224.5609 \\
228.6550 \\
232.7295\end{array}$ & $\begin{array}{l}13.0820 \\
13.0708 \\
13.0591 \\
13.0474 \\
13.0360 \\
13.0247 \\
13.0136 \\
13.0778 \\
13.0663 \\
13.0545 \\
13.0429 \\
13.0366 \\
13.0204\end{array}$ & $\begin{array}{r}3.0500 \\
7.6500 \\
12.4935 \\
17.3100 \\
21.9707 \\
26.5135 \\
30.9793 \\
4.7731 \\
9.5297 \\
14.3842 \\
19.1450 \\
23.7552 \\
28.2700\end{array}$ & $\begin{array}{l}0.9559 \\
0.9559 \\
0.9559 \\
0.9558 \\
0.9558 \\
0.9558 \\
0.9558 \\
0.9559 \\
0.9559 \\
0.9559 \\
0.9558 \\
0.9562 \\
0.9558\end{array}$ & $\begin{array}{l}73.072 \\
73.132 \\
73.195 \\
73.259 \\
73.322 \\
73.384 \\
73.445 \\
73.094 \\
73.156 \\
73.220 \\
73.284 \\
73.346 \\
73.408\end{array}$ & $\begin{array}{l}4.1321 \\
4.2132 \\
4.1793 \\
4.1399 \\
4.1082 \\
4.0855 \\
4.0532 \\
4.2230 \\
4.2228 \\
4.1688 \\
4.1385 \\
4.1329 \\
4.1025\end{array}$ & $\begin{array}{l}312.034 \\
320.209 \\
319.606 \\
318.481 \\
317.836 \\
317.794 \\
316.900 \\
319.696 \\
321.727 \\
319.556 \\
319.085 \\
320.427 \\
319.769\end{array}$ & $\begin{array}{l}166.325 \\
163.022 \\
164.377 \\
165.867 \\
167.054 \\
167.862 \\
169.089 \\
162.877 \\
162.772 \\
164.767 \\
165.810 \\
166.463 \\
167.573\end{array}$ & $\begin{array}{l}14.552 \\
17.261 \\
17.459 \\
17.369 \\
17.268 \\
17.334 \\
17.650 \\
16.463 \\
17.418 \\
17.441 \\
17.311 \\
17.339 \\
17.493\end{array}$ & $\begin{array}{l}91.48 \\
86.94 \\
87.78 \\
88.85 \\
89.63 \\
90.00 \\
90.75 \\
87.30 \\
86.46 \\
88.00 \\
88.62 \\
88.81 \\
89.51\end{array}$ \\
\hline
\end{tabular}

Table 54(b). Experimental liquid heat capacity data for HFC-125.

\begin{tabular}{|c|c|c|c|c|c|c|c|c|c|}
\hline \multirow{2}{*}{$\begin{array}{l}\mathbf{T} \\
\cdot \mathbf{F}\end{array}$} & $\rho$ & \multirow{2}{*}{$\begin{array}{l}\text { P } \\
\text { psia }\end{array}$} & \multirow{2}{*}{$\begin{array}{l}\mathrm{N} \\
1 \mathrm{~b}\end{array}$} & \multirow{2}{*}{$\begin{array}{c}V_{\text {bomb }} \\
i n^{3}\end{array}$} & \multirow{2}{*}{$\begin{array}{l}\Delta T \\
{ }^{\circ} \mathrm{F}\end{array}$} & $Q_{\text {taxe }}$ & $Q / \Delta T$ & \multicolumn{2}{|c|}{$W_{p v, m} \quad C_{v, \exp }$} \\
\hline & $1 b f t^{-3}$ & & & & & Btu & Btu ${ }^{\circ} F^{-1}$ & Btu & $1 b^{-1} \circ F^{-1}$ \\
\hline $\begin{array}{l}-80.720 \\
-73.287 \\
-65.804 \\
-58.344 \\
-50.964 \\
-43.616 \\
-36.367 \\
-77.862 \\
-70.368 \\
-62.890 \\
-55.460 \\
-48.091 \\
-40.757\end{array}$ & $\begin{array}{l}98.02 \\
97.93 \\
97.85 \\
97.76 \\
97.67 \\
97.59 \\
97.51 \\
97.99 \\
97.90 \\
97.81 \\
97.73 \\
97.68 \\
97.56\end{array}$ & $\begin{array}{r}442.4 \\
1109.5 \\
1812.0 \\
2510.6 \\
3186.6 \\
3845.5 \\
4493.2 \\
692.3 \\
1382.2 \\
2086.3 \\
2776.8 \\
3445.4 \\
4100.2\end{array}$ & $\begin{array}{l}0.253 \\
0.253 \\
0.253 \\
0.253 \\
0.253 \\
0.253 \\
0.253 \\
0.253 \\
0.253 \\
0.253 \\
0.253 \\
0.253 \\
0.253\end{array}$ & $\begin{array}{l}4.459 \\
4.463 \\
4.467 \\
4.471 \\
4.474 \\
4.478 \\
4.482 \\
4.460 \\
4.464 \\
4.468 \\
4.472 \\
4.476 \\
4.480\end{array}$ & $\begin{array}{l}7.438 \\
7.584 \\
7.523 \\
7.452 \\
7.395 \\
7.354 \\
7.296 \\
7.601 \\
7.601 \\
7.504 \\
7.449 \\
7.439 \\
7.384\end{array}$ & $\begin{array}{l}0.296 \\
0.304 \\
0.303 \\
0.302 \\
0.301 \\
0.301 \\
0.301 \\
0.303 \\
0.305 \\
0.303 \\
0.303 \\
0.304 \\
0.303\end{array}$ & $\begin{array}{ll}0.088 & 0.0 \\
0.086 & 0.0 \\
0.087 & 0.0 \\
0.087 & 0.0 \\
0.088 & 0.0 \\
0.0888 & 0.0 \\
0.089 & 0.0 \\
0.086 & 0.0 \\
0.086 & 0.0 \\
0.087 & 0.0 \\
0.087 & 0.0 \\
0.088 & 0.0 \\
0.088 & 0.0\end{array}$ & 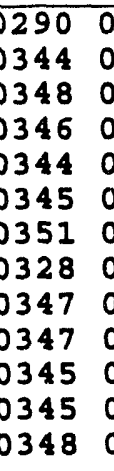 & $\begin{array}{l}0.1822 \\
0.1731 \\
0.1748 \\
0.1769 \\
0.1785 \\
0.1792 \\
0.1807 \\
0.1738 \\
0.1722 \\
0.1752 \\
0.1765 \\
0.1769 \\
0.1782\end{array}$ \\
\hline
\end{tabular}


Table 55(a). Experimental liquid heat capacity data for HFC-125.

\begin{tabular}{|c|c|c|c|c|c|c|c|c|c|}
\hline $\mathbf{T}$ & $\rho$ & $\mathbf{P}$ & $\mathrm{N}$ & $v_{\text {bomb }}$ & $\Delta \mathrm{T}$ & $Q_{\text {taro }}$ & $Q / \Delta T$ & $W_{p v, m}$ & $C_{v, \exp }$ \\
\hline $\mathbf{x}$ & mol $\mathrm{dm}^{-3}$ & MPa & mol & $\mathrm{cm}^{3}$ & $\mathrm{x}$ & $J$ & $J K^{-1}$ & \multicolumn{2}{|c|}{$J \operatorname{mol} 1^{-1} \mathrm{x}^{-1}$} \\
\hline $\begin{array}{l}196.0028 \\
200.2258 \\
204.3906 \\
208.5233 \\
212.6287 \\
216.6938\end{array}$ & $\begin{array}{l}13.5191 \\
13.5056 \\
13.4925 \\
13.4795 \\
13.4667 \\
13.4541\end{array}$ & $\begin{array}{r}5.2271 \\
10.8882 \\
16.4177 \\
21.8243 \\
27.1043 \\
32.2433\end{array}$ & $\begin{array}{l}0.9875 \\
0.9875 \\
0.9875 \\
0.9875 \\
0.9874 \\
0.9874\end{array}$ & $\begin{array}{l}73.048 \\
73.118 \\
73.187 \\
73.256 \\
73.324 \\
73.392\end{array}$ & $\begin{array}{l}4.2523 \\
4.2297 \\
4.2067 \\
4.1592 \\
4.1095 \\
4.0671\end{array}$ & $\begin{array}{l}313.206 \\
313.936 \\
314.487 \\
313.069 \\
311.350 \\
310.052\end{array}$ & $\begin{array}{l}162.021 \\
162.824 \\
163.584 \\
165.412 \\
167.308 \\
168.952\end{array}$ & $\begin{array}{l}18.733 \\
19.488 \\
19.517 \\
19.358 \\
19.328 \\
19.663\end{array}$ & $\begin{array}{l}85.07 \\
85.12 \\
85.31 \\
86.63 \\
88.01 \\
89.06\end{array}$ \\
\hline
\end{tabular}

Table 55(b). Experimental liquid heat capacity data for HFC- 125 .

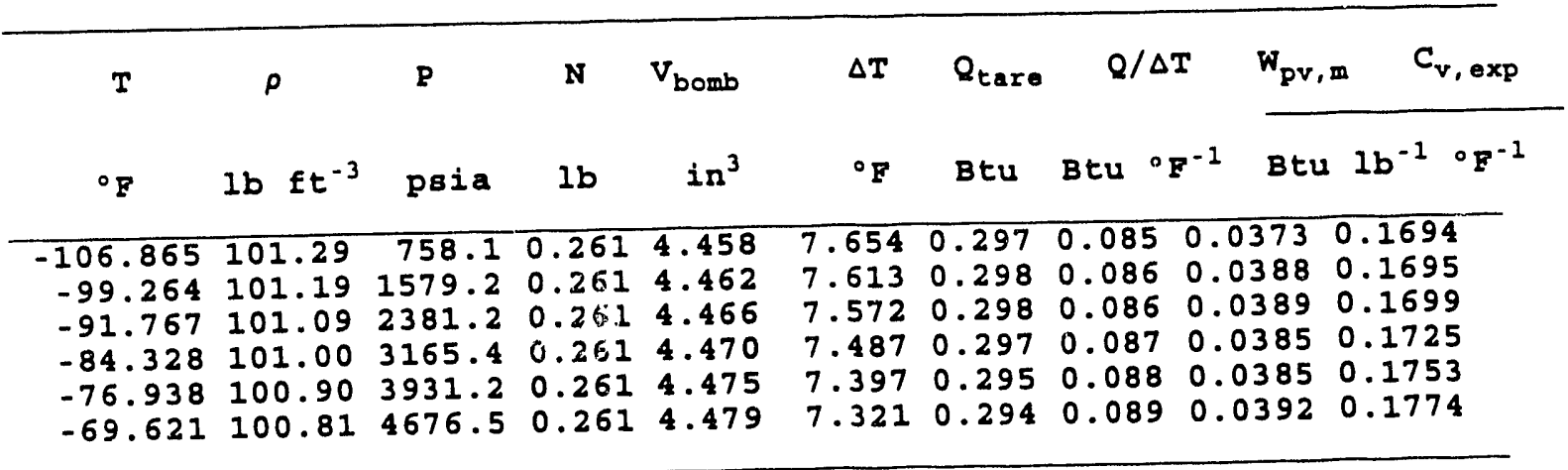


Table 56(a). Experimental two-phase heat capacity data for HFC-125.

\begin{tabular}{|c|c|c|c|c|c|c|c|c|}
\hline $\mathbf{T}$ & $\rho_{\sigma}$ & $\mathbf{P}_{\sigma}$ & $\mathbf{N}$ & $v_{\text {bomb }}$ & $\Delta T$ & $Q / \Delta T$ & $C_{v}{ }^{(2)}$ & $c_{\sigma}$ \\
\hline $\mathbf{K}$ & mol $\mathrm{dm}^{-3}$ & MPa & mol & $\mathrm{cm}^{3}$ & $\mathbf{K}$ & $\mathrm{J} \mathrm{K}^{-1}$ & J mo: & $1^{-1} \mathrm{~K}^{-1}$ \\
\hline $\begin{array}{l}178.2938 \\
184.6476 \\
190.9409 \\
197.1488 \\
203.3100 \\
209.3844 \\
215.4020 \\
221.3770 \\
227.2622 \\
233.1046 \\
238.8647 \\
244.6039 \\
250.2920 \\
255.9238 \\
261.5051 \\
267.0220 \\
272.4828 \\
277.9599\end{array}$ & $\begin{array}{l}13.8035 \\
13.6444 \\
13.4863 \\
13.3285 \\
13.1694 \\
13.0099 \\
12.8486 \\
12.6849 \\
12.5196 \\
12.3511 \\
12.1800 \\
12.0040 \\
11.8235 \\
11.6381 \\
11.4469 \\
11.2498 \\
11.0456 \\
10.8303\end{array}$ & $\begin{array}{l}0.0050 \\
0.0084 \\
0.0135 \\
0.0207 \\
0.0308 \\
0.0442 \\
0.0619 \\
0.0847 \\
0.1131 \\
0.1483 \\
0.1909 \\
0.2421 \\
0.3028 \\
0.3738 \\
0.4559 \\
0.5499 \\
0.6567 \\
0.7787\end{array}$ & $\begin{array}{l}0.77749 \\
0.77749 \\
0.77749 \\
0.77749 \\
0.77749 \\
0.77749 \\
0.77749 \\
0.77749 \\
0.77749 \\
0.77749 \\
0.77749 \\
0.77749 \\
0.77749 \\
0.77749 \\
0.77749 \\
0.77748 \\
0.77748 \\
0.77748\end{array}$ & $\begin{array}{l}72.9435 \\
72.9622 \\
72.9810 \\
72.9998 \\
73.0186 \\
73.0374 \\
73.0561 \\
73.0749 \\
73.9937 \\
73.1124 \\
73.1311 \\
73.1499 \\
73.1687 \\
73.1876 \\
73.2065 \\
73.2254 \\
73.2443 \\
73.2635\end{array}$ & $\begin{array}{l}6.4299 \\
6.3696 \\
6.2842 \\
6.2026 \\
6.1373 \\
6.0793 \\
5.9967 \\
5.9575 \\
5.8976 \\
5.8389 \\
5.7947 \\
5.7298 \\
5.6767 \\
5.6243 \\
5.5446 \\
5.5014 \\
5.4405 \\
5.3900\end{array}$ & $\begin{array}{l}167.884 \\
169.412 \\
171.584 \\
173.704 \\
175.418 \\
176.850 \\
179.207 \\
180.407 \\
181.867 \\
183.548 \\
185.197 \\
187.112 \\
188.745 \\
190.358 \\
192.312 \\
193.629 \\
196.388 \\
197.547\end{array}$ & $\begin{array}{l}124.604 \\
125.274 \\
126.866 \\
128.480 \\
129.643 \\
130.512 \\
132.629 \\
133.308 \\
134.375 \\
135.771 \\
137.172 \\
138.951 \\
140.407 \\
141.874 \\
143.813 \\
144.964 \\
147.997 \\
148.986\end{array}$ & $\begin{array}{l}124.520 \\
125.157 \\
126.713 \\
128.288 \\
129.409 \\
130.240 \\
132.325 \\
132.981 \\
134.041 \\
135.450 \\
136.892 \\
138.749 \\
140.327 \\
141.974 \\
144.162 \\
145.547 \\
149.121 \\
150.692\end{array}$ \\
\hline
\end{tabular}

Table $56(\mathrm{~b})$. Experimental two-phase heat capacity data for HFC-125.

\begin{tabular}{|c|c|c|c|c|c|c|c|c|c|}
\hline \multirow[b]{2}{*}{${ }^{\circ} \mathrm{E}$} & $\rho_{\sigma}$ & \multirow{2}{*}{$\begin{array}{c}\mathbf{P}_{\sigma} \\
\text { psia }\end{array}$} & \multirow{2}{*}{$\begin{array}{l}\text { N } \\
1 b\end{array}$} & \multirow{2}{*}{$\begin{array}{c}v_{\text {bomb }} \\
\text { in }^{3}\end{array}$} & \multirow{2}{*}{$\begin{array}{l}\Delta \mathbf{T} \\
{ }^{\circ} \mathrm{F}\end{array}$} & \multicolumn{2}{|c|}{$Q / \Delta T$} & \multicolumn{2}{|c|}{$C_{v}{ }^{(2)}$} \\
\hline & $1 b f t^{-3}$ & & & & & Btu ${ }^{\circ}$ & $F^{-1}$ & Btu & $1 b^{-1} \cdot a^{-1}$ \\
\hline $\begin{array}{r}-138.741 \\
-127.304 \\
-115.976 \\
-104.802 \\
-93.712 \\
-82.778 \\
-71.946 \\
-61.191 \\
-50.598 \\
-40.082 \\
-29.714 \\
-19.383 \\
-9.144 \\
0.993 \\
11.039 \\
20.970 \\
30.799 \\
40.658\end{array}$ & $\begin{array}{r}103.42 \\
102.23 \\
101.05 \\
99.87 \\
98.67 \\
97.48 \\
96.27 \\
95.04 \\
93.80 \\
92.54 \\
91.26 \\
89.94 \\
88.59 \\
87.20 \\
85.77 \\
84.29 \\
82.76 \\
81.15\end{array}$ & $\begin{array}{r}0.73 \\
1.22 \\
1.96 \\
3.00 \\
4.47 \\
6.41 \\
8.98 \\
12.28 \\
16.40 \\
21.51 \\
27.69 \\
35.11 \\
43.92 \\
54.22 \\
66.12 \\
79.76 \\
95.25 \\
112.94\end{array}$ & $\begin{array}{l}0.206 \\
0.206 \\
0.206 \\
0.206 \\
0.206 \\
0.206 \\
0.206 \\
0.206 \\
0.206 \\
0.206 \\
0.206 \\
0.206 \\
0.206 \\
0.206 \\
0.206 \\
0.206 \\
0.206 \\
0.206\end{array}$ & $\begin{array}{l}4.451 \\
4.452 \\
4.454 \\
4.455 \\
4.456 \\
4.457 \\
4.458 \\
4.459 \\
4.460 \\
4.462 \\
4.463 \\
4.464 \\
4.465 \\
4.466 \\
4.467 \\
4.469 \\
4.470 \\
4.471\end{array}$ & $\begin{array}{l}11.574 \\
11.465 \\
11.312 \\
11.165 \\
11.047 \\
10.943 \\
10.794 \\
10.723 \\
10.616 \\
10.510 \\
10.430 \\
10.314 \\
10.218 \\
10.124 \\
9.980 \\
9.903 \\
9.793 \\
9.702\end{array}$ & $\begin{array}{l}0.088 \\
0.089 \\
0.090 \\
0.092 \\
0.092 \\
0.093 \\
0.094 \\
0.095 \\
0.096 \\
0.097 \\
0.098 \\
0.099 \\
0.099 \\
0.100 \\
0.101 \\
0.102 \\
0.103 \\
0.104\end{array}$ & $\begin{array}{l}0.2 \\
0.2 \\
0.2 \\
0.2 \\
0.2 \\
0.2 \\
0.2 \\
0.2 \\
0.2 \\
0.2 \\
0.2 \\
0.2 \\
0.2 \\
0.2 \\
0.2 \\
0.2 \\
0.2 \\
0.2\end{array}$ & $\begin{array}{l}2481 \\
2495 \\
2526 \\
2559 \\
2582 \\
2599 \\
2641 \\
2655 \\
2676 \\
2704 \\
2732 \\
2767 \\
2796 \\
2825 \\
2864 \\
2887 \\
2947 \\
2967\end{array}$ & $\begin{array}{l}0.2480 \\
0.2492 \\
0.2523 \\
0.2555 \\
0.2577 \\
0.2594 \\
0.2635 \\
0.2648 \\
0.2669 \\
0.2697 \\
0.2726 \\
0.2763 \\
0.2794 \\
0.2827 \\
0.2871 \\
0.2900 \\
0.2970 \\
0.3001\end{array}$ \\
\hline
\end{tabular}


Table 57 (a). Experimental two-phase heat capacity data for HFC-125.

\begin{tabular}{|c|c|c|c|c|c|c|c|c|}
\hline \multirow[b]{2}{*}{$\mathrm{K}$} & $\rho_{\sigma}$ & $\mathbf{P}_{\sigma}$ & & \multirow{2}{*}{$\begin{array}{c}V_{\text {bomb }} \\
\mathrm{cm}^{3}\end{array}$} & \multirow{2}{*}{$\begin{array}{l}\Delta \mathrm{T} \\
\mathrm{K}\end{array}$} & $Q / \Delta T$ & $C_{v}^{(2)}$ & $c_{\sigma}$ \\
\hline & mol $\mathrm{dm}^{-3}$ & MPa & mol & & & $\mathbf{J} \mathbf{K}^{-1}$ & I mol & $1^{-1} \mathrm{~K}^{-1}$ \\
\hline $\begin{array}{l}180.5651 \\
185.5445 \\
190.4684 \\
195.3841 \\
200.2359 \\
205.0443 \\
209.8366 \\
214.5500 \\
219.2416 \\
223.8851 \\
228.4866 \\
233.0650 \\
237.6176 \\
242.1330 \\
246.5986 \\
251.0478 \\
255.4390 \\
259.8416 \\
264.1775\end{array}$ & $\begin{array}{l}13.7461 \\
13.6220 \\
13.4982 \\
13.3735 \\
13.2491 \\
13.1242 \\
12.9979 \\
12.8717 \\
12.7439 \\
12.6150 \\
12.4847 \\
12.3522 \\
12.2175 \\
12.0805 \\
11.9414 \\
11.7990 \\
11.6543 \\
11.5047 \\
11.3525\end{array}$ & $\begin{array}{l}0.0061 \\
0.0090 \\
0.0130 \\
0.0184 \\
0.0253 \\
0.0342 \\
0.0454 \\
0.0591 \\
0.0759 \\
0.0960 \\
0.1199 \\
0.1481 \\
0.1810 \\
0.2189 \\
0.2622 \\
0.3117 \\
0.3672 \\
0.4301 \\
0.4998\end{array}$ & $\begin{array}{l}0.81724 \\
0.81724 \\
0.81724 \\
0.81724 \\
0.81724 \\
0.81724 \\
0.81724 \\
0.81724 \\
0.81724 \\
0.81724 \\
0.81724 \\
0.81724 \\
0.81724 \\
0.81724 \\
0.81724 \\
0.81724 \\
0.81724 \\
0.81724 \\
0.81724\end{array}$ & $\begin{array}{l}72.9501 \\
72.9649 \\
72.9796 \\
72.9945 \\
73.0092 \\
73.0240 \\
73.0388 \\
73.0535 \\
73.0682 \\
73.0829 \\
73.0976 \\
73.1123 \\
73.1270 \\
73.1418 \\
73.1565 \\
73.1712 \\
73.1859 \\
73.2008 \\
73.2156\end{array}$ & $\begin{array}{l}5.0246 \\
4.9883 \\
4.9451 \\
4.8825 \\
4.8602 \\
4.8177 \\
4.7909 \\
4.7407 \\
4.6912 \\
4.6760 \\
4.6160 \\
4.5736 \\
4.5435 \\
4.5288 \\
4.4765 \\
4.4515 \\
4.4080 \\
4.3762 \\
4.3613\end{array}$ & $\begin{array}{l}174.080 \\
174.998 \\
176.490 \\
178.773 \\
179.693 \\
180.974 \\
181.905 \\
183.422 \\
185.258 \\
185.994 \\
187.805 \\
189.619 \\
190.835 \\
191.328 \\
193.487 \\
194.323 \\
196.135 \\
197.403 \\
197.944\end{array}$ & $\begin{array}{l}125.673 \\
125.846 \\
126.781 \\
128.727 \\
129.054 \\
129.865 \\
130.283 \\
131.456 \\
133.049 \\
133.327 \\
134.949 \\
136.600 \\
137.543 \\
137.627 \\
139.775 \\
140.323 \\
142.091 \\
143.208 \\
143.456\end{array}$ & $\begin{array}{l}125.601 \\
125.755 \\
126.670 \\
128.596 \\
128.905 \\
129.699 \\
130.103 \\
131.268 \\
132.862 \\
133.149 \\
134.794 \\
136.483 \\
137.484 \\
137.648 \\
139.901 \\
140.588 \\
142.530 \\
143.868 \\
144.386\end{array}$ \\
\hline
\end{tabular}

Table 57 (b). Experimental two-phase heat capacity data for HFC-125.

\begin{tabular}{|c|c|c|c|c|c|c|c|c|}
\hline \multirow{2}{*}{$\begin{array}{l}\mathbf{T} \\
\circ \mathrm{F}\end{array}$} & $P_{\sigma}$ & \multirow{2}{*}{$\begin{array}{c}\mathbf{P}_{\sigma} \\
\text { psia }\end{array}$} & \multirow{2}{*}{$\begin{array}{l}\text { N } \\
1 b\end{array}$} & \multirow{2}{*}{$\begin{array}{c}V_{\text {bomb }} \\
i n^{3}\end{array}$} & \multirow{2}{*}{$\begin{array}{l}\Delta \mathrm{T} \\
\cdot \mathrm{F}\end{array}$} & $Q / \Delta T$ & $C_{v}{ }^{(2)}$ & $c_{\sigma}$ \\
\hline & $1 b \in t^{-3}$ & & & & & Btu ${ }^{\circ} \mathrm{F}$ & Btu & $1 b^{-1} \circ F^{-1}$ \\
\hline $\begin{array}{r}-134.653 \\
-125.690 \\
-116.827 \\
-107.979 \\
-99.245 \\
-90.590 \\
-81.964 \\
-73.480 \\
-65.035 \\
-56.677 \\
-48.394 \\
-40.153 \\
-31.958 \\
-23.831 \\
-15.793 \\
-7.784 \\
0.120 \\
8.045 \\
15.849\end{array}$ & $\begin{array}{r}102.99 \\
102.06 \\
101.14 \\
100.20 \\
99.27 \\
98.33 \\
97.39 \\
96.44 \\
95.49 \\
94.52 \\
93.54 \\
92.55 \\
91.54 \\
90.51 \\
89.47 \\
88.41 \\
87.32 \\
86.20 \\
85.06\end{array}$ & $\begin{array}{r}0.88 \\
1.31 \\
1.89 \\
2.67 \\
3.67 \\
4.96 \\
6.58 \\
8.57 \\
11.01 \\
13.92 \\
17.39 \\
21.48 \\
26.25 \\
31.75 \\
38.03 \\
45.21 \\
53.26 \\
62.38 \\
72.49\end{array}$ & $\begin{array}{l}0.216 \\
0.216 \\
0.216 \\
0.216 \\
0.216 \\
0.216 \\
0.216 \\
0.216 \\
0.216 \\
0.216 \\
0.216 \\
0.216 \\
0.216 \\
0.216 \\
0.216 \\
0.216 \\
0.216 \\
0.216 \\
0.216\end{array}$ & $\begin{array}{l}4.452 \\
4.453 \\
4.454 \\
4.454 \\
4.455 \\
4.456 \\
4.457 \\
4.458 \\
4.459 \\
4.460 \\
4.461 \\
4.462 \\
4.463 \\
4.463 \\
4.464 \\
4.465 \\
4.466 \\
4.467 \\
4.468\end{array}$ & $\begin{array}{l}9.044 \\
8.979 \\
8.901 \\
8.788 \\
8.748 \\
8.672 \\
8.624 \\
8.533 \\
8.444 \\
8.417 \\
8.309 \\
8.232 \\
8.178 \\
8.152 \\
8.058 \\
8.013 \\
7.934 \\
7.877 \\
7.850\end{array}$ & $\begin{array}{l}0.092 \\
0.092 \\
0.093 \\
0.094 \\
0.095 \\
0.095 \\
0.096 \\
0.097 \\
0.098 \\
0.098 \\
0.099 \\
0.100 \\
0.101 \\
0.101 \\
0.102 \\
0.102 \\
0.103 \\
0.104 \\
0.104\end{array}$ & $\begin{array}{l}0.2503 \\
0.2506 \\
0.2525 \\
0.2563 \\
0.2570 \\
0.2586 \\
0.2594 \\
0.2618 \\
0.2650 \\
0.2655 \\
0.2687 \\
0.2720 \\
0.2739 \\
0.2741 \\
0.2783 \\
0.2794 \\
0.2830 \\
0.2852 \\
0.2857\end{array}$ & $\begin{array}{l}0.2501 \\
0.2504 \\
0.2523 \\
0.2561 \\
0.2567 \\
0.2583 \\
0.2591 \\
0.2614 \\
0.2646 \\
0.2652 \\
0.2684 \\
0.2718 \\
0.2738 \\
0.2741 \\
0.2786 \\
0.2800 \\
0.2838 \\
0.2865 \\
0.2875\end{array}$ \\
\hline
\end{tabular}


Table 58 (a). Experimental two-phase heat capacity data for HFC-125.

\begin{tabular}{|c|c|c|c|c|c|c|c|c|}
\hline \multirow{2}{*}{$\begin{array}{l}\mathbf{T} \\
\mathrm{X}\end{array}$} & $\rho_{\sigma}$ & \multirow{2}{*}{$\begin{array}{l}\mathbf{P}_{\sigma} \\
\mathrm{MPa}\end{array}$} & \multirow{2}{*}{$\begin{array}{c}\mathbf{N} \\
\text { mol }\end{array}$} & \multirow{2}{*}{$\begin{array}{c}V_{\text {bomb }} \\
\mathrm{cm}^{3}\end{array}$} & \multirow{2}{*}{$\begin{array}{l}\Delta \mathbf{T} \\
\mathrm{K}\end{array}$} & \multirow{2}{*}{$\begin{array}{l}Q / \Delta T \\
\mathrm{~J} \mathrm{~K}^{-1}\end{array}$} & \multicolumn{2}{|c|}{$C_{v}^{(2)}$} \\
\hline & mol $\mathrm{dm}^{-3}$ & & & & & & U I & $01^{-1} \mathrm{~K}^{-1}$ \\
\hline $\begin{array}{l}183.1585 \\
189.0687 \\
194.9050 \\
200.6980 \\
206.4265 \\
212.0807 \\
217.6838 \\
223.2474 \\
228.7619 \\
234.2143 \\
239.6168 \\
244.9882\end{array}$ & $\begin{array}{l}13.6816 \\
13.5335 \\
13.3858 \\
13.2372 \\
13.0879 \\
12.9380 \\
12.7866 \\
12.6329 \\
12.4768 \\
12.3185 \\
12.1572 \\
11.9920\end{array}$ & $\begin{array}{l}0.0075 \\
0.0118 \\
0.0178 \\
0.0261 \\
0.0372 \\
0.0516 \\
0.0700 \\
0.0930 \\
0.1215 \\
0.1559 \\
0.1971 \\
0.2459\end{array}$ & $\begin{array}{l}0.86950 \\
0.86950 \\
0.86950 \\
0.86950 \\
0.86950 \\
0.86950 \\
0.86950 \\
0.86950 \\
0.86950 \\
0.86950 \\
0.86950 \\
0.86949\end{array}$ & $\begin{array}{l}72.958 \\
72.975 \\
72.993 \\
73.011 \\
73.028 \\
73.046 \\
73.063 \\
73.081 \\
73.098 \\
73.116 \\
73.134 \\
73.151\end{array}$ & $\begin{array}{l}5.9720 \\
5.9120 \\
5.8399 \\
5.7788 \\
5.7307 \\
5.6586 \\
5.6284 \\
5.5795 \\
5.5135 \\
5.4561 \\
5.4160 \\
5.3738\end{array}$ & $\begin{array}{l}180.801 \\
182.503 \\
184.585 \\
186.463 \\
187.853 \\
189.863 \\
191.289 \\
192.833 \\
194.345 \\
196.306 \\
197.774 \\
199.196\end{array}$ & $\begin{array}{l}125.38 \\
126.31 \\
127.75 \\
129.01 \\
129.77 \\
131.30 \\
132.19 \\
133.26 \\
134.33 \\
135.95 \\
137.04 \\
138.10\end{array}$ & $\begin{array}{l}125.33 \\
126.25 \\
127.68 \\
128.94 \\
129.70 \\
131.23 \\
132.15 \\
133.26 \\
134.39 \\
136.09 \\
137.30 \\
138.52\end{array}$ \\
\hline
\end{tabular}

Table 58 (b). Experimental two-phase heat capacity data for HFC-125.

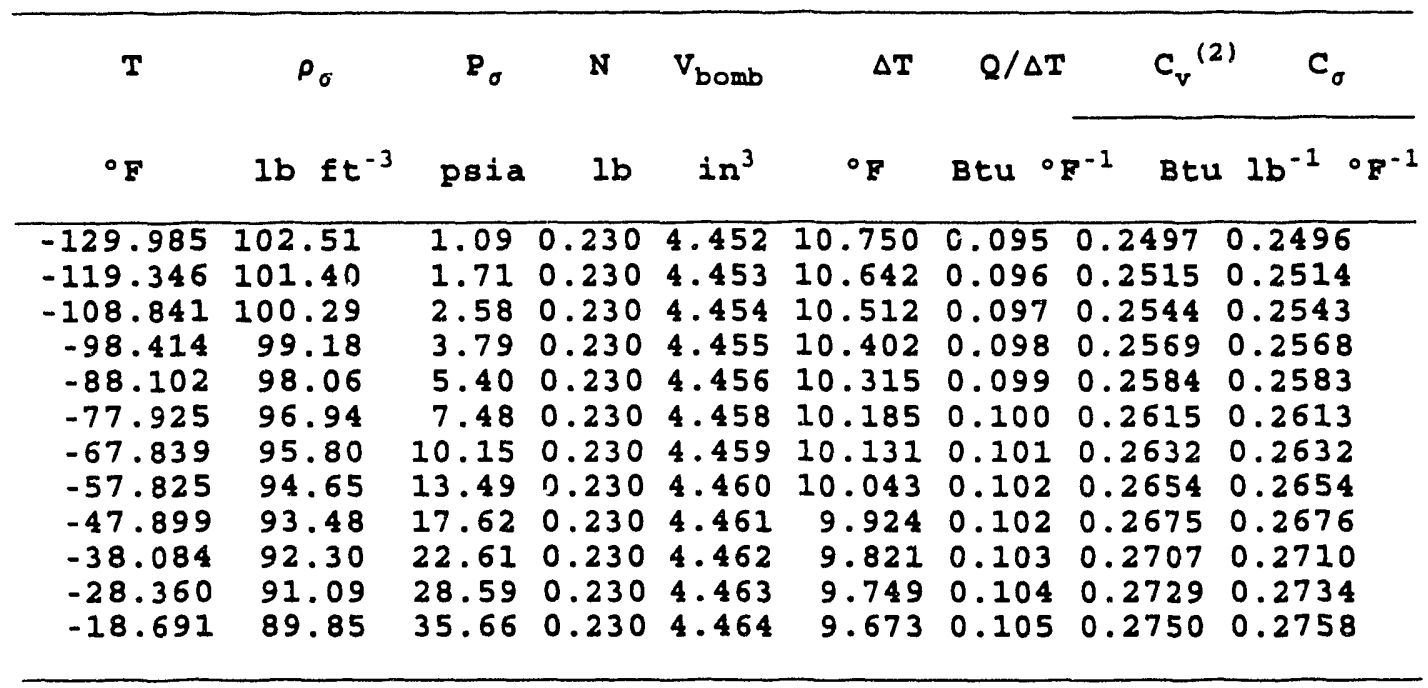


Table 59 (a). Experimental two-phase heat capacity data for HFC-125.

\begin{tabular}{|c|c|c|c|c|c|c|c|c|}
\hline \multirow[b]{2}{*}{$\mathbf{K}$} & $\rho_{\sigma}$ & $\mathbf{P}_{\sigma}$ & $\mathbf{N}$ & \multirow{2}{*}{$\begin{array}{c}V_{\text {bomb }} \\
\mathrm{cm}^{3}\end{array}$} & \multirow{2}{*}{$\begin{array}{l}\Delta \mathbf{T} \\
\mathbf{K}\end{array}$} & \multirow{2}{*}{$\begin{array}{l}Q / \Delta T \\
J K^{-1}\end{array}$} & $C_{v}^{(2)}$ & \multirow{2}{*}{$\frac{C_{\sigma}}{k^{-1}}$} \\
\hline & $\operatorname{mol} \mathrm{dm}^{-3}$ & MPa & mol & & & & $\mathrm{J}$ mol & \\
\hline $\begin{array}{l}175.8792 \\
179.6211 \\
183.3434 \\
187.0326 \\
190.6978 \\
194.3437 \\
197.9625 \\
201.5545 \\
205.1270 \\
208.6716 \\
212.1979 \\
215.7057 \\
219.1862 \\
222.6555 \\
226.0944 \\
229.5116\end{array}$ & $\begin{array}{l}13.8623 \\
13.7696 \\
13.6769 \\
13.5847 \\
13.4925 \\
13.4001 \\
13.3076 \\
13.2150 \\
13.1220 \\
13.0288 \\
12.9349 \\
12.8404 \\
12.7454 \\
12.6494 \\
12.5528 \\
12.4553\end{array}$ & $\begin{array}{l}0.0041 \\
0.0056 \\
0.0076 \\
0.0101 \\
0.0132 \\
0.0171 \\
0.0219 \\
0.0276 \\
0.0344 \\
0.0425 \\
0.0519 \\
0.0630 \\
0.0757 \\
0.0903 \\
0.1070 \\
0.1258\end{array}$ & $\begin{array}{l}0.90400 \\
0.90400 \\
0.90400 \\
0.90400 \\
0.90400 \\
0.90400 \\
0.90400 \\
0.90400 \\
0.90400 \\
0.90400 \\
0.90400 \\
0.90400 \\
0.90400 \\
0.90400 \\
0.90400 \\
0.90400\end{array}$ & $\begin{array}{l}72.9364 \\
72.9474 \\
72.9584 \\
72.9693 \\
72.9803 \\
72.9913 \\
73.0023 \\
73.0133 \\
73.0242 \\
73.0352 \\
73.0461 \\
73.0571 \\
73.0680 \\
73.0790 \\
73.0899 \\
73.1009\end{array}$ & $\begin{array}{l}3.7730 \\
3.7476 \\
3.7279 \\
3.7040 \\
3.6760 \\
3.6510 \\
3.6349 \\
3.6097 \\
3.5867 \\
3.5558 \\
3.5471 \\
3.5142 \\
3.5066 \\
3.4889 \\
3.4605 \\
3.4391\end{array}$ & $\begin{array}{l}183.303 \\
184.473 \\
185.353 \\
186.472 \\
187.776 \\
188.963 \\
189.694 \\
190.893 \\
192.012 \\
193.364 \\
194.264 \\
195.313 \\
196.321 \\
197.217 \\
198.102 \\
199.269\end{array}$ & $\begin{array}{l}124.664 \\
125.275 \\
125.596 \\
126.212 \\
127.059 \\
127.802 \\
128.064 \\
128.867 \\
129.599 \\
130.609 \\
131.137 \\
131.845 \\
132.524 \\
133.092 \\
133.664 \\
134.561\end{array}$ & $\begin{array}{l}124.639 \\
125.246 \\
125.564 \\
126.178 \\
127.024 \\
127.767 \\
128.031 \\
128.839 \\
129.579 \\
130.602 \\
131.146 \\
131.877 \\
132.585 \\
133.189 \\
133.807 \\
134.759\end{array}$ \\
\hline
\end{tabular}

Table 59(b). Experimental two-phase heat capacity data for HFC-125.

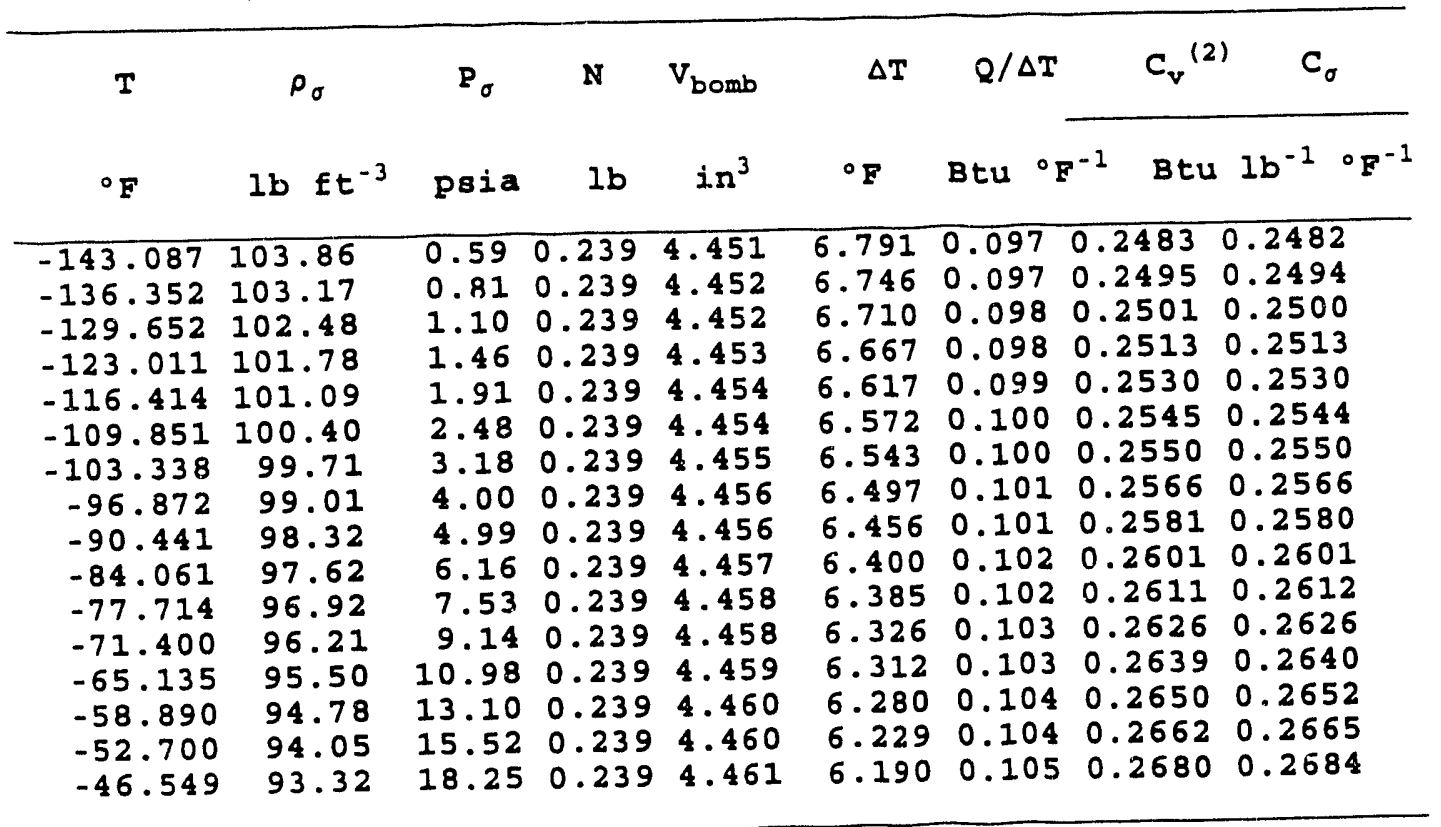


Table $60(a)$. Experimental two-phase heat capacity data for HFC-125.

\begin{tabular}{|c|c|c|c|c|c|c|c|c|}
\hline $\mathbf{T}$ & $\rho_{\sigma}$ & $\mathbf{P}_{\sigma}$ & $\mathbf{N}$ & $V_{\text {bomb }}$ & $\Delta \mathbf{T}$ & $\mathrm{Q} / \Delta \mathrm{T}$ & $C_{v}^{(2)}$ & $c_{\sigma}$ \\
\hline $\mathbf{K}$ & mol $\mathrm{dm}^{-3}$ & MPa & mol & $\mathrm{cm}^{3}$ & $\mathbf{K}$ & $J K^{-1}$ & $\mathrm{~J}$ mol & $\mathrm{I}^{-1} \mathrm{k}^{-1}$ \\
\hline $\begin{array}{l}176.0157 \\
179.6961 \\
183.3471 \\
186.9696 \\
190.5771 \\
194.1524 \\
197.7048 \\
201.2357 \\
204.7404 \\
208.2300 \\
211.7007 \\
215.1432 \\
180.1131 \\
183.8288 \\
187.5018 \\
191.1682 \\
194.8005 \\
198.3545 \\
201.8726 \\
205.3871 \\
208.8727 \\
212.3306 \\
215.7546\end{array}$ & $\begin{array}{l}13.8589 \\
13.7677 \\
13.6769 \\
13.5863 \\
13.4955 \\
13.4049 \\
13.3142 \\
13.2233 \\
13.1321 \\
13.0404 \\
12.9482 \\
12.8556 \\
13.7573 \\
13.6648 \\
13.5729 \\
13.4806 \\
13.3884 \\
13.2975 \\
13.2068 \\
13.1152 \\
13.0234 \\
12.9314 \\
12.8391\end{array}$ & $\begin{array}{l}0.0041 \\
0.0057 \\
0.0076 \\
0.0101 \\
0.0131 \\
0.0169 \\
0.0215 \\
0.0270 \\
0.0336 \\
0.0414 \\
0.0505 \\
0.0611 \\
0.0059 \\
0.0079 \\
0.0105 \\
0.0137 \\
0.0177 \\
0.0224 \\
0.0281 \\
0.0349 \\
0.0430 \\
0.0523 \\
0.0631\end{array}$ & $\begin{array}{l}0.93202 \\
0.93202 \\
0.93201 \\
0.93201 \\
0.93201 \\
0.93201 \\
0.93201 \\
0.93201 \\
0.93201 \\
0.93201 \\
0.93201 \\
0.93201 \\
0.93202 \\
0.93201 \\
0.93201 \\
0.93201 \\
0.93201 \\
0.93201 \\
0.93201 \\
0.93201 \\
0.93201 \\
0.93201 \\
0.93201\end{array}$ & $\begin{array}{l}72.9368 \\
72.9476 \\
72.9584 \\
72.9691 \\
72.9800 \\
72.9907 \\
73.0015 \\
73.0123 \\
73.0230 \\
73.0338 \\
73.0446 \\
73.0553 \\
72.9488 \\
72.9598 \\
72.9707 \\
72.9817 \\
72.9927 \\
73.0035 \\
73.0142 \\
73.0250 \\
73.0358 \\
73.0465 \\
73.0572\end{array}$ & $\begin{array}{l}3.7130 \\
3.6821 \\
3.6585 \\
3.6356 \\
3.6139 \\
3.5951 \\
3.5640 \\
3.5484 \\
3.5214 \\
3.5021 \\
3.4789 \\
3.4632 \\
3.6730 \\
3.6497 \\
3.6374 \\
3.6327 \\
3.5927 \\
3.5647 \\
3.5634 \\
3.5206 \\
3.5065 \\
3.4765 \\
3.4572\end{array}$ & $\begin{array}{l}186.649 \\
187.574 \\
188.722 \\
190.002 \\
191.057 \\
191.862 \\
193.424 \\
194.115 \\
195.475 \\
196.519 \\
197.739 \\
198.584 \\
188.531 \\
189.454 \\
189.986 \\
190.776 \\
192.778 \\
193.445 \\
193.558 \\
195.751 \\
196.540 \\
197.914 \\
199.459\end{array}$ & $\begin{array}{l}124.483 \\
124.825 \\
125.435 \\
126.216 \\
126.780 \\
127.100 \\
128.255 \\
128.495 \\
129.472 \\
130.128 \\
130.987 \\
131.461 \\
125.779 \\
126.141 \\
126.114 \\
126.387 \\
127.987 \\
128.184 \\
127.808 \\
129.681 \\
130.066 \\
131.095 \\
132.325\end{array}$ & $\begin{array}{l}124.467 \\
124.808 \\
125.418 \\
126.200 \\
126.766 \\
127.090 \\
128.252 \\
128.502 \\
129.492 \\
130.165 \\
131.047 \\
131.550 \\
125.762 \\
126.124 \\
126.098 \\
126.374 \\
127.978 \\
128.183 \\
127.817 \\
129.704 \\
130.107 \\
131.160 \\
132.420\end{array}$ \\
\hline
\end{tabular}


Table 60 (b). Experimental two-phase heat capacity data for HFC-125.

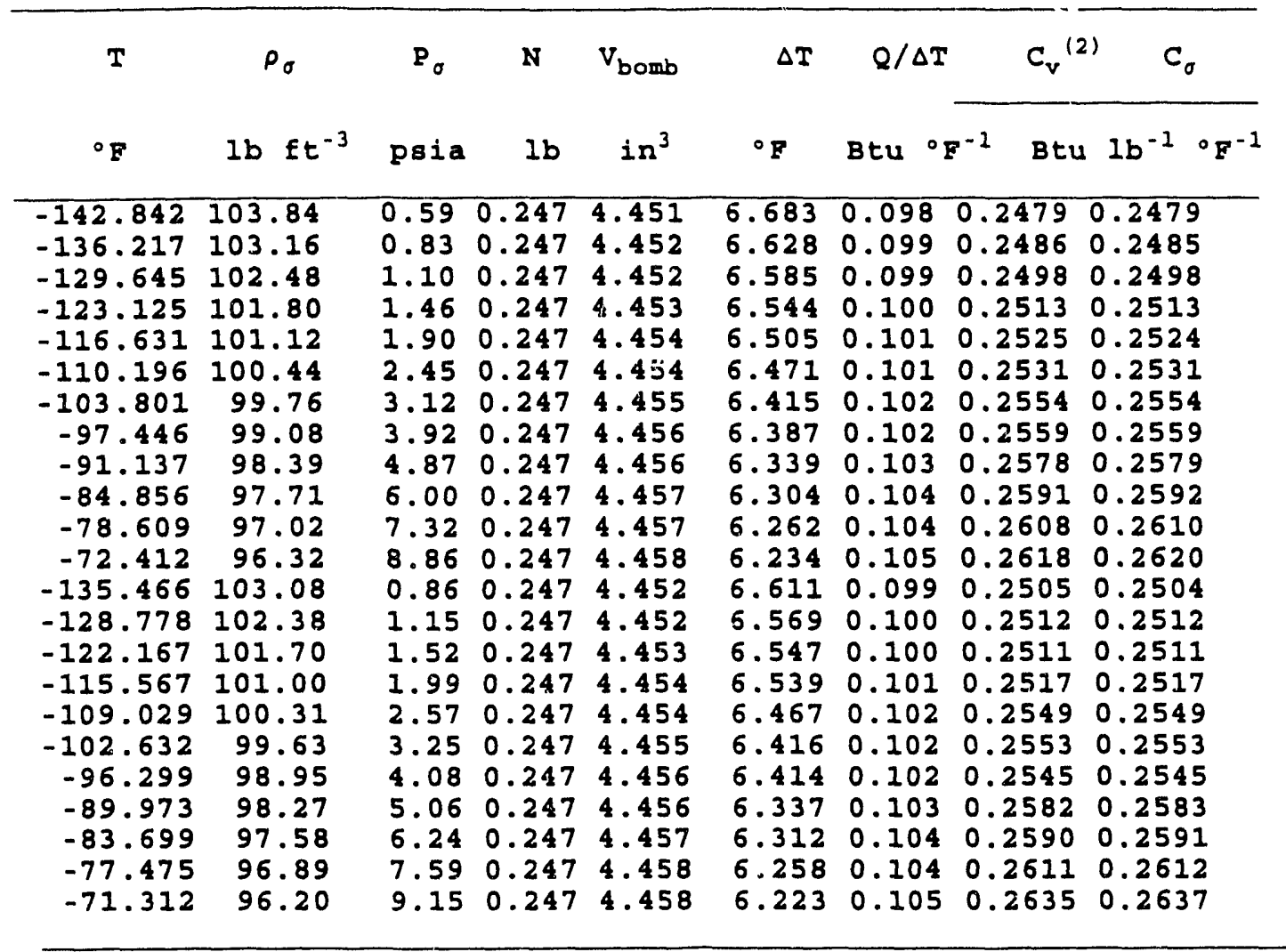


Table 61(a). Experimental two-phase heat capacity data for HFC-125.

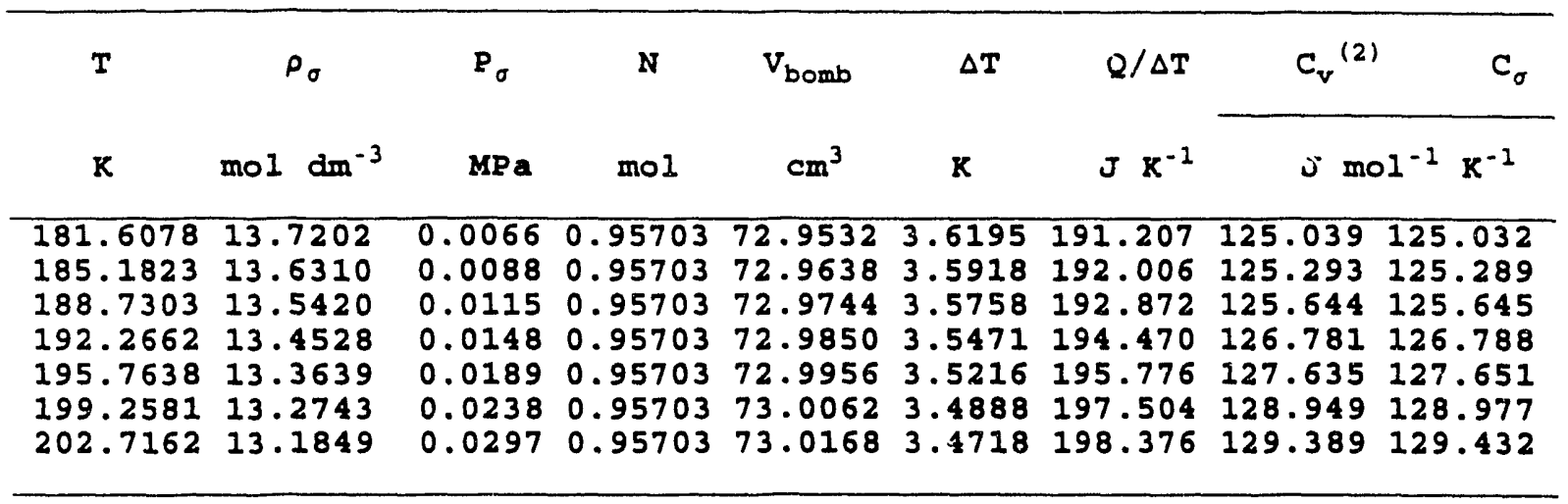

Table $61(\mathrm{~b})$. Experimental two-phase heat capacity data for HFC-125.

\begin{tabular}{|c|c|c|c|c|c|c|c|c|}
\hline $\mathbf{T}$ & $\rho_{\sigma}$ & $\mathbf{P}_{\sigma}$ & $\mathbf{N}$ & $v_{\text {bomb }}$ & $\Delta T$ & $Q / \Delta T$ & $C_{v}{ }^{12}$ & $c_{\sigma}$ \\
\hline${ }^{\circ} \mathrm{F}$ & $1 b E t^{-3}$ & psia & $1 b$ & $i n^{3}$ & ${ }^{\circ} \mathrm{F}$ & Btu ${ }^{\circ} \mathrm{F}^{-1}$ & Btu & $I b^{-1} \circ g^{-1}$ \\
\hline $\begin{array}{r}-132.776 \\
-126.342 \\
-119.955 \\
-113.591 \\
-107.295 \\
-101.005 \\
-94.781\end{array}$ & $\begin{array}{r}102.80 \\
102.13 \\
101.46 \\
100.80 \\
100.13 \\
99.46 \\
98.79\end{array}$ & $\begin{array}{l}0.96 \\
1.28 \\
1.67 \\
2.15 \\
2.74 \\
3.45 \\
4.31\end{array}$ & $\begin{array}{l}0.253 \\
0.253 \\
0.253 \\
0.253 \\
0.253 \\
0.253 \\
0.253\end{array}$ & $\begin{array}{l}4.452 \\
4.453 \\
4.453 \\
4.454 \\
4.454 \\
4.455 \\
4.456\end{array}$ & $\begin{array}{l}6.515 \\
6.465 \\
6.436 \\
6.385 \\
6.335 \\
6.280 \\
6.249\end{array}$ & $\begin{array}{ll}0.101 & 0.2 \\
0.101 & 0.2 \\
0.102 & 0.2 \\
0.102 & 0.2 \\
0.103 & 0.2 \\
0.104 & 0.2 \\
0.105 & 0.1\end{array}$ & $\begin{array}{l}2490 \\
2495 \\
2502 \\
2525 \\
2542 \\
2568 \\
2577\end{array}$ & $\begin{array}{l}0.2490 \\
0.2495 \\
0.2502 \\
0.2525 \\
0.2542 \\
0.2568 \\
0.2578\end{array}$ \\
\hline
\end{tabular}


Table 62 (a). Experimental two-phase heat capacity data for HFC-125.

\begin{tabular}{|c|c|c|c|c|c|c|c|}
\hline $\mathbf{T}$ & $\rho_{\sigma}$ & $\mathbf{P}_{\sigma}$ & $\mathbf{N}$ & $v_{\text {bomb }}$ & $\Delta T$ & $\mathrm{Q} / \Delta \mathrm{T}$ & $C_{v}{ }^{(2)}$ \\
\hline $\mathbf{K}$ & mol $\mathrm{dm}^{-3}$ & M:? & mol & $\mathrm{cm}^{3}$ & $\mathbf{K}$ & $\mathbf{J} \mathbf{K}^{-1}$ & $\mathrm{~J} \operatorname{mol}^{-1} \mathrm{~K}^{-1}$ \\
\hline $\begin{array}{l}176.9515 \\
180.4896 \\
183.9894 \\
187.4907\end{array}$ & $\begin{array}{l}13.8357 \\
13.7480 \\
13.6608 \\
13.5732\end{array}$ & $\begin{array}{l}0.0045 \\
0.0060 \\
0.0080 \\
0.0105\end{array}$ & $\begin{array}{l}0.98866 \\
0.98866 \\
0.98866 \\
0.98866\end{array}$ & $\begin{array}{l}72.9395 \\
72.9499 \\
72.9603 \\
72.9707\end{array}$ & $\begin{array}{l}3.5375 \\
3.5354 \\
3.5122 \\
3.5063\end{array}$ & $\begin{array}{l}195.188 \\
195.493 \\
196.605 \\
196.883\end{array}$ & $\begin{array}{ll}125.829 & 125.831 \\
125.552 & 125.558 \\
126.121 & 126.131 \\
125.865 & 125.883\end{array}$ \\
\hline
\end{tabular}

Table 62 (b). Experimental two-phase heat capacity data for HFC-125.

\begin{tabular}{|c|c|c|c|c|c|c|c|c|}
\hline $\mathbf{T}$ & $\rho_{\sigma}$ & $\mathbf{P}_{\sigma}$ & $N$ & & $\Delta \mathbf{T}$ & $Q / \Delta T$ & $C_{v}^{(2)}$ & $c_{\sigma}$ \\
\hline${ }^{\circ} \mathrm{F}$ & Ib $f t^{-3}$ & poia & $1 b$ & in $^{3}$ & ${ }^{\circ} \mathrm{F}$ & Btu ${ }^{\circ} \mathrm{F}^{-1}$ & Btu & $1 b^{-1} \circ F^{-1}$ \\
\hline $\begin{array}{l}-141.157 \\
-134.789 \\
-128.489 \\
-122.187\end{array}$ & $\begin{array}{l}103.67 \\
103.01 \\
102.35 \\
101.70\end{array}$ & $\begin{array}{l}0.65 \\
0.87 \\
1.16 \\
1.52\end{array}$ & $\begin{array}{l}0.262 \\
0.262 \\
0.262 \\
0.262\end{array}$ & $\begin{array}{l}4.451 \\
4.452 \\
4.452 \\
4.453\end{array}$ & $\begin{array}{l}6.367 \\
6.364 \\
6.322 \\
6.311\end{array}$ & $\begin{array}{ll}0.103 & 0.2 \\
0.103 & 0.2 \\
0.1 C 1 & 0.2 \\
0.104 & 0.2\end{array}$ & $\begin{array}{l}506 \\
500 \\
512 \\
506 \\
506\end{array}$ & $\begin{array}{l}0.2506 \\
0.2500 \\
0.2512 \\
0.2507\end{array}$ \\
\hline
\end{tabular}


Table 63. Coefficients to the MBWR equation of state for HFC-125 (units sie K, bar, L, mol)

$$
\begin{aligned}
& p=\sum_{n=1}^{9} a_{n} \rho^{n}+\exp \left(-\rho^{2} / \rho_{c}^{2}\right) \sum_{n=10}^{15} a_{n} \rho^{2 n-17} \\
& \rho_{\mathrm{c}} \quad=\quad 4.7650 \mathrm{~mol} / \mathrm{L} \\
& \begin{array}{l}
a_{1}=R T \\
a_{2}=b_{1} T+b_{2} T^{0.5}+b_{3}+b_{4} / T+b_{5} / T^{2}
\end{array} \\
& a_{3}=b_{6} T+b_{7}+b_{8} / T+b_{9} / T^{2} \\
& a_{4}=b_{10} T+b_{11}+b_{12} / T \\
& a_{5}=b_{13} \\
& a_{6}=b_{14} / T+b_{15} / T^{2} \\
& a_{7}=b_{16} / T \\
& a_{8}=b_{17} / T+b_{18} / T^{2} \\
& a_{9}=b_{19} / T^{2} \\
& \mathrm{a}_{10}=\mathrm{b}_{20} / \mathrm{T}^{2}+\mathrm{b}_{21} / \mathrm{T}^{3} \\
& a_{11}=b_{22} / T^{2}+b_{23} / T^{4} \\
& a_{12}=b_{24} / T^{2}+b_{25} / T^{3} \\
& a_{13}=b_{26} / T^{2}+b_{27} / T^{4} \\
& \mathrm{a}_{14}=\mathrm{b}_{28} / \mathrm{T}^{2}+\mathrm{b}_{29} / \mathrm{T}^{3} \\
& \mathrm{a}_{15}=\mathrm{b}_{30} / \mathrm{T}^{2}+\mathrm{b}_{31} / \mathrm{T}^{3}+\mathrm{b}_{32} / \mathrm{T}^{4} \\
& \text { i } \quad b_{i} \\
& 1 \quad 0.695150135527 \mathrm{E}-01 \\
& 2-0.109596263920 \mathrm{E}+02 \\
& 3 \quad 0.289171467191 \mathrm{E}+03 \\
& 4-0.510408655996 \mathrm{E}+05 \\
& 5 \quad 0.366753946576 \mathrm{E}+07 \\
& 6 \quad 0.385350808228 \mathrm{E}-01 \\
& 7-0.370988373715 \mathrm{E}+02 \\
& 8 \quad 0.134556555861 \mathrm{E}+05 \\
& 9 \quad 0.371143622964 \mathrm{E}+07 \\
& 10 \quad-0.123685768773 \mathrm{E}-02 \\
& 11 \quad 0.130495983411 \mathrm{E}+01 \\
& 12-0.468463056623 E+03 \\
& 13 \quad 0.511361375061 \mathrm{E}-01 \\
& 14-0.204695459886 \mathrm{E}+02 \\
& 15-0.414622181605 \mathrm{E}+04 \\
& 16 \quad 0.219744136091 \mathrm{E}+01
\end{aligned}
$$


Table 63. Coefficients to the MBWR equation of state for HFC-125 (units are K, bar, L, mol) (continued)

$\begin{array}{cc}17 & -0.637258406198 \mathrm{E}-01 \\ 18 & 0.291220108725 \mathrm{E}+02 \\ 19 & -0.102197580663 \mathrm{E}+01 \\ 20 & -0.560938443772 \mathrm{E}+07 \\ 21 & 0.770104599552 \mathrm{E}+08 \\ 22 & -0.224544749331 \mathrm{E}+06 \\ 23 & 0.183452398750 \mathrm{E}+10 \\ 24 & -0.292476384933 \mathrm{E}+04 \\ 25 & -0.388467529252 \mathrm{E}+05 \\ 26 & -0.339743229627 \mathrm{E}+02 \\ 27 & -0.544169038319 \mathrm{E}+06 \\ 28 & -0.168305711698 \mathrm{E}+00 \\ 29 & 0.115387298598 \mathrm{E}+02 \\ 30 & -0.734893856572 \mathrm{E}-03 \\ 31 & -0.329200834300 \mathrm{E}+00 \\ 32 & -0.403885226023 \mathrm{E}+01\end{array}$




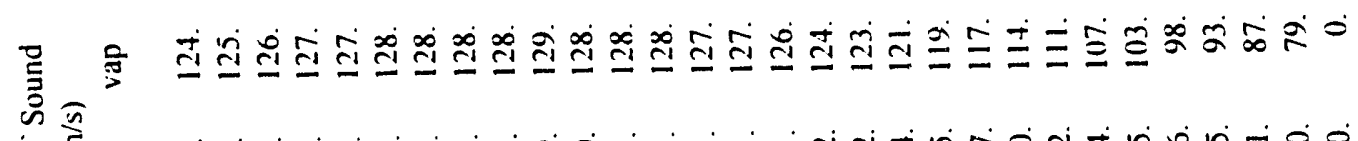

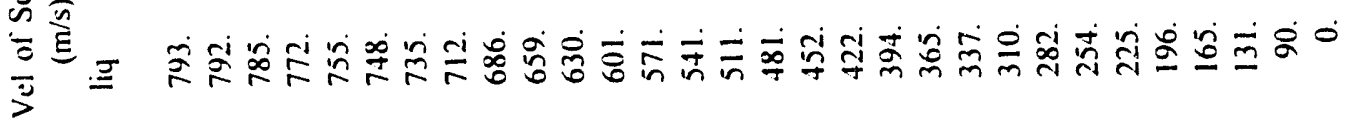

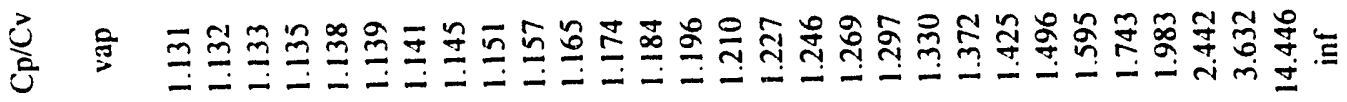

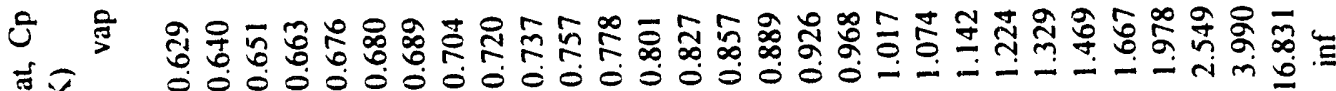

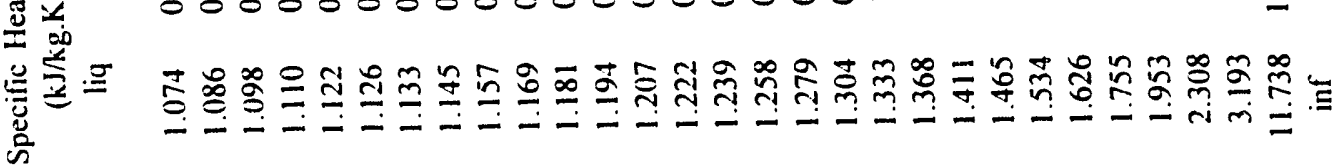

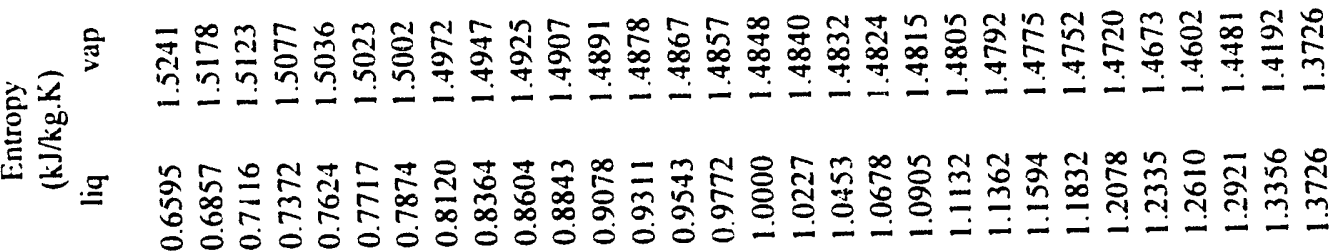

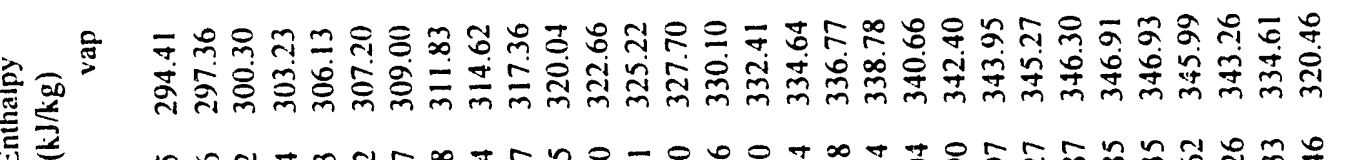

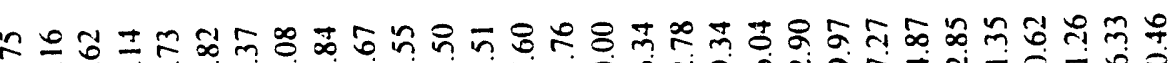

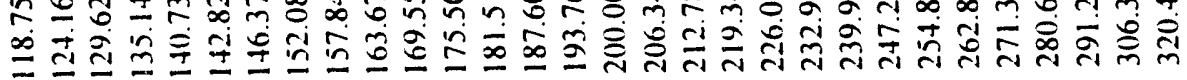

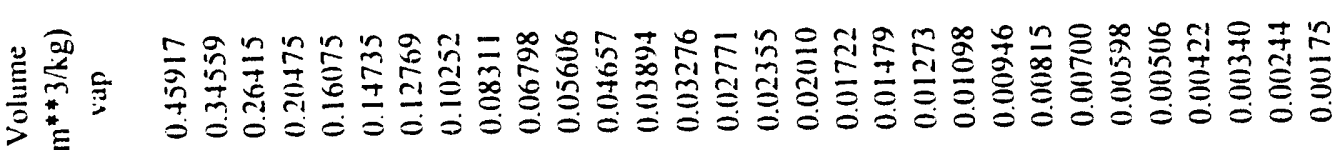

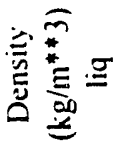

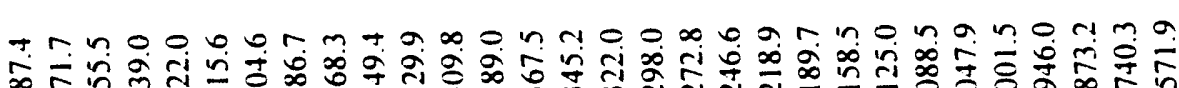

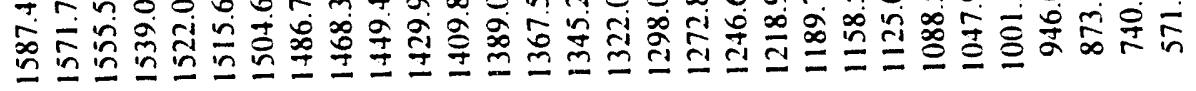

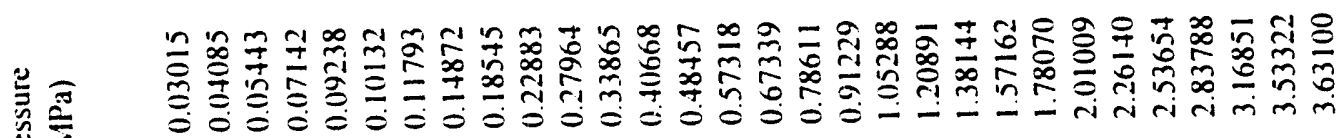

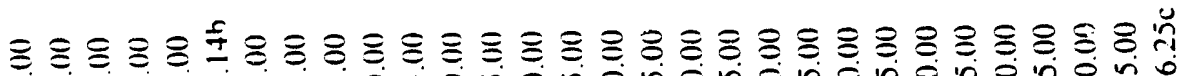

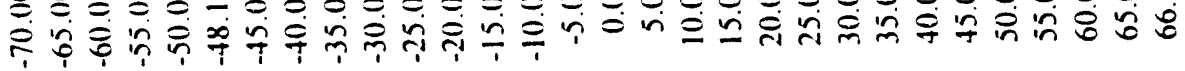




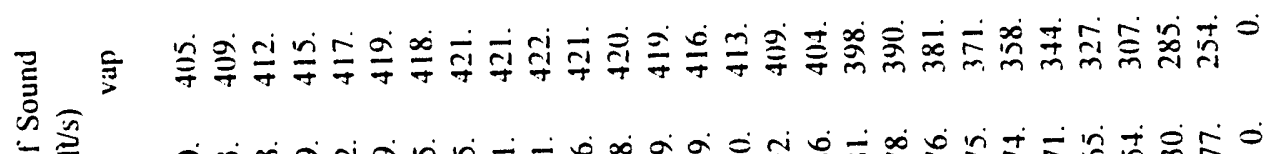

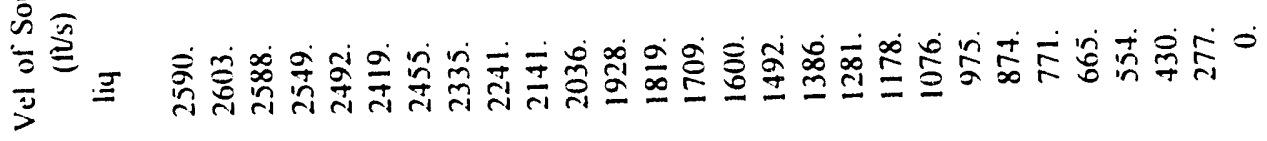

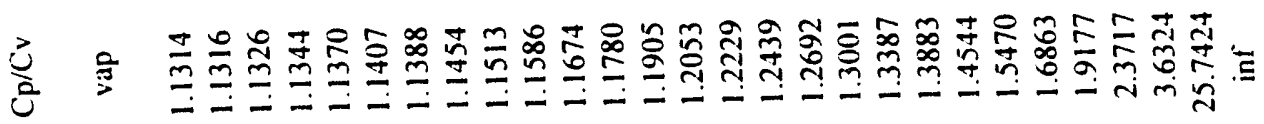

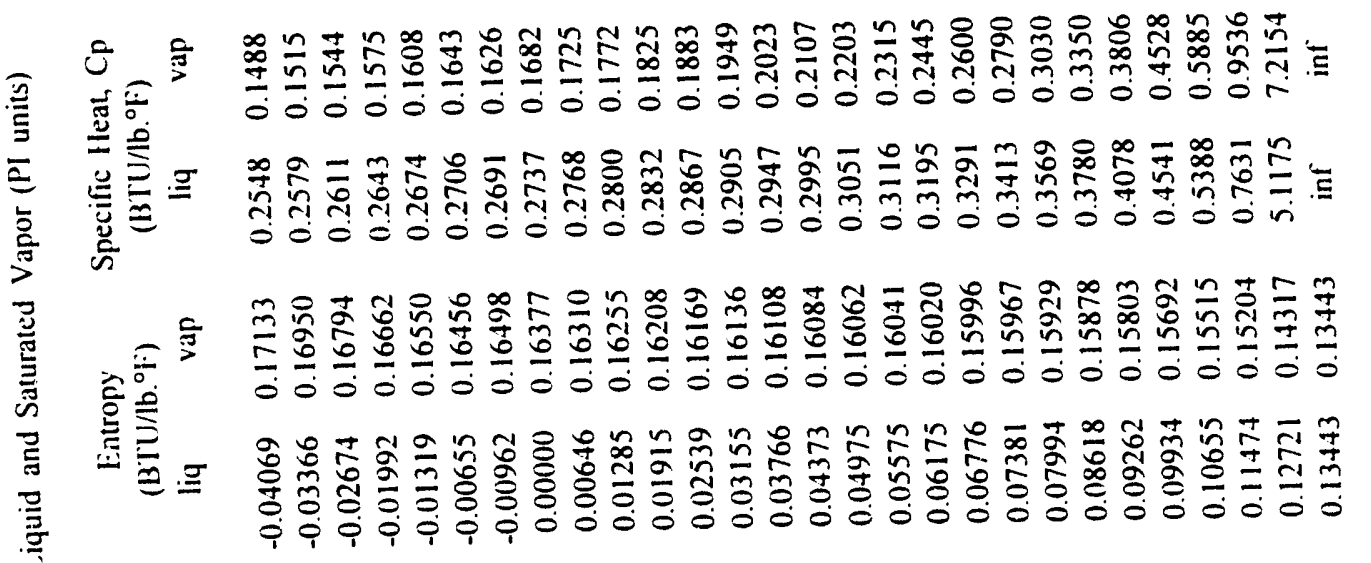

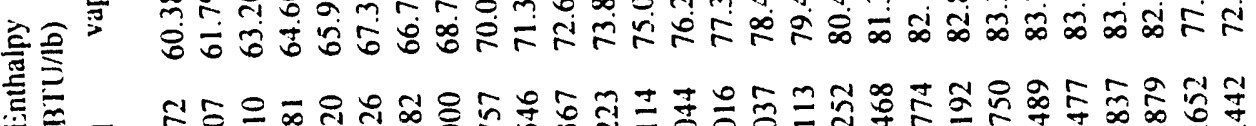

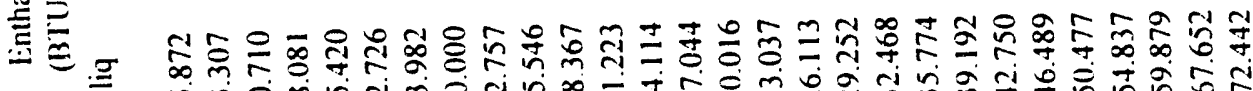

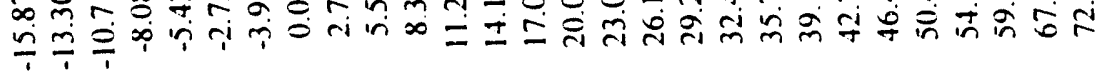

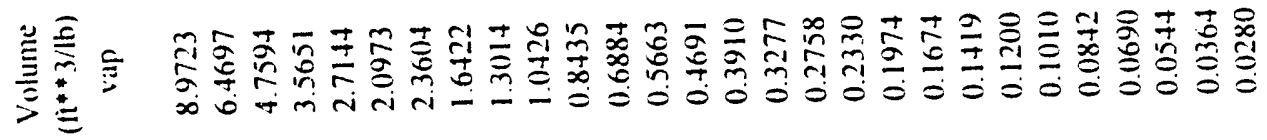

年

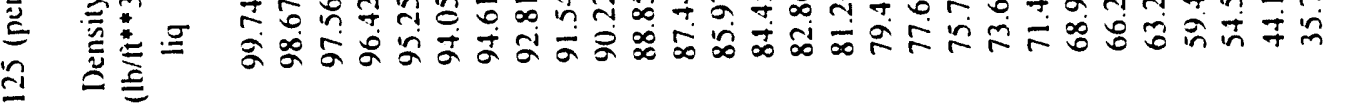

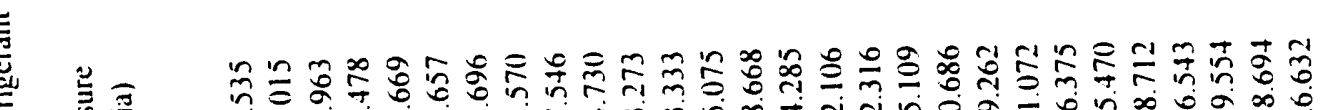

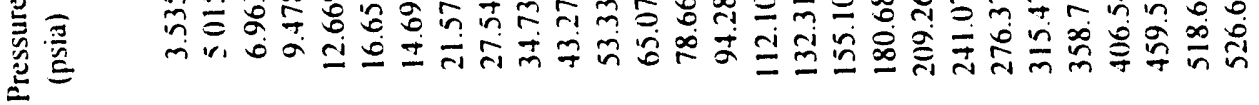

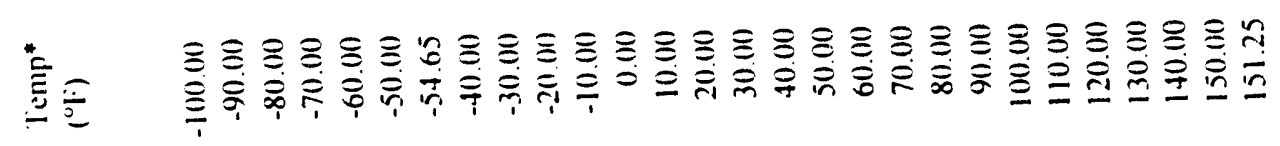


Table 65a. Iiquid PVT Data (Isochoric Apparatus) for HCFC-123 $1 / 3$

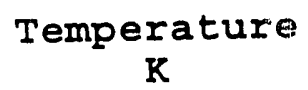

$$
\begin{aligned}
& 176.002 \\
& 178.000 \\
& 180.000 \\
& 182.000 \\
& 184.000 \\
& 186.000 \\
& 188.001 \\
& 190.000 \\
& 191.999
\end{aligned}
$$

189.999

191.999

194.000

195.999

198.001

199.999

202.000

204.000

206.000

207.999

206.000

208.002

210.001

212.001

214.000

216.000

218.000

220.000

222.000

224.001

226.000

225.000

226.002

228.001

230.000

231.999

234.000

236.001

238.000

240.001

241.998

244.001

245.999

248.002

250.001
Pressure $\mathrm{MPa}$

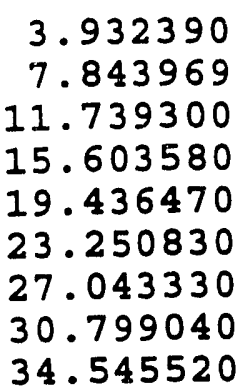

1.769352

5.297392

8.795440

12.283070

15.750070

19.196620

22.624520

26.034240

29.415060

32.774780

2.124055

5.273329

8.411843

11.535470

14.637850

17.722830

20.796100

23.850750

26.891850

29.917650

32.929010

.434245

1.810371

4.549389

7.273252

9.988414

12.691280

15.380230

18.056130

20.727310

23.377690

26.022690

28.650340

31.271920

33.876820
Density

11.45541
11.45294
11.45051
11.44811
11.44575
11.44341
11.44108
11.43877
11.43648

11.23748

11.23513

11.23282

11.23054

11.22828

11.22604

11.22382

11.22161

11.21942

11.21725

11.00750

11.00528

11.00310

11.00093

10.99879

10.99666

10.99455

10.99245

10.99036

10.98828

10.98621

10.71264

10.71159

10.70951

10.70745

10.70543

10.70342

10.70141

10.69942

10.69743

10.69545

10.69348

10.69152

10.68956

10.68760 
Table 65a. Liquid PVT Data (Isochoric Apparatus) for HCFC-123 $2 / 3$

\begin{tabular}{|c|c|c|}
\hline$\underset{\mathrm{K}}{\text { Temperature }}$ & $\begin{array}{l}\text { Pressure } \\
\text { MPa }\end{array}$ & $\begin{array}{l}\text { Density } \\
\text { mol } \cdot \mathrm{dm}^{-3}\end{array}$ \\
\hline $\begin{array}{l}249.001 \\
250.002 \\
252.000 \\
254.000 \\
255.999 \\
258.000 \\
259.999 \\
262.001 \\
263.999 \\
266.000 \\
268.001 \\
270.000 \\
272.000 \\
274.000 \\
276.001 \\
278.000\end{array}$ & $\begin{array}{r}0.840899 \\
2.001339 \\
4.321377 \\
6.631163 \\
8.930861 \\
11.225400 \\
13.508870 \\
15.785470 \\
18.049410 \\
20.311120 \\
22.560400 \\
24.798100 \\
27.035190 \\
29.259860 \\
31.475760 \\
33.681210\end{array}$ & $\begin{array}{l}10.35754 \\
10.35656 \\
10.35464 \\
10.35273 \\
10.35083 \\
10.34895 \\
10.34707 \\
10.34519 \\
10.34333 \\
10.34146 \\
10.33961 \\
10.33776 \\
10.33590 \\
10.33406 \\
10.33221 \\
10.33037\end{array}$ \\
\hline $\begin{array}{l}269.999 \\
272.000 \\
274.001 \\
275.998 \\
278.002 \\
280.002 \\
282.001 \\
284.001 \\
286.002 \\
288.001 \\
290.002 \\
291.998 \\
293.999 \\
296.000 \\
298.001 \\
300.000 \\
302.001\end{array}$ & $\begin{array}{r}2.688618 \\
4.698253 \\
6.704591 \\
8.712440 \\
10.701840 \\
12.696030 \\
14.687050 \\
16.671610 \\
18.649080 \\
20.612280 \\
22.580660 \\
24.538400 \\
26.489790 \\
28.434620 \\
30.368990 \\
32.300790 \\
34.214710\end{array}$ & $\begin{array}{l}10.06031 \\
10.05849 \\
10.05669 \\
10.05489 \\
10.05309 \\
10.05131 \\
10.04952 \\
10.04774 \\
10.04597 \\
10.04420 \\
10.04242 \\
10.04065 \\
10.03889 \\
10.03712 \\
10.03535 \\
10.03358 \\
10.03182\end{array}$ \\
\hline $\begin{array}{l}298.001 \\
300.001 \\
302.000 \\
303.999 \\
306.001 \\
307.998 \\
310.001 \\
312.001 \\
313.999 \\
316.001 \\
320.000 \\
324.000 \\
328.001\end{array}$ & $\begin{array}{r}3.166692 \\
4.822544 \\
6.475250 \\
8.125226 \\
9.771155 \\
11.406540 \\
13.050340 \\
14.683990 \\
16.310520 \\
17.932590 \\
21.172570 \\
24.392040 \\
27.589630\end{array}$ & $\begin{array}{l}9.62839 \\
9.62670 \\
9.62502 \\
9.62334 \\
9.62167 \\
9.62002 \\
9.61832 \\
9.61665 \\
9.61499 \\
9.61332 \\
9.60998 \\
9.60664 \\
9.60332\end{array}$ \\
\hline
\end{tabular}


Table 65a. Liquid PVT Data (Isochoric Apparatus) for HCFC-123 $3 / 3$

Temperature

$\mathrm{K}$

332.001

336.001

332.002

336.000

340.002

344.001

348.000

352.000

356.002

360.000

364.000

368.000

372.001

376.000

379.999
Pressure MPa

30.772140

33.932900

2.588553

5.132132

7.654527

10.190930

12.699880

15.210770

17.703940

20.191730

22.681310

25.146830

27.601790

30.049470

32.487460
Density

$\mathrm{mol} \cdot \mathrm{dm}^{-3}$

9.59997

9.59662

3.04135

9.03826

9.03533

9.03210

9.02904

9.02609

9.02304

9.01995

9.01684

9.01372

9.01061

9.00747

9.00433 
Table 65b. Liquid PVT Data (Isochoric Apparatus) for HCFC-123 $1 / 3$

\begin{tabular}{|c|c|c|}
\hline $\begin{array}{c}\text { Temperature } \\
{ }^{\circ} \mathrm{F}\end{array}$ & $\begin{array}{c}\text { Pressure } \\
\text { psia }\end{array}$ & $\begin{array}{l}\text { Density } \\
\text { lb. } \mathrm{ft}^{-3}\end{array}$ \\
\hline $\begin{array}{l}-142.87 \\
-139.27 \\
-135.67 \\
-132.07 \\
-128.47 \\
-124.87 \\
-121.27 \\
-117.67 \\
-114.07\end{array}$ & $\begin{array}{r}570.346 \\
1137.674 \\
1702.645 \\
2263.112 \\
2819.027 \\
3372.254 \\
3922.310 \\
4467.031 \\
5010.413\end{array}$ & $\begin{array}{l}109.366 \\
109.343 \\
109.320 \\
109.297 \\
109.274 \\
109.252 \\
109.230 \\
109.208 \\
109.186\end{array}$ \\
\hline $\begin{array}{l}-117.67 \\
-114.07 \\
-110.47 \\
-106.87 \\
-103.27 \\
-99.67 \\
-96.07 \\
-92.47 \\
-88.87 \\
-85.27\end{array}$ & $\begin{array}{r}256.623 \\
768.323 \\
1275.673 \\
1781.512 \\
2284.359 \\
2784.239 \\
3281.415 \\
3775.954 \\
4266.301 \\
4753.588\end{array}$ & $\begin{array}{l}107.286 \\
107.263 \\
107.241 \\
107.220 \\
107.198 \\
107.177 \\
107.155 \\
107.134 \\
107.113 \\
107.093\end{array}$ \\
\hline $\begin{array}{l}-88.87 \\
-85.27 \\
-81.67 \\
-78.07 \\
-74.47 \\
-70.87 \\
-67.27 \\
-63.67 \\
-60.07 \\
-56.47 \\
-52.87\end{array}$ & $\begin{array}{r}308.069 \\
764.833 \\
1220.037 \\
1673.081 \\
2123.044 \\
2570.484 \\
3016.225 \\
3459.265 \\
3900.340 \\
4339.196 \\
4775.958\end{array}$ & $\begin{array}{l}105.090 \\
105.069 \\
105.048 \\
105.027 \\
105.007 \\
104.987 \\
104.967 \\
104.947 \\
104.927 \\
104.907 \\
104.887\end{array}$ \\
\hline $\begin{array}{l}-54.67 \\
-52.87 \\
-49.27 \\
-45.67 \\
-42.07 \\
-38.47 \\
-34.87 \\
-31.27 \\
-27.67 \\
-24.07 \\
-20.47 \\
-16.87 \\
-13.27 \\
-9.67\end{array}$ & $\begin{array}{r}62.982 \\
262.573 \\
659.834 \\
1054.898 \\
1448.700 \\
1840.718 \\
2230.718 \\
2618.825 \\
3006.247 \\
3390.653 \\
3774.279 \\
4155.388 \\
4535.617 \\
4913.426\end{array}$ & $\begin{array}{l}102.275 \\
102.265 \\
102.245 \\
102.226 \\
102.206 \\
102.187 \\
102.168 \\
102.149 \\
102.130 \\
102.111 \\
102.092 \\
102.073 \\
102.055 \\
102.036\end{array}$ \\
\hline
\end{tabular}


Table 65b. Iiquid PVT Data (Isochoric Apparatus) for HCFC-123 $2 / 3$

$\underset{\circ F}{\substack{\text { Temperature } \\ \circ}}$

-11.47
-9.67
-6.07
-2.47
1.13
4.73
8.33
11.93
15.53
19.13
22.73
26.33
29.93
33.53
37.13
40.73

26.33

29.93

33.53

37.13

40.73

44.33

47.93

51.53

55.13

58.73

62.33

65.93

69.53

73.13

76.73

80.33

83.93

76.73

80.33

83.93

87.53

91.13

94.73

98.33

101.93

105.53

109.13

116.33

123.53
Pressure
psia

121.962

290.270

626.764

961.771

1295.314

1628.110

1959.299

2289.493

2617.850

2945.884

3272.115

3596.667

3921.130

4243.791

4565.181

4885.055

389.952

681.425

972.420

1263.635

1552.173

1841.407

2130.180

2418.017

2704.825

2989.564

3275.054

3559.000

3842.026

4124.100

4404.657

4684.842

4962.433

459.291

699.452

939.157

1178.466

1417.189

1654.382

1892.795

2129.736

2365.645

2600.907

3070.827

3537.773
Density
Ib $\mathrm{ft}^{-3}$

98.885

98.876

98.857

98.839

98.821

98.803

98.785

98.767

98.749

98.731

98.714

98.696

98.678

98.661

98.643

98.626

96.047

96.030

96.013

95.995

95.978

95.961

95.944

95.927

95.910

95.893

95.876

95.860

95.843

95.826

95.809

95.792

95.775

91.924

91.907

91.891

91.875

91.859

91.844

91.827

91.812

91.796

91.780

91.748

91.716 
Table 65b. Liquid PVT Data (Isochoric Apparatus) for HCFC-123 $3 / 3$

$\begin{array}{ccc}\begin{array}{c}\text { Temperature } \\ { }^{\circ} \text { F }\end{array} & \begin{array}{c}\text { Pressure } \\ \text { psia }\end{array} & \begin{array}{c}\text { Density } \\ \text { lb. } \text { ft }^{-3}\end{array} \\ 130.73 & 4001.545 & 91.684 \\ 137.93 & 4463.129 & 91.652 \\ 145.13 & 4921.560 & 91.620 \\ 137.93 & 375.439 & 86.319 \\ 145.13 & 744.354 & 86.290 \\ 152.33 & 1110.197 & 86.262 \\ 159.53 & 1478.072 & 86.231 \\ 166.73 & 1841.965 & 86.202 \\ 173.93 & 2206.140 & 86.173 \\ 181.13 & 2567.744 & 86.144 \\ 188.33 & 2928.568 & 86.115 \\ 195.53 & 3289.652 & 86.085 \\ 202.73 & 3647.246 & 86.055 \\ 209.93 & 4003.308 & 86.026 \\ 217.13 & 4358.315 & 85.996 \\ 224.33 & 4711.916 & 85.966\end{array}$


Table $66(a)$. Experimental liquid heat capacity data for HCFC-123.

\begin{tabular}{|c|c|c|c|c|c|c|c|c|c|}
\hline$T$ & $\delta \mathbf{T}$ & $P$ & $\delta \rho$ & P & $\delta P$ & $C_{v, \exp }$ & $\delta C_{v}$ & \multirow[t]{2}{*}{$\delta C_{v}, \frac{9}{6}$} & \multirow[t]{2}{*}{ Run } \\
\hline \multicolumn{2}{|c|}{$\mathbf{K}$} & mol & $d m^{-3}$ & \multicolumn{2}{|c|}{$\mathbf{M P a}$} & $\mathrm{J}$ mol & $-1 \mathbf{K}^{-1}$ & & \\
\hline $\begin{array}{l}257.8615 \\
259.8746 \\
261.9246 \\
263.9040 \\
265.9697 \\
267.9195 \\
269.9803 \\
271.9136 \\
273.9567 \\
275.8598 \\
277.9191 \\
279.7909 \\
283.6947 \\
287.5835\end{array}$ & $\begin{array}{l}0.0034 \\
0.0032 \\
0.0035 \\
0.0033 \\
0.0036 \\
0.0031 \\
0.0034 \\
0.0032 \\
0.0033 \\
0.0032 \\
0.0032 \\
0.0032 \\
0.0032 \\
0.0033\end{array}$ & $\begin{array}{l}10.241 \\
10.237 \\
10.232 \\
10.228 \\
10.224 \\
10.220 \\
10.216 \\
10.212 \\
10.208 \\
10.204 \\
10.200 \\
10.196 \\
10.188 \\
10.180\end{array}$ & $\begin{array}{l}0.009 \\
0.010 \\
0.011 \\
0.011 \\
0.012 \\
0.013 \\
0.014 \\
0.014 \\
0.015 \\
0.016 \\
0.017 \\
0.017 \\
0.019 \\
0.020\end{array}$ & $\begin{array}{r}3.163 \\
5.244 \\
7.357 \\
9.390 \\
11.502 \\
13.484 \\
15.565 \\
17.504 \\
19.539 \\
21.420 \\
23.440 \\
25.263 \\
29.024 \\
32.720\end{array}$ & $\begin{array}{l}0.033 \\
0.033 \\
0.033 \\
0.033 \\
0.033 \\
0.033 \\
0.033 \\
0.033 \\
0.033 \\
0.033 \\
0.033 \\
0.033 \\
0.033 \\
0.033\end{array}$ & $\begin{array}{l}103.93 \\
104.12 \\
105.04 \\
105.11 \\
106.26 \\
106.39 \\
106.68 \\
106.51 \\
107.52 \\
107.07 \\
108.29 \\
108.26 \\
109.19 \\
110.20\end{array}$ & $\begin{array}{l}0.64 \\
0.39 \\
0.29 \\
0.25 \\
0.22 \\
0.21 \\
0.20 \\
0.19 \\
0.19 \\
0.18 \\
0.18 \\
0.18 \\
0.17 \\
0.17\end{array}$ & $\begin{array}{l}0.62 \\
0.38 \\
0.28 \\
0.24 \\
0.21 \\
0.19 \\
0.19 \\
0.18 \\
0.17 \\
0.17 \\
0.16 \\
0.16 \\
0.16 \\
0.16\end{array}$ & $\begin{array}{l}a \\
b \\
a \\
b \\
a \\
b \\
a \\
b \\
a \\
b \\
a \\
b \\
b \\
b\end{array}$ \\
\hline
\end{tabular}

Table $66(\mathrm{~b})$. Experimental liquid heat capacity data for HCFC-123.

\begin{tabular}{|c|c|c|c|c|c|c|c|c|c|}
\hline $\mathbf{T}$ & $\delta \mathrm{T}$ & $\rho$ & $\delta p$ & P & $\delta P$ & $C_{v, \exp }$ & $\delta C_{v}$ & $\delta C_{v}, 8$ & Run \\
\hline \multicolumn{2}{|c|}{${ }^{\circ} \mathrm{F}$} & $1 b$ & $f t^{-3}$ & \multicolumn{2}{|c|}{ psia } & Btu & $1 b^{-1}{ }^{\circ} F^{-1}$ & & \\
\hline $\begin{array}{r}4.481 \\
8.104 \\
11.794 \\
15.357 \\
19.075 \\
22.585 \\
26.295 \\
29.774 \\
33.452 \\
36.878 \\
40.584 \\
43.954 \\
50.980 \\
57.980\end{array}$ & $\begin{array}{l}0.006 \\
0.006 \\
0.006 \\
0.006 \\
0.006 \\
0.006 \\
0.006 \\
0.006 \\
0.006 \\
0.006 \\
0.006 \\
0.006 \\
0.006 \\
0.006\end{array}$ & $\begin{array}{l}97.77 \\
97.73 \\
97.69 \\
97.65 \\
97.61 \\
97.57 \\
97.53 \\
97.49 \\
97.46 \\
97.42 \\
97.38 \\
97.34 \\
97.27 \\
97.19\end{array}$ & $\begin{array}{l}0.09 \\
0.10 \\
0.11 \\
0.11 \\
0.11 \\
0.12 \\
0.13 \\
0.13 \\
0.14 \\
0.15 \\
0.16 \\
0.16 \\
0.18 \\
0.19\end{array}$ & $\begin{array}{r}458.8 \\
760.6 \\
1067.0 \\
1361.9 \\
1668.2 \\
1955.7 \\
2257.5 \\
2538.7 \\
2833.9 \\
3106.7 \\
3399.7 \\
3664.1 \\
4209.6 \\
4745.6\end{array}$ & $\begin{array}{l}4.8 \\
4.8 \\
4.8 \\
4.8 \\
4.8 \\
4.8 \\
4.8 \\
4.8 \\
4.8 \\
4.8 \\
4.8 \\
4.8 \\
4.8 \\
4.8\end{array}$ & $\begin{array}{l}0.16243 \\
0.16272 \\
0.16416 \\
0.16427 \\
0.16607 \\
0.16627 \\
0.16672 \\
0.16646 \\
0.16804 \\
0.16733 \\
0.16924 \\
0.16919 \\
0.17065 \\
0.17223\end{array}$ & $\begin{array}{l}0.00100 \\
0.00061 \\
0.00045 \\
0.00039 \\
0.00034 \\
0.00033 \\
0.00031 \\
0.00030 \\
0.00030 \\
0.00028 \\
0.00028 \\
0.00028 \\
0.00027 \\
0.00027\end{array}$ & $\begin{array}{l}0.62 \\
0.38 \\
0.28 \\
0.24 \\
0.21 \\
0.1 .9 \\
0.19 \\
0.18 \\
0.17 \\
0.17 \\
0.16 \\
0.16 \\
0.16 \\
0.16\end{array}$ & $\begin{array}{l}a \\
b \\
a \\
b \\
a \\
i \\
a \\
b \\
a \\
b \\
a \\
b \\
b \\
b\end{array}$ \\
\hline
\end{tabular}


Table 67 (a). Experimental liquid heat capacity data for HCFC-123.

\begin{tabular}{|c|c|c|c|c|c|c|c|c|c|}
\hline$T$ & $\delta \mathrm{T}$ & $\rho$ & $\delta p$ & P & $\delta P$ & $C_{v, \exp }$ & $\delta C_{v}$ & $\delta C_{v}, \frac{8}{\delta}$ & $\overline{\text { Run }}$ \\
\hline \multicolumn{2}{|c|}{ K } & mol & $\mathrm{dm}^{-3}$ & \multicolumn{2}{|c|}{ MPa } & $\mathrm{J}$ mol & $-1 \mathbf{K}^{-1}$ & & \\
\hline $\begin{array}{l}284.2425 \\
286.1803 \\
288.2634 \\
290.1925 \\
292.2663 \\
294.1864 \\
296.2546 \\
298.1617 \\
300.2107 \\
302.1200 \\
304.1621 \\
306.0576 \\
308.0901 \\
309.9691 \\
311.9977 \\
313.8777\end{array}$ & $\begin{array}{l}0.0035 \\
0.0031 \\
0.0037 \\
0.0032 \\
0.0032 \\
0.0032 \\
0.0032 \\
0.0031 \\
0.0032 \\
0.0032 \\
0.0033 \\
0.0031 \\
0.0034 \\
0.0033 \\
0.0033 \\
0.0032\end{array}$ & $\begin{array}{l}9.846 \\
9.843 \\
9.839 \\
9.836 \\
9.832 \\
9.828 \\
9.825 \\
9.821 \\
9.818 \\
9.814 \\
9.810 \\
9.807 \\
9.803 \\
9.800 \\
9.797 \\
9.793\end{array}$ & $\begin{array}{l}0.009 \\
0.009 \\
0.010 \\
0.011 \\
0.011 \\
0.012 \\
0.013 \\
0.013 \\
0.014 \\
0.014 \\
0.015 \\
0.016 \\
0.016 \\
0.017 \\
0.017 \\
0.018\end{array}$ & $\begin{array}{r}3.642 \\
5.340 \\
7.157 \\
8.832 \\
10.624 \\
12.275 \\
14.044 \\
15.668 \\
17.404 \\
19.013 \\
20.725 \\
22.306 \\
23.992 \\
25.542 \\
27.206 \\
28.738\end{array}$ & $\begin{array}{l}0.012 \\
0.012 \\
0.012 \\
0.012 \\
0.012 \\
0.012 \\
0.012 \\
0.012 \\
0.012 \\
0.012 \\
0.012 \\
0.012 \\
0.012 \\
0.012 \\
0.012 \\
0.012\end{array}$ & $\begin{array}{l}108.44 \\
108.36 \\
109.32 \\
109.06 \\
110.08 \\
109.76 \\
110.48 \\
110.60 \\
111.31 \\
111.02 \\
11 . .47 \\
111.52 \\
111.57 \\
111.91 \\
112.82 \\
113.25\end{array}$ & $\begin{array}{l}0.54 \\
0.35 \\
0.28 \\
0.23 \\
0.21 \\
0.19 \\
0.18 \\
0.18 \\
0.17 \\
0.17 \\
0.17 \\
0.16 \\
0.17 \\
0.16 \\
0.16 \\
0.16\end{array}$ & $\begin{array}{l}0.50 \\
0.33 \\
0.25 \\
0.21 \\
0.19 \\
0.18 \\
0.17 \\
0.16 \\
0.16 \\
0.15 \\
0.15 \\
0.15 \\
0.15 \\
0.15 \\
0.15 \\
0.14\end{array}$ & $\begin{array}{l}a \\
b \\
a \\
b \\
a \\
b \\
a \\
b \\
a \\
b \\
a \\
b \\
a \\
b \\
a \\
b\end{array}$ \\
\hline
\end{tabular}

Table 67 (b). Experimental liquid heat capacity data for HCFC-123.

\begin{tabular}{|c|c|c|c|c|c|c|c|c|c|}
\hline$T$ & $\delta \mathbf{T}$ & $\rho$ & $\delta p$ & P & $\delta P$ & $C_{v, \exp }$ & $\delta C_{v}$ & \multirow[t]{2}{*}{$\delta C_{v}, 9$} & $\overline{\text { Run }}$ \\
\hline \multicolumn{2}{|c|}{${ }^{\circ} \mathbf{F}$} & $1 b$ & $f t^{-3}$ & \multicolumn{2}{|c|}{ psia } & Btu & $1 b^{-1} \circ g^{-1}$ & & \\
\hline $\begin{array}{r}51.966 \\
55.455 \\
59.204 \\
62.676 \\
66.409 \\
69.865 \\
73.588 \\
77.021 \\
80.709 \\
84.146 \\
87.822 \\
91.234 \\
94.892 \\
98.274 \\
101.926 \\
105.310\end{array}$ & $\begin{array}{l}0.006 \\
0.006 \\
0.007 \\
0.006 \\
0.006 \\
0.006 \\
0.006 \\
0.006 \\
0.006 \\
0.006 \\
0.006 \\
0.006 \\
0.006 \\
0.006 \\
0.006 \\
0.006\end{array}$ & $\begin{array}{l}94.00 \\
93.97 \\
93.93 \\
93.91 \\
93.87 \\
93.83 \\
93.80 \\
93.76 \\
93.73 \\
93.70 \\
93.66 \\
93.63 \\
93.59 \\
93.56 \\
93.53 \\
93.49\end{array}$ & $\begin{array}{l}0.09 \\
0.09 \\
0.10 \\
0.11 \\
0.11 \\
0.11 \\
0.12 \\
0.12 \\
0.13 \\
0.13 \\
0.14 \\
0.15 \\
0.15 \\
0.16 \\
0.16 \\
0.17\end{array}$ & $\begin{array}{r}528.2 \\
774.5 \\
1038.0 \\
1281.0 \\
1540.9 \\
1780.3 \\
2036.9 \\
2272.5 \\
2524.2 \\
2757.6 \\
3005.9 \\
3235.2 \\
3479.8 \\
3704.6 \\
3945.9 \\
4168.1\end{array}$ & $\begin{array}{l}1.7 \\
1.7 \\
1.7 \\
1.7 \\
1.7 \\
1.7 \\
1.7 \\
1.7 \\
1.7 \\
1.7 \\
1.7 \\
1.7 \\
1.7 \\
1.7 \\
1.7 \\
1.7\end{array}$ & $\begin{array}{l}0.16947 \\
0.16935 \\
0.17085 \\
0.17044 \\
0.17204 \\
0.17154 \\
0.17266 \\
0.17285 \\
0.17396 \\
0.17351 \\
0.17421 \\
0.17429 \\
0.17437 \\
0.17490 \\
0.17632 \\
0.17699\end{array}$ & $\begin{array}{l}0.00084 \\
0.00055 \\
0.00044 \\
0.00036 \\
0.00033 \\
0.00030 \\
0.00028 \\
0.00028 \\
0.00027 \\
0.00027 \\
0.00027 \\
0.00025 \\
0.00027 \\
0.00025 \\
0.00025 \\
0.00025\end{array}$ & $\begin{array}{l}0.50 \\
0.33 \\
0.25 \\
0.21 \\
0.19 \\
0.18 \\
0.17 \\
0.16 \\
0.16 \\
0.15 \\
0.15 \\
0.15 \\
0.15 \\
0.15 \\
0.15 \\
0.14\end{array}$ & $\begin{array}{l}a \\
b \\
a \\
b \\
a \\
b \\
a \\
b \\
a \\
b \\
a \\
b \\
a \\
b \\
a \\
b\end{array}$ \\
\hline
\end{tabular}


Table 68(a). Experimental liquid heat capacity data for HCFC-123.

\begin{tabular}{|c|c|c|c|c|c|c|c|c|c|}
\hline $\bar{T}$ & $\delta T$ & $\rho$ & $\delta \rho$ & P & $\delta P$ & $\bar{C}_{v, \exp }$ & $\delta C_{v}$ & $\delta C_{v}, 8$ & $\overline{R u n}$ \\
\hline \multicolumn{2}{|c|}{$\mathrm{K}$} & mol & $\mathrm{dm}^{-3}$ & \multicolumn{2}{|c|}{$\mathbf{M P a}$} & J mol & $-1 \mathbf{K}^{-1}$ & & \\
\hline $\begin{array}{l}313.2597 \\
315.3753 \\
317.3023 \\
319.3942 \\
321.3426 \\
323.3948 \\
325.3607 \\
327.4009 \\
329.3804 \\
331.3872 \\
333.3849 \\
335.3769 \\
337.3937 \\
339.3594 \\
341.3919\end{array}$ & $\begin{array}{l}0.0033 \\
0.0034 \\
0.0031 \\
0.0031 \\
0.0032 \\
0.0032 \\
0.0031 \\
0.0032 \\
0.0030 \\
0.0033 \\
0.0031 \\
0.0031 \\
0.0032 \\
0.0036 \\
0.0031\end{array}$ & $\begin{array}{l}9.375 \\
9.372 \\
9.369 \\
9.366 \\
9.363 \\
9.360 \\
9.357 \\
9.354 \\
9.351 \\
9.348 \\
9.344 \\
9.341 \\
9.338 \\
9.335 \\
9.332\end{array}$ & $\begin{array}{l}0.008 \\
0.009 \\
0.009 \\
0.010 \\
0.010 \\
0.011 \\
0.011 \\
0.012 \\
0.012 \\
0.013 \\
0.013 \\
0.014 \\
0.014 \\
0.015 \\
0.015\end{array}$ & $\begin{array}{l}3.311 \\
4.819 \\
6.188 \\
7.670 \\
9.046 \\
10.491 \\
11.871 \\
13.298 \\
14.678 \\
16.071 \\
17.452 \\
18.824 \\
20.207 \\
21.548 \\
22.928\end{array}$ & $\begin{array}{l}0.010 \\
0.010 \\
0.010 \\
0.010 \\
0.010 \\
0.010 \\
0.010 \\
0.010 \\
0.010 \\
0.010 \\
0.010 \\
0.010 \\
0.010 \\
0.010 \\
0.010\end{array}$ & $\begin{array}{l}112.31 \\
112.74 \\
112.95 \\
113.07 \\
113.36 \\
114.01 \\
114.06 \\
114.27 \\
114.04 \\
114.69 \\
115.02 \\
115.25 \\
115.36 \\
116.06 \\
115.66\end{array}$ & $\begin{array}{l}0.56 \\
0.36 \\
0.28 \\
0.23 \\
0.21 \\
0.19 \\
0.18 \\
0.17 \\
0.16 \\
0.16 \\
0.16 \\
0.16 \\
0.16 \\
0.16 \\
0.15\end{array}$ & $\begin{array}{l}0.50 \\
0.32 \\
0.25 \\
0.20 \\
0.18 \\
0.17 \\
0.16 \\
0.15 \\
0.14 \\
0.14 \\
0.14 \\
0.14 \\
0.14 \\
0.14 \\
0.13\end{array}$ & $\begin{array}{l}e \\
f \\
e \\
f \\
e \\
f \\
e \\
f \\
e \\
f \\
e \\
f \\
e \\
f \\
e\end{array}$ \\
\hline
\end{tabular}

Table $68(\mathrm{~b})$. Experimental liquid heat capacity data for HCFC-123.

\begin{tabular}{|c|c|c|c|c|c|c|c|c|c|}
\hline$T$ & $\delta T$ & $\bar{\rho}$ & $\delta p$ & $P$ & $\delta P$ & $C_{v, \exp }$ & $\delta C_{v}$ & $\delta C_{v}, 9$ & $\overline{R u n}$ \\
\hline \multicolumn{2}{|c|}{${ }^{\circ} \mathrm{F}$} & $1 b$ & $E t^{-3}$ & \multicolumn{2}{|c|}{ poia } & Btu & $1 b^{-1} \circ F^{-1}$ & & \\
\hline $\begin{array}{l}104.197 \\
108.006 \\
111.474 \\
115.240 \\
118.747 \\
122.441 \\
125.979 \\
129.652 \\
133.215 \\
136.827 \\
140.423 \\
144.008 \\
147.639 \\
151.177 \\
154.835\end{array}$ & $\begin{array}{l}0.006 \\
0.006 \\
0.006 \\
0.006 \\
0.006 \\
0.006 \\
0.006 \\
0.006 \\
0.005 \\
0.006 \\
0.006 \\
0.006 \\
0.006 \\
0.006 \\
0.006\end{array}$ & $\begin{array}{l}89.50 \\
89.48 \\
89.45 \\
89.42 \\
89.39 \\
89.36 \\
89.33 \\
89.30 \\
89.27 \\
89.25 \\
89.21 \\
89.18 \\
89.15 \\
89.12 \\
89.09\end{array}$ & $\begin{array}{l}0.08 \\
0.09 \\
0.09 \\
0.10 \\
0.10 \\
0.11 \\
0.11 \\
0.11 \\
0.11 \\
0.12 \\
0.12 \\
0.13 \\
0.13 \\
0.14 \\
0.14\end{array}$ & $\begin{array}{r}480.2 \\
698.9 \\
897.5 \\
1112.4 \\
1312.0 \\
1521.6 \\
1721.7 \\
1928.7 \\
2128.9 \\
2330.9 \\
2531.2 \\
2730.2 \\
2930.8 \\
3125.3 \\
3325.4\end{array}$ & $\begin{array}{l}1.5 \\
1.5 \\
1.5 \\
1.5 \\
1.5 \\
1.5 \\
1.5 \\
1.5 \\
1.5 \\
1.5 \\
1.5 \\
1.5 \\
1.5 \\
1.5 \\
1.5\end{array}$ & $\begin{array}{l}0.17552 \\
0.17619 \\
0.17652 \\
0.17671 \\
0.17716 \\
0.17818 \\
0.17826 \\
0.17859 \\
0.17823 \\
0.17924 \\
0.17976 \\
0.18012 \\
0.18029 \\
0.18138 \\
0.18076\end{array}$ & $\begin{array}{l}0.00088 \\
0.00056 \\
0.00044 \\
0.00036 \\
0.00033 \\
0.00030 \\
0.00028 \\
0.00027 \\
0.00025 \\
0.00025 \\
0.00025 \\
0.00025 \\
0.00025 \\
0.00025 \\
0.00023\end{array}$ & $\begin{array}{l}0.50 \\
0.32 \\
0.25 \\
0.20 \\
0.18 \\
0.17 \\
0.16 \\
0.15 \\
0.14 \\
0.14 \\
0.14 \\
0.14 \\
0.14 \\
0.14 \\
0.13\end{array}$ & $\begin{array}{l}e \\
f \\
e \\
f \\
e \\
f \\
e \\
f \\
e \\
f \\
e \\
f \\
e \\
f \\
e\end{array}$ \\
\hline
\end{tabular}




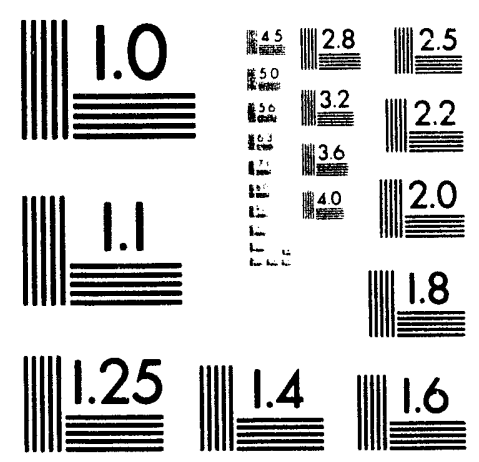



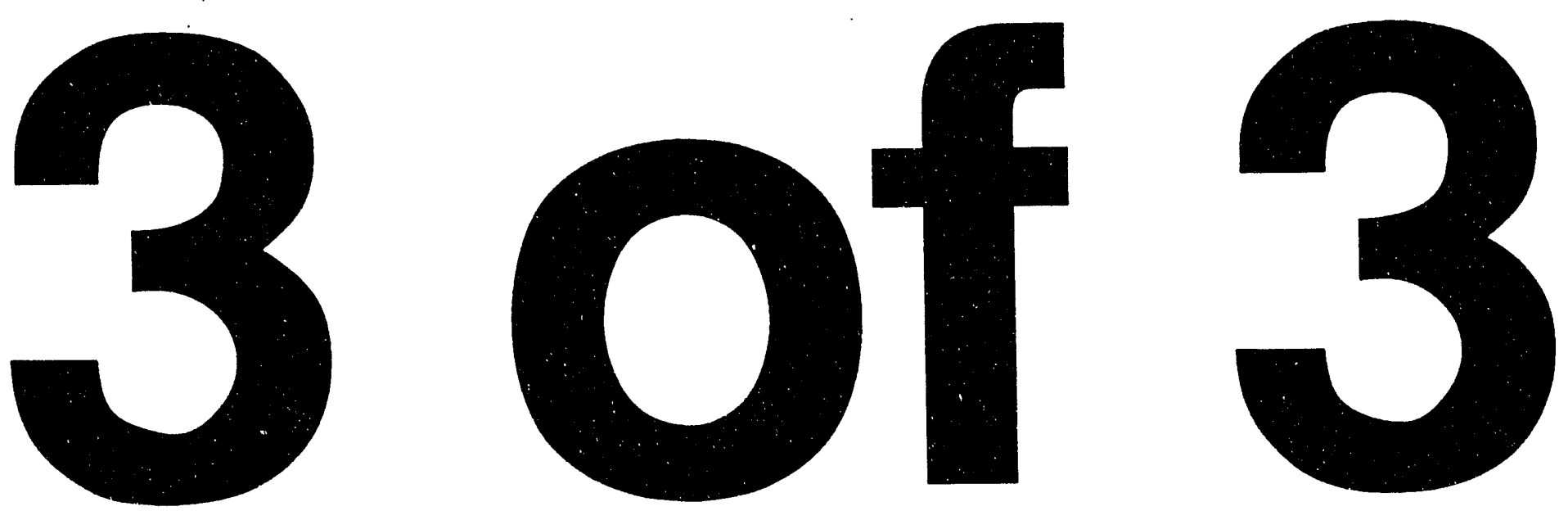
Table $69(a)$. Experimental Iiquid heat capacity data for HCFC-123.

\begin{tabular}{|c|c|c|c|c|c|c|c|c|c|}
\hline$T$ & $\delta \mathbf{T}$ & $\rho$ & $\delta p$ & P & $\delta \mathrm{P}$ & $C_{v, \exp }$ & $\delta \mathrm{C}_{\mathrm{v}}$ & $\delta C_{v}, g_{i}$ & Run \\
\hline \multicolumn{2}{|c|}{$\mathbf{K}$} & mol & $\mathrm{dm}^{-3}$ & \multicolumn{2}{|c|}{ ME a } & $\mathrm{J}$ mol & $-1 \mathrm{~K}^{-1}$ & & \\
\hline $\begin{array}{l}195.5984 \\
197.6426 \\
199.8128 \\
201.8289 \\
203.9834 \\
205.9799 \\
208.1146 \\
210.0943 \\
212.2068 \\
214.1706\end{array}$ & $\begin{array}{l}0.0030 \\
0.0031 \\
0.0031 \\
0.0030 \\
0.0031 \\
0.0030 \\
0.0030 \\
0.0030 \\
0.0032 \\
0.0031\end{array}$ & $\begin{array}{l}11.163 \\
11.157 \\
11.151 \\
11.145 \\
11.139 \\
11.133 \\
11.127 \\
11.122 \\
11.116 \\
11.110\end{array}$ & $\begin{array}{l}0.010 \\
0.011 \\
0.013 \\
0.014 \\
0.015 \\
0.017 \\
0.018 \\
0.019 \\
0.020 \\
0.022\end{array}$ & $\begin{array}{r}4.003 \\
7.174 \\
10.491 . \\
13.538 \\
16.765 \\
19.729 \\
22.872 \\
25.758 \\
28.803 \\
31.596\end{array}$ & $\begin{array}{l}0.068 \\
0.068 \\
0.068 \\
0.068 \\
0.068 \\
0.068 \\
0.068 \\
0.068 \\
0.068 \\
0.068\end{array}$ & $\begin{array}{l}97.54 \\
97.65 \\
98.48 \\
98.44 \\
99.22 \\
99.38 \\
99.82 \\
100.55 \\
100.79 \\
101.74\end{array}$ & $\begin{array}{l}0.56 \\
0.35 \\
0.29 \\
0.25 \\
0.24 \\
0.22 \\
0.22 \\
0.21 \\
0.21 \\
0.21\end{array}$ & $\begin{array}{l}0.57 \\
0.36 \\
0.29 \\
0.26 \\
0.24 \\
0.22 \\
0.22 \\
0.21 \\
0.21 \\
0.20\end{array}$ & $\begin{array}{l}b \\
a \\
b \\
a \\
b \\
a \\
b \\
a \\
b \\
a\end{array}$ \\
\hline
\end{tabular}

Table 69 (b). Experimental liquid heat capacity data for HCrC-123.

\begin{tabular}{|c|c|c|c|c|c|c|c|c|c|}
\hline $\mathbf{T}$ & $\delta \mathrm{T}$ & $p$ & $\delta \rho$ & $\mathbf{P}$ & $\delta \bar{P}$ & $C_{v, \exp }$ & $\overline{\delta C_{v}}$ & $\delta C_{v}, \frac{8}{6}$ & Rus \\
\hline \multicolumn{2}{|c|}{${ }^{\circ} \mathrm{F}$} & $1 b$ & $f t^{-3}$ & \multicolumn{2}{|c|}{ psia } & Btu & $1 b^{-1} \circ p^{-}$ & & \\
\hline $\begin{array}{r}-107.593 \\
-103.913 \\
-100.007 \\
-96.378 \\
-92.500 \\
-88.906 \\
-85.064 \\
-81.500 \\
-77.698 \\
-74.163\end{array}$ & $\begin{array}{l}0.005 \\
0.006 \\
0.006 \\
0.005 \\
0.006 \\
0.005 \\
0.005 \\
0.005 \\
0.006 \\
0.006\end{array}$ & $\begin{array}{l}106.57 \\
106.52 \\
106.46 \\
106.40 \\
106.35 \\
106.29 \\
106.23 \\
106.18 \\
106.13 \\
106.07\end{array}$ & $\begin{array}{l}0.10 \\
0.11 \\
0.12 \\
0.13 \\
0.14 \\
0.16 \\
0.17 \\
0.18 \\
0.19 \\
0.21\end{array}$ & $\begin{array}{r}580.6 \\
1040.5 \\
1521.6 \\
1963.5 \\
2431.6 \\
2861.5 \\
3317.3 \\
3735.9 \\
4177.5 \\
4582.6\end{array}$ & $\begin{array}{l}9.9 \\
9.9 \\
9.9 \\
9.9 \\
9.9 \\
9.9 \\
9.9 \\
9.9 \\
9.9 \\
9.9\end{array}$ & $\begin{array}{l}0.15244 \\
0.15261 \\
0.15391 \\
0.15385 \\
0.15507 \\
0.15532 \\
0.15600 \\
0.15714 \\
0.15752 \\
0.15900\end{array}$ & $\begin{array}{l}0.00088 \\
0.00055 \\
0.00045 \\
0.00039 \\
0.00038 \\
0.00034 \\
0.00034 \\
0.00033 \\
0.00033 \\
0.00033\end{array}$ & $\begin{array}{l}0.57 \\
0.36 \\
0.29 \\
0.26 \\
0.24 \\
0.22 \\
0.22 \\
0.21 \\
0.21 \\
0.20\end{array}$ & $\begin{array}{l}b \\
a \\
b \\
a \\
b \\
a \\
b \\
a \\
b \\
a\end{array}$ \\
\hline
\end{tabular}


Table 70 (a). Experimental liquid heat capacity data for HCFC-123.

\begin{tabular}{|c|c|c|c|c|c|c|c|c|c|}
\hline$T$ & $\delta T$ & $\rho$ & $\delta \rho$ & P & $\delta P$ & $C_{v, \exp }$ & $\delta C_{v}$ & $\delta C_{v}, g$ & $\overline{\text { Run }}$ \\
\hline \multicolumn{2}{|c|}{$\mathbf{K}$} & mol & $d m^{-3}$ & \multicolumn{2}{|c|}{$\mathrm{MPa}$} & $\mathrm{J}$ mol & $-1 k^{-1}$ & & \\
\hline $\begin{array}{l}215.2794 \\
217.3735 \\
219.4251 \\
221.5092 \\
223.5372 \\
225.6081 \\
227.6234 \\
229.6749 \\
231.6692 \\
233.7101 \\
235.6875\end{array}$ & $\begin{array}{l}0.0030 \\
0.0031 \\
0.0030 \\
0.0030 \\
0.0030 \\
0.0031 \\
0.0031 \\
0.0031 . \\
0.0031 . \\
0.0030 \\
0.0030\end{array}$ & $\begin{array}{l}10.874 \\
10.868 \\
10.863 \\
10.858 \\
10.852 \\
10.847 \\
10.842 \\
10.837 \\
10.831 \\
10.826 \\
10.821\end{array}$ & $\begin{array}{l}0.010 \\
0.011 \\
0.012 \\
0.013 \\
0.014 \\
0.015 \\
0.016 \\
0.017 \\
0.018 \\
0.019 \\
0.020\end{array}$ & $\begin{array}{r}3.334 \\
6.184 \\
8.959 \\
11.760 \\
14.464 \\
17.203 \\
19.845 \\
22.511 \\
25.080 \\
27.686 \\
30.188\end{array}$ & $\begin{array}{l}0.020 \\
0.020 \\
0.020 \\
0.020 \\
0.020 \\
0.020 \\
0.020 \\
0.020 \\
0.020 \\
0.020 \\
0.020\end{array}$ & $\begin{array}{r}99.37 \\
99.71 \\
99.79 \\
100.25 \\
100.93 \\
100.90 \\
101.41 \\
101.75 \\
102.09 \\
102.37 \\
102.90\end{array}$ & $\begin{array}{l}0.63 \\
0.38 \\
0.29 \\
0.25 \\
0.23 \\
0.22 \\
0.21 \\
0.21 \\
0.20 \\
0.20 \\
0.20\end{array}$ & $\begin{array}{l}0.64 \\
0.38 \\
0.29 \\
0.25 \\
0.23 \\
0.22 \\
0.21 \\
0.20 \\
0.20 \\
0.19 \\
0.19\end{array}$ & $\begin{array}{l}a \\
b \\
a \\
b \\
a \\
b \\
a \\
b \\
a \\
b \\
a\end{array}$ \\
\hline
\end{tabular}

Table $70(\mathrm{~b})$. Experimental liquid heat capacity data for HCFC-123.

\begin{tabular}{|c|c|c|c|c|c|c|c|c|c|}
\hline$T$ & $\delta \mathrm{T}$ & $\rho$ & $\delta \rho$ & P & $\delta P$ & $C_{v, \exp }$ & $\delta C_{v}$ & $\delta \mathrm{C}_{v}, \bar{q}$ & Run \\
\hline \multicolumn{2}{|c|}{${ }^{\circ} \mathrm{F}$} & $1 b$ & $f t^{-3}$ & \multicolumn{2}{|c|}{ psia } & \multicolumn{3}{|c|}{$\circ z^{-1}$} & \\
\hline $\begin{array}{l}-72.167 \\
-68.398 \\
-64.705 \\
-60.953 \\
-57.303 \\
-53.575 \\
-49.948 \\
-46.255 \\
-42.665 \\
-38.992 \\
-35.433\end{array}$ & $\begin{array}{l}0.005 \\
0.006 \\
0.005 \\
0.005 \\
0.005 \\
0.006 \\
0.006 \\
0.006 \\
0.006 \\
0.005 \\
0.005\end{array}$ & $\begin{array}{l}103.82 \\
103.76 \\
103.71 \\
103.66 \\
103.61 \\
103.56 \\
103.51 \\
103.46 \\
103.40 \\
103.36 \\
103.31\end{array}$ & $\begin{array}{l}0.10 \\
0.11 \\
0.11 \\
0.12 \\
0.13 \\
0.14 \\
0.15 \\
0.16 \\
0.17 \\
0.18 \\
0.19\end{array}$ & $\begin{array}{r}483.6 \\
896.9 \\
1299.4 \\
1705.6 \\
2097.8 \\
2495.1 \\
2878.3 \\
3265.0 \\
3637.6 \\
4015.5 \\
4378.4\end{array}$ & $\begin{array}{l}2.9 \\
2.9 \\
2.9 \\
2.9 \\
2.9 \\
2.9 \\
2.9 \\
2.9 \\
2.9 \\
2.9 \\
2.9\end{array}$ & $\begin{array}{l}0.15530 \\
0.15583 \\
0.15596 \\
0.15667 \\
0.15774 \\
0.15769 \\
0.15849 \\
0.15902 \\
0.15955 \\
0.15999 \\
0.16082\end{array}$ & $\begin{array}{l}0.00098 \\
0.00059 \\
0.00045 \\
0.00039 \\
0.00036 \\
0.00034 \\
0.00033 \\
0.00033 \\
0.00031 \\
0.00031 \\
0.00031\end{array}$ & $\begin{array}{l}0.64 \\
0.38 \\
0.29 \\
0.25 \\
0.23 \\
0.22 \\
0.21 \\
0.20 \\
0.20 \\
0.19 \\
0.19\end{array}$ & $\begin{array}{l}a \\
b \\
a \\
b \\
a \\
b \\
a \\
b \\
a \\
b \\
a\end{array}$ \\
\hline
\end{tabular}


Table 71 (a). Experimental liquid heat capacity data for HCFC-123.

\begin{tabular}{|c|c|c|c|c|c|c|c|c|c|}
\hline$T$ & $\delta \mathrm{T}$ & $\rho$ & $\delta \rho$ & P & $\delta \mathrm{P}$ & $C_{v, \exp }$ & $\delta C_{v}$ & $\delta C_{v}, g$ & Run \\
\hline \multicolumn{2}{|c|}{$\mathbf{K}$} & mol & $d m^{-3}$ & \multicolumn{2}{|c|}{$\mathrm{MPa}$} & $\mathrm{J}$ mol & $\mathbf{k}^{-1}$ & & \\
\hline $\begin{array}{l}234.1386 \\
236.3143 \\
238.2504 \\
240.4017 \\
242.3312 \\
244.4587 \\
246.3745 \\
248.4877 \\
250.3971 \\
252.4928 \\
254.3877 \\
256.4665 \\
258.3437\end{array}$ & $\begin{array}{l}0.0030 \\
0.0030 \\
0.0030 \\
0.0030 \\
0.0031 \\
0.0032 \\
0.0031 \\
0.0031 \\
0.0031 \\
0.0031 \\
0.0031 \\
0.0032 \\
0.0032\end{array}$ & $\begin{array}{l}10.596 \\
10.591 \\
10.586 \\
10.581 \\
10.577 \\
10.572 \\
10.567 \\
10.563 \\
10.558 \\
10.553 \\
10.549 \\
10.544 \\
10.540\end{array}$ & $\begin{array}{l}0.009 \\
0.010 \\
0.011 \\
0.012 \\
0.013 \\
0.014 \\
0.015 \\
0.016 \\
0.017 \\
0.018 \\
0.019 \\
0.019 \\
0.020\end{array}$ & $\begin{array}{r}3.134 \\
5.760 \\
8.085 \\
10.654 \\
12.942 \\
15.449 \\
17.689 \\
20.141 \\
22.339 \\
24.733 \\
26.880 \\
29.215 \\
31.307\end{array}$ & $\begin{array}{l}0.017 \\
0.017 \\
0.017 \\
0.017 \\
0.017 \\
0.017 \\
0.017 \\
0.017 \\
0.017 \\
0.017 \\
0.017 \\
0.017 \\
0.017\end{array}$ & $\begin{array}{l}101.54 \\
101.73 \\
102.35 \\
102.72 \\
103.07 \\
103.50 \\
104.09 \\
104.17 \\
105.08 \\
104.73 \\
105.35 \\
105.76 \\
105.87\end{array}$ & $\begin{array}{l}0.66 \\
0.38 \\
0.29 \\
0.25 \\
0.23 \\
0.21 \\
0.20 \\
0.20 \\
0.19 \\
0.19 \\
0.19 \\
0.19 \\
0.18\end{array}$ & $\begin{array}{l}0.65 \\
0.38 \\
0.29 \\
0.24 \\
0.22 \\
0.21 \\
0.20 \\
0.19 \\
0.18 \\
0.18 \\
0.18 \\
0.18 \\
0.17\end{array}$ & $\begin{array}{l}b \\
a \\
b \\
a \\
b \\
a \\
b \\
a \\
b \\
a \\
b \\
a \\
b\end{array}$ \\
\hline
\end{tabular}

Table 71 (b). Experimental liquid heat capacity data for HCFC-123.

\begin{tabular}{|c|c|c|c|c|c|c|c|c|c|}
\hline$T$ & $\delta T$ & $\bar{\rho}$ & $\delta p$ & P & $\delta P$ & $C_{v, \exp }$ & $\delta C_{v}$ & $\delta C_{v}, \frac{g}{\delta}$ & $\overline{\operatorname{Run}}$ \\
\hline \multicolumn{2}{|c|}{${ }^{\circ} \mathrm{F}$} & $1 b$ & $E t^{-3}$ & \multicolumn{2}{|c|}{ paia } & Btu & $1 b^{-1} \circ \mathbf{F}^{-1}$ & & \\
\hline $\begin{array}{r}-38.221 \\
-34.304 \\
-30.819 \\
-26.947 \\
-23.474 \\
-19.644 \\
-16.196 \\
-12.392 \\
-8.955 \\
-5.183 \\
-1.772 \\
1.970 \\
5.349\end{array}$ & $\begin{array}{l}0.005 \\
0.005 \\
0.005 \\
0.005 \\
0.006 \\
0.006 \\
0.006 \\
0.006 \\
0.006 \\
0.006 \\
0.006 \\
0.006 \\
0.006\end{array}$ & $\begin{array}{l}101.16 \\
101.11 \\
101.07 \\
101.02 \\
100.98 \\
100.93 \\
100.88 \\
100.85 \\
100.80 \\
100.75 \\
100.71 \\
100.66 \\
100.63\end{array}$ & $\begin{array}{l}0.09 \\
0.10 \\
0.11 \\
0.11 \\
0.12 \\
0.13 \\
0.14 \\
0.15 \\
0.16 \\
0.17 \\
0.18 \\
0.18 \\
0.19\end{array}$ & $\begin{array}{r}454.5 \\
835.4 \\
1172.6 \\
1545.2 \\
1877.1 \\
2240.7 \\
2565.6 \\
2921.2 \\
3240.0 \\
3587.2 \\
3898.6 \\
4237.3 \\
4540.7\end{array}$ & $\begin{array}{l}2.5 \\
2.5 \\
2.5 \\
2.5 \\
2.5 \\
2.5 \\
2.5 \\
2.5 \\
2.5 \\
2.5 \\
2.5 \\
2.5 \\
2.5\end{array}$ & $\begin{array}{l}0.15869 \\
0.15899 \\
0.15996 \\
0.16054 \\
0.16108 \\
0.16175 \\
0.16268 \\
0.16280 \\
0.16422 \\
0.16368 \\
0.16465 \\
0.16529 \\
0.16546\end{array}$ & $\begin{array}{l}0.00103 \\
0.00059 \\
0.00045 \\
0.00039 \\
0.00036 \\
0.00033 \\
0.00031 \\
0.00031 \\
0.00030 \\
0.00030 \\
0.00030 \\
0.00030 \\
0.00028\end{array}$ & $\begin{array}{l}0.65 \\
0.38 \\
0.29 \\
0.24 \\
0.22 \\
0.21 \\
0.20 \\
0.19 \\
0.18 \\
0.18 \\
0.18 \\
0.18 \\
0.17\end{array}$ & $\begin{array}{l}b \\
a \\
b \\
a \\
b \\
a \\
b \\
a \\
b \\
a \\
b \\
a \\
b\end{array}$ \\
\hline
\end{tabular}


Table 72 (a). Experimental two-phase heat capacity data for HCFC-123.

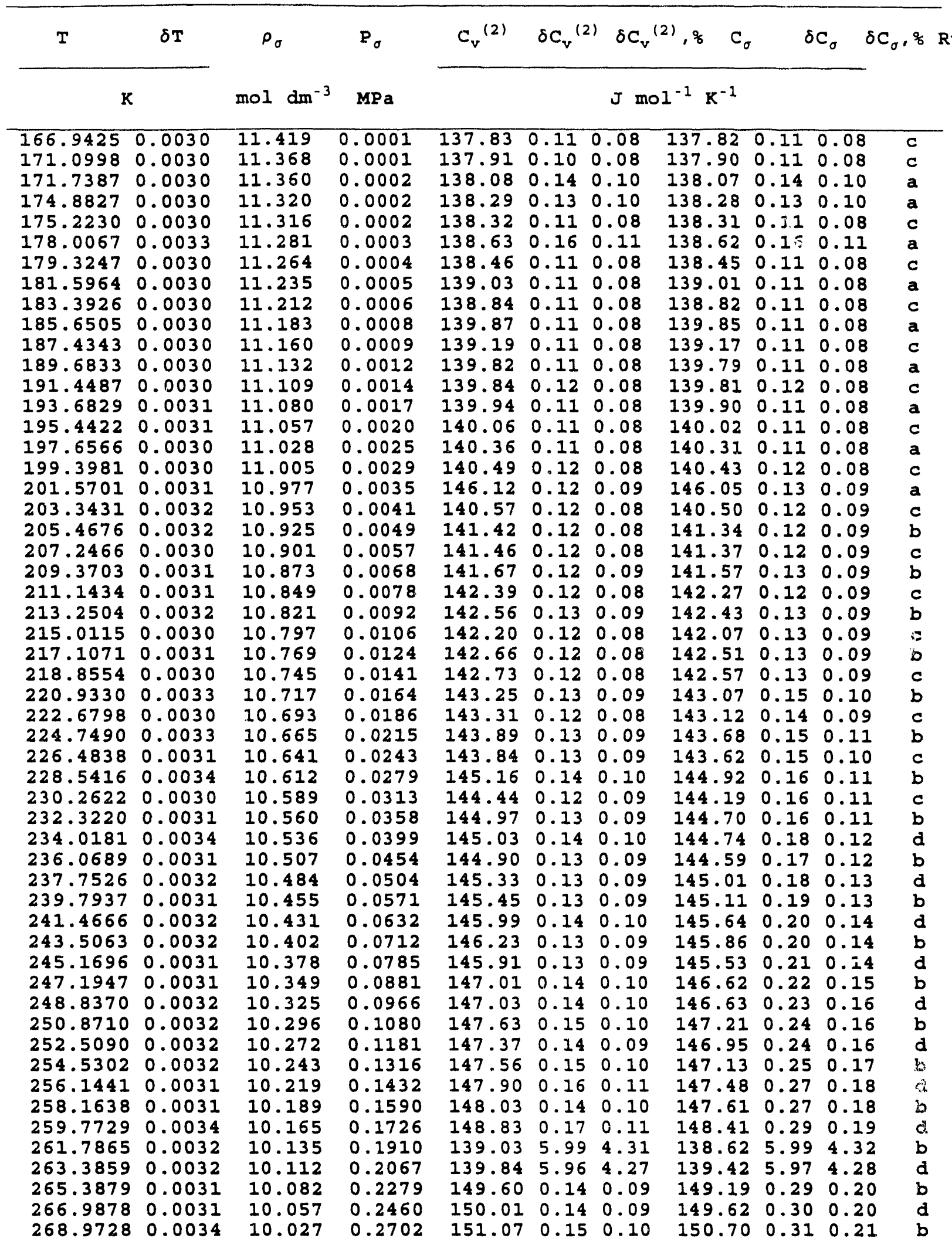


Table 72 (a) (continued). Experimental two-phase heat capacity data for HCFC-123.

\begin{tabular}{|c|c|c|c|c|c|c|c|c|c|c|c|}
\hline $\mathbf{T}$ & $\delta T$ & $\rho_{\sigma}$ & \multirow{2}{*}{$\begin{array}{l}\mathbf{P}_{\sigma} \\
\mathbf{M P a}\end{array}$} & \multirow[t]{2}{*}{$\mathrm{C}_{\mathrm{v}}{ }^{(2)}$} & $\delta C_{v}^{(2)}$ & \multicolumn{2}{|c|}{$\delta C_{v}{ }^{(2)}, 8$} & \multicolumn{2}{|c|}{$\delta C_{\sigma}$} & \multirow[t]{2}{*}{$\delta \mathrm{C}_{\sigma}, \frac{8}{\delta}$} & \\
\hline $\mathrm{K}$ & C & mol $\mathrm{dm}^{-3}$ & & & & $\mathrm{~J}$ mo & $I^{-1} k^{-1}$ & & & & \\
\hline $\begin{array}{l}270.5732 \\
272.5468 \\
274.1380 \\
276.1054 \\
277.6859 \\
279.6487 \\
281.2308 \\
283.1806 \\
284.7545 \\
286.6920 \\
288.2690 \\
290.1942 \\
291.7702 \\
293.6878 \\
295.2590 \\
297.1620 \\
298.7470 \\
300.6368 \\
302.2147 \\
304.0893 \\
307.5811\end{array}$ & $\begin{array}{l}0.0031 \\
0.0032 \\
0.0032 \\
0.0031 \\
0.0031 \\
0.0036 \\
0.0032 \\
0.0032 \\
0.0033 \\
0.0033 \\
0.0032 \\
0.0033 \\
0.0033 \\
0.0035 \\
0.0032 \\
0.0033 \\
0.0032 \\
0.0038 \\
0.0035 \\
0.0034 \\
0.0035\end{array}$ & $\begin{array}{r}10.003 \\
9.973 \\
9.949 \\
9.918 \\
9.894 \\
9.863 \\
9.839 \\
9.808 \\
9.783 \\
9.753 \\
9.728 \\
9.697 \\
9.672 \\
9.641 \\
9.615 \\
9.584 \\
9.558 \\
9.527 \\
9.501 \\
9.470 \\
9.411\end{array}$ & $\begin{array}{l}0.2911 \\
0.3187 \\
0.3424 \\
0.3737 \\
0.4006 \\
0.4361 \\
0.4665 \\
0.5063 \\
0.5404 \\
0.5850 \\
0.6233 \\
0.6729 \\
0.7158 \\
0.7709 \\
0.8185 \\
0.8793 \\
0.9326 \\
0.9994 \\
1.0581 \\
1.1313 \\
1.2783\end{array}$ & $\begin{array}{l}150.28 \\
151.61 \\
150.90 \\
151.86 \\
152.35 \\
152.03 \\
152.98 \\
152.50 \\
153.09 \\
153.25 \\
153.80 \\
154.00 \\
154.68 \\
154.09 \\
155.20 \\
154.61 \\
155.27 \\
155.04 \\
156.55 \\
155.48 \\
151.27\end{array}$ & $\begin{array}{l}0.14 \\
0.15 \\
0.14 \\
0.14 \\
0.14 \\
0.16 \\
0.15 \\
0.15 \\
0.15 \\
0.15 \\
0.15 \\
0.15 \\
0.15 \\
0.16 \\
0.15 \\
0.15 \\
0.15 \\
0.17 \\
0.17 \\
0.16 \\
0.16\end{array}$ & $\begin{array}{l}0.09 \\
0.10 \\
0.10 \\
0.09 \\
0.09 \\
0.11 \\
0.10 \\
0.10 \\
0.10 \\
0.10 \\
0.10 \\
0.10 \\
0.10 \\
0.11 \\
0.10 \\
0.10 \\
0.10 \\
0.11 \\
0.11 \\
0.10 \\
0.11\end{array}$ & $\begin{array}{l}149.95 \\
151.31 \\
150.64 \\
151.65 \\
152.19 \\
151.94 \\
152.95 \\
152.57 \\
153.23 \\
153.51 \\
154.15 \\
154.48 \\
155.28 \\
154.86 \\
156.11 \\
155.71 \\
156.54 \\
156.52 \\
158.24 \\
157.41 \\
153.73\end{array}$ & $\begin{array}{l}0.31 \\
0.32 \\
0.32 \\
0.32 \\
0.33 \\
0.34 \\
0.33 \\
0.33 \\
0.33 \\
0.33 \\
0.33 \\
0.33 \\
0.32 \\
0.32 \\
0.31 \\
0.31 \\
0.30 \\
0.31 \\
0.30 \\
0.29 \\
0.30\end{array}$ & $\begin{array}{l}0.21 \\
0.21 \\
0.21 \\
0.21 \\
0.21 \\
0.22 \\
0.22 \\
0.22 \\
0.22 \\
0.22 \\
0.21 \\
0.21 \\
0.21 \\
0.21 \\
0.20 \\
0.20 \\
0.19 \\
0.20 \\
0.19 \\
0.19 \\
0.20\end{array}$ & $\begin{array}{l}d \\
b \\
d \\
b \\
d \\
b \\
d \\
b \\
d \\
b \\
d \\
b \\
d \\
b \\
d \\
b \\
d \\
b \\
d \\
b \\
b\end{array}$ & \\
\hline
\end{tabular}


Table 72 (b). Experimental two-phase heat capacity data for HCFC-123.

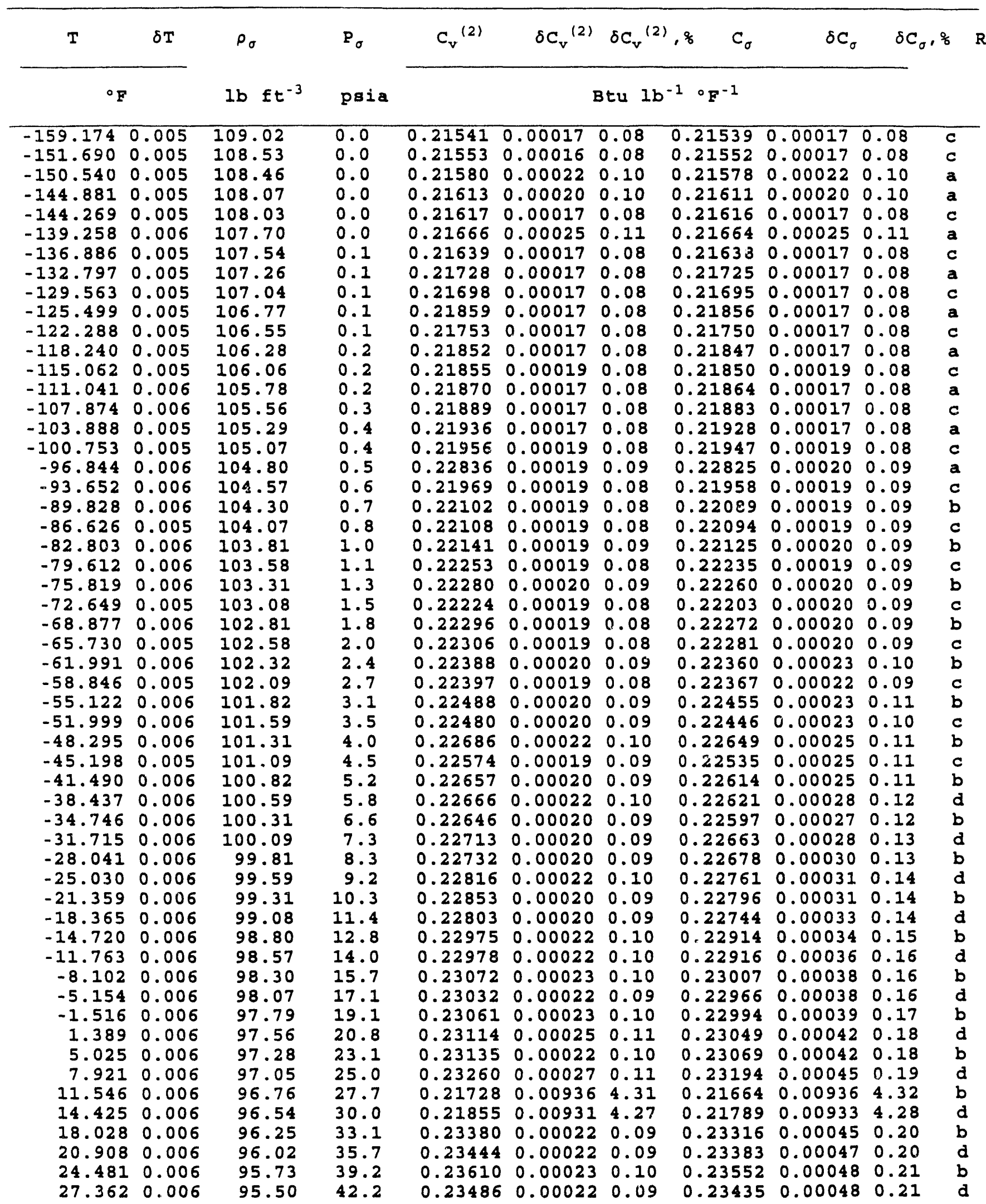


Table 72 (b) (continued). Experimental two-phase heat capacity data for HCFC-123.

\begin{tabular}{|c|c|c|c|c|c|c|c|c|c|c|}
\hline $\mathbf{T}$ & $\delta T$ & $\rho_{\sigma}$ & \multirow{2}{*}{$\begin{array}{l}P_{\sigma} \\
\text { psia }\end{array}$} & $C_{v}{ }^{(2)}$ & $\delta C_{v}^{(2)}$ & $\delta C_{v}{ }^{(2)}$ & $c_{0}$ & $\delta C_{\sigma}$ & \multicolumn{2}{|l|}{$\delta C_{\sigma}, \frac{q}{\sigma}$} \\
\hline${ }^{\circ} \mathrm{F}$ & & $1 b E t^{-3}$ & & & & Btu $1 b^{-}$ & $-1 \circ \mathrm{F}^{-1}$ & & & \\
\hline $\begin{array}{l}30.914 \\
33.778 \\
37.320 \\
40.165 \\
43.698 \\
46.545 \\
50.055 \\
52.888 \\
56.376 \\
59.214 \\
62.680 \\
65.516 \\
68.968 \\
71.796 \\
75.222 \\
78.075 \\
81.476 \\
84.316 \\
87.691 \\
93.976\end{array}$ & $\begin{array}{l}0.006 \\
0.006 \\
0.006 \\
0.006 \\
0.006 \\
0.006 \\
0.006 \\
0.006 \\
0.006 \\
0.006 \\
0.006 \\
0.006 \\
0.006 \\
0.006 \\
0.006 \\
0.006 \\
0.007 \\
0.006 \\
0.006 \\
0.006\end{array}$ & $\begin{array}{l}95.21 \\
94.98 \\
94.69 \\
94.46 \\
94.16 \\
93.93 \\
93.64 \\
93.40 \\
93.11 \\
92.87 \\
92.58 \\
92.34 \\
92.04 \\
91.80 \\
91.50 \\
91.25 \\
90.96 \\
90.71 \\
90.41 \\
89.85\end{array}$ & $\begin{array}{r}46.3 \\
49.7 \\
54.2 \\
58.1 \\
63.3 \\
67.7 \\
73.4 \\
78.4 \\
84.8 \\
90.4 \\
97.6 \\
103.8 \\
111.8 \\
118.7 \\
127.5 \\
135.3 \\
145.0 \\
153.5 \\
164.1 \\
185.4\end{array}$ & $\begin{array}{l}0.23694 \\
0.23583 \\
0.23733 \\
0.23810 \\
0.23760 \\
0.23908 \\
0.23833 \\
0.23926 \\
0.23951 \\
0.24037 \\
0.24068 \\
0.24174 \\
0.24082 \\
0.24255 \\
0.24163 \\
0.24266 \\
0.24230 \\
0.24466 \\
0.24299 \\
0.23641\end{array}$ & $\begin{array}{l}0.00023 \\
0.00022 \\
0.00022 \\
0.00022 \\
0.00025 \\
0.00023 \\
0.00023 \\
0.00023 \\
0.00023 \\
0.00023 \\
0.00023 \\
0.00023 \\
0.00025 \\
0.00023 \\
0.00023 \\
0.00023 \\
0.00027 \\
0.00027 \\
0.00025 \\
0.00025\end{array}$ & $\begin{array}{l}0.10 \\
0.10 \\
0.09 \\
0.09 \\
0.11 \\
0.10 \\
0.10 \\
0.10 \\
0.10 \\
0.10 \\
0.10 \\
0.10 \\
0.11 \\
0.10 \\
0.10 \\
0.10 \\
0.11 \\
0.11 \\
0.10 \\
0.11\end{array}$ & $\begin{array}{l}0.23647 \\
0.23543 \\
0.23700 \\
0.23785 \\
0.23746 \\
0.23904 \\
0.23844 \\
0.23947 \\
0.23991 \\
0.24091 \\
0.24143 \\
0.24268 \\
0.24202 \\
0.24398 \\
0.24335 \\
0.24465 \\
0.24462 \\
0.24730 \\
0.24601 \\
0.24026\end{array}$ & $\begin{array}{l}0.00050 \\
0.00050 \\
0.00050 \\
0.00052 \\
0.00053 \\
0.00052 \\
0.00052 \\
0.00052 \\
0.00052 \\
0.00052 \\
0.00052 \\
0.00050 \\
0.00050 \\
0.00048 \\
0.00048 \\
0.00047 \\
0.00048 \\
0.00047 \\
0.00045 \\
0.00047\end{array}$ & $\begin{array}{l}0.21 \\
0.21 \\
0.21 \\
0.21 \\
0.22 \\
0.22 \\
0.22 \\
0.22 \\
0.22 \\
0.21 \\
0.21 \\
0.21 \\
0.21 \\
0.20 \\
0.20 \\
0.19 \\
0.20 \\
0.19 \\
0.19 \\
0.20\end{array}$ & 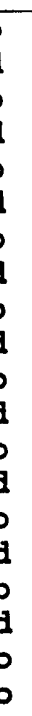 \\
\hline
\end{tabular}


Table 73 (a). Experimental two-phase heat capacity data for HCFC-123.

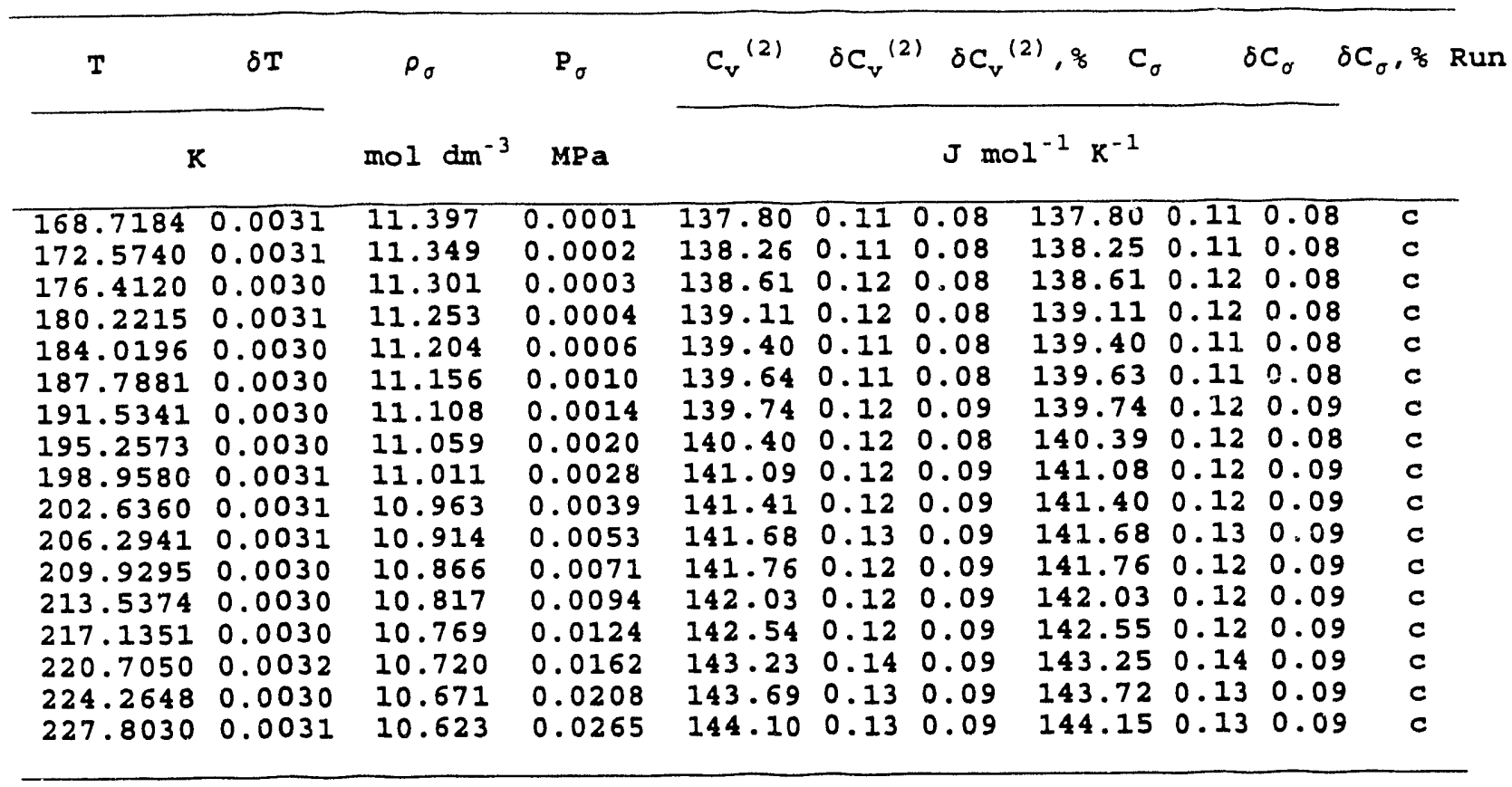

Table 73 (b). Experimental two-phase heat capacity data for HCFC-123.

\begin{tabular}{|c|c|c|c|c|c|c|c|c|c|c|}
\hline $\mathbf{T}$ & & \multirow{2}{*}{$\begin{array}{l}\rho_{\sigma} \\
1 b \in t^{-3}\end{array}$} & \multirow{2}{*}{$\begin{array}{l}E_{\sigma} \\
\text { psia }\end{array}$} & $C_{v}^{(2)}$ & $\delta C_{v}{ }^{(2)}$ & $\delta C_{v}$ & $c_{\sigma}$ & $\delta \mathrm{C}_{\sigma}$ & $\delta C_{\sigma}, 8$ & \\
\hline${ }^{\circ} \mathrm{F}$ & & & & & & Btu $1 b^{-}$ & $-1 \circ \mathrm{F}^{-1}$ & & & \\
\hline $\begin{array}{r}-155.977 \\
-149.037 \\
-142.128 \\
-135.271 \\
-128.435 \\
-121.651 \\
-114.909 \\
-108.207 \\
-101.546 \\
-94.925 \\
-88.341 \\
-81.797 \\
-75.303 \\
-68.827 \\
-62.401 \\
-55.993 \\
-49.625\end{array}$ & $\begin{array}{l}0.006 \\
0.006 \\
0.005 \\
0.006 \\
0.005 \\
0.005 \\
0.005 \\
0.005 \\
0.005 \\
0.006 \\
0.006 \\
0.005 \\
0.005 \\
0.005 \\
0.006 \\
0.005 \\
0.006\end{array}$ & $\begin{array}{l}108.81 \\
108.35 \\
107.89 \\
107.43 \\
106.97 \\
106.51 \\
106.05 \\
105.58 \\
105.12 \\
104.66 \\
104.20 \\
103.74 \\
103.27 \\
102.81 \\
102.34 \\
101.88 \\
101.42\end{array}$ & $\begin{array}{l}0.0 \\
0.0 \\
0.0 \\
0.1 \\
0.1 \\
0.1 \\
0.2 \\
0.3 \\
0.4 \\
0.6 \\
0.8 \\
1.0 \\
1.4 \\
1.8 \\
2.3 \\
3.0 \\
3.8\end{array}$ & $\begin{array}{l}0.21536 \\
0.21608 \\
0.21663 \\
0.21741 \\
0.21786 \\
0.21824 \\
0.21839 \\
0.21942 \\
0.22050 \\
0.22100 \\
0.22142 \\
0.22155 \\
0.22197 \\
0.22277 \\
0.22385 \\
0.22456 \\
0.22521\end{array}$ & $\begin{array}{l}0.00017 \\
0.00017 \\
0.00019 \\
0.00019 \\
0.00017 \\
0.00017 \\
0.00019 \\
0.00019 \\
0.00019 \\
0.00019 \\
0.00020 \\
0.00019 \\
0.00019 \\
0.00019 \\
0.00022 \\
0.00020 \\
0.00020\end{array}$ & $\begin{array}{l}0.08 \\
0.08 \\
0.08 \\
0.08 \\
0.08 \\
0.08 \\
0.09 \\
0.08 \\
0.09 \\
0.09 \\
0.09 \\
0.09 \\
0.09 \\
0.09 \\
0.09 \\
0.09 \\
0.09\end{array}$ & $\begin{array}{l}0.21536 \\
0.21606 \\
0.21663 \\
0.21741 \\
0.21786 \\
0.21822 \\
0.21839 \\
0.21941 \\
0.22049 \\
0.22099 \\
0.22142 \\
0.22155 \\
0.22197 \\
0.22278 \\
0.22388 \\
0.22461 \\
0.22528\end{array}$ & $\begin{array}{l}0.00017 \\
0.00017 \\
0.00019 \\
0.00019 \\
0.00017 \\
0.00017 \\
0.00019 \\
0.00019 \\
0.00019 \\
0.00019 \\
0.00020 \\
0.00019 \\
0.00019 \\
0.00019 \\
0.00022 \\
0.00020 \\
0.00020\end{array}$ & $\begin{array}{l}0.08 \\
0.08 \\
0.08 \\
0.08 \\
0.08 \\
0.08 \\
0.09 \\
0.08 \\
0.09 \\
0.09 \\
0.09 \\
0.09 \\
0.09 \\
0.09 \\
0.09 \\
0.09 \\
0.09\end{array}$ & $\begin{array}{l}c \\
c \\
c \\
c \\
c \\
c \\
c\end{array}$ \\
\hline
\end{tabular}


Table 74. Coefficients to the MBWR equation of state for HCFC-123 (units are K, bar, L, mol)

$$
p=\sum_{n=1}^{9} a_{n} \rho^{n}+\exp \left(-\rho^{2} / \rho_{c}^{2}\right) \sum_{n=10}^{15} a_{n} \rho^{2 n-17}
$$

$$
\rho_{\mathrm{c}} \quad=\quad 3.596417 \mathrm{~mol} / \mathrm{L}
$$

$$
\begin{array}{ll}
\mathrm{a}_{1}=\mathrm{RT} \\
\mathrm{a}_{2}=\mathrm{b}_{1} \mathrm{~T}+\mathrm{b}_{2} \mathrm{~T}^{0.5}+\mathrm{b}_{3}+\mathrm{b}_{4} / \mathrm{T}+\mathrm{b}_{5} / \mathrm{T}^{2} \\
\mathrm{a}_{3}=\mathrm{b}_{6} \mathrm{~T}+\mathrm{b}_{7}+\mathrm{b}_{8} / \mathrm{T}+\mathrm{b}_{9} / \mathrm{T}^{2} \\
\mathrm{a}_{4}=\mathrm{b}_{10} \mathrm{~T}+\mathrm{b}_{11}+\mathrm{b}_{12} / \mathrm{T} \\
\mathrm{a}_{5}=\mathrm{b}_{13} \\
\mathrm{a}_{6}=\mathrm{b}_{14} / \mathrm{T}+\mathrm{b}_{15} / \mathrm{T}^{2} \\
\mathrm{a}_{7}=\mathrm{b}_{16} / \mathrm{T} \\
\mathrm{a}_{8}=\mathrm{b}_{17} / \mathrm{T}+\mathrm{b}_{18} / \mathrm{T}^{2} \\
\mathrm{a}_{9}=\mathrm{b}_{19} / \mathrm{T}^{2} \\
\mathrm{a}_{10}=\mathrm{b}_{20} / \mathrm{T}^{2}+\mathrm{b}_{21} / \mathrm{T}^{3} \\
\mathrm{a}_{11}=\mathrm{b}_{22} / \mathrm{T}^{2}+\mathrm{b}_{23} / \mathrm{T}^{4} \\
\mathrm{a}_{12}=\mathrm{b}_{24} / \mathrm{T}^{2}+\mathrm{b}_{25} / \mathrm{T}^{3} \\
\mathrm{a}_{13}=\mathrm{b}_{26} / \mathrm{T}^{2}+\mathrm{b}_{27} / \mathrm{T}^{4} \\
\mathrm{a}_{14}=\mathrm{b}_{28} / \mathrm{T}^{2}+\mathrm{b}_{29} / \mathrm{T}^{3} \\
\mathrm{a}_{15}=\mathrm{b}_{30} / \mathrm{T}^{2}+\mathrm{b}_{31} / \mathrm{T}^{3}+\mathrm{b}_{32} / \mathrm{T}^{4}
\end{array}
$$

i $\quad b_{i}$

$$
\begin{array}{cc}
1 & -0.193042434973 \mathrm{E}-01 \\
2 & -0.263410206086 \mathrm{E}+00 \\
3 & 0.266439262928 \mathrm{E}+02 \\
4 & -0.102447174272 \mathrm{E}+05 \\
5 & -0.714962376060 \mathrm{E}+06 \\
6 & 0.179594735089 \mathrm{E}-01 \\
7 & -0.106601466621 \mathrm{E}+02 \\
8 & -0.106973465680 \mathrm{E}+04 \\
9 & -0.150556666672 \mathrm{E}+07 \\
10 & -0.126504809410 \mathrm{E}-02 \\
11 & -0.123264787943 \mathrm{E}+00 \\
12 & 0.293238981229 \mathrm{E}+03 \\
13 & 0.134389339775 \mathrm{E}+00 \\
14 & 0.745030119681 \mathrm{E}+01 \\
15 & 0.413916532768 \mathrm{E}+04 \\
16 & -0.212267981526 \mathrm{E}+01
\end{array}
$$


Table 74. Coefficients to the MBWR equation of state for HCFC-123 (units are K, bar, L, mol) (continued)

$\begin{array}{cc}17 & 0.106201732381 \mathrm{E}+00 \\ 18 & -0.401991529370 \mathrm{E}+02 \\ 19 & 0.156703568146 \mathrm{E}+01 \\ 20 & 0.395804226685 \mathrm{E}+07 \\ 21 & -0.490428403406 \mathrm{E}+09 \\ 22 & 0.171175389582 \mathrm{E}+06 \\ 23 & 0.376067424212 \mathrm{E}+10 \\ 24 & 0.719667521763 \mathrm{E}+04 \\ 25 & -0.110348184730 \mathrm{E}+07 \\ 26 & 0.571211837951 \mathrm{E}+02 \\ 27 & 0.64249861788 \mathrm{E}+07 \\ 28 & 0.227383595657 \mathrm{E}+01 \\ 29 & -0.670239087161 \mathrm{E}+03 \\ 30 & -0.162446239669 \mathrm{E}-01 \\ 31 & 0.190850894641 \mathrm{E}+02 \\ 32 & -0.267293932199 \mathrm{E}+04\end{array}$


Table 75. Transient Hot-Wire Thermal Conductivity Data for HCFC-123

\begin{tabular}{|c|c|c|c|c|c|c|}
\hline $\begin{array}{l}\text { Point } \\
\text { Number }\end{array}$ & $\begin{array}{l}T_{\text {Exp. }} \\
(K)\end{array}$ & $\begin{array}{l}\mathrm{P}_{\mathrm{Cell}} \\
(\mathrm{MPa})\end{array}$ & $\underset{(W / m-K)}{\lambda_{\operatorname{Exp}}}$ & $\mathrm{T}_{\text {Exp. }}$ & $\begin{array}{l}\mathrm{P}_{\text {Cell }} \\
\text { (psia) }\end{array}$ & $\begin{array}{c}\lambda_{\text {Exp. }} \\
\text { (BTU/ft-hr-F) }\end{array}$ \\
\hline 17001 & 183.092 & 66.424 & 0.12112 & -130.104 & 9633.98 & 0.07003 \\
\hline 17002 & 182.579 & 66.419 & 0.12132 & -131.028 & 9633.30 & 0.07014 \\
\hline 17003 & 182.120 & 66.423 & 0.12146 & -131.854 & 9633.86 & 0.07023 \\
\hline 17004 & 181.704 & 66.423 & 0.12178 & -132.603 & 9633.83 & 0.07041 \\
\hline 17005 & 183.191 & 52.668 & 0.11908 & -129.926 & 7638.90 & 0.06885 \\
\hline 17006 & 182.635 & 52.687 & 0.11924 & -130.927 & 7641.62 & 0.06894 \\
\hline 17007 & 182.168 & 52.695 & 0.11943 & -131.768 & 7642.81 & 0.06905 \\
\hline 17008 & 181.744 & 52.698 & 0.11960 & -132.531 & 7643.22 & 0.06915 \\
\hline 17010 & 182.705 & 38.682 & 0.11697 & -130.801 & 5610.38 & 0.06763 \\
\hline 17011 & 182.220 & 38.683 & 0.11724 & -131.674 & 5610.48 & 0.06779 \\
\hline 17012 & 181.798 & 38.687 & 0.11740 & -132.434 & 5611.04 & 0.06788 \\
\hline 17013 & 182.913 & 24.866 & 0.11437 & -130.427 & 3606.48 & 0.06613 \\
\hline 17014 & 182.411 & 24.883 & 0.11466 & -131.330 & 3608.96 & 0.06629 \\
\hline 17015 & 181.959 & 24.891 & 0.11494 & -132.144 & 3610.15 & 0.06646 \\
\hline 17016 & 181.554 & 24.898 & 0.11509 & -132.873 & 3611.17 & 0.06654 \\
\hline $1 \% 117$ & 182.984 & 12.494 & 0.11212 & -130.299 & 1812.10 & 0.06483 \\
\hline 17018 & $18 \angle .473$ & 12.498 & 0.11234 & -131.219 & 1812.65 & 0.06495 \\
\hline 17019 & 182.012 & 12.496 & 0.11265 & -132.048 & 1812.41 & 0.06513 \\
\hline 17020 & 181.605 & 12.509 & 0.11293 & -132.781 & 1814.25 & 0.06529 \\
\hline 17021 & 183.029 & 0.827 & 0.10987 & -130.218 & 119.94 & 0.06352 \\
\hline 17022 & 182.505 & 0.847 & 0.11015 & -131.161 & 122.83 & 0.06369 \\
\hline 17023 & 182.048 & 0.828 & 0.11064 & -131.984 & 120.16 & 0.06397 \\
\hline 17024 & 181.614 & 0.822 & 0.11076 & -132.765 & 119.23 & 0.06404 \\
\hline 18002 & 202.672 & 68.629 & 0.11870 & -94.860 & 9953.78 & 0.06863 \\
\hline 18003 & 202.138 & 68.613 & 0.11899 & -95.822 & 9951.46 & 0.06880 \\
\hline 18004 & 201.669 & 68.589 & 0.11911 & -96.666 & 9948.05 & 0.06887 \\
\hline 18005 & 202.925 & 54.558 & 0.11621 & -94.405 & 7912.97 & 0.06719 \\
\hline 18006 & 202.372 & 54.555 & 0.11648 & -95.400 & 7912.56 & 0.06735 \\
\hline 18007 & 201.867 & 54.540 & 0.11668 & -96.309 & 7910.44 & 0.06746 \\
\hline 18008 & 201.428 & 54.536 & 0.11705 & -97.100 & 7909.87 & 0.06768 \\
\hline 18009 & 203.056 & 40.804 & 0.11388 & -94.169 & 5918.09 & 0.06584 \\
\hline 18010 & 202.488 & 40.778 & 0.11420 & -95.192 & 5914.35 & 0.06603 \\
\hline 18011 & 201.976 & 40.771 & 0.11450 & -96.113 & 5913.36 & 0.06620 \\
\hline 18012 & 201.527 & 40.775 & 0.11498 & -96.921 & 5913.97 & 0.06648 \\
\hline 18013 & 203.023 & 27.178 & 0.11084 & -94.229 & 3941.79 & 0.06409 \\
\hline 18014 & 202.443 & 27.176 & 0.11120 & -95.273 & 3941.60 & 0.06429 \\
\hline 18015 & 201.929 & 27.176 & 0.11141 & -96.198 & 3941.51 & 0.06442 \\
\hline 18016 & 201.472 & 27.170 & 0.11183 & -97.020 & 3940.76 & 0.06466 \\
\hline 18017 & 202.683 & 13.327 & 0.10808 & -94.841 & 1932.94 & 0.06249 \\
\hline 18018 & 202.204 & 13.337 & 0.10830 & -95.703 & 1934.34 & 0.06262 \\
\hline 18019 & 201.801 & 13.343 & 0.10903 & -96.428 & 1935.30 & 0.06304 \\
\hline 18020 & 201.368 & 13.349 & 0.10881 & -97.208 & 1936.10 & 0.06291 \\
\hline 18021 & 202.702 & 1.732 & 0.10542 & -94.806 & 251.26 & 0.06095 \\
\hline 18022 & 202.234 & 1.715 & 0.10592 & -95.649 & 248.70 & 0.06124 \\
\hline 18023 & 201.779 & 1.711 & 0.10556 & -96.468 & 248.09 & 0.06103 \\
\hline 18025 & 202.707 & 1.721 & 0.10484 & -94.797 & 249.58 & 0.06062 \\
\hline 18026 & 202.217 & 1.722 & 0.10570 & -95.679 & 249.77 & 0.06111 \\
\hline 18027 & 201.774 & 1.723 & 0.10598 & -96.477 & 249.87 & 0.06128 \\
\hline 18028 & 201.391 & 1.727 & 0.10627 & -97.166 & 250.46 & 0.06144 \\
\hline
\end{tabular}


Table 75. Transient Hot-Wire Thermal Conductivity Data for HCFC-123 (Continued)

\begin{tabular}{|c|c|c|c|c|c|c|}
\hline $\begin{array}{l}\text { Point } \\
\text { Number }\end{array}$ & $\begin{array}{l}T_{\text {Exp. }} \\
(\mathrm{K})\end{array}$ & $\begin{array}{l}\mathrm{P}_{\mathrm{Cell}} \\
(\mathrm{MPa})\end{array}$ & $\begin{array}{c}\lambda_{\text {Exp. }} \\
(W / m-K)\end{array}$ & $\mathrm{T}_{\text {Exp. }}$ & $\begin{array}{l}\mathrm{P}_{\text {Cell }} \\
\text { (psia) }\end{array}$ & $\frac{\lambda_{\text {Exp. }}}{(B T U / f t-h r-F)}$ \\
\hline 19001 & 223.275 & 67.411 & 0.11514 & -57.775 & 9777.21 & 0.06657 \\
\hline 19002 & 222.740 & 67.398 & 0.11543 & -58.738 & 9775.23 & 0.06674 \\
\hline 19003 & 222.249 & 67.405 & 0.11576 & -59.622 & 9776.25 & 0.06693 \\
\hline 19004 & 221.808 & 67.387 & 0.11605 & -60.416 & 9773.68 & 0.06710 \\
\hline 19005 & 223.354 & 56.074 & 0.11287 & -57.633 & 8132.93 & 0.06526 \\
\hline 19006 & 222.806 & 56.065 & 0.11315 & -58.619 & 8131.56 & 0.06542 \\
\hline 19007 & 222.312 & 56.065 & 0.11338 & -59.508 & 8131.62 & 0.06555 \\
\hline 19008 & 221.859 & 56.069 & 0.11385 & -60.324 & 8132.14 & 0.06583 \\
\hline 19009 & 223.43 .5 & 45.413 & 0.11057 & -57.487 & 6586.63 & 0.06393 \\
\hline 19010 & 222.838 & 45.418 & 0.11095 & -58.562 & 6587.35 & 0.06415 \\
\hline 19011 & 222.333 & 45.418 & 0.11119 & -59.471 & 6587.39 & 0.06429 \\
\hline 19012 & 221.877 & 45.426 & 0.11166 & -60.291 & 6588.47 & 0.06456 \\
\hline 19013 & 222.998 & 34.705 & 0.10850 & -58.274 & 5033.58 & 0.06273 \\
\hline 19014 & 222.477 & 34.701 & 0.10877 & -59.211 & 5032.99 & 0.06289 \\
\hline 19015 & 222.002 & 34.699 & 0.10927 & -60.066 & 5032.61 & 0.06318 \\
\hline 19016 & 221.574 & 34.698 & 0.10969 & -60.837 & 5032.55 & 0.06342 \\
\hline 19017 & $2 ? 3.066$ & 23.950 & 0.10592 & -58.151 & 3473.67 & $0.061: 2,4$ \\
\hline 19018 & 222.530 & 23.960 & 0.10646 & -59.116 & 3475.17 & 0.06155 \\
\hline 19019 & 222.044 & 23.968 & 0.10658 & -59.991 & 3476.28 & 0.06162 \\
\hline 19020 & 221.613 & 23.972 & 0.10709 & -60.767 & 3476.79 & 0.06192 \\
\hline 19021 & 223.121 & 13.069 & 0.10321 & -58.052 & 1895.55 & 0.05967 \\
\hline 19022 & 222.573 & 13.078 & 0.10364 & -59.039 & 1896.77 & 0.05992 \\
\hline 19023 & 222.073 & 13.082 & 0.10389 & -59.939 & 1897.37 & 0.06007 \\
\hline 19024 & 221.632 & 13.081 & 0.10444 & -60.732 & 1897.21 & 0.06039 \\
\hline 19025 & 223.144 & 2.049 & 0.10020 & -58.011 & 297.17 & 0.05793 \\
\hline 19026 & 222.610 & 2.059 & 0.10067 & -58.972 & 298.65 & 0.05821 \\
\hline 19027 & 222.100 & 2.067 & 0.10097 & -59.890 & 299.86 & 0.05838 \\
\hline 19028 & 221.649 & 2.070 & 0.10152 & -60.702 & 300.17 & 0.05870 \\
\hline 20001 & 243.187 & 66.560 & 0.11104 & -21.933 & 9653.70 & 0.06420 \\
\hline 20002 & 242.620 & 66.554 & 0.11136 & -22.954 & 9652.87 & 0.06439 \\
\hline 20003 & 242.110 & 66.550 & 0.11165 & -23.872 & 9652.27 & 0.06455 \\
\hline 20004 & 241.656 & 66.541 & 0.11227 & -24.689 & 9651.05 & 0.06491 \\
\hline 20005 & 243.263 & 55.286 & 0.10846 & -21.797 & 8018.65 & 0.06271 \\
\hline 20006 & 242.687 & 55.289 & 0.10882 & -22.833 & 8018.96 & 0.06292 \\
\hline 20007 & 242.168 & 55.290 & 0.10916 & -23.768 & 8019.17 & 0.06311 \\
\hline 20008 & 241.703 & 55.292 & 0.10976 & -24.605 & 8019.45 & 0.06346 \\
\hline 20009 & 243.345 & 44.157 & 0.10548 & -21.649 & 6404.42 & 0.06099 \\
\hline 20010 & 242.757 & 44.160 & 0.10571 & -22.707 & 6404.87 & 0.06112 \\
\hline 20011 & 242.225 & 44.167 & 0.10639 & -23.665 & 6405.90 & 0.06151 \\
\hline 20012 & 241.752 & 44.165 & 0.10701 & -24.516 & 6405.61 & 0.06187 \\
\hline 20013 & $? 42.886$ & 33.502 & 0.10350 & -22.475 & 4859.01 & 0.05984 \\
\hline 20014 & $2 \backsim 2.423$ & 33.503 & 0.10381 & -23.309 & 4859.23 & 0.06002 \\
\hline 20015 & 241.992 & 33.506 & 0.10423 & -24.084 & 4859.65 & 0.06026 \\
\hline 20016 & 241.614 & 33.510 & 0.10471 & -24.765 & 4860.21 & 0.06054 \\
\hline 20017 & 242.948 & 22.767 & 0.10064 & -22.364 & 3302.03 & 0.05819 \\
\hline 20018 & 242.445 & 22.766 & 0.10090 & -23.269 & 3301.99 & 0.05834 \\
\hline 20019 & 242.007 & 22.768 & 0.10143 & -24.057 & 3302.24 & 0.05864 \\
\hline $2002 \mathrm{C}$ & 241.619 & 22.770 & ¿ 10187 & -24.756 & 3302.56 & 0.05890 \\
\hline 20021 & 242.995 & 11.728 & 0.09746 & -22.279 & 1700.96 & 0.05635 \\
\hline
\end{tabular}


Table 75. Transient Hot-Wire Thermal Conductivity Data for HCFC-123 (Continued)

\begin{tabular}{|c|c|c|c|c|c|c|}
\hline $\begin{array}{l}\text { Point } \\
\text { Number }\end{array}$ & $\begin{array}{l}T_{\text {Exp. }} \\
(K)\end{array}$ & $\begin{array}{l}\mathrm{P}_{\mathrm{Cell}} \\
(\mathrm{MPa})\end{array}$ & $\begin{array}{c}\lambda_{\operatorname{Exp}_{.}} \\
(W / m-K)\end{array}$ & $\mathrm{T}_{\text {Exp. }}$ & $\begin{array}{l}\mathrm{P}_{\text {Cell }} \\
\text { (psia) }\end{array}$ & $\stackrel{\lambda_{\text {Exp. }}}{(B T U / f t-h r-F)}$ \\
\hline 20022 & 242.507 & 11.729 & 0.09783 & -23.157 & 1701.20 & 0.05656 \\
\hline 20023 & 242.067 & 11.733 & 0.09826 & -23.949 & 1701.77 & 0.05681 \\
\hline 20024 & 241.655 & 11.733 & 0.09870 & -24.691 & 1701.70 & 0.05707 \\
\hline 20025 & 243.078 & 1.955 & 0.09440 & -22.130 & 283.62 & 0.05458 \\
\hline 20026 & 242.574 & 1.957 & 0.09487 & -23.037 & 283.89 & 0.05485 \\
\hline 20027 & 242.117 & 1.959 & 0.09516 & -23.859 & 284.13 & 0.05502 \\
\hline 20028 & 241.706 & 1.965 & 0.09570 & -24.599 & 284.96 & 0.05533 \\
\hline 21001 & 264.017 & 67.199 & 0.10627 & 15.561 & 9746.46 & 0.06144 \\
\hline 21002 & 263.373 & 67.194 & 0.10679 & 14.401 & 9745.70 & 0.06174 \\
\hline 21003 & 262.799 & 67.194 & 0.10723 & 13.368 & 9745.77 & 0.06200 \\
\hline 21004 & 262.285 & 67.189 & 0.10764 & 12.443 & 9744.97 & 0.06224 \\
\hline 21005 & 264.091 & 55.971 & 0.10374 & 15.694 & 8118.00 & 0.05998 \\
\hline 21006 & 263.433 & 55.973 & 0.10408 & 14.509 & 8118.21 & 0.06018 \\
\hline 21007 & 262.844 & 55.977 & 0.10452 & 13.449 & 8118.84 & 0.06043 \\
\hline 21008 & 262.311 & 55.967 & 0.10500 & 12.490 & 8117.39 & 0.06071 \\
\hline 21009 & 264.164 & 44.816 & 0.10067 & 15.825 & 6499.99 & 0.05821 \\
\hline 21010 & 263.499 & 44.820 & 0.10098 & 14.628 & 6500.59 & 0.05838 \\
\hline 21011 & 262.894 & 44.819 & 0.10142 & 13.539 & 6500.48 & 0.05864 \\
\hline 21012 & 262.356 & 44.821 & 0.10217 & 12.571 & 6500.83 & 0.05907 \\
\hline 21013 & 263.689 & 33.999 & 0.09818 & 14.970 & 4931.13 & 0.05677 \\
\hline 21014 & 263.162 & 34.002 & 0.09843 & 14.022 & 4931.58 & 0.05691 \\
\hline 21015 & 262.676 & 34.000 & 0.09883 & 13.147 & 4931.30 & 0.05714 \\
\hline 21016 & 262.238 & 34.002 & 0.09930 & 12.358 & 4931.63 & 0.05741 \\
\hline 21017 & 263.745 & 23.281 & 0.09505 & 15.071 & 3376.68 & 0.05496 \\
\hline 21018 & 263.202 & 23.292 & 0.09530 & 14.094 & 3378.23 & 0.05510 \\
\hline 21019 & 262.707 & 23.289 & 0.09579 & 13.203 & 3377.83 & 0.05538 \\
\hline 21020 & 262.257 & 23.290 & 0.09598 & 12.393 & 3377.95 & 0.05549 \\
\hline 21021 & 263.799 & 12.524 & 0.09168 & 15.168 & 1816.42 & 0.05301 \\
\hline 21022 & 263.240 & 12.526 & 0.09195 & 14.162 & 1816.76 & 0.05316 \\
\hline 21023 & 262.731 & 12.529 & 0.09240 & 13.246 & 1817.12 & 0.05342 \\
\hline 21024 & 262.268 & 12.526 & 0.09268 & 12.412 & 1816.77 & 0.05359 \\
\hline 21025 & 263.875 & 1.717 & 0.08796 & 15.305 & 249.02 & 0.05086 \\
\hline 21026 & 263.292 & 1.721 & 0.08836 & 14.256 & 249.54 & 0.05109 \\
\hline 21027 & 262.764 & 1.720 & 0.08871 & 13.305 & 249.43 & 0.05129 \\
\hline 21028 & 262.283 & 1.724 & 0.08903 & 12.439 & 249.99 & 0.05148 \\
\hline 22001 & 284.205 & 66.072 & 0.10171 & 51.899 & 9582.93 & $0.0588 \mathrm{I}$ \\
\hline 22002 & 283.491 & 66.071 & 0.10194 & 50.614 & 9582.78 & 0.05894 \\
\hline 22003 & 282.837 & 66.067 & 0.10210 & 49.437 & 9582.26 & 0.05903 \\
\hline 22004 & 282.261 & 66.067 & 0.10273 & 48.400 & 9582.27 & 0.05940 \\
\hline 22005 & 284.286 & 54.558 & 0.09870 & 52.045 & 7913.05 & 0.05707 \\
\hline 22006 & 283.554 & 54.556 & 0.09874 & 50.727 & 7912.74 & 0.05709 \\
\hline 22007 & 282.885 & 54.563 & 0.09890 & 49.523 & 7913.67 & 0.05718 \\
\hline 22008 & 282.291 & 54.562 & 0.09945 & 48.454 & 7913.61 & 0.05750 \\
\hline 22009 & 284.358 & 43.600 & 0.09604 & 52.174 & 6323.63 & 0.05553 \\
\hline 22010 & 283.604 & 43.599 & 0.09610 & 50.817 & 6323.59 & 0.05556 \\
\hline 22011 & 282.924 & 43.604 & 0.09545 & 49.593 & 6324.21 & 0.05519 \\
\hline 22012 & 282.312 & 43.603 & 0.09646 & 48.492 & 6324.15 & 0.05577 \\
\hline 22013 & 284.527 & 32.317 & 0.09225 & 52.479 & 4687.17 & 0.05334 \\
\hline 22014 & 283.743 & 32.320 & 0.09243 & 51.067 & 4687.60 & 0.05344 \\
\hline
\end{tabular}


Table 75. Transient Hot-Wire Thermal Conductivity Data for HCFC-123 (Continued)

\begin{tabular}{|c|c|c|c|c|c|c|}
\hline $\begin{array}{l}\text { Point } \\
\text { Number }\end{array}$ & $\begin{array}{l}T_{\text {Exp. }} \\
(K)\end{array}$ & $\begin{array}{l}\mathrm{P}_{\mathrm{Cell}} \\
(\mathrm{MPa})\end{array}$ & $\underset{(W / m-K)}{\lambda_{\operatorname{Exp}}}$ & $\mathrm{T}_{\text {Exp. }}$ & $\begin{array}{l}\mathrm{P}_{\text {Cell }} \\
\text { (psia) }\end{array}$ & $\begin{array}{c}\lambda_{\text {Exp. }} \\
(B T U / f t-h r-F)\end{array}$ \\
\hline 22015 & 283.039 & 32.319 & 0.09268 & 49.800 & 4687.42 & 0.05359 \\
\hline 22016 & 282.412 & 32.322 & 0.09302 & 48.672 & 4687.90 & 0.05378 \\
\hline 22018 & 283.923 & 21.124 & 0.08905 & 51.391 & 3063.82 & 0.05149 \\
\hline 22019 & 283.301 & 21.119 & 0.08910 & 50.272 & 3062.99 & 0.05152 \\
\hline 22020 & 282.729 & 21.117 & 0.08918 & 49.242 & 3062.78 & 0.05156 \\
\hline 22021 & 282.209 & 21.115 & 0.08987 & 48.306 & 3062.50 & 0.05196 \\
\hline 22022 & 283.968 & 11.339 & 0.08553 & 51.472 & 1644.60 & 0.04945 \\
\hline 22023 & 283.326 & 11.340 & 0.08529 & 50.317 & 1644.70 & 0.04931 \\
\hline 22024 & 282.732 & 11.344 & 0.08562 & 49.248 & 1645.34 & 0.04950 \\
\hline 22025 & 282.195 & 11.349 & 0.08638 & 48.281 & 1646.06 & 0.04994 \\
\hline 22026 & 284.064 & 1.615 & 0.08163 & 51.645 & 234.20 & 0.04720 \\
\hline 22027 & 283.394 & 1.615 & 0.08166 & 50.439 & 234.28 & 0.04721 \\
\hline 22028 & 282.783 & 1.619 & 0.08217 & 49.339 & 234.84 & 0.04751 \\
\hline 22029 & 282.235 & 1.621 & 0.08280 & 48.353 & 235.05 & 0.04787 \\
\hline 8001 & 283.712 & 0.040 & 0.00963 & 51.012 & 5.84 & 0.00557 \\
\hline 8002 & 283.281 & 0.040 & 0.00955 & 50.236 & 5.85 & 0.00552 \\
\hline 8003 & 282.873 & 0.040 & 0.00946 & 49.501 & 5.85 & 0.00547 \\
\hline 8004 & 282.500 & 0.040 & 0.00942 & 48.830 & 5.85 & 0.00545 \\
\hline 8005 & 283.766 & 0.037 & 0.01006 & 51.109 & 5.30 & 0.00582 \\
\hline 8006 & 283.311 & 0.037 & 0.01007 & 50.290 & 5.30 & 0.00582 \\
\hline 8007 & 282.905 & 0.037 & 0.01001 & 49.559 & 5.31 & 0.00579 \\
\hline 8008 & 282.517 & 0.037 & 0.01002 & 48.861 & 5.30 & 0.00579 \\
\hline 8009 & 283.789 & 0.029 & 0.01076 & 51.150 & 4.27 & 0.00622 \\
\hline 8010 & 283.344 & 0.030 & 0.01068 & 50.349 & 4.30 & 0.00617 \\
\hline 8011 & 282.912 & 0.030 & 0.01067 & 49.572 & 4.29 & 0.00617 \\
\hline 8012 & 282.532 & 0.030 & 0.01063 & 48.888 & 4.31 & 0.00615 \\
\hline 8013 & 283.847 & 0.030 & 0.01074 & 51.255 & 4.30 & 0.00621 \\
\hline 8014 & 283.373 & 0.030 & 0.01015 & 50.401 & 4.29 & 0.00587 \\
\hline 8015 & 282.963 & 0.029 & 0.01066 & 49.663 & 4.27 & 0.00616 \\
\hline 8016 & 282.563 & 0.029 & 0.01080 & 48.943 & 4.27 & 0.00624 \\
\hline 8017 & 283.820 & 0.031 & 0.01090 & 51.206 & 4.50 & 0.00630 \\
\hline 8018 & 283.359 & 0.030 & 0.01087 & 50.376 & 4.42 & 0.00628 \\
\hline 8019 & 282.916 & 0.030 & 0.01050 & 49.579 & 4.40 & 0.00607 \\
\hline 8020 & 282.527 & 0.030 & 0.01082 & 48.879 & 4.37 & 0.00626 \\
\hline 8021 & 283.731 & 0.042 & 0.00867 & 51.046 & 6.10 & 0.00501 \\
\hline 8022 & 283.277 & 0.042 & 0.00856 & 50.229 & 6.11 & 0.00495 \\
\hline 8023 & 282.860 & 0.042 & 0.00847 & 49.478 & 6.10 & 0.00490 \\
\hline 8024 & 282.468 & 0.042 & 0.00838 & 48.772 & 6.11 & 0.00485 \\
\hline 9001 & 288.398 & 0.052 & 0.00621 & 59.446 & 7.58 & 0.00359 \\
\hline 9002 & 287.951 & 0.052 & 0.00606 & 58.642 & 7.59 & 0.00350 \\
\hline 9003 & 287.545 & 0.052 & 0.00584 & 57.911 & 7.59 & 0.00338 \\
\hline 9004 & 287.203 & 0.052 & 0.00576 & 57.295 & 7.57 & 0.00333 \\
\hline 9005 & 288.559 & 0.048 & 0.00773 & 59.736 & 7.02 & 0.00447 \\
\hline 9006 & 288.110 & 0.048 & 0.00781 & 58.928 & 7.02 & 0.00452 \\
\hline 9007 & 287.677 & 0.048 & 0.00771 & 58.149 & 7.01 & 0.00446 \\
\hline 9008 & 287.277 & 0.048 & 0.00763 & 57.429 & 7.02 & 0.00441 \\
\hline 9009 & 288.625 & 0.044 & 0.00874 & 59.855 & 6.37 & 0.00505 \\
\hline 9010 & 288.117 & 0.044 & 0.00872 & 58.941 & 6.38 & 0.00504 \\
\hline 9011 & 287.732 & 0.044 & 0.00866 & 58.248 & 6.36 & 0.00501 \\
\hline
\end{tabular}


Table 75. Transient Hot-Wire Thermal Conductivity Data for HCFC-123 (Continued)

\begin{tabular}{|c|c|c|c|c|c|c|}
\hline $\begin{array}{l}\text { Point } \\
\text { Number }\end{array}$ & $\begin{array}{l}\mathrm{T}_{\text {Exp. }} \\
(\mathrm{K})\end{array}$ & $\begin{array}{l}\mathrm{P}_{\mathrm{Cell}} \\
(\mathrm{MPa})\end{array}$ & $\begin{array}{c}\lambda_{\operatorname{Exp}_{.}} \\
(W / m-K)\end{array}$ & ${ }^{\mathrm{T}}$ ( $\left.{ }^{\mathrm{0xp}} \mathrm{F}\right)$ & $\begin{array}{l}\mathrm{P}_{\text {Cell }} \\
\text { (psia) }\end{array}$ & $\begin{array}{c}\lambda_{\text {Exp. }} \\
(B T U / f t-h r-F)\end{array}$ \\
\hline 9012 & 287.311 & 0.044 & 0.00866 & 57.490 & 6.37 & 0.00501 \\
\hline 9013 & 288.676 & 0.038 & 0.00924 & 59.947 & 5.56 & 0.00534 \\
\hline 9014 & 288.206 & 0.038 & 0.00920 & 59.101 & 5.57 & 0.00532 \\
\hline 9015 & 287.758 & 0.038 & 0.00920 & 58.294 & 5.57 & 0.00532 \\
\hline 9016 & 287.353 & 0.038 & 0.00920 & 57.565 & 5.56 & 0.00532 \\
\hline 9017 & 288.684 & 0.033 & 0.00953 & 59.961 & 4.73 & 0.00551 \\
\hline 9018 & 288.244 & 0.033 & 0.00950 & 59.169 & 4.74 & 0.00549 \\
\hline 9019 & 287.836 & 0.033 & 0.00936 & 58.435 & 4.72 & 0.00541 \\
\hline 9020 & 287.387 & 0.033 & 0.00946 & 57.627 & 4.73 & 0.00547 \\
\hline 10001 & 293.214 & 0.066 & 0.00576 & 68.115 & 9.53 & 0.00333 \\
\hline 10002 & 292.772 & 0.066 & 0.00560 & 67.320 & 9.51 & 0.00324 \\
\hline 10003 & 292.362 & 0.066 & 0.00545 & 66.582 & 9.51 & 0.00315 \\
\hline 10004 & 292.019 & 0.066 & 0.00528 & 65.964 & 9.52 & 0.00305 \\
\hline 10005 & 293.333 & 0.063 & 0.00730 & 68.329 & 9.10 & 0.00422 \\
\hline 10006 & 292.908 & 0.063 & 0.00700 & 67.564 & 9.11 & 0.00405 \\
\hline 10007 & 292.492 & 0.063 & 0.00683 & 66.816 & 9.12 & 0.00395 \\
\hline 10008 & 292.100 & 0.063 & 0.00666 & 66.110 & 9.13 & 0.00385 \\
\hline 10009 & 293.434 & 0.058 & 0.00850 & 68.511 & 8.42 & 0.00491 \\
\hline 10010 & 292.982 & 0.058 & 0.00842 & 67.698 & 8.40 & 0.00487 \\
\hline 10011 & 292.561 & 0.058 & 0.00831 & 66.940 & 8.41 & 0.00480 \\
\hline 10012 & 292.171 & 0.058 & 0.00828 & 66.238 & 8.42 & 0.00479 \\
\hline 10013 & 293.500 & 0.051 & 0.00934 & 68.630 & 7.34 & 0.00540 \\
\hline 10014 & 293.041 & 0.051 & 0.00929 & 67.804 & 7.33 & 0.00537 \\
\hline 10015 & 292.612 & 0.051 & 0.00926 & 67.032 & 7.34 & 0.00535 \\
\hline 10016 & 292.173 & 0.051 & 0.00914 & 66.241 & 7.34 & 0.00528 \\
\hline 10017 & 293.558 & 0.043 & 0.00970 & 68.734 & 6.26 & 0.00561 \\
\hline 10018 & 293.090 & 0.043 & 0.00970 & 67.892 & 6.26 & 0.00561 \\
\hline 10019 & 292.657 & 0.043 & 0.00968 & 67.113 & 6.25 & 0.00560 \\
\hline 10020 & 292.247 & 0.043 & 0.00965 & 66.375 & 6.24 & 0.00558 \\
\hline 11001 & 298.278 & 0.080 & 0.00635 & 77.230 & 11.66 & 0.00367 \\
\hline 11002 & 297.894 & 0.080 & 0.00615 & 76.539 & 11.64 & 0.00356 \\
\hline 11003 & 297.494 & 0.080 & 0.00606 & 75.819 & 11.63 & 0.00350 \\
\hline 11004 & 297.116 & 0.080 & 0.00591 & 75.139 & 11.63 & 0.00342 \\
\hline 11005 & 298.440 & 0.076 & 0.00818 & 77.522 & 10.98 & 0.00473 \\
\hline 11006 & 297.997 & 0.076 & 0.00804 & 76.725 & 10.99 & 0.00465 \\
\hline 11007 & 297.587 & 0.076 & 0.00791 & 75.987 & 10.99 & 0.00457 \\
\hline 11008 & 297.166 & 0.076 & 0.00772 & 75.229 & 10.97 & 0.00446 \\
\hline 11009 & 298.484 & 0.070 & 0.00920 & 77.601 & 10.19 & 0.00532 \\
\hline 11010 & 298.042 & 0.070 & 0.00913 & 76.806 & 10.19 & 0.00528 \\
\hline 11011 & 297.624 & 0.070 & 0.00909 & 76.053 & 10.19 & 0.00526 \\
\hline 11012 & 297.235 & 0.070 & 0.00905 & 75.353 & 10.18 & 0.00523 \\
\hline 11013 & 298.523 & 0.064 & 0.00968 & 77.671 & 9.24 & 0.00560 \\
\hline 11014 & 298.069 & 0.064 & 0.00965 & 76.854 & 9.24 & 0.00558 \\
\hline 11015 & 297.636 & 0.064 & 0.00967 & 76.075 & 9.24 & 0.00559 \\
\hline 11016 & 297.238 & 0.064 & 0.00963 & 75.358 & 9.24 & 0.00557 \\
\hline 11017 & 298.560 & 0.057 & 0.00998 & 77.738 & 8.20 & 0.00577 \\
\hline 11018 & 298.110 & 0.057 & 0.00998 & 76.928 & 8.21 & 0.00577 \\
\hline 11019 & 297.683 & 0.056 & 0.00993 & 76.159 & 8.19 & 0.00574 \\
\hline 11020 & 297.239 & 0.057 & 0.00995 & 75.360 & 8.20 & 0.00575 \\
\hline
\end{tabular}


Table 75. Transient Hot-Wire Thermal Conductivity Data for HCFC-123 (Continued)

\begin{tabular}{|c|c|c|c|c|c|c|}
\hline $\begin{array}{l}\text { Point } \\
\text { Number }\end{array}$ & $\begin{array}{l}T_{\text {Exp. }} \\
(\mathrm{K})\end{array}$ & $\begin{array}{l}\mathrm{P}_{\mathrm{Cell}} \\
(\mathrm{MPa})\end{array}$ & $\begin{array}{c}\lambda_{\text {Exp. }} \\
(\mathrm{W} / \mathrm{m}-\mathrm{K})\end{array}$ & $\mathrm{T}_{\text {Exp. }}$ & $\begin{array}{l}P_{\text {Cell }} \\
\text { (psia) }\end{array}$ & $\begin{array}{c}\lambda_{\text {Exp. }} \\
\text { (BTU/ft-hr-F) }\end{array}$ \\
\hline 11021 & 298.625 & 0.049 & 0.01018 & 77.855 & 7.05 & 0.00589 \\
\hline 11022 & 298.163 & 0.049 & 0.01015 & 77.023 & 7.07 & 0.00587 \\
\hline 11023 & 297.738 & 0.049 & 0.01013 & 76.258 & 7.05 & 0.00586 \\
\hline 11024 & 297.336 & 0.049 & 0.01011 & 75.535 & 7.06 & 0.00585 \\
\hline 1001 & 304.488 & 66.312 & 0.09591 & 88.408 & 9617.78 & 0.05545 \\
\hline 1002 & 303.801 & 66.316 & 0.09611 & 87.172 & 9618.32 & 0.05557 \\
\hline 1003 & 303.142 & 66.323 & 0.09645 & 85.986 & 9619.41 & 0.05577 \\
\hline 1004 & 302.571 & 66.331 & 0.09671 & 84.958 & 9620.50 & 0.05592 \\
\hline 1005 & 304.610 & 46.683 & 0.09074 & 88.628 & 6770.86 & 0.05246 \\
\hline 1006 & 303.887 & 46.679 & 0.09082 & 87.327 & 6770.22 & 0.05251 \\
\hline 1007 & 303.220 & 46.677 & 0.09094 & 86.126 & 6769.94 & 0.05258 \\
\hline 1008 & 302.609 & 46.675 & 0.09114 & 85.026 & 6769.67 & 0.05270 \\
\hline 1009 & 304.188 & 29.483 & 0.08543 & $\{77.868$ & 4276.12 & 0.04939 \\
\hline 1010 & 303.485 & 29.488 & 0.08568 & 85.603 & 4276.95 & 0.04954 \\
\hline 1011 & 302.852 & 29.494 & 0.08570 & 85.464 & 4277.69 & 0.04955 \\
\hline 1012 & 302.265 & 29.497 & 0.08563 & 84.407 & 4278.15 & 0.04951 \\
\hline 1013 & 304.356 & 15.106 & 0.08045 & 88.171 & 2191.01 & 0.04651 \\
\hline 1014 & 303.612 & 15.107 & 0.08053 & 86.832 & 2191.10 & 0.04656 \\
\hline 1015 & 302.934 & 15.110 & 0.08093 & 85.611 & 2191.57 & 0.04679 \\
\hline 1016 & 302.328 & 15.113 & 0.08125 & 84.520 & 2191.94 & 0.04698 \\
\hline 1017 & 304.543 & 4.023 & 0.07601 & 88.507 & 583.42 & 0.04395 \\
\hline 1018 & 303.763 & 4.011 & 0.07662 & 87.103 & 581.73 & 0.04430 \\
\hline 1019 & 303.068 & 4.021 & 0.07667 & 85.852 & 583.14 & 0.04433 \\
\hline 1020 & 302.423 & 4.026 & 0.07680 & 84.691 & 583.98 & 0.04440 \\
\hline 12001 & 303.273 & 0.091 & 0.00849 & 86.221 & 13.26 & 0.00491 \\
\hline 12002 & 302.847 & 0.091 & 0.00841 & 85.455 & 13.24 & 0.00486 \\
\hline 12003 & 302.444 & 0.091 & 0.00827 & 84.729 & 13.24 & 0.00478 \\
\hline 12004 & 302.067 & 0.091 & 0.00818 & 84.051 & 13.24 & 0.00473 \\
\hline 12005 & 303.307 & 0.088 & 0.00928 & 86.283 & 12.70 & 0.00537 \\
\hline 12006 & 302.873 & 0.088 & 0.00916 & 85.501 & 12.70 & 0.00530 \\
\hline 12007 & 302.468 & 0.088 & 0.00911 & 84.772 & 12.70 & 0.00527 \\
\hline 12008 & 302.085 & 0.088 & 0.00910 & 84.083 & 12.71 & 0.00526 \\
\hline 12009 & 303.341 & 0.081 & 0.00986 & 86.344 & 11.68 & 0.00570 \\
\hline 12010 & 302.907 & 0.081 & 0.00978 & 85.563 & 11.68 & 0.00565 \\
\hline 12011 & 302.497 & 0.081 & 0.00977 & 84.825 & 11.70 & 0.00565 \\
\hline 12012 & 302.114 & 0.081 & 0.00976 & 84.135 & 11.70 & 0.00564 \\
\hline 12013 & 303.370 & 0.073 & 0.01020 & 86.396 & 10.56 & 0.00590 \\
\hline 12014 & 302.924 & 0.073 & 0.01018 & 85.593 & 10.56 & 0.00589 \\
\hline 12015 & 302.518 & 0.073 & 0.01017 & 84.862 & 10.58 & 0.00588 \\
\hline 12016 & 302.131 & 0.073 & 0.01016 & 84.166 & 10.57 & 0.00587 \\
\hline 12017 & 303.378 & 0.065 & 0.01044 & 86.410 & 9.38 & 0.00604 \\
\hline 12018 & 302.938 & 0.065 & 0.01044 & 85.618 & 9.39 & 0.00604 \\
\hline 12019 & 302.519 & 0.065 & 0.01044 & 84.864 & 9.39 & 0.00604 \\
\hline 12020 & 302.138 & 0.065 & 0.01045 & 84.178 & 9.39 & 0.00604 \\
\hline 12021 & 303.447 & 0.057 & 0.01054 & 86.535 & 8.22 & 0.00609 \\
\hline 12022 & 302.995 & 0.057 & 0.01052 & 85.721 & 8.22 & 0.00608 \\
\hline 12023 & 302.573 & 0.057 & 0.01047 & 84.961 & 8.23 & 0.00605 \\
\hline 12024 & 302.181 & 0.057 & 0.01047 & 84.256 & 8.22 & 0.00605 \\
\hline 13001 & 307.969 & 0.113 & 0.00864 & 94.674 & 16.34 & 0.00500 \\
\hline
\end{tabular}


Table 75. Transient Hot-Wire Thermal Conductivity Data for HCFC-123 (Continued)

\begin{tabular}{|c|c|c|c|c|c|c|}
\hline $\begin{array}{l}\text { Point } \\
\text { Number }\end{array}$ & $\begin{array}{l}\mathrm{T}_{\text {Exp. }} \\
(\mathrm{K})\end{array}$ & $\begin{array}{l}\mathrm{P}_{\mathrm{Cell}} \\
(\mathrm{MPa})\end{array}$ & $\begin{array}{c}\lambda_{\operatorname{Exp}_{.}} \\
(W / m-K)\end{array}$ & $\left.{ }_{\left({ }^{0 \times p}\right.} \times\right)$ & $\begin{array}{l}\mathrm{P}_{\mathrm{Cell}} \\
\text { (psia) }\end{array}$ & $\begin{array}{c}\lambda_{\text {Exp. }} \\
\text { (BTU/ft-hr-F) }\end{array}$ \\
\hline 13002 & 307.562 & 0.113 & 0.00846 & 93.942 & 16.34 & 0.00489 \\
\hline 13003 & 307.167 & 0.113 & 0.00834 & 93.231 & 16.36 & 0.00482 \\
\hline 13004 & 306.806 & 0.113 & 0.00818 & 92.581 & 16.38 & 0.00473 \\
\hline 13005 & 307.995 & 0.109 & 0.00950 & 94.721 & 15.75 & 0.00549 \\
\hline 13006 & 307.580 & 0.109 & 0.00933 & 93.974 & 15.80 & 0.00539 \\
\hline 13007 & 307.193 & 0.109 & 0.00917 & 93.277 & 15.82 & 0.00530 \\
\hline 13008 & 306.833 & 0.109 & 0.00927 & 92.629 & 15.81 & 0.00536 \\
\hline 13009 & 308.021 & 0.105 & 0.00997 & 94.768 & 15.17 & 0.00576 \\
\hline 13010 & 307.641 & 0.105 & 0.00987 & 94.084 & 15.17 & 0.00571 \\
\hline 13011 & 307.216 & 0.105 & 0.00984 & 93.319 & 15.19 & 0.00569 \\
\hline 13012 & 306.891 & 0.106 & 0.00969 & 92.734 & 15.35 & 0.00560 \\
\hline 13013 & 308.017 & 0.106 & 0.00996 & 94.761 & 15.34 & 0.00576 \\
\hline 13014 & 307.651 & 0.106 & 0.00990 & 94.102 & 15.34 & 0.00572 \\
\hline 13015 & 307.260 & 0.106 & 0.00984 & 93.398 & 15.40 & 0.00569 \\
\hline 13016 & 306.893 & 0.106 & 0.00976 & 92.737 & 15.41 & 0.00564 \\
\hline 13017 & 308.116 & 0.095 & 0.01048 & 94.939 & 13.82 & 0.00606 \\
\hline 13018 & 307.686 & 0.096 & 0.01040 & 94.165 & 13.88 & 0.00601 \\
\hline 13019 & 307.293 & 0.096 & 0.01041 & 93.457 & 13.86 & 0.00602 \\
\hline 13020 & 306.915 & 0.096 & 0.01045 & 92.777 & 13.88 & 0.00604 \\
\hline 13021 & 308.140 & 0.088 & 0.01063 & 94.982 & 12.75 & 0.00615 \\
\hline 13022 & 307.713 & 0.088 & 0.01062 & 94.213 & 12.76 & 0.00614 \\
\hline 13023 & 307.308 & 0.088 & 0.01062 & 93.484 & 12.76 & 0.00614 \\
\hline 13024 & 306.940 & 0.088 & 0.01060 & 92.822 & 12.78 & 0.00613 \\
\hline 13025 & 308.115 & 0.079 & 0.01085 & 94.937 & 11.48 & 0.00627 \\
\hline 13026 & 307.723 & 0.079 & 0.01082 & 94.231 & 11.47 & 0.00626 \\
\hline 13027 & 307.320 & 0.079 & 0.01083 & 93.506 & 11.46 & 0.00626 \\
\hline 13028 & 306.947 & 0.079 & 0.01081 & 92.835 & 11.46 & 0.00625 \\
\hline 13029 & 308.207 & 0.069 & 0.01097 & 95.103 & 10.05 & 0.00634 \\
\hline 13030 & 307.768 & 0.069 & 0.01096 & 94.312 & 10.07 & 0.00634 \\
\hline 13031 & 307.354 & 0.070 & 0.01095 & 93.567 & 10.09 & 0.00633 \\
\hline 13032 & 306.974 & 0.070 & 0.01094 & 92.883 & 10.10 & 0.00633 \\
\hline 13033 & 308.199 & 0.058 & 0.01106 & 95.088 & 8.44 & 0.00639 \\
\hline 13034 & 307.754 & 0.058 & 0.01103 & 94.287 & 8.37 & 0.00638 \\
\hline 13035 & 307.340 & 0.058 & 0.01104 & 93.542 & 8.34 & 0.00638 \\
\hline 13036 & 306.953 & 0.058 & 0.01103 & 92.845 & 8.35 & 0.00638 \\
\hline 13037 & 308.294 & 0.049 & 0.01116 & 95.259 & 7.04 & 0.00645 \\
\hline 13038 & 307.794 & 0.049 & 0.01118 & 94.359 & 7.06 & 0.00646 \\
\hline 13039 & 307.377 & 0.049 & 0.01113 & 93.609 & 7.08 & 0.00644 \\
\hline 13040 & 306.986 & 0.049 & 0.01110 & 92.905 & 7.09 & 0.00642 \\
\hline 14001 & 312.962 & 0.132 & 0.01072 & 103.662 & 19.09 & 0.00620 \\
\hline 14002 & 312.563 & 0.132 & 0.01062 & 102.943 & 19.10 & 0.00614 \\
\hline 14003 & 312.183 & 0.132 & 0.01059 & 102.259 & 19.13 & 0.00612 \\
\hline 14004 & 311.838 & 0.132 & 0.01054 & 101.638 & 19.11 & 0.00609 \\
\hline 14005 & 312.984 & 0.123 & 0.01116 & 103.701 & 17.85 & 0.00645 \\
\hline 14006 & 312.576 & 0.123 & 0.01104 & 102.967 & 17.86 & 0.00638 \\
\hline 14007 & 312.193 & 0.123 & 0.01102 & 102.277 & 17.85 & 0.00637 \\
\hline 14008 & 311.851 & 0.123 & 0.01088 & 101.662 & 17.88 & 0.00629 \\
\hline 14009 & 312.975 & 0.114 & 0.01134 & 103.685 & 16.54 & 0.00656 \\
\hline 14010 & 312.583 & 0.114 & 0.01120 & 102.979 & 16.57 & 0.00648 \\
\hline
\end{tabular}


Table 75. Transient Hot-Wire Thermal Conductivity Data for HCFC-123 (Continued)

\begin{tabular}{|c|c|c|c|c|c|c|}
\hline $\begin{array}{l}\text { Point } \\
\text { Number }\end{array}$ & $\begin{array}{l}\mathrm{T}_{\text {Exp. }} \\
(\mathrm{K})\end{array}$ & $\begin{array}{l}\mathrm{P}_{\mathrm{Cell}} \\
(\mathrm{MPa})\end{array}$ & $\underset{(W / m-K)}{\lambda_{\operatorname{Exp}}}$ & $\mathrm{T}_{\text {Exp. }}$ & $\begin{array}{l}\mathrm{P}_{\text {Cell }} \\
\text { (psia) }\end{array}$ & $\begin{array}{c}\lambda_{\text {Exp. }} \\
\text { (BTU/ft-hr-F) }\end{array}$ \\
\hline 14011 & 312.166 & 0.114 & 0.01120 & 102.229 & 16.56 & 0.00648 \\
\hline 14012 & 311.857 & 0.114 & 0.01121 & 101.673 & 16.59 & 0.00648 \\
\hline 14013 & 313.021 & 0.106 & 0.01138 & 103.768 & 15.39 & 0.00658 \\
\hline 14014 & 312.605 & 0.106 & 0.01141 & 103.019 & 15.40 & 0.00660 \\
\hline 14015 & 312.216 & 0.106 & 0.01136 & 102.319 & 15.40 & 0.00657 \\
\hline 14016 & 311.859 & 0.106 & 0.01131 & 101.676 & 15.42 & 0.00654 \\
\hline 14017 & 313.060 & 0.092 & 0.01151 & 103.838 & 13.28 & 0.00665 \\
\hline 14018 & 312.637 & 0.092 & 0.01150 & 103.077 & 13.30 & 0.00665 \\
\hline 14019 & 312.239 & 0.092 & 0.01147 & 102.360 & 13.29 & 0.00663 \\
\hline 14020 & 311.882 & 0.092 & 0.01153 & 101.718 & 13.31 & 0.00667 \\
\hline 14021 & 313.126 & 0.071 & 0.01176 & 103.957 & 10.25 & 0.00680 \\
\hline 14022 & 312.697 & 0.071 & 0.01173 & 103.185 & 10.24 & 0.00678 \\
\hline 14023 & 312.292 & 0.071 & 0.01168 & 102.456 & 10.26 & 0.00675 \\
\hline 14024 & 311.924 & 0.071 & 0.01173 & 101.793 & 10.27 & 0.00678 \\
\hline 14025 & 313.211 & 0.052 & 0.01200 & 104.110 & 7.47 & 0.00694 \\
\hline 14026 & 312.771 & 0.052 & 0.01197 & 103.318 & 7.47 & 0.00692 \\
\hline 14027 & 312.357 & 0.052 & 0.01197 & 102.573 & 7.48 & 0.00692 \\
\hline 14028 & 311.975 & 0.052 & 0.01195 & 101.885 & 7.47 & 0.00691 \\
\hline 15001 & 317.811 & 0.165 & 0.01118 & 112.390 & 23.86 & 0.00646 \\
\hline 15002 & 317.401 & 0.165 & 0.01112 & 111.652 & 23.86 & 0.00643 \\
\hline 15003 & 317.089 & 0.165 & 0.01112 & 111.090 & 23.86 & 0.00643 \\
\hline 15004 & 316.758 & 0.165 & 0.01105 & 110.494 & 23.86 & 0.00639 \\
\hline 15005 & 317.845 & 0.151 & 0.01216 & 112.451 & 21.84 & 0.00703 \\
\hline 15006 & 317.458 & 0.151 & 0.01214 & 111.754 & 21.85 & 0.00702 \\
\hline 15007 & 317.099 & 0.151 & 0.01205 & 111.108 & 21.83 & 0.00697 \\
\hline 15008 & 316.764 & 0.151 & 0.01211 & 110.505 & 21.85 & 0.00700 \\
\hline 15009 & 317.853 & 0.139 & 0.01231 & 112.465 & 20.16 & 0.00712 \\
\hline 15010 & 317.458 & 0.139 & 0.01224 & 111.754 & 20.20 & 0.00708 \\
\hline 15011 & 317.104 & 0.139 & 0.01230 & 111.117 & 20.20 & 0.00711 \\
\hline 15012 & 316.770 & 0.139 & 0.01228 & 110.516 & 20.22 & 0.00710 \\
\hline 15013 & 317.891 & 0.117 & 0.01255 & 112.534 & 17.00 & 0.00726 \\
\hline 15014 & 317.489 & 0.117 & 0.01245 & 111.810 & 17.00 & 0.00720 \\
\hline 15015 & 317.131 & 0.117 & 0.01246 & 111.166 & 17.01 & 0.00720 \\
\hline 15016 & 316.798 & 0.117 & 0.01241 & 110.566 & 16.99 & 0.00718 \\
\hline 15017 & 317.918 & 0.105 & 0.01259 & 112.582 & 15.21 & 0.00728 \\
\hline 15018 & 317.527 & 0.105 & 0.01247 & 111.879 & 15.21 & 0.00721 \\
\hline 15019 & 317.151 & 0.105 & 0.01248 & 111.202 & 15.24 & 0.00722 \\
\hline 15020 & 316.805 & 0.105 & 0.01251 & 110.579 & 15.25 & 0.00723 \\
\hline 15021 & 317.968 & 0.088 & 0.01265 & 112.672 & 12.72 & 0.00731 \\
\hline 15022 & 317.580 & 0.088 & 0.01258 & 111.974 & 12.72 & 0.00727 \\
\hline 15023 & 317.203 & 0.088 & 0.01260 & 111.295 & 12.72 & 0.00729 \\
\hline 15024 & 316.846 & 0.088 & 0.01256 & 110.653 & 12.72 & 0.00726 \\
\hline 15025 & 318.086 & 0.070 & 0.01290 & 112.885 & 10.14 & 0.00746 \\
\hline 15026 & 317.667 & 0.070 & 0.01285 & 112.131 & 10.13 & 0.00743 \\
\hline 15027 & 317.285 & 0.070 & 0.01282 & 111.443 & 10.11 & 0.00741 \\
\hline 15028 & 316.925 & 0.070 & 0.01276 & 110.795 & 10.09 & 0.00738 \\
\hline 15029 & 318.121 & 0.054 & $0.0133 !$ & 112.948 & 7.82 & 0.00770 \\
\hline 15030 & 317.700 & 0.054 & 0.01330 & 112.190 & 7.82 & 0.00769 \\
\hline 15031 & 317.339 & 0.054 & 0.01323 & 111.540 & 7.81 & 0.00765 \\
\hline
\end{tabular}


Table 75. Transient Hot-Wire Thermal Conductivity Data for HCFC-123 (Continued)

\begin{tabular}{|c|c|c|c|c|c|c|}
\hline $\begin{array}{l}\text { Point } \\
\text { Number }\end{array}$ & $\begin{array}{l}\mathrm{T}_{\text {Exp. }} \\
(\mathrm{K})\end{array}$ & $\begin{array}{l}\mathrm{P}_{\mathrm{Cell}} \\
(\mathrm{MPa})\end{array}$ & $\begin{array}{c}\lambda_{\operatorname{Exp}} \\
(W / m-K)\end{array}$ & $\mathrm{T}_{\text {Exp. }}$ & $\begin{array}{l}\mathrm{P}_{\text {Cell }} \\
\text { (psia) }\end{array}$ & $\begin{array}{c}\lambda_{\text {Exp }} \\
(B T U / f t-h r-F)\end{array}$ \\
\hline 15032 & 316.960 & 0.054 & 0.01325 & 110.858 & 7.81 & 0.00766 \\
\hline 15033 & 318.234 & 0.038 & 0.01393 & 113.151 & 5.58 & 0.00805 \\
\hline 15034 & 317.794 & 0.038 & 0.01387 & 112.359 & 5.58 & 0.00802 \\
\hline 15035 & 317.383 & 0.039 & 0.01386 & 111.619 & 5.59 & 0.00801 \\
\hline 15036 & 317.008 & 0.038 & 0.01385 & 110.944 & 5.58 & 0.00801 \\
\hline 16001 & 322.859 & 0.188 & 0.01046 & 121.476 & 27.32 & 0.00605 \\
\hline 16002 & 322.468 & 0.188 & 0.01030 & 120.772 & 27.32 & 0.00596 \\
\hline 16003 & 322.105 & 0.188 & 0.01021 & 120.119 & 27.32 & 0.00590 \\
\hline 16004 & 321.769 & 0.188 & 0.01017 & 119.514 & 27.32 & 0.00588 \\
\hline 16005 & 322.881 & 0.176 & 0.01131 & 121.516 & 25.51 & 0.00654 \\
\hline 16006 & 322.482 & 0.176 & 0.01130 & 120.798 & 25.51 & 0.00653 \\
\hline 16007 & 322.118 & 0.176 & 0.01123 & 120.142 & 25.51 & 0.00649 \\
\hline 16008 & 321.785 & 0.176 & 0.01125 & 119.543 & 25.48 & 0.00650 \\
\hline 16009 & 322.885 & 0.166 & 0.01164 & 121.523 & 24.04 & 0.00673 \\
\hline 16010 & 322.488 & 0.166 & 0.01156 & 120.808 & 24.03 & 0.00668 \\
\hline 16011 & 322.133 & 0.166 & 0.01149 & 120.169 & 24.02 & 0.00664 \\
\hline 16012 & 321.779 & 0.166 & 0.01161 & 119.532 & 24.05 & 0.00671 \\
\hline 16013 & 322.946 & 0.148 & 0.01182 & 121.633 & 21.42 & 0.00683 \\
\hline 16014 & 322.543 & 0.148 & 0.01180 & 120.907 & 21.43 & 0.00682 \\
\hline 16015 & 322.177 & 0.148 & 0.01186 & 120.249 & 21.46 & 0.00686 \\
\hline 16016 & 321.834 & 0.148 & 0.01185 & 119.631 & 21.46 & 0.00685 \\
\hline 16017 & 322.952 & 0.131 & 0.01192 & 121.644 & 18.94 & 0.00689 \\
\hline 16018 & 322.557 & 0.131 & 0.01188 & 120.933 & 18.97 & 0.00687 \\
\hline 16019 & 322.212 & 0.131 & 0.01197 & 120.312 & 18.93 & 0.00692 \\
\hline 16020 & 321.847 & 0.131 & 0.01194 & 119.655 & 18.96 & 0.00690 \\
\hline 16021 & 323.020 & 0.110 & 0.01219 & 121.766 & 15.98 & 0.00705 \\
\hline 16022 & 322.616 & 0.110 & 0.01207 & 121.039 & 15.96 & 0.00698 \\
\hline 16023 & 322.231 & 0.110 & 0.01204 & 120.346 & 15.98 & 0.00696 \\
\hline 16024 & 321.864 & 0.110 & 0.01214 & 119.685 & 15.99 & 0.00702 \\
\hline 16025 & 323.049 & 0.095 & 0.01217 & 121.818 & 13.82 & 0.00704 \\
\hline 16026 & 322.632 & 0.096 & 0.01210 & 121.068 & 13.86 & 0.00700 \\
\hline 16027 & 322.245 & 0.096 & 0.01226 & 120.371 & 13.88 & 0.00709 \\
\hline 16028 & 321.874 & 0.096 & 0.01211 & 119.703 & 13.89 & 0.00700 \\
\hline 16029 & 323.195 & 0.066 & 0.01204 & 122.081 & 9.58 & 0.00696 \\
\hline 16030 & 322.752 & 0.066 & 0.01201 & $! 21.284$ & 9.59 & 0.00694 \\
\hline 16031 & 322.343 & 0.066 & 0.01201 & 120.547 & 9.58 & 0.00694 \\
\hline 16032 & 321.960 & 0.066 & 0.01200 & 119.858 & 9.59 & 0.00694 \\
\hline 16033 & 323.298 & 0.047 & 0.01218 & 122.266 & 6.77 & 0.00704 \\
\hline 16034 & 322.842 & 0.047 & 0.01216 & 121.446 & 6.78 & 0.00703 \\
\hline 16035 & 322.416 & 0.047 & 0.01217 & 120.679 & 6.78 & 0.00704 \\
\hline 16036 & 322.030 & 0.047 & 0.01211 & 119.984 & 6.78 & 0.00700 \\
\hline 2001 & 327.403 & 66.509 & 0.09183 & 129.655 & 9646.41 & 0.05309 \\
\hline 2002 & 326.633 & 66.508 & 0.09218 & 128.269 & 9646.14 & 0.05330 \\
\hline 2003 & 325.911 & 66.505 & 0.09232 & 126.970 & 9645.77 & 0.05338 \\
\hline 2004 & 325.289 & 66.504 & 0.08989 & 125.850 & 9645.68 & 0.05197 \\
\hline 2005 & 326.749 & 48.679 & 0.08674 & 128.478 & 7060.24 & 0.05015 \\
\hline 2006 & 325.992 & 48.675 & 0.08710 & 127.116 & 7059.70 & 0.05036 \\
\hline 2007 & 325.326 & 48.680 & 0.08709 & 125.917 & 7060.43 & 0.05035 \\
\hline 2008 & 324.709 & 48.681 & 0.08742 & 124.806 & 7060.61 & 0.05054 \\
\hline
\end{tabular}


Table 75. Transient Hot-Wire Thermal Conductivity Data for HCFC-123 (Continued)

\begin{tabular}{|c|c|c|c|c|c|c|}
\hline $\begin{array}{l}\text { Point } \\
\text { Number }\end{array}$ & $\begin{array}{l}\mathrm{T}_{\text {Exp. }} \\
(\mathrm{K})\end{array}$ & $\begin{array}{l}\mathrm{P}_{\mathrm{Cell}} \\
(\mathrm{MPa})\end{array}$ & $\begin{array}{c}\lambda_{\text {Exp. }} \\
(W / m-K)\end{array}$ & $\mathrm{T}_{\text {Exp. }}$ & $\begin{array}{l}P_{\text {Cell }} \\
\text { (psia) }\end{array}$ & $\begin{array}{c}\lambda_{\text {Exp. }} \\
(B T U / f t-h r-F)\end{array}$ \\
\hline 2009 & 326.935 & 33.881 & 0.08198 & 128.813 & 4914.09 & 0.04740 \\
\hline 2010 & 326.144 & 33.882 & 0.08216 & 127.389 & 4914.19 & 0.04750 \\
\hline 2011 & 325.424 & 33.885 & 0.08218 & 126.093 & 4914.65 & 0.04751 \\
\hline 2012 & 324.783 & 33.885 & 0.08191 & 124.939 & 4914.65 & 0.04736 \\
\hline 2013 & 326.328 & 19.664 & 0.07683 & 127.720 & 2852.03 & 0.04442 \\
\hline 2014 & 325.564 & 19.665 & 0.07739 & 126.345 & 2852.21 & 0.04475 \\
\hline 2015 & 324.893 & 19.665 & 0.07751 & 125.137 & 2852.21 & 0.04481 \\
\hline 2016 & 324.286 & 19.665 & 0.07759 & 124.045 & 2852.21 & 0.04486 \\
\hline 2017 & 326.508 & 8.749 & 0.07277 & 128.044 & 1268.89 & 0.04207 \\
\hline 2018 & 325.721 & 8.749 & 0.07307 & 126.628 & 1268.89 & 0.04225 \\
\hline 2019 & 325.005 & 8.747 & 0.07314 & 125.339 & 1268.61 & 0.04229 \\
\hline 2020 & 324.371 & 8.748 & 0.07321 & 124.198 & 1268.80 & 0.04233 \\
\hline 24002 & 332.102 & 0.262 & 0.01158 & 138.114 & 37.95 & 0.00670 \\
\hline 24003 & 331.673 & 0.262 & 0.01137 & 137.341 & 37.97 & 0.00657 \\
\hline 24004 & 331.278 & 0.262 & 0.01141 & 136.630 & 37.99 & 0.00660 \\
\hline 24005 & 332.588 & 0.238 & 0.01208 & 138.988 & 34.55 & 0.00698 \\
\hline 24006 & 332.152 & 0.238 & 0.01204 & 138.204 & 34.49 & 0.00696 \\
\hline 24007 & 331.719 & 0.238 & 0.01202 & 137.424 & 34.52 & 0.00695 \\
\hline 24008 & 331.320 & 0.237 & 0.01198 & 136.706 & 34.42 & 0.00693 \\
\hline 24009 & 332.662 & 0.202 & 0.01208 & 139.122 & 29.28 & 0.00698 \\
\hline 24010 & 332.190 & 0.201 & 0.01214 & 138.272 & 29.21 & 0.00702 \\
\hline 24011 & 331.754 & 0.201 & 0.01210 & 137.487 & 29.14 & 0.00700 \\
\hline 24012 & 331.352 & 0.201 & 0.01200 & 136.764 & 29.11 & 0.00694 \\
\hline 24013 & 332.729 & 0.167 & 0.01212 & 139.242 & 24.24 & 0.00701 \\
\hline 24014 & 332.267 & 0.167 & 0.01220 & 138.411 & 24.18 & 0.00705 \\
\hline 24015 & 331.826 & 0.167 & 0.01215 & 137.617 & 24.15 & 0.00702 \\
\hline 24016 & 331.421 & 0.166 & 0.01213 & 136.888 & 24.08 & 0.00701 \\
\hline 24017 & 332.799 & 0.143 & 0.01235 & 139.368 & 20.67 & 0.00714 \\
\hline 24018 & 332.304 & 0.142 & 0.01223 & 138.477 & 20.66 & 0.00707 \\
\hline 24019 & 331.853 & 0.142 & 0.01226 & 137.665 & 20.64 & 0.00709 \\
\hline 24020 & 331.435 & 0.142 & 0.01224 & 136.913 & 20.62 & 0.00708 \\
\hline 24021 & 332.872 & 0.117 & 0.01246 & 139.500 & 16.90 & 0.00720 \\
\hline 24022 & 332.381 & 0.117 & 0.01240 & 138.616 & 16.90 & 0.00717 \\
\hline 24023 & 331.917 & 0.116 & 0.01241 & 137.781 & 16.89 & 0.00718 \\
\hline 24024 & 331.472 & 0.117 & 0.01273 & 136.980 & 16.91 & 0.00736 \\
\hline 3001 & 343.532 & 66.812 & 0.08933 & 158.688 & 9690.25 & 0.05165 \\
\hline 3002 & 342.781 & 66.810 & 0.08947 & 157.336 & 9689.97 & 0.05173 \\
\hline 3003 & 342.063 & 66.800 & 0.08954 & 156.043 & 9688.52 & 0.05177 \\
\hline 3004 & 341.422 & 66.800 & 0.08954 & 154.890 & 9688.52 & 0.05177 \\
\hline 3005 & 343.685 & 51.197 & 0.08457 & 158.963 & 7425.53 & 0.04890 \\
\hline 3007 & 342.149 & 51.201 & 0.08479 & 156.198 & 7426.08 & 0.04902 \\
\hline 3008 & 341.495 & 51.204 & 0.08497 & 155.021 & 7426.54 & 0.04913 \\
\hline 3009 & 343.038 & 36.309 & 0.07982 & 157.798 & 5266.13 & 0.04615 \\
\hline 3010 & 342.269 & 36.312 & 0.08004 & 156.414 & 5266.68 & 0.04628 \\
\hline 3011 & 341.586 & 36.314 & 0.08015 & 155.185 & 5266.87 & 0.04634 \\
\hline 3012 & 340.959 & 36.316 & 0.08014 & 154.056 & 5267.23 & 0.04634 \\
\hline 3013 & 343.238 & 23.308 & 0.07515 & 158.158 & 3380.53 & 0.04345 \\
\hline 3014 & 342.438 & 23.311 & 0.07522 & 156.718 & 3380.99 & 0.04349 \\
\hline
\end{tabular}


Table 75. Transient Hot-Wire Thermal Conductivity Data for HCFC-123 (Continued)

\begin{tabular}{|c|c|c|c|c|c|c|}
\hline $\begin{array}{l}\text { Point } \\
\text { Number }\end{array}$ & $\mathrm{T}_{\text {Exp. }}$ & $\begin{array}{l}\mathrm{P}_{\mathrm{Cell}} \\
(\mathrm{MPa})\end{array}$ & $\begin{array}{c}\lambda_{\text {Exp. }} \\
(\mathrm{W} / \mathrm{m}-\mathrm{K})\end{array}$ & $\mathrm{T}_{\text {Exp. }}$ & $\begin{array}{l}\mathrm{P}_{\text {Cell }} \\
\text { (psia) }\end{array}$ & $\begin{array}{c}\lambda_{\text {Exp. }} \\
\text { (BTU/ft-hr-F) }\end{array}$ \\
\hline 3016 & 341.038 & 23.313 & 0.07557 & 154.198 & 3381.27 & 0.04369 \\
\hline 3017 & 343.460 & 12.875 & 0.07074 & 158.558 & 1867.37 & 0.04090 \\
\hline 3018 & 342.611 & 12.876 & 0.07086 & 157.030 & 1867.46 & 0.04097 \\
\hline 3019 & 341.830 & 12.870 & 0.07109 & 155.624 & 1866.71 & 0.04110 \\
\hline 3020 & 341.138 & 12.873 & 0.07116 & 154.378 & 1867.09 & 0.04114 \\
\hline 3021 & 342.811 & 5.010 & 0.06507 & 157.390 & 726.64 & 0.03762 \\
\hline 3022 & 341.960 & 5.011 & 0.06735 & 155.858 & 726.83 & 0.03894 \\
\hline 3023 & 341.257 & 5.012 & 0.06768 & 154.593 & 726.92 & 0.03913 \\
\hline 3024 & 340.615 & 5.014 & 0.06765 & 153.437 & 727.21 & 0.03911 \\
\hline 25001 & 342.899 & 0.355 & 0.01219 & 157.548 & 51.56 & 0.00705 \\
\hline 25002 & 342.519 & 0.355 & 0.01222 & 156.864 & 51.55 & 0.00707 \\
\hline 25004 & 342.133 & 0.355 & 0.01217 & 156.169 & 51.55 & 0.00704 \\
\hline 25004 & 341.800 & 0.355 & 0.01217 & 155.570 & 51.55 & 0.00704 \\
\hline 25005 & 342.942 & 0.306 & 0.01262 & 157.626 & 44.36 & 0.00730 \\
\hline 25006 & 342.527 & 0.306 & 0.01262 & 156.879 & 44.34 & 0.00730 \\
\hline 25007 & 342.136 & 0.306 & 0.01265 & 156.175 & 44.31 & 0.00731 \\
\hline 25008 & 341.815 & 0.305 & 0.01258 & 155.597 & 44.28 & 0.00727 \\
\hline 25009 & 342.998 & 0.259 & 0.01264 & 157.726 & 37.61 & 0.00731 \\
\hline 25010 & 342.592 & 0.259 & 0.01266 & 156.996 & 37.57 & 0.00732 \\
\hline 25011 & 342.195 & 0.259 & 0.01268 & 156.281 & 37.53 & 0.00733 \\
\hline 25012 & 341.836 & 0.258 & 0.01260 & 155.635 & 37.49 & 0.00729 \\
\hline 25013 & 343.044 & 0.224 & 0.01279 & 157.809 & 32.47 & 0.00739 \\
\hline 25014 & 342.633 & 0.224 & 0.01287 & 157.069 & 32.47 & 0.00744 \\
\hline 25015 & 342.242 & 0.224 & 0.01276 & 156.366 & 32.47 & 0.00738 \\
\hline 25016 & 341.859 & 0.224 & 0.01254 & 155.676 & 32.42 & 0.00725 \\
\hline 25017 & 343.106 & 0.177 & 0.01293 & 157.921 & 25.68 & 0.00748 \\
\hline 25018 & 342.678 & 0.177 & 0.01298 & 157.150 & 25.63 & 0.00750 \\
\hline 25019 & 342.277 & 0.176 & 0.01289 & 156.429 & 2.5 .56 & 0.00745 \\
\hline 25020 & 341.899 & 0.176 & 0.01288 & 155.748 & 25.54 & 0.00745 \\
\hline 25022 & 343.223 & 0.135 & 0.01304 & 158.131 & 19.63 & 0.00754 \\
\hline 25022 & 342.776 & 0.135 & 0.01312 & 157.327 & 19.57 & 0.00759 \\
\hline 25023 & 342.370 & 0.134 & 0.01310 & 156.596 & 19.50 & 0.00757 \\
\hline 25024 & 341.979 & 0.134 & 0.01299 & 155.892 & 19.44 & 0.00751 \\
\hline 25025 & 343.287 & 0.101 & 0.01329 & 158.247 & 14.58 & 0.00768 \\
\hline 25026 & 342.840 & 0.100 & 0.01319 & 157.442 & 14.53 & 0.00763 \\
\hline 25027 & 342.431 & 0.100 & 0.01324 & 156.706 & 14.54 & 0.00766 \\
\hline 25028 & 342.036 & 0.100 & 0.01320 & 155.995 & 14.55 & 0.00763 \\
\hline 26001 & 353.222 & 0.456 & 0.01333 & 176.130 & 66.12 & 0.00771 \\
\hline 26002 & 352.815 & 0.456 & 0.01277 & 175.397 & 66.18 & 0.00738 \\
\hline 26003 & 352.487 & 0.456 & 0.01316 & 174.807 & 66.19 & 0.00761 \\
\hline 26004 & 352.165 & 0.456 & 0.01327 & 174.227 & 66.21 & 0.00767 \\
\hline 26005 & 353.241 & 0.425 & 0.01336 & 176.164 & 61.60 & 0.00772 \\
\hline 26006 & 352.859 & 0.425 & 0.01319 & 175.476 & 61.65 & 0.00763 \\
\hline 26007 & 352.491 & 0.425 & 0.01324 & 174.814 & 61.63 & 0.00766 \\
\hline 26008 & 352.166 & 0.425 & 0.01322 & 174.229 & 61.63 & 0.00764 \\
\hline 26009 & 353.297 & 0.372 & 0.01332 & 176.265 & 53.99 & 0.00770 \\
\hline 26010 & 352.881 & 0.372 & 0.01320 & 175.516 & 53.99 & 0.00763 \\
\hline 26011 & 352.524 & 0.372 & 0.01320 & 174.873 & 53.96 & 0.00763 \\
\hline 26012 & 352.169 & 0.372 & 0.01326 & 174.234 & 53.95 & 0.00767 \\
\hline
\end{tabular}


Table 75. Transient Hot-Wire Thermal Conductivity Data for HCFC-123 (Continued)

\begin{tabular}{|c|c|c|c|c|c|c|}
\hline $\begin{array}{l}\text { Point } \\
\text { Number }\end{array}$ & $\begin{array}{l}T_{\text {Exp. }} \\
(\mathrm{K})\end{array}$ & $\begin{array}{l}\mathrm{P}_{\mathrm{Cell}} \\
(\mathrm{MPa})\end{array}$ & $\underset{(W / m-K)}{\lambda_{\operatorname{Exp}}}$ & $\mathrm{T}_{\text {Exp. }}$ & $\begin{array}{l}\mathrm{P}_{\text {Cell }} \\
\text { (psia) }\end{array}$ & $\begin{array}{c}\lambda_{\text {Exp. }} \\
\text { (BTU/ft-hr-F }\end{array}$ \\
\hline 26013 & 353.383 & 0.316 & 0.01338 & 176.419 & 45.87 & 0.00774 \\
\hline 26014 & 352.978 & 0.316 & 0.01338 & 175.690 & 45.83 & 0.00774 \\
\hline 26015 & 352.592 & 0.316 & 0.01335 & 174.996 & 45.79 & 0.00772 \\
\hline 26016 & 352.236 & 0.316 & 0.01327 & 174.355 & 45.78 & 0.00767 \\
\hline 26017 & 353.432 & 0.261 & 0.01342 & 176.508 & 37.91 & 0.00776 \\
\hline 26018 & 353.023 & 0.261 & 0.01344 & 175.771 & 37.90 & 0.00777 \\
\hline 26019 & 352.616 & 0.261 & 0.01345 & 175.039 & 37.88 & 0.00778 \\
\hline 26020 & 352.239 & 0.261 & 0.01343 & 174.360 & 37.86 & 0.00776 \\
\hline 26021 & 353.470 & 0.219 & 0.01358 & 176.576 & 31.75 & 0.00785 \\
\hline 26022 & 353.053 & 0.218 & 0.01356 & 175.825 & 31.68 & 0.00784 \\
\hline 26024 & 352.655 & 0.218 & 0.01350 & 175.109 & 31.63 & 0.00781 \\
\hline 26024 & 352.309 & 0.218 & 0.01353 & 174.486 & 31.60 & 0.00782 \\
\hline 26025 & 353.542 & 0.173 & 0.01371 & 176.706 & 25.12 & 0.00793 \\
\hline 26026 & 353.128 & 0.173 & 0.01367 & 175.960 & 25.02 & 0.00790 \\
\hline 26027 & 352.726 & 0.172 & 0.01369 & 175.237 & 24.97 & 0.00792 \\
\hline 26027 & 352.362 & 0.172 & 0.01360 & 174.582 & 24.90 & 0.00786 \\
\hline 26029 & 353.637 & 0.133 & 0.01390 & 176.877 & 19.32 & 0.00804 \\
\hline 26030 & 353.174 & 0.131 & 0.01397 & 176.043 & 18.93 & 0.00808 \\
\hline 26031 & 352.773 & 0.130 & 0.01383 & 175.321 & 18.90 & 0.00800 \\
\hline 26032 & 352.400 & 0.130 & 0.01389 & 174.650 & 18.87 & 0.00803 \\
\hline 26033 & 353.710 & 0.101 & 0.01402 & 177.008 & 14.59 & 0.00811 \\
\hline 26034 & 353.241 & 0.101 & 0.01403 & 176.164 & 14.60 & 0.00811 \\
\hline 26035 & 352.839 & 0.101 & 0.01397 & 175.440 & 14.61 & 0.00808 \\
\hline 26036 & 352.469 & 0.101 & 0.01410 & 174.774 & 14.66 & 0.00815 \\
\hline 4001 & 363.200 & 66.074 & 0.08557 & 194.090 & 9583.26 & 0.04947 \\
\hline 4002 & 362.398 & 66.063 & 0.08607 & 192.646 & 9581.62 & 0.04976 \\
\hline 4003 & 361.721 & 66.058 & 0.08640 & 191.428 & 9580.89 & 0.04995 \\
\hline 4004 & 361.073 & 66.053 & 0.08620 & 190.261 & 9580.26 & 0.04984 \\
\hline 4005 & 363.172 & 51.570 & 0.08142 & 194.040 & 7479.58 & 0.04708 \\
\hline 4006 & 362.372 & 51.570 & 0.08163 & 192.600 & 7479.67 & 0.04720 \\
\hline 4007 & 361.654 & 51.565 & 0.08183 & 191.307 & 7478.95 & 0.04731 \\
\hline 4008 & 360.974 & 51.565 & 0.08184 & 190.083 & 7478.95 & 0.04732 \\
\hline 4009 & 363.334 & 38.124 & 0.07675 & 194.331 & 5529.43 & 0.04438 \\
\hline 4010 & 362.482 & 38.125 & 0.07676 & 192.798 & 5529.52 & 0.04438 \\
\hline 4011 & 361.721 & 38.124 & 0.07715 & 191.428 & 5529.43 & 0.04461 \\
\hline 4012 & 361.034 & 38.123 & 0.07745 & 190.191 & 5529.25 & 0.04478 \\
\hline 4013 & 363.521 & 26.603 & 0.07237 & 194.668 & 3858.43 & 0.04184 \\
\hline 4014 & 362.630 & 26.603 & 0.07264 & 193.064 & 3858.43 & 0.04200 \\
\hline 4015 & 361.838 & 26.603 & 0.07283 & 191.638 & 3858.52 & 0.04211 \\
\hline 4016 & 361.109 & 26.605 & 0.07310 & 190.326 & 3858.71 & 0.04226 \\
\hline 4017 & 362.925 & 16.858 & 0.06844 & 193.595 & 2445.07 & 0.03957 \\
\hline 4018 & 362.067 & 16.857 & 0.06846 & 192.051 & 2444.97 & 0.03958 \\
\hline 4019 & 361.316 & 16.859 & 0.06879 & 190.699 & 2445.25 & 0.03977 \\
\hline 4020 & 360.645 & 16.861 & 0.06881 & 189.491 & 2445.53 & 0.03978 \\
\hline 4021 & 363.081 & 9.276 & 0.06480 & 193.876 & 1345.44 & 0.03747 \\
\hline 4022 & 362.207 & 9.278 & 0.06500 & 192.303 & 1345.72 & 0.03758 \\
\hline 4023 & 361.423 & 9.278 & 0.06521 & 190.891 & 1345.72 & 0.03770 \\
\hline 4024 & 360.708 & 9.278 & 0.06537 & 189.604 & 1345.72 & 0.03780 \\
\hline 4025 & 363.250 & 3.236 & 0.06149 & 194.180 & 469.40 & 0.03555 \\
\hline
\end{tabular}


Table 75. Transient Hot-Wire Thermal Conductivity Data for HCFC-123 (Continued)

\begin{tabular}{|c|c|c|c|c|c|c|}
\hline $\begin{array}{l}\text { Point } \\
\text { Number }\end{array}$ & $\begin{array}{l}\mathrm{T}_{\text {Exp. }} \\
(\mathrm{K})\end{array}$ & $\begin{array}{l}\mathrm{P}_{\mathrm{Cell}} \\
(\mathrm{MPa})\end{array}$ & $\begin{array}{c}\lambda_{\operatorname{Exp}} \\
(W / m-K)\end{array}$ & $\mathrm{T}_{\text {Exp. }}$ & $\begin{array}{r}P_{\text {Cell }} \\
\text { (psia) }\end{array}$ & $\begin{array}{c}\lambda_{\text {Exp. }} \\
\text { (BTU/ft-hr-F) }\end{array}$ \\
\hline 4026 & 362.325 & 3.236 & 0.06163 & 192.515 & 469.31 & 0.03563 \\
\hline 4027 & 361.508 & 3.237 & 0.06186 & 191.044 & 469.49 & 0.03577 \\
\hline 4028 & 360.774 & 3.238 & 0.06193 & 189.723 & 469.68 & 0.03581 \\
\hline 27001 & 362.516 & 0.488 & 0.01385 & 192.859 & 70.81 & 0.00801 \\
\hline 27002 & 362.105 & 0.489 & 0.01397 & 192.119 & 70.87 & 0.00808 \\
\hline 27003 & 361.762 & 0.489 & 0.01390 & 191.502 & 70.93 & 0.00804 \\
\hline 27004 & 361.418 & 0.489 & 0.01371 & 190.882 & 70.95 & 0.00793 \\
\hline 27005 & 362.572 & 0.421 & 0.01389 & 192.960 & 61.04 & 0.00803 \\
\hline 27006 & 362.179 & 0.421 & 0.01375 & 192.252 & 61.03 & 0.00795 \\
\hline 27007 & 361.825 & 0.421 & 0.01383 & 191.615 & 61.02 & 0.00800 \\
\hline 27008 & 361.500 & 0.420 & 0.01382 & 191.030 & 60.97 & 0.00799 \\
\hline 27009 & 362.668 & 0.317 & 0.01404 & 193.132 & 46.03 & 0.00812 \\
\hline 27010 & 362.259 & 0.317 & 0.01402 & 192.396 & 45.92 & 0.00811 \\
\hline 27011 & 361.898 & 0.316 & 0.01404 & 191.746 & 45.88 & 0.00812 \\
\hline 27012 & 361.568 & 0.316 & 0.01402 & 191.152 & 45.85 & 0.00811 \\
\hline 27013 & 362.750 & 0.248 & 0.01409 & 193.280 & 35.95 & 0.00815 \\
\hline 27014 & 362.360 & 0.248 & 0.01423 & 192.578 & 35.90 & 0.00823 \\
\hline 27015 & 361.966 & 0.247 & 0.01418 & 191.869 & 35.84 & 0.00820 \\
\hline 27016 & 361.624 & 0.247 & 0.01401 & 191.253 & 35.79 & 0.00810 \\
\hline 27017 & 362.885 & 0.171 & 0.01438 & 193.523 & 24.77 & 0.00831 \\
\hline 27018 & 362.425 & 0.170 & 0.01446 & 192.695 & 24.71 & 0.00836 \\
\hline 27019 & 362.034 & 0.170 & 0.01444 & 191.991 & 24.63 & 0.00835 \\
\hline 27020 & 361.684 & 0.169 & 0.01434 & 191.361 & 24.53 & 0.00829 \\
\hline 27021 & 362.987 & 0.122 & 0.01468 & 193.707 & 17.72 & 0.00849 \\
\hline 27022 & 362.518 & 0.122 & 0.01454 & 192.862 & 17.71 & 0.00841 \\
\hline 27023 & 362.104 & 0.122 & 0.01462 & 192.117 & 17.70 & 0.00845 \\
\hline 27024 & 361.736 & 0.122 & 0.01452 & 191.455 & 17.72 & 0.00840 \\
\hline 28001 & 373.082 & 0.750 & 0.01391 & 211.878 & 108.73 & 0.00804 \\
\hline 28002 & 372.726 & 0.750 & 0.01391 & 211.237 & 108.80 & 0.00804 \\
\hline 28003 & 372.405 & 0.750 & 0.01386 & 210.659 & 108.80 & 0.00801 \\
\hline 28004 & 372.087 & 0.750 & 0.01397 & 210.087 & 108.84 & 0.00808 \\
\hline 28005 & 373.155 & 0.641 & 0.01459 & 212.009 & 92.98 & 0.00844 \\
\hline 28006 & 372.772 & 0.64 .1 & 0.01461 & 211.320 & 93.02 & 0.00845 \\
\hline 28007 & 372.451 & 0.641 & 0.01457 & 210.742 & 93.03 & 0.00842 \\
\hline 28008 & 372.130 & 0.641 & 0.01458 & 210.164 & 93.01 & 0.00843 \\
\hline 28009 & 373.241 & 0.548 & 0.01459 & 212.164 & 79.42 & 0.00844 \\
\hline 28010 & 372.860 & 0.547 & 0.01448 & 211.478 & 79.40 & 0.00837 \\
\hline 28011 & 372.494 & 0.547 & 0.01455 & 210.819 & 79.39 & 0.00841 \\
\hline 28012 & 372.198 & 0.547 & 0.01456 & 210.286 & 79.38 & 0.00842 \\
\hline 28013 & 373.331 & 0.440 & 0.01466 & 212.326 & 63.84 & 0.00848 \\
\hline 28014 & 372.957 & 0.440 & 0.01460 & 211.653 & 63.85 & 0.00844 \\
\hline 28015 & 372.586 & 0.440 & 0.01463 & 210.985 & 63.86 & 0.00846 \\
\hline 28016 & 372.297 & 0.440 & 0.01465 & 210.465 & 63.83 & 0.00847 \\
\hline 28017 & 373.469 & 0.276 & 0.01489 & 212.574 & 40.06 & 0.00861 \\
\hline 28018 & 373.055 & 0.276 & 0.01494 & 211.829 & 40.03 & 0.00864 \\
\hline 28019 & 372.685 & 0.276 & 0.01479 & 211.163 & 40.01 & 0.00855 \\
\hline 28020 & 372.333 & 0.275 & 0.01488 & 210.529 & 39.94 & 0.00860 \\
\hline 28021 & 373.657 & 0.146 & 0.01528 & 212.913 & 21.17 & 0.00883 \\
\hline 28022 & 373.236 & 0.146 & 0.01541 & 212.155 & 21.12 & 0.00891 \\
\hline
\end{tabular}


Table 75. Transient Hot-Wire Thermal Conductivity Data for HCFC-123 (Continued)

\begin{tabular}{|c|c|c|c|c|c|c|}
\hline $\begin{array}{l}\text { Point } \\
\text { Number }\end{array}$ & $\begin{array}{l}\mathrm{T}_{\text {Exp. }} \\
(\mathrm{K})\end{array}$ & $\begin{array}{l}\mathrm{P}_{\mathrm{Cell}} \\
(\mathrm{MPa})\end{array}$ & $\begin{array}{c}\lambda_{\operatorname{Exp}} \\
(W / m-K)\end{array}$ & $\mathrm{T}_{\text {Exp. }}$ & $\begin{array}{l}\mathrm{P}_{\text {Cell }} \\
\text { (psia) }\end{array}$ & $\begin{array}{c}\lambda_{\text {Exp. }} \\
\text { (BTU/ft-hr-F) }\end{array}$ \\
\hline 28023 & 372.851 & 0.145 & 0.01536 & 211.462 & 20.99 & 0.00888 \\
\hline 28024 & 372.476 & 0.145 & 0.01526 & 210.787 & 20.97 & 0.00882 \\
\hline 5001 & 382.556 & 67.276 & 0.08326 & 228.931 & 9757.58 & 0.04814 \\
\hline 5002 & 381.708 & 67.273 & 0.08345 & 227.404 & 9757.12 & 0.04825 \\
\hline 5003 & 380.947 & 67.268 & 0.08360 & 226.035 & 9756.49 & 0.04834 \\
\hline 5004 & 380.263 & 67.242 & 0.08384 & 224.803 & 9752.67 & 0.04847 \\
\hline 5005 & 382.691 & 52.358 & 0.07864 & 229.174 & 7593.84 & 0.04547 \\
\hline 5006 & 381.803 & 52.355 & 0.07867 & 227.575 & 7593.48 & 0.04549 \\
\hline 5007 & 381.011 & 52.355 & 0.07898 & 226.150 & 7593.48 & 0.04566 \\
\hline 5008 & 380.283 & 52.356 & 0.07872 & $224.8^{79}$ & 7593.57 & 0.04551 \\
\hline 5009 & 382.009 & 37.199 & 0.07323 & 227.946 & 5395.31 & 0.04234 \\
\hline 5010 & 381.153 & 37.201 & 0.07359 & 226.405 & 5395.59 & 0.04255 \\
\hline 5011 & 380.435 & 37.202 & 0.07062 & 225.113 & 5395.68 & 0.04083 \\
\hline 5012 & 379.727 & 37.203 & 0.07387 & 223.839 & 5395.86 & 0.04271 \\
\hline 5013 & 352.029 & 37.209 & 0.07329 & 227.982 & 5396.78 & 0.04237 \\
\hline 5014 & 381.173 & 37.211 & 0.07343 & 226.441 & 5397.05 & 0.04246 \\
\hline 5015 & 380.424 & 37.207 & 0.07367 & 225.093 & 5396.50 & 0.04259 \\
\hline 5016 & 379.769 & 37.212 & 0.07392 & 223.914 & 5397.14 & 0.04274 \\
\hline 5017 & 382.211 & 28.571 & 0.06995 & 228.310 & 4143.94 & 0.04044 \\
\hline 5018 & 381.317 & 28.566 & 0.07024 & 226.701 & 4143.20 & 0.04061 \\
\hline 5019 & 380.537 & 28.566 & 0.07015 & 225.297 & 4143.20 & 0.04056 \\
\hline 5020 & 379.845 & 28.562 & 0.07056 & 224.051 & 4142.55 & 0.04080 \\
\hline 5021 & 382.395 & 20.339 & 0.06645 & 228.641 & 2949.99 & 0.03842 \\
\hline 5022 & 381.500 & 20.352 & 0.06654 & 227.030 & 2951.75 & $0.0384^{\prime}$ \\
\hline 5023 & 380.662 & 20.356 & 0.06669 & 225.522 & 2952.40 & 0.03856 \\
\hline 5024 & 379.951 & 20.355 & 0.06701 & 224.242 & 2952.31 & 0.03874 \\
\hline 5025 & 382.598 & 13.320 & 0.06307 & 229.006 & 1931.97 & 0.03647 \\
\hline 5026 & 381.637 & 13.319 & 0.06319 & 227.277 & 1931.78 & 0.03654 \\
\hline 5027 & 380.792 & 13.318 & 0.06332 & 225.756 & 1931.60 & 0.03661 \\
\hline 5028 & 380.063 & 13.319 & 0.06343 & 224.443 & 1931.69 & 0.03667 \\
\hline 5029 & 381.940 & 6.690 & 0.05950 & 227.822 & 970.25 & 0.03440 \\
\hline 5030 & 381.021 & 6.690 & 0.05972 & 226.168 & 970.25 & 0.03453 \\
\hline 5031 & 380.229 & 6.692 & 0.05997 & 224.742 & 970.53 & 0.03467 \\
\hline 5032 & 379.523 & 6.692 & 0.06008 & 223.471 & 970.62 & 0.03474 \\
\hline 5033 & 382.068 & 2.760 & 0.05686 & 228.052 & 400.37 & 0.03288 \\
\hline 5034 & 381.124 & 2.760 & 0.05718 & 226.353 & 400.28 & 0.03306 \\
\hline 5035 & 380.277 & 2.760 & 0.05724 & 224.829 & 400.28 & 0.03310 \\
\hline 5036 & 379.588 & 2.760 & 0.05747 & 223.588 & 400.37 & 0.03323 \\
\hline 29001 & 382.543 & 0.913 & 0.01425 & 228.907 & 132.48 & 0.00824 \\
\hline 29002 & 382.216 & 0.914 & 0.01405 & 228.319 & 132.51 & 0.00812 \\
\hline 29003 & 381.900 & 0.914 & 0.01374 & 227.750 & 132.51 & 0.00794 \\
\hline 29004 & 381.581 & 0.914 & 0.01333 & 227.176 & 132.53 & 0.00771 \\
\hline 29005 & 382.638 & 0.835 & 0.01529 & 229.078 & 121.07 & 0.00884 \\
\hline 29006 & 382.277 & 0.835 & 0.01525 & 228.429 & 121.11 & 0.00882 \\
\hline 29007 & 381.947 & 0.835 & 0.01518 & 227.835 & 121.04 & 0.00878 \\
\hline 29008 & 381.628 & 0.835 & 0.01527 & 227.260 & 121.06 & 0.00883 \\
\hline 29009 & 382.730 & 0.718 & 0.01522 & 229.244 & 104.10 & 0.00880 \\
\hline 29010 & 382.355 & 0.718 & 0.01528 & 228.569 & 104.15 & 0.00883 \\
\hline 29011 & 382.042 & 0.718 & 0.01508 & 228.006 & 104.12 & 0.00872 \\
\hline
\end{tabular}


Table 75. Transient Hot-Wire Thermal Conductivity Data for HCFC-123 (Continued)

\begin{tabular}{|c|c|c|c|c|c|c|}
\hline $\begin{array}{l}\text { Point } \\
\text { Number }\end{array}$ & $\begin{array}{l}\mathrm{T}_{\text {Exp. }} \\
(\mathrm{K})\end{array}$ & $\begin{array}{l}\mathrm{P}_{\mathrm{Cell}} \\
(\mathrm{MPa})\end{array}$ & $\begin{array}{c}\lambda_{\operatorname{Exp}_{.}} \\
(W / m-K)\end{array}$ & $\mathrm{T}_{\mathrm{Exp}}$ & $\begin{array}{l}\mathrm{P}_{\text {Cell }} \\
\text { (psia) }\end{array}$ & $\begin{array}{c}\lambda_{\text {Exp. }} \\
\text { (BTU/ft-hr-F) }\end{array}$ \\
\hline 29012 & 381.718 & 0.718 & 0.01504 & 227.422 & 104.13 & 0.00870 \\
\hline 29013 & 382.817 & 0.618 & 0.01520 & 229.401 & 89.65 & 0.00879 \\
\hline 29014 & 382.436 & 0.618 & 0.01515 & 228.715 & 89.66 & 0.00876 \\
\hline 29015 & 382.076 & 0.618 & 0.01509 & 228.067 & 89.66 & 0.00872 \\
\hline 29016 & 381.734 & 0.618 & 0.01519 & 227.451 & 89.64 & 0.00878 \\
\hline 29017 & 382.876 & 0.519 & 0.01528 & 229.507 & 75.32 & 0.00883 \\
\hline 29018 & 382.536 & 0.519 & 0.01524 & 228.895 & 75.32 & 0.00881 \\
\hline 29019 & 382.154 & 0.519 & 0.01514 & 228.2 .07 & 75.26 & 0.00875 \\
\hline 29020 & 381.827 & 0.519 & 0.01514 & 227.619 & 75.29 & 0.00875 \\
\hline 29021 & 383.016 & 0.397 & 0.01539 & 229.759 & 57.56 & 0.00890 \\
\hline 29022 & 382.630 & 0.397 & 0.01529 & 229.064 & 57.54 & 0.00884 \\
\hline 29033 & 382.266 & 0.397 & 0.01536 & 228.409 & 57.53 & 0.00888 \\
\hline 29024 & 381.890 & 0.397 & 0.01523 & 227.732 & 57.52 & 0.00881 \\
\hline 29025 & 382.695 & 0.312 & 0.01545 & 229.181 & 45.31 & 0.00893 \\
\hline 29026 & 382.303 & 0.312 & 0.01532 & 228.475 & 45.27 & 0.00886 \\
\hline 29027 & 381.932 & 0.312 & 0.01547 & 227.808 & 45.24 & 0.00894 \\
\hline 29028 & 381.633 & 0.311 & 0.01550 & 227.269 & 45.16 & 0.00896 \\
\hline 29029 & 382.819 & 0.193 & 0.01581 & 229.404 & 27.98 & 0.00914 \\
\hline 29030 & 382.423 & 0.193 & 0.01585 & 228.691 & 27.98 & 0.00916 \\
\hline 29031 & 382.049 & 0.192 & 0.01587 & 228.018 & 27.91 & 0.00918 \\
\hline 29032 & 381.623 & 0.192 & 0.01578 & 227.251 & 27.90 & 0.00912 \\
\hline 29033 & 383.014 & 0.106 & 0.01624 & 229.755 & 15.40 & 0.00939 \\
\hline 29034 & 382.605 & 0.106 & 0.01621 & 229.019 & 15.42 & 0.00937 \\
\hline 29035 & 382.196 & 0.106 & 0.01601 & 228.283 & 15.42 & 0.00926 \\
\hline 29036 & 381.796 & 0.106 & 0.01625 & 227.563 & 15.41 & 0.00940 \\
\hline 30001 & 392.949 & 1.1 .23 & 0.01541 & 247.638 & 162.82 & 0.00891 \\
\hline 30002 & 392.622 & 1.123 & 0.01530 & 247.050 & 162.85 & 0.00885 \\
\hline 30003 & 392.307 & 1.123 & 0.01508 & 246.483 & 162.89 & 0.00872 \\
\hline 30004 & 392.020 & 1.123 & 0.01491 & 245.966 & 162.86 & 0.00862 \\
\hline 30005 & 393.076 & 0.951 & 0.01593 & $247.86^{7}$ & 137.89 & 0.00921 \\
\hline 30006 & 392.716 & 0.951 & 0.01593 & 247.219 & 137.90 & 0.00921 \\
\hline 30007 & 392.388 & 0.951 & 0.01603 & 246.628 & 137.92 & 0.00927 \\
\hline 30008 & 392.117 & 0.951 & 0.01579 & 246.14 & 137.93 & 0.00913 \\
\hline 30009 & 393.109 & 0.869 & 0.01593 & $247.9: 26$ & 126.10 & 0.00921 \\
\hline 30010 & 392.791 & 0.869 & 0.01587 & 247.354 & 126.08 & 0.00918 \\
\hline 30011 & 392.429 & 0.869 & 0.01578 & 246.702 & 126.09 & 0.00912 \\
\hline 30012 & 392.116 & 0.869 & 0.01583 & 246.139 & 126.10 & 0.00915 \\
\hline 30013 & 393.207 & 0.735 & 0.01595 & 248.103 & 106.63 & 0.00922 \\
\hline 30014 & 392.848 & 0.735 & 0.01589 & 247.456 & 106.60 & 0.00919 \\
\hline 30015 & 392.489 & 0.735 & 0.01573 & 246.810 & 106.64 & 0.00909 \\
\hline 30016 & 392.173 & 0.735 & 0.01590 & 246.241 & 106.67 & 0.00919 \\
\hline 30017 & 393.300 & 0.616 & 0.01592 & 248.270 & 89.37 & 0.00920 \\
\hline 30018 & 392.934 & 0.616 & 0.01582 & 247.611 & 89.32 & 0.00915 \\
\hline 30019 & 392.558 & 0.616 & 0.01583 & 246.934 & 89.31 & 0.00915 \\
\hline 30020 & 392.248 & 0.616 & 0.01582 & 246.376 & 89.33 & 0.00915 \\
\hline 30021 & 393.423 & 0.494 & 0.01595 & 248.491 & 71.63 & 0.00922 \\
\hline 30022 & 393.035 & 0.494 & 0.01604 & 247.793 & 71.59 & 0.00927 \\
\hline 30023 & 392.651 & 0.494 & 0.01597 & 247.102 & 71.60 & 0.00923 \\
\hline 30024 & 392.354 & 0.493 & 0.01600 & 246.567 & 71.55 & 0.00925 \\
\hline
\end{tabular}


Table 75. Transient Hot-Wire Thermal Conductivity Data for HCFC-123 (Continued)

\begin{tabular}{|c|c|c|c|c|}
\hline $\begin{array}{l}\mathrm{T}_{\text {Exp. }} \\
(\mathrm{K})\end{array}$ & $\begin{array}{l}\mathrm{P}_{\text {Cell }} \\
(\mathrm{MPa})\end{array}$ & $\lambda_{(\mathrm{W} / \mathrm{Exp}}$ & $T_{\text {Exp. }}$ & $\begin{array}{l}P_{\text {Cell }} \\
\text { (psia) }\end{array}$ \\
\hline 393.097 & 0.390 & 0.01603 & 247.905 & 56.53 \\
\hline 392.738 & 0.389 & 0.01606 & 247.258 & 56.46 \\
\hline 392.379 & 0.389 & 0.01614 & 246.612 & 56.42 \\
\hline 392.066 & 0.388 & 0.01588 & 246.049 & 56.34 \\
\hline 393.229 & 0.291 & 0.0162 .7 & 248.142 & 42.20 \\
\hline 392.803 & 0.290 & 0.01622 & 247.375 & 42.08 \\
\hline 392.449 & 0.290 & 0.01637 & 246.738 & 42.01 \\
\hline 392.113 & 0.290 & 0.01638 & 246.133 & 42.00 \\
\hline 393.292 & 0.216 & 0.01658 & 248.256 & 31.27 \\
\hline 392.874 & 0.215 & 0.01653 & 247.503 & 31.21 \\
\hline 392.488 & 0.214 & 0.01649 & 246.808 & 31.08 \\
\hline 392.174 & 0.213 & 0.01644 & 246.243 & 30.95 \\
\hline 393.469 & 0.106 & 0.01700 & 248.574 & 15.43 \\
\hline 393.029 & 0.106 & 0.01701 & 247.782 & 15.41 \\
\hline 392.654 & 0.106 & 0.01709 & 247.107 & 15.37 \\
\hline 392.280 & 0.106 & 0.01704 & 246.434 & 15.43 \\
\hline 402.024 & 67.179 & 0.08114 & 263.973 & 9743.53 \\
\hline 401.157 & 67.166 & 0.08109 & 262.413 & 9741.71 \\
\hline 400.397 & 67.168 & 0.08100 & 261.045 & 9741.89 \\
\hline 399.718 & 67.168 & 0.08113 & 259.822 & 9741.98 \\
\hline 402.114 & 54.020 & 0.07647 & 264.135 & 7835.00 \\
\hline 401.229 & 54.022 & 0.07660 & 262.542 & 7835.19 \\
\hline 400.447 & 54.023 & 0.07663 & 261.135 & 7835.46 \\
\hline 399.751 & 54.023 & 0.07691 & 259.882 & 7835.37 \\
\hline $402.32 i$ & 41.889 & 0.07239 & 264.508 & 6075.53 \\
\hline $40 \mathrm{i} .411$ & 41.890 & 0.07242 & 262.870 & 6075.62 \\
\hline 400.570 & 41.892 & 0.07239 & 261.356 & 6075.90 \\
\hline 399.837 & 41.893 & 0.07258 & 260.037 & 6076.08 \\
\hline 401.618 & 31.198 & 0.06826 & 263.242 & 4524.90 \\
\hline 400.724 & 31.199 & 0.06858 & 261.633 & 4525.08 \\
\hline 399.945 & 31.198 & 0.06810 & 260.231 & 4524.90 \\
\hline$j 97.265$ & 31.199 & 0.06845 & 259.007 & 4525.08 \\
\hline 401.597 & 31.202 & 0.06849 & 263.205 & 4525.45 \\
\hline 400.719 & 31.206 & 0.06848 & 261.624 & 4526.00 \\
\hline 399.966 & 31.209 & 0.06822 & 260.269 & 4526.46 \\
\hline 399.254 & 31.211 & 0.06840 & 258.987 & 4526.83 \\
\hline 401.810 & 23.290 & 0.06468 & 263.588 & 3377.94 \\
\hline 400.880 & 23.293 & 0.06472 & 261.914 & 3378.32 \\
\hline 400.071 & 23.293 & 0.06480 & 260.458 & 3378.32 \\
\hline 399.342 & 23.291 & 0.06520 & 259.146 & 3378.13 \\
\hline 402.223 & 16.258 & 0.06110 & 264.331 & 2358.01 \\
\hline 402.196 & 16.253 & 0.06111 & 264.283 & 2357.27 \\
\hline 401.238 & 16.252 & 0.06131 & 262.558 & 2357.18 \\
\hline 400.374 & 16.252 & 0.06131 & 261.003 & 2357.18 \\
\hline 399.590 & 16.255 & 0.06158 & 259.592 & 2357.55 \\
\hline 402.368 & 10.752 & 0.05825 & 264.592 & 1559.49 \\
\hline $40 i .367$ & 10.752 & 0.05822 & 262.791 & 1559.49 \\
\hline 400.467 & 10.752 & 0.05843 & 261.171 & 1559.40 \\
\hline 399.667 & 10.754 & 0.05844 & 259.731 & 1559.67 \\
\hline
\end{tabular}


Table 75. Transient Hot-Wire Thermal Conductivity Data for HCFC-123 (Continued)

\begin{tabular}{|c|c|c|c|c|c|c|}
\hline $\begin{array}{l}\text { Point } \\
\text { Number }\end{array}$ & $\begin{array}{l}T_{\text {Exp. }} \\
\text { (K) }\end{array}$ & $\begin{array}{l}\mathrm{P}_{\mathrm{Cell}} \\
(\mathrm{MPa})\end{array}$ & $\begin{array}{c}\lambda_{\operatorname{Exp}_{\dot{ }}} \\
(\mathrm{W} / \mathrm{m}-\mathrm{K})\end{array}$ & $\mathrm{T}_{\text {Exp. }}$ & $\begin{array}{l}P_{\text {Cell }} \\
\text { (psia) }\end{array}$ & $\begin{array}{l}\lambda_{\text {Exp. }} \\
\text { (BTU/ft-hr-F) }\end{array}$ \\
\hline 6033 & 401.480 & 7.125 & 0.05573 & 262.994 & 1033.45 & 0.03222 \\
\hline 6034 & 400.561 & 7.125 & 0.05601 & 261.340 & 1033.45 & 0.03238 \\
\hline 6035 & 399.746 & 7.126 & 0.05618 & 259.873 & 1033.54 & 0.03248 \\
\hline 6036 & 399.032 & 7.127 & 0.05632 & 258.588 & 1033.64 & 0.03256 \\
\hline 6037 & 401.665 & 3.690 & 0.05322 & 263.327 & 535.19 & 0.03077 \\
\hline 6038 & 400.710 & 3.690 & 0.05354 & 261.608 & 535.19 & 0.03096 \\
\hline 6039 & 399.850 & 3.691 & 0.05360 & 260.060 & 535.28 & 0.03099 \\
\hline 6040 & 399.115 & 3.692 & 0.05394 & 258.737 & 535.47 & 0.03119 \\
\hline 6041 & 401.785 & 1.578 & 0.05170 & 263.543 & 228.85 . & 0.02989 \\
\hline 6042 & 400.792 & 1.579 & 0.05184 & 261.756 & 228.95 & 0.02997 \\
\hline 6043 & 399.887 & 1.577 & 0.05204 & 260.127 & 228.76 & 0.03009 \\
\hline 6044 & 399.150 & 1.578 & 0.05219 & 258.800 & 228.85 & 0.03018 \\
\hline 31001 & 403.215 & 1.334 & 0.01693 & 266.117 & 193.55 & 0.00979 \\
\hline 31002 & 402.906 & 1.335 & 0.01690 & 265.561 & 193.60 & 0.00977 \\
\hline 31003 & 402.607 & 1.335 & 0.01685 & 265.023 & 193.69 & 0.00974 \\
\hline 31004 & 402.317 & 1.336 & 0.01682 & 264.501 & 193.70 & 0.00972 \\
\hline 31005 & 403.284 & 1.215 & 0.01682 & 266.241 & 176.21 & 0.00972 \\
\hline 31006 & 402.969 & 1.215 & 0.01680 & 265.674 & 176.23 & 0.00971 \\
\hline 31007 & 402.683 & 1.215 & 0.01674 & 265.159 & 176.24 & 0.00968 \\
\hline 31008 & 402.387 & 1.215 & 0.01676 & 264.627 & 176.23 & 0.00969 \\
\hline 31009 & 403.382 & 1.079 & 0.01668 & 266.418 & 156.54 & 0.00964 \\
\hline 31010 & 403.050 & 1.079 & 0.01666 & 265.820 & 156.52 & 0.00963 \\
\hline 31011 & 402.790 & 1.079 & 0.01658 & 265.352 & 156.51 & 0.00959 \\
\hline 31012 & 402.475 & 1.079 & 0.01658 & 264.785 & 156.52 & 0.00959 \\
\hline 31013 & 403.547 & 0.881 & 0.01658 & 266.715 & 127.78 & 0.00959 \\
\hline 31014 & 403.186 & 0.881 & 0.01650 & 266.065 & 127.73 & 0.00954 \\
\hline 31015 & 402.841 & 0.880 & 0.01643 & 265.444 & 127.70 & 0.00950 \\
\hline 31016 & 402.551 & 0.880 & 0.01647 & 264.922 & 127.68 & 0.00952 \\
\hline 31017 & 403.638 & 0.753 & 0.01657 & 266.878 & 109.28 & 0.00958 \\
\hline 31018 & 403.288 & 0.753 & 0.01652 & 266.248 & 109.22 & 0.00955 \\
\hline 31019 & 402.945 & 0.753 & 0.01649 & 265.631 & 109.20 & 0.00953 \\
\hline 31020 & 402.615 & 0.753 & 0.01645 & 265.037 & 109.15 & 0.00951 \\
\hline 31021 & 403.323 & 0.638 & 0.01663 & 266.311 & 92.48 & 0.00962 \\
\hline 31022 & 402.993 & 0.637 & 0.01655 & 265.717 & 92.43 & 0.00957 \\
\hline 31023 & 402.656 & 0.637 & 0.01654 & 265.111 & 92.44 & 0.00956 \\
\hline 31024 & 402.380 & 0.637 & 0.01652 & 264.614 & 92.43 & 0.00955 \\
\hline 31025 & 403.420 & 0.502 & 0.01662 & 266.486 & 72.87 & 0.00961 \\
\hline 31026 & 403.090 & 0.502 & 0.01665 & 265.892 & 72.79 & 0.00963 \\
\hline 31027 & 402.745 & 0.501 & 0.01666 & 265.271 & 72.71 & 0.00963 \\
\hline 31028 & 402.437 & 0.501 & 0.01662 & 264.717 & 72.68 & 0.00961 \\
\hline 31029 & 403.517 & 0.385 & 0.01684 & 266.661 & 55.81 & 0.00974 \\
\hline 31030 & 403.163 & 0.385 & 0.01686 & 266.023 & 55.77 & 0.00975 \\
\hline 31031 & 402.829 & 0.384 & 0.01698 & 265.422 & 55.75 & 0.00982 \\
\hline 31032 & 402.493 & 0.384 & 0.01677 & 264.817 & 55.72 & 0.00970 \\
\hline 31033 & 403.611 & 0.273 & 0.01716 & 266.830 & 39.55 & 0.00992 \\
\hline 31034 & 403.256 & 0.272 & 0.01716 & 266.191 & 39.50 & 0.00992 \\
\hline 31035 & 402.933 & 0.273 & 0.01724 & 265.609 & 39.54 & 0.00997 \\
\hline 31036 & 402.559 & 0.272 & 0.01711 & 264.936 & 39.44 & 0.00989 \\
\hline 31037 & 403.839 & 0.132 & 0.01789 & 257.240 & 19.11 & 0.01034 \\
\hline
\end{tabular}


Table 75. Transient Hot-Wire Thermal Conductivity Data for HCFC-123 (Continued)

\begin{tabular}{|c|c|c|c|c|c|c|}
\hline $\begin{array}{l}\text { Point } \\
\text { Number }\end{array}$ & $\begin{array}{l}\mathrm{T}_{\text {Exp. }} \\
(\mathrm{K})\end{array}$ & $\begin{array}{l}\mathrm{P}_{\text {Cell }} \\
(\mathrm{MPa})\end{array}$ & $\begin{array}{c}\lambda_{\operatorname{Exp}} \\
(W / m-K)\end{array}$ & $\mathrm{T}_{\text {Exp. }}$ & $\begin{array}{l}\mathrm{P}_{\text {Cell }} \\
\text { (psia) }\end{array}$ & $\frac{\lambda_{\text {Exp. }}}{(B T U / f t-h r-F)}$ \\
\hline 31038 & 403.433 & 0.131 & 0.01787 & 266.509 & 19.03 & 0.01033 \\
\hline 31039 & 403.060 & 0.131 & 0.01784 & 265.838 & 18.97 & 0.01031 \\
\hline 31040 & 402.717 & 0.130 & 0.01791 & 265.221 & 18.91 & 0.01036 \\
\hline 32001 & 411.953 & 1.585 & 0.01820 & 281.845 & 229.87 & 0.01052 \\
\hline 32002 & 411.702 & 1.584 & 0.01839 & 281.394 & 229.70 & 0.01063 \\
\hline 32003 & 411.472 & 1.583 & 0.01811 & 280.980 & 229.63 & 0.01047 \\
\hline 32004 & 411.242 & 1.583 & 0.01807 & 280.566 & 229.60 & 0.01045 \\
\hline 32005 & 412.078 & 1.451 & 0.01818 & 282.070 & 210.46 & 0.01051 \\
\hline 32006 & 411.796 & 1.451 & 0.01796 & 281.563 & 210.45 & 0.01038 \\
\hline 32007 & 411.560 & 1.451 & 0.01799 & 281.138 & 210.46 & 0.01040 \\
\hline 32008 & 411.314 & 1.451 & 0.01783 & 280.695 & 210.46 & 0.01031 \\
\hline 32000 & 412.174 & 1.299 & 0.01784 & 282.243 & 188.38 & 0.01031 \\
\hline 32009 & 412.185 & 1.299 & 0.01763 & 282.263 & 188.35 & 0.01019 \\
\hline 32010 & 411.918 & 1.299 & 0.01745 & 281.782 & 188.38 & 0.01009 \\
\hline 32011 & 411.643 & 1.299 & 0.01776 & 281.287 & 188.38 & 0.01027 \\
\hline 32012 & 411.400 & 1.299 & 0.01750 & 280.850 & 188.38 & 0.01012 \\
\hline 32013 & 412.306 & 1.150 & 0.01745 & 282.481 & 166.75 & 0.01010 \\
\hline 32014 & 411.992 & 1.150 & 0.01748 & 281.916 & 166.79 & 0.01011 \\
\hline 32015 & 411.718 & 1.150 & 0.01746 & 281.422 & 166.79 & 0.01010 \\
\hline 32016 & 411.470 & 1.150 & 0.01731 & 280.976 & 166.79 & 0.01001 \\
\hline 32017 & 412.413 & 0.997 & 0.01735 & 282.673 & 144.61 & 0.01003 \\
\hline 32018 & 412.109 & 0.998 & 0.01745 & 282.126 & 144.75 & 0.01009 \\
\hline 32019 & 411.815 & 0.999 & 0.01733 & 281.597 & 144.93 & 0.01002 \\
\hline 32020 & 411.556 & 1.000 & 0.01729 & 281.131 & 145.00 & 0.01000 \\
\hline 32021 & 412.508 & 0.823 & 0.01729 & 282.844 & 119.30 & 0.01000 \\
\hline 32022 & 412.194 & 0.822 & 0.01727 & 282.279 & 119.23 & 0.00999 \\
\hline 32023 & 411.901 & 0.822 & 0.01724 & 281.752 & 119.16 & 0.00997 \\
\hline 32024 & 411.600 & 0.821 & 0.01722 & 281.210 & 119.14 & 0.00996 \\
\hline 32025 & 412.592 & 0.649 & 0.01731 & 282.996 & 94.17 & 0.01001 \\
\hline 32026 & 412.278 & 0.649 & 0.01736 & 282.430 & 94.11 & 0.01004 \\
\hline 32027 & 411.978 & 0.648 & 0.01744 & 281.890 & 94.03 & 0.01008 \\
\hline 32028 & 411.688 & 0.648 & 0.01731 & 281.368 & 94.01 & 0.01001 \\
\hline 32029 & 412.721 & 0.497 & 0.01735 & 283.228 & 72.13 & 0.01003 \\
\hline 32030 & 412.402 & 0.496 & 0.01754 & 282.654 & 72.01 & 0.01014 \\
\hline 32031 & 412.063 & 0.496 & 0.01745 & 282.043 & 71.94 & 0.01009 \\
\hline 32032 & 411.786 & 0.495 & 0.01737 & 281.545 & 71.84 & 0.01004 \\
\hline 32033 & 412.836 & 0.371 & 0.01765 & 283.435 & 53.80 & 0.01020 \\
\hline 32034 & 412.495 & 0.370 & 0.01773 & 282.821 & 53.70 & 0.01025 \\
\hline 32035 & 412.165 & 0.370 & 0.01768 & 282.227 & 53.65 & 0.01022 \\
\hline 32036 & 411.847 & 0.370 & 0.01762 & 281.655 & 53.69 & 0.01019 \\
\hline 32037 & 412.997 & 0.221 & 0.01811 & 283.725 & 32.08 & 0.01047 \\
\hline 32038 & 412.631 & 0.221 & 0.01815 & 283.066 & 32.00 & 0.01049 \\
\hline 32039 & 412.262 & 0.220 & 0.01821 & 282.402 & 31.91 & 0.01053 \\
\hline 32040 & 411.954 & 0.220 & 0.01826 & 281.847 & 31.96 & 0.01056 \\
\hline 32041 & 413.197 & 0.119 & 0.01879 & 284.085 & 17.29 & 0.01086 \\
\hline 32042 & 412.805 & 0.121 & 0.01854 & 283.379 & 17.50 & 0.01072 \\
\hline 32043 & 412.439 & 0.124 & 0.01880 & 282.720 & 17.94 & 0.01087 \\
\hline 32044 & 412.073 & 0.125 & 0.01875 & 282.061 & 18.08 & 0.01084 \\
\hline 42001 & 423.161 & 69.189 & 0.08065 & 302.020 & 10035.06 & 0.04663 \\
\hline
\end{tabular}


Table 75. Transient Hot-Wire Thermal Conductivity Data for HCFC-123 (Continued)

\begin{tabular}{|c|c|c|c|c|c|c|}
\hline $\begin{array}{l}\text { Point } \\
\text { Number }\end{array}$ & $\begin{array}{l}T_{\text {Exp. }} \\
(K)\end{array}$ & $\begin{array}{l}\mathrm{P}_{\mathrm{Cell}} \\
(\mathrm{MPa})\end{array}$ & $\begin{array}{c}\lambda_{\operatorname{Exp}_{.}} \\
(W / m-K)\end{array}$ & $\mathrm{T}_{\text {Exp. }}$ & $\begin{array}{l}{ }_{\text {(psia) }} \\
{ }_{\text {Cell }}\end{array}$ & $\begin{array}{c}\lambda_{\text {Exp. }} \\
(B T U / f t-h r-F)\end{array}$ \\
\hline 42002 & 422.751 & 69.186 & 0.08066 & 301.282 & 10034.56 & 0.04664 \\
\hline 42003 & 422.373 & 69.186 & 0.08070 & 300.601 & 10034.56 & 0.04666 \\
\hline 42004 & 422.030 & 69.179 & 0.08064 & 299.984 & 10033.59 & 0.04662 \\
\hline 42005 & 423.072 & 55.832 & 0.07572 & 301.860 & 8097.77 & 0.04378 \\
\hline 42006 & 422.638 & 55.835 & 0.07570 & 301.078 & 8098.17 & 0.04377 \\
\hline 42007 & 422.248 & 55.838 & 0.07555 & 300.376 & 8098.61 & 0.04368 \\
\hline 42008 & 421.892 & 55.842 & 0.07584 & 299.736 & 8099.25 & 0.04385 \\
\hline 42009 & 423.046 & 45.258 & 0.07187 & 301.813 & 6564.09 & 0.04155 \\
\hline 42010 & 422.601 & 45.262 & 0.07169 & 301.012 & 6564.79 & 0.04145 \\
\hline 42011 & 422.191 & 45.269 & 0.07169 & 300.274 & 6565.77 & 0.04145 \\
\hline 42012 & 421.817 & 45.272 & 0.07166 & 299.601 & 6566.24 & 0.04143 \\
\hline 42013 & 423.091 & 35.851 & 0.06704 & 301.894 & 5199.70 & 0.03876 \\
\hline 42014 & 422.622 & 35.855 & 0.06698 & 301.050 & 5200.29 & 0.03873 \\
\hline 42015 & 422.189 & 35.860 & 0.06723 & 300.270 & 5201.01 & 0.03887 \\
\hline 42016 & 421.795 & 35.859 & 0.06685 & 299.561 & 5200.98 & 0.03865 \\
\hline 42017 & 423.131 & 27.874 & 0.06322 & 301.966 & 4042.84 & 0.03655 \\
\hline 42018 & 422.634 & 27.877 & 0.06327 & 301.071 & 4043.28 & 0.03658 \\
\hline 42019 & 422.175 & 27.874 & 0.06311 & 300.245 & 4042.83 & 0.03649 \\
\hline 42020 & 421.762 & 27.871 & 0.06318 & 299.502 & 4042.34 & 0.03653 \\
\hline 42021 & 422.799 & 21.545 & 0.05981 & 301.368 & 3124.82 & 0.03458 \\
\hline 42022 & 422.306 & 21.546 & 0.05992 & 300.481 & 3124.97 & 0.03464 \\
\hline 42023 & 421.859 & 21.547 & 0.05971 & 299.676 & 3125.13 & 0.03452 \\
\hline 42024 & 421.450 & 21.549 & 0.06000 & 298.940 & 3125.49 & 0.03469 \\
\hline 42025 & 422.832 & 15.917 & 0.05662 & 301.428 & 2308.64 & 0.03274 \\
\hline 42026 & 422.319 & 15.918 & 0.05668 & 300.504 & 2308.69 & 0.03277 \\
\hline 42027 & 421.852 & 15.918 & 0.05652 & 299.664 & 2308.71 & 0.03268 \\
\hline 42028 & 421.423 & 15.920 & 0.05662 & 298.891 & 2309.05 & 0.03274 \\
\hline 42029 & 422.962 & 8.321 & 0.05151 & 301.662 & 1206.85 & 0.02978 \\
\hline 42030 & 422.404 & 8.320 & 0.05148 & 300.657 & 1206.68 & 0.02976 \\
\hline 42031 & 421.898 & 8.319 & 0.05165 & 299.746 & 1206.51 & 0.02986 \\
\hline 42032 & 421.439 & 8.317 & 0.05166 & 298.920 & 1206.23 & 0.02987 \\
\hline 42033 & 422.989 & 11.649 & 0.05390 & 301.710 & 1689.50 & 0.03116 \\
\hline 42034 & 422.449 & 11.650 & 0.05387 & 300.738 & 1689.69 & 0.03115 \\
\hline 42035 & 421.958 & 11.650 & 0.05391 & 299.854 & 1689.76 & 0.03117 \\
\hline 42036 & 421.510 & 11.648 & 0.05397 & 299.048 & 1689.47 & 0.03120 \\
\hline 42037 & 422.677 & 5.393 & 0.04915 & 301.149 & 782.16 & 0.02842 \\
\hline 42038 & 422.119 & 5.393 & 0.04910 & 300.144 & 782.26 & 0.02839 \\
\hline 42039 & 421.625 & 5.395 & 0.04918 & 299.255 & 782.52 & 0.02843 \\
\hline 42040 & 421.180 & 5.396 & 0.04902 & 298.454 & 782.62 & 0.02834 \\
\hline 42041 & 422.727 & 3.797 & 0.04762 & 301.239 & 550.73 & 0.02753 \\
\hline 42042 & 422.168 & 3.798 & 0.04772 & 300.232 & 550.81 & 0.02759 \\
\hline 42043 & 421.667 & 3.799 & 0.04761 & 299.331 & 551.05 & 0.02753 \\
\hline 42044 & 421.205 & 3.800 & 0.04783 & 298.499 & 551.10 & 0.02765 \\
\hline 42045 & 422.800 & 2.343 & 0.04608 & 301.370 & 339.86 & 0.02664 \\
\hline 42046 & 422.225 & 2.344 & 0.04611 & 300.335 & 339.95 & 0.02666 \\
\hline 42047 & 421.707 & 2.345 & 0.04606 & 299.403 & 340.05 & 0.02663 \\
\hline 42048 & 421.239 & 2.345 & 0.04594 & 298.560 & 340.14 & 0.02656 \\
\hline 33001 & 423.816 & 2.007 & 0.01890 & 303.199 & 291.10 & 0.01093 \\
\hline 33002 & 423.513 & 2.009 & 0.01842 & 302.653 & 291.40 & 0.01065 \\
\hline
\end{tabular}


Table 75. Transient Hot-Wire Thermal Conductivity Data for HCFC-123 (Continued)

\begin{tabular}{|c|c|c|c|c|c|c|}
\hline $\begin{array}{l}\text { Point } \\
\text { Number }\end{array}$ & $\begin{array}{l}\mathrm{T}_{\text {Exp. }} \\
(\mathrm{K})\end{array}$ & $\begin{array}{l}\mathrm{P}_{(} \\
(\mathrm{MPa})\end{array}$ & $\begin{array}{c}\lambda_{\operatorname{Exp}_{.}} \\
(\mathrm{W} / \mathrm{m}-\mathrm{K})\end{array}$ & $\mathrm{T}_{\text {Exp. }}$ & $\begin{array}{l}\mathrm{P}_{\text {Cell }} \\
\text { (psia) }\end{array}$ & $\begin{array}{c}\lambda_{\text {Exp. }} \\
(\text { BTU/ft-hr-F) }\end{array}$ \\
\hline 33003 & 423.253 & 2.009 & 0.01803 & 302.185 & 291.42 & 0.01042 \\
\hline 33004 & 422.965 & 2.010 & 0.01773 & 301.667 & 291.49 & 0.01025 \\
\hline 33005 & 423.769 & 1.939 & 0.01942 & 303.114 & 281.16 & 0.01123 \\
\hline 33006 & 423.485 & 1.938 & 0.01946 & 302.603 & 281.11 & 0.01125 \\
\hline 33007 & 423.216 & 1.938 & 0.01953 & 302.119 & 281.15 & 0.01129 \\
\hline 33008 & 422.951 & 1.938 & 0.01916 & 301.642 & 281.14 & 0.01108 \\
\hline 33009 & 423.867 & 1.886 & 0.01920 & 303.291 & 273.53 & 0.01110 \\
\hline 33010 & 423.526 & 1.886 & 0.01983 & 302.677 & 273.56 & 0.01147 \\
\hline 33011 & 423.250 & 1.886 & 0.01955 & 302.180 & 273.56 & 0.01130 \\
\hline 33012 & 423.005 & 1.886 & 0.01947 & 301.739 & 273.58 & 0.01126 \\
\hline 33013 & 423.923 & 1.764 & 0.01937 & 303.391 & 255.84 & 0.01120 \\
\hline 33014 & 423.621 & 1.764 & 0.01935 & 302.848 & 255.87 & 0.01119 \\
\hline 33015 & 423.366 & 1.764 & 0.01941 & 302.389 & 255.88 & 0.01122 \\
\hline 33016 & 423.118 & 1.764 & 0.01916 & 301.942 & 255.85 & 0.01108 \\
\hline 33017 & 423.873 & 1.672 & 0.01903 & 303.301 & 242.49 & 0.01100 \\
\hline 33018 & 423.555 & 1.672 & 0.01888 & 302.729 & 242.52 & 0.01092 \\
\hline 33019 & 423.280 & 1.672 & 0.01891 & 302.234 & 242.50 & 0.01093 \\
\hline 33020 & 423.026 & 1.672 & 0.01893 & 301.777 & 242.53 & 0.01094 \\
\hline 33021 & 423.943 & 1.558 & 0.01879 & 303.427 & 226.01 & 0.01086 \\
\hline 33023 & 423.347 & 1.558 & 0.01873 & 302.355 & 225.96 & 0.01083 \\
\hline 33022 & 423.642 & 1.558 & 0.01873 & 302.886 & 225.95 & 0.01083 \\
\hline 33024 & 423.066 & 1.558 & 0.01865 & 301.849 & 225.91 & 0.01078 \\
\hline 33025 & 423.919 & 1.369 & 0.01835 & 303.384 & 198.60 & 0.01061 \\
\hline 33026 & 423.614 & 1.369 & 0.01832 & 302.835 & 198.59 & 0.01059 \\
\hline 33027 & 423.315 & 1.369 & 0.01832 & 302.297 & 198.51 & 0.01059 \\
\hline 33028 & 423.047 & 1.369 & 0.01836 & 301.815 & 198.54 & 0.01062 \\
\hline 33029 & 423.995 & 1.265 & 0.01824 & 303.521 & 183.41 & 0.01055 \\
\hline 33030 & 423.681 & 1.264 & 0.01827 & 302.956 & 183.39 & 0.01056 \\
\hline 33031 & 423.361 & 1.264 & 0.01829 & 302.380 & 183.37 & 0.01057 \\
\hline 33032 & 423.080 & 1.264 & 0.01822 & 301.874 & 183.37 & 0.01053 \\
\hline 33033 & 424.038 & 1.148 & 0.01816 & 303.598 & 166.44 & 0.01050 \\
\hline 33034 & 423.718 & 1.147 & 0.01807 & 303.022 & 166.41 & 0.01045 \\
\hline 33035 & 423.460 & 1.148 & 0.01801 & 302.558 & 166.44 & 0.01041 \\
\hline 33036 & 423.121 & 1.148 & 0.01800 & 301.948 & 166.49 & 0.01041 \\
\hline 33037 & 424.138 & 1.021 & 0.01798 & 303.778 & 148.09 & 0.01040 \\
\hline 33038 & 423.808 & 1.020 & 0.01795 & 303.184 & 148.01 & 0.01038 \\
\hline 33039 & 423.494 & 1.021 & 0.01797 & 302.619 & 148.06 & 0.01039 \\
\hline 33040 & 423.193 & 1.021 & 0.01797 & 302.077 & 148.03 & 0.01039 \\
\hline 33041 & 423.861 & 0.899 & 0.01800 & 303.280 & 130.44 & 0.01041 \\
\hline 33042 & 423.541 & 0.899 & 0.01803 & 302.704 & 130.38 & 0.01042 \\
\hline 33043 & 423.233 & 0.899 & 0.01791 & 302.149 & 130.35 & 0.01036 \\
\hline 33044 & 422.951 & 0.899 & 0.01800 & 301.642 & 130.40 & 0.01041 \\
\hline 33045 & 423.927 & 0.795 & 0.01791 & 303.399 & 115.33 & 0.01036 \\
\hline 33046 & 423.575 & 0.796 & 0.01799 & 302.765 & 115.44 & 0.01040 \\
\hline 33047 & 423.258 & 0.796 & 0.01788 & 302.194 & 115.52 & 0.01034 \\
\hline 33048 & 422.977 & 0.797 & 0.01796 & 301.689 & 115.59 & 0.01038 \\
\hline 33049 & 423.595 & $0.69 \mathrm{I}$ & 0.01870 & 302.801 & 100.18 & 0.01081 \\
\hline 33050 & 423.293 & 0.691 & 0.01847 & 302.257 & 100.23 & 0.01068 \\
\hline 33051 & 422.989 & 0.691 & 0.01830 & 301.710 & 100.21 & 0.01058 \\
\hline
\end{tabular}


Table 75. Transient Hot-Wire Thermal Conductivity Data for HCFC-123 (Continued)

\begin{tabular}{|c|c|c|c|c|c|c|}
\hline $\begin{array}{l}\text { Point } \\
\text { Number }\end{array}$ & $\begin{array}{l}T_{\text {Exp. }} \\
(K)\end{array}$ & $\begin{array}{l}\mathrm{P}_{\mathrm{Cell}} \\
(\mathrm{MPa})\end{array}$ & $\begin{array}{c}\lambda_{\text {Exp }} \\
(W / m-K)\end{array}$ & $\mathrm{T}_{\text {Exp. }}$ & $\begin{array}{l}\mathrm{P}_{\text {Cell }} \\
\text { (psia) }\end{array}$ & $\begin{array}{c}\lambda_{\text {Exp. }} \\
(B T U / f t-h r-F)\end{array}$ \\
\hline 33052 & 422.734 & 0.691 & 0.01818 & 301.251 & 100.23 & 0.01051 \\
\hline 33053 & 423.685 & 0.557 & 0.01827 & 302.963 & 80.78 & 0.01056 \\
\hline 33054 & 423.359 & 0.557 & 0.01837 & 302.376 & 80.79 & 0.01062 \\
\hline 33055 & 423.057 & 0.557 & 0.01828 & 301.833 & 80.76 & 0.01057 \\
\hline 33056 & 422.766 & 0.557 & 0.01815 & 301.309 & 80.73 & 0.01049 \\
\hline 33057 & 423.788 & 0.390 & 0.01848 & 303.148 & 56.59 & 0.01068 \\
\hline 33058 & 423.464 & 0.390 & 0.01846 & 302.565 & 56.54 & 0.01067 \\
\hline 33059 & 423.145 & 0.389 & 0.01837 & 301.991 & 56.45 & 0.01062 \\
\hline 33060 & 422.863 & 0.389 & 0.01849 & 301.483 & 56.46 & 0.01069 \\
\hline 33061 & 423.925 & 0.266 & 0.01882 & 303.395 & 38.54 & 0.01088 \\
\hline 33062 & 423.585 & 0.266 & 0.01889 & 302.783 & 38.52 & 0.01092 \\
\hline 33063 & 423.261 & 0.265 & 0.01872 & 302.200 & 38.48 & 0.01082 \\
\hline 33064 & 422.947 & 0.265 & 0.01865 & 301.635 & 38.47 & 0.01078 \\
\hline 33065 & 423.982 & 0.119 & 0.01961 & 303.498 & 17.24 & 0.01134 \\
\hline 33066 & 423.622 & 0.119 & 0.01975 & 302.850 & 17.26 & 0.01142 \\
\hline 33067 & 423.277 & 0.120 & 0.01988 & 302.229 & 17.38 & 0.01149 \\
\hline 33068 & 422.934 & 0.121 & 0.01964 & 301.611 & 17.52 & 0.01136 \\
\hline 34025 & 433.111 & 2.069 & 0.02011 & 319.930 & 300.11 & 0.01163 \\
\hline 34026 & 432.829 & 2.041 & 0.01996 & 319.422 & 295.97 & 0.01154 \\
\hline 34027 & 432.573 & 2.098 & 0.02000 & 318.961 & 304.23 & 0.01156 \\
\hline 34028 & 432.320 & 2.069 & 0.01980 & 318.506 & 300.11 & $0: 01145$ \\
\hline 34029 & 432.890 & 1.993 & 0.01956 & 319.532 & 289.08 & 0.01131 \\
\hline 34030 & 432.624 & 1.963 & 0.01966 & 319.053 & 284.76 & 0.01137 \\
\hline 34031 & 432.380 & 1.991 & 0.01966 & 318.614 & 288.83 & 0.01137 \\
\hline 34032 & 432.124 & 1.963 & 0.01963 & 318.153 & 284.72 & 0.01135 \\
\hline 34033 & 432.931 & 1.900 & 0.01964 & 319.606 & 275.62 & 0.01136 \\
\hline 34034 & 432.658 & 1.899 & 0.01963 & 319.114 & 275.50 & 0.01135 \\
\hline 34035 & 432.397 & 1.899 & 0.01946 & 318.645 & 275.42 & 0.01125 \\
\hline 34036 & 432.159 & 1.899 & 0.01961 & 318.216 & 275.39 & 0.01134 \\
\hline 34037 & 432.977 & 1.838 & 0.01937 & 319.689 & 266.55 & 0.01120 \\
\hline 34038 & 432.706 & 1.838 & 0.01943 & 319.201 & 266.52 & 0.01123 \\
\hline 34039 & 432.439 & 1.837 & 0.01936 & 318.720 & 266.50 & 0.01119 \\
\hline 34040 & 432.195 & 1.837 & 0.01937 & 318.281 & 266.49 & 0.01120 \\
\hline 34041 & 433.047 & 1.732 & 0.01909 & 319.815 & 251.19 & 0.01104 \\
\hline 34042 & 432.769 & 1.732 & 0.01914 & 319.314 & 251.17 & 0.01107 \\
\hline 34043 & 432.494 & 1.732 & 0.01921 & 318.819 & 251.20 & 0.01111 \\
\hline 34044 & 432.228 & 1.732 & 0.01916 & 318.340 & 251.16 & 0.01108 \\
\hline 34045 & 433.108 & 1.645 & 0.01905 & 319.924 & 238.65 & 0.01101 \\
\hline 34046 & 432.808 & 1.645 & 0.01904 & 319.384 & 238.65 & 0.01101 \\
\hline 34047 & 432.542 & 1.645 & 0.01887 & 318.906 & 238.63 & 0.01091 \\
\hline 34048 & 432.278 & 1.645 & 0.01913 & 318.430 & 238.62 & 0.01106 \\
\hline 34049 & 433.171 & 1.540 & 0.01890 & 320.038 & 223.42 & 0.01093 \\
\hline 34050 & 432.873 & 1.540 & 0.01878 & 319.501 & 223.43 & 0.01086 \\
\hline 34051 & 432.604 & 1.541 & 0.01900 & 319.017 & 223.44 & 0.01099 \\
\hline 34052 & 432.322 & 1.540 & 0.01876 & 318.510 & 223.41 & 0.01085 \\
\hline 34053 & 433.250 & 1.420 & 0.01874 & 320.180 & 205.92 & 0.01084 \\
\hline 34054 & 432.940 & 1.419 & 0.01886 & 319.622 & 205.85 & 0.01090 \\
\hline 34055 & 432.653 & 1.419 & 0.01885 & 319.105 & 205.78 & 0.01090 \\
\hline 34056 & 432.384 & 1.418 & 0.01874 & 318.621 & 205.69 & 0.01084 \\
\hline
\end{tabular}


Table 75. Transient Hot-Wire Thermal Conductivity Data for HCFC-123 (Continued)

\begin{tabular}{|c|c|c|c|c|c|c|}
\hline $\begin{array}{l}\text { Point } \\
\text { Number }\end{array}$ & $\begin{array}{l}\mathrm{T}_{\text {Exp. }} \\
(\mathrm{K})\end{array}$ & $\begin{array}{l}\mathrm{P}_{\mathrm{Cell}} \\
(\mathrm{MPa})\end{array}$ & $\begin{array}{c}\lambda_{\operatorname{Exp}} \\
(\mathrm{W} / \mathrm{m}-\mathrm{K})\end{array}$ & $\mathrm{T}_{\text {Exp. }}$ & $\begin{array}{l}\mathrm{P}_{\text {Cell }} \\
\text { (psia) }\end{array}$ & $\begin{array}{l}\lambda_{\text {Exp }} \\
\text { (BTU/ft-hr-F) }\end{array}$ \\
\hline 34057 & 433.292 & 1.339 & 0.01864 & 320.256 & 194.21 & 0.01078 \\
\hline 34058 & 432.987 & 1.339 & 0.01869 & 319.707 & 194.15 & 0.01081 \\
\hline 34059 & 432.682 & 1.339 & 0.01857 & 319.158 & 194.14 & 0.01074 \\
\hline 34060 & 432.415 & 1.338 & 0.01855 & 318.677 & 194.12 & 0.01073 \\
\hline 34061 & 433.037 & 1.223 & 0.01857 & 319.797 & 177.34 & 0.01074 \\
\hline 34062 & 432.744 & 1.222 & 0.01865 & 319.269 & 177.28 & 0.01078 \\
\hline 34063 & 432.447 & 1.222 & 0.01843 & 318.735 & 177.28 & 0.01066 \\
\hline 34064 & 432.194 & 1.221 & 0.01850 & 318.279 & 177.15 & 0.01070 \\
\hline 34065 & 433.189 & 1.072 & 0.01862 & 320.070 & 155.46 & 0.01077 \\
\hline 34066 & 432.887 & 1.072 & 0.01865 & 319.527 & 155.43 & 0.01078 \\
\hline 34067 & 432.594 & 1.071 & 0.01839 & 318.999 & 155.40 & 0.01063 \\
\hline 34068 & 432.324 & 1.072 & 0.01847 & 318.513 & 155.44 & 0.01068 \\
\hline 34069 & 433.245 & 0.954 & 0.01845 & 320.171 & 138.31 & 0.01067 \\
\hline 34070 & 432.922 & 0.951 & 0.01857 & 319.590 & 137.96 & 0.01074 \\
\hline 34071 & 432.639 & 0.949 & 0.01841 & 319.080 & 137.71 & 0.01064 \\
\hline 34072 & 432.386 & 0.951 & 0.01858 & 318.625 & 137.90 & 0.01074 \\
\hline 34073 & 433.330 & 0.793 & 0.01868 & 320.324 & 115.00 & 0.01080 \\
\hline 34074 & 433.017 & 0.793 & 0.01851 & 319.761 & 115.00 & 0.01070 \\
\hline 34075 & 432.697 & 0.793 & 0.01845 & 319.185 & 114.98 & 0.01067 \\
\hline 34076 & 432.414 & 0.792 & 0.01839 & 318.675 & 114.94 & 0.01063 \\
\hline 34077 & 433.063 & 0.661 & 0.01870 & 319.843 & 95.82 & 0.01081 \\
\hline 34078 & 432.748 & 0.658 & 0.01856 & 319.276 & 95.50 & 0.01073 \\
\hline 34079 & 432.458 & 0.656 & 0.01860 & 318.754 & 95.17 & 0.01075 \\
\hline 34080 & 432.180 & 0.654 & 0.01847 & 318.254 & 94.79 & 0.01068 \\
\hline 34081 & 433.097 & 0.563 & 0.01878 & 319.905 & 81.71 & 0.01086 \\
\hline 34082 & 432.759 & 0.563 & 0.01864 & 319.296 & 81.61 & 0.01078 \\
\hline 34083 & 432.465 & 0.562 & 0.01873 & 318.767 & 81.53 & 0.01083 \\
\hline 34084 & 432.188 & 0.561 & 0.01893 & 318.268 & 81.42 & 0.01094 \\
\hline 34085 & 433.218 & 0.384 & 0.01905 & 320.122 & 55.64 & 0.01101 \\
\hline 34086 & 432.889 & 0.385 & 0.01918 & 319.530 & 55.77 & 0.01109 \\
\hline 34087 & 432.573 & 0.386 & 0.01912 & 318.961 & 55.92 & 0.01105 \\
\hline 34088 & 432.274 & 0.386 & 0.01903 & 318.423 & 55.97 & 0.01100 \\
\hline 34089 & 433.374 & 0.235 & 0.01974 & 320.403 & 34.12 & 0.01141 \\
\hline 34090 & 433.041 & 0.235 & 0.01988 & 319.804 & 34.06 & 0.01149 \\
\hline 34091 & 432.699 & 0.235 & 0.01968 & 319.188 & 34.05 & 0.01138 \\
\hline 34092 & 432.385 & 0.235 & 0.01965 & 318.623 & 34.03 & 0.01136 \\
\hline 34093 & 433.584 & 0.117 & 0.02047 & 320.781 & 16.98 & 0.01184 \\
\hline 34094 & 433.207 & 0.119 & 0.02028 & 320.103 & 17.19 & 0.01173 \\
\hline 34095 & 432.859 & 0.120 & 0.02026 & 319.476 & 17.34 & 0.01171 \\
\hline 34096 & 432.530 & 0.122 & 0.02016 & 318.884 & 17.70 & 0.01166 \\
\hline 38001 & 430.901 & 2.250 & 0.02113 & 315.952 & 326.33 & 0.01222 \\
\hline 38003 & 430.643 & 2.250 & 0.02094 & 315.487 & 326.39 & 0.01211 \\
\hline 38005 & 430.420 & 2.251 & 0.02093 & 315.086 & 326.47 & 0.01210 \\
\hline 38007 & 430.194 & 2.251 & 0.02098 & 314.679 & 326.52 & 0.01213 \\
\hline 38009 & 430.196 & 2.252 & 0.02085 & 314.683 & 326.64 & 0.01206 \\
\hline 38011 & 429.985 & 2.252 & 0.02071 & 314.305 & 326.67 & 0.01197 \\
\hline 38013 & 429.789 & 2.253 & 0.02078 & 313.950 & 326.70 & 0.01201 \\
\hline 38015 & 429.607 & 2.252 & 0.02054 & 313.623 & 326.68 & 0.01188 \\
\hline 38017 & 430.779 & 2.105 & 0.01995 & 315.732 & 305.30 & 0.01153 \\
\hline
\end{tabular}


Table 75. Transient Hot-Wire Thermal Conductivity Data for HCFC-123 (Continued)

\begin{tabular}{|c|c|c|c|c|c|c|}
\hline $\begin{array}{l}\text { Point } \\
\text { Number }\end{array}$ & $\begin{array}{l}\mathrm{T}_{\text {Exp. }} \\
(\mathrm{K})\end{array}$ & $\begin{array}{l}\mathrm{P}_{\mathrm{Cell}} \\
(\mathrm{MPa})\end{array}$ & $\underset{(W / m-K)}{\lambda \operatorname{Exp}_{.}}$ & ${ }_{(\text {Exp. }}$ & $\begin{array}{l}\mathrm{P}_{\text {Cell }} \\
\text { (psia) }\end{array}$ & $\begin{array}{c}\lambda_{\text {Exp. }} \\
\text { (BTU/ft-hr-F) }\end{array}$ \\
\hline 38019 & 430.542 & 2.105 & 0.01994 & 315.306 & 305.30 & 0.01153 \\
\hline 38021 & 430.307 & 2.105 & 0.01988 & 314.883 & 305.30 & 0.01149 \\
\hline 38023 & 430.090 & 2.105 & 0.01981 & 314.492 & 305.31 & 0.01145 \\
\hline 38025 & 430.906 & 1.954 & 0.01936 & 315.961 & 283.34 & 0.01119 \\
\hline 38027 & 430.651 & 1.954 & 0.01934 & 315.502 & 283.37 & 0.01118 \\
\hline 38029 & 430.418 & 1.954 & 0.01930 & 315.082 & 283.37 & 0.01116 \\
\hline 38031 & 430.183 & 1.954 & 0.01938 & 314.659 & 283.41 & 0.01121 \\
\hline 38033 & 430.998 & 1.818 & 0.01915 & 316.126 & 263.61 & 0.01107 \\
\hline 38035 & 430.742 & 1.818 & 0.01901 & 315.666 & 263.61 & 0.01099 \\
\hline 38037 & 430.495 & 1.818 & 0.01903 & 315.221 & 263.65 & 0.01100 \\
\hline 38039 & 430.260 & 1.818 & 0.01888 & 314.798 & 263.66 & 0.01092 \\
\hline 38041 & 431.116 & 1.619 & 0.01873 & 316.339 & 234.88 & 0.01083 \\
\hline 38043 & 430.838 & 1.619 & 0.01879 & 315.838 & 234.82 & 0.01086 \\
\hline 38045 & 430.584 & 1.618 & 0.01870 & 315.381 & 234.69 & 0.01081 \\
\hline 38047 & 430.338 & 1.617 & 0.01853 & 314.938 & 234.59 & 0.01071 \\
\hline 38049 & 431.260 & 1.386 & 0.01841 & 316.598 & 201.04 & 0.01064 \\
\hline 38051 & 430.975 & 1.386 & 0.01844 & 316.085 & 200.99 & 0.01066 \\
\hline 38053 & 430.707 & 1.386 & 0.01837 & 315.603 & 201.01 & 0.01062 \\
\hline 38055 & 430.447 & 1.386 & 0.01824 & 315.135 & 201.00 & 0.01055 \\
\hline 38057 & 431.052 & 1.187 & 0.01831 & 316.224 & 172.13 & 0.01059 \\
\hline 38059 & 430.772 & 1.181 & 0.01827 & 315.720 & 171.26 & 0.01056 \\
\hline 38061 & 430.514 & 1.175 & 0.01831 & 315.255 & 170.41 & 0.01059 \\
\hline 38063 & 430.259 & 1.169 & 0.01838 & 314.796 & 169.57 & 0.01063 \\
\hline 38065 & 431.049 & 1.078 & 0.01824 & 316.218 & 156.42 & 0.01055 \\
\hline 38067 & 430.764 & 1.079 & 0.01825 & 315.705 & 156.48 & 0.01055 \\
\hline 38069 & 430.492 & 1.079 & 0.01814 & 315.216 & 156.54 & 0.01049 \\
\hline 38071 & 430.233 & 1.079 & 0.01830 & 314.749 & 156.55 & 0.01058 \\
\hline 38073 & 431.144 & 0.906 & 0.01835 & 316.389 & 131.36 & 0.01061 \\
\hline 38075 & 430.851 & 0.906 & 0.01823 & 315.862 & 131.37 & 0.01054 \\
\hline 38077 & 430.571 & 0.905 & 0.01822 & 315.358 & 131.31 & 0.01053 \\
\hline 38079 & 430.302 & 0.905 & 0.01811 & 314.874 & 131.31 & 0.01047 \\
\hline 38081 & 431.243 & 0.688 & 0.01844 & 316.567 & 99.72 & 0.01066 \\
\hline 38083 & 430.948 & 0.677 & 0.01847 & 316.036 & 98.23 & 0.01068 \\
\hline 38085 & 430.665 & 0.668 & 0.01823 & 315.527 & 96.90 & 0.01054 \\
\hline 38087 & 430.389 & 0.659 & 0.01846 & 315.030 & 95.61 & 0.01067 \\
\hline 38089 & 431.371 & 0.510 & 0.01855 & 316.798 & 73.99 & 0.01073 \\
\hline 38091 & 431.055 & 0.510 & 0.01860 & 316.229 & 73.99 & 0.01075 \\
\hline 38093 & 430.751 & 0.510 & 0.01843 & 315.682 & 73.97 & 0.01066 \\
\hline 38095 & 430.467 & 0.510 & 0.01861 & 315.171 & 73.96 & 0.01076 \\
\hline 38097 & 431.542 & 0.299 & 0.01921 & 317.106 & 43.31 & 0.01111 \\
\hline 38099 & 431.205 & 0.299 & 0.01924 & 316.499 & 43.30 & 0.01112 \\
\hline 38101 & 430.887 & 0.299 & 0.01904 & 315.927 & 43.30 & 0.01101 \\
\hline 38103 & 430.588 & 0.298 & 0.01908 & 315.388 & 43.28 & 0.01103 \\
\hline 38105 & 431.406 & 0.143 & 0.01998 & $3 i 6.861$ & 20.76 & 0.01155 \\
\hline 38107 & 431.066 & 0.142 & 0.01993 & 316.249 & 20.61 & 0.01152 \\
\hline 38109 & 430.743 & 0.142 & 0.01989 & 315.667 & 20.53 & 0.01150 \\
\hline 38111 & 430.442 & 0.141 & 0.01984 & 315.126 & 20.44 & 0.01147 \\
\hline 38113 & 430.618 & 2.287 & 0.02153 & 315.442 & 331.67 & 0.01245 \\
\hline 38115 & 430.378 & 2.287 & 0.02146 & 315.010 & 331.77 & 0.01241 \\
\hline
\end{tabular}


Table 75. Transient Hot-Wire Thermal Conductivity Data for HCFC-123 (Continued)

\begin{tabular}{|c|c|c|c|c|c|c|}
\hline $\begin{array}{l}\text { Point } \\
\text { Number }\end{array}$ & $\begin{array}{l}\mathrm{T}_{\text {Exp. }} \\
(\mathrm{K})\end{array}$ & $\begin{array}{l}\mathrm{P}_{\mathrm{Cell}} \\
(\mathrm{MPa})\end{array}$ & $\underset{(W / m-K)}{\lambda_{\operatorname{Exp}}}$ & $\mathrm{T}_{\mathrm{Exp}}$ & $\begin{array}{l}\mathrm{P}_{\text {Cell }} \\
\text { (psia) }\end{array}$ & $\begin{array}{c}\lambda_{\text {Exp. }} \\
\text { (BTU/ft-hr-F) }\end{array}$ \\
\hline 38117 & 430.157 & 2.288 & 0.02138 & 314.613 & 331.84 & 0.01236 \\
\hline 38119 & 429.940 & 2.288 & 0.02106 & 314.222 & 331.90 & 0.01218 \\
\hline 38121 & 430.803 & 2.124 & 0.02003 & 315.775 & 308.12 & 0.01158 \\
\hline 38123 & 430.563 & 2.123 & 0.02007 & 315.343 & 307.93 & 0.01160 \\
\hline 38125 & 430.332 & 2.122 & 0.01998 & 314.928 & 307.78 & 0.01155 \\
\hline 38127 & 430.107 & 2.121 & 0.01997 & 314.523 & 307.66 & 0.01155 \\
\hline 38129 & $43 n .718$ & 1.959 & 0.01954 & 315.622 & 284.19 & 0.01130 \\
\hline 38131 & 430.466 & 1.960 & 0.01935 & 315.169 & 284.25 & 0.01119 \\
\hline 38133 & 430.235 & 1.959 & 0.01946 & 314.753 & 284.19 & 0.01125 \\
\hline 38135 & 430.005 & 1.960 & 0.01935 & 314.339 & 284.25 & 0.01119 \\
\hline 38137 & 430.820 & 1.832 & 0.01914 & 315.806 & 265.76 & 0.01107 \\
\hline 38139 & 430.560 & 1.832 & 0.01904 & 315.338 & 265.76 & 0.01101 \\
\hline 38141 & 430.314 & 1.833 & 0.01894 & 314.895 & 265.79 & 0.01095 \\
\hline 38143 & 430.089 & 1.832 & 0.01897 & 314.490 & 265.77 & 0.01097 \\
\hline 38145 & 430.923 & 1.680 & 0.01885 & 315.991 & 243.73 & 0.01090 \\
\hline 38147 & 430.656 & 1.681 & 0.01883 & 315.511 & 243.76 & 0.01089 \\
\hline 38149 & 430.404 & 1.681 & 0.01879 & 315.057 & 243.75 & 0.01086 \\
\hline 38151 & 430.159 & 1.681 & 0.01886 & 314.616 & 243.75 & 0.01090 \\
\hline 38153 & 430.995 & 1.556 & 0.01861 & 316.121 & 225.74 & 0.01076 \\
\hline 38155 & 430.715 & 1.556 & 0.01865 & 315.617 & 225.65 & 0.01078 \\
\hline 38157 & 430.457 & 1.556 & 0.01849 & 315.153 & 225.66 & 0.01069 \\
\hline 38159 & 430.212 & 1.556 & 0.01860 & 314.712 & 225.69 & 0.01075 \\
\hline 38161 & 431.080 & 1.393 & 0.01843 & 316.274 & 202.00 & 0.01066 \\
\hline 38163 & 430.796 & 1.392 & 0.01848 & 315.763 & 201.91 & 0.01068 \\
\hline 38165 & 430.520 & 1.392 & 0.01845 & 315.266 & 201.93 & 0.01067 \\
\hline 38167 & 430.269 & 1.392 & 0.01836 & 314.814 & 201.93 & 0.01062 \\
\hline 40002 & 444.924 & 68.492 & 0.07808 & 341.193 & 9934.01 & 0.04514 \\
\hline 40003 & 444.474 & 68.485 & 0.07808 & 340.383 & 9932.97 & 0.04514 \\
\hline 40004 & 444.045 & 68.479 & 0.07816 & 339.611 & 9932.12 & 0.04519 \\
\hline 40005 & 445.526 & 57.608 & 0.07388 & 342.277 & 8355.38 & 0.04272 \\
\hline 40006 & 445.009 & 57.606 & 0.07394 & 341.346 & 8355.01 & 0.04275 \\
\hline 40007 & 444.528 & 57.603 & 0.07398 & 340.480 & 8354.68 & 0.04277 \\
\hline 40008 & 444.096 & 57.601 & 0.07395 & 339.703 & 8354.40 & 0.04276 \\
\hline 40009 & 445.662 & 46.977 & 0.06963 & 342.522 & 6813.51 & 0.04026 \\
\hline 40010 & 445.128 & 46.979 & 0.06957 & 341.560 & 6813.68 & 0.04022 \\
\hline 40011 & 444.636 & 46.978 & 0.06962 & 340.675 & 6813.64 & 0.04025 \\
\hline 40012 & 444.179 & 46.978 & 0.06974 & 339.852 & 6813.58 & 0.04032 \\
\hline 40013 & 445.841 & 37.614 & 0.06551 & 342.844 & 5455.46 & 0.03788 \\
\hline 40014 & 445.284 & 37.615 & 0.06570 & 341.841 & 5455.61 & 0.03799 \\
\hline 40015 & 444.762 & 37.617 & 0.06556 & 340.902 & 5455.85 & 0.03791 \\
\hline 40016 & 444.284 & 37.618 & 0.06577 & 340.041 & 5456.11 & 0.03803 \\
\hline 40017 & 445.180 & 30.594 & 0.06234 & 341.654 & 4437.23 & 0.03604 \\
\hline 40018 & 444.654 & 30.592 & 0.06235 & 340.707 & 4437.04 & 0.03605 \\
\hline 40019 & 444.170 & 30.591 & 0.06249 & 339.836 & 4436.82 & 0.03613 \\
\hline 40020 & 443.728 & 30.591 & 0.06239 & 339.040 & 4436.88 & 0.03607 \\
\hline 40021 & 445.318 & 23.886 & 0.05892 & 341.902 & 3464.45 & 0.03407 \\
\hline 40022 & 444.766 & 23.887 & 0.05904 & 340.909 & 3464.60 & 0.03414 \\
\hline 40023 & 444.262 & 23.889 & 0.05893 & 340.002 & 3464.80 & 0.03407 \\
\hline 40024 & 443.800 & 23.890 & 0.05905 & 339.170 & 3464.94 & 0.03414 \\
\hline
\end{tabular}


Table 75. Transient Hot-Wire Thermal Conductivity Data for HCFC-123 (Continued)

\begin{tabular}{|c|c|c|c|c|c|c|}
\hline $\begin{array}{l}\text { Point } \\
\text { Number }\end{array}$ & $\begin{array}{l}\mathrm{T}_{\text {Exp. }} \\
(\mathrm{K})\end{array}$ & $\begin{array}{l}\mathrm{P}_{\mathrm{Cell}} \\
(\mathrm{MPa})\end{array}$ & $\begin{array}{c}\lambda_{\operatorname{Exp}} \\
(\mathrm{W} / \mathrm{m}-\mathrm{K})\end{array}$ & $\mathrm{T}_{\text {Exp. }}$ & $\begin{array}{l}\mathrm{P}_{\mathrm{Cell}} \\
\text { (psia) }\end{array}$ & $\begin{array}{c}\lambda_{\text {Exp. }} \\
(B T U / f t-h r-F)\end{array}$ \\
\hline 40025 & 444.694 & 19.680 & 0.05650 & 340.779 & 2854.28 & 0.03267 \\
\hline 40026 & 444.188 & 19.681 & 0.05652 & 339.868 & 2854.51 & 0.03268 \\
\hline 40027 & 443.723 & 19.681 & 0.05663 & 339.031 & 2854.52 & 0.03274 \\
\hline 40028 & 443.306 & 19.681 & 0.05659 & 338.281 & 2854.54 & 0.03272 \\
\hline 40029 & 444.786 & 15.411 & 0.05409 & 340.945 & 2235.22 & 0.03127 \\
\hline 40030 & 444.259 & 15.412 & 0.05391 & 339.996 & 2235.35 & 0.03117 \\
\hline 40031 & 443.774 & 15.414 & 0.05403 & 339.123 & 2235.64 & 0.03124 \\
\hline 40032 & 443.349 & 15.415 & 0.05410 & 338.358 & 2235.72 & 0.03128 \\
\hline 40033 & 444.904 & 11.817 & 0.05137 & 341.157 & 1713.91 & 0.02970 \\
\hline 40034 & 444.351 & 11.818 & 0.05138 & 340.162 & 1714.03 & 0.02971 \\
\hline 40035 & 443.856 & 11.819 & 0.05155 & 339.271 & 1714.20 & 0.02981 \\
\hline 40036 & 443.408 & 11.820 & 0.05135 & 338.464 & 1714.31 & 0.02969 \\
\hline 40037 & 445.009 & 8.932 & 0.04909 & 341.346 & 1295.50 & 0.02838 \\
\hline 40038 & 444.434 & 8.933 & 0.04903 & 340.311 & 1295.57 & 0.02835 \\
\hline 40039 & 443.919 & 8.933 & 0.04920 & 339.384 & 1295.61 & 0.02845 \\
\hline 40040 & 443.449 & 8.933 & 0.04918 & 338.538 & 1295.69 & 0.02843 \\
\hline 40041 & 444.687 & 6.961 & 0.04726 & 340.767 & 1009.59 & 0.02732 \\
\hline 40042 & 444.134 & 6.961 & 0.04736 & 339.771 & 1009.57 & 0.02738 \\
\hline 40043 & 443.642 & 6.962 & 0.04729 & 338.886 & 1009.70 & 0.02734 \\
\hline 40044 & 443.196 & 6.962 & 0.04744 & 338.083 & 1009.79 & 0.02743 \\
\hline 40045 & 444.770 & 5.550 & 0.04579 & 340.916 & 804.98 & 0.02647 \\
\hline 40046 & 444.208 & 5.550 & 0.04577 & 339.904 & 805.03 & 0.02646 \\
\hline 40047 & 443.694 & 5.551 & 0.04593 & 338.979 & 805.07 & 0.02656 \\
\hline 40048 & 443.240 & 5.551 & 0.04607 & 338.162 & 805.12 & 0.02664 \\
\hline 40049 & 444.827 & 4.357 & 0.04439 & 341.019 & 631.90 & 0.02567 \\
\hline 40050 & 444.250 & 4.357 & 0.04430 & 339.980 & 631.89 & 0.02561 \\
\hline 40051 & 443.727 & 4.356 & 0.04433 & 339.039 & 631.84 & 0.02563 \\
\hline 40052 & 443.264 & 4.357 & 0.04453 & 338.205 & 631.93 & 0.02575 \\
\hline 40053 & 444.869 & 3.319 & 0.04298 & 341.094 & 481.45 & 0.02485 \\
\hline 40054 & 444.281 & 3.319 & 0.04285 & 340.036 & 481.42 & 0.02478 \\
\hline 40055 & 443.747 & 3.320 & 0.04281 & 339.075 & 481.47 & 0.02475 \\
\hline 40056 & 443.274 & 3.320 & 0.04291 & 338.223 & 481.55 & 0.02481 \\
\hline 44009 & 442.552 & 2.797 & 0.02335 & 336.924 & 405.74 & 0.01350 \\
\hline 44011 & 442.382 & 2.798 & 0.02330 & 336.618 & 405.75 & 0.01347 \\
\hline 44013 & 442.220 & 2.797 & 0.02327 & 336.326 & 405.71 & 0.01345 \\
\hline 44015 & 442.062 & 2.798 & 0.02322 & 336.042 & 405.77 & 0.01343 \\
\hline 44017 & 442.651 & 2.753 & 0.02299 & 337.102 & 399.30 & 0.01329 \\
\hline 44019 & 442.480 & 2.753 & 0.02286 & 336.794 & 399.23 & 0.01322 \\
\hline 44021 & 442.309 & 2.753 & 0.02293 & 336.486 & 399.27 & 0.01326 \\
\hline 44023 & 442.149 & 2.753 & 0.02291 & 336.198 & 399.32 & 0.01325 \\
\hline 44025 & 442.513 & 2.722 & 0.02270 & 336.853 & 394.79 & 0.01312 \\
\hline 44027 & 442.337 & 2.722 & 0.02260 & 336.537 & 394.75 & 0.01307 \\
\hline 44029 & 442.169 & 2.722 & 0.02269 & 336.234 & 394.74 & 0.01312 \\
\hline 44031 & 442.016 & 2.722 & 0.02251 & 335.959 & 394.73 & 0.01301 \\
\hline 44033 & 442.544 & 2.679 & 0.02235 & 336.909 & 388.56 & 0.01292 \\
\hline 44035 & 442.364 & 2.679 & 0.02234 & 336.585 & 388.60 & 0.01292 \\
\hline 44037 & 442.204 & 2.679 & 0.02245 & 336.297 & 388.60 & 0.01298 \\
\hline 44039 & 442.048 & 2.679 & 0.02235 & 336.016 & 388.58 & 0.01292 \\
\hline 44041 & 442.577 & 2.623 & 0.02211 & 336.969 & 380.50 & 0.01278 \\
\hline
\end{tabular}


Table 75. Transient Hot-Wire Thermal Conductivity Data for HCFC-123 (Continued)

\begin{tabular}{|c|c|c|c|c|c|c|}
\hline $\begin{array}{l}\text { Point } \\
\text { Number }\end{array}$ & $\begin{array}{l}\mathrm{T}_{\text {Exp. }} \\
(\mathrm{K})\end{array}$ & $\begin{array}{c}\mathrm{P}_{\mathrm{Cell}} \\
(\mathrm{MPa})\end{array}$ & $\begin{array}{c}\lambda_{\operatorname{Exp}} \\
(\mathrm{W} / \mathrm{m}-\mathrm{K})\end{array}$ & $\mathrm{T}_{\text {Exp. }}$ & $\begin{array}{l}\mathrm{P}_{\text {Cell }} \\
\text { (psia) }\end{array}$ & $\begin{array}{c}\lambda_{\text {Exp. }} \\
(B T U / f t-h r-F)\end{array}$ \\
\hline 44043 & 442.386 & 2.623 & 0.02198 & 336.625 & 380.47 & 0.01271 \\
\hline 44045 & 442.217 & 2.623 & 0.02212 & 336.321 & 380.44 & 0.01279 \\
\hline 44047 & 442.055 & 2.623 & 0.02206 & 336.029 & 380.43 & 0.01275 \\
\hline 44049 & 442.588 & 2.578 & 0.02188 & 336.988 & 373.98 & 0.01265 \\
\hline 44051 & 442.404 & 2.578 & 0.02167 & 336.657 & 373.98 & 0.01253 \\
\hline 44053 & 442.223 & 2.578 & 0.02178 & 336.331 & 373.96 & 0.01259 \\
\hline 44055 & 442.040 & 2.578 & 0.02165 & 336.002 & 373.95 & 0.01252 \\
\hline 44057 & 442.664 & 2.531 & 0.02163 & 337.125 & 367.07 & 0.01251 \\
\hline 44059 & 442.473 & 2.531 & 0.02150 & 336.781 & 367.07 & 0.01243 \\
\hline 44061 & 442.289 & 2.531 & 0.02152 & 336.450 & 367.11 & 0.01244 \\
\hline 44063 & 442.129 & 2.531 & 0.02171 & 336.162 & 367.09 & 0.01255 \\
\hline 44065 & 442.512 & 2.472 & 0.02134 & 336.852 & 358.53 & 0.01234 \\
\hline 44067 & 442.329 & 2.472 & 0.02146 & 336.522 & 358.58 & 0.01241 \\
\hline 44069 & 442.152 & 2.472 & 0.02135 & 336.204 & 358.57 & 0.01234 \\
\hline 44071 & 442.005 & 2.472 & 0.02164 & 335.939 & 358.60 & 0.01251 \\
\hline 44073 & 442.562 & 2.419 & 0.02113 & 336.942 & 350.92 & 0.01222 \\
\hline 44075 & 442.259 & 2.420 & 0.02131 & 336.576 & 350.95 & 0.01232 \\
\hline 44077 & 442.192 & 2.420 & 0.02113 & 336.276 & 350.99 & 0.01222 \\
\hline 44079 & 442.017 & 2.420 & 0.02120 & 335.961 & 351.01 & 0.01226 \\
\hline 44081 & 442.592 & 2.357 & 0.02093 & 336.996 & 341.85 & 0.01210 \\
\hline 44083 & 442.404 & 2.357 & 0.02110 & 336.657 & 341.88 & 0.01220 \\
\hline 44085 & 442.222 & 2.357 & 0.02101 & 336.330 & 341.90 & 0.01215 \\
\hline 44087 & 442.054 & 2.358 & 0.02094 & 336.027 & 341.93 & 0.01211 \\
\hline 44089 & 442.626 & 2.301 & 0.02082 & 337.057 & 333.70 & 0.01204 \\
\hline 44091 & 442.431 & 2.302 & 0.02067 & 336.706 & 333.84 & 0.01195 \\
\hline 44093 & 442.243 & 2.303 & 0.02068 & 336.367 & 333.96 & 0.01196 \\
\hline 44095 & 442.066 & 2.304 & 0.02052 & 336.049 & 334.10 & 0.01186 \\
\hline 44097 & 442.642 & 2.208 & 0.02053 & 337.086 & 320.21 & 0.01187 \\
\hline 44099 & 442.451 & 2.208 & 0.02058 & 336.742 & 320.25 & 0.01190 \\
\hline 44101 & 442.253 & 2.209 & 0.02038 & 336.385 & 320.35 & 0.01178 \\
\hline 44103 & 442.085 & 2.209 & 0.02036 & 336.083 & 320.39 & 0.01177 \\
\hline 44105 & 442.254 & 2.147 & 0.02027 & 336.387 & 311.38 & 0.01172 \\
\hline 44107 & 442.081 & 2.146 & 0.02040 & 336.076 & 311.31 & 0.01179 \\
\hline 44109 & 441.906 & 2.146 & 0.02015 & 335.761 & 311.30 & 0.01165 \\
\hline 44111 & 441.748 & 2.146 & 0.02002 & 335.476 & 311.29 & 0.01158 \\
\hline 39090 & 444.265 & 2.186 & 0.02068 & 340.007 & 317.04 & 0.01196 \\
\hline 39092 & 444.140 & 2.186 & 0.02063 & 339.782 & 317.03 & 0.01193 \\
\hline 39094 & 444.012 & 2.186 & 0.02062 & 339.552 & 317.04 & 0.01192 \\
\hline 39096 & 444.427 & 2.114 & 0.02045 & 340.299 & 306.63 & 0.01182 \\
\hline 39098 & 444.295 & 2.114 & 0.02052 & 340.061 & 306.62 & 0.01186 \\
\hline 39100 & 444.166 & 2.114 & 0.02053 & 339.829 & 306.62 & 0.01187 \\
\hline 39102 & 444.038 & 2.115 & 0.02044 & 339.598 & 306.69 & 0.01182 \\
\hline 39104 & 444.490 & 2.011 & 0.02025 & 340.412 & 291.66 & 0.01171 \\
\hline 39106 & 444.355 & 2.011 & 0.02026 & 340.169 & 291.67 & 0.01171 \\
\hline 39108 & 444.225 & 2.011 & 0.02027 & 339.935 & 291.67 & 0.01172 \\
\hline 39110 & 444.095 & 2.011 & 0.02026 & 339.701 & 291.65 & 0.01171 \\
\hline 39112 & 444.540 & 1.921 & 0.02010 & 340.502 & 278.55 & 0.01162 \\
\hline 39114 & 444.394 & 1.920 & 0.02006 & 340.239 & 278.50 & 0.01160 \\
\hline 39116 & 444.261 & 1.920 & 0.02007 & 340.000 & 278.44 & 0.01160 \\
\hline
\end{tabular}


Table 75. Transient Hot-Wire Thermal Conductivity Data for HCFC-123 (Continued)

\begin{tabular}{|c|c|c|c|c|c|c|}
\hline $\begin{array}{l}\text { Point } \\
\text { Number }\end{array}$ & $\begin{array}{l}T_{\text {Exp. }} \\
(K)\end{array}$ & $\begin{array}{l}\mathrm{P}_{\mathrm{Cell}} \\
(\mathrm{MPa})\end{array}$ & $\begin{array}{c}\lambda_{\operatorname{Exp}_{.}} \\
(W / m-K)\end{array}$ & $\mathrm{T}_{\text {Exp. }}$ & $\begin{array}{r}\mathrm{P}_{\text {Cell }} \\
\text { (psia) }\end{array}$ & $\begin{array}{c}\lambda_{\text {Exp. }} \\
\text { (BTU/ft-hr-F) }\end{array}$ \\
\hline 39118 & 444.130 & 1.920 & 0.02005 & 339.764 & 278.45 & 0.01159 \\
\hline 39120 & 444.301 & 1.804 & 0.01991 & 340.072 & 261.66 & 0.01151 \\
\hline 39122 & 444.037 & 1.804 & 0.01994 & 339.597 & 261.66 & 0.01153 \\
\hline 39124 & 443.787 & 1.804 & 0.01982 & 339.147 & 261.65 & 0.01146 \\
\hline 39126 & 443.553 & 1.804 & 0.01986 & 338.725 & 261.59 & 0.01148 \\
\hline 39128 & 444.340 & 1.687 & 0.01973 & 340.142 & 244.65 & 0.01141 \\
\hline 39130 & 444.071 & 1.687 & 0.01978 & 339.658 & 244.63 & 0.01144 \\
\hline 39132 & 443.814 & 1.686 & 0.01981 & 339.195 & 244.55 & 0.01145 \\
\hline 39134 & 443.572 & 1.686 & 0.01964 & 338.760 & 244.58 & 0.01136 \\
\hline 39136 & 444.366 & 1.594 & 0.01960 & 340.189 & 231.19 & 0.01133 \\
\hline 39138 & 444.095 & 1.594 & 0.01957 & 339.701 & 231.16 & 0.01131 \\
\hline 39140 & 443.839 & 1.594 & 0.01961 & 339.240 & 231.13 & 0.01134 \\
\hline 39142 & 443.584 & 1.594 & 0.01968 & 338.781 & 231.12 & 0.01138 \\
\hline 39144 & 444.441 & 1.444 & 0.01956 & 340.324 & 209.37 & 0.01131 \\
\hline 39146 & 444.161 & 1.443 & 0.01960 & 339.820 & 209.34 & 0.01133 \\
\hline 39148 & 443.899 & 1.443 & 0.01950 & 339.348 & 209.34 & 0.01127 \\
\hline 39150 & 443.645 & 1.443 & 0.01933 & 338.891 & 209.35 & 0.01118 \\
\hline 39152 & 444.488 & 1.322 & 0.01948 & 340.408 & 191.76 & 0.01126 \\
\hline 39154 & 444.209 & 1.322 & 0.01952 & 339.906 & 191.80 & 0.01129 \\
\hline 39156 & 443.940 & 1.322 & 0.01947 & 339.422 & 191.77 & 0.01126 \\
\hline 39158 & 443.681 & 1.322 & 0.01940 & 338.956 & 191.75 & 0.01122 \\
\hline 39160 & 444.252 & 1.194 & 0.01952 & 339.984 & 173.17 & 0.01129 \\
\hline 39162 & 443.975 & 1.193 & 0.01926 & 339.485 & 173.06 & 0.01114 \\
\hline 39164 & 443.715 & 1.193 & 0.01949 & 339.017 & 173.00 & 0.01127 \\
\hline 39166 & 443.463 & 1.193 & 0.01945 & 338.563 & 172.96 & 0.01125 \\
\hline 39168 & 444.320 & 0.992 & 0.01950 & 340.106 & 143.95 & 0.01127 \\
\hline 39170 & 444.042 & 0.992 & 0.01946 & 339.606 & 143.85 & 0.01125 \\
\hline 39172 & 443.771 & 0.992 & 0.01958 & 339.118 & 143.82 & 0.01132 \\
\hline 39174 & 443.515 & 0.991 & 0.01952 & 338.657 & 143.77 & 0.01129 \\
\hline 39176 & 444.391 & 0.821 & 0.01960 & 340.234 & 119.09 & 0.01133 \\
\hline 39178 & 444.102 & 0.821 & 0.01953 & 339.714 & 119.01 & 0.01129 \\
\hline 39180 & 443.816 & 0.820 & 0.01944 & 339.199 & 118.96 & 0.01124 \\
\hline 39182 & 443.560 & 0.820 & 0.01962 & 338.738 & 118.93 & 0.01134 \\
\hline 39184 & 444.156 & 0.681 & 0.01956 & 339.811 & 98.74 & 0.01131 \\
\hline 39186 & 443.863 & 0.681 & 0.01970 & 339.283 & 98.71 & 0.01139 \\
\hline 39188 & 443.592 & 0.680 & 0.01958 & 338.796 & 98.67 & 0.01132 \\
\hline 39190 & 443.344 & 0.680 & 0.01967 & 338.349 & 98.64 & 0.01137 \\
\hline 39192 & 444.232 & 0.517 & $0.0 i 985$ & 339.948 & 74.99 & 0.01148 \\
\hline 39194 & 443.941 & 0.516 & 0.01987 & 339.424 & 74.86 & 0.01149 \\
\hline 39196 & 443.659 & 0.516 & 0.01972 & 338.916 & 74.79 & 0.01140 \\
\hline 39198 & 443.394 & 0.515 & 0.01968 & 338.439 & 74.74 & 0.01138 \\
\hline 39200 & 444.343 & 0.348 & 0.02042 & 340.147 & 50.47 & 0.01181 \\
\hline 39202 & 444.034 & 0.347 & 0.01995 & 339.591 & 50.40 & 0.01153 \\
\hline 39204 & 443.747 & 0.347 & 0.02013 & 339.075 & 50.33 & 0.01164 \\
\hline 39206 & 443.464 & 0.347 & 0.02007 & 338.565 & 50.30 & 0.01160 \\
\hline 39208 & 444.587 & 0.126 & 0.02158 & 340.587 & 18.33 & 0.01248 \\
\hline 39210 & 444.254 & 0.125 & 0.02140 & 339.987 & 18.14 & 0.01237 \\
\hline 39212 & 443.938 & 0.124 & 0.02153 & 339.418 & 18.03 & 0.01245 \\
\hline 39214 & 443.640 & 0.123 & 0.02142 & 338.882 & 17.91 & 0.01238 \\
\hline
\end{tabular}


Table 75. Transient Hot-Wire Thermal Conductivity Data for HCFC-123 (Continued)

\begin{tabular}{|c|c|c|c|c|c|}
\hline $\begin{array}{l}T_{\text {Exp. }} \\
(K)\end{array}$ & $\begin{array}{l}\mathrm{P}_{\mathrm{Cell}} \\
(\mathrm{MPa})\end{array}$ & $\begin{array}{c}\lambda_{\operatorname{Exp}_{.}} \\
(\mathrm{W} / \mathrm{m}-\mathrm{K})\end{array}$ & $\mathrm{T}_{\text {Exp. }}$ & $\begin{array}{l}\mathrm{P}_{\text {Cell }} \\
\text { (psia) }\end{array}$ & $\begin{array}{c}\lambda_{\text {Exp. }} \\
(B T U / f t-h r-F)\end{array}$ \\
\hline 480.638 & 69.367 & 0.07683 & 405.478 & 10060.80 & 0.04442 \\
\hline 480.219 & 69.369 & 0.07677 & 404.724 & 10061.12 & 0.04439 \\
\hline 479.832 & 69.365 & 0.07684 & 404.028 & 10060.61 & 0.04443 \\
\hline 479.484 & 69.366 & 0.07707 & 403.401 & 10060.71 & 0.04456 \\
\hline 480.740 & 59.192 & 0.07304 & 405.662 & 8585.16 & 0.04223 \\
\hline 480.311 & 59.195 & 0.07294 & 404.890 & 8585.48 & 0.04217 \\
\hline 479.919 & 59.197 & 0.07296 & 404.184 & 8585.78 & 0.04218 \\
\hline 479.561 & 59.197 & 0.07296 & 403.540 & 8585.84 & 0.04218 \\
\hline 480.944 & 50.366 & 0.06936 & 406.029 & 7304.93 & 0.04010 \\
\hline 480.498 & 50.365 & 0.06933 & 405.226 & 7304.87 & 0.04009 \\
\hline 480.094 & 50.367 & 0.06936 & 404.499 & 7305.17 & 0.04010 \\
\hline 479.716 & 50.371 & 0.06936 & 403.819 & 7305.72 & 0.04010 \\
\hline 480.956 & 50.393 & 0.06928 & 406.051 & 7308.97 & 0.04006 \\
\hline 480.510 & 50.395 & 0.06936 & 405.248 & 7309.20 & 0.04010 \\
\hline 480.103 & 50.398 & 0.06953 & 404.515 & 7309.68 & 0.04020 \\
\hline 479.732 & 50.400 & 0.06949 & 403.848 & 7309.94 & 0.04018 \\
\hline 480.814 & 43.264 & 0.06647 & 405.795 & 6274.87 & 0.03843 \\
\hline 480.377 & 43.263 & 0.06653 & 405.009 & 6274.85 & 0.03847 \\
\hline 479.973 & 43.263 & 0.06618 & 404.281 & 6274.85 & 0.03826 \\
\hline 479.611 & 43.262 & 0.06635 & 403.630 & 6274.63 & 0.03836 \\
\hline 480.991 & 37.083 & 0.06366 & 406.114 & 5378.47 & 0.03681 \\
\hline 480.526 & 37.085 & 0.06350 & 405.277 & 5378.69 & 0.03671 \\
\hline 480.110 & 37.084 & 0.06353 & 404.528 & 5378.60 & 0.03673 \\
\hline 479.734 & 37.085 & 0.06358 & 403.851 & 5378.71 & 0.03676 \\
\hline 480.840 & 30.492 & 0.06036 & 405.842 & 4422.53 & 0.03490 \\
\hline 480.396 & 30.493 & 0.06039 & 405.043 & 4422.65 & 0.03492 \\
\hline 479.988 & 30.493 & 0.06017 & 404.308 & 4422.61 & 0.03479 \\
\hline 479.621 & 30.493 & 0.06009 & 403.648 & 4422.71 & 0.03474 \\
\hline 480.971 & 26.937 & 0.05842 & 406.078 & 3906.87 & 0.03378 \\
\hline 480.512 & 26.938 & 0.05826 & 405.252 & 3907.04 & 0.03368 \\
\hline 480.094 & 26.937 & 0.05845 & 404.499 & 3906.90 & 0.03379 \\
\hline 479.702 & 26.939 & 0.05848 & 403.794 & 3907.19 & 0.03381 \\
\hline 481.107 & 23.074 & 0.05613 & 406.323 & 3346.67 & 0.03245 \\
\hline 480.642 & 22.801 & 0.05601 & 405.486 & 3306.96 & 0.03238 \\
\hline 480.206 & 22.800 & 0.05600 & 404.701 & 3306.91 & 0.03238 \\
\hline 479.818 & 22.801 & 0.05606 & 404.002 & 3306.99 & 0.03241 \\
\hline 481.231 & 19.421 & 0.05400 & 406.546 & 2816.83 & 0.03122 \\
\hline 480.740 & 19.420 & 0.05398 & 405.662 & 2816.68 & 0.03121 \\
\hline 480.288 & 19.421 & 0.05380 & 404.848 & 2816.72 & 0.03111 \\
\hline 479.888 & 19.421 & 0.05375 & 404.128 & 2816.74 & 0.03108 \\
\hline 481.334 & 16.720 & 0.05214 & 406.731 & 2425.04 & 0.03015 \\
\hline 480.825 & 16.721 & 0.05207 & 405.815 & 2425.18 & 0.03011 \\
\hline 480.360 & 16.723 & 0.05191 & 404.978 & 2425.51 & 0.03001 \\
\hline 479.937 & 16.724 & 0.05191 & 404.217 & 2425.61 & 0.03001 \\
\hline 481.051 & 14.596 & 0.05041 & 406.222 & 2116.98 & 0.02915 \\
\hline 480.558 & 14.598 & 0.05030 & 405.334 & 2117.29 & 0.02908 \\
\hline 480.118 & 14.599 & 0.05046 & 404.542 & 2117.46 & 0.02917 \\
\hline 479.719 & 14.600 & 0.05041 & 403.824 & 2117.53 & 0.02915 \\
\hline 481.151 & 12.591 & 0.04878 & 406.402 & 1826.11 & 0.02820 \\
\hline
\end{tabular}


Table 75. Transient Hot-Wire Thermal Conductivity Data for HCFC-123 (Continued)

\begin{tabular}{|c|c|c|c|c|c|c|}
\hline $\begin{array}{l}\text { Point } \\
\text { Number }\end{array}$ & $\begin{array}{l}\mathrm{T}_{\text {Exp. }} \\
(\mathrm{K})\end{array}$ & $\begin{array}{l}\mathrm{P}_{\mathrm{Cell}} \\
(\mathrm{MPa})\end{array}$ & $\begin{array}{c}\lambda_{\operatorname{Exp}} \\
(\mathrm{W} / \mathrm{m}-\mathrm{K})\end{array}$ & $\mathrm{T}_{\text {Exp. }}$ & $\begin{array}{l}P_{\text {Cell }} \\
\text { (psia) }\end{array}$ & $\begin{array}{c}\lambda_{\text {Exp. }} \\
(B T U / f t-h r-F)\end{array}$ \\
\hline 43051 & 480.643 & 12.591 & 0.04872 & 405.487 & 1826.17 & 0.02817 \\
\hline 43052 & 480.191 & 12.591 & 0.04887 & 404.674 & 1826.22 & 0.02826 \\
\hline 43053 & 479.773 & 12.593 & 0.04874 & 403.921 & 1826.44 & 0.02818 \\
\hline 43054 & 481.239 & 10.923 & 0.04730 & 406.560 & 1584.29 & 0.02735 \\
\hline 43055 & 480.714 & 10.924 & 0.04717 & 405.615 & 1584.38 & 0.02727 \\
\hline 43056 & 480.249 & 10.925 & 0.04711 & 404.778 & 1584.49 & 0.02724 \\
\hline 43057 & 479.832 & 10.925 & 0.04692 & 404.028 & 1584.49 & 0.02713 \\
\hline 43058 & 481.326 & 9.839 & 0.04615 & 406.717 & 1426.96 & 0.02668 \\
\hline 43059 & 480.797 & 9.840 & 0.04605 & 405.765 & 1427.14 & 0.02663 \\
\hline 43060 & 480.317 & 9.840 & 0.04608 & 404.901 & 1427.14 & 0.02664 \\
\hline 43061 & 479.889 & 9.840 & 0.04596 & 404.130 & 1427.18 & 0.02657 \\
\hline 43062 & 481.388 & 8.788 & 0.04491 & 406.828 & 1274.63 & 0.02597 \\
\hline 43063 & 480.840 & 8.788 & 0.04485 & 405.842 & 1274.61 & 0.02593 \\
\hline 43064 & 480.353 & 8.788 & 0.04488 & 404.965 & 1274.63 & 0.02595 \\
\hline 43065 & 479.914 & 8.789 & 0.04483 & 404.175 & 1274.71 & 0.02592 \\
\hline 43066 & $481.41^{7}$ & 8.001 & 0.04393 & 406.881 & 1160.52 & 0.02540 \\
\hline 43067 & 480.854 & 8.002 & 0.04381 & 405.867 & 1160.58 & 0.02533 \\
\hline 43068 & 480.363 & 8.002 & 0.04378 & 404.983 & 1160.58 & 0.02531 \\
\hline 43069 & 479.912 & 8.002 & 0.04375 & 404.172 & 1160.60 & 0.02530 \\
\hline 43070 & 481.461 & 7.190 & 0.04284 & 406.960 & 1042.83 & 0.02477 \\
\hline 43071 & 480.903 & 7.190 & 0.04272 & 405.955 & 1042.82 & 0.02470 \\
\hline 43072 & 480.401 & 7.190 & 0.04288 & 405.052 & 1042.83 & 0.02479 \\
\hline 43073 & 479.937 & 7.190 & 0.04270 & 404.217 & $10<2.86$ & 0.02469 \\
\hline 43074 & 481.064 & 6.825 & 0.04214 & 406.245 & 989.90 & 0.02436 \\
\hline 43075 & 480.547 & 6.826 & 0.04226 & 405.315 & 989.97 & 0.02443 \\
\hline 43076 & 480.073 & 6.825 & 0.04211 & 404.461 & 989.92 & 0.02435 \\
\hline 43077 & 479.656 & 6.825 & 0.04232 & 403.711 & 989.91 & 0.02447 \\
\hline 43078 & 481.084 & 6.623 & 0.04199 & 406.281 & 960.61 & 0.02428 \\
\hline 43079 & 480.563 & 6.623 & 0.04192 & 405.343 & 960.60 & 0.02424 \\
\hline 43080 & 480.074 & 6.623 & 0.04166 & 404.463 & 960.64 & 0.02409 \\
\hline 43081 & 479.663 & 6.623 & 0.04192 & 403.723 & 960.65 & 0.02424 \\
\hline 43082 & 481.105 & 6.449 & 0.04168 & 406.319 & 935.36 & 0.02410 \\
\hline 43083 & 480.571 & 6.449 & 0.04169 & 405.358 & 935.41 & 0.02410 \\
\hline 43084 & 480.081 & 6.449 & 0.04160 & 404.476 & 935.38 & 0.02405 \\
\hline 43085 & 479.673 & 6.449 & 0.04168 & 403.741 & 935.42 & 0.02410 \\
\hline 43086 & 480.727 & 6.272 & 0.04136 & 405.639 & 909.65 & 0.02391 \\
\hline 43087 & 480.231 & 6.272 & 0.04127 & 404.746 & 909.62 & 0.02386 \\
\hline 43088 & 479.789 & 6.272 & 0.04124 & 403.950 & 909.65 & 0.02384 \\
\hline 43089 & 479.404 & 6.272 & 0.04121 & 403.257 & 909.67 & 0.02383 \\
\hline 43090 & 480.679 & 6.133 & 0.04125 & 405.552 & 889.53 & 0.02385 \\
\hline 43091 & 480.184 & 6.133 & 0.04106 & 404.661 & 889.58 & 0.02374 \\
\hline 43092 & 479.742 & 6.133 & 0.04107 & 403.866 & 889.59 & 0.02375 \\
\hline 43093 & 479.349 & 6.134 & 0.04130 & 403.158 & 889.66 & 0.02388 \\
\hline 43094 & 480.345 & 6.005 & 0.04110 & 404.951 & 870.97 & 0.02376 \\
\hline 43095 & 479.888 & 6.005 & 0.04085 & 404.128 & 871.01 & 0.02362 \\
\hline 43096 & 479.478 & 6.005 & 0.04080 & 403.390 & 871.02 & 0.02359 \\
\hline 43097 & 479.142 & 6.006 & 0.04106 & 402.786 & 871.04 & 0.02374 \\
\hline 43098 & 480.357 & 5.889 & 0.04080 & 404.973 & 854.15 & 0.02359 \\
\hline 43099 & 479.892 & 5.890 & 0.04070 & 404.136 & 854.21 & 0.02353 \\
\hline
\end{tabular}


Table 75. Transient Hot-Wire Thermal Conductivity Data for HCFC-123 (Continued)

\begin{tabular}{|c|c|c|c|c|c|c|}
\hline $\begin{array}{l}\text { Point } \\
\text { Number }\end{array}$ & $\begin{array}{l}T_{\text {Exp. }} \\
(\mathrm{K})\end{array}$ & $\begin{array}{l}\mathrm{P}_{\mathrm{Cell}} \\
(\mathrm{MPa})\end{array}$ & $\underset{(W / m-K)}{\lambda_{\text {Exp. }}}$ & $\mathrm{T}_{\text {Exp. }}$ & $\begin{array}{l}\mathrm{P}_{\text {Cell }} \\
\text { (psia) }\end{array}$ & $\begin{array}{c}\lambda_{\text {Exp. }} \\
(B T U / f t-h r-F)\end{array}$ \\
\hline 43100 & 479.487 & 5.890 & 0.04046 & 403.407 & 854.21 & 0.02339 \\
\hline 43101 & 479.135 & 5.890 & 0.04082 & 402.773 & 854.27 & 0.02360 \\
\hline 43102 & 480.351 & 5.796 & 0.04067 & 404.962 & 840.57 & 0.02351 \\
\hline $43 ! 03$ & 479.885 & 5.796 & 0.04055 & 404.123 & 840.59 & 0.02345 \\
\hline 43104 & 479.483 & 5.795 & 0.04040 & 403.399 & 840.54 & 0.02336 \\
\hline 43105 & 479.140 & 5.795 & 0.04053 & 402.782 & 840.51 & 0.02343 \\
\hline 43106 & 480.346 & 5.699 & 0.04057 & 404.953 & 826.54 & 0.02346 \\
\hline 43107 & 479.889 & 5.699 & 0.04027 & 404.130 & 826.59 & 0.02328 \\
\hline 43108 & 479.475 & 5.699 & 0.04011 & 403.385 & 826.55 & 0.02319 \\
\hline 43109 & 479.127 & 5.699 & 0.04048 & 402.759 & 826.61 & 0.02340 \\
\hline 43110 & 480.331 & 5.623 & 0.04043 & 404.926 & 815.51 & 0.02338 \\
\hline 43111 & 479.876 & 5.622 & 0.04044 & 404.107 & 815.46 & 0.02338 \\
\hline 43112 & 479.463 & 5.622 & 0.04004 & 403.363 & 815.45 & 0.02315 \\
\hline 43113 & 479.120 & 5.622 & 0.04042 & 402.746 & 815.40 & 0.02337 \\
\hline 43114 & 480.346 & 5.548 & 0.04037 & 404.953 & 804.61 & 0.02334 \\
\hline 43115 & 479.883 & 5.548 & 0.04018 & 404.119 & 804.61 & 0.02323 \\
\hline 43116 & 479.475 & 5.548 & 0.04013 & 403.385 & 804.63 & 0.02320 \\
\hline 43117 & 479.122 & 5.548 & 0.03998 & 402.750 & 804.62 & 0.02312 \\
\hline 43118 & 480.234 & 5.465 & 0.04051 & 404.751 & 792.58 & 0.02342 \\
\hline 43119 & 479.771 & 5.465 & 0.04034 & 403.918 & 792.62 & 0.02332 \\
\hline 43120 & 479.367 & 5.465 & 0.04008 & 403.191 & 792.60 & 0.02317 \\
\hline 43121 & 479.004 & 5.465 & 0.03997 & 402.537 & 792.61 & 0.02311 \\
\hline 43122 & 480.254 & 5.420 & 0.04052 & 404.787 & 786.09 & 0.02343 \\
\hline 43123 & 479.794 & 5.420 & 0.04003 & 403.959 & 786.11 & 0.02314 \\
\hline 43124 & 479.390 & 5.420 & 0.03970 & 403.232 & 786.08 & 0.02295 \\
\hline 43125 & 479.033 & 5.420 & 0.03984 & 402.589 & 786.06 & 0.02303 \\
\hline 43126 & 480.266 & 5.359 & 0.04035 & 404.809 & 777.27 & 0.02333 \\
\hline 43127 & 479.802 & 5.359 & 0.03998 & 403.974 & 777.27 & 0.02312 \\
\hline 43128 & 479.389 & 5.359 & 0.03982 & 403.230 & 777.32 & 0.02302 \\
\hline 43129 & 479.041 & 5.360 & 0.03992 & 402.604 & 777.34 & 0.02308 \\
\hline 43130 & 480.291 & 5.316 & 0.04039 & 404.854 & 771.06 & 0.02335 \\
\hline 43131 & 479.821 & 5.316 & 0.03991 & 404.008 & 771.07 & 0.02308 \\
\hline 43132 & 479.414 & 5.316 & 0.03975 & 403.275 & 771.08 & 0.02298 \\
\hline 43133 & 479.058 & 5.316 & 0.03979 & 402.634 & 771.08 & 0.02301 \\
\hline 43134 & 480.294 & 5.271 & 0.04038 & 404.859 & 764.56 & 0.02335 \\
\hline 43135 & 479.826 & 5.271 & 0.03982 & 404.017 & 764.54 & 0.02302 \\
\hline 43136 & 479.424 & 5.271 & 0.03963 & 403.293 & 764.55 & 0.02291 \\
\hline 43137 & 479.072 & 5.271 & 0.03944 & 402.660 & 764.55 & 0.02280 \\
\hline 43138 & 480.303 & 5.224 & 0.04030 & 404.875 & 757.64 & 0.02330 \\
\hline 43139 & 479.843 & 5.224 & 0.03978 & 404.047 & 757.64 & $0.0 \check{\angle 3} 300$ \\
\hline 43140 & 479.429 & 5.224 & 0.03939 & 403.302 & 757.66 & 0.02277 \\
\hline 43141 & 479.064 & 5.224 & 0.03960 & 402.645 & 757.66 & 0.02290 \\
\hline 43142 & 480.178 & 5.183 & 0.04002 & 404.650 & 751.77 & 0.02314 \\
\hline 43143 & 479.783 & 5.183 & 0.03969 & 403.939 & 751.77 & 0.02295 \\
\hline 43144 & 479.433 & 5.183 & 0.03929 & 403.309 & 751.80 & 0.02272 \\
\hline 43145 & 479.125 & 5.183 & 0.03933 & 402.755 & 751.76 & 0.02274 \\
\hline 43146 & 480.180 & 5.147 & 0.04013 & 404.654 & 746.51 & 0.02320 \\
\hline 43147 & 479.788 & 5.147 & 0.03958 & 403.948 & 746.51 & 0.02288 \\
\hline 43148 & 479.429 & 5.147 & 0.03926 & 403.302 & 746.50 & 0.02270 \\
\hline
\end{tabular}


Table 75. Transient Hot-Wire Thermal Conductivity Data for HCFC-123 (Continued)

\begin{tabular}{|c|c|c|c|c|c|c|}
\hline $\begin{array}{l}\text { Point } \\
\text { Number }\end{array}$ & $\begin{array}{l}\mathrm{T}_{\text {Exp. }} \\
(\mathrm{K})\end{array}$ & $\begin{array}{l}\mathrm{P}_{\text {Cell }} \\
(\mathrm{MPa})\end{array}$ & $\underset{(\mathrm{V} / \mathrm{Exp}-\mathrm{K})}{\lambda_{\text {Exp }}}$ & $\mathrm{T}_{\text {Exp. }}$ & $\begin{array}{l}\mathrm{P}_{\text {Cell }} \\
\text { (psia) }\end{array}$ & ${ }_{(B T U / f t-h r-F)}^{\lambda_{\text {Exp. }}}$ \\
\hline 43149 & 479.116 & 5.147 & 0.03891 & 402.739 & 746.52 & 0.02250 \\
\hline 43150 & 480.178 & 5.102 & 0.03985 & 404.650 & 739.96 & 0.02304 \\
\hline 43151 & 479.779 & 5.102 & 0.03952 & 403.932 & 739.92 & 0.02285 \\
\hline 43152 & 479.428 & 5.102 & 0.03904 & 403.300 & 739.94 & 0.02257 \\
\hline 43153 & 479.126 & 5.102 & 0.03919 & 402.757 & 739.92 & 0.02266 \\
\hline 43154 & 480.177 & 5.061 & 0.03993 & 404.649 & 733.99 & 0.02309 \\
\hline 43155 & 479.784 & 5.061 & 0.03943 & 403.941 & 734.04 & 0.02280 \\
\hline 43156 & 479.427 & 5.061 & 0.03882 & 403.299 & 734.00 & 0.02244 \\
\hline $4315^{\circ}$ & 479.117 & 5.061 & 0.03889 & 402.741 & 734.05 & 0.02249 \\
\hline 43158 & 480.196 & 5.026 & 0.03966 & 404.683 & 728.92 & 0.02293 \\
\hline 43159 & 479.791 & 5.026 & 0.03901 & 403.954 & 728.94 & 0.02255 \\
\hline 43160 & 479.438 & 5.026 & 0.03884 & 403.318 & 728.91 & 0.02246 \\
\hline 43161 & 479.126 & 5.026 & 0.03902 & 402.757 & 728.90 & 0.02256 \\
\hline 43162 & 480.217 & 4.986 & 0.03959 & 404.721 & 723.12 & 0.02289 \\
\hline 43163 & 479.812 & 4.986 & 0.03884 & 403.992 & 723.13 & 0.02246 \\
\hline 43164 & 479.462 & 4.986 & 0.03855 & 403.362 & 723.13 & 0.02229 \\
\hline 43165 & 479.142 & 4.986 & 0.03845 & 402.786 & 723.12 & 0.02223 \\
\hline 43166 & 480.039 & 4.953 & 0.03908 & 404.400 & 718.38 & 0.02260 \\
\hline 43167 & 479.656 & 4.953 & 0.03865 & 403.711 & 718.38 & 0.02235 \\
\hline 43168 & 479.314 & 4.953 & 0.03832 & 403.095 & 718.39 & 0.02216 \\
\hline 43169 & 479.012 & 4.953 & 0.03796 & 402.552 & 718.36 & 0.02195 \\
\hline 43170 & 480.078 & 4.907 & 0.03868 & 404.470 & 711.73 & 0.02236 \\
\hline 43171 & 479.685 & 4.907 & 0.03791 & 403.763 & 711.73 & 0.02192 \\
\hline 43172 & 479.333 & 4.907 & 0.03764 & 403.129 & 711.76 & 0.02176 \\
\hline 43173 & 479.038 & 4.908 & 0.03742 & 402.598 & 711.78 & 0.02164 \\
\hline 43174 & 480.113 & 4.868 & 0.03809 & 404.533 & 705.99 & 0.02202 \\
\hline 43175 & 479.713 & 4.868 & 0.03753 & 403.813 & 705.98 & 0.02170 \\
\hline 43176 & 479.359 & 4.867 & 0.03708 & 403.176 & 705.95 & 0.02144 \\
\hline 43177 & 479.059 & 4.867 & 0.03733 & 402.636 & 705.96 & 0.02158 \\
\hline 43178 & 480.046 & 4.822 & 0.03773 & 404.413 & 699.33 & 0.02181 \\
\hline 43179 & 479.639 & 4.822 & 0.03717 & 403.680 & 699.31 & 0.02149 \\
\hline 43180 & 479.276 & 4.822 & 0.03692 & 403.027 & 699.34 & 0.02135 \\
\hline 43181 & 478.969 & 4.822 & 0.03681 & 402.474 & 699.36 & 0.02128 \\
\hline 43182 & 480.116 & 4.782 & 0.03714 & 404.539 & 693.64 & 0.02147 \\
\hline 43183 & 479.704 & 4.782 & 0.03648 & 403.797 & 693.62 & 0.02109 \\
\hline 43184 & 479.332 & 4.782 & 0.03643 & 403.128 & 693.60 & 0.02106 \\
\hline 43185 & 479.008 & 4.782 & 0.03591 & 402.544 & 693.58 & 0.02076 \\
\hline 43186 & 480.185 & 4.737 & 0.03658 & 404.663 & 687.08 & 0.02115 \\
\hline 43187 & 479.761 & 4.737 & 0.03589 & 403.900 & 686.99 & 0.02075 \\
\hline 43188 & 479.388 & 4.736 & 0.03545 & 403.228 & 686.92 & 0.02050 \\
\hline 43189 & 479.049 & 4.735 & 0.03572 & 402.618 & 686.80 & 0.02065 \\
\hline 43190 & 480.251 & 4.692 & 0.03577 & 404.782 & 680.57 & 0.02068 \\
\hline 43191 & 479.814 & 4.692 & 0.03522 & 403.995 & 680.55 & 0.02036 \\
\hline 43192 & 479.425 & 4.692 & 0.03500 & 403.295 & 680.54 & 0.02024 \\
\hline 43193 & 479.081 & 4.692 & 0.03477 & 402.676 & 680.53 & 0.02010 \\
\hline 43194 & 480.309 & 4.645 & 0.03513 & 404.886 & 673.69 & 0.02031 \\
\hline 43195 & 479.864 & 4.645 & 0.03447 & 404.085 & 673.68 & 0.01993 \\
\hline 43196 & 479.458 & 4.645 & 0.03392 & 403.354 & 673.69 & 0.01961 \\
\hline $4 \overline{3} 19 \overline{7}$ & 479.117 & 4.6045 & 0.03396 & 402.741 & 673.68 & $0.0 ! 963$ \\
\hline
\end{tabular}


Table 75. Transient Hot-Wire Thermal Conductivity Data for HCFC-123 (Continued)

\begin{tabular}{|c|c|c|c|c|c|c|}
\hline $\begin{array}{l}\text { Point } \\
\text { Number }\end{array}$ & $\begin{array}{l}T_{\text {Exp. }} \\
(\mathrm{K})\end{array}$ & $\begin{array}{l}\mathrm{P}_{\mathrm{Cell}} \\
(\mathrm{MPa})\end{array}$ & $\begin{array}{c}\lambda_{\text {Exp }} \\
(W / m-K)\end{array}$ & $\mathrm{T}_{\mathrm{Exp}}$ & $\begin{array}{l}\mathrm{P}_{\text {Cell }} \\
\text { (psia) }\end{array}$ & $\frac{\lambda_{\text {Exp. }}}{(B T U / f t-h r-F)}$ \\
\hline 43198 & 480.387 & 4.579 & 0.03415 & 405.027 & 664.16 & 0.01974 \\
\hline 43199 & 479.920 & 4.579 & 0.03355 & 404.186 & 664.16 & 0.01940 \\
\hline 43200 & 479.503 & 4.579 & 0.03329 & 403.435 & 664.17 & 0.01925 \\
\hline 43201 & 479.149 & 4.579 & 0.03339 & 402.798 & 664.19 & 0.01931 \\
\hline 43202 & 480.455 & 4.524 & 0.03338 & 405.149 & 656.19 & 0.01930 \\
\hline 43203 & 479.979 & 4.524 & 0.03288 & 404.292 & 656.18 & 0.01901 \\
\hline 43204 & 479.547 & 4.524 & 0.03270 & 403.515 & 656.19 & 0.01891 \\
\hline 43205 & 479.180 & 4.524 & 0.03222 & 402.854 & 656.19 & 0.01863 \\
\hline 43206 & 480.373 & 4.449 & 0.03214 & 405.001 & 645.27 & 0.01858 \\
\hline 43207 & 479.894 & 4.449 & 0.03169 & 404.139 & 645.30 & 0.01832 \\
\hline 43208 & 479.453 & 4.449 & 0.03139 & 403.345 & 645.31 & 0.01815 \\
\hline 43209 & 479.107 & 4.449 & 0.03176 & 402.723 & 645.30 & 0.01836 \\
\hline 43210 & 480.424 & 4.396 & 0.03154 & 405.093 & 637.57 & 0.01824 \\
\hline 43211 & 479.933 & 4.396 & 0.03110 & 404.209 & 637.55 & 0.01798 \\
\hline 43212 & 479.502 & 4.396 & 0.03087 & 403.434 & 637.54 & 0.01785 \\
\hline 43213 & 479.126 & 4.396 & 0.03098 & 402.757 & 637.58 & 0.01791 \\
\hline 43214 & 480.507 & 4.319 & 0.03077 & 405.243 & 626.39 & 0.01779 \\
\hline 43215 & 479.995 & 4.319 & 0.03035 & 404.321 & 626.43 & 0.01755 \\
\hline 43216 & 479.550 & 4.319 & 0.03017 & 403.520 & 626.41 & 0.01744 \\
\hline 43217 & 479.151 & 4.319 & 0.03006 & 402.802 & 626.46 & 0.01738 \\
\hline 43218 & 480.558 & 4.247 & 0.02983 & 405.334 & 616.04 & 0.01725 \\
\hline 43219 & 480.048 & 4.247 & 0.02978 & 404.416 & 616.01 & 0.01722 \\
\hline 43220 & 479.578 & 4.247 & 0.02942 & 403.570 & 615.98 & 0.01701 \\
\hline 43221 & 479.172 & 4.247 & 0.02946 & 402.840 & 615.99 & 0.01703 \\
\hline 43222 & 480.650 & 4.170 & 0.02924 & 405.500 & 604.83 & 0.01691 \\
\hline 43223 & 480.105 & 4.170 & 0.02891 & 404.519 & 604.82 & 0.01672 \\
\hline 43224 & 479.620 & 4.170 & 0.02877 & 403.646 & 604.87 & 0.01663 \\
\hline 43225 & 479.213 & 4.170 & 0.02861 & 402.913 & 604.87 & 0.01654 \\
\hline 43226 & 480.751 & 4.064 & 0.02842 & 405.682 & 589.45 & 0.01643 \\
\hline 43227 & 480.192 & 4.064 & 0.02817 & 404.676 & 589.48 & 0.01629 \\
\hline 43228 & 479.688 & 4.064 & 0.02835 & 403.768 & 589.45 & 0.01639 \\
\hline 43229 & 479.254 & 4.064 & 0.02811 & 402.987 & 589.46 & 0.01625 \\
\hline 43230 & 480.828 & 3.985 & 0.02785 & 405.820 & 578.03 & 0.01610 \\
\hline 43231 & 480.253 & 3.985 & 0.02778 & 404.785 & 577.98 & 0.01606 \\
\hline 43232 & 479.746 & 3.985 & 0.02788 & 403.873 & 577.99 & 0.01612 \\
\hline 43233 & 479.300 & 3.985 & 0.02770 & 403.070 & 577.96 & 0.01602 \\
\hline 43234 & 480.958 & 3.795 & 0.02692 & 406.054 & 550.49 & 0.01556 \\
\hline 43235 & 480.357 & 3.795 & 0.02683 & 404.973 & 550.48 & 0.01551 \\
\hline 43236 & 479.824 & 3.795 & 0.02682 & 404.013 & 550.46 & 0.01551 \\
\hline 43237 & 479.354 & 3.795 & 0.02690 & 403.167 & 550.47 & 0.01555 \\
\hline 43238 & 480.752 & 3.677 & 0.02629 & 405.684 & 533.26 & 0.01520 \\
\hline 43239 & 480.263 & 3.677 & 0.02623 & 404.803 & 533.25 & 0.01517 \\
\hline 43240 & 479.812 & 3.676 & 0.02605 & 403.992 & 533.22 & 0.01506 \\
\hline 43241 & 479.420 & 3.676 & 0.02645 & 403.286 & 533.17 & 0.01529 \\
\hline 43242 & 480.850 & 3.527 & 0.02573 & 405.860 & 511.52 & 0.01488 \\
\hline 43243 & 480.338 & 3.527 & 0.02568 & 404.938 & 511.59 & 0.01485 \\
\hline 43244 & 479.876 & 3.527 & 0.02578 & 404.107 & 511.57 & 0.01491 \\
\hline 43245 & 479.464 & 3.527 & 0.02558 & 403.365 & 511.59 & 0.01479 \\
\hline 43246 & 480.929 & 3.387 & 0.02522 & 406.002 & 491.29 & 0.01458 \\
\hline
\end{tabular}


Table 75. Transient Hot-Wire Thermal Conductivity Data for HCFC-123 (Continued)

\begin{tabular}{|c|c|c|c|c|c|c|}
\hline $\begin{array}{l}\text { Point } \\
\text { Number }\end{array}$ & $\begin{array}{l}\mathrm{T}_{\text {Exp. }} \\
(\mathrm{K})\end{array}$ & $\begin{array}{l}{ }^{P_{C e l l}} \\
(\mathrm{MPa})\end{array}$ & $\begin{array}{c}\lambda_{\text {Exp. }} \\
(W / m-K)\end{array}$ & ${ }^{T_{\text {Exp. }}}$ & $\begin{array}{l}P_{\text {Cell }} \\
\text { (psia) }\end{array}$ & $\begin{array}{c}\lambda_{\text {Exp. }} \\
(\mathrm{BTU} / \mathrm{ft}-\mathrm{hr}-\mathrm{F})\end{array}$ \\
\hline 43247 & 480.408 & 3.388 & 0.02519 & 405.064 & 491.32 & 0.01456 \\
\hline 43248 & 479.934 & 3.388 & 0.02517 & 404.211 & 491.34 & 0.01455 \\
\hline 43249 & 479.506 & 3.388 & 0.02518 & 403.441 & 491.38 & 0.01456 \\
\hline 43250 & 480.821 & 3.213 & 0.02478 & 405.808 & 466.04 & 0.01433 \\
\hline 43251 & 480.293 & 3.213 & 0.02472 & 404.857 & 466.00 & 0.01429 \\
\hline 43252 & 479.819 & 3.213 & 0.02464 & 404.004 & 466.06 & 0.01425 \\
\hline 43253 & 479.398 & 3.213 & 0.02448 & 403.246 & 466.00 & 0.01415 \\
\hline 43254 & 480.893 & 3.046 & 0.02439 & 405.937 & 441.81 & 0.01410 \\
\hline 43255 & 480.356 & 3.046 & 0.02439 & 404.971 & 441.80 & 0.01410 \\
\hline 43256 & 479.873 & 3.046 & 0.02439 & 404.101 & 441.77 & 0.01410 \\
\hline 43257 & 479.443 & 3.046 & 0.02438 & 403.327 & 441.76 & 0.01410 \\
\hline 43258 & 480.949 & 2.880 & 0.02413 & 406.038 & 417.64 & 0.01395 \\
\hline 43259 & 480.398 & 2.879 & 0.02407 & 405.046 & 417.58 & 0.01392 \\
\hline 43260 & 479.901 & 2.879 & 0.02399 & 404.152 & 417.59 & 0.01387 \\
\hline 43261 & 479.452 & 2.879 & 0.02393 & 403.344 & 417.57 & 0.01384 \\
\hline 43262 & 481.024 & 2.650 & 0.02368 & 406.173 & 384.29 & 0.01369 \\
\hline 43263 & 480.443 & 2.649 & 0.02365 & 405.127 & 384.24 & 0.01367 \\
\hline 43264 & 479.935 & 2.649 & 0.02359 & 404.213 & 384.22 & 0.01364 \\
\hline 43265 & 479.479 & 2.649 & 0.02350 & 403.392 & 384.23 & 0.01359 \\
\hline 43266 & 480.851 & 2.445 & 0.02350 & 405.862 & 354.61 & 0.01359 \\
\hline 43268 & 480.396 & 2.445 & 0.02330 & 405.043 & 354.57 & 0.01347 \\
\hline 43270 & 479.988 & 2.445 & 0.02337 & 404.308 & 354.58 & 0.01351 \\
\hline 43272 & 479.610 & 2.445 & 0.02325 & 403.628 & 354.60 & 0.01344 \\
\hline 43274 & 480.909 & 2.257 & 0.02323 & 405.966 & 327.39 & 0.01343 \\
\hline 43276 & 480.452 & 2.257 & 0.02325 & 405.144 & 327.31 & 0.01344 \\
\hline 43278 & 480.019 & 2.257 & 0.02310 & 404.364 & 327.28 & 0.01336 \\
\hline 43280 & 479.629 & 2.256 & 0.02313 & 403.662 & 327.24 & 0.01337 \\
\hline 43282 & 481.020 & 1.950 & 0.02307 & 406.166 & 282.79 & 0.01334 \\
\hline 43285 & 480.530 & 1.949 & 0.02291 & 405.284 & 282.66 & 0.01325 \\
\hline 43288 & 480.082 & 1.948 & 0.02278 & 404.478 & 282.57 & 0.01317 \\
\hline 43291 & 479.680 & 1.948 & 0.02266 & 403.754 & 282.56 & 0.01310 \\
\hline 43294 & 481.069 & 1.661 & 0.02289 & 406.254 & 240.92 & 0.01323 \\
\hline 43297 & 480.569 & 1.662 & 0.02280 & 405.354 & 241.00 & 0.01318 \\
\hline 43300 & 480.108 & 1.662 & 0.02282 & 404.524 & 241.04 & 0.01319 \\
\hline 43303 & 479.693 & 1.663 & 0.02265 & 403.777 & 241.15 & 0.01310 \\
\hline 43306 & 480.892 & 1.430 & 0.02277 & 405.936 & 207.35 & 0.01317 \\
\hline 43310 & 480.403 & 1.429 & 0.02281 & 405.055 & 207.32 & 0.01319 \\
\hline 43314 & 479.951 & 1.429 & 0.02270 & 404.242 & 207.29 & 0.01312 \\
\hline 43318 & 479.543 & 1.429 & 0.02275 & 403.507 & 207.30 & 0.01315 \\
\hline 43322 & 480.850 & 0.814 & 0.02299 & 405.860 & 118.04 & 0.01329 \\
\hline 43326 & 480.346 & 0.814 & 0.02305 & 404.953 & 118.10 & 0.01333 \\
\hline 43330 & 479.883 & 0.815 & 0.02286 & 404.119 & 118.18 & 0.01322 \\
\hline 43334 & 479.475 & 0.815 & 0.02292 & 403.385 & 118.20 & 0.01325 \\
\hline 43338 & 481.390 & 0.156 & 0.02533 & 406.832 & 22.62 & 0.01465 \\
\hline 43342 & 480.800 & 0.155 & 0.02525 & 405.770 & 22.45 & 0.01460 \\
\hline 43346 & 480.254 & 0.154 & 0.02507 & 404.787 & 22.36 & 0.01449 \\
\hline 43350 & 479.774 & 0.154 & 0.02515 & 403.923 & 22.28 & 0.01454 \\
\hline
\end{tabular}


Table 76. Thermal conductivity correlation parameters for HCFC-123, with thermal conductivity in $\mathrm{W} / \mathrm{m}-\mathrm{K}$, temperature in $\mathrm{K}$, and density in $\mathrm{mol} / \mathrm{L}$. The parameters are explained in Appendix B.

Dilute Gas Terms

$\begin{array}{lr}a_{1} & 3.45987 \times 10^{-3} \\ a_{2} & -3.49292 \times 10^{-6} \\ a_{3} & 8.68962 \times 10^{-8}\end{array}$

Excess Function Terms

$\begin{array}{ll}b_{1} & -2.71484 \times 10^{-1} \\ b_{2} & 0.00 \\ b_{3} & 5.89917 \times 10^{-1} \\ b_{4} & 0.00 \\ b_{5} & -2.19701 \times 10^{-2} \\ b_{6} & 0.00 \\ b_{7} & 4.10742 \times 10^{-3} \\ b_{8} & 0.00\end{array}$

Critical Enhancement Parameters

$$
\begin{aligned}
& T_{c} \\
& P_{c} \\
& \rho_{c} \\
& q_{D}
\end{aligned}
$$

$456.94 \mathrm{~K}$

$36.74 \mathrm{bar}$

$3.597 \mathrm{~mol} / \mathrm{L}$

$3.0 \times 10^{9} \mathrm{~m}^{-1}$ 


\section{APPENDIX B: THERMAL CONDUCTIVITY DATA CORRELATION}

\section{TRANSIENT HOT-WIRE TECHNIQUE}

The transient hot-wire instruments used in this work have been described in detail previously [1,2]. The low-temperature instrument operates from 30 to $330 \mathrm{~K}$ at pressures to 70 $\mathrm{MPa}$. The high-temperature instrument operates from 300 to $750 \mathrm{~K}$ at pressures to $70 \mathrm{MPa}$. Both instruments are absolute, with dual platinum hot wires to eliminate errors due to axial conduction. The temperature region studied in this work required the use of both instruments and allows a consistency check between the two instruments near $300 \mathrm{~K}$. These instruments measure thermal conductivity with a repeatability of $\pm 0.3 \%$ and a nominal uncertainty of less than $\pm 1 \%$ in nonpolar fluids [1,2]. Accurate measurements of the liquid-phase thermal conductivity require modification of the cell grounding [3] in order to eliminate measurement errors due to the polarization of ionic impurities around the bare platinum hot-wires. It is shown in Ref. 3 that introducing a fixed dc polarization voltage between the cell walls and the hot-wires eliminates this measurement error with liquid refrigerant samples.

The working equation for the temperature rise at the surface of an idealized wire is

$$
\Delta T_{\mathrm{id}}\left(r_{0}, T\right)=\frac{q}{4 \pi \lambda} \ln \left(\frac{4 a t}{r_{0}{ }^{2} C}\right)=\frac{q}{4 \pi \lambda} \ln \left(\frac{4 a}{r_{0}^{2} C}\right)+\frac{q}{4 \pi \lambda} \ln (t) .
$$

Here, $q$ is the power input per unit length of wire. $t$ is the elapsed time from the start of the step power input, $\lambda$ is the fluid thermal conductivity, $a=\lambda \rho C_{\mathrm{p}}$ is the fluid thermal diffusivity, $r_{0}$ is the radius of the wire, $\rho$ is the fluid density, $C_{\mathrm{p}}$ is the fluid isobaric heat capacity, and $C$ is the exponential of Euler's constant. We obtain the thermal conductivity from the slope of a line fit through the temperature rise versus $\ln (t)$. The thermal diffusivity is obtained from the intercept of this line but is not reported here.

in practice there are several corrections to this ideal temperature rise to account for nonidealities in the heat transfer from the wires. These small corrections. $\delta T_{i}$, are applied to the measured temperature rise. $\Delta T_{\mathrm{w}}$, according to

$$
\Delta T_{\mathrm{id}}=\Delta T_{\mathrm{w}}+\sum_{i} \delta T_{i}
$$

All of the applicable corrections are discussed in detail elsewhere [1-5]. In this work we have applied corrections for the finite wire heat capacity [5], finite outer boundary [5], truncation error [5], and thermal radiation [2]. All of the other corrections have been found to be negligible for these measurements [1-5]. 


\section{THERMAL CONDUCTIVITY SURFACES}

The surface is developed by breaking the thermal conductivity into three terms

$$
\lambda(T, \rho)=\lambda_{0}(T)+\lambda_{\mathrm{ex}}(T, \rho)+\lambda_{\mathrm{cr}}(T, \rho) .
$$

The thermal conductivity of the dilute gas, $\lambda_{0}$, is a function of the fluid temperature only. The thermal conductivity excess function, $\lambda_{\mathrm{ex}}$, and critical enhancement, $\lambda_{\mathrm{cr}}$, are functions of the fluid temperature and density.

The thermal conductivity surface is developed by fitting the low-density data to obtain the dilute-gas thermal conductivity. Next, a reasonable value for the thermal conductivity critical enhancement parameter, $q_{\mathrm{D}}$, is used in the crossover theory (see below) to obtain the thermal conductivity critical enhancement. The dilute-gas and critical enhancement contributions are subtracted from each experimental data point to leave the excess thermal conductivity. The excess thermal conductivity data are then fit to a polynomial in temperature and density. We then alternate between guesses for the critical enhancement parameter and regression of the excess function polynomial until the deviations are minimized.

\section{Dilute-Gas Thermal Conductivity}

The dilute-gas thermal conductivity is given by

$$
\lambda_{0}(T)=a_{1}+a_{2} T+a_{3} T^{2} .
$$

where $\lambda_{0}$ is in $\mathrm{W} \cdot \mathrm{m}^{-1} \mathrm{~K}^{-1}$ and $T$ is in $\mathrm{K}$.

\section{Excess Thermal Conductivity}

The excess thermal conductivity is given by

$$
\lambda_{\mathrm{ex}}(T . \rho)=\left(b_{1}+b_{2} T\right) \rho+\left(b_{3}+b_{4} T\right) \rho^{2}+\left(b_{5}+b_{6} T\right) \rho^{3}+\left(b_{7}+b_{8} T\right) \rho^{4}
$$

where $\lambda_{\text {ex }}$ is in $\mathrm{W} \cdot \mathrm{m}^{-1} \mathrm{~K}^{-1}, \mathrm{~T}$ is in $\mathrm{K}$. and $\rho$ is in $\mathrm{mol} \cdot \mathrm{L}^{-1}$.

\section{Critical Enhancement}

Olchowy and Sengers have proposed a solution to the mode-coupling equations which allows calculation of the thermal conductivity enhancement throughout the fluid state [6.7]. We have shown that this theory provides an excellent description of the thermal conductivity critical enhancement for fluids, such as nitrogen and argon [8.9], where accurate equations of state are available. The approach involves the approximate solution of coupled integral equations with a wave number cutoff $\left(q_{\mathrm{D}}\right)$ to limit the momentum-space range over which critically driven fluctuations can contribute to dynamic critical phenomena. Thus. in addition to a knowledge of thermodynamic properties and background values of the viscosity and thermal conductivity, the single fluid-dependent parameter $q_{\mathrm{D}}$ must be optimized to describe the critical enhancement. 
The thermal conductivity enhancement can be described by [6]

$$
\lambda_{\mathrm{cr}}=\frac{R_{\mathrm{c}} k_{\mathrm{B}} T \rho C_{\mathrm{p}}}{6 \pi \eta \xi}\left(\Omega-\Omega_{0}\right) \text {. }
$$

where the amplitude $R_{\mathrm{c}}$ has been set to $1.01, k_{\mathrm{B}}$ is Boltzmann's constant. $\eta$ is the shear viscosity, $\xi$ is the correlation length, and $\Omega$ and $\Omega_{0}$ are complicated functions of $T$ and $\rho$ as briefly described below.

The correlation length $\xi$ has been approximated by relating it to the critical part of the dimensionless compressibility as in Refs. 6 and 7. Thus, we write

$$
\xi=\xi_{0}\left[\frac{P_{\mathrm{c}} \rho}{\Gamma \rho_{\mathrm{c}}^{2}}\right]\left(\frac{v}{\gamma}\right)\left[\left.\frac{\partial \rho(\rho . T)}{\partial P}\right|_{T}-\left.\left(\frac{T_{\mathrm{r}}}{T}\right) \frac{\partial \rho\left(\rho . T_{\mathrm{r}}\right)}{\partial P}\right|_{T}\right]\left(\frac{v}{\gamma}\right) .
$$

Here, $v=0.63$ and $\gamma=1.2415$ are universal scaling exponents. while $\Gamma$ and $\xi_{0}$ are fluid specific amplitudes. The temperature at which the background compressibility has been identified with the total compressibility, so that the critical contribution vanishes, is selected as $T_{\mathrm{r}}=2.5 T_{\mathrm{c}}$. The correlation length for critical fluctuations, and hence the critical enhancement, vanishes above this temperature: thus, both $\xi$ and $\lambda_{\mathrm{cr}}$ should be set to zero for temperatures above $2.5 T_{\mathrm{c}}$. The choice of $T_{\mathrm{r}}$ is somewhat arbitrary, and affects the calculated enhancement only at temperatures far removed from the critical temperature.

The function $\Omega$ can be evaluated using the parameters

$$
\begin{aligned}
& y_{\mathrm{D}}=q_{\mathrm{D}} \xi . \\
& y_{\gamma}=C_{\mathrm{v}} /\left(C_{\mathrm{p}}-C_{\mathrm{v}}\right), \\
& y_{\alpha}=\left(k_{\mathrm{B}} T M \rho\right) /\left(8 \pi \eta^{2} \xi\right), \\
& y_{\beta}=\left[\left(\lambda_{0}+\lambda_{\mathrm{ex}}\right) M\right] /\left[\eta\left(C_{\mathrm{p}}-C_{\mathrm{v}}\right)\right] . \\
& y_{\delta}=\left\{\tan ^{-1}\left[q_{\mathrm{D}} \xi /\left(1+q_{\mathrm{D}}^{2} \xi^{2}\right)^{1 / 2}\right]-\tan ^{-1}\left(q_{\mathrm{D}} \xi\right)\right\} /\left(1+q_{\mathrm{D}} \xi^{2}\right)^{1 / 2} .
\end{aligned}
$$

where $\mathrm{C}_{\mathrm{y}}$ is the fluid isochoric heat capacity. $M$ is the molar mass, and he other variables have been defined above. The final expression for $\Omega$ from Refs. 6.7 has been simplified [ 8.9 ] by evaluating the mode-coupling integral in closed algebraic form: the matrix-inversion algorithm. required to use the results reported by Sengers and Olchowy [6,7], is no longer necessary. We can write 


$$
\left.\Omega=\frac{2}{\pi\left(1+y_{\gamma}\right)}\left[y_{\mathrm{D}}+\sum_{i=1}^{4}\left[\frac{g\left(z_{i}\right)\left(1-z_{i}^{2}\right)^{-\frac{1}{2}}}{\prod_{i \neq j=1}^{4}\left(z_{i}-z_{j}\right)} \times \ln \left(\frac{1-z_{i}+\left(1-z_{i}^{2}\right)^{\frac{1}{2}} \tan ^{-1}\left(\frac{y_{\mathrm{D}}}{2}\right)}{1-z_{i}-\left(1-z_{i}^{2}\right)^{\frac{1}{2}} \tan ^{-1}\left(\frac{y_{\mathrm{D}}}{2}\right)}\right]\right)\right]\right]
$$

The auxiliary function $g(z)$ is defined by

$$
g(z)=-y_{\mathrm{D}} y_{\alpha} z^{3}+\left(y_{\gamma}-y_{\beta}-y_{\alpha} y_{\delta}\right) z^{2}-y_{\gamma} y_{\mathrm{D}} y_{\alpha} z+y_{\gamma}^{2}-y_{\gamma} y_{\delta} y_{\alpha},
$$

and $z_{\mathrm{i}}$ are the roots of the quartic equation

$$
\prod_{i=1}^{4}\left(z-z_{i}\right)=z^{4}+y_{\alpha} y_{D} z^{3}+\left(y_{\gamma}+y_{\beta}+y_{\delta} y_{\alpha}\right) z^{2}+y_{\alpha} y_{D}^{y} z+y_{\alpha} y_{\delta} y_{\gamma}=0 \text {. }
$$

The roots can be found in closed algebraic form by standard procedures such as that described in Sec. 3.8.3 of the handbook of Abramowitz and Stegun [10]; in that case. the first 2 roots are typically real and the final 2 roots are complex conjugates. The expression for $\Omega$ is real, although the arguments may be complex. Our definition of the $z_{\mathrm{i}}$ differs by a minus sign from that published in Refs. 6,7.

The final term $\Omega_{0}$ represents the contribution of dynamical fluctuations to the thermal conductivity, which is caused by the long-time tail of their correlations [6.7]. This term must be subtracted so that the experimental thermal conductivity well away from the critical region can be identified with the background thermal conductivity; these contributions are thus included in the background correlation rather than in the enhancement term $\lambda_{c r}$. We have retained the empirical term proposed by Olchowy and Sengers [6.7], but have slightly revised the denominator appearing in $\Omega_{0}$. The term $\Omega_{0}$ is defined by

$$
\Omega_{0}=\frac{2\left[1-\exp \left(\frac{-q_{D} \xi}{1+\frac{q_{D}^{3} \xi^{3} p_{c}^{2}}{3 p^{2}}}\right)\right]}{\pi\left[1+y_{\alpha^{\prime}{ }_{D}}+\frac{y_{\beta}}{\left(1+y_{\gamma}\right)}\right]} .
$$

The term $\Omega_{0}$ rigorously cancels $\Omega$ to lowest order in an expansion about $q_{\mathrm{D}} \xi=0$, that is, well away from the critical point. We have removed the term in the denominator of $\Omega_{0}$ which does not explicitly contribute to the term cancellation; other higher order terms remain in the exponential and the denominator. Our revision of $\Omega_{0}$ has only a small effect on the calculated 
enhancement since its contribution is important only to points far from the critical point where the total enhancement is negligible.

In our work on HFC-32 and HCFC-123, we have used the critical parameters from the MBWR equations of state for these fluids and the same equilibrium critical amplitudes as for HFC-134a, which we obtained from Tang, et al. [11], i.e.. $\Gamma=0.0498$, and $\xi_{0}=0.2019 \mathrm{~nm}$. The viscosity is calculated with the extended corresponding states formulation of Huber and Ely [12]. With $T_{\mathrm{r}}=2.5 T_{\mathrm{c}}$, we find the optimum value for $q_{\mathrm{D}}^{-1}$ which satisties our critical enhancement data.

\section{REFERENCES}

[1] C.A. Nieto de Castro and H.M. Roder, J. Res. Natl. Bur. Stand. (U.S.) 86:293 (1981).

[2] R.A. Perkins, H.M. Roder, and C.A. Nieto de Castro, J. Res. Natl. Inst. Stand. and Tech. (U.S.) 96:247 (1991).

[3] R.A. Perkins, A. Laesecke, and C.A. Nieto de Castro, Fluid Phase Equilibria (1992), in press.

[4] J.J. Healy, J.J. de Groot, and J. Kestin, Physica 82C:392 (1976).

[5] C.A. Nieto de Castro, B. Taxis, H.M. Roder, and W.A. Wakeham. Int. J. Thermophys. 9:293 (1988).

[6] G.A. Olchowy and J.V. Sengers, Phys. Rev. Lett. 61:15 (1988).

[7] G.A. Olchowy, Crossover from Singular to Regular Behavior of the Transport Properties of Fluids in the Critical Region, Ph.D. Thesis (University of Maryland. College Park, MD 1989).

[8] R.A. Perkins, H.M. Roder, D.G. Friend, and C.A. Nieto de Castro, Physica A 173:332 (1991).

[9] R.A. Perkins, D.G. Friend, H.M. Roder, and C.A. Nieto de Castro. Int. J. Thermophys. 12:965 (1991).

[10] M. Abramowitz and I.A. Stegun. Handbook of Mathematical Functions, Applied Mathematics Series 55 (U.S. National Bureau of Standards, Washington D.C., 1972), p. 17-18.

[11] S. Tang. G.X. Gin, and J.V. Sengers, Int. J. Thermophys. 12: 515 (1991).

[12] M.L. Huber, and J.F. Ely, Fluid Phase Equil. 80:239 (1992). 

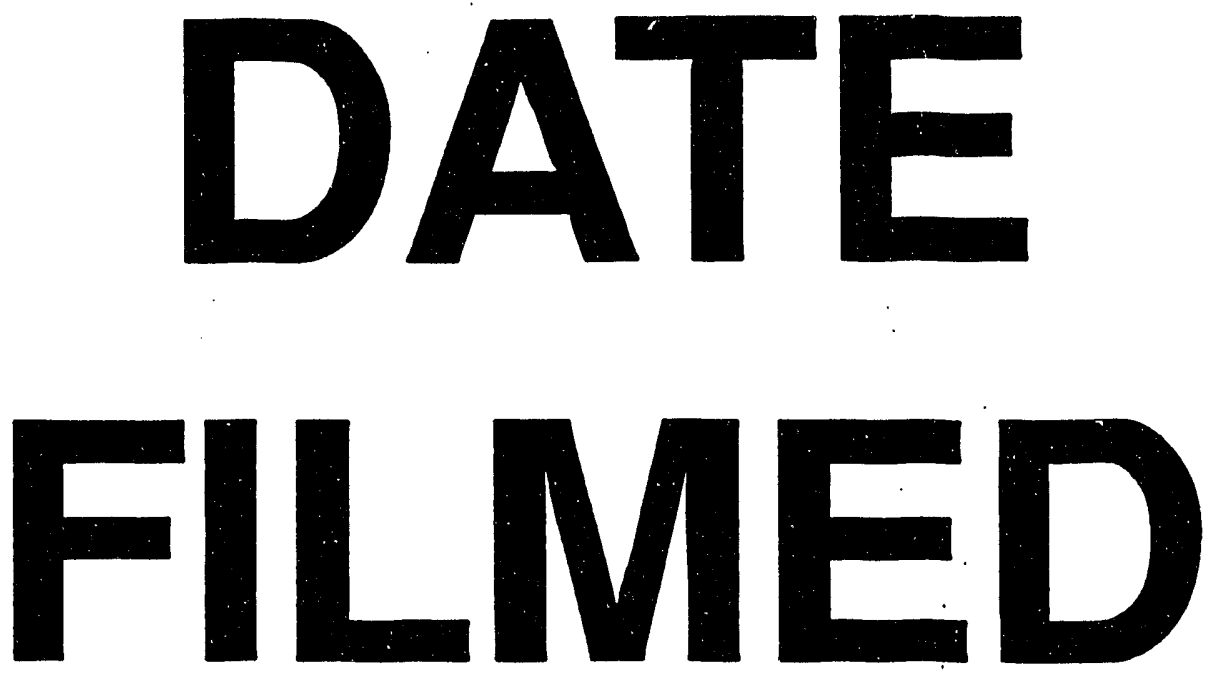

$9 / 29 / 93$
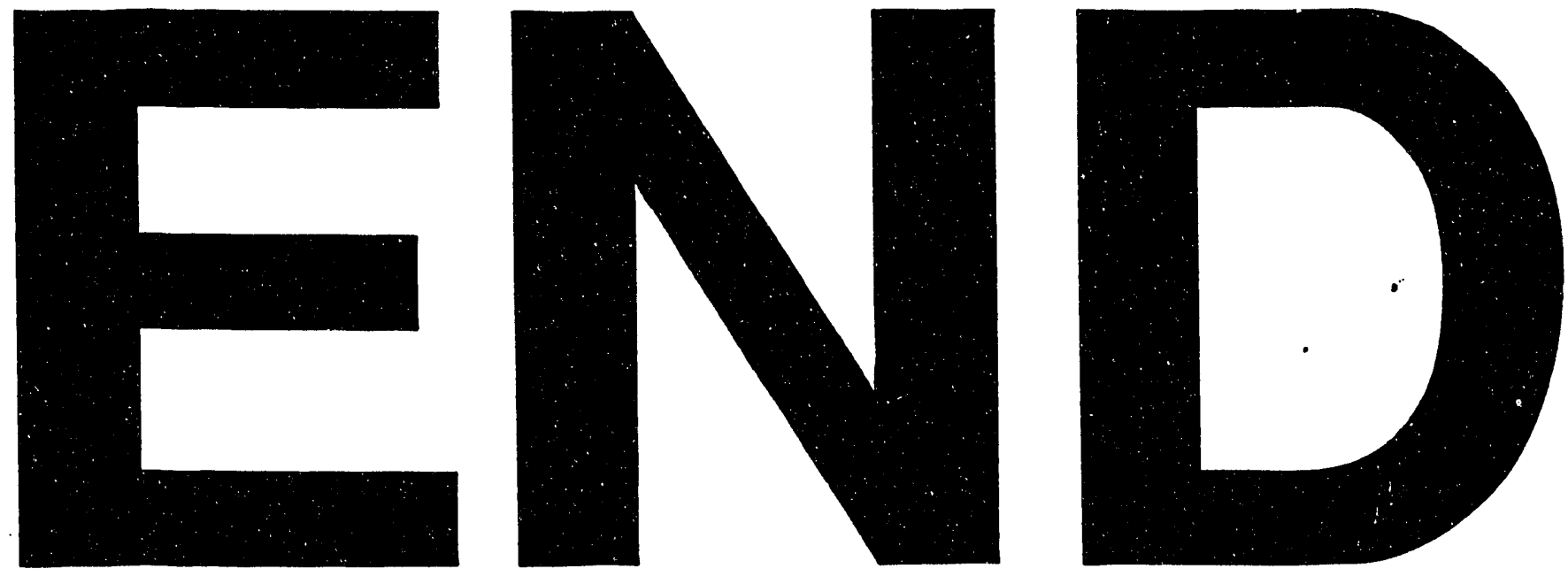
